INIFRNATLNAL SERIES OF MONOGRAPHS GN PURE AND APRLIED BIOLOGY 


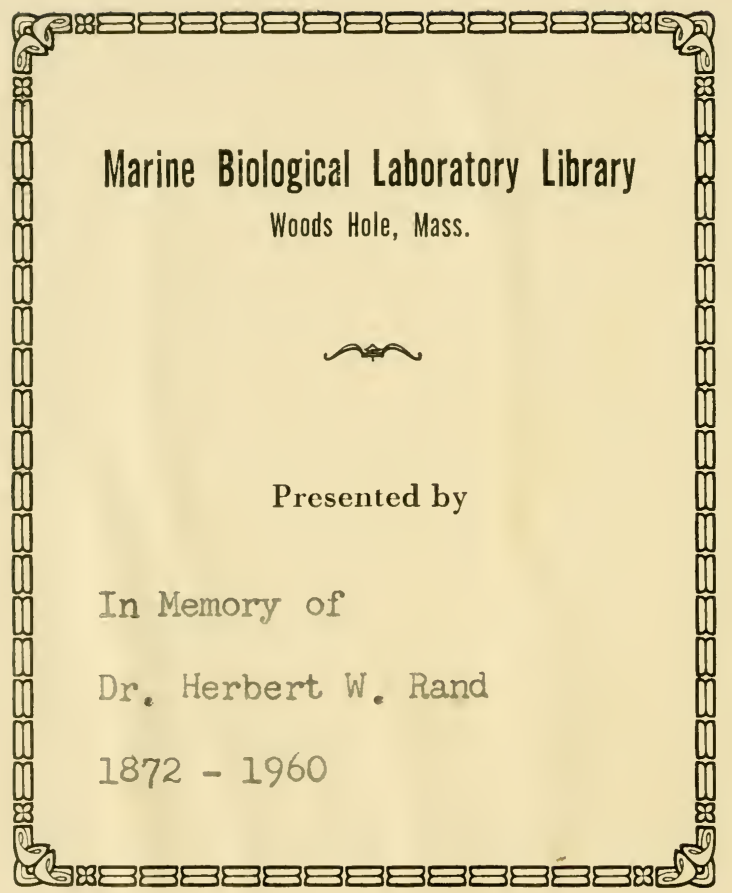




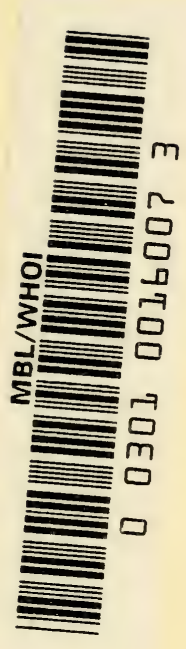



INTER NATIONAL SERIES OF MONOGRAPHS ON PURE AND APPLIED BIOLOGY

\author{
Division: ZOOLOGY
}

General Editor: G. A. Kerkut

Volume i6

THE

EVOLUTION OF THE

METAZOA 
OTHER TITLESIN THE ZOOLOGY DIVISION

General Editor G. A. Kerkut

Vol. I. Raven - An Outline of Developmental Pbysiology

Vol. 2. RAven - Morphogenesis: The Analysis of Molluscan Development

Vol. 3. SAVORY - Instinctive Living

Vol. 4. KERKUT - Implications of Evolution

Vol. s. TARTAR - Biology of Stentor

Vol. 6. Jenkins - Animal Hormones

Vol. 7. Conliss - The Ciliated Protozoa

Vol. 8. George - The Brain as a Computer

Vol. 9. Arthur - Ticks and Disease

Vol. Io. RaVEN - Oogenesis

Vol. I I. ManN - Leeches

Vol. I 2. Sleigh - The Biology of Cilia and Flagella

Vol. I3. Pitelka - Electron-Microscopic Structure of Protozoa

Vol. I4. Fingerman - The Control of Chromatophores

Vol. I s. Laverack - The Physiology of Earthworms

OTHER DIVISIONSIN THE SERIESON PURE AND APPLIED BIOLOGY

B IOCHE M ISTRY

BOTANY

MODERN TRENDS

IN PHYSIOLOGICAL SCIENCES

PLANT PHYSIOLOGY 


\title{
THE EVOLUTION OF THE
} METAZOA

\author{
PROFESSOR JOVAN HADŽI \\ Slovenska Akademija Znanosti in Umetnosti \\ L jubljana
}

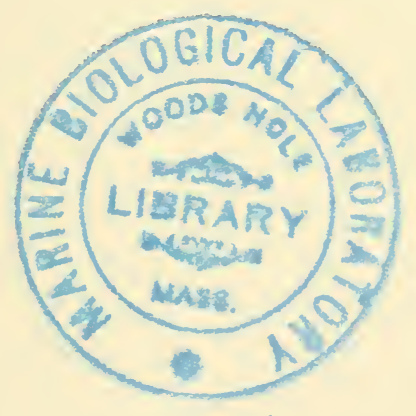

A Pergamon Press Book

\author{
THE MACMILI,AN COMPANY \\ NEW YORK \\ 1963
}


THE MACMILLAN COMPANY 60 Fifth Avenue New York 1/, N. Y.

This book is distributed by

THE MACMILLAN COMPANY pursuant to a special arrangement with PERGAMON PRESS LIMITED Oxford, England Copyright (C) 1963
PERGAMON PRESS LTD.

Library of Congress Card Number 63-10129 


\section{CONTENTS}

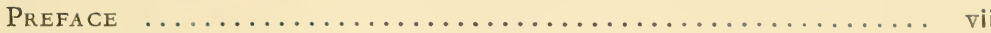

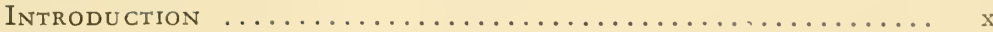

Chapter 1. Cnidaria as the Only Coelenterata............ 1

The systematic position of the Spongiae ............... 1

The systematic position of the Ctenophora ............ 8

Cnidaria as the only Coelenterata .................. 23

Something about the methods that can be used in connection with the construction of the natural animal system .......... 28

The significance of the sessile way of life ............ 47

The role of the polymerization and of subsequent oligomerization

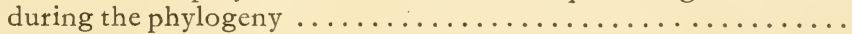

The validity of Watson's rule for the Invertebrates ........66 68

Chapter 2. The Previous Interpretations of Cnidaria ....... 73

The origin of Cnidaria $\ldots \ldots \ldots \ldots \ldots \ldots \ldots \ldots \ldots \ldots \ldots \ldots$

The right sequence of the Cnidaria groups ............. 95

Why is there no medusa form in Anthozoa? ............. 103

The primarily solitary polyps appear in Anthozoa only ..... 106

Polymorphism has reached its climax in Hydrozoa ........... 109

The regressive development of the intermediate layer in Cnidaria 112

The morphologic proofs of the new interpretation of the phylogeny

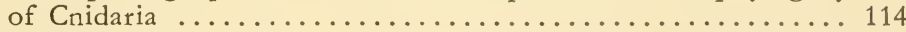

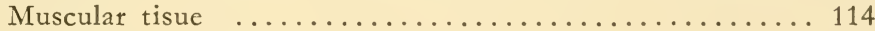

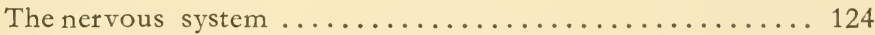

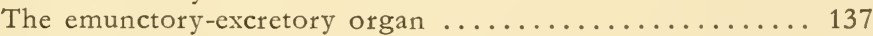

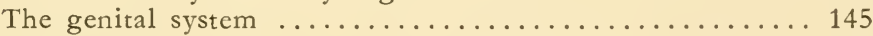

The alternation of generations in Cnidaria .......... 153

The digestive system ...................... 163

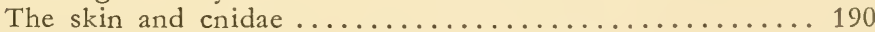

Ontogeny (The development of the embryo) ......... 204

Asexual reproduction and the regeneration $\ldots \ldots \ldots \ldots 213$

The endosymbiotic mononuclear algae ............ 222

Chapter 3. The Consequences of the New Interpretation of

C

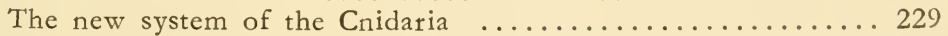

The rank and the position of Cnidaria in the animal system .. 243

Discussion of the new interpretation of the evolution of Cnidaria 245

The probable origin of Cnidaria .................. 248

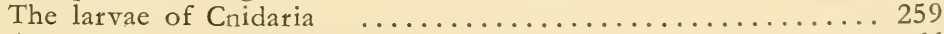

A comparison of Cnidaria larvae with other larvae ........ 266

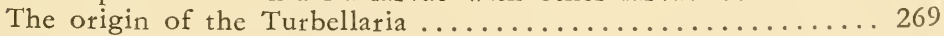

The origin of the acoelous Turbellaria .............. 273 
The relationship between conjugation and copulation $\ldots \ldots \ldots 280$

The point of separation of the evolutions of Infusoria and

Eumetazoa .............................. 298

The origin of the complex, hypercellular individualities ...... 305

Is the plasmodial state of the Acoela a primary clement? . . . . 314

An attempt to reconstruct the initial state of the Eumetazoa... 319

The origin of the middle body layer of the so-called mesoderm 321

Two difficultics of the theory of polykaryonts .......... 329

Can cleavage be really considered as a proof of the colonial theory? 335

The basic principles that should be used in a reform of the whole

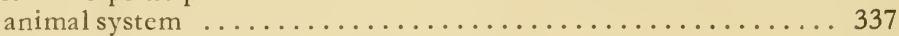

Evolution generally had a progressive trend ............ 346

Chapter 4. The Classification of the Animal World and the

New Genealogical Tree ................... 352

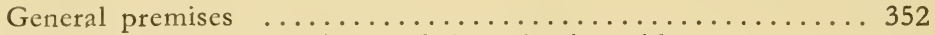

The limit between the plant and the animal worlds ....... 352

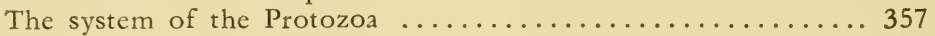

The transition from the Protozoa to the metazoan state ..... 361

There are no well-warranted Mesozoa ................ 366

The classification of Metazoa .................... 368

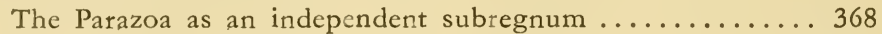

The Eumetazoa and their subdivisions ........... 372

The elimination of the terms Coelenterata-Coelomata ..... 372

The Coelomata as a taxonomic unit ............. 384

The first phylum of the Eumetazoa: The Ameria ..... 391

The transition from the amerous to the polymerous state . . . 397

The problem of justification of the high taxons: Protostomia-

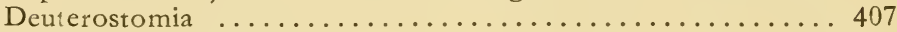

The classes of the Polymeria ................... 411

The phylum Oligomeria and its classes ............. 421

The sphere of the phylum Oligomeria ............. 423

The problem of the inclusion of the Sipunculida into the phylum

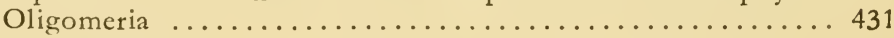

The Oligomeria as the initial form for the evolution of the

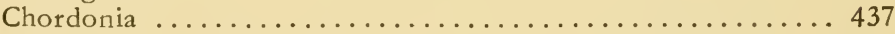

The fou th and the last phylum of the Eumetazoa: Chordonia 441

The probable origin of the Chordonia ............. 447

A comparison of the present attempt to derive the Chordonia from

the ancestral form resembling the present day Enteropneusta

with the similar attempt made by Hans Steiner ......... 452

The genealogical tree of the Chordonia ............ 455

The gerealogical tree of the entire animal world ........ 457

An attempt to unify the animal system $\ldots \ldots \ldots \ldots \ldots \ldots . \ldots 46$

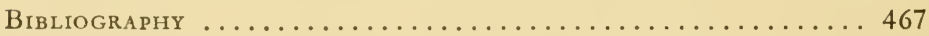

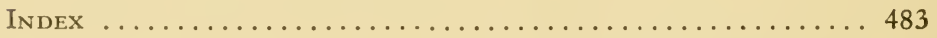




\section{PREFACE}

It was with great pleasure that I accepted the invitation of the Editor of the Pergamon Press Zoological Series to publish in book form and in English, as an international language, the results of my research on the Cnidaria, the interpretations of this research, and the final effects of these interpretations. I was all the more pleased with this invitation because my first general study of this problem appeared 17 years ago during the turmoils of the Second World War, when I tried to save the existence of the Slovene Academy of Arts and Sciences at Ljubljana which was threatened by the occupying powers.

My book, The Turbellarian Theory of the Cnidaria; The Phylogeny of the Cnidaria and Their Position in the Animal System was published in a Slav language, in the language of the small yet progressive nation of Slovenes. Added to this text was a detailed summary written in an international language which could help various scholars to get acquainted with the contents of the book. The summary was written in German because I myself completed my studies at Vienna University.

Since the publication of this book many new elements have become known in the field of biology, and above all in zoology. Neither have I ceased with my studies: a whole series of special papers have been written by me in the meantime which are closely connected with problems that had only been touched on in the book. In the international literature there were a number of reactions to my book and to my subsequent shorter publications; this was especcially true for my article which appeared in the American review, Sy'stematic Zoology. The publication of this article was made possible due to the generous help of an excellent translator, Piofessor A. Petrunkewitch. American and the English zoologists have shown particularly vii 
great interest in my interpretations and ideas. I have, therefore, now a welcome chance to answer all the critical observations that have so far been made as regards my suggestions.

The present volume must not be simply considered as a second revised edition of the first volume even if I deal in both these books with the same or at least similar problems. This book is a completely new work: the foundations only of the two books have remained the same.

To these few words of preface I wish to add a few more words dictated by my feelings of indebte Iness. Above all, I wish to thank all those critics of my earlier studies who have helped me to clarify many points that had previously been not clear. They must excuse me if I have occasionally used too vehement words that have been dictated "by the heat of the struggle." My wish has always been to convince them. I can not accept the suggestion made by a prominent scholar that such revolutionary novelties have to wait the till present generation dies out and the next generation becomes convinced of the correctness of the new concept.

My special thanks go to my dear friend, Professor Dr. Otto Steinböck (Innsbruck, Austria). He has not only critically read my manuscript; he has also always excelled as a noble colleague. Without any previous knowledge of our researches, we both came to the same conclusions regarding the origin of the Eumetazoa. Each of us has made his researches in his special group of animals: Professor Steinböck has mainly worked on the Turbellaria, and I myself mainly on the Cnidaria. Otto Steinböck came to the conclusion that the Turbellaria, i.e. the acoelous Turbellaria, must be the most primitive Eumetazoa, even before I was able to formulate the results of my studies. As a consequence of this discovery, Steinböck had correctly made important conclusions especially as regards the way the Eumetazoa had evolved, and as regards the previously firmly held theory of the germ layers. He did not, however, succeed in publishing the results of his research and his ideas. Just before the outbreak of the Second World War, Steinböck gave 
a lecture on this subject at the International Congress of Cyto$\operatorname{logy}$ at Stockholm. Only the title of this lecture has ever been published. This is how it happened that I was the first scholar to publish these ideas, in spite of the fact that I developed the same ideas later than Professor Steinböck: this has been due to various circumstances, among others, also to the outbreak of the Second World War. This fact, however, is not ultimately so important for the progress of the science itself; it is much more important that two researchers have reached quite independently the same conclusions; and that they continue to collaborate in a friendly way to prepare the way for the final reception of their ideas. It is unimportant that we differ in several unessential points; it seems that we will soon reach an agreement even on these points. The difficulties we have to overcome are great; we must struggle against interpretations and ideas which were considered inviolate since the days of the struggle for the victory of Darwinism and which were supported by such an authority as Haeckel, and by many other most prominent names. We must struggle against interpretations which have been considered as laws, i.e. as truths, and replace them by new suggestions.

I also wish to express my warmest thanks to the distinguished publishing house of Pergamon Press which has made possible the publication of this book in such a full presentation. I am also grateful to Dr. Janez Stanonik, the translator of the present text, the original of which was written in German ; he has completed his task, which has certainly not been easy, to my full satisfaction. 


\section{INTRODUC'TION}

It was in 1903, 58 years ago, that I, then a young man who had just left the classical grammar school at Zagreb, went to Vienna to study Natural Sciences and above all my beloved Zoology at Vienna University. For this study I was well prepared. Whilst at school, I had made a large collection of zoological objects. I had learnt the richness of forms and the ways of life of the animal world through my diligent study of Brehm's work on the Lives of Animals as well as through my field studies. At Vienna my teachers were the two professors of zoology, Carl Grobben and Berthold Hatschek, particularly the latter under whose guidance I also worked on my dissertation thesis.

During my second year at university, a public aquarium was purchased in Prater by some young Austrian biologists supported by their prosperous parents; in it they established a modern biological research institute-The Prater Biological Experimental Testing Station (Die biologische Versuchsanstalt im Prater) - and I applied as one of the first to this place to learn there the methods of scientific research. Dr. Hans Przibram, then Privatdocent, was the head of the department; he is the author of the well known synoptic work on Experimental Zoology. Hydra was my first object of study. There were numerous green Hydra in a half-darkened concrete basin; their exterior looked rather pale. This induced me to transfer the same Hydra into a completely darkened place. When these Hydra began to procreate I could observe what I had actually expected: their ova were without the symbiotic green algae so that finally there were at least some colourless "green" Hydra. Other tests and researches were made in the same place. In 1906 my first results were published in Roux's Archiv für Entwicklungsmechanik. 
From here I went to the Zoological Institute of Vienna University which was at that time headed by the famous zoologist, Councillor Prof. Dr. Berthold Hatschek. Besides him there were also on the staff Prof. Dr. Karl Camillo Schneider, a well known comparative histologist of Invertebrata, Prof. Dr. Heinrich Joseph, a comparative cytologist, and Dr. R. Zwicklitzer who worked as assistant. I remained faithful to Hydra and so I chose "The Nervous System of Hydra" as my theme for the doctoral thesis. I succeeded in using methylene blue as an intra-vitam colouring of the complete nervous system of Hydra. In spring 1908, when I was in the eighth semester of my studies, I became a Doctor of Philosophy. In 1909 the results of my thesis were published in the Institute's journal Arbeiten der zoologischen Institute Wien und Triest. This work has never been surpassed by any other study in the same field, it has been quoted everywhere, and the pictures published in it have often been reproduced. Because of the early appearance of this work and because of the other works written by me on Hydra and other hydroids (gemmation, migration of nematocysts, etc.) that soon followed, it was not strange that after a lapse of years I have been looked upon at various congresses of zoologists and other meetings as being a son or even a grandson of the "Old Hydra-Hadži."

After the completion of my studies and after I had passed in 1907 the state examination, I returned to my country (Zagreb in Croatia, now Yugoslavia). Here I came, after a short stay at the local Zoological Museum, to the University Institute of Comparative Anatomy, headed by Prof. Dr. Lazar Car. I still continued to remain faithful to hydroids as well as to other Cnidaria and Coelenterata. It was only when I was called, at the end of the First World War, to the newly founded Ljubljana University (Slovenia, Yugoslavia) that I extended the sphere of my interest to some groups of land Arthropoda (Chelicerata), particularly those which inhabit underground caves.

For more than 40 years I have collected experience and knowledge about Cnidaria and Coelenterata, constantly thinking about 
their real nature. Though dogmas are characteristic for religious systems, yet the same can also be found in science. One such dogma was, and it still is, the belief in the primary simplicity of Hydra, and even more so of Hydrozoa, and so too with Cnidaria and Coelenterata. It is well known how difficult it is to fight against dogmas, particularly when they have been accepted for so long, when they have been supported by renowned scholars, and when they appear to be well founded. My initial researches have shown that Hydra is in no way so simply organized as the simple structure of its body would seem to indicate when considered from a crudely anatomical point of view. Soon I saw the first pillar of the proud structture of Coelenterata collapse. It has been almost generally accepted that Hydra and its closest relatives are not primarily solitary animals but that, instead, they are derived from ancestors that were able to form cormi. Naturally, this discovery alone could not suffice to make all Hydrozoa the secondarily simplified Eumetazoa. Nevertheless, with this a good beginning was made. 


\section{CNIDARIA AS THE ONLY COELENTERATA}

\section{The Systematic Position of the Spongiae}

The next step, apparently without connection with the former, was made when some well-known researchers especially Sollas and Delage, came to the conclusion that the Spongiae must not only be placed far from the immediate vicinity of Cnidaria, but also that they must be completely separated from the Coelenterata and even from all the remaining real Metazoa, the Eumetazoa; they must appear completely isolated as an independent type, as Parazoa or Enantiozoa. We must consider this separation as being definite, and, in my opinion, nothing can be changed in this respects by recent attempts made particularly by Miss $O$. Tuzet and her collaborators.

On the basis of works of older irvestigators, especially the researchers into the ontogeny of Spongiae (Metschnikoff, Delage, etc.) it is no longer necessary to defer d the special position that the Spongiae have as Parazoa (Sollas) in the animal classification. Recently, however, a tendency has arisen which aims at a revision of this point of view and tries to bring Spongiae back amongst the other Metazoa. The French school in particular, which created the term Enantiozoa (Delage), attempts to cancel out the progress that has been achieved; Tuzet points in this connection mainly to the following evidence: (1) According to her School, the Spongiae have a nervous system; (2) the choanocytes are found in the other Metazoa; (3) the stretching out and the pulling in of a part of the blastoderm observed in the 
embryos of Spongiae is not a completely isolated case ( Volvox). On the basis of all this, Tuzet comes to the conclusion that Spongiae are genuine Coelenterata, and are thus Eumetazoa and not Parazoa. In this she is followed by many zoologists, even by scholars who are not French (Remane, Jägersten, Alvarado).

Considered critically, one can see that the arguments proposed by Tuzet in favour of the old thesis which holds that Spongiae are Coelenterates, have a rather weak foundation. The existence of a nervous system is in no way proved for Spongiae, yet it is of great importance in this connection and Tuzet tries hard to prove the contrary. The pictures of thin sections that have been shown by Tuzet and her collaborators, have been seen by many investigators and I, too, have able been to see them. Yet no one-with one exception that will be mentioned later-has classified these cells of the central stratum with plasmatic processes, as genuine ganglion-cells or even nerve cells. They have always been identified as simple cells of the connective tissue from the central stratum which is filled up with jelly. In all probability these cells are as much, or perhaps slightly more, irritable and able to conduct excitement much as any other animal cell. In order to prove that these cells, or at least some of them, really have a nervous character it would be necessary to apply successfully special selective stains, as for example methylene blue which colours the nerve cells selectivily intra-vitam. If this has not been done, then at least it would be necessary to carry out some neurophysiological experiments, and achieve a positive result.

The general behaviour of Spongiae as well as the kind of reactions they show to various stimuli, make it completely improbable that Spongiae could possess a real net of nerves, let alone sensory nerves, as these can with certainty be considered to exist, e.g. in Hydra. Some time ago, von Lendenfeld sketched such nerve cells in pictures published by him, yet nobody believed his allegations or his beautiful pictures. And even, if there were some of these cells that exist-not 
that they do!-they would be a luis a non lucendo, since they would be physiologically meaningless.

Lévi (1956) made, after the publications of the school of Tuzet had become known, an extensive and critical histological study of Spongiae, and did not report any finding of a nervous system or of nerve cells in Spongiae. Neither could such a finding be confirmed by others, e.g. G. Eberl (Eberl, 1950), an excellent histologist from Vienna who used numerous staining techniques in her work. Yet in spite of all this the propositions as well as pictures published by Tuzet have found acceptance in many schoolbooks, especially in France.

Neither did Miss Tuzet succeed with her-it can be truly said-attempt to disprove the law of the supposedly inversed sequence of body layers in Spongiae. As is well known, Metschnikoff and many other famous zoologists after him, among them particularly Yres Delage, have shown that in the ontogenies of Spongiae, the early morphogeny proceeds along very particular lines. We get the impression that during the morphogeny of Spongiae a process takes place that is opposite to the process which can be observed in Eumetazoa. The primitive "ectoderm," i.e. the foremost part of the spongula, develops into the definite "entoderm," and the primitive "entoderm" into skin, this into the "ectoderm." For this reason Spongiae have been called Enantiozoa (i.e. animals with the two strata of body inversed) by Delage. Naturally, this inversed character is relative only i.e. in comparison to the development that can be observed in Eumetazoa. In fact, we have here a particular characteristic of Spongiae which has been developed during the course of their own phylogeny. It is therefore better not to speak in connection with Spongiae about an ecto- or an entoderm but rather to use special names, e.g. pinacoderm and choanoderm. The facts themselves cannot be changed. Yet Tuzet gives a description of an isolated case among Calcispongiae where the extroversion of the spongula blastoderms can be observed. A comparison with the only slightly similar case in the 
ontogeny of Volvox, is in this connection completely out of place. Volvox, is in fact a real alga in spite of its slight resemblance to an animal (its spheric form, free swimming ability). The very rare appearance of an organelle in some Eumetazoa which resembles more or less the "collar" of the choanocytes in Spongiae is too insignificant to be used as a proof to support the thesis that Spongiae are really Coelenterata, and it can be explained by the fact that both Parazoa as well as Eumetazoa arise from the ancestors of Flagellata (i.e. the scattered appearance of similar genes).

Disregarding the fact that the arguments brought forward by Tuzet cannot be considered sound, we must emphasize that the principal mistake in this kind of reasoning lies in the wrong belief that individual morphological or morphogenetic features and characteristics can represent the special type of organisation of Spongiae; in reality, this is represented by their special "nature," so as to say by the whole spirit of the organisation of Spongiae, and by their way of life. Again and again it is necessary to point out that the Spongiae have neither a digestive canal nor an oral opening. To this one could remark that in this respect Spongiae do not represent a unique case; even among the genuine Eumetazoa there are some in which we can find neither an oral opening nor a digestive canal, e.g. among the extreme endoparasites, or in the strongly aberrant Pogonophora. Nevertheless, there is a basic difference between Spongiae and these cases observed in Eumetazoa. It is beyond any doubt that in the case of the parasitic Eumetazoa and the freely living Pogonophora the absence of the oral opening and of the digestive canal is nothing but a secondary phenomenon. Spongiae, on the other hand, indubitably have primarily neither an oral opening nor a digestive canal, and this is a consequence of their special organisation. The feeding system with its numerous pores, the canal system with layers of choanocytes in chambers separated from each other that serve to maintain the flow of water, the larger aperture for the discharge of surplus water, and many other things, all this is 
unparalleled in other animals. In addition to this comes the special kind of ontogeny.

Finally, when we study attempts to construct the phylogeny of Spongiae as if they had the nature of Coelenterata (Remane, Jägersten)-these attempts must be considered as quite unsuccessful-then it actually becomes clear that Spongiae are really something "exceptional," and that they have developed independently from Protozoa, most probably out of Choanoflagellata. (Hadži, 1917). Yet even here it remains impossible to get ahead without a grain of imagination; we must use it to construct the phylogenetic processes of sponge evolution since it is completely hopeless to expect that we will ever run into any new documents which will help us to construct an actual phylogeny of Spongiae. Naturally enough, such an artificial construction, too, must be based on the actual facts that are now accessible. The scheme must be as close as possible to reality.

Since the Spongiae with their structure, their way of life, and their individual development represent something different from all other Metazoa, it must be expected, and actually reckoned with, that their phylogenetic evolution, too, was a special one. It is therefore quite improbable that the ancestors of Spongiae could be similar to gastraea. Here we run into very great difficulties when we try to construct the origin of Spongiae. We cannot expect any palaeontological evidence. We must limit ourselves to comparative morphology where, too, we are completely unable to make any progress without some imagination. Nevertheless, our scientific imaginationlet us call it so, though it is usually called speculation-must be kept in check and within the limits of probability. The reconstruction must correspond to the factual material that is available.

On the basis of my studies on Spongiae (Hadži, 1917) I constructed a phylogeny of Spongiae and submitted this to the International Congress of Zoologists at Budapest in Hungary (Hadži, 1929). This construction was later somewhat improved 
and is reproduced in the present volume (Fig. 1). According to this hypothesis the evolution of Spongiae progressed completely independent from that of Eumetazoa. The starting point was probably the freely swimming colonies of Mastigophora of the subtype Choanoflagellata. The subindividuals were
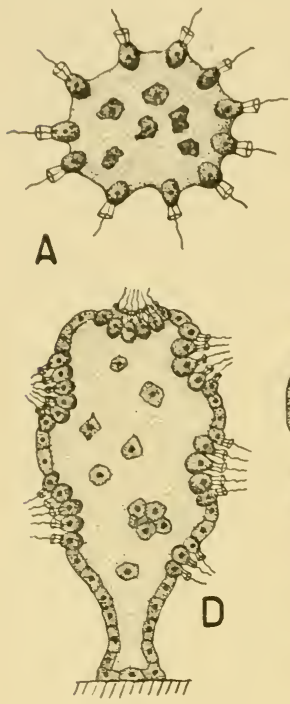
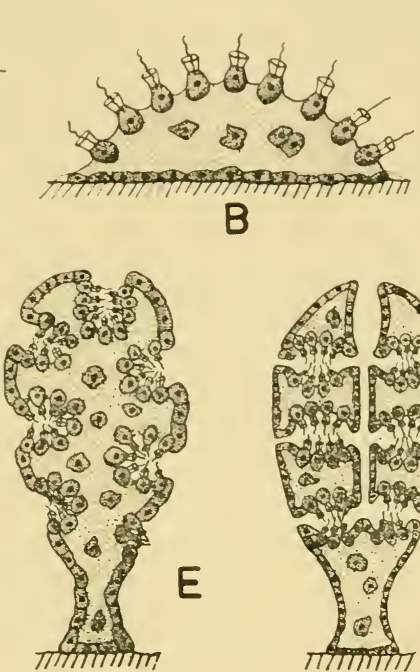
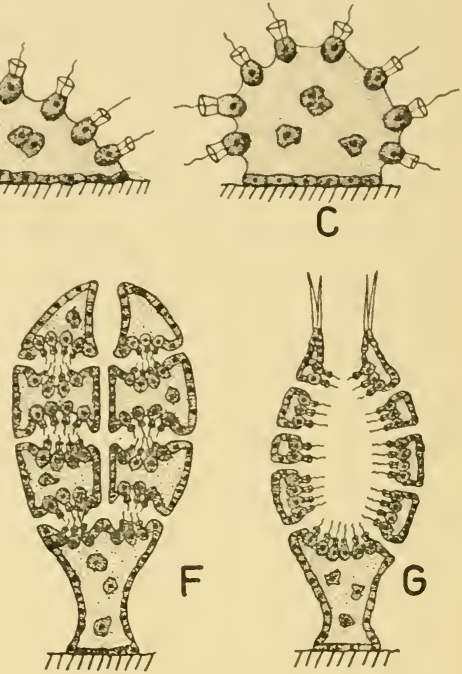

Fig. 1. Hypothetical construction of the evolution of the sponges. $\mathrm{A}$, free swimming phase resembling Proterospongia sp.; $\mathrm{B}-\mathrm{C}$, the beginning of the sessile phase; $\mathrm{D}-\mathrm{E}$, erect phase with the formation of chambers; F, primitive phase of the heterocoelic state; $\mathrm{G}$, homocoelic phasc. (After Hadži, somewhat altered.)

kept together by means of a richly secreted jelly in a way which can even now be observed among Choanoflagellata in the genus Proterospongia. The existence of this form of Choanoflagellata, which was first described by Saville Kent, was disbelicved and it was considered that we did not have here an independent animal but rather a surviving fragment of a real sponge. In the meantime, another species of the same genus has been described, so that now we have not only the old species Proterospongia baeckelii S. Kent, (Gröntved, 1956; Lackey, 
1959). In this way the existence of freely living colonies of Choanoflagellata can no longer te doubted. Of course, this can only be an example in our attempt to reconstruct the phylogeny of Spongiae. When an organism which was similar to Proterospongia changed to a sessile way of life, the duty to stick to the floor of the sea was taken over by a small number of basal choanocytes losing their character as choanocytes. Later, some of the surface cells that could stretch out freely into water took over the form of flattened amoebocytes and the latter developed the peculiarities of pinacocytes. Depressions developed in several parts of the surface when groups of choanocytes sank deeper into the jelly. It was probably in this way that several chambers with pores developed on the surface; the remaining surface was covered with finacocytes. The pore of the highest placed chamber developed into an osculum when the internal connection of chambers was evolved. The water flow through individual chambers was united into a common draining system. The amoebocytes in the jelly developed partly into the skeleton cells and partly into the primitive sex cells.

As a result of such an interpretation we find that the homocoelous type of Spongiae, the Ascon or Olynthus according to Haeckel, cannot be primordial but rather a result of a secondary development due to a secondary union of chambers. This makes it clear that ascon cannot be a gastraea. The freely living larva, the spongula, which is characteristic for Spongiae and which actually has a very manifold structure, developed independently and thus it cannot be considered to be a recapitulated form. There were no primitive Spongiae whose form and structure resembled those of the spongulae. The adoption of a freely living, usually planktonic juvenile stage of Spongiae, and, herewith, their prolonged ontogeny, corresponds to a general phenomenon and is a consequence of the transition to the sessile way of life by the adult form. The sexual stage together with the development (cleavage, etc.) which follows fertilization cannot be considered as a proof of the common derivation of all Metazoa from the colonies of Flagellata. The 
former has been inherited from the protozoic ancestors, the latter corresponds to a disposition which is common to all metabionts including metaphytes to develop a multicellular organism from a zygote (see the conditions in Volvox, a green alga).

In this way the second pillar of the old concept about Coelenterata collapsed with the definite removal of Spongiae from the relationship with Coelenterata. This was initially made by Leuckart (1859). The first pillar was the removal of Hydra from the basis of Hydrozoa.

\section{The Position of Ctenophora in the Animal Classification}

It was comparatively easy to show that the removal of Spongiae from the vicinity of Cnidaria could be convincingly upheld; such was their isolation (as Parazoa) from all other Metazoa. The great differences that exist between Spongiae and the remaining Coelenterata - the latter taken in the old sense of the word-are evident since they are both considerable as well as numerous. As has been already emphasized, we have here two completely different "spirits of organization" which force us to consider a different origin. It is clear that though there are some similarities between Spongiae and Cnidaria, that these similarities are purely external and can be mostly traced back to a sessile way of life and to a generally low stage of organisation.

Considerably greater difficulties have to be overcome if we try to exclude Ctenophora from the triangle Spongiae-Cnidaria-Ctenophora. It is clear that Ctenophora stand much closer to Cnidaria. Both are animals whose digestive organs have reached the aproctous stage of development; they have one opening only, the oral opening. In both, the digestive tract shows an inclination to the formation of the so-called gastrovascular system. Due to their life as plankton, a similarity of forms developed, particularly in medusae and Cteno- 
phora, with a rich development of a jelly-like intermediate substance. Though cnidae are, on the whole, developed in Cnidaria only, they have nevertheless been found also in a genuine ctenophore, in Euchlora rubra. Furthermore, a coelenterate has been described which has been considered as an intermediate form between the two subtypes of Coelenterata (Hydroctena salenskii Dawydoff). Finally, not without significance is the discovery of ctenophores which, though genuine, have begun to resemble Turbellaria due to their transition to a benthonic way of life; they have been explained as a transition form to Turbellaria, and in this way a connection of Colenterata with "higher forms" has been won.

All these facts have helped to support zoologists in their belief that Ctenophora are real Coelenterata and as such the closest relatives of Cnidaria. It is surprising to see how the numerous and fundamental differences that actually exist between the two groups of animals and touch not only their structures but even their ontogenies, make no deep impression on the majority of zoologists. At the most, the absence of cnidae in Ctenophora has been considered as an important mark of difference and for this reason they have been called Acnidaria. In our discussion we will limit ourselves to the essential points only; we shall try to show that Cnidaria are basically different from Ctenophora and that this difference is due to the fact that both these types of animals have a different origin, even if these origins are not widely separated from each other. For this reason we will base our discussion on selected properties that can be observed in Ctenophora.

One of the principal characteristics of Ctenophora is their bisymmetry. This quality pervades the whole organization of Ctenophora to such an extent that it can be observed already in the fine structure of their ovum and also in their ontogeny. Something identical-or even anything similarcannot be found in any medusa. The basically quadri-radial symmetry which can be secondarily multiplied through the process of polymerization is typical for medusae. On the other 
hand one can observe a development towards a secondary oligomerization of individual pairs of antimeres or of individual antimeres so that finally we see either a single pair preserved, or a marginal tentacle only, or even none whatever. In this way a purely external similarity with Ctenophora can natur.lly develop. One such case was formerly described by Ernst Haeckel who called an Anthomedusa with one pair only of marginal tentacles, Ctenaria ctenophora (see Fig. 2); by this he wished to express the palpable similırity that exists with a typical Ctenophora and in this way point to the possibility that Ctenophora had perhaps developed from Hydromedusae. For some time this comparison had a very great influence upon zoologists; a more precise comparison, however, has finally shown that these are inessential external similarities only. Ctenaria remains a typical Anthomedusa and it does not stand in the slightest degree closer to Ctenophora than any other Hydromedusa. The symmetry of the body is not a property which can be considered unchangeable. On the contrary, it shows comparatively rapid changes, e.g. when the sessile way of life has been adopted, or, vice versa, during the transition from a sessile way of life to that of a free movement. Many instances could be mentioned in this connection yet it should suffice to point to the case of echinoderms only. We shall later return to this question to discuss it in greater details.

It is in no way difficult to show how the bisymmetry has been developed in Ctenophora. It is a result of the transition from a benthonic to a pelagic way of life; during this transition the difference between the ventral and dorsal sides has disappeared. Thus we have here a combination of bilateral and radial symmetries. The internal organs remained true to the original bilateral symmetry while those situated on the periphery leaned towards the radial symmetry.

The second main difference between Ctenophora and Cnidaria is in their tentacles. It is well known that both a majority of Cnidaria as well as Ctenophora possess tentacles which are strongly contractile and serve mainly for the capture of the 
living prey. In those cases where there are no tentacles it seems more probable that their absence is a secondary phenomenon. The fact is noteworthy that Ctenophora never form more than one pair of tentacles, and that they show no leaning to polymerization which nevertheless can be observed to have

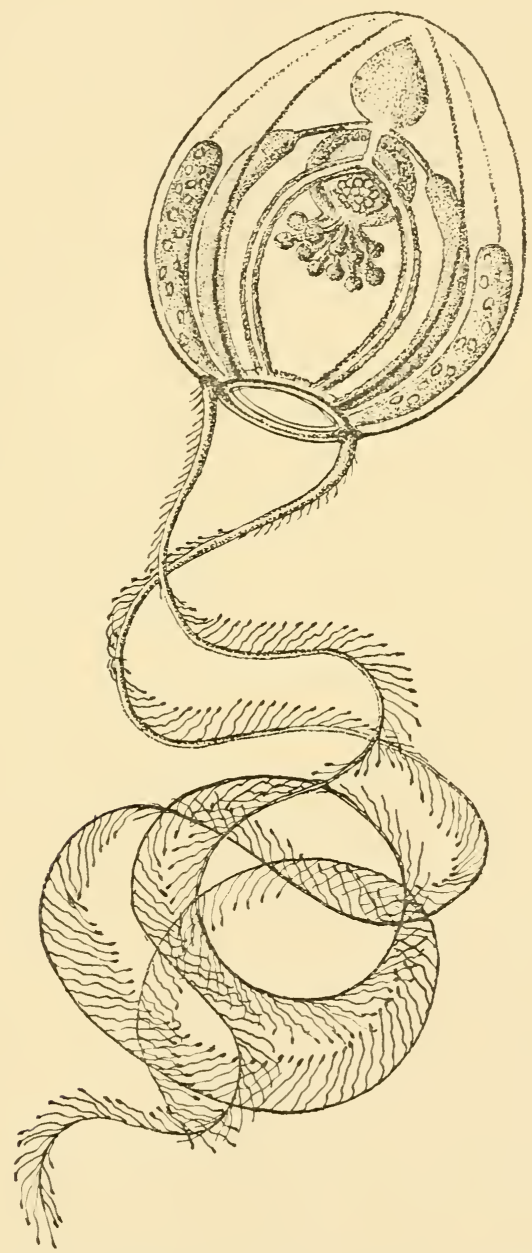

Fig. 2. An anthomedusa species: Ctenaria ctenophora Haeckel. (After Haeckel.) 
taken place in several other organs (intestinal diverticulum, swimming plates).

In both groups we find their tentacles equipped with particular specialized monocellular gland cells; on one hand with colloblasts, and on the other with cnidae. The two forms are so widely different from each other that they cannot be considered to have developed one from the other. It seems, however, as if Eucblora rubra, a ctenophore, stood in apparent contradiction to these observations. This ctenophore can rarely be observed and it does not have colloblasts in its two tentacles, but instead genuine cnidae; these are not only "drawn up" (i.e. ready to be used) but also kept in reserve, as is the case with medusae which are genuine Cnidaria. A detailed analysis of this case (Hadži, 1951, 1959, 1959a), however, has shown that we do not have here a real exception, but that instead this phenomenon is not limited to Euchlora only. In all probability this is a case of the so-called kleptocnidae, i. e. cnidae that have remained alive and that have been stolen by the predator from prey which had been devoured. Taku Komai $(1935,1951)$, a well-known Japanese zoologist, who has recently had the good luck to be able to make a detailed study of Euchlora and who in his work first expressed his opinion that these cnidae actually belong to the animal, has later become convinced that we really have here a case of kleptocnidae. Euchlora subsists mainly on Narcomedusae and it seems that it has become so specialized that it has ceased to produce colloblasts, its original weapons, and instead "works" exclusively with cnidae taken over from its usual prey. This certainly is a very interesting case and it is at the same time at least one more fact which supports our thesis that the Ctenophora cannot be directly related to Cnidaria.

Besides the differences which have been already mentioned, between the tentacles of Ctenophora and medusae, there is one more which makes it completely impossible-in spite of their great external similarities - to consider the two organs as homologous or descending from the same origin, or even to 
be brought forward as a proof of the direct relationship between Ctenophora and Cnidaria. The tentacles of medusae have always and without exception, an intestinal diverticulum in their axes, i.e. a continuation of the intestine with an entodermal coat and with an intestinal lumen. It is not rare for the intestinal lumen to be secondarily obliterated. In this case the entoderm forms a solid entodermal axis which is not unlike a rouleau of money or a primitive chorda dorsalis. In this way the entodermal axis develops into an internal skeleton. The structure of the tentacles of Cnidaria is usually described as if they were composed of an ectoderm and of an entoderm only. Later we shall try to show in more detail and in connection with the whole body of Cnidaria that the intermediate layer of the body participates also in the structure of the tentacles. This layer usually bears the unsuitable name of a mesoderm or mesogloea which again creates the impression that we have here something different from the intermedial layer of the body of other Eumetazoa. I have therefore proposed for it the name mesobyl. It is better to speak about the mesoderm only in connection with early ontogenies.

A typical feature of all Ctenophora is the fact that their tentacles never have an intestinal diverticulum or any entoderm whatever. As important as this difference is, it is, however, by no means the only one. Even the way that these tentacles grow is completely different in the two cases. In Ctenophora the tentacles actually grow constantly during their whole life time; their growth continually takes place at the considerably thickened bases deeply sunk into a cutaneous follicle, while at the same time the free ends slowly break away. In connection with this we find in Ctenophora the base is organized in a completely different way from that of Cnidaria. Without finding it necessary to enter into further details we have the full right to maintain and conclude on the basis of what has already been mentioned, that the tentacles of Ctenophora and Cnidaria do not have the same origin and that we have here a case of functionally similar, and therefore analogous, organs. 
The difference between the tentacles of Ctenophora and Cnidaria would be perfectly clear if we could show the origin of tentacles in Ctenophora as well as in Cnidaria. In both cases the tentacles are, in all probability, not completely new formations. We presuppose that in both cases the tentacles have developed out of paired excrescences at the anterior part of the body (at the "head") of their turbellarian ancestors and that on both occasions this development was independent from each other. It should be mentioned in this connection that paired organs of touch (this in connection with the rilateral symmetry of the animals) can already be found among Turbellaria, once with the inclusion of the intestinal diverticulum, another time without it. Cnidaria must therefore have descended from those turbellarian ancestors whose palpi had an intestinal diverticulum, and Ctenophora from those whose palpi had none. We can see that in Temnocephala, i.e. in Turbellaria which have adopted a half-sessile way of life, the polymerization of these excrescences has already taken place; this has become even more developed in Cnidaria which have similarly adopted first a half-sessile and later a completely sessile way of life. Ctenophora, however, as freely moving animals, retained the original paired system of tentacles which became specialized in another direction. In the first case (Cnidaria) the turkellarian ancestors belonged to a rhabdocoelian subtype while in the second case (Ctenophora) they belonged to the subtype of Polycladida, and especially to their larvae that became neotenic and remained in the plankton during the whole of their life.

In this connection I would like to point to the fact that in the course of phylogeny, tentacles have been repeatedly evolved and have either a horseshoe-shaped (the younger phyletic stage) or crown-shaped form. This pattern has always stood in connection with the transition to a sessile way of life (Entoprocta among the Ameria, in nearly all Oligomeria, and even in the "lower" Chordata: in Tunicata).

The third peculiarity and at the same time the third point in which Ctenophora differ sharply from the Cnidarian medusae 
is their combined aboral sensory organ (see Fig. 3). Anything identical or even similar is completely absent in medusae. Hydroctena has been mentioned again and again as if it was an exception and at the same time an intermediate link between those Cnidaria which resemble Narcomedusae and Ctenophora.

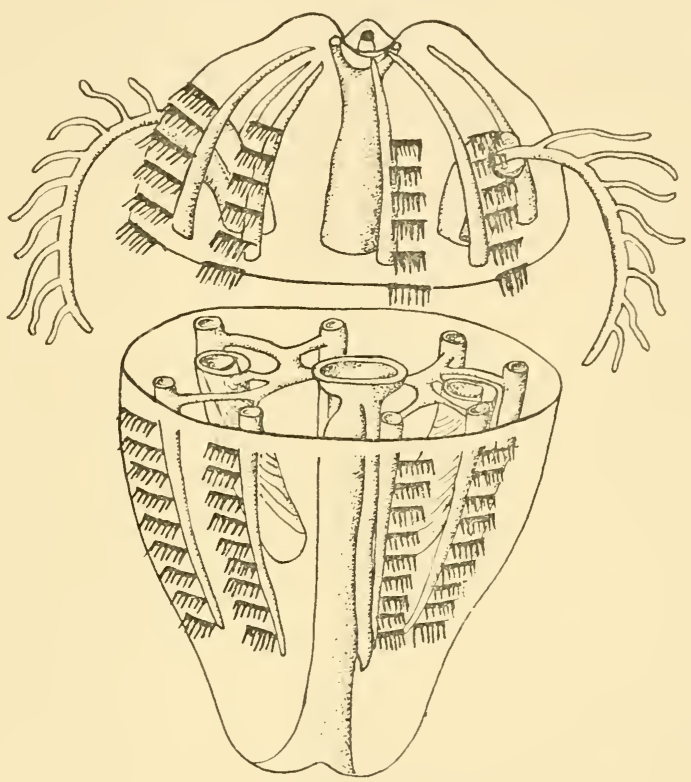

Fig. 3. Diagram of the Ctenophore organization. (After Hertwig and Kaestner.)

Hydroctena, however, as this will be shown in the ensuing pages of the present study, cannot be considered to have such a role. The absence of a static organ in the exumbrella of medusae is not a mere charce. Its absence in medusae and its presence in Ctenophora can be easily and convincingly explained.

We shall later see how the part of the body which is turned upwards in a ctenophore that is freely poised in water corresponds to its anterior part. In Turbellaria, a static organ situated close under the skin and in the centre of their central nervous 
system has been developed in the same place. This organ has been inherited by the Ctenophora from their turbellarian ancestors, and during the transition it came completely to the surface. This agrees perfectly with the orientation as well as with the way of life of Ctenophora. We must not forget in this connection that the Ctenophora must in all probability be considered to have evolved by means of neoteny from the organizationally simpler planktonic larvae. Naturally, evolution has proceeded until the present forms of Ctenophora have been reached. The oral opening was transplaced from the vicinity of the aboral pole (first the anterior pole, and in Ctenophora the upper pole) to the vegetative pole (in the creeping Turbellaria the posterior pole, and in Ctenophora which are poised in water the lower pole) (see Fig. 2).

Medusae, too, regardless of whether they are Hydromedusae or Scyphomedusae, live as planktonic animals poised in water, not with their "head turned upwards" as in the case with Ctenophora, but rather with their "head turned downwards," i.e. with their vegetative pole turned upwards. Thus the oral opening of medusae is turned downwards; its place corresponds to that of the animal pole since they have developed from polyps either by means of gemmation budding, (Hydromedusae), or by means of transverse fission (Scyphomedusae), or secondarily, directly from ova. The polyp, the initial form of Cnidaria, "stands" upright and is attached with its vegetative or posterior pole to the floor of the sea. The medusa, which has evolved from the oral part of the polyp, underwent a reversion when it became a freely-swimming animal and turned its originally anterior part upwards. Yet this uppermost part of the medusa is no longer a part of its head as it has remained in the ancestors of polyps, but instead the oral part has been removed and is now, so to speak, only at the end of a stem. We find therefore the surface of the so-called exumbrella turned upwards when the animal rests, and forward when the animal moves. The exumbrella is genetically and morphogenetically empty because it is covered with one layer only of the ectoderm 
and it is not able to form-even if this were useful-a nerve centre, a brain ganglion, or even a sensory organ of the type of statocyst. The facts show that this is really so; it becomes particularly clear in those cases-which are by no means rare-where a secondary, the so-called hypogenetic development takes place, i.e. where the original generation of polyps is left out so that the form of medusa develops as the only type from the egg, and where, in spite of all this, the

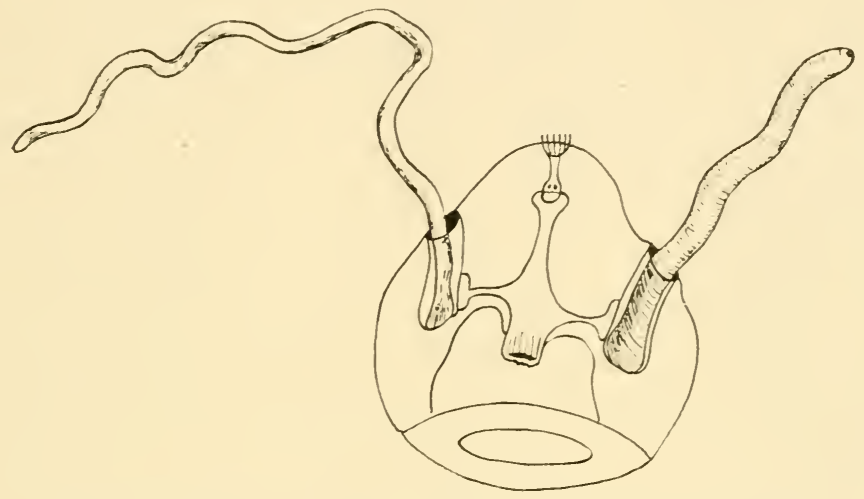

Fig. 4. Hydroctena salenskii Dawydoff. (After Dawydoff.)

exumbrella remains empty and does not possess a vertical sensory organ. This can be understood only if we take into consideration the fact that in medusae their physiological anterior pole is not their vertical pole, as is, on the other hand, the case with Ctenophora.

We will now pass to Hydroctena salenskii which has been discussed by C. Dawydoff. The name itself of this remarkable animal shows that it was considered as an intermediate link between medusae and Ctenophora. Hydroctena has been observed once and in three specimens only; these were neither sexually ripe nor, as it seems, particularly well preserved. It has been thought that it represents a mixture of elements characteristic for Ctenophora and Hydromedusae, that is Hydromedusae of the type of Narcomedusae (see Fig. 4). Various authors 
proposed different opinions regarding Hydroctena. Some have thought it to be an aberrant type of Narcomedusae, others have considered that it is a ctenophore yet without the swimming apparatus in the form of eight meridional bands of plates which is so characteristic for Ctenophora; the least accepted interpretation has been that it represents a transition form. I have tried to submit all the known factual data (which are very incomplete) to a renewed scrutiny (Hadži, 1958 b). In this way I have come to the conclusion that in all probability we have here ctenophores which have developed crippled forms because their embryos had been damaged by an external event (storm). In this connection I have referred to observations, especially those made by $\mathrm{C}$. Chun, according to which the damaged embryos of Ctenophora continued to develop into partial forms because of the strong determinism in their ontogeny. Dawydoff maintained that Hydroctina had no colloblasts on its tentacles and that instead cnidae had been found much as can be observed in Narcomedusae. On the basis of this I have proposed that the supposed Hydroctena could have developed from the embryos of Haeckelia rubra. I have therefore suggested that Hydroctena should be set aside.

In the meantime C. Dawydoff has published a short notice where, on the basis of serial sections of Hydroctena made by another zoologist, he comes to the conclusion that we have here a genuine Narcomedusan. The vertical sensory organ, which was the main point in this revision, has been explained as a form sui generis that has nothing in common with the aboral sensory organ in Ctenophora, but instead corresponds to a pair of club-shaped marginal sensory organs of Narcomedusae: this was first transplaced to the vertex of the umbrella and later sunk into the interior of the same. This interpretation seems to me completely improbable and at the same time incompatible with the original description of the structure of the supposed Hydroctena as it had been given by the same author (cf. the conditions of tentacles and of the 
so-called gastrovascular system). I consider that I am justified in deciding my interpretation as the more probable.

Many other arguments and evidence could be given to support the thesis that the Ctenophora cannot be directly related to Cnidaria. The ontogenies of the two groups in particular are completely different from each other. It may be useful to mention the so-called ciliary rosettes in Ctenophora for which there is no homologue in Cnidaria. I think that these ciliary rosettes developed out of the terminal organs of the protonephridial system in Turbellaria. The examples which have been given so far should suffice to prove that there are important differences between Ctenophora and Cnidaria; yet at the same time there are also some similarities which are such that they make it probable that the two groups did not develop one from the other, but instead independently from two ancestors that had been closely related to each other and that had evolved from the same primitive form. This interpretation could perhaps be also expressed with the dictum: si duo faciunt idem-non est idem. In both of them the evolution took a new direction: it was the same in both cases and it tried to adopt the characteristics of animals living a pure planktonic life. In the case of medusae, the evolution proceeded from a bilateral symmetric and mobile stage, through a second stage which was radial symmetric and sessile, to a third stage which was radial symmetric and planktonic. In Ctenophora the development went from a bilateral symmetric and mobile stage through that of a planktonic larva influenced by the neoteny into the final planktonic stage. In both cases the ancestors were bilateral symmetric Turbellaria, but they belonged to two different subgroups (see Fig. 5).

To the best of my knowledge no hypothesis that is more probable, and therefore better, has been proposed to explain the origin of Ctenophora which takes into consideration so many of the numerous peculiarities of this small and now isolated animal group, and which would make it possible to understand them. Yet even if we do not bother about this 


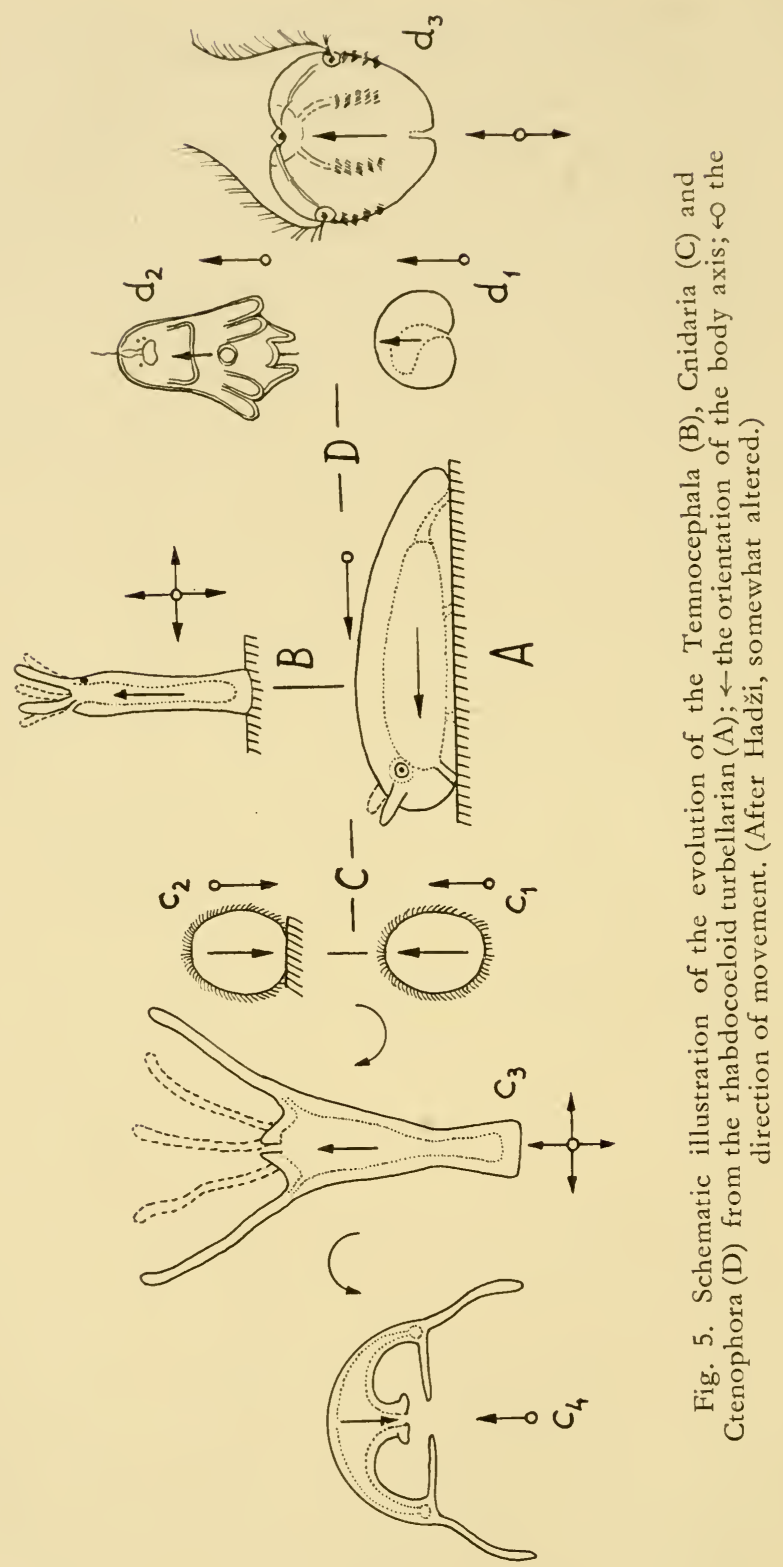


origin of Ctenophora which has now been accepted by numerous zoologists, e.g. Sir Gavin de Beer (1954 a), it has now become completely clear that Ctenophora cannot be simply considered as Coelenterata that have no cnidae (Acnidaria). Nevertheless, such an interpretation can be frequently encountered. The Ctenophora are not Coelenterata and they are not directly related to Cnidaria even if there is some relationship between the two. Later we shall try to show in more detail that the Turbellaria, the original form out of which all Eumetazoa have developed, are also the starting point of many other Ameria (lower invertebrates), even of molluscs. All these groups represent independent units as is true also for Cnidaria and Ctenophora. Yet it will not do to establish special phyla for these groups; the highest systematic category (taxon) must be reserved for large groups that are basically different from each other. I will return to the discussion of this problem later in the present study.

It should be remarked in passing that unknown to me, I was not the only person who tried to derive Ctenophora by way of neoteny from the planktonic polyclad larvae. A similar interpretation of the origin of Ctenophora has been proposed by W. Garstang (cf. J. I. Brooks, 1954) at approximately the same time that my thesis was published. Yet the form in which that interpretation appeared is somewhat unusual for a scientist: it was in a poem (Comic Verses) which was first published in 1951 by A. C. Hardy (1954). Nevertheless, this does not change the fact that an idea has been conceived according to which the origin of Ctenophora must be sought in Müller's larvae that remain in the plankton and whose evolution is influenced by neoteny.

It has been now generally accepted by all zoologists that the Spongiae represent a blind side branch of the animal kingdom, which means that no other higher type of animal has been developed from them. We have, therefore, no further interest in the Spongiae. We shall return to discuss them only when we deal with the problem of the origin of Metazoa. On the other 
hand, however, attempts have been made to see in the Ctenophora an initial stage for a higher development. This was suggested in connection with the discovery of certain species of Ctenophora which do not live in the plankton as is typical for Ctenophora but rather on the sea bottom, and for this reason have been correspondingly changed, especially in their external forms (Platyctenea). They have been thought in this connection as a possible transition to Turbellaria: but the specialists have been able to show that these are genuine, though specialized, Ctenophora. In this way, the Ctenophora, too, as the third element of the old group of Coelenterata represent a blind side branch of the animal kingdom: no higher type of animals has been developed out of them. Though there are some connections between Ctenophora and Turbellaria, these connections are in my opinion exactly opposite to those as viewed by the old interpretation. The Ctenophora should be considered to have evolved out of a turbellarian form due to their transition from a life on the bottom of the sea, when they had a pure bilateral symmetry, to a life in the plankton, with a change of their symmetrical structure to that of bisymmetry. A detailed comparison of Ctenophora and Turbellaria brings to light a large number of common characteristics and peculiarities, particularly if in connection with the latter we take into consideration the planktonic larvae of Polycladida. The difference, however, can be explained on one hand as a result of the neoteny, and, on the other, as being due to the adoption of a freely moving life in water. This latter specialization makes it particularly impossible to regard the Turbellaria as having evolved from the Ctenophora. A small but welcome contribution which supports my hypothesis of the development of the Ctenophora from the Turbellaria has been the recently proposed theory (Hadži, 1955) which tries to find the origin of the so-called ciliary rosette organs of Ctenophora in the turbellarian protonephridia. There are present-day zoologists who have assumed a critical attitude towards all attempts which try to explain the formation of 
new animal types by way of neoteny. This scepticism, does not seem to be justified in spite of the fact that not all such attempts can be considered to have been very successful. New animal forms-and not only new animal types-have in numerous cases been developed by way of neoteny (cf. de Beer, 1958).

\section{Cnidaria as the Only Coelenterata}

Now that we have succeeded in isolating the Cnidaria, the concept of the Coelenterata which has been so widely used during the last hundred years has been reduced to one group only. In this connection the question arises whether it is still wise to continue to use this concept as well as the name Coelenterata as opposed to Coelomata. The fact that we have reduced the contents of the Coelenterata to a single group, can certainly not be considered as sufficient to allow us to question its existence. As a matter of fact, classifications can occasionally be found where "Coelenterata" appear in which Coelenterata are represented by the Cnidaria only. It can be easily imagined that Coelenterata-considered as a necessary stage in the evolution-are represented by one subtype only, by Cnidaria. Yet in this case it would be necessary to make it probable, if not proved, that the Cnidaria have developed as the first Eumetazoa from the Protozoa; a supposition which again, if taken alone, could not be sufficient because it would be necessary to show further that the next higher animal group which, according to the present opinion, are Tubellaria, have developed from the Cnidaria.

I will try now to prove or to show it more probable that the Cnidaria are neither the first (i.e. the lowermost) Eumetazoa, nor the ancestors of the next higher developed Eumetazoa, the Turbellaria. Since 1944, I have defended this thesis and supported it in numerous articles (Hadži, 1946-1959.). The fact that my book appeared during the Second World War and in Slovene, a language of a small though progressive 3* 
Slavic nation, has been of considerable disadvantage even though the Slovene text appeared together with an extensive German summary. This book was followed by later publications in international languages. The thesis has become best known and discussed through an article published in the United States in the journal Systematic Zoology; the article was beautifully translated into English by Professor A. Petrunkevitch for whose translation I wish to express here my warmest thanks. There was no visible reaction to my short lecture given at the Fourteenth International Congress of Zoologists held at Paris. A further essential contribution to the propagation of my ideas was made by Sir Gavin de Beer's Evolution as a Process which contained an article written from a sympathetic point of view by G. S. Carter (1949, 1954.), and in the German-speaking area by Professors Ferdinand Pax, (1954) Dr. Otto Steinböck, and by the palaeontologist Müller (1958), among others. An important contribution to the development of my ideas has been made by Hanson (1958) who discussed the consequences my theory would have for the estimation of the true nature of Cnidaria. It had been expected as well as desired that there would be several critics of my thesis (among them some well-known scholars, e.g. Beklemischew [1958], Laubenfels [1955], Carter [1949, 1954], A. Remane [1957], L. H. Hyman [1940-1958]). No criticism has yet been published which I consider to the point and which I would be forced to accept; there have been no critiques which have weakened my conviction or even forced me to abandon my theory. The majority of remarks do not even touch the core of my concept, i. e. my hypothesis about the origin and the position of Cnidaria in the system of the animal classification, but instead they discuss the numerous and important consequences of this hypothesis. These consequences undermine the basic pillars of the old concept. It is, therefore, not surprising that they have provoked sharp and occasionally even agressive criticism. 
The three types of Metazoa, Spongiaria, Cnidaria, and Ctenophora, have not been united into the higher unit of Coelenterata not only for the reason that they lack a secondary body cavity, the coelom (I prefer to call it perigastrocoel), since there are many other groups of lower Coelomata where the same coelom is absent; but rather because they represent with their general organization a lower stage of development. This, however, does not suffice, as has been demonstrated, to unite them all into one group. The organizations of all these animal groups are different from each other. The thing above all which has to be done if we wish to place them into one common higher systematic category, would be to prove a common origin of all these three groups. This was not necessary in the days of Leuckart. After the general acceptance of the Theory of Evolution the problem was solved first by E. Haeckel, and later by numerous other zoologists (E. Metschnikoff, B. Hatschek, C. Claus, L. v. Graff, the two brothers Hertwig, R. Lankester, A. Balfour, and many other zoologist up to the present day for example, A. Sachwatkin, A. Remane, L. H. Hyman, etc.), in a very simple and quite obvious, way. There was a simply-built primitive form which, though variously called (gastraea according to Haeckel, placula according to v. Graff, parenchymella according to Metschnikoff, and planula according to Lankester), and differing in details from one another, is, nevertheless, essentially the same. This primitive form has been used in connection with the ontogenetic stages and declared to be the common primitive form of the recapitulation theory as it appears in the "fundamental biogenetic law" proposed by Haeckel. According to this theory three different types of animals have been developed from the one polarized bistratal being which resembled a gastrula. The development diverged in three different directions, and as a result of it we have the Spongiae and Cnidaria which have adopted a sessile way of life, and the Turbellaria which creep over the surface of the sea bottom. The Ctenophora, however, have been usually 
considered-as it has been already mentioned-to have evolved from the Cnidaria, and not directly from the blastea. The Tubellaria, finally, have been thought to have developed from the Ctenophora. The main idea has been to derive the lowest Metazoa from a ciliated gastrula-like primitive form, which itself has been supposed to go back to an organism resembling a blastula, and this finally to a colony of Flagellata. Even nowadays this same theory is proposed with an admirable ardour by A. A. Sachwatkin (1956) on the basis of Metschnikoff's parenchymella theory.

Since in a classification which should be natural, i.e. phylogenetically founded, a similar or even identical level of organization cannot be considered sufficient to bring together several groups into a higher unit; it is also necessary to prove that they have descended from the same ancestral form. In the case of the Coelenterata it has been attempted to prove again and again-or at least to make it seem probable - that they have a common origin. These attempts, however, cannot be considered to have been successful. The completely artificial constructions such as those proposed by Remane (1950) and by those who followed him, e.g. by Alvarado and Jägersten-to mention three recent attempts only-have been completely unconvincing.

The isolation of Cnidaria alone, after the withdrawal of Spongiae and Ctenophora, would not suffice to cause the abandonment of the higher category of Coelenterata. It would now be necessary to prove that there is a basic difference between Cnidaria and the animal groups that stand closest to them, and, then, all other animal groups (the so-called Coelomata, or, according to Ray Lankester, Coelocoela), as has been the case with Spongiae. This difference was believed to have been found in the fact that Cnidaria are real "Gastraeadae," i.e. animals with their body built of two body layers. For this reason they have been called Diblastica, Didermique, etc. Furthermore, and in connection with this, the Cnidaria have no coelom cavities and in particular no 
perigastrocoel. Later I will show that the assertion according to which Cnidaria are built of two body layers does not agree with actual fact. The true coelom, which I call perigastrocoel, and which alone would allow us to call the bearer of such a coelom, a coelomate, does not occur earlier than in Annelida, the lowest subgroup of Polymeria.

Even if the Cnidaria remain, on the basis of their actual situation and not on that of a hypothesis, isolated from the two other supposed members of Coelenterata (Spongiae and Ctenophora), they are not yet distinguished from other lower invertebrates, i.e. from my Ameria or from the primarily unsegmented animals. We must search for their connection downwards, i.e. for the point where they have branched off; that is, we must search for it among the freely moving bilaterally symmetric ancestors, that lived on the bottom of the sea and whose organization shows a somewhat higher development. This must be proved, or at least made probable, and it will be my duty to try to do this in the present study.

A detailed comparison of Cnidaria with Ctenophora shows that these two groups of animals, which for a long time have been grouped together with Spongiae into the phylum Coelenterata, do not even stand in a direct mutual relationship. In my opinion they have evolved independently, each from its own ancestor. In this way Cnidaria are the only animals that remain in the large group of Coelenterata.

Yet before we begin to tackle our main problem, to try to identify the origin and the true nature of Cnidaria, it may be useful to discuss first those methods which have been used in connection with the solution of our problem. 


\section{Something About the Methods That Can Be Used in Connection with the Construction of the Natural Animal System}

It should first be mentioned that we are discussing here a phylogenetic construction which should form a basis of an attempted reform of the system. This touches the very roots of the concepts that have so far prevailed with regard to classification, and the consequences can be of considerable importance. Furthermore, it should be mentioned, even if it seems superfluous, that I base my work constantly on the neo-Darwinian principles of evolution. I find any idealistic morphology, or any system based on it, and any purely idealistic taxonomy, unacceptable; they are both out of date as well as unscientific. Modern zoologists are endeavouring to construct a natural animal classification which, in agreement with this, must be based on the supposed phylogenetic development. The results, for better or worse as they are achieved, depend above all on our present positive knowledge-which is an objective element-and, naturally, to a certain extent on the scientific worker-which is an unavoidable subjective element. We can make no progress either with agnosticism or with an ultraconservatism, if it is linked with a complete absence of the creative imagination. It is therefore necessary to form a realistic concept even in those cases where the obstacles we have to overcome are due to our present insufficient knowledge. In this connection we must not be afraid to dare to conjecture or to use our imagination. Yet at the same time we have to be aware of the fact that these are hypothetical constructions only. They have not been made for all eternity.

It would be detrimental to our progress is we reject on principle and without discussion, any more or less daring construction; if we declare any work of this kind to be a pure waste of time; and if we look upon it disparagingly as is frequently the case. The numerous and mutually different attempts to construct the animal system that we have met even 
in the present day, prove that we are still far from the ideal solution. Many difficulties arise from this situation, especially in the field of teaching. I tried to seek a redress to this situation at the Fourteenth International Congress of Zoologists which was held at Copenhagen, and I proposed to find a unified or compromissary animal classification which could serve our practical purposes (Hadži, 1956 a). Due to my proposition a committee and president have been elected, as yet without any further results. It is possible that this attempt was made too early, or that it was discarded because it was considered as being impracticable.

Fortunately enough, the interest in the problem of phylogeny and, in connection with this, of the construction of a truly natural animal classification has been recently reawakened. As an illustration I can mention the reaction which occurred when my article appeared in Systematic Zoology (Hadži, 1953). It is perfectly natural that such reactions were not always favourable, or, even constructive in regard to the new proposition. It is not easy to convince those zoologists who have been trained in long established concepts which they firmly accept, that these old concepts are actually untenable. Max Planck, if I am not mistaken, once stated that one is more likely to see the defenders of the old concepts die out before becoming converted to new interpretations. It is now time for the new generation, one free of tradition, to proceed critically and make the decision.

Some progress has nevertheless been made in the field of phylogeny; this can be seen in the fact that the "variation width" of hypotheses and theories, and thus of concepts, has finally become somewhat narrower. An objective, even if sharp, discussion can always be useful.

In my opinion, in this area of biology one can arrive at a new concept, at a new evaluation which has a synthetic character, mainly along two lines. Either we succeed in making a new discovery, a discovery of a brand new fact, e.g. of a new fossil or of a living animal which has been unknown 
so far ("a living fossil"), or we succeed in establishing new facts-facts which had not been known previously-through our study of the structure and of the ontogeny of a known animal species. The new fact must throw a new light on our present concepts. The second way that can be taken which, even if harder, is in reality much less dependent on a mere chance, is that of a profounder comparison or of a juxtaposition of facts that are already known. It enables us to discover new relationships. Frequently we find this second way to be connected with the discovery of mistakes made by earlier authors when they had formulated their final conclusions or in relation to methods which they had incorrectly used. This second way has been pursued in our investigations.

In our case, that is in the study of the origin of Cnidaria, it is impossible to expect that we will be able either to find the corresponding fossil-material or to make direct observations; and so it is clear that we must rely on the comparative method as applied to the present living species. The fossil documents that have been found belong exclusively to the skeleton-building Cnidaria: with a few exceptions they are all impressions of fully-developed medusae. We can see, however, that even in those cases where a much richer fossil-material stands at our disposal (e.g. in Vertebrata) and where we are able, on the basis of the fossil-remains of skeletons, to make far-reaching conclusions with regard to the structure of the "soft" parts of their bodies, that even here we must use the comparative method in its full extent in order to be able to make correct interpretations of facts i.e. to reconstruct the way the evolution had taken.

It should be mentioned in passing that only humble results have been achieved so far by means of the biochemical method in our study of the degrees of relationship, even if considerable expectations had been originally placed in this method. The chemical processes in question are certainly very complicated and the special serum methods that have been used till now 
are not yet fully developed. In the future we can with certainty expect more important results from this method. My invitation to biochemists to check, by means of the serum method, my hypothesis of the closer relationship between Cnidaria and Turbellaria has remained, to the best of my knowledge, so far without any response in the international literature on the subject.

It has been already mentioned that we cannot expect to find fossil remains of the early stages in the evolution of Cnidaria, since possibly no species belonging to them had existed at that time which could have left some fossilized traces. Yet even those traces which go back to more recent times are not unimportant for our purposes. Somewhat doubtful kinds of remains belonging to Cnidaria are known, that date from the Middle Cambrian. They belong to the now extinct Tetracoralla. No remains that can with certainty be attributed to Hydrozoa can be found earlier than in the Upper Triassic; some partly uncertain forms that resemble the present-day Campanulariae date from the Silurian. Impressions of Scyphomedusae that can be easily identified go back to the Middle Cambrian.

According to Schindewolf (1950), who in his studies paid special attention to the evolution of corals, the earliest remains that can with certainty be attributed to Madreporaria, and thus to Anthozoa, belong to the Upper Ordovician (which was formerly usually called Silurian). They evolved rapidly and differentiated into numerous species, some lived a solitary life (this is very important in our connection!), and some which formed colonies or cormi and which began to build coral reefs. Similar earlier reefs had been built by the Archaeocyathidae, which are now considered to have been a kind of silicious sponges. Fortunately enough, we find sclerosepta even in the earliest stone-corals; this enables us to study their internal structures, i.e. the distribution of the sarcosepta. It has even been possible to trace the ontogenetical development of their septal apparatus. In this way Schindewolf 
has been able to prove not only that in pterocorals the distribution of septa is bilaterally symmetric, but also that even the first anlage of the septal apparatus was bilateral " the six protosepta show a distinct bilaterally symmetric development" Schindewolf, 1950, p. 179). Later, a secondary radial (hexamerous) symmetry has been developed from the former. It must be emphasized that according to Schindewolf (1950, p. 181-2) "the Palaeozoic pterocorals form the ancestral forms of the now living stone-corals. Together with the latter they must be included in the order of Madreporaria which had been previously limited to the recent forms only."

The fact is established by Schindewolf that this orthogenetic course of evolution which goes from an internal bilateral symmetry to a full cyclomeric radial symmetry has not been completely carried through even in the present-day Madreporaria. It will be later seen that this has been first achieved in Scyphozoa, and particularly perfectly in Hydrozoa. Here we meet with a phenomenon which is especially important to a phylogenist: it enables him to make well-founded conclusions about to the interrelationships on the basis of a comparison of recent forms only.

Again it can be stated that fortunately enough for phylogenists, the phyletic evolution of animals did not take such a course that it evolved along a single progressively advancing front so that the present day fauna would consist of the most advanced vertebrata only, actually of only one species: man. Even now we can find, so to say, in one and the same pool, various forms living together: Flagellata, amoebae, Turbellaria, snails, Annelida, insects, Bryozoa, fishes, frogs, etc. Thus we have old types which themselves have undergone further development and which in this way have become more differentiated, together with new types which are constantly emerging yet which are not necessarily always the more progressive forms. The primitive characteristics have not been preserved in the older types of structures only, but also in structures of some younger forms. These latter 
forms are particularly interesting to a phylogenist. It is only necessary to identify these primitive characteristics which had been typical also for their ancestors, and to interpret them correctly. Comparative morphology leads us to find these true homologies.

In our morphological comparisons we take into consideration above all the stages reached by the grown-up animals. This is a point that has been accepted by all morphologists. In Protista we consider the so-called vegetative stage in their cycles of development to correspond to the grown-up stage in metabionts. Secondarily, we have to take into consideration the succeeding stages in the individual, or ontogenetic, development, starting with the zygote (the fertilized egg) or even with gametes and with the gametogenesis. The comparison can be made either between various grown-up forms, or between various stages of its development, or, finally, between the grown-up forms and their stages of development. Naturally the latter kind of comparison is to be preferred.

Here, however, we meet with a problem which has been widely discussed and which has provoked the sharpest controversies among the zoologists. This is the question of the relation between ontogenies and phylogenies. The way we understand this relationship will be decisive to a large extent for the kind of conclusions we shall reach in individual cases. Here we cannot possibly start to discuss this whole problem; it should suffice to refer to the book by Sir Gavin de Beer, Embryos and Ancestors. Purely as a result of my own research in Cnidaria, and not as a result of the study of the corresponding literature, I came to the conclusion that the now prevailing concept of this relationship which finds its culmination in the well-known "fundamental biogenetic law" as it has been proposed by Ernst Haeckel, must be basically wrong. The facts that can be established on the basis of a study of the structure as well as of the individual development of Cnidaria disprove decisively the very spirit of this rule (it is better not to speak about laws within the 
field of biology) which wishes to see a recapitulation of the phyletic stages (i.e. the characteristics of the grown-up forms) in the ontogenies of their descendants, i.e. of the recent animal species. Garstang, was the first scholar to make it clear that the ontogenetic morphogeneses which are constantly repeated in each succeeding generation are also constantly changing.

The changes in the ontogenetic process, whether the introduction of new characteristics or the loss of characteristics and peculiarities which had previously existed, cannot possibly be considered to originate in an adult stage. It is only if we accept the standpoint of Lamarckism, that we could take a possible recapitulation of characteristics of the grown up individual into consideration.

In this way, and to the great regret of the comparative morphologists, the recapitulations which can take place during the ontogenies will be found to be limited, even if not completely eliminated. It is not true when it is said-as this can be frequently heard- "that Haeckel's law is not generally valid but that instead its validity is only limited;" in reality this supposed law is basically wrong. Nevertheless, there are recapitulations in ontogenies and these recapitulations are such that they can be of good use and service to a phylogenist. It is necessary to proceed very cautiously when in search of these recapitulations. One must be constantly aware of the fact that these are really ontogenetic characteristics that are recapitulated. This, however, does not exclude the possibility that these same characteristics eventually appeared in the grown up stages of their ancestors. These characteristics remain, even if they can be observed only under special circumstances of morphogeny, in spite of the fact that they had long been lost in their grown-up stages because of suppression due to the natural selection. In this way the phylogenist is fully justified in using those characteristics that have developed during ontogeny for his conclusions regarding the interrelationships between such animal 
types which he wishes to compare; but this must be done cum grano salis.

Even those peculiarities of different ontogenetic stages for which the unsuitable name of Caenogeneses (foreign or tampered peculiarities) has been invented by Haeckel, could be profitably used. Yet in this connection it is necessary to be even more cautious. I think that at this point morphologists have gone much too far, especially when they tried to develop the classification of animal types into large units on the basis of similarities that can only be observed during the earlier ontogenetic stages, e.g. during the segmentation of the egg, during the formation of the entoderm, etc. To demonstrate the above statement we may take the systematic category of Spiralia. A special type of cleavage, of the formation of the blastomere-the spiral type-is common to all animal types (this of course is not without exceptions) even if they show great varieties in their structures. Will this type of cleavage prove the common origin of all the animal species in which it appears, and should we therefore in a natural system combine all of them into one large group? I completely agree with Steinböck's suggestion to look upon these types of division from the point of view of the evolutionary mechanics (I like to speak in this connection about an "ontogenetic technique"). The type of cleavage usually depends on the quantity of the reserve food and so it appears in various forms within the narrow frame of a systematic category. Sacarão (1952) has descrited many examples in this connection which he explained in the same way. The phylogenist finds it useless to speak about Spiralia because among these there are whole groups that are no "Spiralia" whatever.

A special treatment is needed in the case of those ontogenetic stages that live freely and-as larvae-have had a progressive development which has been, say, in other surroundings and with another way of life than the corresponding grown-up forms. These cases are important for phylogenetic purposes. 
The situation is usually such that the grown-up animal types-actually with widely different kinds of structure-live on the floor of the sea, lead a benthonic life, while at the same time their embryos have developed into planktonic larvae. The way that this case is presented here shows that we consider the life on the floor of the sea to be primary, and that in the open water, i.e. in the plankton, to be a secondary result of the development. In fact, the larval stage represents a prolongation of the ontogeny. In no way can we consider these larvae to be ancestors of any recent animals that live on the floor of the sea. We have no recapitulation of some stage reached by their ancestors, as is the case with some other stages in ontogeny In spite of this the larval stages can be of good use in our study of phylogeny. Larvae of some animal groups, e.g. those of Crustacea, have very characteristic forms and structures (in this instance the socalled nauplius). These forms are so firmly established in the whole that they are present even when the grown-up forms had been much changed so that they can hardly be identifiedwhether this change be due to an adaptation of the benthonic way of life or to a strong parasitism. A similar case can be observed in Tunicata (Kowalevski). In such cases it is our right to consider larvae as evidence that species do belong together whose larvae have the same forms and structures. It would, however, be completely wrong to conclude on the basis of these facts that an ancestral form is being recapitulated in a nauplius. The example of Crustacea itself shows that it is not impossible that the larval stage, the nauplius, disappears. In the transition from the sea to fresh water, the free larval stage - the nauplius-becomes embryonalized. Frequently, this change is hardly noticeable. The free living stage of ontogeny had possibly originated in various levels which the evolution of the animal had reached, and it can also depart more or less from the "normal" type. Not infrequently (e.g. in insects) we find the main part of the individual existence transferred to the larval stage. In these instances the 
life of the adult stage can become very short: particularly in the case of male animals, so short that it just suffices for propagation. In these instances, the differences between a larva and its imago have become so considerable, both with regard to their structures as well as their ways of life (the type of feeding, the sphere within which the animal lives) that finally a great crisis occurs and the ontogeny which can only be overcome by way of an extremely radical method i.e. that of the total metamorphosis. These instances have the character of true catastrophies which, however, can fortunately enough be mastered. In the case of strongly developed parasitism we can frequently observe that a larva which has preserved a free mode of life, reaches a higher level of development than its grown-up form.

Beginning with Turbellaria and ending with Amphibia among the Vertebrata, larvae have had an important role during the levels reached in evolution. It is therefore not surprising that attempts have been made to use larvae as a basic element in the construction of a natural (i.e. evolutionistic) animal system. This is particularly true for those zoologists who came under the influence of the supposed "fundamental biogenetic law" as it had been formulated by Haeckel. It was all too easy to accept the possibility that in larvae, an ancestral form is being recapitulated and that for this reason all smaller groups whose larvae show a certain degree of similarity must have (1) a common ancestor, and (2) the form of this common ancestor must have been basically the same as that of the recent larva. In spite of the fact that these theses have been found to be essentially untrue, they have proved useful in the struggle for the recognition of the theory of evolution. This particularly in the sense that they have numerous well-known zoologists to make "embryological" studies of such species and groups of animals where natural relationships are not clear. It had been hoped that the better known ontogenies could prove useful in attempts to unravel these connections. The facts which have 
been discovered in this way have had an enduring value in spite of the fact that they have been arrived at by way of some wrong initial presumptions.

It was soon found that the larval stages, as well as complete ontogenies, proved useful for the construction of the natural system even if one did not employ the "fundamental biogenetic law;" it is only necessary to change the basis of such comparisons and to make these comparisons exclusively between various stages of development, even if the results reached in this way seem to be less important and certain. We can now state-and this with considerable certainty-that in a comparison of ontogenetic stages the chances of success are the greater, when the older stages are compared. It must be initially expected that in comparison to the primitive forms, the greatest changes will be observed in the youngest or initial stages of the ontogeny, since these are not only phyletically the oldest but also in their organization, the simplest stages. Here Caenogeneses may often take place. This is particularly true in the case of those animals whose development occurs from the very beginning in free water. It is therefore better to omit the early phases of ontogenesis from phyletic constructions, or, as is frequently heard, from our speculations.

Let us bypass the intermediate ontogenetic phases and discuss the phyletically oldest larvae. The planula of Cnidaria has generally been considered to be such a larva partly because the Cnidaria are believed to be the oldest Eumetazoa and partly because the planula has the simplest structure, i.e. it corresponds to Haeckel's gastraea. It consists of two layers only and it has neither an intestine nor an oral opening. The next form which follows the planula is the famous trochophore. This development frequently takes place by way of an intermediate form of protrochophora.

Because of its simplicity the planula has given rise to conjectures regarding the primitive form of all Metazoa. The planula has generally been considered as a very primitive 
form even if it does not have a feeding or a digestive system and even if it lives for a while on the reserve food it had obtained from its maternal organism. The structure, however, of this planula is not quite so simple even if it does not yet possess organs; it is well differentiated both histologically and cytologically. The tissue that can be observed in its interior does not correspond to an anlage of an intestine, to an entoderm; it is rather an "entomesoderm."

The planula, however, is a pure larva and by no means corresponds to a primitive form of the ancestors of Cnidaria; it is impossible to imagine the existence of a freely living Eumetazoa without an active digestive apparatus. As is the case with other animals that live on the floor of the sea, and particularly those that lead a sessile way of life, so it was with Cnidaria when they developed their specific larva which lived in the sea plankton and which was characteristic of them. We can speak here of a metamorphosis in Cnidaria even if it is not a very radical one. In numerous Cnidaria, with the exception of the Scyphozoa, the next higher ontogenetic stage, which is called the actinula, develops from the planula during its free life in the plankton. This can be explained as a case of paedogenesis and here we can see a formerly older phase, which had developed on the floor of the sea, transferred into the larval period. It is my belief that it would be possible to find early stages in the ontogenies of animal groups that stand higher in the animal system, where the same stages would resemble that of the planula in Cnidaria. It would only be necessary to make the effort, though this seems hardly worth while. It is perfectly clear that it would be wrong to make any conclusions about relationships from this. Neither would it be sensible to speak here about some modified planulae.

The same is true for the trochophore, the next higher stage in the ontogenetic development. It is by no means difficult to show that all those stages that have been called trochopphores, are neither identical nor homologous. A modified 
trochophore can occur only within the limits of an animal group which is uniform both phyletically as well as from the point of view of the comparative morphology. Yet in this case it would be senseless to speak about a modified trochophore. In my opinion we should dispense with special new names for each different larval form and drop those that have been already accepted. These names were given in former days when specimens were found swimming in the sea plankton; the puzzled scholars did not know where these animals belonged and since each child must have its own name, names were given to these specimens as if they were independent organisms. It was later found that these specimens were really larvae of some known or even unknown species. The names, however, have been preserved together with their suggestive influence, particularly in those cases where there is some similarity between several larval forms which have for this reason previously received a common name.

As long as the name trochophore continues to be used it should be limited to the planktonic larvae of Annelida only. Larvae of animal groups that stand either below or above Annelida in the animal system should not be called trochophores even in cases where they resemble the latter; these similarities are due first to analogous ways of life which lead to convergences, and, secondly, to an approximately equally low stage of organization (the unsegmented stage). Even the orientation of animals, e.g. the poised position in free water with its longitudinal axis in the direction of the earth's centre of gravity can lead to a general similarity in the habit of these larvae.

Certainly there won't be many objections if we do not call the planktonic larvae of aproctous animals (Ameria) trochophores, even if they look somewhat similar to the trochophore of Polygordius which is considered as the most typical form of all trochophores. These larvae are not numerous. Boas in his excellent Lebrbuch der Zoologie which was widely used in his day, had already stated for good reasons that trochophores 
were convergently developed pelagic larval forms, i.e. they developed independently from each other in completely different animal groups, beginning with Turbellaria and continuing up to the very last Oligomeria, or close to the very threshold of Chordonia.

Unfortunately, there have been numerous zoologists not only in former days (e.g. Hatschek, Cori) but even, now (e.g. Carter, Marcus) who have been induced to attribute great phyletic significance to these larval forms. This led finally to an unnecessary increase in the number of proposed animal systems. One example should suffice to show how this exaggerated appreciations of similarities between various larvae-in this case between trochophores - led to wrong conclusions. We think here of Mollusca. The veliger larva of Mollusca has been rather generally considered as a kind of trochophore. Because of an external similarity, yet frequently not for this reason only, it has been thought that there is a close relationship between Mollusca and Annelida. According to this interpretation the primitive Mollusca have been believed to have developed from primitive Annelida by way of an omission of their metamerism. In the not very distant past, earnest attempts have been made to find in the ontogeny of Mollusca, in their veliger larva, traces of a former metamerism with the cavity of the former perigastrocoel (eucoelom, as it is called by American authors). It has been thought that these have been found, in the Mollusca, in much the same way that they have been described for the Echiuroidea. The Mollusca, however, are genuine and primary Ameria, i.e. they are from their very beginning unsegmented Eumetazoa, and it can be proved that in their phyletic past they have never been segmented animals. They represent the climax in the progressive development within the phylum of Ameria. They are therefore not directly related to Annelida, either in the sense that we see in Annelida a development from the primitive Mollusca, or, vice versa as a result of a partly regressive development due to a secondary omission of the segmentation. 
I am fully convinced that this, my interpretation, is right in spite of a recent discovery of Neopilina which has been found by Lemche (1958) to be a segmented animal with five body segments. As in the case of several other Ameria we can observe a partial polymerization in a certain number of Mollusca, and therefore not only in Neopilina and in the related fossil species. It occurs, however, in Neopilina to a stronger and fuller degree than, for example in Tetrabranchia among the Cephalopoda.

As far as is known, no pelagic larvae have been developed by the acoelous Turbellaria in spite of the fact that they live (with a few exceptions in planktonic species) on the sea bottom. Unfortunately, our present knowledge of the ontogeny of Acoela is still very unsatisfactory. Surprisingly enough, we do not find pelagic larvae in Turbellaria earlier than in Polycladida (e.g. Müller's larva). It is aproctous like the grown-up Turbellaria-it has no anal orifice-and for this reason it cannot be compared to a trochophore but rather to a protrochophore, unless as it has even been thought, we consider the aproctous condition to be secondary. This larva has developed its own swimming apparatus in the form of an irregular circle which consists of eight lobular excrescences that are rimmed with cilia. This larva is poised "vertically" in water. It develops gradually and without a real metamorphosis into a polyclad that lives on the sea bottom. It is not my intention to discuss here any other larval groups that live in plankton; the only form I would like to mention is the so-called pilidium of Nemertinea. In spite of the fact that Nemertinea stand close to Turbellaria, they have nevertheless become euproctous; there is a very great difference between the pelagic larvae of the two groups which can be observed not only in their external forms. The Pilidium is aproctous-in agreement with its ontogenetic stage-and it has a completely different type of development to the Müller's larva. On the basis of these facts we can come to the conclusion that the two larvae developed independently from each other, and, furthermore, that they 
are no recapitulation of some grown-up ancestors. In the pilidium the first genuine metamorphosis can be observed: in this "catastrophic" metamorphosis the worm develops out of special and quite separate Anlagen and in an entirely different orientation so that finally a considerable part of the body is destroyed, (see Fig. 6).

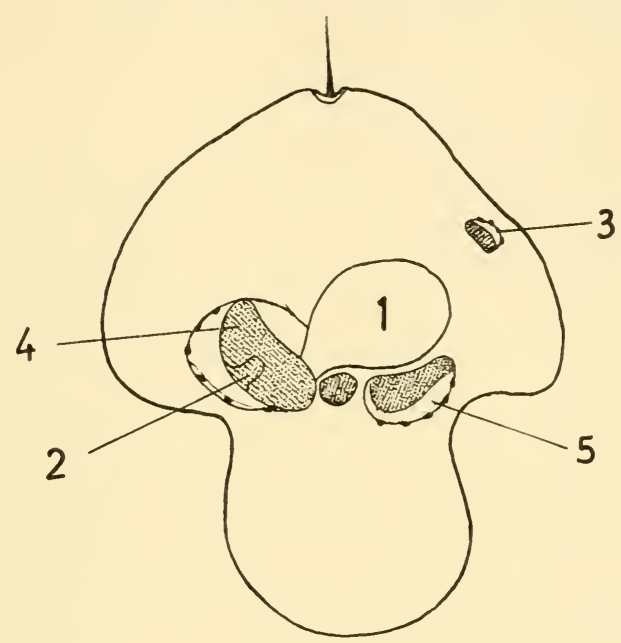

Fig. 6. Nemertinan planktonic larva, called the pilidium; (1), the gut, (2), primordium (Anlage) of the proboscis; (3-5), primordia of the "worm-body".

I repeat that comparative morphology which tries to find homologies and evaluate relationship connections on the basis of such homologies can with full right make use of ontogenetic morphogeny and thus the larval stages. Yet, first, it must not seek in these some recapitulation of the morphogeny of their grown-up ancestors, and, secondly, it should not lay too great an emphasis on the ontogenetic facts and on the larval stages. It should not base the whole animal classification on the larval stages or even on the type of cleavage. The main emphasis should be on comparisons of grown-up forms both of the fossil as well as of recent animal species. 
I will not enter here into the discussion of phylembryogenetic problems which have been already touched on. However, since we are speaking about larvae, I wish to mention two other extreme cases of such changes because they are important from a methodological point of view. In one case which I have already mentioned, the larval phase is simply omitted; it has been cancelled. It seems that this takes place comparatively rapidly.

The best known condition under which the larval phase has become lost is that of the transition from sea to fresh water (e.g. in Hydra, numerous Crustacea, Mollusca). To this should also be added perhaps migration into great depths, and life in the far northern regions with their cold waters (a transition to the nursing of the progeny taking place). Not infrequently, however, traces of a former larval stage can in these cases be observed in the development of the embryo.

The opposite case is represented on one hand by the phenomenon of neoteny and on the other by the complete prevalence of the larval phase. The essential difference between these two cases can be seen in the fact that in the former the adult stage is simply omitted, the development of gonads and maturation are transferred to the larval stage. Dissogeny represents a transition to this stage; this is the double sexual maturation which takes place once during the grown-up stage and once in larva (it has been observed in Ctenophora). This change, too, is connected with a change of the environment, usually in the way that the animal remains in the plankton. Something similar to neoteny can be found in animals with metagenesis (the alternation of generations) when either one or the other generation is preserved as this can be frequantly observed in Cnidaria; here the sexual function which had passed over from the polypoid to the newly formed medusoid generation is later transferred back to the polypoid generation; in case, however, of an omission of the polypoid generation, the sexual function remains with the medusoid generation which is then the only generation preserved (the so-called hypogenetic species). In these 
cases, too, the changes are connected with a change of the environment.

In the other extreme case, the main role in the life cycle is taken over by the larval phase. The animal feeds during this phase only. It lives in the larval environment for most of its life. The imago stage is limited to a very short period of time; it serves mainly the purpose of propagation and it's digestive organs are frequently lost. Such cases are best known to occur among insects.

In our study of phylogeny and in our attempt to reconstruct the phylogenetic development, we frequently meet with the problem of whether a simple structure can be taken as a sign of primitivism. This leads us to the important problem of the progressivity of evolution. In a not too distant past it could be frequently seen, and sometimes this occurs even now, that a simple structure is taken simply as something primary, primitive. Completely wrong constructions have been made because of this mistake. It will be seen later that it was especially so in the interpretation of the evolution of Cnidaria. It must be confessed that it is not always very easy to differentiate between a primary primitive and a secondary simplicity of a structure. Here one has to proceed very carefully; one must make investigations by means of intensive and extensive comparisons, taking particularly into consideration the way of life and its changes. One must especially have a clear understanding of general conditions. Here it should suffice to state that there certainly are both primary as well as secondary simple phenomena, as has been proved by verified facts. This is due to the fact that the evolution does not always and exclusively show a progressive tendency; its tendency can also be in the opposite direction, it can be regressive, and this is quite often the case. This regressive development (which is usually and unfortunately called a degeneration) does not take place to a minor extent only, i.e. in the lower categories of the animal system. In my opinion, it has to be reckoned with even in the so-called macro-evolution, or in the evolution of the 
highest categories which are characterized by phyla. The whole phylum of Oligomeria with its numerous subtypes (situated between Polymeria and Chordonia) is in my opinion a product of a generally regressive development. In all probability such a type of development has occured in the Cnidaria, as is also the case of other animal types that have adopted a sessile way of life.

We speak here of circumstances which usually accompany regressive development, or actually cause it. In a sessile way of life Natural Selection favours the minus mutanta which gradually reduce and eliminate all those organs and parts of the structure that are causally or functionally connected with the free mobility of the mobile or swimming animal types.

Besides the sessile way of life in which the animal becomes immobile, there is also the parasitic way of life which regularly leads to a regressive development, particularly in the case of internal parasites. It is well known that due to parasitism, characteristic form can be reduced so much that it is past recognition. It should be mentioned that it is often the larval stage, so far as this is preserved, which enables zoologists in cases of a well advanced regression ("degeneration") to determine the history of a species and thus the membership in a certain animal group.

All this is well known and nothing new has really been said here. Nevertheless, it has been necessary to discuss this point because it was in connection with Coelenterata, and also with Cnidaria, that many mistakes have been made. The rule-it is better not to speak about laws in biology-according to which a constantly sessile way of life leads to a simplification of the body structure has been believed to be true in connection with other anımal groups; these are by no means few; yet it has not been accepted in connection with Coelenterata, i.e. Cnidaria. Later it will be seen that, due to historical reasons, particularly to our deficient knowledge of the fine structure of Cnidaria, the Cnidaria have been believed to be primarily simple animals which are just on the border between animals 
and plants (Zoophyta). The conservatism and the authority of prominent scholars, both basically useful to the science, can become detrimental to it and an obstacle to the progress when they exceed all bounds. This has been the case with Cnidaria. The freshwater Hydra has for a long time been considered to be the most primitive of all Eumetazoa, and consequently also of Cnidaria. Even at the present time, Hydra is often described as a primitive simply built animal, a description which is made for didactic purposes if for no other reason. Finally, when the idea has been accepted that the solitary character of Hydra is a secondary phenomenon, and that Hydra is without its medusoid generation, progress has stopped here and the scholars did not try to draw the necessary consequences. This situation has continued even after it had been proved by palaeozoologists that the Anthozoa actually have an internal bilaterally symmetric structure.

\section{The Significance of the Sessile Way of Life}

In our study of phylogenetic problems we must not be satisfied with an exclusive use of comparative morphology even in those cases where a rich fossil material has been available. We shall completely understand and comprehend a species of animals-and this is true also for a systematic group of animals-when we also know their way of life, and the environment in which they live. Even if this is an old and generally accepted truth one can never emphasize enough its great, and decisive, importance. In this connection mistakes have again been made, with regard to Cnidaria, which persist down to the present day. It is therefore necessary to discuss this problem at some length. All Cnidaria live in water, and allwith a few exceptions-live in the sea; we shall therefore limit ourselves to the study of conditions that prevail in this largest and oldest of all environments in which the life of organisms, and thus also of animals, has been developed. 
The first and the most important problem we meet here is which of the two opposite large biotopes of the sea is primary; benthos (the bottom of the sea), or the pelagic zone (the zone of the free water)?

It can soon be found if we make a physicochemical comparison of the sea properties, that it was in all probability the sea bottom where the first life came into being. This was the only place where life was initially able to develop further. It must have been in the shallow water that sunrays could penetrate down to the sea bottom and where the early life of animals could develop and be sustained on algae and on bacteria. Individual types of animals could later get used, by way of special adjustments, to the life on a deeper sea bottom, or they could begin to inhabit as plankton the free water area. It is very probable that the life on the deeper sea bottom of the abyssal zone developed much later than that in the plankton. In the plankton, life reached an exuberant growth and sank as an organic rain to the unproductive sea bottom.

Purely inductive thinking alone allows us to suppose that in free water the animal life began to develop in a similar way. First it was necessary that phytoplankton appeared which synthesized the organic substances and served as a basic food for animal consumers. The substance of a living organism has a greater specific gravity than the sea water (in the remote past this difference was probably even greater than it is now); organisms had therefore to depend on the sea bottom until finally individual small algae-it was easier for these to do sosucceeded in developing mechanisms which helped them to overcome the effects of gravity, i.e. to lower their specific gravity, as was done, for example, by undulipodia (flagella, drops of oil, gelatinous coverings lighter than sea water, excrescences and similar forms of the body that increased the friction between the body and water).

It is certainly not a mere chance that we find the phytoplankton of the sea to be composed exclusively of monocellular small algae and that no higher type of plants had been able 
to develop in the sea plankton. We can safely maintain that this was so because, rebus sic stantibus, it could simply not begin to develop here. It was only in a calm and shallow fresh water where Volvox, and Anthophyta like Lemna, could begin to develop, either in free water or on its surface, and without a contact with the bottom.

Protozoa were in all probability the first animals that began to live in the water of the free sea and continued to exist in it. They evolved as Zooflagellata out of Phytoflagellata and further continued their evolution into the planktonic Protozoa, i.e. the rhizopodan Radiolaria. It is a priori improbable that Metazoa developed out of planktonic Protozoa either such as had formed colonies or those that consisted of several nuclei (polyenergida). The reason is the same which prevented metaphytes from developing in the sea plankton.

The first Metazoa that inhabited the free water of the sea were certainly the freely developing ontogenetic stages of animals that lived on the sea bottom, first, because they had evolved out of eggs that had been left freely swimming in water, secondly, because they were small and light with a comparatively large surface, and thirdly, because they were covered with flagella and cilia which they could use, even if to a smaller degree, as a means of locomotion. Slight vertical streams were sufficient to bring these future larvae into the upper strata of water where they could find a rich food and other conditions of life. This is the way we have to explain the origin of planktonic larvae. It was only later that these larvae were followed by grown-up animal species into these strata of free water, and these again had to make special adaptations to new conditions. Or some larvae which belonged to animals that lived on the sea bottom, remained constantly in the plankton so that they changed by way of neoteny into planktonic animals. The feeding of the first metazoic planktonic animals must have been microphagous; it was only later when animal types developed, that macrophages began to feed on the former (Cephalopoda, Vertebrate). 
It is conspicuous that no new animal types have developed in the plankton of the free water zone of the sea. All the major types of animals have developed on the sea bottom. Neither have any of the main types (phyla) developed by way of neoteny.

For methodological reasons and because of its special connection with Cnidaria we must deal here with one more problem. We must differentiate between the two types of relationship that exist between the stage that lives on the sea bottom and the stage that lives in the plankton. On one hand we have the transition which takes place within the frame of one and the same generation. First we have to imagine a case in which the phase of the grown-up form which lives on the sea bottom is primary, and the planktonic phase of a juvenile, usually larval, stage secondary. In another case the form which lives on the sea bottom is primary; naturally, it is able to propagate sexually and it can eventually develop its own juvenile planktonic form; and there is a secondary generation which had developed by way of gemmation or fission - in an asexual waybefore the sexual propagation has been adopted by the phase which lives on the sea bottom. The generation which had been created by way of an asexual reproduction evolves into plankton and adopts sexual reproduction while it becomes adjusted to life in the free water. This is the classical case of the alternation of generations, or metagenesis, where an originally single phase which was for some time also the main phase becomes, so to speak, degraded and it continues to live a vegetative life only.

The subsequent development can pursue two or three different ways. Either we find the originally primary generation withering away (this is the generation which lives on the sea bottom, in our case the generation of polyps), so that the secondary, planktonic, form remains as the only one that has survived (in our case the form of medusae); or, vice versa we find that the secondary planktonic form and generation, i.e. that of medusae, is withering away, while the primary, kenthonic generation, i.e. the generation of polyps, 
is really the only form which survives. Thus we can return by way of an indirect course to the initial situation. Finally, the generation of medusae as the only form that had survived, can develop into an animal that resembles polyps and lives as such on the sea bottom (Armobydra see Fig. 7, Halammobydra see Fig. 8, Otobydra see Fig. I8.)
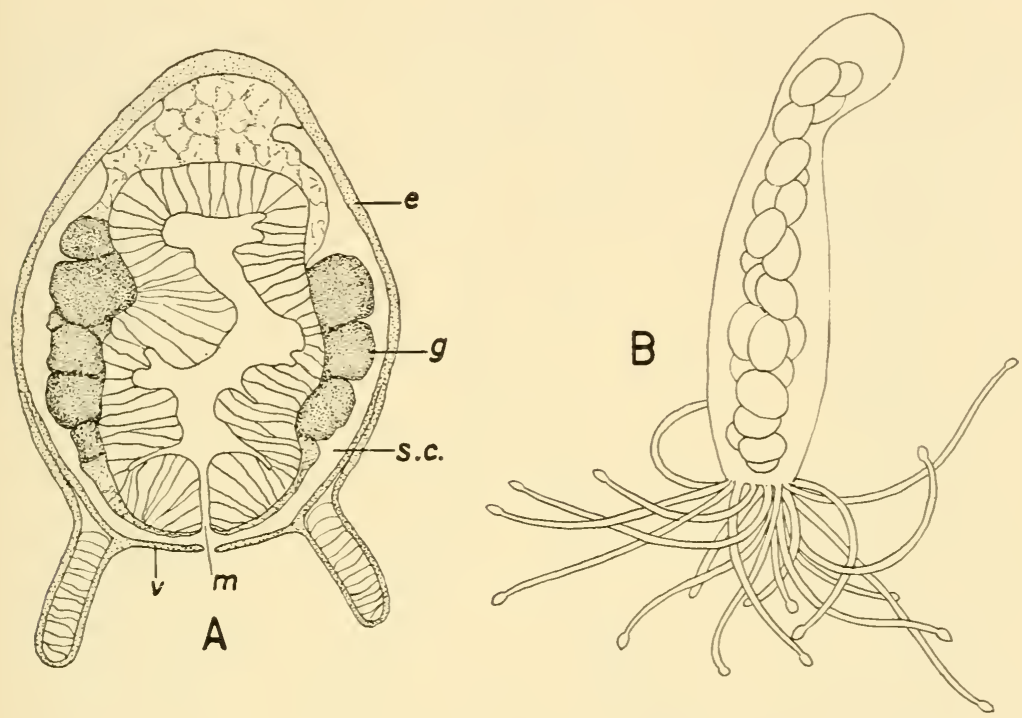

Fig. 7. A reduced trachyline medusa Armobydra janowiczi Swedmark \& Tesnier; A, longitudinal section; B, habit; g, gonad; e, exumbrella; m, mouth; sc, subumbrellar cavity; v, velum; (after Swedmark and Tesnier).

In connection with all these developments the question necessarily arises how can we in a given case differentiate and correctly interpret the path evolution had taken? A broader and deeper comparison can help us here while we take into consideration on one hand the ecologic conditions and on the other Dollo's rule of the irreversibility of evolution. Many mistakes have been made in this connection. Many zoologists, among them even specialists in Cnidaria, are inclined to see in the planktonic form of medusae the original, 
the primary, or the main form out of which the polyps can be considered to have evolved. On the other hand, the solitary forms of the Hydrozoa polyps, and of polyps that have no generation of medusae have also been believed to be the primitive forms. Both these interpretations are equally wrong; both of them are based on pre-suppositions that are methodologically wrong.

One more point can be of good service in our phylogenetic studies. This is the relationship between locomotion on one hand and the environment and the kind of feeding on the other. As is well known, three stages in the evolution of the active forms of locomotion must be taken into consideration (cf. the study by L. Zenkewitsch). We disregard two forms that occur in Protozoa: the amoeboid movement and the movement effected by the individual flagella (it is true that in Spongiae the amoeboid type of movement does occur, even if to a small degree only; cf. Hadži, 1917). In the small and primitive groups of Ameria the type of movement corresponds mainly to conditions that can be found among Protozoa, in the Infusoria; this is movement by means of cilia and this type of movement is preserved in planktonic larvae, so to say, right through one classificatory system. The ciliary pulsation produced by animals while they are exposed to the rhythmical swell of waves enables them not only to glide over a firm ground, which is true for the majority of Turbellaria, but also to swim in free water, as can be observed in Infusoria and Rotatoria. The formation of undulipodia (flagella and cilia) by individual cells belongs to a general inventory of all animals. There have been a few specialized animals however, e.g. Nematoda among Ameria and insects among Polymeria, which have completely dispensed with the action of undulipodia because the surface of these animals had been strongly cuticularized.

Undulipodia which cover the free upper surface do not serve the purpose of locomotion only; they can also be transplaced, together with their maternal cells, into the interior of the 
animal by way of the invagination of the surface stratum of the body. This is how we have to understand the origin of nephridia which serve as excretory and emunctory organs. Another point to be mentioned here is that Dollo's rule does not seem to be valid in connection with the formation of cilia, i.e. even after they had been lost during the phyletic development of
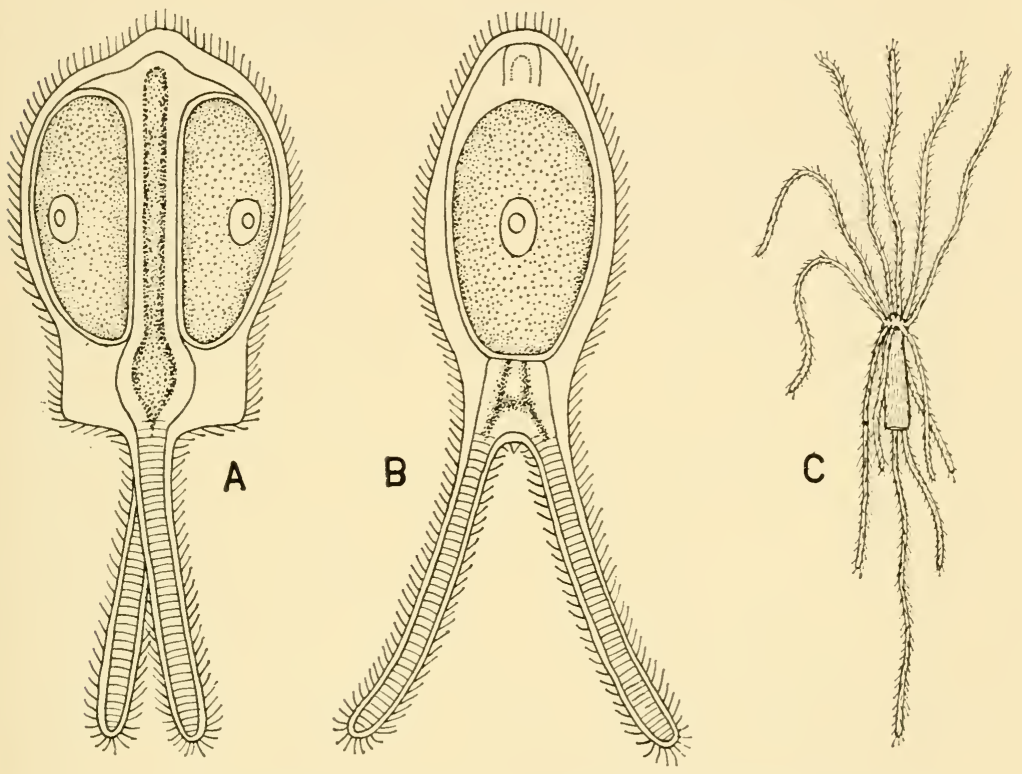

Fig. 8. Secondarily ciliated Hydrozoa; $\mathrm{A}-\mathrm{B}$, sporosac of Dicoryne conferta Alder (after Allman); C, Halammobydra octopodides Remane; (after Remane).

an animal they can be later reintroduced. This can be proved by some clear instances that can be observed among Hydrozoa. It has been known since Allman's days that in the species Dicoryne conferta Allman, which belongs to Hydroidea (Athecata), completely retrograded medusoids which lived otherwise as completely immobile forms as its cormus, regain their ability to swim again by means of a secondary development of cilia (the so-called sporosacs, seeFig. 8 A-B). Halammobydra, a Hydrozoon which has been recently discovered by A. Remane (1937), 
and which lives in the sea sand, has a polyp form; its surface is covered with cilia which enable the animal to glide actively and move in this way among small grains of sand (see Fig. 8C). I have succeeded in showing (Hadži 1959) that Halammobydra actually corresponds to a strongly modified Narcomedusa whose muscular swimming apparatus has been much reduced while at the same time secondary cilia have developed. It is certain that something similar could also be found in other animal groups.

The second type of locomotion is based on contractions of muscular fibres which had developed within the cellular cytoplasm. This specialized form of a general ability of the cytoplasm to contract has already been "invented" by Protozoa. Metazoa have inherited it fully developed from their protozoan ancestors. These contractile fibres served first to make possible movement on a fixed spot, bowing, the contraction of stem-shaped forms, the constriction of cavities, e.g. of the peristomial ciliary apparatus in the peritrichous and epitrichial Infusoria (also in sphincters), etc. As a secondary result and parallel to an increase of the size of the body, locomotion by means of cilia is given up, and the muscular movements of the body begin to be used to effect locomotion. This can be observed as early as in the larger flat Turbellaria, particularly in Polycladida. The highest level in the development of the means of locomotion in free water is reached in medusae which possess special transverse stripes in their muscular fibres: in these, movement is effected by means of regular rhythmic contractions which are controlled and led by nerves and which remind us already of the pulsation of the heart (the latter is first developed in Mollusca).

A higher type of locomotion by means of contraction of muscles has been developed with the formation of extremities. Here we are less interested in this type because it has not been developed in Ameria.

The type of locomotion used by an animal is very important and the symmetrical conditions in animals depend on it to 
a large extent. We meet with this problem immediately when we compare the first opposing pair: the mobility in contact with the firm ground (vagility), and the immobility, the permanent attachment to the ground, and a stronger contractility (sessility). Whenever we see that the free mobility has been abandoned, a phenomenon which can be observed quite often in Protozoa as well as in Metazoa, we can regularly observe how a simultaneous change of the carriage of the animal takes place. The consequences of this change are great. A characteristic property of animals which move or glide over a firm surface is the bilateral symmetry of their bodies. The animal moves forward with its anterior end placed in the direction of the movement; this is the way the head region begins to develop and with it, the great contrast between the anterior and posterior ends of the body. The contacts of the two ends with the environment are widely different. An almost equally great difference can be observed in the contacts of the dorsal and ventral sides and their environments: the flattened ventral side touches the ground over which the animal moves while the slightly vaulted dorsal side has contact with free water only. The contacts of the two lateral sides (the left and the right sides) are basically identical and this is why these two sides are similar. In this way the bilateral organization and form, and thus the bilateral symmetry, have been evolved.

Bilateral symmetry exists in the whole animal world and it must be considered as primitive All the other types of symmetry can be deduced from the bilateral symmetry. The changes in this symmetry are always due to the changes in the relationship between the type of locomotion and the environment; they can all be explained as modifications of the bilateral symmetry.

The most frequent change, which occurs as a more or less distinct disturbance, is that of asymmetry. It is rather rare that we find the assymetry to be conspicuous and characteristic for larger groups of animals. It occurs, for example, in Infusoria when they swim freely but not far from the sea 
bottom by means of beating of cilia, and among Gastropoda where the origin of their asymmetry is a special problem which cannot be discussed here at any length. In certain groups of fishes the asymmetry is due to their lying constantly on one side of their body, similarly as in the case of Branchiostoma (Amphioxus).

Most frequently, however, we see that a secondary radial symmetry develops out of the primary bilateral symmetry. Always, and without an exception, this change stands in causal relationship to the transition to a secondary, sessile, way of life, i.e. to the fact that the active locomotion had been given up. These changes of symmetrical conditions occur in varying degrees of intensity in all the large groups of the animal world, beginning with Flagellata among Protozoa and continuing up to Chordonia. Radial symmetry can occur even without a trace of the former bilateral symmetry or of the asymmetry; the degree of evolution of this radial symmetry depends on the final structural conditions, the way of life, the type of feeding, as well as on the phyletic age of the transition to a semisessile or finally to a fully sedentary way of life. This radial symmetry can be so completely without a trace of the former bilateral symmetry that it was believed, especially by older zoologists (and unfortunately it still is accepted by some modern scholars, ) that this radial symmetry is an original property of Metazoa. We cannot blame the idealistically minded pre-Darwinian morphologists that they put together Coelenterata (actually Cnidaria) and Echinodermata, i.e. two animal groups with the most highly developed radial symmetry, and created for them the taxonomic unit of Radiata. Even in the postDarwinian days there were zoologists-and there are some even now-who, following Cuvier, try to preserve this artificial unit. As a matter of fact, however, Cnidaria are in no way whatever phyletically related to Echinodermata.

As a preliminary stage in the development towards the radial symmetry we can observe first a change in the general carriage of the animal and in connection with this, the disappearance 
of the difference between the dorsal and ventral sides of its body. As a result of this, the form of the transverse section of the animal becomes circular. In some animal groups, whose body is otherwise completely bilaterally symmetrical, the inclination to accept the radial symmetry can be developed in one part of its body only, e.g. in its anterior part. This depends on how much these organs are used by the animal to stick to the ground or to adhere to it by means of suction. Clear examples of such a development can be seen in Cestoda (scolex) and in Nematoda (the coroniform distribution of various papillae and of sensory organs of the skin) (see Fig. 9.). A complete radial symmetry can only be reached in the aproctous types of animals, e.g. in Cnidaria, or in such euproctous animals where the absence of the anal orifice is a secondary phenomenon in their development (some Echinodermata, especially among the Ophiuroidea). In Infusoria, a complete radial symmetry can rarely, if ever, be observed, even if in these the anal orifice has frequently not developed. This is due to their strong inclination to adopt an asymmetrical structure; the latter has also been inherited in their digestive apparatus by animals that live a sessile way of life (epitrichial Infusoria).

The combination of a primary bilateral symmetry and of a secondary radial symmetry can lead to a bisymmetry. This can be observed in the interior of numerous anthopolyps and of other animals, and it is best and most purely developed in Ctenophora. In my opinion, the origin of this bisymmetry can be explained rationally in one way only if we consider the bilaterally symmetric form as primary, and we presuppose that there had been a development caused by the loss of contact with the firm ground which made the ventral and dorsal sides similar. The two lateral sides preserve their original similarity with each other, yet they are more rounded off and therefore better developed. Moreover, they still continue to be different from the ventral and dorsal sides, though the latter have become similar with each other. In Ctenophora this change of symmetrical conditions occurs in connection with their 

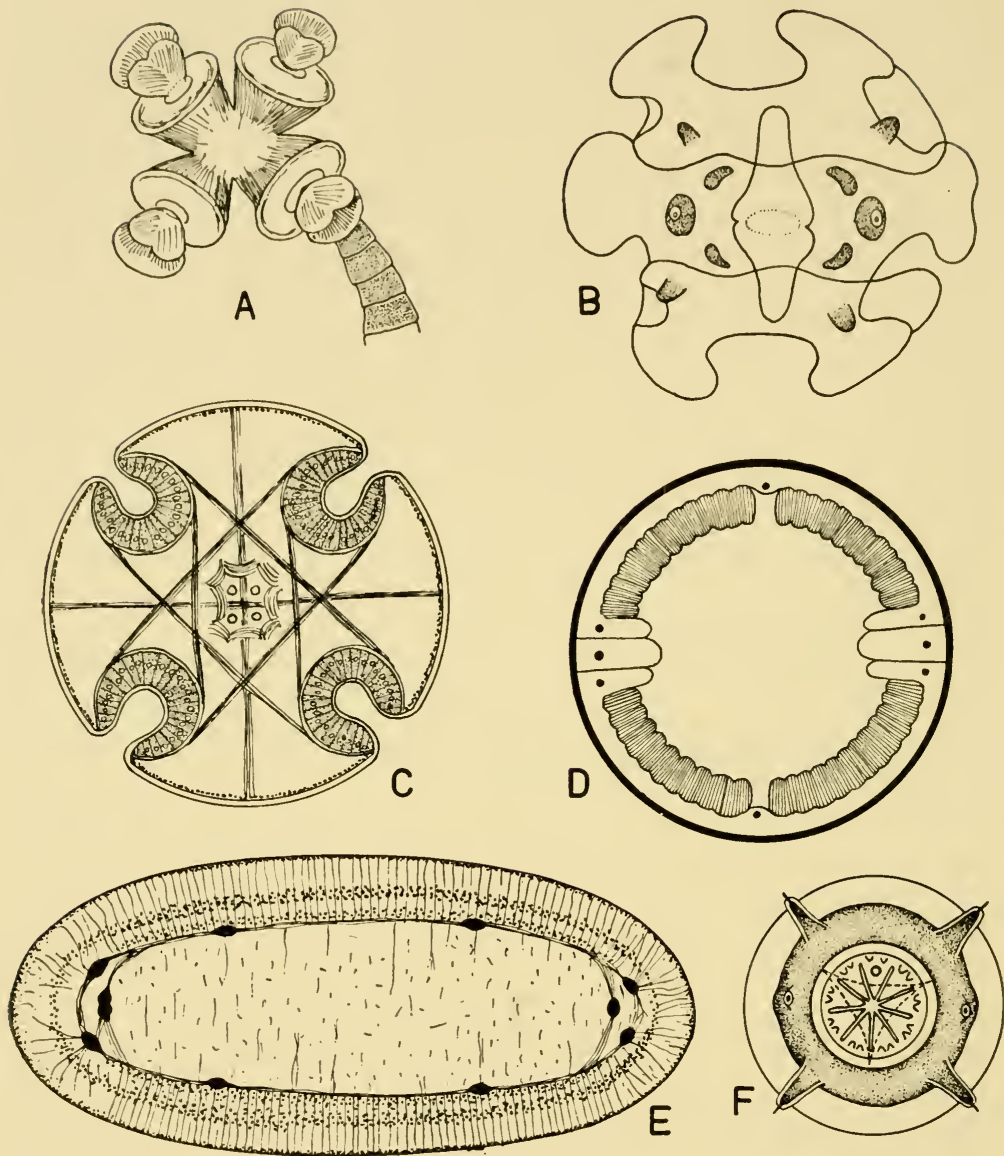

Fig. 9. Some cases of transition from bilateral to radial symmetry of entoparasitic "worms" but only on the "head"; A, scolex of Carpobothrium sp., (after Shipley and Hornell); B, anterior view of the nematode Parabronema sp., (after Baylis); C, cross-section through the scolex of Nematobothrium sp., (after Fuhrmann); D, crosssection of a polymerian nematode (after Filipjev); E, cross-section of a proglottis of Anoplacephala sp. (after Becker); F, anterior view of the nematode Oesophagostomum sp., (after Goodey). 
transition from a life in benthos to a life in the pelagic zone, i.e. to the zone of the free water (see Fig. 10).

In this connection we are able to ascertain a very welcome regularity; it enables us to find out the way along which the change takes place. The rule is that this change occurs first in the peripheral parts of the body of the animal, and only later in its internal organs. The eight "antimeres" or ribs that

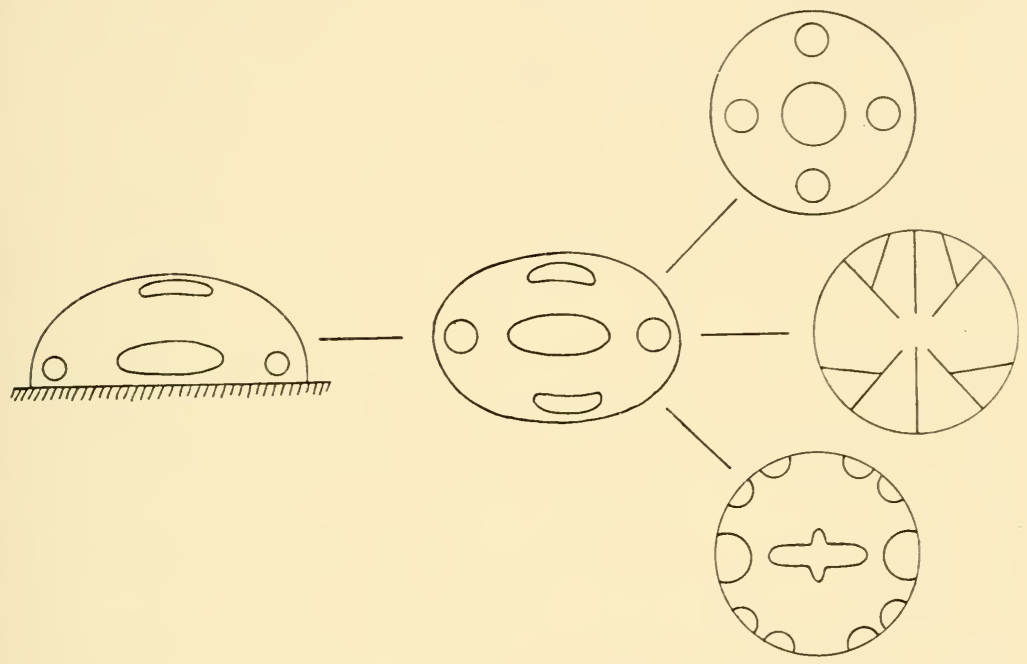

Fig. 10. Scheme illustrating the transition of primarily bilateral symmetry to other symmetries as consequence of a changed way of life.

can be found in an otherwise swivel-round body of Ctenophora show a nearly perfect radially symmetrical distribution, and yet at the same time some well-preserved traces of a former bilateral symmetry can be observed in the central parts of their digestive organs which are frequently called the gastrovascular system (see Fig. 3.). Similar conditions exist in numerous anthozoan polyps as was first noticed by palaeozoologists.

In our study of Ctenophora, we meet with the problem how life in the free zone, i.e. in the pelagic zone, influenced the 
general form of the body of animals. If we review all the planktonic animals the impression we finally get is that there has been no general regularities in respect. In these animals all possible symmetrical conditions can be found exactly as in the animals that live on the ground. And still there are differences which are due, as we shall see later, to the fact that in free water there is no contact between the firm ground and the animal, i.e. its ventral side. In the Ctenophora we can observe how life in free water promotes the development of radial symmetry; this is due to the similarity of contacts between the milieu and all parts of the animal body. If there is, however, an eventual change of symmetrical conditions, then this change is largely due to the type of movement. In types of animals that move only slowly and mainly by means of cilia or organs which have developed out of these (e.g. the swimming plates in Ctenophora, the circlets of cilia in trochophores), and in types that hang vertically in water, the inclination to adopt the radial symmetry must always be expected, and, as a matter of fact, it will be found in them. Bilateral symmetry, however, remains preserved a long time after the transition from a life in the benthos to a life in the pelagic zone had taken place, if the movement of the animal is more energetic and stronger and if it is effected by means of muscles and particularly by means of limbs. This can be best observed in the case of Chaetognatha. I derive them, in agreement with some other zoologists, from the planktonic larvae of Brachiopoda. These brachiopod larvae hang in water or they move slowly by means of a pulsation of cilia. They are basically bilaterally symmetric animals but they show a clear inclination to adopt radial symmetry. In Chaetognatha, however, which are predatory animals with strong contractions of muscles and with an ability to move rapidly head first, bilateral symmetry had been developed in spite of their life in free water. Radiolaria, an extremely old protozoan group which for a long time has been living exclusively in the pelagic zone or in plankton, contain all possible forms among 
their very numerous species that live in various depths of the sea, yet they never have a typical bilaterally symmetric form with a difference between ventral and dorsal sides. The impression we get in the face of these immense riches of forms of Radiolaria is that the life in free water is indifferent to the symmetric conditions of basic forms and that it therefore "allows" all kind of forms.

In Cnidaria, as animals that primarily live on the sea bottom, the radial symmetry has already been developed because of their sessile way of life; after their transition, as medusae, to a life in plankton, this radial symmetry becomes consideratly intensified. A more or less expressed bisymmetry can be occasionally developed due to a reduction of their tentacles, and in some cases even a state of asymmetry can be approached if only one marginal tentacle has been preserved. The bilaterally symmetric and bisymmetric medusae can be found as medusoids in the cormi of Siphonophora only; it is only here that a polymorphism of medusoids can be observed. The individual swimming medusae have never developed bilateral symmetry in spite of the fact that they are excellent swimmers.

The example of Echinodermata shows how long the old symmetric conditions can be preserved during life in plankton. Echinodermata are considered to have evolved out of some bilaterally symmetrical ancestors that had crept over the surface of the sea bottom. Because of a later adoption of a sessile way of life, these Echinodermata have subsequently developed more or less radially symmetric forms. Their larvae, however, are purely bilaterally symmetric animals in spite of the fact that they still continue to go into free water and that they move slowly and only by means of cilia. This shows that here we have a kind of conservatism; similar to the case of starfishes which have adopted a secondary vagile way of life while at the same time they still preserve an obvious radial symmetry because of the slowness of their movements. On the other hand, sea urchins which burrow in the soft sea bottom have adopted a secondary bilateral symmetry. 


\section{The Role of the Polymerization and of the Subsequent Oligomerization During the Phylogeny}

A very important and fruitful criterion for the evaluation of phylogenetic problems has been recently discussed at great length by the well-known Russian scholar, the now unfortunately deceased zoologist V. Dogiel. This is the phenomenon of the polymerization of smaller and also of larger morphologic units in the body of the animal. The subsequent oligomerization is regularly accompanied by differentiation (V. Dogiel, 1954). We must imagine it as a doubling and as a further multiplication of units which takes place in the complex of genes and which is later, during the development of an individual, materialized in the corresponding morphologic "parts". By way of this process of polymerization we can explain the development of monoflagellate and mononuclear Flagellata into Poly- and Hypermastigida as well as into the polynuclear species, a development which has finally led also to the formation of Ciliata. Various ciliary organelles of a complex nature have evolved by way of a specialization of cilia from this polymerization; the polymerized nuclei have become differentiated into macro and micronuclei by way of oligomerization. The ribs of Ctenophora have been developed by way of oligomerization and differentiation. The single pair of tentacles as it occurs in Turbellaria has developed into circlets which frequently consist of multiplied tentacles as in the Cnidaria. A subsequent oligomerization led in some cases observed in Hydrozoa, to a reduction of tentacles to the number of two, or even to their complete disappearance. The polymerization followed by oligomerization and differentiation finally led to the formation of various forms of tentacles in one and the same individual (e.g. in polyps of Eleutheria and of medusae see Fig. 11 and 12).

Naturally, we must be careful to differentiate, when we take into consideration this regularity, between a primary simplicity and a secondary simplicity which has developed by way 

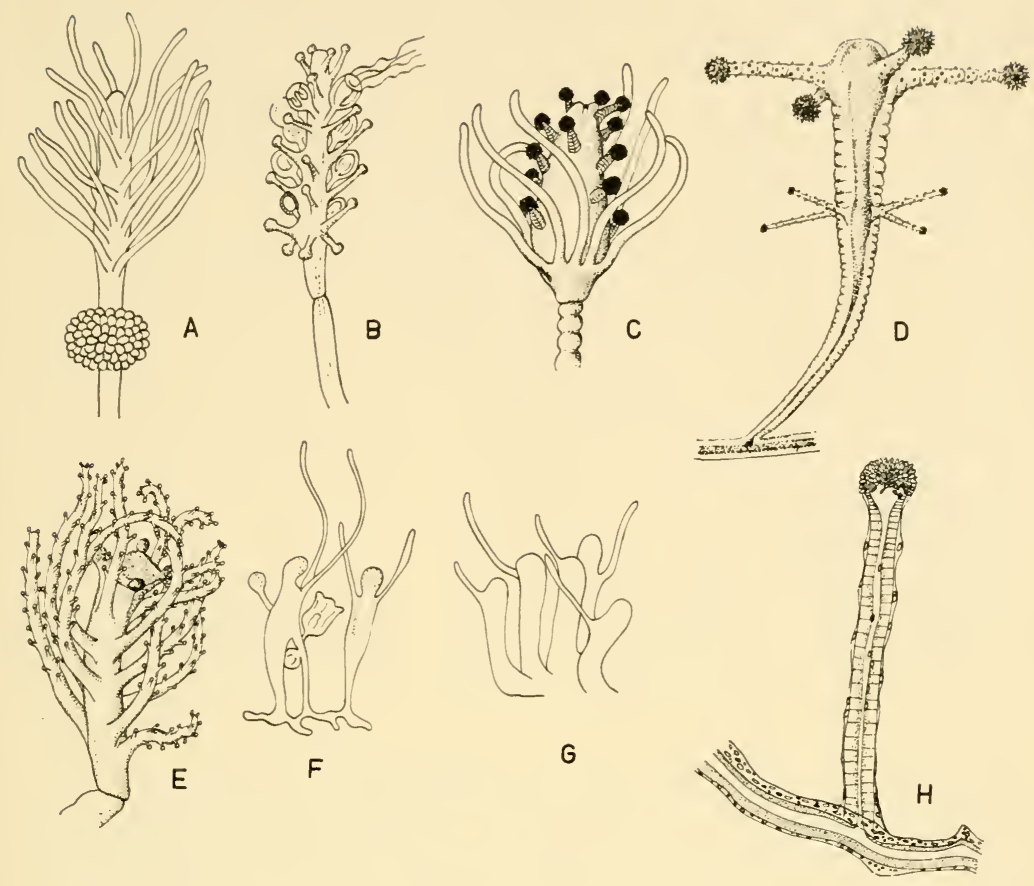

Fig. 11. Some cases of polymerization and oligomerization of tentacles of hydroid polyps; A, Clava sp. (after Hyman). B, Syncoryne sp. (from Dogiel). C, Pennaria cavolinii, (from Broch). D, Cladonema sp. (from Hincks). E, Cladocoryne floccosa (orig.). F, Lar. sabellarum (from Dogiel). G, Monobracbium parasiticum (from Dogicl).

H, Halocoryne epizoica (after Hadži).

of oligomerization. As a rule, this will not be difficult when we make detailed comparisons. We meet in our study of the animal world, at every turn, with the phenomena of polymerization and oligomerization. In this connection some regularities have been observed; above all the fact that in case of oligomerization a fixed number of multiplied, and thus homologous and homodynamous, parts is found preserved (e.g. in Cnidaria four, six, eight, and their products; eight in Ctenophora; five in Echinodermata; etc.). 

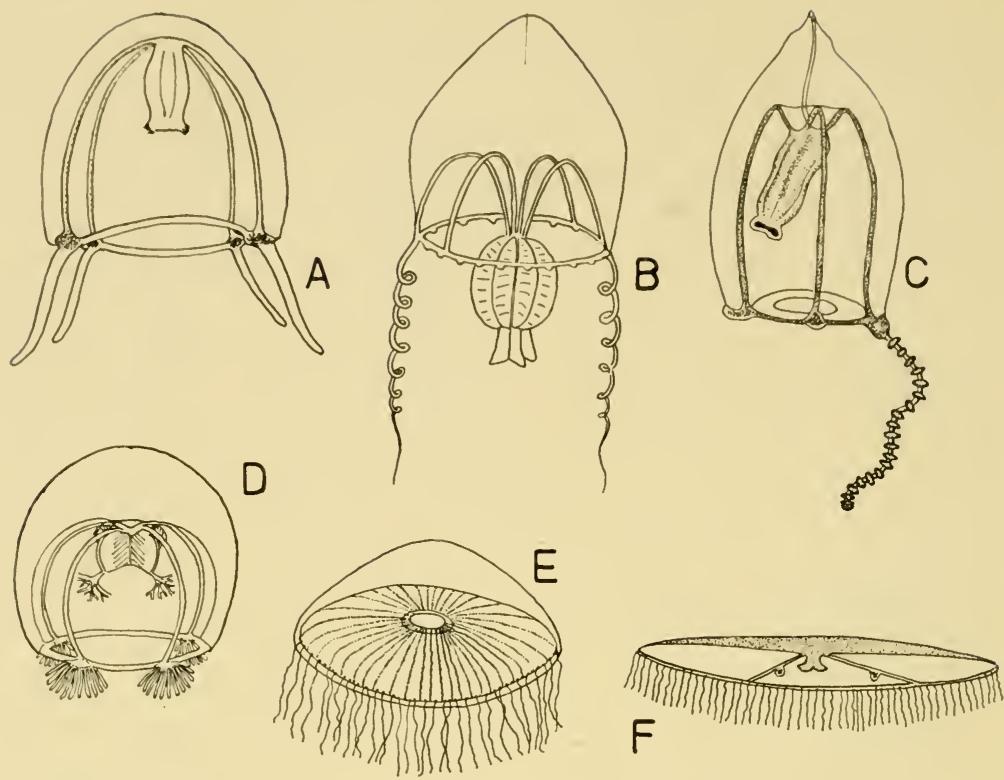

Fig. 12. Oligomerization and polymerization of tentacles in hydromedusae, A, Podocoryne carnea (after Grobben). B, Stomotoca sp. (from Hyman); C, Steenstrupia sp. (after Mayer); D, Bougainvil.ea sp. (after Broch); E, Aequorea sp. (after Hyman); F, Obelia sp. (after Colosi).

The origin of the metamerization, one of the most important problems of the comparative morphology of the metazoa, is closely connected with that of the polymerization and of the oligomerization. The question naturally arises; can we consider that the all-important metamerization has been developed out of the polymery, by way of a multiplication of whole regions of the animal body; or, conversely, by way of an initial polymerization of individual organs (this is usually called the pseudomerization)? This is followed first by a "genuine metamerization," and afterwards either by a wholesale oligomerization with formation of regions, or, by a general oligomerization and corresponding change of the way of life (the origin of the large type of Oligomeria). We will later return to discuss this problem. 
There is one more method which can prove useful in our search to find the relationship connections and herewith the creation of a truly natural animal system. This is my study, the comparison of the so-called parallelisms (they are not identical with analogies!). It has been proved by way of intensive observations that there are certain properties and peculiarities which are easily repeated. We have here, if looked at from the standpoint of genetics, some more or less identical mutations, or complexes of mutations. As an example we can mention the appearance of cnidae. These are complex, not living, but nevertheless organic products of specialized gland cells which are composed of firm (solid) and thickly liquid components. The thickly liquid secretion of cnidocytes is able to liquefy instantly, almost like an explosion with which it can be rightly compared. Here we are particularly interested in the morphological side of this remarkable differentiation in the monocellular glands.

This organelle, which itself is not alive, can be found in varying stages of evolution in various recent animal groups. The highest level in their progressive evolution can certainly be observed in the cnidae of Cnidaria-and this is why the latter have been given their name. If we make a search "downwards" we shall find cnida-like formations in various Protista, even in Protophyta, especially those that stand close to Protozoa (Polykrikos and Pouchetia among the Dinoflagellata). It seems that these cnidoids-as I called these cnida-like and yet completely special formations (Hadži, 1951) - had been developed repeatedly and independently from each other as early as in Protista; later they have evolved in special directions. The foundation, however, the original mutation which had led to their formation seems to have always been the same. Upwards, i.e. above the level which is taken by Cnidaria in the animal system, no real cnidae can any longer be found. The conditions that prevail in Nemertinea have not been completely cleared up. As for Mollusca (Gastropoda) where cnidae have also been found, it is beyond any doubt that in this case we 
have really the so-called kleptocnidae, i.e. cnidae which have been taken over from Cnidaria which had been devoured by Mollusca - a certainly very remarkable case. It is very probable that Nemertinea, too, possess kleptocnidae. A special place among all the animal groups that possess cnidoids is occupied by Infusoria-Turbellaria-Cnidaria; to these we must perhaps add Nemertinea. It has been with intent that Ctenophora have been omitted here, even if the presence of genuine cnidae can be frequently found mentioned in the special literature in connection with at least one species of Ctenophora (Haeckelia or Euchlora rubra). As has been mentioned earlier, I think that it is very probable (Hadži, 1951) that only kleptocnidae occur in this ctenophore and that they have been taken over from a species of Narcomedusae which serve as the main food of this ctenophore.

There are, besides the typical cnidae, some less specialized forms that occur in the large groups of animals which have been mentioned above. They are essentially glandular; their secretion is partly solid, and partly a fluid that can absorb water and swell. They have been described under various names (trichocysts, rhabdites, sagittocysts).

The form of medusa must be considered as a kind of parallelism; it is neither homologous, nor analogous, nor a result of convergences (see Fig. 13). We shall omit here those Protista which show only a purely external similarity with medusae (among Flagellata e.g. Medusochloris, Craspedotella, and Leptodiscus); these have been called the "types of the forms of life" by Remane (1944). The genuine medusae must be considered to have evolved beyond any doubt at least twice, independently from each other; both times they, have developed from a polyp by way of an adoption of the vagile way of life: the first time from a scyphopolyp, and the second time from a hydropolyp. I will show later that this hydropolyp had been developed later than the scyphopolyp, and in all probability from just this same scyphopolyp. A similar way of life (natural selection!) and a close enough relationship (the same direction 


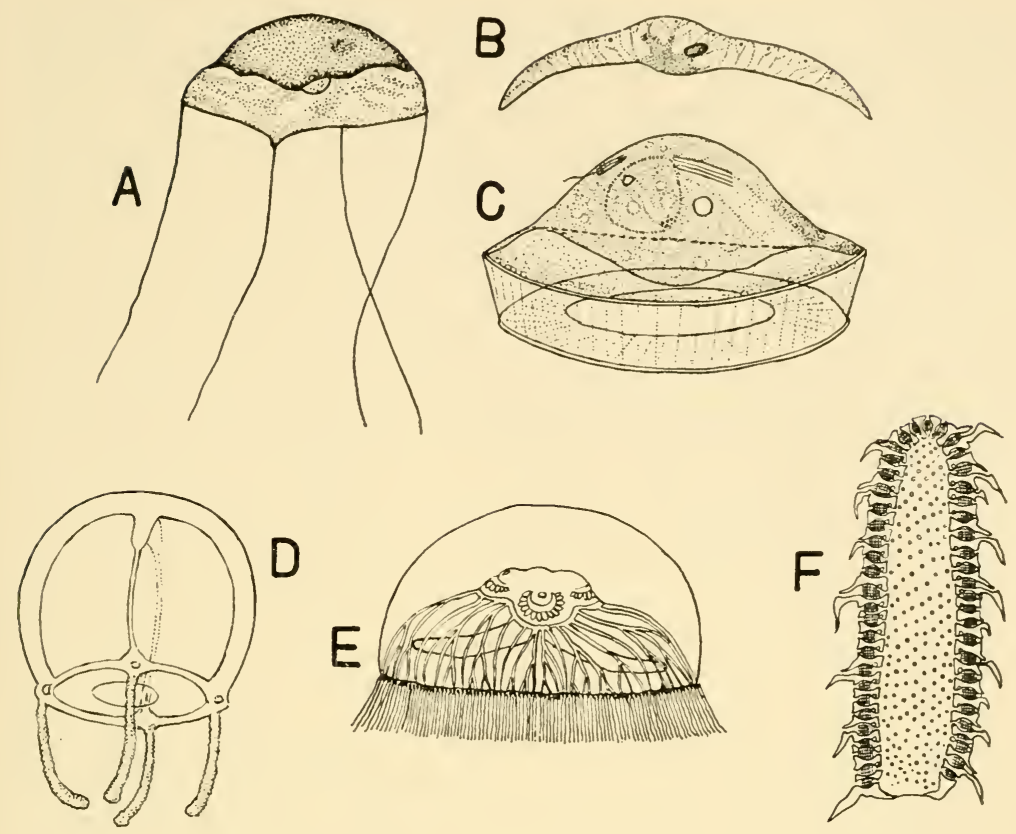

Fig 13. Medusoid forms as cases of parallelism. A, a phytoflagellate, Medusocbloris pbiale (after Pascher). B-C, two heterotrophic dinoflagellates: Leptodiscus medusoides (from Hertwig) and Craspedolella pileolus (after Kofoid). D, an anthomedusa; E. a scyphomedusa; F, Pyrosoma sp. a colony (cormus) of tunicates.

of mutations) have given similar results. Perhaps we could speak of a medusoid form in Pyrosoma among Chordata, yet in this case we have actually a cormus and not an individual. Something similar can be observed in Chondrophora among the Siphonophora. The same is actually true for the polypean form too, yet with the difference that the polypean form occurs much more frequently in the animal world since it is connected with a semi-sessile or to a completely sessile way of life. The form of polyps is an oblong rotund form which is set upright on a firm ground. The oral opening occurs, with the exception of Protozoa and Spongiae, at its free end. The tentacle-like excrescences grow regularly all around this oral opening in the 
form of one or several circlets. It would take us too long to give here a complete survey of all animal groups which have polypean forms. The species of whole large groups can show polypean forms (this is the case with Cnidaria and Entoprocta among the Ameria), or the same thing is true for at least a large part of them (Annelida). Oligomeria are mainly polypoid animals. Even among the Chordonia we can find in the benthonic Ascidia a clear inclination to develop polypean characters. Important from our standpoint, however, is the knowledge that a sessile radially symmetric polypean form had been repeatedly developed from a freely moving bilaterally symmetric form, and that this polypean form itself has been later frequently changed into a freely moving form, either one that creeps over the sea bottom, e.g. Echinodermata, or one that can swim freely in water, e.g. medusae of the Cnidaria.

\section{The Validity of Watson's Rule for the Invertebrates}

In our attempt to place the origin of Cnidaria on a new, rational basis we can use the experiences of researchers in Vertebrata who have certainly had to solve much easier problemseven if we do not intend to enter here in to the whole enormous problem of the origin of new animal types, i.e. macro-evolution. The question is whether Watson's rule (de Beer, 1954) of a mosaic-like evolution can be considered valid also in the case of the "lower" invertebrates. A detailed study of the Archaeopteryx (a third specimen of this animal has recently been found) has led de Beer to develop the ideas which had been first formulated by D. M. S. Watson and according to which there is a divergent evolution in the subclasses (types) of Vertebrata and a simultaneous mosaic-like mixing of old and new characteristics. In this way a new combined form can be created with a simultaneous change of the way of life, which is stil able to live in the milieu. Thus, there had been no general and parallel change of characteristics, (in this case of the 
reptilian characteristics) which finally led by way of all transitions to the new type, the birds; but instead there was an accession, or an admixture, of new bird characteristics to the already existing reptilian characteristics, as this can be seen in the remains of Archaeopteryx. De Beer believes that in the latter the "reptilian-characters" are still prevalent. In such a case the old characteristics can be preserved, yet they are naturally outweighed by the new, progressive, and specialized characteristics. It has been possible to show that other classes of Vertebrata, too, had probably developed along a similar divergent and mosaic-like way.

It has been possible to find this type of evolution in connection with Vertebrata because their fossil remains which have been found go back to a more or less recent past when this process of transformation had been taking place. The fortunate case of the Archaeopteryx has been particularly welcome. Yet unfortunately no such intermediate forms belonging to invertebrate types have been found, nor is there a prospect that they will ever be found; this is particularly true for the lowest, and therefore phylogenetically oldest, invertebrates whose manifold and numerous types had been developed even before the beginning of the era of the formation of the fossil-bearing earth strata. In this naturally disadvantageous situation the researchers in invertebrates have to use methods that are less reliable. Above all, it is a priori quite probable that Watson's rule has a general validity as we cannot see the reason why it should be limited to Vertebrata only. Secondly, if Watson's rule can be considered valid at all, then it must consequently be expected that even if the mosaic-like appearance of characteristics be most manifest during the phase of transition-because here the characteristics of ascendants and of their descendants appear most concentrated in individual species, in this case in Archaeopteryx-we have nevertheless to expect the same characteristics and properties of the two subsequent types to appear, even if somewhat "thinned down," in the parent as well as in the descendant species. It is 
natural that in the parents the old characteristics will strongly prevail, and in the descendants the new characteristics which are distinctive for the new type.

We shall find without any difficulty, if we limit ourselves to the group Cnidaria-Turbellaria-Ctenophora, and if we make a comparison of their recent forms, taking simultaneously into
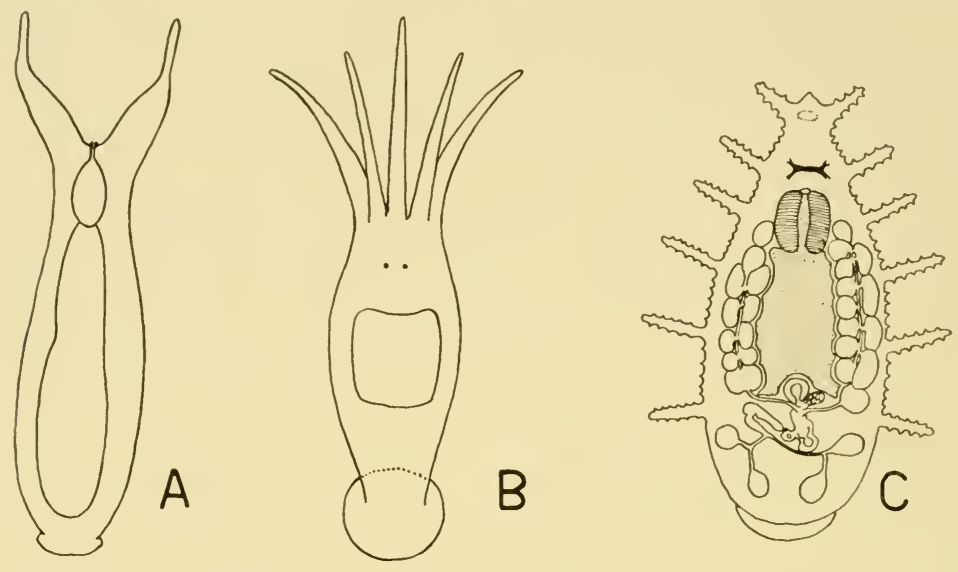

Fig. 14. Three species of Temnocephala; illustrating the polymerization of tentacles like excrescences. A, Bubalocerus pretneri (from Matjašič). B, Temnocephala cljilensis (after Wacke). C, Actinodactylus blanchardi (after Haswell).

consideration Watson's rule, that the validity of this rule extends also to the lower invertebrates, and, consequently, over all the animal groups. Here we shall limit ourselves to mention a few characteristic examples only. Thus we can find in various Turbellaria: (1) the body set upright while mobility is at the same time temporarily preserved (this led to a complete sessility and immobility, to the formation of cormi and of colonies, and finally to a polymorphism connected with the formation of medusae); (2) the tentacle-like excrescences at their anterior ends which show an inclination to polymerization (Temnocephala, see Fig. 14), and the growth of the intestinal diverticula into the interior of these tentacles (in polyps 
this led to the typical circlet of tentacles which serves as the main appliance with which they catch their food); (3) the specialization of the monocellular skin glands with their solid or outflowing secretion (here rhabdites and sagittocysts; cnidae in cnidaria); (4) the tendency of the aproctous intestine to develop diverticula (excrescences) and folds and thus to increase the internal surface (this is so characteristic and manifold in Turbellaria that it has been used as a criterion on which, their classification has been founded: Acoela, Rhabdocoela, Tricladida, Polycladida; in Anthozoa it is best developed in the polyps of Cnidaria in the form of sarcosepta, or of longitudinal folds, yet here we can observe a later gradual regression with exception of the diverticula which grow into the tentacles; an even richer development can be observed in medusae); (5) the inclination to form a cuticle; in Turbellaria this can be observed occasionally only and it is usually strictly localized; it is only in Temnocephala that it covers almost the whole surface of the body; in Anthozoa its occurrence is less extensive; in Hydrozoa which have adopted a completely sessile way of life, it becomes a generally accepted property (here it is known as the periderm formation); (6) the same is true also for the impregnation of the cuticle (periderm) with calcium salts; this can also take place, even if to a lesser degree, in the mesenchymal cells; in Turbellaria this happens to a very limited degree, a fact which can be explained as being due to their free mobility; (7) the inclination to transversal division; in Turbellaria this has been inherited from their supposed infusorial ancestors; even if this is - as well as several other properties that have been mentioned-not a quality that generally appears, it is nevertheless a common property, i.e. it depends on the same gene or on the same complex of genes; in Scyphozoa it has led to the development of a form of medusae, and in Anthozoa to the first step in the same direction (Fungia). Many other facts could be mentioned in this connection, yet the above points should suffice.

Whatever is generally valid for the relationship TurbellariaCnidaria, the same can also be proved valid for the evolutionary $6 *$ 
trends between the three classes of Cnidaria. It can be generally stated that the farther away from Anthozoa we move in the direction of Scyphozoa and Hydrozoa, the fewer properties of Turbellaria we meet; all this is due to a divergent development and to the prevalence of new properties and peculiarities which are typical of Cnidaria. Yet while we can observe that there are also some side branches which have developed out of Turbellaria into several new directions, either in the direction of animal types which have adopted a parasitic way of life (Trematoda, Cestoda), or such that have adopted the free mobility and the free way of life (Nemertinea, etc.; most probably after the transition to an euproctous state, also in the direction of Mollusca), nothing similar can be observed in Cnidaria. On the basis of all this we can conclude-and rightly I think-that these Cnidaria represent a blind sidebranch on the tree of Ameria. We can see the climax of a partly progressive development in the strongly individualized cormi of Hydrozoa, in the Siphonophora. It is possible, though it is difficult to prove, that Cnidosporidia, which are attributed to Protozoa, had possibly been developed from the parasitic Narcomedusae. This hypothesis seems to be supported by the so-called polar capsulae, thus cnidae, and by the polycaryontic (polyenergetid) state of their vegetative phase connected with the phenomenon of cellulation.

Parasites have occasionally been used-and this successfully-to explain the phylogenetic conditions of their host animals. This can be considered to be a special method in phylogenetics. If this is valid for parasites then its validity could also be extended to symbionts. It is probably not a mere chance that a frequent symbiosis of monocellular algae that live intracellularly (e.g. Zoochlorella and Zooxantbella) has been observed on one hand in certain Infusoria, and on the other in the Turbellaria and Cnidaria. In the two last mentioned cases this symbiosis can be so strong that it becomes strictly obligatory and that in connection with this, systems have been developed which secure the "inheritance" of their symbionts. 


\section{THE PREVIOUS INTERPRETATIONS OF CNIDARIA}

We shall now pass on-after this preparatory introduction which has been necessarily rather extensive-to our main problem: what Cnidaria actually are and what is their origin? We shall be better able to understand our new answer to these questions if we first make a critical survey of the interpretations of the nature of Cnidaria which have been proposed till now. It will be sufficient to divide the history of the study of Cnidaria into two parts only, i.e. the first part which begins with Aristotle, and the second part which commences with Leuckart (ca. 1847) and which covers the Darwinian period.

From the very beginning the larger Cnidaria, i.e. those that can be observed with the naked eye and therefore more easily accessible (medusae, Siphonophora, Actiniae, corals) have given the impression that they were a kind of organism that resembled the flowering plants. Firmly attached to the ground or moving so slowly that this can hardly be noticed, frequently of rich colours, appearing in shrubs, covered with parts that are similar to flowers, and finally stinging like a nettle when touched-all these are properties that are usually attributed only to more highly developed flowering plants. It is therefore not surprising to find acalephs and cnidae mentioned as early as in Aristotle, and that these same organisms were called sea anemones, sea nettles, phytozoa, etc., by the fishing nations that inhabited the shores of the seas. Later there were various other natural scientists who for a long time saw in these organisms, like Aristotle, something that is at least between an animal and a plant. This is why the name 
Zoopbyta had been created. It was much later that it has finally become clear that these Zoophyta are really animals, and not plants, or something that is between a plant and an animal. It was not till 1723 that it was first suggested, by Peysonell, that these are really the lowest or the most primitive animals. The external forms of these animals, have always been particularly emphasized and many zoologists have become especially impressed by their radial symmetry. This had been important when the first attempts were made to construct a classification of the animal world on the basis of a comparative method. In place of the name Zoophyta, the name Radiata was introduced, with some simultaneous changes of their spheres and of the subgroups they were supposed to include: a possibly worse solution. The name Zoophyta itself (this name was used for a long time) included all kind of animals, or, as this has been said by L. Hyman, "A variety of softbodied animals, from sponges to ascidians." Under the banner of Radiata, Cnidaria were again connected with completely different types of animals, especially with the Echinodermata; this was done because of their radial symmetry only (Cuvier). Lamarck himself had tried to separate the medusae from polyps. Many zoologists, especially the French scholars, adhered strictly to this wrong concept of Radiata even a long time after Darwinism had been firmly established and when attempts had been made to create a natural animal system built on the principles of evolution; the same mistakes can be partly encountered even to the present day.

In the mean time great progress has been made in the field of the fine morphology of the lower invertebrates. The progress made in the ontogenetics or in embryology-especially of Vertebrata-has been of considerable importance for the evaluation of Cnidaria; it was in this way that the theory of germ layers was developed. Even the bodies of adult animals have repeatedly been divided into layers: first there was one stratum, then two, and finally three strata or dermas (ecto-, ento-, and finally mesoderm). Even if it was likely that 
the third stratum, the mesoderm, had been first laid out as a thin leaf which has later lost its leaf-like form, it has nevertheless remained a mesoderm, or something that has been added later, as a final layer. The knowledge which had been first arrived at in connection with Vertabrata was soon extended to invertebrates. And here a great mistake was made: this was in the case of those lower Metazoa where no leaf-like mesoderm could be found in their ontogenies and where for this reason the presence of a third body layer has simply been denied. In this way the notion of Diploblastica was created, i.e. Metazoa with two germ layers, in contrast to the higher developed Triploblastica. Later I will try to show that there is no real justification to believe in the existence of Diploblastica.

There has been a clarification of concepts during the second period which developed under the influence of the theory of evolution, due to the theory of recapitulation. It was very soon (in 1859, when the notion of Coelenterata was created by Leuckart) that the interpretation of these problems became stabilized; and they have been preserved, with few changes only, down to the present day. It is because of this generally accepted situation that it is so difficult to weaken the erroneous basis of this view and to propose something new in its stead. This stabilized interpretation which sees in Cnidaria, and herewith in all Coelenterata, primarily simple animals that consist of two germ layers has been accepted by all the great authorities in the field of zoology, beginning with Ernst Haeckel.

It is certain that it was Ernst Haeckel who developed in detail the basis of the interpretation of Cnidaria and of their gradation. He accepted Leuckart's interpretation of Coelenterata because it seemed suitable to the principle of evolution: whatever shows a simple structure is primary, and it is followed by a progressive evolution. In this way it was not only the sequence: Spongiaria-Cnidaria-Ctenophora which seemed to agree with the "natural evolution," but there was even within the group of Cnidaria the "natural" sequence Hydro- 
zoa-Scyphozoa-Anthozoa which has seemed to indicate a progressive development. Frequently the latter two classes have been united under the name Scyphozoa; for a long time it was believed that both Anthozoa and Scyphozoa have an ectodermal gullet (pharyx). It is worth mentioning that Haeckel himself was partly irresolute whether the border line between Coelenterata and the more highly developed Coelomata should be placed between Ctenophora and the lower Turbellaria, or somewhat higher so that Turbellaria could be included among Coelenterata; it was clear that there was a relationship between Ctenophora (particularly those that lived on the sea bottom) and Turbellaria whose structure seems to be quite close to the structure of the former-and for this reason these lower "worms" could not be genuine Coelomata. On the other hand such an extension of the sphere of Coelenterata could lead to a muddle and to a system which it would be difficult to survey. Carl Börner (1923) later proposed to associate Platyhelminthes and Acanthocephala more closely with Coelenterata.

Still, some changes were proposed in spite of the fact that this part of the natural system of zoology appeared very stable and that it had been generally accepted. T. H. Huxley (1875) already expressed his doubts that there was a close relationship between Spongiae and other Coelenterata, and generally to Metazoa. It was Sollas (1884) who succeeded in transforming this doubt into a fact, into a truth; he made a definite separation of Spongiae, as Parazoa, from Eumetazoa as the genuine Metazoa. This radical step was well supported by numerous and fundamental differences that occur both in the structures and in the ways of life of Spongiae and of other Metazoa. Even greater differences have later been observed in connection with their ontogenies. Delage tried to emphasize this difference when he invented the name Enantiozoa for Spongiae, i.e. Spongiae are Metazoa in whose ontogenies the layers of their bodies become inverted: that layer of cells, which in an embryo or in a larva occurs in their external or anterior parts, develops into the 
intestine (entoderm) of Eumetazoa, and the internal or posterior layers of cells into their skin (ectoderm).

Such is the situation that appears when we try to make a comparison between two items which are, as a matter of fact, incomparable: we are now certain that Parazoa and Eumetazoa, i.e. all the remaining polycellular animals, cannot have the same origin. It is therefore necessary to separate the Spongiae definitely and for ever from Cnidaria and Ctenophora, and they can not be included among Coelenterata. Even the most recent attempts to try and halt the progress made by Sollas and Delage cannot change this situation. These attempts have been made, as it has already been mentioned, by Tuzet and her collaborators who try to find a justification for this mainly in a supposed discovery of nerve cells in Spongiae, and by Sachwatkin (1956) who tries to overcome the unbridgeable differences that exist in their ontogenies.

Nobody has followed the attempt made by K. C. Schneider (1902) who proposed to connect phylogenetically Spongiae and Ctenophora, with a simultaneous exclusion of Cnidaria from this group, which he had called Diskyneta. This has been, to my knowledge, the only attempt which has endeavoured radically to separate the Cnidaria from Ctenophora and in this way to give up the group of Coelenterata; the attempt, however, remained unsuccessful. Till now the Cnidaria and Ctenophora have been looked upon as closely connected with each other. The main difference which has been mentioned in this connection has been the presence, or the absence, of cnidae (it has been due to this that the names Cnidaria and Acnidaria have been introduced): yet this difference is not by far of the most decisive importance. Even if Ctenophora quite generally possessed their own genuine cnidae, as this has repeatedly been supposed to be the case in the species Haeckelia (Euchlora) rubra, it would still be necessary to make a clear distinction between Ctenophora and Cnidaria. Cnidae, however, such as they occur in Euchlora, could actually be of an extraneous origin, as this has already been mentioned above. 
Jürgen W. Harms (1924) also separated Cnidaria from Ctenophora in his somewhat bizarre system of the animal world; this separation was less radical than the former and it is based on other reasons. The first of his three circles was subdivided into three subcircles; it is defined well enough because it includes all the animal groups which have primarily - as modified Gastraeadae - no anal orifice (aproctous animals; here he should have omitted Spongiae); he uses for his subdivisions the criterion of stability. Harms included in his first subcircle Porifera, Cnidaria, and Turbellaria as labile and regulative forms ("Regulationsformen"). The second subcircle includes the parasitic Platyhelminthes as semi-stabile forms with a partly constant number of cells. The Ctenophora and Acanthocephala, as blindly ending branches of the animal world, with a constant number of cells (this, however, is not even true for Ctenophora) and their stability, have been placed by Harms into the third subcircle; yet, as a matter of fact the Acanthocephala are not primarily aproctous animals: the absence of the whole digestive apparatus, and thus also of the anal orifice, is in these animals a secondary phenomenon since the loss of these organs has been caused by their parasitism.

Among the almost innumerable attempts to construct a natural animal system there are a few who have tried to abandon the notion and the category of Coelenterata (Hyman, Beklemischew, Perrier, Dudich, etc.). Here we are able to mention a few such cases only. Such authors have usually gone back to the old notion and name of Radiata or Radialia, excluding from them Spongiae and Echinodermata. This was made for example, by L. H. Hyman (she uses them as a contrast to Bilateria, adding "Cnidaria [or] Coelenterata"); Beklemischew (1958) divides Metazoa first into Enantiozoa (after Delage, for Porifera) and Enterozoa; the latter are subdivided into Radialia and Bilateria; Radialia, finally, consist of Cnidaria and Acnidaria. Dudich (1957) divides Eumetazoa into Acoelomata (i.e. Cnidaria and Ctenophora) and Coelomata (Proto- and Deuterostomia). Dogiel (1947) takes over the old division into Diplo- 
blastica or Radiata - which consist of Spongiae and Coelenterata-and Triploblastica (Bilateria). The influence of the historical moment (or of conservatism?) can be seen in the fact that the old notion of Radiata reappears again and again to be used for systematic-taxonomic purposes, even if in the mean time it has become clear that it is not in Cnidaria only that radial symmetry can be found (in Cnidaria this symmetry is not even completely developed), and that it is arbitrary (even if correct) to exclude Echinodermata from Radiata. It was due to this influence that it has not been perceived that the radially symmetric distribution of various parts (the formation of antimeres) is not connected with any definite type of structure, and that instead it can appear at any level of organization, even if the more or less perfect development of this symmetry can vary: the only condition for it is the adoption of a sessile way of life. Exactly as it has finally become necessary to accept the fact that, phyletically, Cnidaria have nothing in common with Echinodermata even if they are both (secondarily!) more or less radially symmetric, it will be necessary to get used to the fact that Ctenophora must be separated from Cnidaria-like Echinodermata-even if they are not widely different from them: each has developed out of its own "root" and it was the ancestors only of these two groups which were closely related to each other; in both cases the ancestors were turbellarians, yet of different subtypes. The same is naturally true-yet to a considerably greater degree-for the mutual connections between Porifera and Cnidaria.

For historical reasons and because of their external appearance Cnidaria have always been-justly, as it has seemedconnected with Ctenophora. The latter are occasionally called Acnidaria (Bütschli) in order particularly to emphasize in this way their close connection with Cnidaria. The names of these animals have been repeatedly changed in the more distant past as this can be seen in Krumbach (1928); frequently they were called Acalepha, i.e. medusae. Their jelly-like, even watery, consistency, their life in plankton, the strong contractility of 
their tentacles (even if it is developed in one pair of tentacles only), the inclination to adopt the radial symmetry, and many other minor morphologic phenomena which Haeckel could find in his Hydromedusa Ctenaria ctenophora (with a very suggestive name!), all these facts have helped to preserve the idea firmly and faithfully which connects Ctenophora closely with Cnidaria. And what has been the attitude towards those differences that are even more numerous and all-important? These have simply been pushed aside while at the same time attempts have constantly been made to connect these two animal types by way of a common origin; yet these attempts have always remained fruitless. When finally Haeckel's attempt with his Ctenaria had completely failed, new assays were made in the search for the ancestor in the hope to find it in the most remote past. Thus Goette (1912) proposed, to derive the Ctenophora from a common primitive form which was constructed ad boc and which he called a "Scyphula;" this "Scyphula" was freely swimming and "binumeral" ("qweizäblig") (A fine example of a "suitcase theory" ["Koffertheorie"]. Those scyphulae which had remained in plankton developed into Ctenophora, and those that had sunk to the sea bottom evolved into the sessile scyphopolyps, first without a medusa stage. This artificial construction could find no acceptance. Not very different from it was the interpretation proposed by Heider (1913) who has tried to derive Ctenophora-surprisingly enough by way of neoteny-from the anthozoan larvae that had lived in the pelagic zone. Heider believed that there was some larval element in Ctenophora.

It has been a sign of emergency when attempts have been made to go even further back into an already completely indifferent past and when the most simply built planula, a modified "gastraea" which had lived in the plankton began to be considered as a starting point for the evolution of all the lowest invertebrates. In this way the question of the origin has been completely watered down. This is how the problem of the origin of Ctenophora, and thus of their relationship connec- 
tions to Cnidaria, remained unsolved until a new hypothesis was proposed by me, 39 years ago (Hadži, 1923 a), according to which Ctenophora had been evolved from planktonic larvae of the polyclad Turbellaria which had been further developed in plankton by way of neoteny. On the basis of this hypothesis we can best explain the much discussed problem of the genetic relationship between Turbellaria and Ctenophora. It has been generally recognized and accepted that there were such close relationship connections, particularly as regards the Polycladida among Turbellaria. In this way we can make a completely satisfactory explanation both of their general forms, as well as of their symmetric conditions, of their internal structures, and of their ontogenies. Th. Krumbach (1928) who made a detailed study of Ctenophora for his Handbuch der Zoologie (edited Kükenthal, and later by Krumbach) has given a critical evaluation of all hypotheses which have tried to explain their origin; he has come closest to the best solution when he wrote, "Die Ctenophora stehen den Turbellarien nahe, stehen keiner Bilateriengruppe so nahe wie den Turbellarien" (p. 978). In the same book E. Bresslau and E. Reisinger (1913) have emphasized how my hypothesis "die eigenartige biradiale Symmetrie der Rippenquallen auf ein Kompromiss zwischen der ererbten bilateralen Symmetrie der Protrochula und der Tendenz zur Radialsymmetrie infolge der planktonischen Lebensweise zurückzuführen sucht."

L. H. Hyman (I: 693-695) refuses to derive Ctenophora from the Cnidaria, especially out of Hydrozoa (Hydroctena!); she prefers to go further back to the very primitive, i.e. completely indifferent ancestors which she supposes to be common to all the "trachyline-hydrozoan, scyphozoan, and anthozoan lines." We have already characterized such an attempt to be simply an auxiliary solution. Hyman does not even mention my hypothesis; it is possible that this hypothesis was not known to her even if it is mentioned - though not in the chapter on Ctenophora-in the extensive Handbuch der Zoologie. Hyman, however, finally returns to the problem of Ctenophora in a 
supplement to the fifth volume of her book The Invertebrates where my hypothesis can also be found mentioned under the title "Retrospect" (p. 730). She refuses straightforwardly to accept my hypothesis which she calls "the fantastic theory of Hadži," and adds, "Hadži gives no real grounds for this view, only theoretical vaporizing." This opinion, or rather judgment, is not only unjust, it is also wrong. The way I proceeded to found my hypothesis has been exactly the same as has been done by other zoologists, Hyman included. I referred to wellestablished facts which I tried to bring into a new focus and to give them new interpretations. In this connection Hyman has omitted to mention that in the meantime, P. Us (1932), one of my students, succeeded in proving the older data about the development of the mesoderm in Ctenophora as it had been published by Metschnikoff to be essentially correct (Fig. 15). One would expect in an objective criticism, to be able to find at least some arguments quoted and an honest statement that there have been zoologists who have considered my hypothesis to be feasable, at least before the author begins a straightforward refusal of my thesis. In this connection Hyman could have remembered what she read in the article "Evolution of Metazoa" by Sir Gavin de Beer (in the book "Evolution as a Process," 1954). In it a paragraph from the book by MacBride is quoted which refers to Lang, and which states that the ciliary seams of the eight lobes that occur in Müller's larva are "jointed edgewise so as to form combs." Anyhow, on the basis of the numerous similarities that exist between Ctenophora and Polycladida, MacBride, according to de Beer, comes to the conclusion "that Müller's larva represents a pelagic Ctenophore-like adult ancestor of Polyclada, and that the Platyhelminthes were evolved from the Ctenophora." De Beer accepts my hypothesis because he obviously thinks it to be better founded and more probable than other previous hypotheses. In the introduction to the present study it has been mentioned that in our research in the phylogenetic problems no progress can be made without some imagination, or rather 

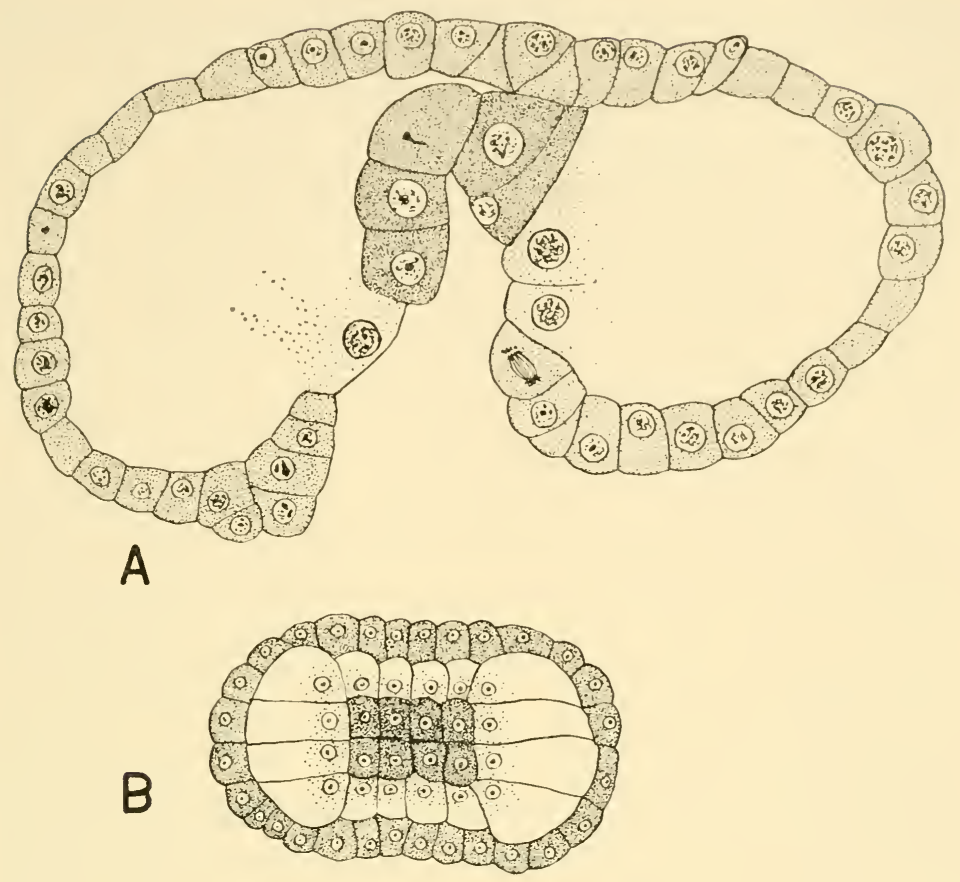

Fig. 15. Young ontogenetic phase of Ctenophora; the dense dotted cells are the primordium of the mesohyl (mesoderm). A, longitudinal section. B, oral view of the embryo (after Us).

without an esprit, particularly in those cases where no fossil documents can be found, yet we should realize that this is far from a "tantastic theory" and "theoretical vaporizing."

It should be mentioned in this connection that it is wrong to speak about a "degradation of ctenophores from polyclad larvae;" on the contrary, in my opinion, the evolution of Ctenophora as animals that live in plankton has been progressive; their starting point, the supposed polyclad larva, has also reached a much higher level of evolution than any constructed planuloid form of an "Urcoclenterate" from which Hyman derives both Cnidaria and Ctenophora by way of an equally constructed medusa, a construction which itself 
is not without some fantasy (cf. the "Hypothetical Diagram" in vol. I., p. 38 of Hyman's work). The reader should also then compare how I explain and understand the origin of bisymmetry in Ctenophora and how this is done by Hyman; she simply writes, "The biradial symmetry of ctenophores is obviously derivative of tetramerous radial symmetry, as seen in Hydrozoan medusae." I should only like to ask why the bisymmetry should be developed from a radial symmetry during the life of the animal in the plankton? We must be aware of the fact that in Ctenophora the paired system can be observed not only in their tentacles.

Here it should be emphasized that no facts are availableas far as I can see them-which disprove the validity of my hypothesis. For some time it seemed that there was an essential difference between Ctenophora and Turbellaria; this difference was thought to be found in the ontogenetic development of the mesoderm (mesohyl). Ctenophora have, according to Metschnikoff, a "genuine mesoderm," such as it also occurs in Turbellaria, particularly in Polycladida among the latter. Hatschek, however, who later investigated the development of Ctenophora maintained that Metschnikoff had made a mistake and that those groups of cells which had been considered by Metschnikoff to be an Anlage of a genuine mesoderm belonged in reality to the entoderm. This study by Hatschek which had been completed and illustrated was unfortunately never published. The two authors of the standard work on the ontogeny of invertebrates (Korschelt and Heider, 1936. p. 265) were able to see Hatschek's pictures and they were convinced that his explanations were right. In this they have been followed by Hyman. If no further progress had later been made in this field then the close connection between Ctenophora and Cnidaria would have seemed to be a firmly established fact. I selected this theme for a doctoral thesis to be investigated by one of my students, Peter Us; he made a new investigation of this point in the ontogeny of Ctenophora. This investigation was constantly made under my 
guidance and supervision. Us (1932) succeeded in showing that Metschnikoff's results correspond fully to the actual facts. In this way the difficulty has been removed, the Ctenophores have a mesoderm and this helps to prove the accuracy of my hypothesis which tries to derive Ctenophora from the planktonic polyclad larvae.

Much later, I found that I was neither the first nor the only person who formulated this idea about the origin of Ctenophora. Garstang developed his idea to explain the origin of Ctenophora from the Müller's larva of Polycladida by way of neoteny; this idea was proposed in an ingenious poetical work whose form, however, did not correspond to that of a serious scholarly interpretation. It was published by Hardy in 1954. I have briefly mentioned this case in the introduction to the present study.

The origin of Turbellaria, and especially of Polycladida, from the Ctenophora has been discussed much more than the origin of Ctenophora. The reason for this can be found in the large number of similarities that exist between Ctenophora and Polycladida; it has been much more obvious to explain these similarities as being due to a development of Polycladida with their complicated genital mechanisms from the simply built Ctenophora, than vice versa. This interpretation has seemed to be supported by the discovery of benthonic, and therefore flattened, species of Ctenophora, e.g. Coeloplana, Platyctena, etc. Lang (1880) who had proposed this theory, has been followed by numerous zoologists. Nevertheless it soon became clear that these species of benthonic Ctenophora cannot be anything but aberrant species which can lead to no new forms except such as for example the sessile Tjalfiella. In this way the Ctenophora must definitely be excluded from the line of evolution of the so-called Coelomata or Bilateria. They only represent a blindly ending sidebranch on the tree of the animal world.

Finally, it should be mentioned that there have even been some zoologists who have tried to find a solution of the 
problem of the origin of Ctenophora in the nebulous idea of Gastraeadae which they consider to be the common origin of Spongiae, Cnidaria, and Ctenophora, while at the same time they wish to give up the old taxon of Coelenterata. This should be done, according to this interpretation, first, because their supposed common origin goes too far back into a vague past, and, secondly, because in the mean time these three types (Spongiae, Cnidaria, Ctenophora) have had such widely diverging evolutions that it is now senseless to try to bring them into one unit. E. Perrier (1921 pp. 284-5), for example wrote in connection with his statement that Ctenophora had a genuine mesoderm, "... font douter qu'on doive rattacher réellement les Cténophores à l'embranchement des Coelentérés." In spite of this E. Perrier (1921) divides Metazoa into Phytozoaires and Artiozoaires, and he mentions as "embranchements" of Phytozoaires: (1) Coelentérés, (2) Spongiaires, and (3) Echinodermes.

We are fully convinced that sooner or later, the taxon Coelenterata will be completely abandoned and that Cnidaria will be the only group which will survive within the frame of Coelenterata; so that finally the names Cnidaria and Coelenterata will become synonymus. In this way Cnidaria will become isolated, yet this isolation will be an apparent isolation only, because at the same time it will become possible to connect them, by way of a supposed derivation from Turbellaria, to a higher category, i.e. to the large group (phylum s. lat.) of Ameria.

Here it should suffice briefly to touch the problem of the connection between Cnidaria and Echinodermata. This problem has repeatedly appeared in the literature since the days of Lamarck and Cuvier and it is connected with the names Radiata or Radialia. The name itself indicates that it is the similarity of symmetric condition only which is here constantly being taken into consideration; yet the conditions are not identical: in Echinodermata a pure radial symmetry is really an exception due to a secondary omission of their anal orifice 
as is the case in Ophiuroidea; while at the same time the primarily aproctous Cnidaria usually have-with exceptions that can be found above all in the Anthozoa- a pure radial symmetry. We have earlier pointed out that the radial symmetry does not depend on a certain type of the structure and that it is entirely and exclusively influenced by the type of the contact with the environment. The sessile way of life will produce the radial symmetry whatever the type of the structure may be. In a natural system we are therefore not allowed to base a taxonomic unit mainly on such a factor. The names, however, of Radiata or Radialia have been widely compromised and so it is better to avoid them altogether whenever we discuss systematic categories, groups, or taxons. In the zoological literature one can even find cases where Mesozoa, too, can be found attributed to Radiata, either directly (D'Ancona 1955), or as a kind of an appendix to the latter (Bütschli 1921). The Echinodermata, however, do not belong even near the vicinity of Cnidaria; their true position is far away from the latter, among the Oligomeria.

It is interesting to see in this connection how a faithful adherent to the taxon Radiata (E. Perrier) explains the origin of the two groups of Metazoa: the Phytozoaires (i.e. Radiata) and the Artozoaires: According to him the freely swimming Gastraeadae were the first Metazoa. These began to settle on the sea bottom where they grew larger and it was in this way that the radially symmetric Radiata together with Echinodermata developed; or they began to creep over the substratum, and this was the way how the bilaterally symmetric "Artiozoaires" evolved.

\section{The Origin of Cnidaria}

Before we begin to discuss the main problem of the present study, i.e. the true nature and the origin of Cnidaria, we have first to touch on the question, "what is the primary or fundamental form of Cnidaria?" It is well known that 7* 
two forms exist in Cnidaria, and at the first sight they appear to be rather different; above all they differ considerably in their ways of life. These two forms are that of the polyp and that of the medusa. In the literature three possible explanations can be found supported by various scholars: (1) The polyp is the initial form which has been further developed into the medusa as a secondary form; this type of evolution can frequently be observed in recent species. (2) The form of medusa is the primary form; out of this form the polyp has been developed as a larval or post-larval ontogenetic stage; it is possible that this polyp finally takes over the reproductive function. (3) The forms of polyps and of medusae have been evolved in parallell out of an indifferent form; in this way we can imagine "Polyp und Meduse (seien) essentiell ... für den Typus des Nesseltieres," as this has been expressed by E. Reisinger (1957, p. 695).

The last (neutral) of the above mentioned three possibilities seems to be the one least probable. It cannot be proved and it is not supported by any facts. The existence of numerous species of Cnidaria with a complete metagenesis which can be observed particularly among Hydrozoa, somewhat less among Scyphozoa, and not at all among Anthozoa, can be explained by way of any of the above three possibilities. As regards their origin, the conditions in Lucernariidae among Scyphozoa, and in Narcomedusae among Hydrozoa seem to support the third interpretation. Yet these two cases can also be explained on the basis of the first variant, as will be later explained in more detail. Neither Lucernariidae nor Narcomedusae can with certainty be considered to be primitive forms. On the contrary, they are both highly specialized and their position is at the periphery of the groups they belong to. In this way the two opposite possibilities finally remain; polyp or medusa as the primitive form. Let us first take into consideration the second possibility.

There are some zoologists-even if they do not represent a majority of them-who have investigated the Cnidaria and 
who have firmly accepted the interpretation (or who have been more or less inclined to accept it) which considers the form of the medusa to be the primary form. The adherents to this theory refer above all to its complete radial symmetry and to its life in plankton which are both considered to be a sign of primitiveness. Moreover, there are many species of Cnidaria which occur in the form of medusa only, where this form is therefore directly developed out of a planktonic planula. All the elements which occur in Siphonophora can be traced back to a modified form of medusa (according to the medusa theory). The Anlagen of medusoids appear very early in their ontogenies. Yet all these and many other facts which are referred to by those who support the theory of the primacy of the medusa form can be explained even more easily and probably, on the basis of the first interpretation. Even if we disregard the fact that a polyp has never been developed from a medusa (either by way of proliferation or by way of fission) and that the development has always been exactly in the opposite direction so that medusae have been developed out of polyps, we are hardly able to imagine how a highly developed creature with a complicated structure as can be observed in medusae could have evolved out of a planuloid form. One of the most recent advocates of the primacy of the medusa form is L. H. Hyman (I: $634 \mathrm{ff}$ ); her arguments can be used here as an illustration of this thesis. Initially, she admits that "nothing definitive can be said" about the origin of Cnidaria. Yet in spite of this she later expresses the following opinion, "It seems unavoidable to assume that they come from a gastrea type..." Then she tries to help herself with Naef's "Metagastraea" as the next higher stage; this had been further evolved into an "Actinea" which corresponds, according to her, to the present-day actinula larva. What, however, is an actinula? It is clearly a larval stage of a polyp, and it is already highly specialized so that it can be found in Hydroidea only, e.g. only in the highly specialized Tubulariidae. The Actinula descends, so to speak, to the bottom and develops 
into a sessile hydropolyp. It is in the Trachylinae only whose hypogenetic character is a secondary phenomenon because they have secondarily lost their polyp generation (which had been their first and primitive generation) that a polypoid larva had been developed due to their adaptation to the life in plankton. The evolution of this truly polypoid larva of Trachylinae has been - in connection with its parasitism-progressive and it led finally to a new asexually produced generation.

The rigid adherence to a wrong interpretation of the polyp form in general-and not of Trachylinae only as secondary larval forms - has induced Hyman to the following absurd conclusion, "The hydroid colony thus represents a persistent larval state." In the same way we could be justified to call the medusoid form a persistent larval form of the polyp. Hyman believes that "something like the present actinula larva" was the universal starting point even of Siphonophora, and this was, at a very early point. The Scyphozoa, which could be explained even more easily as exclusively medusoid forms, have been evolved, according to Hyman, from the Trachylinae. The Anthozoa, the third subtype of Cnidaria can then be easily deduced from a scyphistoma which is, naturally enough, again a larvoid form. It is surprising how Hyman acknowledges, and this without any explanation, that in these, "all trace of the medusa is lacking."

The interpretation which considers the medusa form to be the primary form can be defended only if we accept the proposition that the phylogenetic development of Cnidaria began with the Hydrozoa and that it was progressive because no medusa can be found in Anthozoa. This proposition has been generally defended as the correct interpretation. Yet even if we disregard this we can find facts which support the idea of the primacy of the polyp form. We have here, besides others, the fact that medusae develop regularly out of polyps; in those cases where a medusa is developed directly out of its egg-a phenomenon which has been given a special 
name (hypogenetic development according to Haeckel)-we find that the circumstances (especially the closer relationship) indicate that this direct development is a secondary phenomenon only. Here it should be mentioned that the planktonic way of life must be considered as secondary in comparison to the benthonic way of life. On the basis of this view we can explain that the polyp which became secondarily free, has been developed into the medusoid form as a secondary form
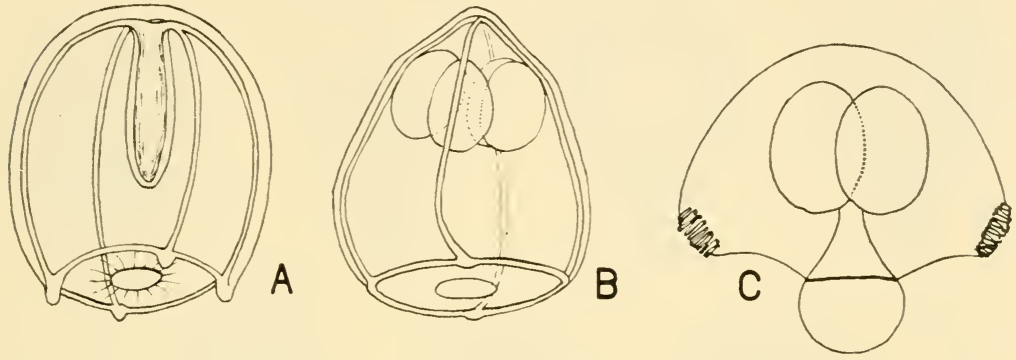

Fig. 16. Three examples of reduced free swimming hydromedusae. A, Pennaria sp. (without tentacles, after Hyman). B, Eugymnanthea inquilina $\mathrm{Palombi}$ (without tentacles and manubrium, after Palombi). C, Millepora sp. (after Delage \& Hérouard).

whose organization is much higher because it is connected with the free movement. It must be admitted, however, that it is possible to imagine a retrogressive development by way of which the form of medusa evolves into something that resembles a polyp form. Such processes have actually taken place both among Scyphozoa (Lucernariidae) as well as among Hydrozoa (very numerous instances). Yet in all appearance no genuine polyp has ever been developed in this way, but only some polypoidal forms. In the Lucernariidae this has led to a polypo-medusoid form which can be easily recognized as such; in Hydrozoa we can find medusae which are in this way strongly degenerated (resimplified) inasmuch as they have remained freely swimming animals, (see Fig. 16) or they have been degraded into the organs of cormi by becoming attached 
as buds to the latter (see Fig. 17); and, finally, they have completely disappeared when they have abandoned the state of cormi (the form of a colony). This has been more than probably the case with the freshwater Hydrae.

Again and again the adherents to the idea of the primacy of the medusa form refer to the hypogenetic trachylinae Hydromedusae (Narco- and Trachy-medusae); yet these schol-
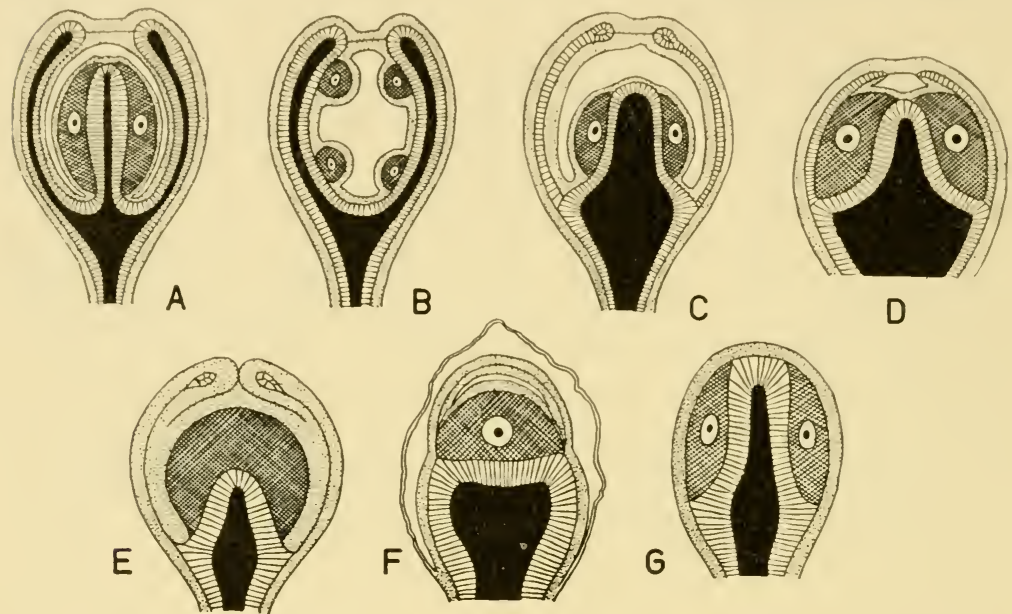

D

Fig. 17. Progressive reduction of sessile medusae (medusoids). A, B, Eumedusoids of Tubularia and Campanularia. C, D, cryptomedusoid (Gonothyrea, Clava, etc.). E, heteromedusoid (Grammaria sp.).

F, G, styloids (Eudendrium, etc.). (After Kühn and Broch.)

ars are inconsistent because they do not place Trachylinae in a classification, which is supposed to be natural, at the beginning of Cnidaria, but rather-which is actually quite correct-close to their end, usually as the penultimate group, followed by Siphonophora, i.e. they classify them as one of the most specialized groups (cf. the system proposed by Hyman).

The hypothesis of an actinula as a primitive form and as an ancestor of Narcomedusae as well as of Hydroidea, and thus of all Hydrozoa, and in the last line of all Cnidaria, had 
been earlier defended by Hjalmar Broch, the author of articles on Hydroidea and Narcomedusae in the extensive German work Handbuch der Zoologie (1924), edited by KükenthalKrumbach. Broch considered budding to be a common and a very old property which had thus been introduced independently in the planktonic Narcomedusae. This violates the generally accepted rule that budding is due to the sessile way of life and that it can be transferred afterwards to descendants which have later adopted the pelagic way of life (e.g. parasitic larvae of some Narcomedusae). Another difficulty the adherents to the actinula hypothesis meet is the question of the origin of Hydromedusae in the benthonic cormi (colonies). One can refer here to the proposition made by Goette (1912) who tried to derive the metagenetic forms of Hydromedusae from the sessile medusoids.

Here we must briefly touch one of the extreme and least probable hypotheses which try to derive the Cnidaria from the primitive forms that live in the pelagic zone. This is the so-called medusom theory which has been proposed by F. Moser (1921-1924). During her study of Siphonophora she came to the idea that the larvae of Siphonophora might correspond exactly to the primitive form of Hydrozoa and, in connection with this-even if she does not state it explicitly-of all Cnidaria. First numerous medusoid organs have developed which have later evolved into full members i.e. persons (subindivides) of colonies. In the benthonic colonies of the Hydroidea which are a result of a later development, hydromedusae and polyps have again evolved on their own account. Hyman (1940 I: 636), too, considers this hypothesis to be "not very convincing," and I have submitted it to a severe criticism (Hadži, 1926) in which I have shown its complete untenability. It is certainly absurd to try to find the origin of Hydrozoa in Siphonophora, even in their most primitive forms, since there can be no doubt that they really represent the climax of a progressive and specializing evolution of the colony of Hydrozoa. The very first premise in the 
concept proposed by Moser-according to which the form of the ancestors of Cnidaria is recapitulated in the siphonula, or in the larva of Siphonophora-is unacceptable and wrong; and the consequences of this supposition are even more so. An individual can never be developed out of an organ, neither can a polyp be evolved out of a medusa. According to this hypothesis proposed by Moser, the Hydromedusae had been developed four times independently from each other, both in plankton as well as in the benthos. It can be truly said that, fortunately enough, Moser has never been followed by anybody else in this interpretation she had proposed. It must be regretted that such an obviously improbable construction could have found admittance into a representative manual on zoology. The construction itself has a purely historical interest now as an entirely erroneous attempt.

Finally we must mention here also the case of Halammobydra, an aberrant Narcomedusa (see Hadži, 1959 b), Halammobydra is actually not the only Hydromedusa that has readopted a benthonic way of life, yet in their evolution they have developed farthest away from the typical medusa form because they have become adapted to a life in sand on the sea bottom (Fig. 18). One could expect that they would evolve into a polyp form. Yet here the validity of Dollo's rule of irreversibility has again been proved. Though Halammobydra has undergone a very considerable change, this change, has not been so intensive (fortunately enough for zoologists!) that their nature as Narcomedusae could not be clearly recognized. The supposed polypoid characteristics which have been thought to have been identified by A. Remane, the discoverer of this aberrant form, and by other scholars, are in reality the excessively changed medusoid properties only, the general polypoid shape is due to a strong reduction of the umbrella and to a considerable lengthening of the manubium. It is interesting to notice that a general ciliation of its skin has secondarily reappeared in this medusa, a case which has been explained by A. Remane as an indication of a neoteny. Thus 

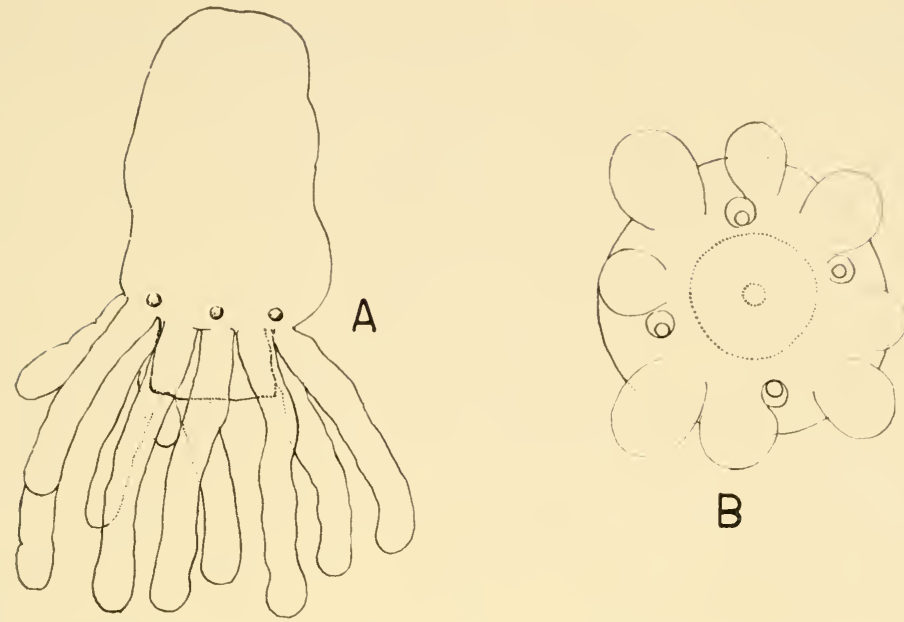

Fig. 18. Aberrant benthonic narcomedusa: Otobydra vagans Swedmark \& Teissier, with preserved otocysts; A, side-view, B, oralview. (After Swedmark and Teissier.)

Dollo's rule (which was proposed, according to L. Cuénot, first by Edgar Quinet, [1870]) does not seem to be valid for individual characteristics which are probably due to the mutation of a single gene. We can conclude, as a result of this discussion, that without any doubt the polyp form is the primitive, the initial form in all Cnidaria, and that this form has been secondarily developed into the medusa form. Later we shall try to show how this development took place twice and independently from each other.

\section{The Right Sequence of the Cnidaria Groups}

The second major problem we meet in our study of Cnidaria is whether we should arrange the three generally accepted subgroups of Cnidaria which have been given the title of classes, as one after the other, i.e. Hydrozoa-Scyphozoa -Anthozoa, or conversely. It should be remarked in passing that repeated attempts have been made, particularly by 
Goette, to prove the close relationship between Scyphozoa and Anthozoa and to unite them into one class. The main reason for these attempts has been the supposed presence of an ectodermal pharynx both in Scyphozoa as well as in Anthozoa. There can be no doubt that an ectodermal gullet does really occur in Anthozoa. On the other hand, I succeeded in showing several years ago (Hadži, 1911), that the Scyphozoa do not possess an ectodermal gullet. It has even been found that the opposite is really true, i.e. the oral area of the scyphopolyp has been raised to an oral cone so that the limit between the ectoderm and the entoderm runs along the oral margin. Even if the taeniolae of scyphopolyps represent the remains of the sarcosepta that can be found in Anthozoa, they have nevertheless been developed in their own direction. In this way the division of the two subgroups of Cnidaria into two independent classes seems to be fully justified.

If we disregard the form of medusa and if we take into consideration the polyp form only, it could seem at first sight to be justified to see in the sequence Hydrozoa-Scyphozoa--Anthozoa a progressive line of development. This would fully correspond to the principle of evolution of a generally progressive development. It is therefore understandable that this sequence has generally and incontestably been regarded as the sequence which agrees with the line that evolution had taken; we can say that it has been accepted as a postulate which nobody has even tried to touch or doubt.

To this should be added the fact that in connection with Hydrozoa, and with the solitarily living Hydra,-because of the recapitulation theory - we have an example of a metazoan that embodies an ideal transition from Flagellata among Protozoa to Metazoa and the ideal pattern of a gastraea composed of two body layers. The difference between Hydra and gastraea consists in its circlet of tentacles and in its life on the bottom only. It was easy to derive first the scyphopolyp and later the anthopolyp form Hydra as the primitive form of all Hydrozoa. Everything seemed to agree well. Nothing 
amiss was seen in the fact that in Anthozoa there is one polyp form only and no medusa form. Even among Hydrozoa as well as among Scyphozoa there are species that possess no medusa form, and it was possible that the Anthozoa, which have no medusa form either, had completely lost this form and generation.

An important mistake was made in the evaluation of the conditions that prevail in the Cnidaria. The generally valid rule that progressive evolution takes place in connection with the normal way of life, with free movement, and the type of feeding, had been forgotten or at least neglected. There is always a more or less distinct retrogression in the organization - and thus a regressive development-when the free movement is abandoned and the sessile or the sedentary way of life is adopted. This, however, does not exclude the possibility that in spite of a general retrogression of the organization a progressive development could be observed in some details, i.e. in the new developed strictly adaptive characteristics. Thus this rule whose general validity has already been proved in connection with the study of Protozoa (compare Euciliata with Suctoria!) would not be valid in connection with Cnidaria!

The regressive, or, as it has also been less fortunately called, degenerative development of Cnidaria refers to their basic form, i.e. to the form of polyp. Yet the secondary form of medusa supports, if it is interpreted correctly, the idea of a reirogressive evolution in Cnidaria because the emergence of the medusa form (which has actually twice taken place, first in Scyphozoa and later in Hydrozoa) agrees with this rule since the release of a polyp represents the beginning of a new progressive development. The medusa as a freely living form which swims actively in plankton has reached an unquestionably higher level in its organization. The same medusae, however-and this is true both for Scyphomedusae as well as for Hydromedusae-can retrogress so that they cannot even be discerned any longer. They even become "degenerated" (in this case the usage of this word is approp- 
riate), to the level of an organ and, finally, they disappear without any trace when the animal adopts the colonial status.

It would suffice to take into consideration only the unavoidable consequences of the sedentary way of life in order to show the untenability of the concept which has been proposed of a progressive evolution in Cnidaria. Further proofs of the accuracy of our interpretation can be made by a morphological comparison together with a simultaneous consideration of the ecological condition.

First we shall discuss here the symmetrical conditions; they are the most conspicuous element and they have actually been the first reason why scholars have begun to doubt the accuracy of the idea of a progressive line of evolution in Cnidaria. The palaeczoologists were the first who became aware of this fact; in connection with a detailed analysis of the internal skeleton of Madreporaria among Anthopolyps they have discovered that the sclerosepta do not agree with the radial symmetry of these animals as one would expect particularly if we consider the Anthozoa as the most highly developed Cnidaria. It was Otto H. Schindewolf (1930) who has especially discussed this fact in great detail. We can read in his book Grundlagen der Paläontologie (1950, Stuttgart, p. 179), "Die erste Anlage des Septalapparates bei den Pterokorallen ist bilateral; die sechs Protosepten werden ausgesprochen zweiseitig-symmetrisch herausgebildet. Erst nach ihrer vollen Ausgestaltung tritt erstmal eine radiale Symmetrieform hervor, und zwar ist diese hexamer, sechszählig." To this he continues, "Die Radialität beschränkt sich jedoch nur auf ein vorübergehendes Stadium." Similar conditions can be observed in numerous recent Anthozoa, particularly those that live as solitary animals.

The palaeozoologist O. Kuhn (1939) has been lead by this important fact to state, in spite of his reserved attitude with regard to the "Wurzel der Coelenteraten," that the ancestral form of Cnidaria must be imagined as a bilaterally built, creeping, or freely swimming organism. 
We can observe in Cnidaria, as this has already been the case with Ctenophora, that they also adhere to the rule according to which the arrangement of the internal organs or parts towards symmetrical conditions, is much more definite than that of the peripheral organs. The external form soon becomes changed to a radially symmetrical construction due to the sessile way of life or posture when the animal is firmly attached at one end of its body; this has taken place in such a way that the body which was initially more or less flattened, took on a cylindric form. The earlier ventral and dorsal sides which had previously slightly differed from each other have thus obtained an identical form; the tentacles which in all probability had been earlier arranged in the form of a bow or of a horse-shoe and whose number has been increased by way of polymerization, have now developed into regular circlets. Yet at the same time we find that in the digestive organs of anthopolyps-and this is actually the septal apparatus-the remains of an earlier bilateral symmetry have been preserved. In ontogeny such traces have also been preserved in the process of the formation of tentacles. Some insignificant remains of this old bilateral symmetry can also be found in the young scyphopolyps (the so-called scyphistomae). Any trace of a former bilateral symmetry, however, is completely lost in the adult Scyphozoa and particularly in the Hydrozoa.

There have been some neo-zoologists who did not take easily to the fact of an internal bilateral symmetry observed in Anthozoa. As early as 1879 it has been proposed by Haake (according to Pax 1954, 288), that the primitive form of "Zoophyta" must have been bilaterally symmetric and that it has later become radially symmetrical because of their later transition to a sessile way of life. Professor Dr. Ferdinand Pax, an expert on the Anthozoa, pointed to the fact, in 1914, "dass viele der primitivsten Aktinien, die noch einer Fusscheibe entbehren, durch eine bilaterale Symmetrie ausgezeichnet sind" (Pax, 1954:2889). On the basis of this Pax concluded, "Man könnte daher geneigt sein, die Aktinien von fusslosen, 
kriechenden oder grabenden Formen mit bilateraler Symmetrie abzuleiten, die später mit dem Übergange zur festsitzenden Lebensweise eine radiale Symmetrie erworben haben...". However this was as far as Pax could go. In the extensively planned Handbuch der Zoologie where Pax contributed the section on Hexactiniaria the old classification has been preserved: following the example of Kückenthal we find the Anthozoa as usually placed at the end of the system of Cnidaria.

A similar statement was made by the palaeozoologist $O$. Kuhn (1939) whom I have already mentioned above, according to which all theories must be rejected which "von den Radialien (Coelenterata) die Bilaterien ableiten und in der Ontogenie von Larven den Übergang von der Radialität zur Bilateralität suchen". This statement has had practically no effect whatever (cf. the books by Hyman, Kaestner, etc.) on subsequent authors.

It is interesting to observe the attitude of neo-zoologists, in their general treatments on Cnidaria, towards the fact of internal bilateral symmetry or bisymmetry in these animals. A. Kaestner (1954) in his recent work on zoology does not even take into consideration this sufficiently important circumstance. At the beginning of his book we can only read (1954:9) that "die Coelenterata (9,000 Arten) sind äusserlich gekennzeichnet durch ihren radiären Bau." In the special part (1954:118) we find the brief explanation, "Ihre Anordnung (sc. of mesenteria) bringt wie der spaltförmige Mund einen Zug bilateraler Symmetrie in die radiäre Anlage der Anthozoa," and, slightly later (1954:121), "die bilaterale Symmetrieachse der Seerose" is briefly mentioned. No explanation is given where this disturbance (as it can be justly called) of an otherwise radial symmetry comes from, and of its significance.

Something else can be found in the manual by Hyman. She does not ignore the actual situation, instead she tries to find some explanation of the internal bilateral symmetry of Anthozoa-a most unpleasant fact for all the adherents to the 
old interpretation - of a planula-like primitive form of Cnidaria and of the primitiveness of the Hydrozoa. Yet at the same time Hyman does not draw the necessary consequences. First she brings forward some older attempts which had tried to explain this bilateral symmetry. When she first mentions this phenomenon (Hyman I. p. 539) she makes the correct statement that the internal bilatera! symmetry of Anthozoa must be a primary property and that it could not have been later developed out of a radial symmetry, a fact which appears especially clearly in their embryology. However, Hyman explains at the end of her discussion that this bilateral symmetry is a secondary phenomenon.

On page 640 Hyman discusses this problem in more detail. She mentions as the first possible interpretation the opinion that the bilateral symmetry could have been developed secondarily as a consequence of the "colonial life." Hyman is right when she rejects this interpretation because the same symmetry occurs also in Actiniaria and Ceriantharia which are primarily solitary animals. To this we could add that in the "colonial" Hydroidea and Siphonophora cases are not rare where the externally developed bilateral symmetry is clearly a secondary phenomenon. The second interpretation mentioned by Hyman and which is, in my opinion, the right one, proposes that the present Anthozoa must go back to ancestors that had crept over the sea bottom, because bilateral symmetry appears in such animals. It can be easily understood that Hyman cannot consider this interpretation to be a good one because it stands in opposition to her concept of the origin of Cnidaria. A third interpretation which has been especially supported by some palaeontologists is based on the idea that the old Anthozoa which had otherwise "stood" erect, had lain down on one side, as must be supposed for some Tetracoralla, and it was in this way that the bilateral symmetry has been developed. Jägersten (1955:331), however, believes that this does not explain the origin of the bilateral symmetry because, "the eccentric attachment was simply a part of the bilateral symmetry." 
Hyman finally proposes her own interpretation which she naturally thinks to be the best and the one most acceptable. Bilateral symmetry is a result of a secondary development and it is due to the increased size of anthopolyps and, in connection with this, to an increased need to send water necessary for respiration into the interior of the body by means of a quick water current which is produced by siphonoglyphs. A normal round oral opening was not suitable for this purpose; it was for this reason that the mouth has become elongated. The bilateral symmetry in the arrangement of sarcosepta is a consequence of such a change. Even if we could accept as probable this explanation of the origin of the elongated oral form and of the siphonoglyphs, we must nevertheless reject it in view of the fact that the bisymmetry (or the bilateral symmetry) does not occur only in the arrangement of the sarcosepta but it can also be found in the arrangement of the septal muscles which, are not brought into connection with the water current-a fact which has already been pointed out by Jägersten.

Thus we can conclude that so far nobody has succeeded in explaining the origin of the internal bilateral symmetry observed in the Anthozoa on the basis of the presumption that it is a secondary phenomenon.

Recently G. Jägersten (1955) has come to the conviction that in the Anthozoa the bilateral symmetry must be a primary property inherited from their ancestors. Jägersten, however, has come to this conviction along a different way from the one I have taken and, as he maintains (Jägersten, 1955:333), independently of me. In spite of this Jägersten uses the same arguments I have used, and proposes to reverse the evolutionary sequence which has so far been considered as valid within the system of the group of Cnidaria.

Above all Jägersten was interested in the origin of Metazoa, and especially of Coelomata. He believed that this problem could be solved best if he modernized the old gastraea theory invented by Haeckel. For the purposes of such a modernization he constructed a hypothetical intermediate stage which he 
called a Bilaterogastraea (Fig. 55). He placed it between the blastea which had lived in plankton and those Metazoa that actually exist. He believed that this was an animal which had crept over the sea bottom, and in this it differed from the classical planktonic gastraea. Jägersten's theory of a bilaterogastraea is essentially a variant of the old theory of Enterocoela which had been proposed by Leuckart and Sedgwick. The only difference is that here the Anlagen of the three pairs of the future coelom cavities are connected with a somewhat higher stage of development than the old gastraea had been. This is a typical suitcase theory (Koffertheorie) which is so general and allembracing that with only a small modification it will explain anything! I have examined the opinions proposed by Jägersten (Hadži 1955), and I have rejected them because I have found this interpretation improbable and unacceptable. Jägersten's ideas will necessarily have the same fate as the ideas of the blastea and of the gastraea which had been proposed by Haeckel. These ideas have finally had to be abandoned and there is no modernization which could help them. In Jägersten's concept, there are individual points only that are correct, e.g. his opinion that the Cnidaria had been originally bilaterally symmetric animals, that the Anthozoa are the oldest form of Cnidaria, that primarily they had no medusoid form, and, finally, that cnidae had been progressively developed within all the group of Cnidaria. All this was already published by me years ago. Later we will return to Jägersten's proposal to derive Metazoa from the Protista.

\section{Why Is There No Medusoid Form in Anthozoa?}

So far scholars have carelessly bypassed the fact that there is no medusoid generation in Anthozoa. This has simply been taken for granted, an attitude that is partly understandable on behalf of the adherents to the thesis that the Hydrozoa are the $8^{*}$ 
oldest Cnidaria. It has been quietly accepted that there is no longer any medusoid form in Anthozoa and that such a form had existed only in their ancestors. Yet in spite of this, this "simple" fact should have been thought over. How is it that we see Hydrozoa appear suddenly with both generations so that they immediately develop the two "essential" forms; that the Scyphozoa had been developed out of the Hydrozoa while at the same time it is supposed that they possess only the medusoid form so that they have been called Scyphomedusae, and their scyphistoma considered to be a larval form; and, finally, in the supposed subsequent evolution into the Anthozoa, the highest developed Cnidaria, we suddenly see the polyp form now as the only form in existence! One cannot help feeling that here something must be wrong. How can we explain the complete absence of the medusa form in Anthozoa, or, at least, how can we make it probable?

One would expect, if the Anthozoa had secondarily lost their medusoid generation, that at least some traces of it could still be found. Yet, as a matter of fact, not the slightest trace of a medusoid generation can be identified. There have been numerous enough opportunities for its preservation. The Anthozoa, too, have larvae (actinulae) that live in the plankton and that partly show, like those of Echinodermata, an inclination to bilateral symmetry. They do not show, however, any trace whatever of a recapitulation of the medusoid formation. Planktonic Actiniae are known (Minyidae) which even possess a special apparatus that enables them to float more easily in water and which, in spite of this, do not show any common element with a medusa. Thus the name of "Anthomedusae" has been used for a subtype of Hydromedusae without fear lest it should be understood as a medusoid form of a subtype of Anthozoa. If there were any gene or complex of genes (any "trend") among the inherited properties of Anthozoa to form medusae this would have been apparent in their abundant cormi (colonies) as traces of the polymorphism. In these cormi both budding as well as fission can be observed, 
yet we always find that pure anthopolyps emerge as a result of such a development.

We come now to the phenomenon of transfission which the Cnidaria, and above all the Anthozoa, have inherited, in our opinion, from their turbellarian ancestors. The conditions in Gonactinia, a solitary animal that possess no skeleton, are very simple and resemble to a surprising degree those that can be observed in several rhabdocoelous Turbellaria. One species among the Madreporaria that is most prone to form cormi, has excited a great interest of zoologists. This is Fungia. In it the alternation of generations connected with transfission has been introduced, in spite of its well developed and comparatively heavy calcareous skeleton (Fig. 28 p. 155). It closely resembles the alternation of generations that can be observed in Hydrozoa and in Scyphozoa, particularly in Scyphozoa, because of the unequal transfission (which is close to the axial budding). The distal half of the maternal animal becomes considerably broadened and flattened; it liberates itself and, though completely passive, it is caught up by the water current and it settles down with its flattened side on the sea bottom not far from the maternal polyp. This anthopolyp, which is in its form only slightly changed, takes over the sexual function. The maternal polyp by way of an unequal transfission produces another sexual polyp, which is again separated from its maternal body.

Though we are reminded strongly by this situation of conditions that prevail in the metagenetic Hydro- and Scypho-zoa, we find nevertheless that in Fungia the sexual generation corresponds to that of an anthopolyp, and there is therefore no trace, or no trend, (with the exception of its flattening) to a medusa form. Here we can raise the question: could Fungia perhaps be a very primitive species of Anthozoa so that it could be considered to be the beginning of the formation of medusae which has finally led, in the remotest geological past, to the metagenetic subtype of Scyphozoa with medusa as their sexual generation? This is completely improbable, and on the contrary, Fungia appears to be a specialist among Madreporaria. 
Its solitary state is probably a secondary phenomenon, developed out of living in cormi, a fact which seems to be supported by its budding which exists to a slight degree only and which no longer leads to the formation of a colony. The main element of the asexual reproduction, or, rather, multiplication, is a transfission with a simultaneous transmission of the sexual function to the freely living (not freely moving!) generation. It is clear that such a situation must be considered as a special "invention" made by Fungia. Yet it nevertheless represents an element of the formation of medusae-all this in the sense of Watson's mosaic theory.

It seems much more probable, even if we do not take into consideration the basic problem of the origin of the Anthozoa, that there is no primary medusa form in Anthozoa. If we combine this with the conclusion that in the Anthozoa the internal bilateral symmetry is a primary property, we come to the more probable conclusion that the Anthozoa are the most primitive Cnidaria. In case one would like to stick to the old "belief" in the primitiveness of the Hydrozoa, it would be necessary to take refuge in the comfortable supposition that the Anthozoa had been separated very early from an indifferent ancestor of the Cnidaria. This auxiliary solution, however, is improbable and it does not offer a rational explanation.

\section{The Primarily Solitary Polyps Appear in Anthozoa Only}

The next piece of evidence of the primitiveness of the Anthozoa is the fact that it is in the Anthozoa only that the primarily solitary species can with certainty be found. So far, too little significance has been attributed to this fact, or it has not even been taken into consideration. Everywhere, in all the three classes of Cnidaria, solitary species have been found (here the polyp form can only be considered!), and they all have usually been thought of as primarily solitary species. 
Hydra was considered as a prototype of all Cnidaria; it usually appears solitary, and it forms only small ephemeral cormi (colonies), a phenomenon which is usually interpreted as a tendency towards the evolution of colonies. In spite of the fact that it can now be shown to be proved that the solitary character of Hydra is a secondary phenomenon and that it is far from being a primitive form, there are even now numerous zoologists who find it difficult to accept this fact. An interesting parallel to Hydra can be found in the species Monobryozoon ambulans Remane (Remane, 1936), which until recently has been thought to be the only solitary ectoproctan (recently, however, another such species has been described); yet in this case the only difference has been that the discoverer of Monobryozoans had immediately been able to establish that in this species the solitary state was a secondary phenomenon. This was not difficult to establish in view of the fact that in this form it shows that it goes back to corm-forming ancestors. The number of solitary species of Hydroidea is comparatively high. A closer comparison with the related species or genera enables us to discover again and again that this solitary life is a secondary phenomenon. Not infrequently we can find all kind of transitions, from species with typical cormi (colonies), and those that, though solitary, still show, clear traces of a former formation of cormi, up to the completely solitary species (e.g. in the hydroid family Tubulariidae). The last remaining trace of a former formation of cormi is the formation of podocysts (Hadži, 1912), i.e. the parts of coenosarcs or of the cellular mass which are covered by the periderm (i.e. cuticule) and which otherwise form the impersonal part of a cormus, above all the hydrorhiza. In our case they take over the role of a resting stage and serve the purposes of asexual reproduction (Fig. 19).

As early as in the Anthozoa, as well as in all the truly sessile animal types up to Chordonia, we can observe an inclination either to an imperfect transfission or to the formation of buds with a simultaneous preservation of an organic contact between the secondary zooids and the maternal polyp. Thus we 
can see that in the whole large group of Octactiniaria among Anthozoa, not a single primarily (in this case not even secondarily) solitary species does appear. Those species that have occasionally been described as solitary (e.g. Haimea, Monoxenia, etc.) were primary zooids (or oozooids) only before they began to develop further into colonies. This situation in Oct-
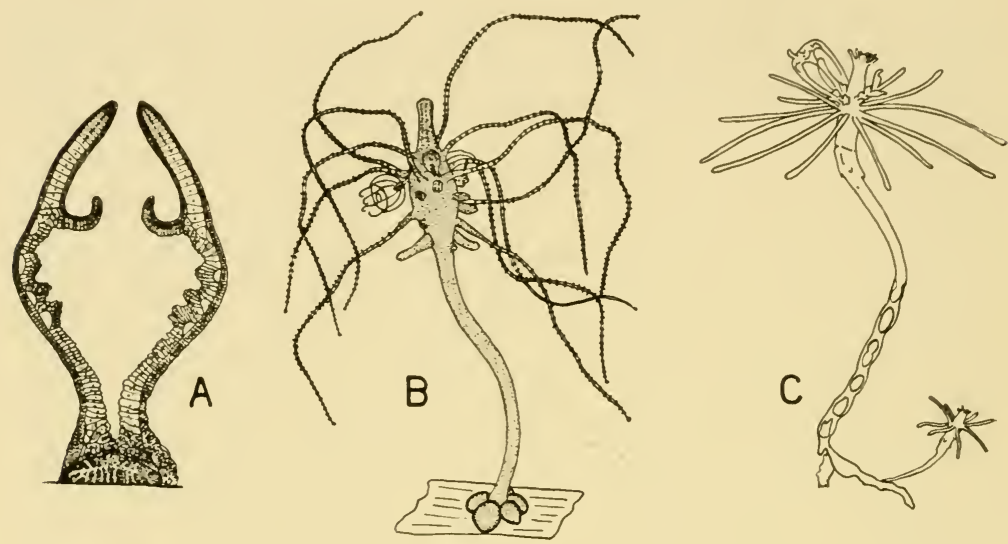

Fig. 19. Podocysts or resting bodies as remains of rhizome. A, scyphopolyp of Cbrysaora byoscela (after Hadži). B, hydroidpolyp of Ostroumovia barii (after Uchida and Nagao). C, Hypolytus sp. (after Miles).

actiniaria, naturally in connection with other properties and characteristics, proves that Octactiniaria do not correspond to the primitive subtype of Anthozoa as this has frequently been believed. The Actiniaria can with all certainty only be considered as primarily solitary animals (with, as it seems, one species only which forms colonies), as can also, in addition to these, the Ceriantharia with their partly rounded off and partly flattened aboral end.

In the Scyphozoa the abolition of the colonies of polyps has gone furthest. In these the species with cormi, which have a completely irregular growth, are a real rarity now (the socalled Stephanoscyphus, the polyp generation of Nausithoë); there 
are, however, numerous species where the polyps have already become solitary individuals, yet with a periderm which they have still preserved as their external skeleton; the periderm must be interpreted as a remnant from the former formation of colonies; in numerous other species the scyphopolyps are completely "naked," i.e. they have no periderm, yet in spite of this they still continue to reproduce by way of budding or by means of the formation of stolons. In not one single case in the Scyphozoa has polymorphism been developed.

\section{Polymorphism Has Reached Its Climax in Hydrozoa}

Polymorphism (dimorphism when it is least developed) in the cormi of polyps is another piece of evidence which supports our thesis. It is a sign that a higher stage in evolution has been reached when this polymorphism appears in combination with the individualization of the cormus (colony). In the Anthozoa the dimorphism of polyps has been developed among the Octactiniaria only, a fact which can again be considered as a proof of their higher condition in comparison with Hexactiniaria. Siphonozooids, whose number has been rather reduced, appear either in species that form irregular cormi, e.g. in the red coral (Corallium rubrum L.), or in species with regularly, and thus individually, formed cormi e.g. Pennatulacea. No cases of polymorphism have been known in the Scyphozoa and this can be easily understood since they form, with a few exceptions, no cormi.

The highest level in differentiation, and thus of specialization, has been reached in the Hydrozoa, a clear indication that in them the Cnidaria have reached a climax in their evolution. In these polymorphism can even be found in the medusoid form, i.e. in the mixed polypo-medusoid cormi of Siphonophora that live in plankton, with a simultaneous individualization of cormi. Nothing similar can be observed in 
Scyphozoa. In Hydroidea the polymorphous cormi usually appear with one medusoid form only and they are mostly irregularly built; the only regular form is that of a plume (Pennaria) among Athecata and in the numerous species of Plumulariidae among the Thecata (Fig. 20.).

There are many other properties and characteristics of Cnidaria which can be understood only if we interpret their phy-
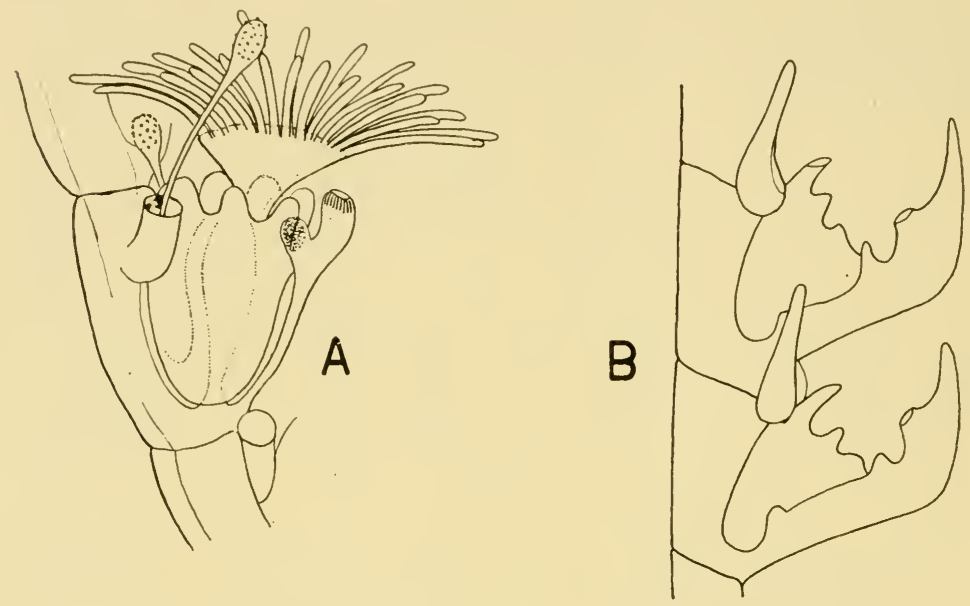

Fig. 20. Polymorphic hydroidpolyps organized as a subcormus or cormidium, composed of a gastropolyp and a few heterozooids (machozooids). A, Aglaophenia sp. (after Kühn). B, Halicornaria cornuta Allman (after Allman).

logeny as having followed the direction Anthozoa-Scyphozoa-Hydrozoa. In our analysis of these properties we must be constantly aware of the fact that two parallel processes are taking place in connection with the unique specialization which the sessile way of life entails. One process is that of regression, the retrograde development which has also been unsuitably called, as has already been mentioned, degeneration (cf. Laubenfels [1955], and the reply by M. Zalokar [1955]). During such a process all characteristics and peculiarities which serve the purpose of a free movement are gradually lost. 
The change in symmetry of the form from a bilateral to a radial symmetry, and simultaneously to the general polyp form of the entire body, is connected with this. On the other hand, we can find progressive morphogenetic processes in individual organs. Here we have first to mention the progressive development of the cnidae together with the tentacles; the strong extensibility connected with an increased contractibility (a special development of the cutaneous muscle tube), connected with the ability to move in all directions (nutations). We must certainly attribute the evolution of secondary freely swimming medusae, and, finally, the liberation of whole cormi (Siphonophora) and the formation of a special impersonal floating apparatus, the pneumatophore (cf. Hadži, 1954), to such progressive properties.

Both regressive as well as progressive developments have reached their climax in the Hydrozoa which can be easily understood if viewed from our standpoint. So far it has been the regressive element in particular which has been taken into consideration, and it has been wrongly interpreted as a sign of a primary simplicity. This has been especially done because of the epithelization of the three body layers which is a characteristic of all the sedentary groups of Metazoa; the intermediate body layer can be strongly reduced by way of such an epithelization so that locally it can almost completely disappear. It was in this way that T. H. Huxley concluded (1875) with regard to medusae, and Allman with regard to Hydroidea, that these animals consisted of two body layers only, an ectoderm and an entoderm. This circumstance, together with events that take place during their ontogenies, was used by Haeckel when he formulated his gastraea theory. As a matter of fact, however, there is not a single species in Cnidaria which would really consist of two "skins" or epithelia only. If we disregard sex cells which cannot be properly attributed to any body layer even in those cases where they adhere closely either to the ectoderm or to the entoderm, there have always been found other cells between these two 
epithelia which have been given various names and which have been variously interpreted (indifferent, interstitial, mesenchymal, mesogloeal cells). These are frequently hidden between the somewhat dehiscent basal ends either of the one or of the other, or, finally, of the two epithelium layers. Neither during their ontogenies, nor in their fully developed state do these cells represent a compact layer or an epithelial organisation, and so the name mesoderm which is frequently used in this connection is wrong. I have therefore proposed a new name instead of the former: mesobyl.

\section{The Regressive Development of the Intermediate Layer in Cnidaria}

I must emphasize, when we first make a brief study of the mesohyl, that it is most developed in the Anthozoa where it often contains even muscle cells. This layer can be found well developed in the tentacles of anthopolyps. In the Scyphozoa and in the Hydrozoa it has almost completely disappeared and it is only occasionally revived by the actively moving cnidocytes (Hadži 1907, 1911 b, c). In the Cnidaria among others a jelly-like hyaline substance which plays an important role is secreted into this middle layer (Fig. 21). This so-called mesogloeal layer has usually been wrongly interpreted as a limit between the entodermal and ectodermal epithelia. Whatever occurs on one side of this layer has been attributed to the entoderm, and whatever appears on the other side has been attributed to the ectoderm, and in this no exception has been made even in connection with gonocytes. Attempts have even been made (Hertwig) to use this as a basis. for a systematic division of Cnidaria into Ectocarpa and Endocarpa. We really get the impression that Nature herself had tried to make it easier for zoologists to differentiate between the two body layers which alone have been supposed to exist (Diblastica, according to Ray Lankester). 
The layer of the jelly-like substance partly takes over the role of an extensible internal skeleton as early as in Anthozoa, and even more so in the Scyphozoa and in the Hydrozoa, concordantly with its new position: the appendices of the muscular tissue of the two epithelia, which find support in this skeleton,

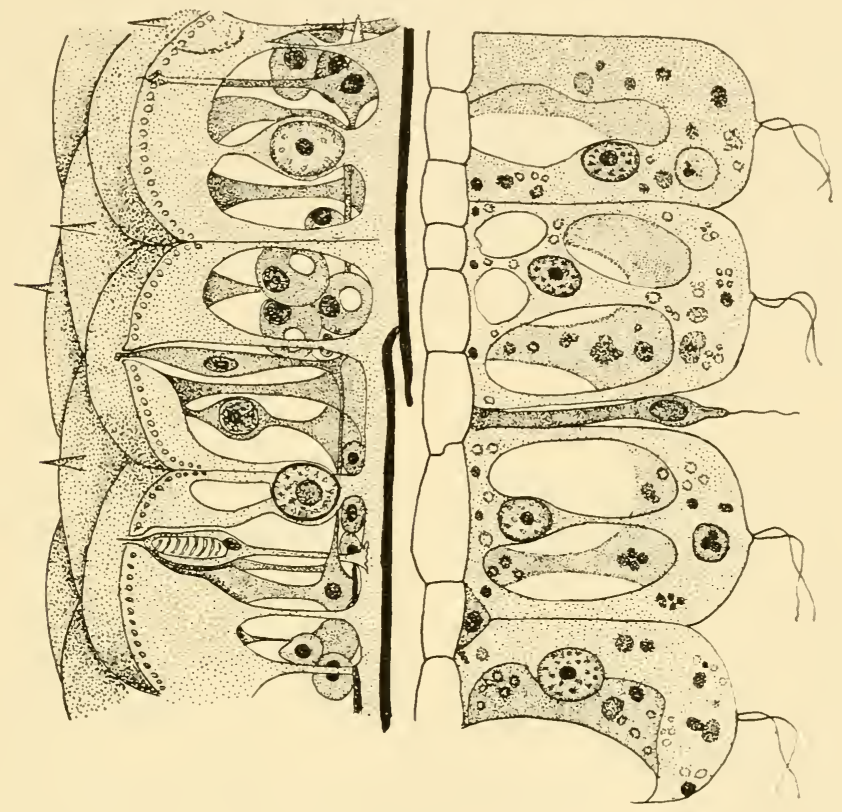

Fig. 21. Half schematic longitudinal section of the body wall of Hydra; left, the skin, right, the gut; centre, the mesohyl (after Hadži).

passively share the stretching and the shrinking which takes place during the thickening and the flattening of the body. This is true for the actively moving parts of polyps. In medusae this layer of a jelly-like substance can occasionally be of considerable size; here it represents a firm elastic substance which takes over the role of an antagonist to the rhythmically contracting subumbrellar muscles. In the Anthozoa and in the Scyphozoa this layer of a jelly-like substance contains individual, usually amoeboid, mesohyl cells, while in the Hydromedusae 
no such cells can any longer be found. Thus we see that this intermediate layer represents a property which has been developed in Cnidaria partly progressively and partly regressively.

In this connection it is interesting to observe that these mesohyl cells-whether they are cnidocytes, or genital cells, or I-cells-do not heed the imaginary limit between the two epithelia. We can observe how these cells freely pass this "limit" in their amoeboid movements (Hadži, 1907, 1911). I have been able to show this both during the formation of buds in Hydra (Hadži, 1909 a, b) and in other Hydrozoa (the medusa buds at the manubrium of Hydromedusae, Hadži, 1912 d), as well as during the widely practiced migrations of cnidocytes (Hadži, 1907 a), and of the primitive sex cells, as had been formerly shown by Weisman (1883).

\section{The Morphological Proof of the New Interpretation of the Phylogeny of Cnidaria}

\section{Muscular Tissue}

Let us study now the muscles of Cnidaria. We must attribute these to the middle layer even in those cases where the contractile fibres do not form an independent middle layer, so that it appears only as an appendix either of the ectodermal or of the entodermal epithelia. Such a situation is generally considered as a very primitive one. We wish to show here that the same is true for the muscles as has been said for the whole middle layes, i.e. their development has been progressive or regressive, concordant with the changes of the type of movement. The consequence of the sessile way of life has been a simplification of the cutaneous muscle tube, and, parallel to this, a specialization of the body muscles in the movements on a given spot. A new specialization has taken place during the secondary transition to the free movement, which applies especially to the medusoid form, yet this specialization again 
follows Dollo's rule and it does not represent a return to those conditions that had been observed in the movements of their creeping ancestors.

We will maintain, even if this may appear surprising, that it is right to attribute the muscular tissue of Cnidaria to the middle layer even in those cases where it appears as a basal appendix of the epithelium cells of the ecto- or entoderm and where it does not possess its own nuclei, i.e. where it does not consist of independent cells. In numerous anthopolyps we can observe how this muscular tissue subsequently develops locally into independent cells with their own nuclei. Chester (1912:755) observed in the species Psendoplexaura crassa how the epithelial cells (they were classified as entodermal cells) that occur in mesenteries and that had each previously produced a single differentiated muscle fibre, leave the epithelial group and sink into the subepithelium, i.e. into the intermediate layer, and in this way develop into the pure muscular cells.

We can obtain a good understanding of the muscular conditions in Cnidaria only if we derive them from conditions that can be observed in Turbellaria, and if we pass from the situation that can be observed in Anthozoa to those that occur in the Scyphozoa and in the Hydrozoa. A very misleading idea of the primitive nature of the so-called neuro-muscular-epithelial cells has been proposed by Nikolaus Kleinenberg. According to this interpretation such a cell has differentiated in three directions, into the pure epithelium cells on the surface, and into the muscle and nerve cells in the interior of the body. With regard to the nerve cells it soon became necessary to relinquish this concept after the nerve cells had been discovered in Hydra. Yet in spite of this, the idea of the primitive character of the muscle epithelium cells has been preserved down to the present day. H. Wolf (1903) developed this hypothesis for the nerve cells and supported it with beautiful, even if completely imaginary, pictures to show that they originate, like the muscle cells, in the immersed epithelium cells. It has seemed that this interpretation was supported by the 
fact that during the ontogeny, the Anlage of the nerve system emerges in close connection with the Anlage of the ectoderm, a fact which can especially be observed in the higher developed animals.

The destiny of the muscular system of Cnidaria has been completely determined by the transition to the sessile way of life and that in two directions. Due to the formation of colonies (cormogony) which they had early begun to develop, the polyps as dependent "persons" or subindividuals have grown smaller and smaller (a progressive diminution). It is because of this fact that the smallest subindividuals can be found, as this can be expected, in the Hydrozoa. It is evident that the regressive development of the whole muscular system has been conditioned by this fact. On the other hand, and because of the emerging restriction and the change of the role of the muscular system, a cutaneous muscle tube has been formed which is typical of Cnidaria ; a layer of longitudinal muscular fibres under the skin, and another layer of circularly placed muscular fibres under the intestinal epithelium. We have here actually two types of movement only: retraction which is followed by extension, and nutation (curving), due to the contraction of individual sectors of the layer of longitudinal fibres. In species whose solitary life is a secondary phenomenon, e.g. in Hydra, this has led to a new type of movement, such as can be observed in the loop-worm or in Hirudinea.

The anthopolyps have inherited a richly developed muscular system from their turbellarian ancestors. Besides a strongly developed cutaneous muscle tube which consists of three body layers there are also "internal" ("gastrodermal") muscular fibres that show a particularly strong development in the sarcosepta and as the sphincter of the oral area, and finally the dorsoventral and the obliquely placed lateral muscle fibres. In the primitive, solitary anthopolyps which stand erect and glide slowly over the sea bottom, it was the middle layer with its diagonally placed muscular fibres which was first lost because it had become superfluous. Thus two external layers only have 
been preserved here; these have lost their independent character, yet they have become specifically differentiated, and they have been wholly preserved in the active parts of polyps. These layers, have disappeared in the impersonal colonial parts (solenia, caulus, rhiza). It is only among the pelagic individualized cormi of the Siphonophora with their well developed hydrocauli (hydrosomes) that the layer with muscles can be found well developed even in the impersonal parts, a fact which can be easily understood.

Several remains of an internal muscle system have been preserved in the anthopolyps of the solitary Actiniaria. These are the basally situated transversal muscles which are active when the animal creeps forward; a whole system of the socalled septal muscles that occur in sarcosepta and whose fibres are placed longitudinally because they function as retractors; and, finally, the circular muscles that can be found close to the oral disc and which function as an oral sphincter. Here, it becomes clear how little it corresponds to the facts, if we speak about entodermal or ectodermal muscles for the sole reason that sometimes these muscular fibres appear to be more closely connected with the skin epithelium, another time with the intestinal epithelium, and as the third possibility they become independent because they had been separated from these epithelia. Scholars of the old school have frequently run into difficulties when they have tried to decide to which of the two body layers (either ectoderm or entoderm) they should have attributed a certain group of muscular fibres. Yet, as a matter of fact, all these muscular fibres belong to the middle layer only, regardless whether we call this third middle layer a mesogloea, mesoderm, mesoblast, mesenchyme, or, as I have called it, a mesohyl; it is always one and the same part of the body.

In the Scyphozoa, the polyps have inherited as their inner muscles from their anthozoan ancestors the poorly preserved taeniolan bundles of muscles. There are, with few exceptions (Nausithö̈-Stephanoscyphus), four such retractors which used 
to be called ectodermal, even when the taeniolae of Scyphopolyps (the retrograded sarcosepta) were found to be built of an entodermal epithelium, together with some "mesenchyme". Ontogenetically, however, these retractors are formed from the basis of cutaneous infundibula which lie in the oral disc and which are therefore of the "ectodermal origin". These septal retractors have lost their function during the formation of medusae and they have therefore disappeared. On the other hand, we frequently find in Scyphomedusae the layer of longitudinally placed muscular fibres to be progressively developed and specialized, in agreement with their swimming type of movement. As this was the case in anthopolyps we also find here the function of the layer of muscular fibres considerably increased, which has led to fold formation in this layer. It is not rare for "mountains" of folds to become separated, obviously secondarily, from the skin epithelium and it is in this way that they develop into the mesogloeal muscles. The outer layer of muscular fibres shows a particularly strong development under the oral disc of Scyphopolyps. Here it has been given a special function: it helps to open the oral orifice in order to make it possible for the predator to swallow its prey, and for this reason the arrangement of these fibres is radially symmetric. I pointed out, some time ago (Hadži, 1907) that these muscular fibres can be found cross-striated as early as in Scyphopolyps, a fact which has been little noticed, but which I have found, however, to be of considerable importance in view of the fact that the subumbrellar muscles, which are so important for the swimming ability of these animals, had been evolved in both medusoid forms (Scypho- and Hydro-medusae) out of this layer of muscular fibres. In those Scyphomedusae which have reached a larger size these muscles have become progressively widely differentiated (radial, coronal, delta muscles).

In Hydropolyps that have become very small (there are a few species only that have become secondarily solitary and whose size is therefore slightly larger) the subcutaneous layer of 
longitudinal fibres has been sufficient for the quick and complete retraction of the hydranths; and this was the reason why, besides the taeniolae, the inner retractors of Scyphopolyps have also been lost. The same situation has also been preserved in their medusae even in those cases when these have grown larger (e.g. Aequorea). Contrary to this situation in Scyphomedusae, we can find that the exumbrella of Hydromedusae does not show any layer of muscular fibres while at the same time these are strongly developed in the subumbrella where they even begin to form folds (e.g. in Gonionemus); in this they are helped by the velum, a speciality of Hydromedusae, as well as by the firm stratum of a jelly-like substance that can be found in the umbrella.

According to our interpretation, a thin longitudinal stratum is the only thing that has been preserved in Cnidaria, that have adopted the sessile way of life, of the cutaneous muscle tube which had consisted of three layers in their turbellarian ancestors. The inner transversal layer of fibres which is attached to the entodermal epithelium obviously goes back to the muscularis of the intestinal tract of Turbellaria, regardless whether in Turbellaria this stratum of muscular fibres (Bresslau, 1933, K.C. Schneider, 1902) have a transverse or a longitudinal and transverse position, or whether these be independent muscle cells, or only appendices of the entodermal epithelium cells. We have here, moreover, a change of the function inasmuch as in Turbellaria the muscularis exclusively serves the intestinal peristalsis, and in Cnidaria where it works as a part of the general system of the body muscles, in coordination with the external longitudinally placed layer of fibres. In contractions of the animal body - which are very important for animals that have adopted a sessile way of life because they are enabled by such contractions to liberate themselves quickly from contact with a foe or from an unfavourable effect of an environment-the main function lies in the longitudinally placed muscle fibres. During the stretching or extension which follows a contraction when the animal searches for food, 9* 
the main function is taken by the transversally placed layer of muscular fibres which adjoins the entodermal epithelium.

The muscular conditions that can be observed in Cnidaria - not less than those that can be observed in Turbellaria - show clearly that so far it has been much exaggerated when attempts have been made to establish a sharp division between the skin and the intestine on one hand which both show a strong inclination to the epithelization, and the mesohyl on the other as the layer which is situated between them. We are justified in maintaining, on the basis of known facts, that in Cnidaria and in Turbellaria the cytoplasma has the ability to develop special strongly contractile fibres (the muscle fibres), regardless of whether it has become differentiated as skin cells (ectoderm), intestinal cells (entoderm), or mesenchyme cells (mesoderm or mesohyl), as well as the ability to grow undulipodia (cilia or flagella) on its free surfaces (either external, or internal, or intermediate). This process is reversible, i.e. the ability can be lost again, and under special conditions it can again become active.

The mesenchymal muscle system which plays a major role in Turbellaria has been lost in Cnidaria, and the "mesenchyme" itself has become strongly reduced. These muscles, however, have been preserved in Ctenophora, while in Cnidaria which have adopted the sessile way of life they have become more closely connected with the two body epithelia and it has been only secondarily that they have occasionally again liberated themselves from the epithelium cells and sunk under the epithelium.

It should be mentioned here that in some primitive Turbellaria (e.g. in the aloeocoelous species Prorbynchus baswelli according to Steinböck and Reisinger; Bresslau, 1933) the contractile muscle fibres have been developed in the skin stratum itself, i.e. in the cytoplasm of the skin epithelium, and thus intraepithelially. This development has been so rich that here they form even four strata, i.e. in the distal zone (in the "cover 
stratum") one longitudinally and one transversally placed stratum, and similarly in the basal zone.

The hydrosome of some Siphonophora (Apolemia waria, according to K. C.Schneider, and Halistem ma tergestimum, according to Claus) can be mentioned as an example of newly formed muscle fibres. This so-called hydrosome of Siphonophora is nothing else but the hydrocaulus of the Hydroidea which has developed no muscle fibres because the slight general contractility of the cytoplasm has been clearly sufficient, and because of the periderm which as an external skeleton covers the "coenosarc." Yet a strong contractility of the "stem," as the bearer of the whole colony, has been useful for the planktonic Siphonophora and we can therefore observe here not only muscle fibres that have been developed as appendices to the skin layer, but also that the epithelium muscle cells which had been developed in this way have sunk into the "mesogloea." It is natural that K.C. Schneider speaks here of "... in die Tiefe gesunkenen Deckmuskelzellen" which must be interpreted "also als eine Vorstufe echter Muskelzellen" (K.C. Schneider, 1902:607). According to our interpretation we have here, not muscle cells in the process of development, but rather parts of the so-called Deckmuskelzellen which have been secondarily developed into the former.

The subepithelial cells are frequently mentioned in the histology of Cnidaria. They lie close-wedged between the basal epithelium cells and they are usually attributed to the skin layer or to the ectoderm. This, however, is wrong. Everything that lies under the skin epithelium, whether migrated to this place from the epithelium through immersion, or that originated here, belongs to the middle layer ("mesoderm"), and it must be attributed to it even if there is no sharply delineated and clear limit in between. Cnidocytes can serve here as an example; they are recruited from indifferent "mesenchymal cells," and, after their further development, they are ranged into the skin epithelium either "on the spot" or after a shorter or longer wandering; they must therefore be 
attributed to the skin layer in their fully developed state only. Recently the theory of an active wandering of cnidocytes has been doubted by some authors (Brien and Reniers-Decoin, 1950). To this it can be answered that it is easy to observe this wandering, which is frequently very complicated, in a living object (cf. the most recent work by Lenhoff, 1959).

In conclusion, we can state that conditions that can be observed in the muscular system of Cnidaria show (in comparison with conditions that can be observed in the freely living Turbellaria) a generally retrograde development in polyps as the primary form of Cnidaria, a fact due to their sessile way of life. Yet at the same time they have been reorganized and specialized, concordantly with their mode of life. These changes which are partly progressive and partly regressive can be clearly identified as early as in Anthozoa. Here the development followed the direction, solitary Hexactiniaria-colonial Hexactiniaria-colonial Octactiniaria. It is therefore wrong to put the Octactiniaria (as has been done for example by Hyman) as a supposedly more primitive form into the first place of the system of Anthozoa. The Octactiniaria with their sarcosepta (whose number is constantly eight) follow the rule; first polymerization with an unfixed number of multiplied "parts," followed by an oligomerization with a reduced and fixed number of "parts." In the extremely specialized Antipatharia, among Hexactiniaria, whose anthopolyps have a strongly decreased size and which are, moreover, very short, the septal muscles have been mainly lost, while at the same time other strata of the muscular tissue also show a very poor development; the skin, however, has again become covered with cilia. Thus the changes in the muscle system have gone much further in the colonial Hexacorallia than in the typical Octactiniaria.

The muscular conditions that can be observed in medusa of Scyphoza and Hydrozoa which are even more specialized can be deduced from those of polyps, and not conversely as this has been done by numerous zoologists, Hyman among them. Neither the septal muscles nor sphincters can be found 
in the two main forms of medusae(Scypho- and Hydromedusae), and so there is no trace left of the primary internal muscles. On the other hand, we can observe a strengthening of the longitudinal muscular tissue which lies under the skin epithelium. This strengthening is appropriate to the function these muscles have in certain areas, especially close to the marginal tentacles, so that these muscles frequently show a higher level of specialization and they become transversally striped (e.g. according to Krasińska, in the subumbrella of the trachyline hydromedusa Carmarina bastata), or they can even appear as independent myoblasts (e.g. according to Krasińska, in the subumbrella of the hydromedusa Neoturris).

The opponents to the view that considers the polyp form as primary in comparison to the medusoid form and which sees in Anthopolyps the oldest form Cnidaria, should compare the muscular conditions that can be found in Cnidaria with those that can be found in other sessile animal types; thus to compare those in Turbellaria with those in Endoprocta (Camptozoa), those in Annelida with those in Phoronidea and further with those in Ectoprocta, and finally those in Branchiostoma with those in Tunicata. It is wrong to require or to expect a progressive development in the general muscle system of an animal body which adheres to the sessile way of life, a supposition which one has to accept if one tries to derive the Scypho- and Anthozoa from the Hydrozoa.

The unique case of Stauromedusae, or better, say, Lucernariidae is very interesting in this connection. These are not simply medusae as they have frequently been depicted, but real polypomedusae instead (Fig. 22). These Scyphozoa are secondary solitary animals and they usually have readopted the ability to move slowly over the sea bottom. At the same time they have also abandoned the alternation of generations; their medusoid generation which was formed by way of transverse division or strobilation is not severed any longer but instead it remains with the polyp with which it forms a double animal, a genuine polypomedusa, or a combination of a basal 

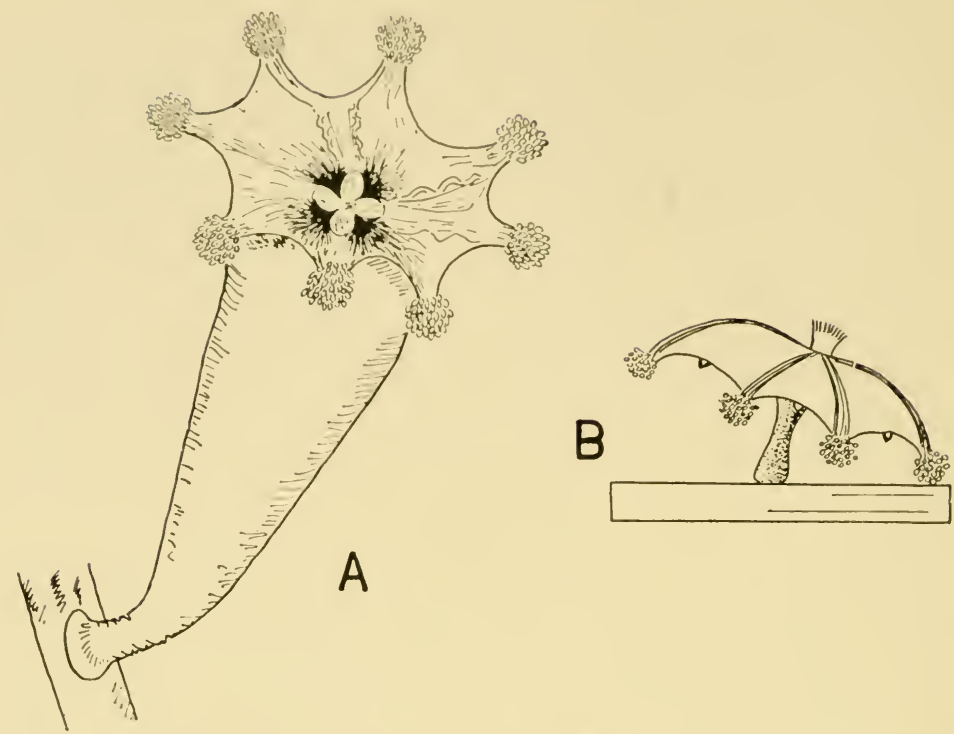

Fig. 22. Scyphozoa, Lucernariidae. A, Lucernaria sp. (from Hyman). B, Haliclystus auricula (after H.J. Cla rk).

scyphopolyp and of a distal scyphomedusa. The same combination can also be found in its muscle system; in one and the same individual we can find the peculiarities of a polyp as well as those of a medusa, i.e. on one hand, the taeniolan or septal muscles, and the coronal muscles in the form of bands, as well as the radially oriented muscular tissue of the oral disc (manubrium).

\section{The Nervous System}

Cnidaria have played a special role in the history of the development of neurology. Nobody has succeeded so far in finding either individual nerve cells or, even less so, a real nerve system in Spongiae; this is the true situation in spite of the contrary assertions made by Tuzet and by her collaborators. On the other hand it has been possible to identify in Cnidaria, and especially in Hydra which has been intensively studied, not 
only individual nerve cells but also whole coherent nerve systems and sensory cells. These results have been arrived at first by means of usual methods (serial sections, and the staining of sections with the iron haematoxylin) which have been used by O. and R. Hertwig (1878), K. C. Schneider (1890), M. Miyashima (1898) and finally by means of the specific intra vitam colouring with the methylene blue (Hadži, 1908). This has disproved Kleinenberg's theory of nerve-muscle-epithelium cells which has been mentioned above, in spite of the attempts made by Eimer to support it. They, and numerous other scholars after them (among these I mention especially P. Grošelj [1909] who has suceeded in intra vitam staining the elements and mutual relationships of the nerve systems of anthopolyps) have established the generally valid fact that there can be no doubt that Cnidaria possess a nervous system, though in the form of a nerve net which can be locally denser and which can even lead, though in medusae only, to the formation of nerve chains and to the local accumulation of ganglion cells into small ganglia. Yet, on the other hand no typical nervous system with one centre, the brain ganglion, and with regular nerve trunks can be found anywhere in Cnidaria.

It has been easy to conclude-and such a conclusion has really been made and it is still generally accepted-that in Cnidaria we can find the primary primitive conditions of the nervous system which has been further developed by way of a progressive evolution into the typical nervous system on a higher level of evolution. G. Wolf (1903), as has already been mentioned, has even tried to construct the origin of the nervous system in the following way: nerve cells have been developed, according to this interpretation, from the epithelially placed sensory cells by way of the latter sinking into the subepithelium; in this way it has been believed that the theory of synapses, or of a secondary conjugation of primarily individual nerve cells, has been confirmed.

It cannot be doubted that the nervous system found in the polyps of Cnidaria (we will first discuss the primary form and 
the basic generation of Cnidaria, i.e. the polypoids as a morphological and a physiological whole) really represent the simplest organization that can be found in the entire animal world. The only problem is whether this simplicity be primary, or a result of a retrogressive development from an earlier higher level of organization. In an analysis like ours we must take into consideration the function of the nervous system as that of an intermediary between the sensory cells or sensory organs as receptors, and the muscles as effectors. I have already shown with regard to the muscle system, that it had gone through a generally retrogressive development, with the exception of that specialization which is appropriate to the sessile way of life. To my knowledge not a single complex sensory organ has been found so far anywhere among the polyps of Cnidaria, nor has there been published a description of such an organ. There have always been only individual sensory cells ot served in all the exposed parts of the skin stratum or even in the gut epithelium. It is therefore not surprising if the absence of a distinct nerve centre had been formerly attributed by the brothers Hertwig (1878:185) to the absence of sensory organs. They were right, with the exception that they should have added that this absence of sensory organs has been a result of the sessile way of life, similarly as has been the case with the simplification of the muscular system.

In my opinion there are two facts which have not been satisfactorily taken into consideration in the interpretation of the nervous system that can be observed in the polyps of Cnidaria. That idea occurred to me when I succeeded in selectively intra vitam staining the nervous system together with the sensory cells that occur in green Hydra. This system appears to be cytologically-histologically strongly differentiated, in spite of its actually quite simple general structure. On the basis of this fact alone one could conclude that the general simplicity of this nervous system cannot be a primary phenomenon.

The second fact that should be mentioned in this connection is that the "nerve net" of the polyps of Cnidaria is not 
developed in one layer, or in the ectoderm, only, as we are now used to saying (thus subepithelially or under the skin), but it also occurs deeper, in the intermediate layer (C.A. Pantin, 1952). Furthermore, the nerve processes do not respect in this connection the limit between the two layers (the supporting lamellae or interstitial lamellae). This fact, taken separately, does not seem to be very surprising, yet it becomes clear if we make a comparison of the nervous system of Cnidaria with the one that can be found in Turbellaria.

Yet before we begin to deal with this problem, let us first make a cursory survey of the nervous systems that exist in othet animal types that have also adopted the sedentary way of life. It can be observed in such sedentary animal types that have evolved from freely living ancestors that already possessed a well developed central nervous system, that the main centre, the brain ganglion, has been preserved with obstinate tenacity, long after their transition to the sessile way of life, and it can still be found when other parts, especially the ventral nerve cord, had disappeared, and when the peripheral nervous system only has been kept. A centre has been preserved even in those cases where sensory organs have not been developed in great abundance. As a rule we find the supraoesophageal ganglion preserved. This centre has disappeared, as it seems, in Endoprocta only and this was probably due to the part of the body which was used by these animals when they began to attach themselves to the bottom. On the other hand, we find the suboesophageal ganglion preserved and it functions as a brain ganglion. In addition to these, the brain ganglion with its ventral extension (the nerve cord) has been lost in Echinodermata when they definitely adopted the sedentary way of life; in these a peripheral nerve net only has been preserved. We can observe, as a fine analogy to the medusae of Cnidaria, that no younger types of Echinodermata have developed a nerve centre similar to a brain ganglion, not even in those cases where they have evolved secondary ambulatory abilities. We can find in the freely moving Echinodermata, as well as in 
medusae, a secondary thickening or condensation of nerve elements (nerve cells and fibres, neurites) into a nerve ring which cannot therefore be homologous to the nerve ring together with the upper gullet and lower gullet ganglia of Polymera, because they do not possess real ganglia. The ambulacral nerve trunks spread radially out of the nerve ring.

When zoologists abandon the accepted idea of a supposedly primary nerve net in Hydra they will have to search for the solution of the problem of the origin of the nervous system somewhere else. In my opinion we must consider the Turbellaria. This suggestion stands in opposition to what has been taught by many comparative morphologists (cf. Hanström, 1928). The simplest conditions of the nervous system can be found in Acoela and in the small Turbellaria that are related to the former. There is no real foundation for the supposition which has been frequently proposed that the Turbellaria are secondarily simplified animals, an interpretation which seems to be supported by the fact that their size has decreased. The Turbellaria are freely moving, and they search actively both for food as well as (even though hermaphroditic animals) for their partners. Their primitive nervous system is situated close under the skin and it forms a primitive nerve net without any real nerve centres, without ganglia or nerve trunks. Without being afraid that the future will prove me wrong (so far no factual data about this have been available) we can expect that this nerve net does not develop during the ontogeny by way of an invagination of the skin epithelium cells (ectocytium), but rather "on the spot," out of the "mesenchyme" cells. We will return to this when we discuss the problem of the origin of Eumetazoa.

During the second stage of the development of Turbellaria a condensation of nerve cells and of nerve fibres into longitudinal bundles that are mutually connected by way of transverse commissures (the orthogon, according to Reisinger) can be observed. At the same time a second plexus is being developed more deeply in the interior of the animal 
body. During the third stage a brain is formed in the anterior part of the body, at the place where the longitudinal bundles develop a mutual contact; this development is aided by sensory organs that had evolved in the front part of the arimal. Here we are not interested in the subsequent developmert of the nervous system during which the polymerization (the increase of the number of the longitudinal nerve trunks) is followed by an oligomerization (two pairs of longitudinal trunks, a ventral pair, the merging of this pair with the nerve cord).

Fortunately enough there exist even now species of Turbellaria that have preserved the primitive conditions of their nervous system. O. Steinböck has given a description of the species Nematoderma batbycola, which has been found in the deep regions of the polar sea, close to Greenland; Steinböck says about this species, "Ein Gehirn in gewöhnlichem Sinne, wie wir es bei Turbellarien gewohnt sind anzutreffen, besitzt unser Tier nicht" (Steinböck, 1931). On the other hand there is in Nematoderma a denser and, as it seems, less regularly formed nerve plexus which is situated close under the skin and which shows indications of some thickenings or of condensation in the longitudinal direction. Hanström is inclined to attribute the main role in the formation of the brain to the emergence of sensory organs. We are convinced, however, that the influence of the function of the muscle system during the active forward movement, with the anterior end orientated forwards, has been at least as important. It is even possible that the static organ, the light receptors, and even more probably the special chemoreceptors have been developed only after the Anlage of the brain had been founded.

If we stick obstinately to the thesis of the evolution of Cnidaria in the direction Hydrozoa-Scyphozoa-Anthozoa and to the belief that Cnidaria have nothing in common with Turbellaria, we are forced to suppose that the nervous system of Eumetazoa has developed at least twice, thus diphyletically, and so to speak, out of nothing. The opinion cannot be taken 
seriously (though it is still occasionally met) which considers the Turbellaria together with all Acoelomata and "Pseudocoelomata" (we call them Ameria) to be a result of a retrogressive development ("degeneration"), and according to which the simple nervous system of small species of Turbellaria must be a secondary element. The specialists in Turbellaria all disagree with such an interpretation. This interpretation has been accepted only by those zoologists who would like to derive, on the basis of the enterocoele theory, the perigastrocoele from the intestinal cavities of anthopolyps, a plan which cannot be condemned sharply enough.

In view of the fact that in the Anthozoa the polyps certainly have a more highly developed nervous system than the one that can be found, in Hydra, we would be obliged, if we adhered to the old interpretation of the development of Cnidaria, to accept the proposition that they had progressively developed their nervous system in spite of their unchanged sedentary way of life. This contradicts all that is known about "the influence of the sessile way of life." In a certain sense there has really been a progressive development, as in the case of the muscle system, yet only inasmuch it has led to a specialization which stands in a causal relationship to the sedentary way of life. With regard to the whole structure, however, we can observe in the nervous system of Cnidaria indications of a progressive retrogression. It is only in the case of the primarily solitary and, even if slightly, moving Actiniae that a clear condensation of nerve elements can be identified at their aboral ends (the foot plate) (P. Grošelj, and recently Pantin and his school). A reduction of the nervous system can be observed as early as in Anthozoa that have become colonial animals. This reduction goes parallel to the reduction of the muscle system and we can see, as the final result, the nerve net limited to the oral region only.

In Scyphopolyps and in Hydropolyps the condensation of the nervous system disappears completely. In connection with the secondarily solitary Hydra which has again become an 
actively moving animal I have been able to show that the nervous system has again become locally dense, especially in the aboral foot plate, with a clear direction (radial or circular) of nerve fibres (Hadži, 1908).

A distinctly progressive tendency can be observed in the stem (siphonosome) of Siphonophora, among Hydrozoa. They have developed, as polypersonal cormi, the ability to move freely. This development of nervous system is parallel to the development of muscles in this strongly contractile stem of cormus (according to K. C. Schneider, 1896-1898).

Now let us pass over to the medusoid form. Since we derive the Cnidaria fiom the Turbellaria we must consequently consider the medusoid form as secondary and as evolved from the polypoid form. The same, however, has also been considered as the correct interpretation by numerous experts in Cnidaria who have believed at the same time that the Hydrozoa were the most primitive Cnidaria because there were such numerous and convincing proofs which seem to support such an interpretation. The development of the medusoid form, which has twice taken place (Scypho- and Hydro-medusae), is above all a secondary liberation, or a readoption of mobility with a simultaneous transition of the sexual function from one form to the other. This is the quintessence of the metagenesis, of the alternation of generations. It is senseless to deny the existence of an alternation of generations in Cnidaria, as has been recently attempted by Hyman: this is a consequence of an inco:rect interpretation of the polyp form and of the polyp generation as if these were a larval stage which had not been developed into an adult form earlier than in Anthozoa. And what are Hydra and other Hydrozoa that have become secondarily solitary animals under a simultaneous loss of their medusa generation, in spite of the fact that they become sexually ripe? Is this a case of neoteny? This cannot possibly be so, in view of the fact that in the Hydroidea we can find all possible degrees of reduction of the medusoid generation; we must therefore have here a secondary omission of the medusoid generation. 
If we disregard a clearly secondary retrogression of the medusoid form that can be observed in Hydrozoa, and if we disregard the case of Lucernariidae among Scyphozoa, we can see all the remaining developments to have been clearly progressive, a fact which can be easily understood because it is connected with a secondary adaptation of free life, in this case in the free water zone and with a simultaneous preservation of the essential characteristics of the older polyp form. On the basis of this standpoint we are also enabled to understand the conditions that can be found in the nervous system of medusae. The progressive development of the whole nervous system went parallel to the progressive development of the muscular system and of the marginal sensory organs. In this connection two regularities can be observed. This progressive development did not lead back to the old conditions that had existed in their freely moving ancestors (Turbellaria). The radially symmetrical form has now been preserved by medusae which developed the ability to swim freely and to creep over the sea bottom. When they swim, they orientate their aboral end forwards. In this way the development did not lead to a cephalization; the new anterior end remained empty, i.e. it did not develop sensory organs. A new centre of sensory and nervous functions has been developed around the margin of the swimming bell, and in this way the nerve rings have been evolved. The fact that there are two such nerve rings can be understood if we take into consideration that there are two nerve nets which are situated one above the other in the original polyp form. The formation of nerve rings is simply due to an intensive local thickening or condensation of the two nerve nets.

The second regularity (in the past people preferred to call them laws), is the fact that the evolution of the polyp into medusa has led to essentially identical results though it had taken place twice. Thus both times a double ring of nervous tissue has been developed along the margin of the umbrella, and both times this evolution has led to similar marginal sensory 
organs. In spite of this, however, there are differences between the two medusoid forms, even if we disregard such differences as, for example, the presence of a velum (only in Hydromedusae), or its absence (primary in all Scyphomedusae, secondary, and rare, in Hydromedusae, e.g. in Obelia species), and the completely different way the two medusoid forms develop; Scyphomedusae are formed by way of transverse division or strobilation, and Hydromedusae by way of budding. As for the velum, I have succeeded in showing that this auxiliary swimming organ developed genetically from the bud, i.e., out of the embryonic protective stratum, with a simultaneous change of its function (Hadži, 1909 b).

Various sense organs, that in the two medusoid forms appear as new formations, can be found developed as early as in Turbellaria (statocysts, light receptors [eyes], and chemoreceptors); as in the Turbellaria we can find in the medusae variants that show considerable differences. The main changes that can be observed, above all in the mechanoreceptors, are due to the transition to the new, specialized type of movement, i.e. to the rhythmic contractions of the umbrella muscles; this, however, is not true for the movement of the manubrium which had previously been found in the oral cone of polyps.

In their progressive evolution the Scyphomedusae have made greater advances than the Hydromedusae; this can be partly attributed to their greater size. This, however, must not be interpreted as if the Scyphomedusae had been developed out of the Hydromedusae; in reality their developments have been parallel and they had proceeded from two completely separate initial stages. The Scyphomedusae are, in my opinion, phylogenetically older, because the Scyphozoa themselves are older and must be interpreted as animals that had evolved from the Anthozoa. In the Scyphomedusae a localized agglomeration of various sensory organs (rhopalia) can be observed to have taken place in certain parts of the umbrella margin that are separated from each other at regular distances. As a consequence of this we can see an accumulation or 10 
condensation of nerve cells in the same spots of the nerve ring which has led to the formation of (imperfect though they are) ganglia (rhopalium ganglia). Something similar took place, it seems to me, in the Mollusca in the case of an assymetrically distorted bilateral symmetry. Data is available (Horridge, 1956) which shows a nerve net to have been developed in the exumbrellar subepithelium of the Scyphomedusae (nothing similar is known to me in the Hydromedusae). It has already been pointed out that no sensory organs, and, in connection with this, no nerve cell aggregates have been evolved in the pole of the exumbrella, in spite of expectations that these should be developed in the anterior part of the body which comes into most contact with the surrounding milieu when the animal swims. It is therefore impossible that the so-called "Hydroctena salenskii" (Dawydoff) could be a Hydromedusa (Hadži, 1959). In the Hydromedusae, a combination of two different sensory organs can sometimes be observed at the margin of their umbrellas (e.g. Tiaropsis), a fact which has been used for an attempted division of Hydromedusae into Ocellatae and Vesiculatae. It should be also noted that in the subumbrella of Hydromedusae an agglomeration of the nerve tissue can also take place along their radial canals. It agrees with our interpretation of the origin of the velum that no nerve net has been developed in it; yet in spite of this the velum is able to perform rhythmic contractions.

The sense organs of medusae, if compared with those of polyps, are, as this has already been stated, new formations. Yet if we try to pursue the development further back we see that those sense organs that occur in medusae had also been essentially developed in their free living ancestors, in the Turbellaria (tango-, stato- [or tono-], chemo-, and light-receptors). The main difference is that in the Turbellaria that creep actively over firm surfaces, these sense organs show a bilaterally symmetric distribution (distribution in pairs) in the anterior end of their body which represents a primitive head, while in medusae they are distributed in groups and, in agree- 
ment with their radial symmetry and bell shape, at regular intervals along the margin of their umbrellas.

If we consider polyps as the primary form of Cnidaria, and if we do not find in them any complex sense organs, we must come to the conclusion that these sense organs had been lost due to their sessile way of life. This indicates that in medusae, which in Scyphozoa and Hydrozoa had been secondarily evolved out of polyps, these sense organs have been newly developed-probably only after they have long been adapted to the free life in water. We must here point to the fact-without any intention to enter into greater details-that there is nothing unusual in the reappearance of complex sense organs in the two subtypes of medusae. These sense organs represent, according to Grošelj, "extremely variable values," and they reappear again and again whenever they are required by the circumstances in which an animal lives; they frequently appear in the most unusual parts of the body, e.g. in the tentaclelike processes of the cloak margin of Pecten, or in the minute feathers of the tentacle crown of some Polychaeta. The consequences of considerable changes in the way of life are first, a reduction of those sense organs that had become useless and finally a complete loss of these; this can also be observed in those species that live in underground caves, regardless of the fact that they can belong to different systematic groups. With the same facility such sense organs can again and again be reintroduced. The explantation of this may be that the mutations that are necessary for the development of these sense organs return, even in those cases when such organs do not appear to be very useful. If again they are ("something to be desired"), useful because of the change of environment, the corresponding mutations win the protection and guidance of Natural Selection and they can again be further progressively developed.

It is therefore not a contradiction of facts when we maintain, on one hand, that Cnidaria have inherited their ability to develop complex sense organs from their turbellarian 10 * 
ancestors, and, on the other hand, when we declare these organs of Cnidaria to be new formations. In details the structures of these sense organs can show considerable differences from each other, which does not surprise us because of the complex nature of these organs and because they have been developed under different circumstances.

It is just in connection with the sense organs of medusae that we can observe numerous and interesting examples of the variability of these organs. Thus, the statocysts, as organs of equilibration and stimulation, show considerable differences in their structures not only if we compare the way they occur in Hydromedusae on one hand, and in Scyphomedusae on the other; there are also considerable differences in statocysts even within Hydromedusae themselves. Sometimes, such organs are developed with participation of the entoderm (as, for example, in tentacles); at other times, however, they are of a purely ectodermal origin; they can lie exposed, or they can be surrounded by other tissue, they can be free or immersed, etc. In Scyphomedusae, these marginal organs (rhopalia) are very complex, i.e. they are composed of several different partial organs. The same is true for the light receptors that can appear in very different stages of development and which have clearly been evolved several times and independently from each other.

It should be mentioned, with regard to the retrogressive development of the nervous system of polyps, that in hydropolyps this process has gone so far that we are hardly justified to speak any longer of a nervous system because it appears as a very sparse net only, and frequently we find even this last remnant of a former nervous system completely absent in many parts of the animal body. It should be added here that parallel to this sparsity of the nerve net, the "nervous tissue" is moved from the intermediate layer into the skin layer, i.e. to the base of the skin epithelium cells.

A reversion of this process can be observed in the medusoid forms-inasmuch as these themselves do not tend to retrogress (Hydromedusae that have adopted the sessile way of life). 
Parallel to the development of their muscle system and of their sense organs, which is connected with an increased density of the nervous tissue, we can observe a sinking of the latter into the skin epithelium, thus into the jelly-like basic substance of the intermediate layer. This development can be observed along the margin of their umbrella and also in their tentacles, as has been proved in Pelagia by Krasińska (1914).

In principle, it would be wrong to suppose the presence of such an advanced nervous system in the sessile polyps.

\section{The Emunctory-Excretory Organ}

As is well known, no visible special excretory organ of a type which would resemble a protonephridium, for instance, can be found in the Cnidaria. On the other hand, we can often find a well developed protonephridial excretory system in the Turbellaria. Those who adhere to the old interpretation, find an easy explanation of this difference: they suppose that in Cnidaria the absence of protonephridia is a primary characteristic, because the Cnidaria are, so they believe, as far as their primary organization is concerned, at a lower level of evolution. Yet, we too can find an explanation of this difference between Cnidaria and Turbellaria, which at the first glance seems to be so important, on the basis of the following arguments:

(1) There is a considerable uncertainty about the origin of protonephridia that have developed later into other nephridial organs, above all into the metanephridia of molluscs among the Ameria and Polymeria. While there are no protonephridia in "Coelenterata," they appear, as it seems, suddenly in the lowest "Coelomata," in the Platyhelminthes. They have, without any previous intermediate stage, a well-developed tube system with specialized terminal cells equipped with beating undulipodia; internally they end blindly, while externally they end in pores. It cannot be supposed that they have developed as such out of nothing. Krumbach (in his Handbuch der Zoo- 
logie) has tried to avoid this situation by attempting to derive the protonephridia from the invaginated and immersed "ribs" of Ctenophora. Fortunately, this interpretation did not find any favourable reception among other zoologists because such an evolution is completely devoid of probability. No other attempt to interpret the origin of these protonephridia has been known to me. We will approach this problem from another standpoint.

Our starting point is the fact that as early as in the Protista an apparatus can be found for the excretion of the superfluous water which constantly enters from the watery environment into the cytoplasm, where it absorbs the dissolved products of the metabolism. This is the contractile vacuole which may have developed from the small intermediate cavities that occur in the cytoplasm. A high level in the evolution of these vacuoles has been reached in the swimming Euciliata so that in this case we are entitled to speak of a special excretoryemunctory organelle (Fig. 23). This organelle is particularly well developed in species that live in fresh water; it shows a considerable lability because it can easily disappear. This frequently takes place in species that live in the sea and in those whose size has been greatly diminished.

From this point of view we can come to the conclusion that the ancestors of the Turbellaria had inherited from their Euciliata ancestors (apart from numerous other things) an emunctory system also as well as the middle layer which also contains the body muscles. It should be remembered in this connection that as early as in Euciliata this emunctory system had been developed into a canal which later becomes its regular form in the Eumetazoa. From the orifice of these excretory canals, i.e. from the porus excretorius, the skin layer (actually quite similarly to the margin of the cytostome where it has led to the formation of the cytopharynx), together with its cilia, has moved by way of invagination into the interior of the body, and in this way the ciliated and pulsating terminal and canal wall cells have been developed. In this connection we can 
observe in the ontogenies of numerous Eumetazoa that their nephridia are developed at least partly by way of the invagination of the skin layer. Later these protonephridia have been influenced first by the polymerization, and subsequently, ac-
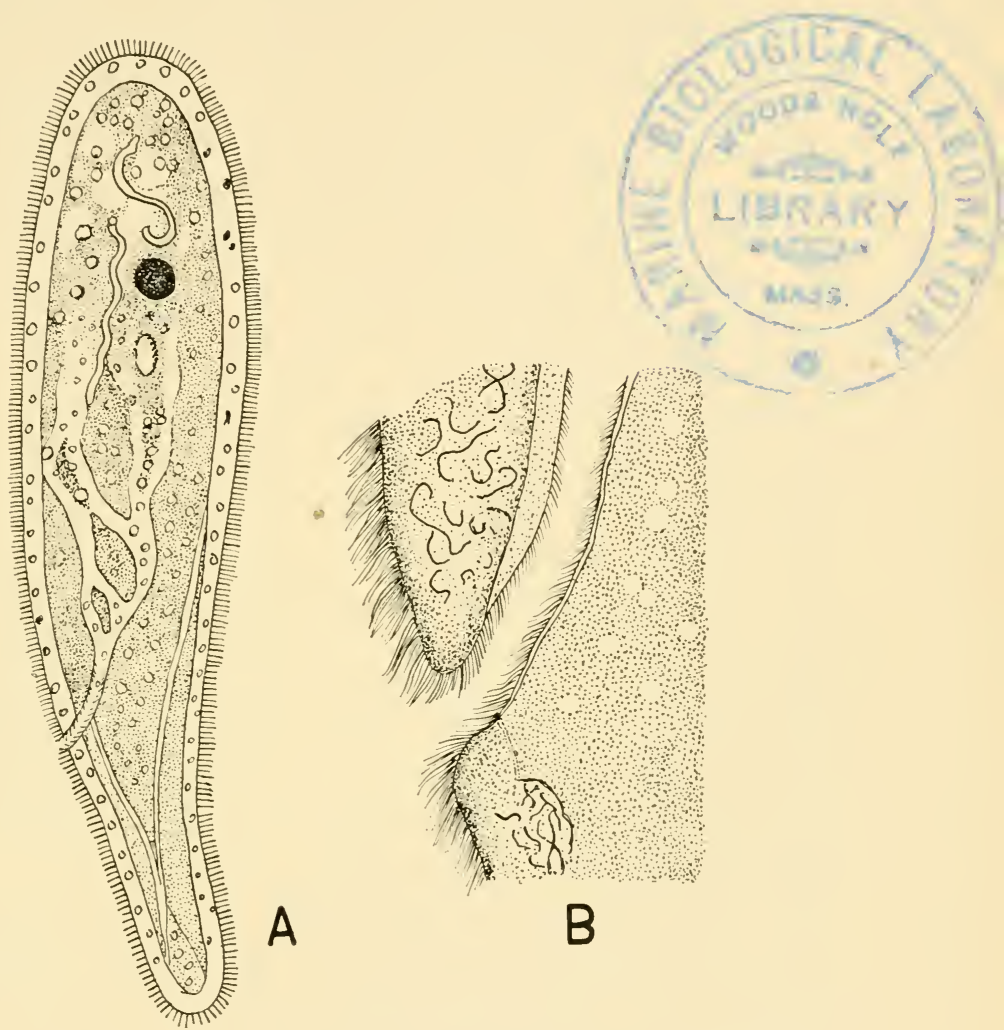

Fig. 23. Excretorial-emunctorial organelle of a ciliate (Pycnotbrix monocystoides). A, habit. B, the ciliated end of the canal. (From Dogiel.)

cording to circumstances, by the oligomerization which has frequently led to a complete disappearance of the nephridial organs. Here we are interested in the fact that, as far as we can judge on the basis of our present knowledge, the small acoelous Turbellaria do not possess-and this without an 
exception-any protonephridia. On the other hand we cannot find, according to Lang, any protonephridia in the rather large Polycladida that live exclusively in the sea, a fact which must be explained as being due to their secondary disappearance. Many other species of Turbellaria, especially those that have adopted the parasitic way of life, have lost their protonephridia, while at the same time these are preserved in the small Temnocephala that live epizoically in the fresh water. In my opinion we must attribute a greater importance to the older data provided by v. Graff (1904-1908, in Bronn's Classen und Ord. des Tierreiches), and b.y Löhners (1911), as well as to those that have been published more recently by Westblad (1948), according to which there can be found an accumulation of excretory products in the form of cellular and plasmatic inclusions in the external body layer, in the so-called ectocyte, which, however, is not equivalent to the ectoepithelium and thus to the epidermis (e.g. in Paraphanostoma crassum, $P$. trianguliferum, the Convoluta species, etc.). This is the same body layer which in Infusoria regularly contains the contractile vacuoles. If the functioning of these contractile vacuoles ceases because of the retrogression of the emunctory function, the excrement is deposited as firm bodies (eventually as crystalloids) in the cytoplasm either of the mesenchymal or of the digestive cells.

(2) It is therefore not surprising, in view of the fact that the protonephridia had been so labile both during their development as well as in their occurrence, that they have been lost during the evolution from the turbellarian ancestors to the Cnidaria, at first semi-sessile and slowly creeping, and afterwards to the completely sessile and immobile animals. In addition to this, first Cnidaria were in all probability small animals. In these circumstances, the protonephridia became superfluous and they did not stand any longer under the protection of Natural Selection. It is quite possible that in the period of the separation of Cnidaria from their turbellarian ancestors the formation of protonephridia had been very labile. 
The following factors had supposedly contributed to the complete disappearance of special emunctory-excretory organs: (a) life in the sea; (b) the abandonment of active movement; (c) the progressive diminution of the size of polyps in connection with the formation of cormi; (d) the relative enlargement of free surfaces (external as well as internal); and, (e) the epithelization of the tissue.

It is characteristic that during the subsequent evolution of the Cnidaria, protonephridia have never reappeared, either in the secondarily solitary polyps whose size has also been simultaneously increased, or in the secondarily free medusae that have also reached a considerable size. In all probability this can be attributed to Dollo's rule of irreversibility. The functions of the abandoned organs have been taken over by the parts of the body that have been preserved, occasionally with a partially progressive development of the latter (the pores in numerous medusae, the so-called liver in Chondrophores among Siphonophora).

(3) We can see, if we look around at a somewhat broader circle, especially among the numerous subtypes of Ameria, how frequently under special conditions protonephridia have been reduced or even abandoned. Protonephridia are extremely reduced in the Endoprocta (Kamptozoa, according to Cori) that live mostly in the sea and that have become sessile animals. Two very short canals, each with one ciliated cell at its internal end and with one pore, is all that has been preserved of all the variously twisted canals with their numerous terminal organs. The protonephridia have completely disappeared in the species of two orders, out of the three, of endoparasitic Acanthocephala. In a majority of Nemertinea that live in the sea, the nephridial apparatus, which is otherwise considerably specialized, is strongly reduced, and it is completely abandoned in numerous bathypelagic species.

Most interesting in this connection are the Ctenophora, Zoologists are usually of the opinion that the Ctenophora as Coelenterata, do not primarily possess any protonephridia. 
I have shown it to be very probable that in the Ctenophora the last remains of protonephridia have still been preserved, even if their condition as well as their function have been considerably changed. These remains have developed the form of the so-called "ciliate rosettes" and they correspond, in my opinion, to the terminal organs of the typical protonephridia which have been grown over by the intestinal wall (Hadži, 1957, see Fig. 24). In this way they have re-established their contact with the external wcrld ky way of an intestinal lumen instead of the lost contact by way of pores.

A very interesting case is that of Nematoidea. They are characterized by the fact that they can no longer produce undulipodia, not even in connection with androgametes. Correspondingly there are no terminal organs in Nematoidea so that here the excretory function has been taken over by a device which consists of two cells. An eventual homology of this device with protonephridia is more than questionable; it is in all probability a new substitute form.

It should be briefly mentioned that in the Annelida among Polymeria, where the general polymerization has also influenced the excretory organs that developed into metanephridia, the nephridia have been reduced either to a sole pair or to an unpaired nephridium. This has been partly due to their transition to a semi-sessile or sedentary way of life, and partly to their extensive diminution. This is true even to a greater extent for Oligomeria that have adopted the sessile way of life; in these we can find developed either individual nephridia or none whatever. Even among the sessile Chordata, the Tunicata, no real nephridia have been preserved.

If we presuppose, though cannot, strictly speaking prove it, that protonephridia have been secondarily lost in Cnidaria because of their sessile way of life, we will find such an interpretation strongly supported by analogies to numerous other cases, where it is immediately clear that protonephridia had been reduced or abandoned under similar circumstances. In all these cases we find the emunctory part of the nephridial 
function considerably reduced as is particularly true for types whose bodies are covered with a cuticle. The real excretory function is always taken over by other organs or tissues because this function remains unavoidably necessary due to the metabolism of the animals, regardless of the way in which these animals feed. On one hand we can see nephrocytes (mesohyl

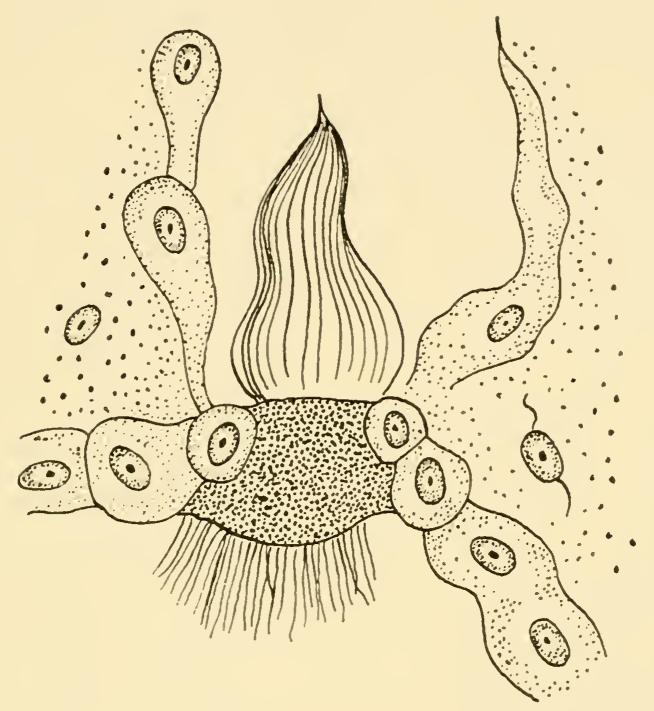

Fig. 24. "Ciliate rosette" of the ctenophore species Coeloplana sp. (after Komai).

cells), i.e. cells of the same origin as protonephridial cells, and on the other hand the intestinal cells, take over the function of the lost protonephridia. This is also true for Cnidaria: the second type of substitution is especially characteristic of the rapidly moving medusae which have developed, as has already been mentioned, excretory pores at ring canals as special auxiliary organs.

Finally we must not forget that it was aeons ago that Cnidaria (as the first Eumetazoa to have adopted the sessile way of life) were separated from Turbellaria. There has therefore been plenty of time available for the reduction and 
for the final elimination of the canal system which had become superfluous. Thus the protonephridia were replaced by an auxiliary apparatus a development which has later also taken place in other groups of Eumetazoa.

A question could perhaps appear justified: is it more probable that the protonephridia of Turbellaria had been developed from the nephrocytes as probably the most primitive excretive organ of Cnidaria? Has anything at all been known which could point to such a direction?

The Spongiae appear, as in so many other instances, in connection with the nephridial system, as standing apart from all other genuine Metazoa, the Eumetazoa. In the Spongiae we find that not only are nephridia absent (this absence is in all probability a primary characteristic), but we cannot even find a substitute for them. The monocellular protozoic ancestors of Spongiae possessed, as this is also the case with other Flagellata, contractile vacuoles that served as special emunctory-excretory organelles. No special excretory organs, no nephridia, developed during the transition from the colonies of Flagellata into the polycellular Spongiae. It seems to us that in the Spongiae the formation of special excretory organs was not necessary, because the Spongiae have been able to live easily without them, first, due to the fact that even after the integration their individual cells have preserved a considerable degree of independence; secondly, because their bodies have considerable external as well as internal surfaces; and, thirdly, because the Spongiae have preserved a very slow life rhythm, and because they have generally remained, with a few exceptions only, marine animals.

On the basis of all this we come to the conclusion that the Cnidaria secondarily do not possess any protonephridia because they have adopted the sessile way of life and as such they can dispense with them or they can replace them with other simpler forms. Furthermore, it seems that Dollo's rule is valid for protonephridia as complex excretory organs; those organs which had completely disappeared cannot be later re-developed. 
The Genital System

Of all the differences that exist between the Turbellaria and Cnidaria the differences that can be found between their genital systems seem to be the greatest. As a matter of fact these differences at the first glance seem to be so great that any attempt to derive the Cnidaria from the Turbellaria appears as a completely hopeless venture. Yet if we study the conditions that can be found in these two groups, and if we compare them with those that can be found in other more closely related groups of Ameria, we find the situation changed so that it does not seem that such a deduction is hopeless. It is certain that the Turbellaria, whose organisation shows in all other respects a lower stage of development, possess at the same time a genital system which has a complex structure and such a variety of forms, that cannot be surpassed by any other animal group, and we do not exclude even the most highly developed Vertebrata. As is well known, the Turbellaria are, with a few exceptions hermaphrodites. I will return later to discuss the probable origin of this hermaphroditism. Without any intention to go into details that are well known to zoologists (frequently the Turbellaria are systematically grouped and identified on the basis of their complicated genital systems), I wish only to mention here that these complexities affect both the gonads themselves (e.g. the division of female gonads into an germarium and a vitellarium), as well as their gonoducts, various auxiliary glands, and, finally, the organs of copulation. Due to their mobility these animals not only search for their food but also seek out their sexual partners. The advantage of the hermaphroditic state consists in the fact that any meeting with a member of the same species makes possible copulation and thus also reproduction.

It has been too little considered that though a hermaphroditic genital apparatus with a complicated structure can be found in a large majority of Turbellaria, there are 
among the primitive Turbellaria species, a quite simple genital apparatus (Fig. 25) which is similar to the condi:ion found in the Cnidaria. Yet even if there were in recent times no such primitive species of Turbellaria, if they had all died out, we would still be justified in defending the thesis (in view of other

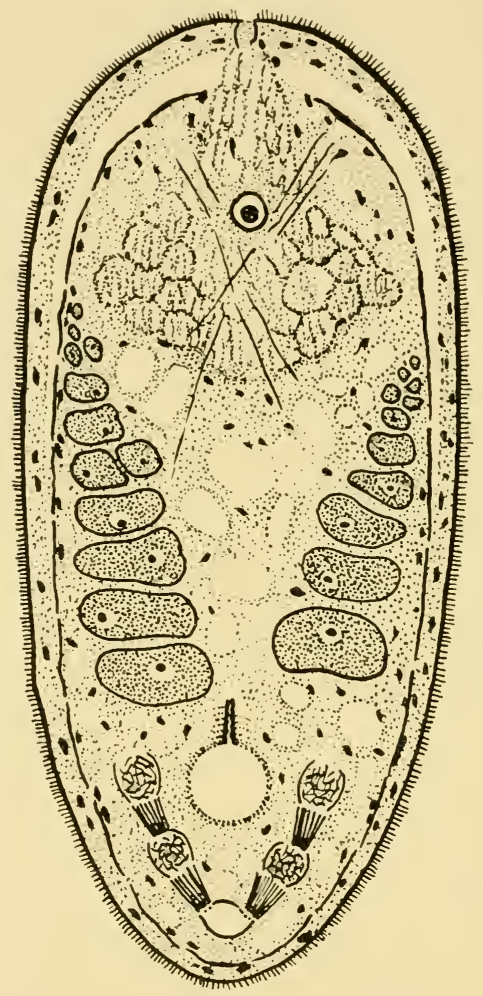

Fig. 25. An acoeleus turbellarian with its simple genital apparatus (Tetrapostbis colymbetes). (From An der Lahn.)

circumstances) that the genital organization of Cnidaria had been developed out of that which had existed in Turbellaria.

It is illuminating to observe in this connection the destiny of the complicated genital system of Turbellaria as they 
evolved. The whole system has been taken over from their tubellarian ancestors by two types which have both developed into parasites; by the Trematoda and by the Cestoda. In all other cases we can observe a very radical reduction of this complex, frequently with a transition to gonochorism. In the Nemertinea which are now quite generally considered as being direct descendants from the Turbellaria and thus their closest relatives, we find all the richly developed "decoration" lost, and of all the oppulence of their auxiliary organs there is hardly the formation of a conduit = canal left. Though hermaphrodites can still be found among the Nemertinea, the latter have nevertheless abandoned the pairing associated with internal fertilization. It is in the pelagic species Phallonemertes only that simple copulatory organs can be found in the male animals (yet even this case is problematic because we do not know whether these organs are a remnants of a primary apparatus, or whether they are secondarily developed organs, due to the secondary pelagic way of life). In Carcinonemertes even the direct contact of gonads with the external world has been relinquished, and the gametes are secreted into the digestive organ. The Rotifera, however, have preserved more numerous elements of the complex structure of the turbellarian genital system the female animals have the division of the ovary into a germarium and a vitellarium, and the male animals have developed a special copulation organ. The generally hermaphroditic Gastrostricha do not differ widely in their genital apparatus from that found in Rotatoria. The turbellarian genital apparatus has been somewhat more preserved in the parasitic Acanthocephala. On the other hand, the auxiliary organs show a complete retrogression in Endoprocta which have adopted a sessile way of life; in these the situation resembles that which can be observed in Cnidaria with the difference that in the former the canals of gonads are always developed while in the polyps of Cnidaria the remains of these canals can be found only in Anthopolyps, just as in Ctenophora which have developed 
secondarily into pelagic animals. Finally we must mention here the Mollusca. In the primitive Mollusca an advanced retrogression can be observed. In these, only the canals can be observed but it is not clear if we are concerned with genuine gonoducts or with coelomoducts, or with metanephridia; it is natural that under such conditions no pairing can take place. Subsequently, however, a secondary complication of the genital system appears in Mollusca, especially in the dioecious species of gastropods.

With regard to their genital system, there is in Cnidaria on one hand a progressive simplification, and, on the other, an increased complexity. The simplification does not only affect the auxiliary organs but also the gonads themselves; the complications can be observed to take place in the bearers of gonads and in their surroundings in the cormus, which is clearly a consequence of the sedentary way of life.

With regard to the distribution of the two sexes there is a clear trend towards the dioecious state. The hermaphroditic state had been inherited at least partly by Anthozoa from their turbellarian ancestors. It is not rare for this state to become hidden and develop into a protandry; the protandrous individuals are therefore usually considered as monoecious. It should be mentioned here that an inclination to protandry appears as early in Turbellaria. On the other hand, we can observe, according to Bresslau (1933:115), that in such Turbellaria, with a lower status of general organization as in the Acoela, Hofstenia among the Alloeocoela and Polyclads also, as the genital apparatus shows a lower stage of organization. This trend in their evolution was inherited by the Cnidaria from their turbellarian ancestors.

The hermaphroditic Anthozoa occur above all among the primarily solitary, and therefore, according to our interpretation, primitive species (Ceriantharia, Actiniaria) with polymerized gonads. In the Anthozoa, just as in Ctenophora, we can find the gonads of both sexes in one and the same sarcoseptum (this name is better than that of mesentery); each gonad is 
bisexual in the species Boloceroides bermaphroditicus, as in the garden-snail; their centre is the testis, and the periphery is the ovary. "Stephanosyphus racemosus" (Komai) is a scyphozoan which we consider as a primitive one and which is, according to Komai (1935) still hermaphroditic in its polyp generation. In its medusa generation (Nausitbö), however, we can observe a division of sexes. The same is true for the whole group Ephyropsidia where during strobilation the sex cells of only one of the two types of sex cells enter the Anlage of ephyrae by migrating from the basis of taeniolae (sarcosepta). During the retrogression or even the complete loss of the polyp generation, gonochorism becomes prevalent and as a consequence of this the Lucernariidae which have secondarily developed into purely sessile animals become monoecious.

On the other hand, we can again find some hermaphroditic species among the generally gonochoristic Hydrozoa, e.g. among the extremely retrogressed and secondarily solitary Hydra. The hermaphroditic state is quite rare among Hydrozoa (e.g. in the medusoid generation of Eleutheria which, however, has become "degenerated" so that it has been changed into an animal that lives on the sea bottom (Fig. 26).

As for the structure of gonads (the word "gland" instead of "gonad" is not suitable for the gonads of the lowest Eumetazoa; gonads can contain gland cells yet they do not always have them; as a rule they are built of sex cells which after all are not gland cells) we can observe, in agreement with our thesis, that it is in the Anthozoa, which have evolved as the first (the word "lowermost" would not be suitable here) group of Cnidaria from the Turbellaria, that the gonads are compact and that they possess at least a few auxiliary devices; to be more precise this is true for the primarily solitary Actiniaria only. There occasionally appear follicular forms with traces of spermoducts which are produced by their testes; their oöcytes show a type of feeding device which was correctly interpreted by F. Pax (1924:787) as a remnant 
of a former oviduct whose function had been changed. It is therefore probable that we have here the remains of an earlier stage in which evolutionary progress had reached a much higher level.

No regular sack gonad, i.e. a gonad with a central cavity or with a gonocoele and with a gonoduct, can be found among the Cnidaria. It is in accordance with the progressive

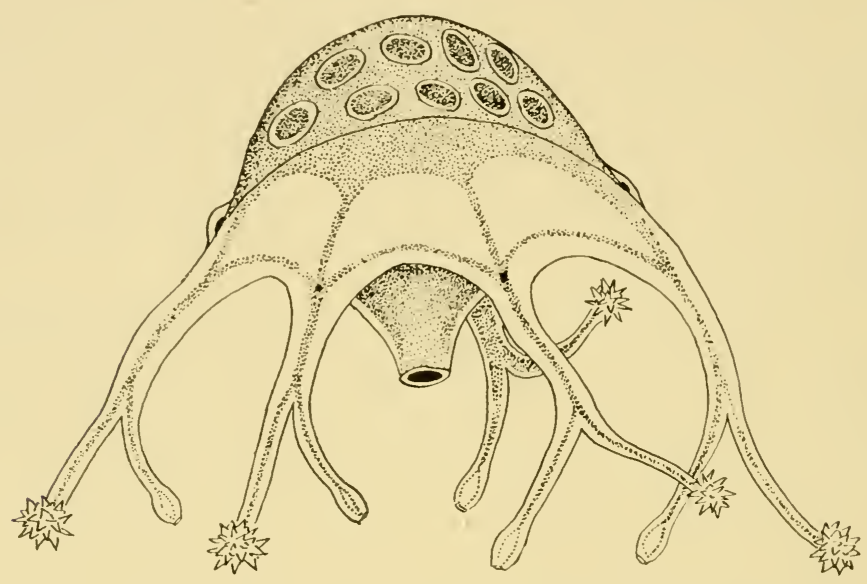

Fig. 26. A benthonic hydromedusa (Eleutheria dichotoma), from Hincks.

retrogression of the middle layer (mesohyl) that the gonad first loses its sharply circumscribed massive character. It develops more and more into a simple agglomeration of gametocytes. Those gonads which in the Turbellaria had been situated in the depth of the parenchyma are transplaced in the Anthozoa into the sarcosepta which is more usefull for the feeding of gametocytes. In this way the gonads have completely lost their contact with the skin layer and they have entered into a closer union with the intestinal epithelium. This, however, should not be understood as a transition to an endocarpic state, as was formerly interpreted 
by experts in Cnidaria. The gonads have remained in the cnidaria in the intermediate layer and then had developed as such into a corpus separatum.

We can observe, as a consequence of the progressive retrogression of sarcosepta (which had been developed from the longitudinal folds of the intestine), as early as in Scyphomedusae and quite generally in Hydrozoa, the transition of gonads into the body wall itself, thus into the very narrow area between the skin and the intestine. In the Anlage the gonad comes into the subepithelium either of the skin or of the intestine. In the ripe state the gonad causes a corresponding swelling either of the skin surface (e.g. in Hydra) during which gametes, that had become free, move into freedom through a dehiscence, or the gonad presses in the direction of the intestinal epithelium and passes over into the gastral space, and the gametes finally leave by way of the oral opening (this is the case, for example, in the supposedly primitive Protobydra, Boreobydra, etc.). The older data, which show the primitive sex cells to have developed out of the functioning skin (or intestinal epithelium cells), need revision during which it will be necessary to pay special attention to the subepithelial elements, this data is valid for a few species only of the Hydroids.

In the metagenetic Hydrozoa the gonads were transferred to the medusoid generation after they had been allotted certain areas of the animal body either along the radial canals, or on the manubrium. As a rule, the primitive sex cells become active as early as in the corm-building polyp generation and frequently far from the place where the medusa buds had been formed, so that the primitive sex cells have to move actively over considerable distances, as was formerly shown especially by A. Weismann. The same seems to be true, even if to a considerably smaller extent, for those Scyphozoa which have still preserved a corm-forming polyp generation (e.g. Nausithö̈). This wandering of the primitive sex cells reminds one of the wandering of cnidocytes. 
In the Hydrozoa the location of gonads can again be changed with a secondary retrogression of their medusoid generation. In this connection we must mention the unique case of Eleutheria medusae where the gonads have been transferred into the exumbrella with a simultaneous development of a brooding cavity during their transition to the benthonic way of life (Fig. 27). We have, furthermore, to mention here the Aeginidae, a group of Narcomedusae, which no longer possess

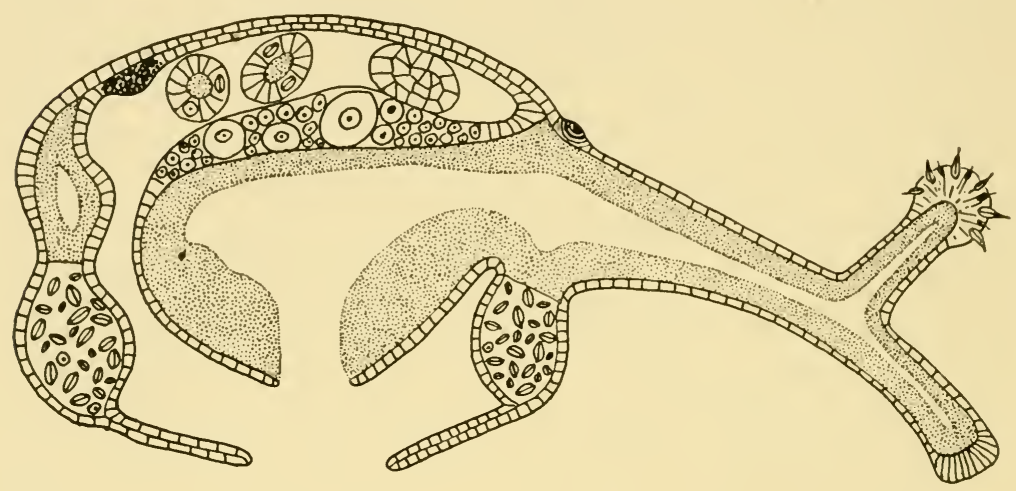

Fig. 27. Half schematic longitudinal section of a benthonic hydromedusa (Eleutheria radiata) with brood pouch in its umbrella (after Lengerich).

any distinct gonads and whose sex cells are scattered all over the subumbrella. In Hydra it is a trace of former conditionswhen sex cells still had to move and when they were therefore amoeboid-that the young egg-cells still move like amoebae, simultaneously eating food cells. There are some authorities who deny that an active migration of gametocytes really takes place. All these singularities can be found in Hydrozoa; they represent, in our interpretation, the final level that retrogression of the sexual apparatus has reached in Cnidaria, and not a primarily primitive condition.

Parallel to this retrogressive development of the sexual apparatus that can be observed in Cnidaria and in clear causal 
connection with the sessile way of life, another process takes place which, however, follows a progressive line of evolution. This process does not affect the gonads themselves but rather their bearers and the immediate surroundings in the cormus. Events have been created by these gonads which have led to far-reaching morphogenetic processes typical of Cnidaria but which, however, can also be found in a similar form in many other animal types. Due to polymorphism, special individuals had evolved which have later developed into specific bearers of gonads. This has finally led evolution to the complex gonosomes besides the medusoid generation (Fig. 31).

\section{The Alternation of Generations in Cnidaria}

It is in agreement with our thesis of the origin of Cnidaria that we find in the Anthozoa the most primitive conditions that can be observed in connection with the alternation of generations. The primarily solitary species of Anthozoa develop into sexually ripe individuals directly from larvae. In some species as, in Gonactinia prolifera, we can observe that an asexual reproduction by way of transverse division takes place before the animal becomes sexually mature; this is also the case in numerous Turbellaria. The transverse division is followed by sexual maturation and the only difference that exists between the two generations is that one had been reproduced sexually and the other asexually. In this case we can already speak of an alternation of generations if we attach greater importance to the type of reproduction than to the morphological or eventually ecological differences. It is my firm conviction that it is senseless to declare a form to be a larva when it is produced sexually and when itself reproduces asexually. Only a form which had been produced sexually can further develop through a pelagic larva. Finally in the solitary species all individuals become, in all probability, sexually ripe regardless of the way that 
they had been produced. Thus we get a simple growth cycle by way of an alternation of generations and without a sharp difference between the two generations.

Subsequent to this we find in a majority of Anthozoa the formation of cormi where the incomplete transverse division developed into longitudinal division and even more so into reproduction by way of buds. The conditions were initially the same as those that can be found in the solitary species that reproduce asexually. There is always a larger number of asexually produced generations. We find occasionally in the formation of cormi that not all asexually produced individuals (they are now subindividuals or persons) become sexually active. In many species of the genus Corallium there are, surprisingly enough, only the changed siphonozooids that become sexually active; the two generations live together as sessile animals in one cormus.

An interesting and, as it seems, unique case among Anthozoa is represented by Fungia which has been already mentioned above (Fig. 28). This is an apparently secondarily and not completely solitary madreporarian. Its sexually produced anthopolyp reproduces in two parallel ways; one by way of lateral budding which can lead to the formation of a cormus (colony). In this Fungia resembles other species of Madreporaria. The maternal polyp, however, can reproduce asexually, by way of transverse division; yet this division is unequal. The destiny of the two halves as well as their forms are different. The distal part which is slightly broadened and which takes over the circlet of tentacles and corresponds to the so-called anthocodium of anthopolyps descends, after its separation from the basal part, the former polypid, to the sea bottom. It grows into a large "coral" which continues to lie on its flat side and develops into sexual maturity. Special names have been given to the two generations which have thus been developed by way of transverse division. The basal part has been called an "anthocaulus," and the distal sexually ripe individual an "anthocyathus." There can be no doubt 
that in Fungia we have a typical case of the alternation of generations (metagenesis) where the two generations are first a typically polypoid generation, and then the sexual generation which does not at all reproduce asexually. We do not find acceptable Hyman's description of the sexual generation as an "adult disc" (I.:611). Due to the transition of gonads
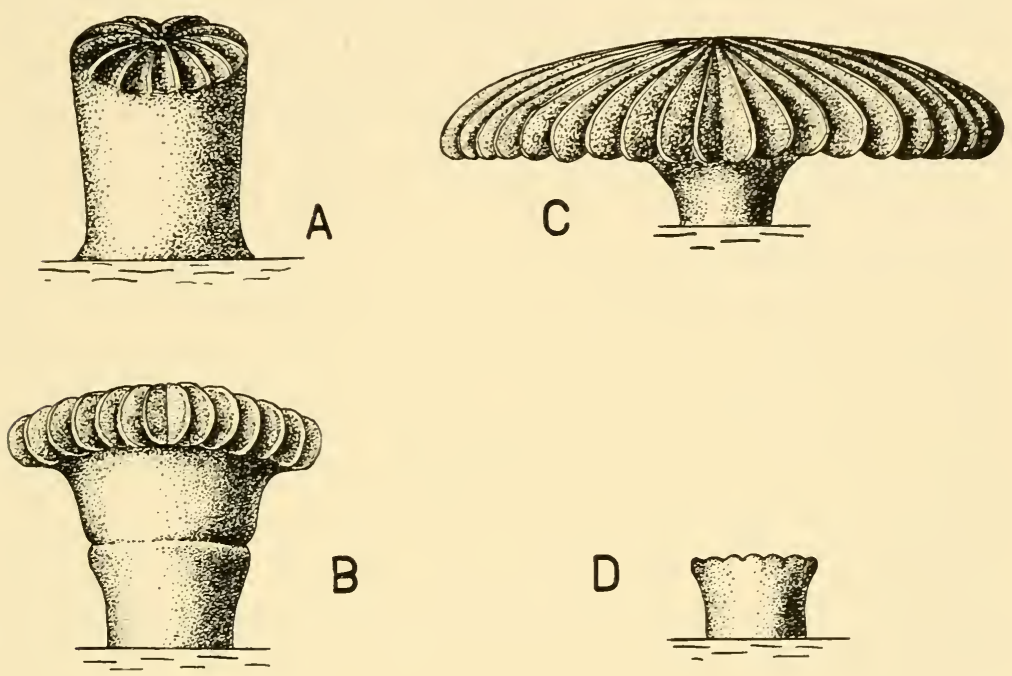

Fig. 28. The life cycle of Fungia sp. A, a young coral polyp. B, the beginning of transverse scission. C, anthocyathus is fully developed. D, after separation of the anthocyathus, the anthocaulus remains (after Delage and Hérouard).

into the second generation which has been developed by way of asexual reproduction, this generation has been considerably changed, morphologically, ecologically and biologically under the influence of these gonads.

In Fungia, and quite generally in the Anthozoa, we cannot speak of a medusoid generation. It is improbable that the case Fungia represents has in any way contributed to the formation of the medusoid generation, in spite of the fact that in it we can see something parallel, a certain common "trend." 
Even the word "medusoid" does not seem to be suitable here, it should continue to be used in connection with the anthocyathus, if it is necessary at all to have a special name in this case. The luxuriously developed inner calcareous skeleton had probably been a very important factor of the case of Fungia. With regard to the life cycle, there exists a deep chasm between the recent Anthozoa and Scyphozoa. A closer union of these two classes into one of Anthozoa, a suggestion which goes back to Goette, is a mistake which has fortunately now been completely rejected. In the Scyphozoa a special freely swimming generation has been developed by way of the transverse division which they had inherited from their anthozoan ancestors and because of the absence of a heavy inner calcareous skeleton. This generation, i.e. the Scyphomedusae, hare taken over the sexual function and later it has assumed the leading role. In this way metagenesis becomes even more clearly perceptible. To the primary polyp form (it is now called a scyphopolyp in opposition to the anthopolyp) which reproduces only asexually has been given a special name, i.e. a scyphistoma or a scyphostoma, and this again has been a reason for unnecessary misunderstandings. Scholars have frequently tried to represent the polyp generation of Scyphozoa as a purely juvenile form, and thus to degrade it to a larva. They have referred in this connection to a completely special and abnormal case which occurs under special circumstances, i.e. when a scyphopolyp transmutes in toto into a scyphomedusa, or an ephyra (i.e. a medusan larva), instead of developing a "medusan larva" by way of transverse division (strobilation).

Fortunately enough, there have survived many species of Scyphozoa that have preserved conditions that were formerly typical of them. Nausithö punctata is one such species which has already been mentioned. Its polyp generation produces irregularly formed cormi (colonies) with a rich development of the "periderm." Though gonocytes still continue to be produced in the basal part of the polyps, they migrate now 
into their distal part (corresponding to the anthocyathus of Fungia; one could therefore speak here of a "scyphocyathus" which, however, would be completely superfluous) which produces the larvae (ephyrae) of Scyphomedusae by way of a repeated transverse division (i.e. strobilation). It is in these ephyrae that the gonads arrive at their sexual maturity. I maintain that originally individual ephyrae only were developed (the primarily monodiscal strobilation); it was only later that the transverse division began to be repeated at certain regular intervals. The genuine strobilation, however,

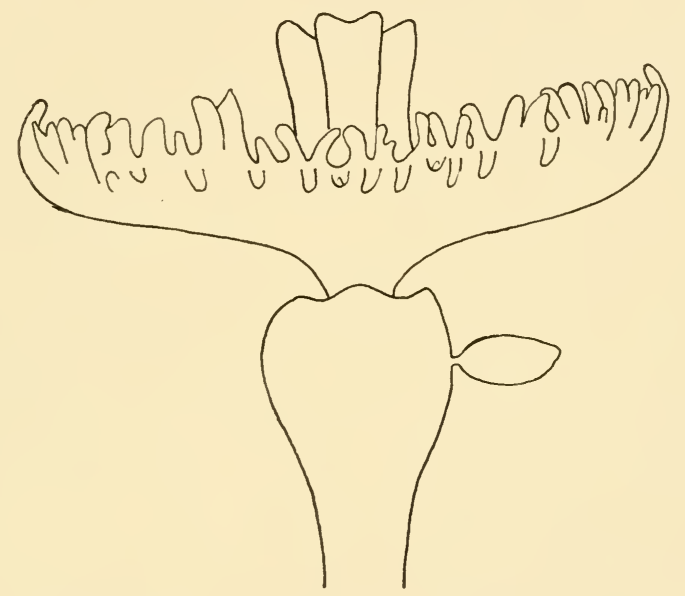

Fig. 29. Monodisc strobilation of Cassiopea sp., with a bud, (after Bigelow).

has been developed only secondarily, i.e. when the transverse division became a multiple one (the appearance of several simultaneous transverse divisions, or the polydiscal strobilation). This again has further led to a secondary single and unequal transverse division (the secondary monodiscal strobilation which is actually no longer a strobilation because it does not lead to the formation of a strobila-like form [Fig. 29]). This has finally led to a complete abandonment of the transverse division. 
Parallel to this we find in the Scyphozoa a characteristic retrogression of the polyp generation. As a consequence of this retrogression the whole class has been given the name of Scyphomedusae, because in this class the medusoid generation has become the prevalent one. No primarily solitary polyps have been known so far. The solitarization of the polyp generation took place by way of a secondary reduction of cormi. The periderm which was originally preserved by scyphopolyps was the same as had been inherited by the corm-forming species from their anthozoan ancestors of the type of the recent Stolonifera, among Octocorallia (e.g. Cornularia cornucopiae, [Fig. 42]). The cuticular external skeleton had been reduced during the solitarization and the elimination of the polyp generation so that finally there are only resting states, as for example podocysts (Hadži, 1912) that have remained covered with this cuticle. The sexual function, the formation of gonads, has been entirely transfered to the freely living medusoid generation. The completely weakened primary generation has finally been completely relinquished, so that it is the very progressively developed medusoid generation only which has been preserved. It has already been mentioned that a side branch has also been evolved in the Scyphozoa which has become completely benthonic so that cilia do not even occur in its planula (Fig. 35). These are the Lucernariidae whose medusoid generation remains hanging on the maternal polyp and is united with it into a new whole which represents a special case of hypogenesis (polypomedusae).

As among Scyphozoa, there are no primarily solitary species of polyps among Hydrozoa which should naturally also be sexually mature, and we dare maintain that in the Hydrozoa such species of polyps have really never existed. In all probability they were corm-forming from the very beginning. The sexual function has been taken over by the medusoid generation which has become secondarily free. We have already mentioned that in the more primitive species the primitive sex cells develop first in the cormus, after which they migrate actively 
into the Anlagen of special sex individuals; these finally develop into independent freely swimming individuals, into the second generation.

In this the Hydrozoa resemble the Scyphozoa, with the only difference that in the Hydrozoa the medusoid generation does not develop by way of a transverse division but rather by way of lateral budding so that they are therefore further developed from an independent root. We can observe, moreover, in Hydrozoa a secondary disappearance of the primary polyp generation and the prevalence of the secondary medusa form, hypogenesis (Trachylina), without a development of special auxiliary sex organs.

It is clearly in connection with this that in Hydrozoa the second sexual medusoid generation develops from "heads" of polyps (hydranths) which had been developed by way of budding, on the bearer of these polyps. This has frequently led to further complications. The colonial or cormus state which made it possible that those zooids in which the budding of medusae takes place, abandon their normal feeding function and develop into pure bearers (which are without tentacles and without a mouth) of medusae or of reduced medusae- of gonophores. They have been given a special name, that of a blastostyle. This again has led to a polymorphism (e.g. Podocoryne carnea). It is frequently difficult to determine whether we have in a certain case a strongly retrogressed polyp type bearer of gonophores or whether we have perhaps a special form, e.g. a stem of a bud (Fig. 30).

Frequently the development does not stop here, and an even greater influence can be exerted in a cormus by the Anlage of a gonad upon its whole environment. Thus in the case of Aglaopheniidae, among the Thecata, where medusae had become strongly retrogressed and where they remain attached to the cormus, we can observe the transformation of a whole branch of the cormus together with its minute twigs so that they form a protective receptacle around the 
"gonangia;" this is called a corbula because of its similarity with a basket. Simultaneously the polypoid individuals have been strongly modified and they have become in this way polymorphous. It was originally thought that this was a case of other species living epizoically on the cormi of Hydroidea, and for this reason these groups of accumulated gonophores and of transformed other zooids have been given special
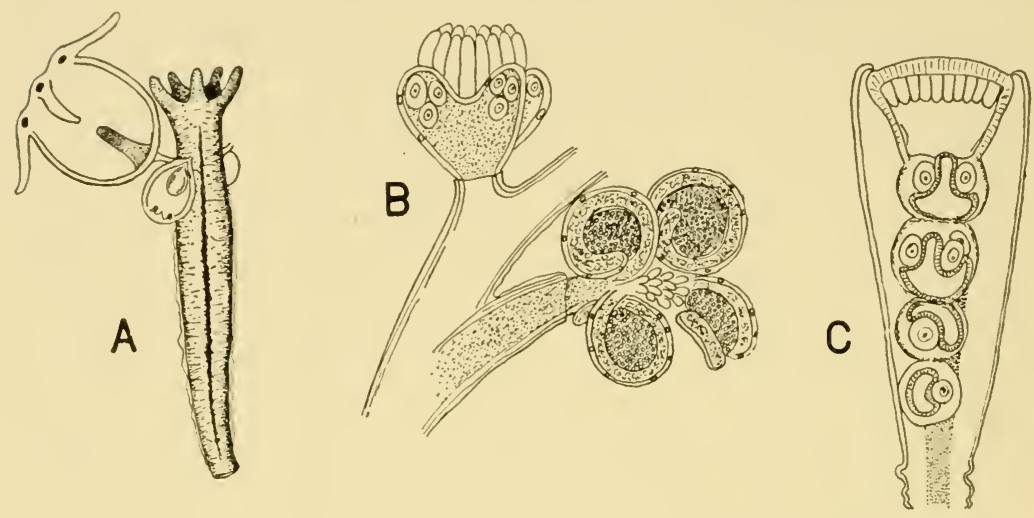

Fig. 30. Reduction of hydropolyps as bearers of gonophores. A, Podocoryne carnea (after Brooks). B, Eudendrium sp., (from Hyman); C, gonangium of Gonothyrea sp., with gonotheca, blastostyl and medusoid-buds (after Hyman).

names, e.g. Coppinia in Lafoëa pygmaea Alder (Hebellidae), or Scapus in Lictorella pinnata (Fig. 31).

In the Hydrozoa the Siphonophora form an interesting case. Contrary to the recent attempts made by A. K. Totton (1954) to separate part of the Siphonophora-Disconanthes or Chondrophora from the remaining Siphonophora and to establish them as a special taxon, I am of the opinion that the Siphonophora had evolved monophyletically and that they must therefore be considered as an indivisible unit, i.e. as an order of the Hydrozoa. The old discussion on how to interpret the organisation of the Siphonophora and their origin has not come to an end yet, still I think that the most probable inter- 
pretation is the suggestion which I have already proposed in one of my studies (Hadži, 1954), i.e. that the Siphonophora had evolved from the sessile cormi of the athecate Hydroidea by way of a transition to a life in plankton; they therefore represent an order of very polymorphous individuals partly of a polypoid and partly of a medusoid character. In all cases the basis of the cormus is an impersonal stock (siphonosome) which corresponds to the hydrocaulus of the Hydroidea. The orientation of the cormus had been turned similarly
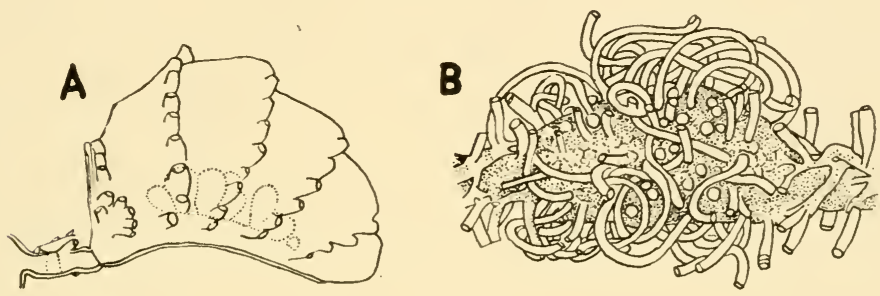

Fig. 31. Two examples of gonosomas of the hydroids. A, corbula of Aglaophenia pluma and B, coppinia of Lictorella pinnata; (after Broch).

as in the case of medusae. In part of the Siphonophora (Pneumatophorae Chun 1897 or Siphonanthes) we can find at the root of the stock which can secondarily be flattened and which can resemble externally a medusoid form, that a pneumatophore had been developed as an organ of the cormus and it therefore does not correspond to a medusoid individual. The Calycophorae (Leuckart 1854) and Pneumatophorae (Chun 1987), the two suborders of the Siphonophora, had separated phylogenetically quite early so that the species belonging to the first subgroup had not developed their own pneumatophore (or is it possible that they had originally possessed it and had lost it only subsequently?) and they use as their hydrostatic and swimming apparatus, the more or less modified swimming medusoids only. The Disconanthes are the most specialized subgroup of the Pneumatophorae. 
We cannot accept Garstang's thesis (W. Garstang, 1946) that the Siphonophora are "bud communities." This thesis is quite untenable because buds are in fact the asexual primordia of these subindividuals. Thus the "bud communities" are in fact true cormi (usually called colonies) which is also true for the chondrophore Siphonophora; Garstang, however, considers them as polypoid individuals.

With regard to the origin of the Siphonophora I again maintain that it is wrong to point in this connection to the planktonic larvae (planula and actinula) and to try to deduce the Siphonophora from them as has recently been done by E. Leloup (1929) and Hyman (1940). We can easily understand that as a consequence of a long progressive evolution within the frame of the subtype of the Siphonophora the ontogenetic process had also been modified and that the siphonula had evolved as a special larval form from the planula (and the ratarula as an even more specialized form which had been developed by the Chondrophora) so that it had developed into an Anlage of the cormus (Fig. 46 a). It can easily be understood why a primary polyp (i.e. a primary gastrozooid) develops as a kind of an Anlage of an oozooid on the larva of the Siphonophora and why it later grows as a monopodium into the Anlage of the cormus, i.e. into the basis of the cormus, into the impersonal stock on which the first members of the family bud as subindividuals either as the first swimming bell which is true for the subgroup Cylycophorae, or as an Anlage of the pneumatophores which is true for the Pneumatophorae.

The Siphonophora must be considered as the climax in the evolution not only of the Hydrozoa but also of the Cnidaria as a whole.

Because of our imperfect knowledge of the whole development cycle of a large number of the Hydroidea we must for the time being still accept the existence of a double system, i.e. that of the hydropolyps and that of the hydromedusae. This certainly is an anomaly which will soon have 
to disappear. The polypoid form (generation) must serve as basis for the construction of the definitive system of the Hydroidea. We cannot begin to discuss here the details of such a system and we must limit ourselves to a few general observations only.

We have here a progressive trend in evolution which has reached its climax in Hydroidea. All these phenomena can be completely understood only if we accept the standpoint which considers Anthozoa to be "the most primitive," and Hydrozoa as the most specialized, i.e. phylogenetically the youngest Cnidaria.

\section{The Digestive System}

While the external form of all three classes of Cnidaria remains essentially identical with their basic form, i.e. with the form of polyps (a cylindrical or a cup-shaped form) we find such differences in the form of the digestive system (of the internal layer or of the entoderm) that by means of these differences we are able to make clear-cut distinctions between the three classes. It was under the influence of the thesis of a generally progressive evolution of all living beings that the polyps of Cnidaria with a simple and smooth intestinal tube generally considered as the most primitive ones, those which show the formation of longitudinal septa in their "initial" stages as the next more highly developed ones, and finally those with strongly developed sarcosepta as the most highly developed polyps, an interpretation which agrees with the generally supposed line of evolution Hydrozoa $\rightarrow$ Scyphozoa $\rightarrow$ Anthozoa.

In addition to this there is one more complication in their digestive systems. On one hand we can observe in cormi that the digestive organs of zooids develop mutual communications; on the other hand, in medusae the tubes grew out of the central part of their digestive system, thus out of the "stomach," and this has finally led to the formation of a 
complete gastrovascular system. Since at the first glance similar complications were also known to occur in Spongiae and Ctenophora, the three animal types were united into the group of Coelenterata (Leuckart). In addition to this there developed the idea that the two principal body cavities (the intestine and the coelom) which appear in the "more highly" developed animal types, are in these animals mutually combined. This, however, has been proved to be wrong. It is almost unbelievable how obstinately the idea has been preserved according to which there should be a genetic relationship between the intestinal cavity and the coelomic cavities (i.e. the part of the coelomic cavities which surrounds the intestine and which we have called a perigastrocoel); this has led to the formulation of the so-called enterocoele theory which is doubtlessly wrong and which has been the cause of numerous controversies.

We have to make here a sharp distinction between three facts; first, between the digestive system of a single individual and that of cormi. It is only in the cormi of Cnidaria that the digestive organs of zooids that form a cormus are directly connected into a uniform canal system. The connecting canals (solenia, as they are frequently called) are impersonal, i.e. they are purely the organs of the cormi. The conditions that exist in the Spongiae are basically different from those that occur in Cnidaria (the Ctenophora, on the other hand, do not form cormi), and it is therefore clear that the Spongiae are completely disassociated from Coelenterata and also from Eumetazoa.

Secondly, in the Cnidaria we must make a sharp distinction between the digestive system of polyps-whether these be primarily or secondarily solitary animals, or whether the digestive system belongs to a zooid-and the "gastrovascular" system of medusae. The peripheral part of the digestive system of medusae with exception of the tentacular canals is a new formation and it has no homologue in the polyp. The principal part of the gastrovascular system is not a continuation of the peripheral part of the digestive canal of the anthopolyps. 
Thirdly, the digestive system of Ctenophora seems at the first glance to resemble that of medusae; in reality, however, it is quite different from the latter: and is, in our opinion, not a new formation but rather a transformation of conditions that had existed in their turbellarian ancestors, in Polycladida. It has been repeatedly emphasized that Ctenophora are not Coelenterata. The similarities that exist in their digestive systems are due to the fact that both Cnidaria as well as Ctenophora come from Turbellaria whose intestine shows a characteristic inclination to form diverticula or excrescences. The roots of the two groups, however, are separate. It was quite wrong when Haeckel tried to deduce the "gastrovascular conditions" of Ctenophora from those that occur in the hydromedusa (anthomedusa) Ctenaria ctenophora. On the basis of this wrong hypothesis we should assume that Turbellaria, as descendants from Ctenophora, had inherited from the latter the tendency of their intestine to form diverticula. In reality the development went in just the opposite direction.

When we pass to the discussion of the digestive organs of individual polyps of the Cnidaria (Fig. 32) we meet the generally accepted opinion that they followed a progressive line of development. If this were true, then it would be necessary to consider both the ectodermal gullet (pharynx) as well as sarcosepta as newly acquired complications. We must here raise the question whether we are allowed to suppose that the intestinal apparatus has been progressively developed in individuals in spite of their consistently sedentary way of life and the constant diminution of the size of the corm-forming zooids? If we take into consideration our experiences with other sessile animal groups we must straight away deny the probability of such a supposition; we must do this especially because this supposition is contradicted by our analyses of other systems of organs.

The situation becomes much more clear if we reverse the interpretation and if we derive the conditions that can be observed in the digestive organ of Anthozoa from those of 
Turbellaria. The two typical properties of this organ system in Anthozoa, the ectodermal gullet and sarcosepta, had been inherited at least as primordia from their turbellarian ancestors. This standpoint explains many facts which could not be understood till now. Both the pharynx as well as the tendency to form
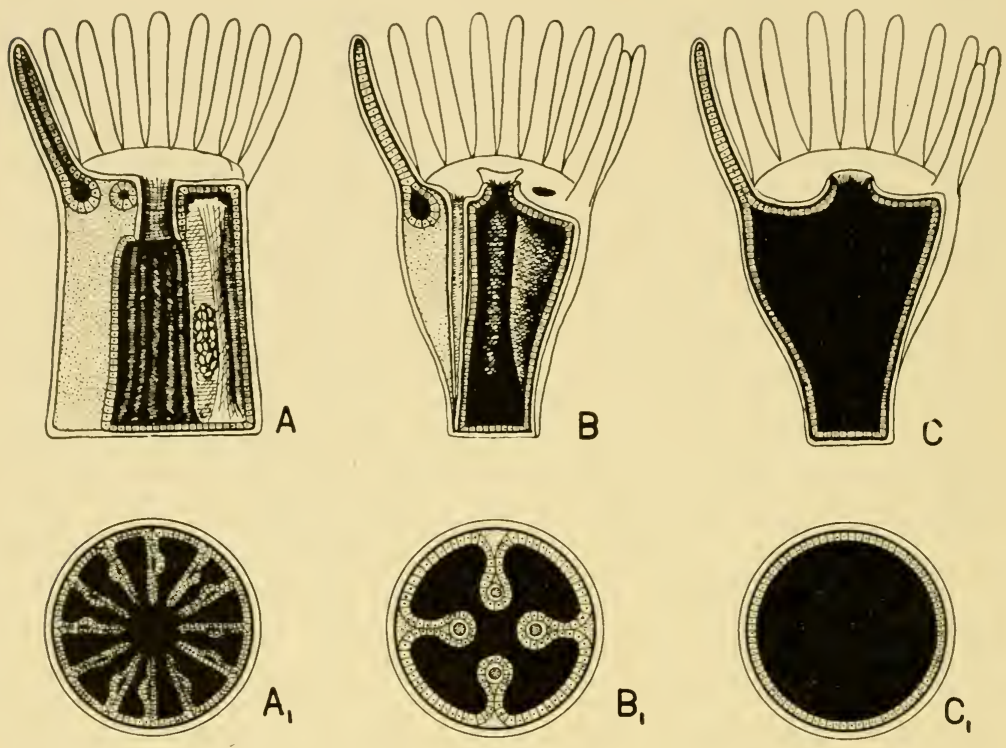

Fig. 32. Digestive systems of the three cnidarian polyps (A, anthopolyp, B, scyphopolyp, C, hydropolyp); above longitudinal sections, below, crossections (after Remane, somewhat altered).

variously shaped diverticula are properties that occur generally in Turbellaria. The formation of a gullet is phylogenetically even older than the tendency of the intestine to form diverticula, because the former had been inherited by Turbellaria from their protozoan ancestors, as we will try to prove later.

Among the Cnidaria we find a pharynx as an invagination of the external body layer and of the muscles that surround the oral opening only in the Anthozoa. Previously it was quite generally believed that an ectodermal gullet was also 
found in Scyphozoa, and this lead Goette to unite the two classes into one taxon of Scyphozoa. There were some zoologists who were opposed to such a classification. I finally succeeded in showing (Hadži, 1907) that there is no ectodermal gullet in scyphopolyps, neither is there one in Scyphomedusae (Heric, 1907), and this has now been generally accepted. It seems that in the Anthozoa both a functional as well as genetic correlation had been developed between the formation of sarcosepta and the formation of the gullet so that they are simultaneously subject to a progressive reduction when the retrogression starts. We must atribute to the formation of retractors in the taeniolae the fact that we can neverthless find remainder of earlier richly developed septal apparatus in the scyphopolyps. The formation of a gullet is extremely characteristic of Turbellaria. Organs with a very complicated structure have been developed in several directions from a simple invagination of the skin around the oral opening (pharynx simplex). Special names have been given to these organs. The pharynx became protractile due to the formation of circular folds so that finally it developed into an important organ by means of which animals that adopted the vagile and predatory way of life were able to catch their prey. This gullet has been called an Ergreifungsorgan (organ of gripping) by Ludwig Graff de Pancsova, who came from the region of present day Yugoslavia, formerly the leading expert in Turbellaria (v. Graff, 1878: 199). This organ has been developed progressively, in the same way as the hermaphroditic copulation organ. It may not be superfluous to mention here that the formation of a pharynx had been adopted by all the numerous descendants from Turbellaria, either in its complex form as a genuine muscular pharynx (e.g. in many classes of Ameria), or in its reduced form as a stomodaeum. We can find the stomodaeum everywhere up to the Chordata. This stomodaeum has been preserved even in those animal types which have entirely adopted the sessile way of life, as in Endoprocta, among Ameria, and in nearly all Oligomeria 
(it is in the Pogonophora and in the entoparasitic Acanthocephala that the stomodaeum together with the whole digestive system has been lost). Not infrequently the gullet has preserved its ability to protract, or if it had lost this ability, it has been secondarily regained.

It is therefore not surprising to see that the Anthozoa, too, had inherited their ectodermal gullet from their turbellarian ancestors, and this in the form of a pharynx simplex. Here I should like to call attention to some older observations which previously did not appear so important, but whose importance has been increased due to my interpretation. This is the use of the gullet of anthopolyps as a gripping organ similarly as observed in the Turbellaria. The first of such observations were made, as it seems, by C. Claus (1864) in a pelagic larva belonging to Actiniae (Claus believed that it resembled a ctenophore). Hertwig considered it to be a larva of Edwardsia which had a "protrusile stomach tube" ("ein vorstülpbares Magenrohr"). Young Halcampia that are ectoparasites and live as such on medusae behave like Turbellaria and they are even able to adopt their form. Carlgren (1904) reports about the species Halcampogeton papillosus that it can evaginate its "actinopharynx." The pharynx has an active role also in other Anthozoa, especially in the adult individuals. According to the brothers Hertwig (1879) gullet "fungiert als Magen, da in seinem Inneren die von den Tentakeln ergriffenen und nach dem Munde befördeten Thiere, kleine Mollusken und Crustacean, längere Zeit verweilen und dem Verdauungsprocess unterworfen werden."

Cases have been known where the pharynx has been reduced or even lost in Turbellaria in spite of the fact that in these it has been progressively developed. Such a reduction or loss has naturally taken place under the corresponding ecological and biological circumstances. The parasitic Vorticidae show, according to v. Graff $(1882: 181)$ a distinct inclination to reduce their gullet; it has been known that during its adult state the parasitic Fecampia does not possess a gullet. 
Quite generally we can reconstruct the history of the pharyngeal tube as follows. The primitive Turbellaria (there are such species even among the recent Turbellaria) do not yet possess a constantly developed pharynx. Palmenia has, according to Bresslau (1909), an oral opening in its surface through which the animal can stretch its digestive plasmodium (a real intestine has not yet been developed) in order to pick up particles of food. There is no reason to doubt that these are really the primitive conditions which had been inherited by the acoelous Turbellaria from their supposed infusorial ancestors. During the next higher stage in the evolution of Turbellaria, the invagination of the skin at the oral opening took place - with simultaneous active locomotion and macrophagiathis development took place also as early as in Infusoria; it led afterwards to the development of a special protrusile pharynx. The ability to form a gullet tube had been inherited by Cnidaria, that is specifically the Anthozoa, from their turbellarian ancestors. We are unable to determine the relationship between the structure of the present-day Anthozoan pharynx, the primitive Anthozoan pharynx, and the primitive Turbellarian pharynx. The pharynx had anyhow been developed in the Anthozoa in a progressive and independent direction, and that in connection with the evolution of the septal apparatus. Even if there are at present whole families among the recent primitive rhabdocoelous Turbellaria which have a pharynx simplex (e.g. Catenulidae, Microstomidae), this does not necessarily mean that the turbellarian ancestors of Anthozoa had only a pharynx simplex. The possibility must also be taken into consideration that at best the present state could be a result of a retrogressive development, especially in view of the fact that the sessile way of life leads to a reduction of the general organization.

In the Anthozoa a diversity can be observed in the subsequent destiny of their gullet tube (pharynx); whether it occurs in solitary species which are capable, however slightly, of locomotion, or in species which form cormi and which are 
attached to the bottom. In the first case, the evolution has followed a somewhat progressive line even if in a different direction to the one that can be observed in Turbellaria. In the second case, there was a strong retrogression which finally led to a complete loss of the gullet. The form of the gullet tube changed in accordance with the change of the form of the mouth(it is only rarely that the round opening has been preserved) into an oblong or oval opening which can be laterally or transversally closed. It is not easy to determine which corner of the mouth is dorsal and which is ventral. The two corners frequently continue in a long tip which extends deep into the interior of the body. A well-known groove, the siphonoglyph or the sulcus, which is densely covered with cilia, develops along one corner of the mouth (called the "ventral" corner), and frequently also along the two corners of the oral opening. Furthermore, a rich longitudinal folding can appear in this oral tube, a sphincter at its lower end, and special, even if poorly developed, muscles which usually extend in the longitudinal direction, a so-called concha, etc. Polymerization can be observed, surprisingly enough, in siphonoglyphs, this reminds us at least partly of the polymerization of whole pharynxes of some planarian species. A further complication appears due to the linkage of the gullet tube with the proximal parts of sarcosepta. Because of this, the gullet tube can reach deeply into the interior of the animal's body. Parallel to the morphological changes, which have led to a further specialization, physiological changes also took place (e.g. respiration by means of a circulation of water).

A reduction of the gullet tube appears as early as in Anthozoa; it is frequently a consequence of the formation of cormi and, in connection with this, of a considerable diminution of subindividuals, combined with microphagy. The gullet tube becomes shorter and shorter, the siphonoglyphs and muscles disappear, and finally there develops an oral cone which surmounts the oral surface, as can be observed in some Madreporaria of the solitary species Balanopbylia. In this way 
conditions had been prepared as early as in the Anthozoa which have become generally characteristic of Scyphozoa and Hydrozoa. It is in agreement with the way of life and with the type of feeding that the peristome evaginates into an oral cone, a development which begins to prevail over the opposite trend, i.e. over the invagination of the oral area.

The oral cone, as a process of the oral area, possesses a welldeveloped muscle system (externally these muscles are placed longitudinally, internally they are placed transversally); by means of these muscles the oral cone is enabled to move in all directions, as well as to contract and to extend, always with the oral opening at its top. The oral cone, or the manubrium of medusae, develops again into a gripping organ; frequently it can even take over the function of tentacles which makes macrophagy possible and this can finally lead to a total loss of tentacles. Not a single trace of an ectodermal gullet can be found either in Scyphozoa or in the Hydrozoa. The entoderm of the anterior intestine-which passes over into an ectoderm exactly at the oral margin-is richly equipped with gland cells which help swallowing and digestion.

It has already been mentioned that among the Cnidaria an ectodermal gullet as well as true sarcosepta can be found in Anthozoa only as special elements of their digestive system. Neither the one nor the other of these two elements can be found in the most primitive Turbellaria, even if both of them can be found to a certain degree in a large number of Turbellaria. Certainly, we do not find here sarcosepta but rather a general and distinct trend of their intestine to form diverticula. The oldest fossilized Anthozoa possessed well developed sarcosepta, even if these cannot be seen; the fact, however, that they possessed sclerosepta which alone could be fossilized is a certain indication that they also possessed sarcosepta which could not be preserved by fossilization. The supposed presence of sarcosepta allows us to consider that these primitive anthopolyps had already had a pharynx. The supposed transition from Turbellaria - with intestinal diverticula-to antho- 
polyps with primitive forms that do not yet deserve the name of sarcosepta but which resemble rather some longitudinal folds, apparently took place in the period when no mineralized skeletons had yet been developed; we can therefore not expect that we will ever be able to find such transitional fossils. This makes it necessary for us to resort to a reconstruction.

We can see considerable differences between the two forms when we compare sarcosepta such as they occur now in Anthozoa with the intestinal diverticula of Turbellaria. These differences seem to support the old interpretation of the evolution of Cnidaria, and they have actually been used by those who adhere to the old interpretation in their critiques of my new thesis. This new thesis, however, becomes clearer if we make a detailed comparison, taking simultaneously into consideration the radical change in the way of life, i.e. the transition from creeping movements to the sessile way of life, and the erection of the body. By means of this new interpretation many things can now be explained which could not be explained on the basis of the old theory.

The tendency of the intestine to form folds that finally leads to the formation of excrescences - the diverticula - which is so generally prevalent among Turbellaria (with the exception of the primitive groups of Acoela and the majority of Rhabdocoela) must be considered as the starting point for the development of sarcosepta which are characteristic of Anthozoa. Here we will first study that part of this tendency which is connected with the formation of excrescences of the body skin at the anterior end of a somewhat cephalized body. This tendency is not so marked in Turbellaria as it is in Cnidaria. We meet here the case of the Ctenophora which we derive from the pelagic larvae of Polycladida, obviously from such where no intestinal diverticula had yet grown into the pair of tentacles. This has led to one of the most conspicuous differences that exist between Cnidaria and Ctenophora. We must suppose that the ancestors of Cnidaria had already developed the tentacular diverticula, and this is the reason why this property 
has become a general and unexceptional characteristic of Cnidaria. The polymerization did not remain limited to the tentacles only, it was also extended to the intestinal diverticula. The only change which took place in these intestinal diverticula of tentacles was a secondary obliteration of the lumen of these diverticula; as a consequence of this change the axis of tentacles became solid and the function of the diverticulum changed. It has now been developed into a supporting organ whose function has become more mechanical. This development has not occured, as it seems, even in single cases in the Anthozoa: their tentacles are always hollow, i.e. the lumen of the diverticula of these tentacles has been preserved as a part of the so-called gastrovascular system. It is only in Scyphozoa, and even more so in Hydrozoa, that the cavities of the intestinal diverticula of tentacles have become obliterated.

In this connection, I cannot silently pass the fact that this morpho-physiological specialization of the intestinal excrescences, which were originally hollow in order to make possible the distribution of the fluid food, into solid organs with a purely mechanical function can be repeatedly observed in the phylogeny of Metazoa (Fig. 33). Here I must limit myself to a few remarks only. Even among the Cnidaria we can find, besides the solid entodermal axes of tentacles, a case in Tubularidae, among Hydrozoa, where a cushion in the form of a circlet and consisting of vacuolized entodermal cells had been developed around the stomach. Other cases have been described in connection with the Turbellaria. I wish particularly to mention here the so-called Hemichordata (Enteropneusta and Pterobranchia) with their "bucal diverticulm," and finally the chorda dorsalis which has given its name to the whole highest phylum, to Chordata. These are certainly not homologous forms in the strict sense of the classical comparative anatomy; they are, nevertheless, parallel organs. We have here a morphogenetic inclination of the intestinal tube locally to take over functions which are not connected with the digestion; this has finally led to the formation of new organs. 

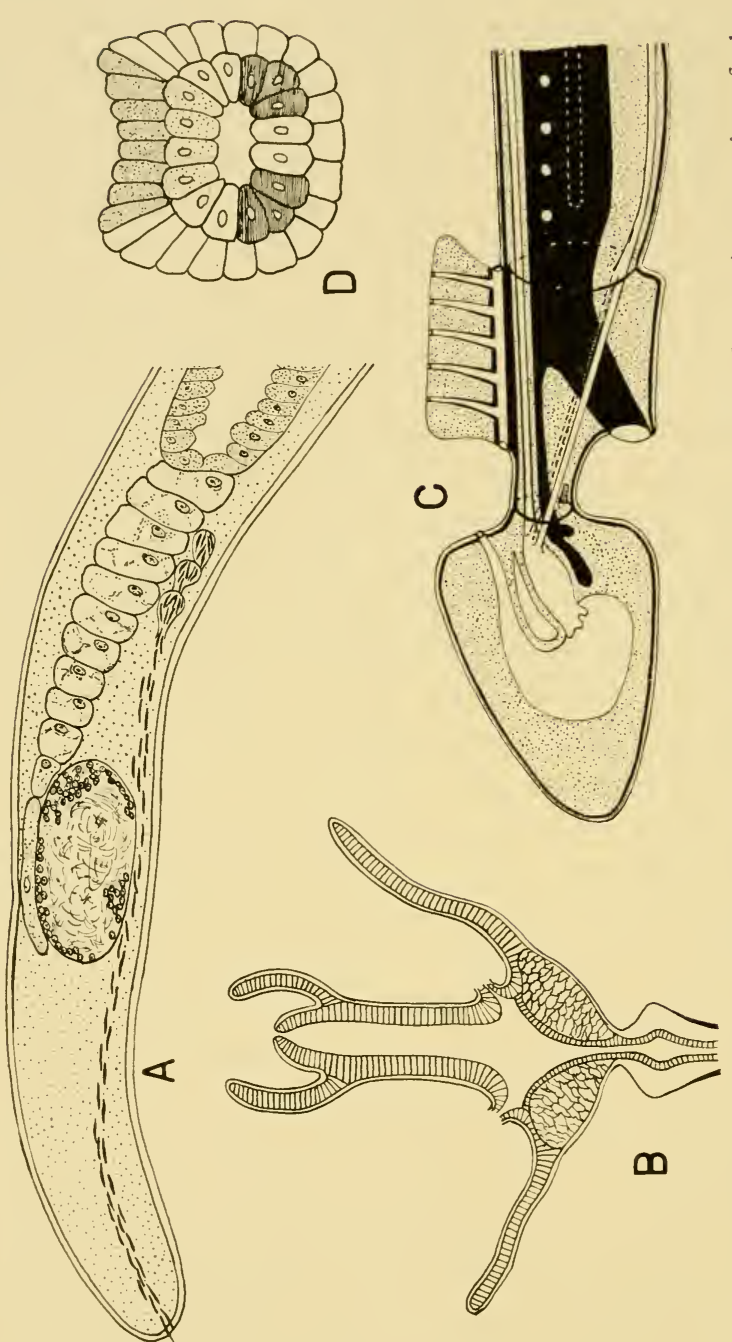

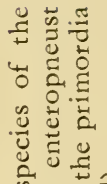

के

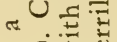
$<\stackrel{\dot{0}}{\dot{\Delta}}$ $\dot{\varepsilon} \cdot \hat{0} \cdot \hat{0}$

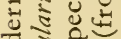
券 G हี. 屯웡

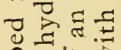
可岁 엉 프 过 䗆 สำ है tै + $\dot{x} . \Xi$ 出 J

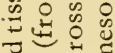
人ิ ¿ี่

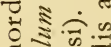
प्रि

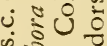
पू कु है के कु ลे है है कि घ क. 동 थे त्ञ क्षे 
When we now pass to the discussion which has taken place within the field of the comparative morphology about the intestinal tube itself, we have first to state that according to the old, and now prevalent, interpretation there can be no homology between the intestinal diverticula of Turbellaria and the sarcosepta of Anthozoa and Scyphozoa. The intestinal tube of hydropolyps has a smooth cylindrical form. A new complication, i.e. four simple sarcosepta or longitudinal folds of the intestine, does not develop earlier than in scyphopolyps (these are usually considered as larvae or young forms of Scyphomedusae). According to Goette, these four sarcosepta have been developed in such a way that there first appeared two intestinal pouches situated one against the other which were followed by another transverse pair; four taeniolae which disappear downwards were developed between these pouches. In the higher level, i.e. Anthozoa, sarcosepta with a complex structure are formed by way of polymerization with a simultaneous formation of a gullet tube. These sarcosepta are usually depicted, quite statically, under the influence of what we see when we make a transverse section through the body of an anthopolyp, as septa which extend longitudinally into the gastral space. W. Kükenthal, an expert in anthopolyps, does not mention, surprisingly enough, "mesenteries" in his general characterization of Anthozoa which was published in the extensive Handbuch der Zoologie (1924:688) in the additional characterization, however, he reports as follows, "Der Gastralraum des Korallenpolypen wird durch Längscheidewände, die vom Maueblatt nach innen vorspringen, in eine Anzahl periphärer Kammern und einen gemeinsamen zentralen Teil zerlegt." In the same work the following definition of sarcosepta has been made by F. Pax (1924:783), "Die Mesenterien (Sarcosepten) sind bindegewebliche, an ihrer Aussenseite hauptsächlich mit Entoderm bekleidete Stützlamellen, die von der Körperwand gegen den Gastralraum radiär vorspringen." The supporting lamellae are, according to this definition, the most important part of sarcosepta. The characterization of 
sarcosepta that occur in scypho- and anthopolyps as longitudinal folds of the "Darmblatt" (intestinal layer) is not so frequent; it was made, by Grobben and Kühn in the well known Lebrbuch der Zoologie (1932:461), "Die Septen ... sind senkrechte von der Seitenwand, der Fuss- und Mundscheibe entspringenden Falten des Darmes..." A. Kästner, in the recent "Lebrbuch der speziellen Zoologie," avoids defining the sarcosepta of Anthozoa; he mentions, however, in connection with scyphopolyps, four folds of the entodermal wall which are directed centralwards (1954:109). Hyman, in her Manual of the Zoology of Invertebrata (1954:367), tries to combine the two standpoints and writes as follows, "Primitively consisting of a simple tube, the digestive system displays through the phylum a tendency to complication by putting out branches and pockets and in the Scyphozoa and Anthozoa is divided into compartments by gastrodermal-mesogloeal projections."

The septa of scyphopolyps (earlier they were frequently called taeniolae) were previously considered by zoologists as homologous to the sarcosepta of Anthozoa; these were previously called mesenteries, in spite of the fact that the conditions that can be found in scyphopolyps give the impression of longitudinal folds which are developed due to the formation of peripheral sacks. On the other hand, there was nobody who thought of a possible homology of these septa, together with the intestinal pockets that occur between them, with the intestinal diverticula of Turbellaria. It was considered that the two forms had been developed quite independently from each other. There is not yet a distinct intestinal tube in Acoela, in Rhabdocoela this tube is smooth, approximately as it is in hydropolyps, and it is only in the "higher" Turbellaria (Polycladida, Tricladida, etc., according to the old system) that a gastrovascular system has been developed which becomes increasingly complex, whose ramifications can finally develop into a whole net, similar to that observed in some Ctenophora. It was therefore quite impossible to believe that Cnidaria could be deduced from Turbellaria, or vice versa. 
When finally the idea has been given up, quite correctly, by modern experts in Turbellaria to derive the Turbellaria from the Cnidaria by way of Ctenophora, they have endeavoured, together with von Graff, to go back to some common planuloid ancestors and wait cautiously until the last word of the origin of Cnidaria was said by the experts in Cnidaria.

Here the question appears of what the anthozoan sarcosepta actually are if we take into consideration their origin? What is their function? How do they appear if compared with the intestinal diverticula of Turbellaria, as well as of Ctenophora? We can get a clear idea if we study the morphogeny of sarcosepta during ontogeny. Sarcosepta have developed due to a longitudinal folding of the intestinal tube. We can say that the first active step (similarly as in the formation of the Anlagen of radial canals in the buds of Hydromedusae) is made by the wall of the intestine by way of a stripe-like allometry. During the next step the mesenchyme (mesogloea) grows into the longitudinal folds in the interstices between these folds; thus from the periphery and towards the centre, while at the same time the growth of the longitudinal folds goes in the opposite direction. We can therefore say that sarcosepta as fully developed forms have been evolved due to a longitudinal folding of the intestinal tube. This longitudinal folding is, from the physiological standpoint, in reality the same thing as the formation of diverticula by the intestine of Turbellaria; both represent an increase of the internal digestive surface of the entodermal epithelium and they both make possible the transportation of the fluid food to the periphery of a body whose size has been increased. A connective tissue (mesohyl) grows between these diverticula and the longitudinal folds; gonads and muscular fibres are later transplaced into it.

At the first glance it does not seem to be important whether in connection with the formation of sarcosepta we speak about longitudinal folds, or about an ingrowth of septa. This difference, however, is fundamental. It is the effect only, the final static state, which are similar. 
We must again emphasize that old as well as the modern embryologists who have studied the ontogeny of Anthozoa and of scyphopolyps agree that sarcosepta (frequently these have been less fortunately called mesenteries) develop by way of a longitudinal folding of the intestinal wall. Kowalevsky (1873) already had established this and he made fine illustrations of it. Korschelt and Heider (1936:198) described this process in their large Lebrbuch der Embryologie der Avertebrata, "Die Mesenterialsepten entstehen an der Wand als Längsfalten des Entoderms." In Actiniaria, as a rule, the first pair of sarcosepta which shows a strictly bilaterally symmetric distribution develops in this way. L. Faurot (1913:283) is right when he speaks of "deux plissements entodermiques." In scyphopolyps this process has been described as a formation of the peripheral pockets of the intestine; and this has actually the same meaning. This formation of folds starts regularly closer to the oral pole and develops in the direction of the lower end of the body until it finally more or less disappears.

Even if it is admitted by those who criticize the interpretation which derives Anthozoa from Turbellaria that the sarcosepta are a result of a longitudinal folding of the intestinal wall (e.g., Luther in lit.), there still remains a great difference from the conditions that can be found in Turbellaria. In the Turbellaria we do not have, they state, septa or a longitudinal folding but rather the formation of intestinal diverticula. These are placed transversally to the longitudinal axis of these animals and they follow each other bilaterally. This objection is certainly true. It is true that in the more or less flattened and bilaterally symmetrical Turbellaria the intestinal diverticula appear, as a rule, as excrescences that are placed transversally to the longitudinal axis of the animal, they are therefore on the right and on the left sides of the intestinal tube, and not around the intestine as this is the case with the cylindric Actiniae which stand erect. If we tried to derive by all means (which would be certainly wrong) one extreme case, an Actinia with its numerous partly perfect and partly imperfect sarcosepta which appear in a bi- 
symmetric distribution, out of another extreme case, e.g. out of a polyclad turbellarian, whose body is maximally flattened and extended with a possibly large number of diverticula or which can even form a net of digestive canals we would have to accept, first, that diverticula had grown additionally in other transversal directions and not only on the right and left sides, and, secondly, that diverticula grew together which appeared successively in lines parallel to the intestine, and that this has finally led to the formation of longitudinal sarcosepta. The first possibility is not only probable; it actually takes place even if to slight degree and only in such species which are not flattened but show instead in their transverse sections the form of a square, and which possess four or even more (instead of two) longitudinal series of intestinal diverticula (e.g. the polyclad species Thysanoplana indica Plehn and Pseudocerops periphaeus Bock). The second possibility is theoretically probable, but it has not been supported by observations and we will therefore prefer not to take this possibility into consideration.

We will rather consider it more probable that in Anthozoa, the formation of sarcosepta had been newly developed by them; yet this formation goes back to a general tendency of the intestine to form excrescences, diverticula, and folds. This tendency had been inherited by the Cnidaria from their turbellarian ancestors and it has finally led to the formation of the gastrovascular system. The formation of longitudinal folds agrees better than the formation of diverticula (which has been preserved in the development of the circlet of tentacles!) with the transition to the polyp form which stands erect and whose size has been constantly increased. It should be mentioned here that the progressive development of subtypes of Actiniaria led to the formation of species which have remained solitary and whose size can be considered as comparatively very large (up to $1 \mathrm{~m}$ in diameter). Sarcosepta have evolved both quantitatively (under the influence of polymerization) as well as qualitatively (differentiation) in those 
groups of Anthozoa which have remained solitary animals. The subsequent evolution took a retrogressive direction after they had passed over to cormogony, a simultaneous decrease of the size of individual zooids and, in connection with this, of the internal surfaces.

I think it useless to discuss whether the intestinal diverticula of Turbellaria be homologous or homotypic to the intestinal pockets of Anthozoa that can be found between their sarcosepta. It is certain, however, that both these forms are due to a tendency of these animals to increase the digestive or resorptive surface of their intestinal tubes; these forms diverged in their evolution which in these animals also pursued different directions: on one hand we have (in Turbellaria) a flattened form of an animal which creeps over the ground, and on the other hand (solitary anthopolyps) a cylindric form which stands erect and which can move actively only slightly, or not at all.

On the other hand, we can find that even in Turbellaria an inclination to develop longitudinal folds in their intestinal tubes is not completely unknown to them. What other than a longitudinal folding can finally be the forked form of that part of the intestine of Tricladida which can be observed in the posterior half of their body? One could expect, if more extensive investigations had been made in this respect, that the formation of such longitudinal folds could be found even more trequently in small species of Turbellaria whose transverse sections show a rounded-off form. It would be permissible, I think, to classify the longitudinal folds and herewith the septal apparatus under the notion of the intestinal diverticule and also the terminal diverticula (prochorda) as well as the chorda dorsalis. The fact is not without a significance that during the transition from anthopolyp to scyphopolyp the sarcosepta which had been reduced into taeniolae had clearly obtained the form of longitudinal folds. It should be added that the wrong interpretation of the true nature of sarcosepta is partly due to the fact that sclerosepta, which are externally similar to sarco- 
septa, really grow from the outside inwards. Yet these two forms are two completely different things if viewed from the standpoint of the comparative morphology, as well as that of physiology. In both cases, in Turbellaria as well as in Cnidaria, a vascularization of the periphery takes place by means of which fluid food can be brought to any part of body. The experts in Turbellaria are inclined to accept such an interpretation. The middle layer contains powerful strings of muscular tissue; some experts in Turbellaria, particularly O. Steinböck (1924:495-502), think that these strings of muscles that usually extend dorsoventrally have a major role in the formation of intestinal lobes and of intestinal diverticula, much as the sex apparatus dominates the middle part of the posterior half of the body. Yet the tendancy of the intestine to form diverticula is not mentioned in this connection. I believe it probable that here the intestinal wall has an active role, pressing forward the diverticula into the tentacles as can be observed in some species, or into some other excrescences, as can be observed in the species belonging to the genus Thysanozoon. It is possible that the strings of muscles that extend in the dorsoventral direction were developed later or almost simultaneously with the formation of diverticula. Obviously, we have to distinguish between the formation of limbs and intestinal diverticula.

A progressive trend can be observed in the evolution of sarcosepta in the primarily solitary anthopolyps. A strong, locally developed system of longitudinal muscles has been developed in their interior; these muscles can be observed in transverse sections as the well-known flags of muscles. Besides these muscles other, weaker, muscular systems have also been developed which extend in other directions. Gonads are transferred into the mesenchyme of sarcosepta (frequently into certain pairs of septa only) which has influenced scholars so that they have begun to speak about entodermal gonads (Endocarpa), similarly as in the Ctenophora: gonads, however, should not be attributed to any body layer. A special histologic-cytological differentiation took place in the free margins of 
sarcosepta which protrude into the gastrovascular space. These free margins have become thickened (in a transverse section they appear completely round), and in the epithelium we find not only gland cells but also cnidae. The curled margins can grow locally into long filaments which can be pressed into the free water through special openings that develop in the body wall (cynclidae) to be used there as weapons (acontia).

The transition to cormogony (the formation of colonies), connected with the decrease of the size of the polyp individual has led to a reduction of sarcosepta. This reduction was both quantitative (the reduction and simultaneously the fixation of the number of sarcosepta to six or eight), as well as qualitative (small acontia, fewer filaments, etc.). In the skeleton forming Madreporaria, the digestive surface has been secondarily increased by means of sclerosepta which grow from below into the gastral space. This reduction is coordinated with the reduction of the gullet pharynx tube. Sarcosepta are not present at all in the solenia of corm-forming species and here we therefore have the opportunity to observe all possible transitions in one and the same object, from a typically anthozoan intestinal tube, to the kind that can be found in Scyphozoa, and finally to a perfectly smooth intestinal tube, a state that has been reached in Hydrozoa.

The reduction of the septal apparatus has gone even further in scyphopolyps, parallel to the decrease of their size and to their sessile way of life. In this way this reduction can now be understood. The number of strongly diminished and cytologically simplified sarcosepta (they are also called taeniolae) has been reduced to the possible minimum, i.e. to four, in an increasingly developed radial symmetry. If we take the old view of a progressive evolution along the line Hydrozoa $\rightarrow$ Scyphozoa $\rightarrow$ Anthozoa, we find it impossible to explain how the sessile polyp of Cnidaria could suddenly begin to form sarcosepta. This situation becomes even more difficult to understand if we deny to the scyphopolyp the character of a special generation, as was done by Hyman. 
It will be easier to understand the actual conditions if we begin our comparison of anthopolyps and scyphopolyps with such forms as Antipatharia among Anthozoa, and with Nausithoë whose polyp generation has been described under the name Stepbanoscyphus or Spongicola. The polyp generation of Nausithö̈ (probably also of several other genera) forms genuine cormi (colonies) and an organic exoskeleton (periderm). There are four sarcosepta only which are richly equipped with filaments, and a large quantity of longitudinal strings of muscular tissue (they were called taeniolae by Komai) which occur under the skin in the wall layer. Gonads appear in the sarcosepta of the scyphopolyp of Nausitbö, a phenomenon which cannot be observed in a typical scyphystoma (a completely unnecessary name); this has been partly the cause of the erroneous interpretation that we have here a medusa larva, even if gonads do not come to their sexual maturity in it, but only later in the medusoid generation which in Scyphozoa takes over the sexual function.

Nobody can, in view of all these facts, doubt the homology between the sarcosepta of Anthozoa and the so-called taeniolae of scyphopolyps. We must only give up the supposition that sarcosepta had been developed from taeniolae. In the ontogeny of scyphopolyps we can observe even more clearly than in the ontogeny of anthopolyps, the development of sarcosepta as longitudinal folds or excrescences of the "stomach pockets." This is actually the same as the formation of intestinal diverticula. In the literature (Goette) we can even find data which show that there is first one pair of such intestinal diverticula, so that for a short time bisymmetry prevails, just as in the ontogenies of Actiniae.

One of the greatest differences between the sarcosepta of anthopolyps and of scyphopolyps can be found in their muscle systems. It is an important and unexplainable difference for those who adhere to the outdated and erroneous gastraea theory and to the theory of two body layers that are separated from each other by a Chinese Wall, when in Anthozoa the flag 
shaped strings of muscles that can be observed in the sarcosepta have developed from entoderm, while the muscles of the taeniolae of scyphopolyps have developed from the ectoderm. A similar situation can also be found in connection with gonads (endocarpous-ectocarpous). As a matter of fact, however, this is not a fundamental difference. Neither the muscle fibres nor the sex cells should be considered as entodermal or ectodermal, even if they appear one time as appendages of the skin epithelium, and at another time as those of the intestinal epithelium. It should be added that in the large and certainly primarily solitary Cerianthidae no concentration of longitudinal muscle fibres into flags can be observed (cf. brothers Hertwig, 1879:117). Longitudinal muscles appear under the skin in the body wall; muscle fibres that extend somewhat transversally can be found in the proximal parts of their numerous sarcosepta, and it is only in the thickened free ends of sarcosepta where stronger strings of longitudinal muscles appear. With regard to the latter it could be stated that they are in all probability "ectodermal" in their origin because it is supposed that the ectodermal tissue sinks downwards from the intestinal tube along the free ends of sarcosepta. We find, conspicuously enough, in Ceriantbus and in the polyp of Nausithö a tendency of the free ends of their septa to fork when they grow into filaments.

The strings of longitudinal muscles of antho- and scyphopolyps were in all probability even better developed in the now extinct Tetracorallia (e.g. in Calceola); they helped the animal to retract.

Here may be the right place to try to get a clear understanding of the fact that a strong tendency to tetramerous radial symmetry can be observed quite generally in the interior (and partly also in the exterior) of Cnidaria, and particularly in Scyphozoa and in Hydrozoa. First I wish to emphasize that this is not a general characteristic of sessile animals as such. There is, to give an example, a pentamerous radial symmetry in Echinodermata which had certainly evolved 
from some sessile ancestors; this pentamerous symmetry, however, is not a property which occurs 100 per cent in all Echinodermata; neither is the tetramerous radial symmetry such a property in Cnidaria.

Among the recent Cnidaria the tetramerous radial symmetry can be found especially strongly developed in Scyphozoa and in Hydrozoa, both in their polyp as well as in their medusa forms. It is least developed in Anthozoa. This could lead us to a conclusion, if we accept the view that the evolution took the course Anthozoa $\rightarrow$ Scyphozoa $\rightarrow$ Hydrozoa, that this inclination to the tetramerous radial symmetry had not been developed earlier than during the phylogeny of Cnidaria. On the other hand, if we consider the opposite interpretation to be true we are obliged to accept that in Hydrozoa the tetraradial symmetry had been developed for an unknown reason, and that it had been inherited from them by Scyphozoa. We will not find this problem so simple if we accept the interpretation which sees in Anthozoa the most primitive Cnidaria, yet the problem is still such that it can be rationally solved.

No recent subgroup of Anthozoa with a tetramerous radial symmetry has been known. Neither can such a group be found among the fossil species, even if one large subgroup which is now extinct bears the name Tetracorallia or Rugosa (palaeozoologists prefer now to call this subgroup Pterocorallia). The number four does not refer here to the individual scelerosepta, but rather to their groups (see Fig. 10). All this is due to the fact that those forms only have been preserved as fossils whose bodies had already reached a considerable degree of specialization and a well-developed set of sclerosepta. This state (which was reached during the Ordovician) was preceded by a long evolution, when anthopolyps did not yet possess a calcareous skeleton and when in all probability they still continued to live as mainly solitary animals. Stages can be observed in the ontogenies of both extinct as well as of the still living Anthozoa which are equipped 
with the first four septa. This can rightly be considered as a recapitulation of a state reached in their ancestors.

The supposition that the primary tetraradial symmetry represents the first stage reached in the transition from a creeping way of life with an explicit bilateral symmetry, is justified in view of the fact that during such a transition it is first the difference between the ventral and dorsal sides which disappears, and, in connection with this, the difference between the two lateral sides and the median sides. In this way a natural basis, or precondition, has been developed, we may say, for the formation of the tetraradial (as the first possible) symmetry. Soon the polymerization of the so-called antimeres took place in anthopolyps which have grown larger and larger, and which have become more and more sessile and solitary animals. This led to a polymerous radial symmetry with clear traces of a primary bilateral symmetry. This was followed by the formation of cormi and, in connection with this, by a secondary diminution of the polypoid individuals; oligomerization finally occured as a consequence of this development, so that ultimately the hexa- and octo-merous states have been developed in Anthozoa. Simultaneous with the disappearance of the tendency to the old bilateral symmetry and with the decrease of the size of polyps went the reduction until the number of antimeres was reduced to four and a complete radial symmetry has been developed. It can be easily understood that this reduction of the polymerous parts was least effective in the tentacles; it is even possible that the number of tentacles has been considerably increased as can be seen in the case of the Nausithö polyp among Scyphozoa, and in numerous other instances among hydropolyps that had inherited their tendency to tetraradial symmetry from their scyphozoic ancestors.

When we pass over to hydropolyps we must first state that in these the last trace of a formation of sarcosepta has disappeared while simultaneously their size has been further decreased. Not infrequently we can find in the oral part of the 
hydranth a contour of the surface of the intestinal epithelium which resembles that of folds; this is not, however, due to a genuine formation of folds but rather to the tact that epithelium cells grow in unequal lengths which leads to the formation of longitudinal stripes. The last traces of the former longitudinal folds can be found in buds and occasionally in the fully developed hydranths (see Fig. 34 with the transverse sections of the hydranth of Clava squamata and of the manubrium
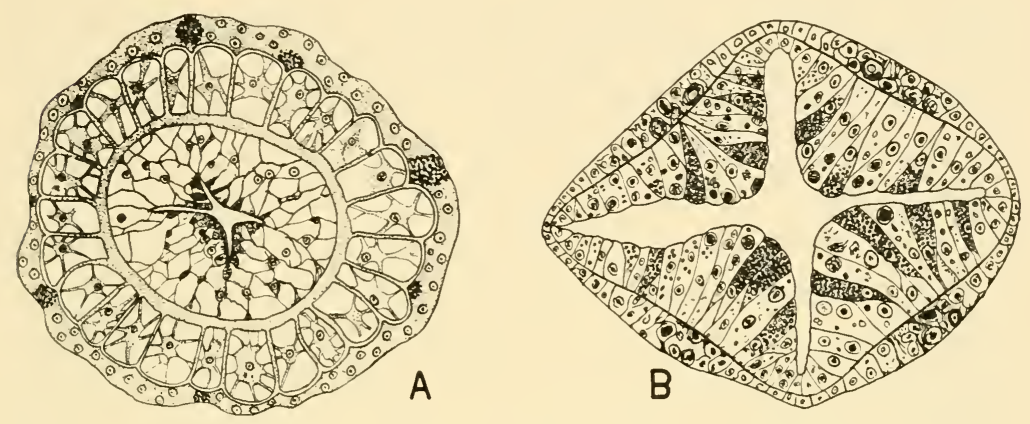

Fig. 34. Transverse sections of the oral region of two hydrozoans to demonstrate the false gutfolds. A, Hebella parasitica. B, medusa of Obelia sp. (after Hadži).

of an Obelia sp.). We think it unimportant whether there is a participation of the mesenchyme in these last traces of longitudinal folds or not. On the other hand, the intestinal diverticula which had grown into tentacles have been preserved till the very end, regardless of whether these tentacles have remained hollow or whether they have become secondarily solid as can be observed in scyphopolyps and very frequently in hydroids.

It is interesting to observe the behaviour of the gastral cavity during the period when Scyphozoa began to form medusae, and the later similar development which took place in Hydrozoa independently from the former. We can immediately state that this development has led to conditions that 
resemble closely those that can be observed in Turbellaria and in Ctenophora that have become more and more flat and large, i.e. to the formation of a richly branched system of peripheral pockets and canals which correspond to the general (here medusoid) form of a spheric calotte. A basis for this can be found in the conditions that occur in the corresponding polyp forms. The conditions in the primitive scyphomedusae whose height is greater than their width (e.g. in Cubomedusae) are still very primitive; they are similar to those that exist in scyphopolyps. In these no canals can yet be found, and in particular there is no circular canal along the periphery of their umbrellas. Their central part (stomach) is connected with the gastral pockets by way of four ostia; these are separated from each other $\mathrm{t} y$ four broad septa and they do not yet contain a funnel-shaped cavity. The gonads grow out of the bases of septa into the cavities of the gastral pockets. The peripheral gastral cavities have been developed later, after a further flattening and extension of the umbrella; they are due either to a further growth of the intestine, or to the fact that the two gastral walls have grown together locally. There are frequently whole bunches of septal filaments growing into the gastral cavity in those places where septa (taeniolae) had been reduced. It is unnecessary to discuss further details here.

Additional complications appear in the oral lobes of the most highly developed scyphomedusae (Rhizostomae); in these the original oral openings have grown together while at the same time "suctorial mouths" have been formed at the very numerous fringes of the oral lobes. The intestinal diverticula of tentacles that have become solid in scyphopolyps (as has already been mentioned) can again become hollow in scyphomedusae, or they can completely disappear together with the tentacles while at the same time their function is taken over by the excrescences of the oral lobes. All this development took place while microphagy was strongly advanced. Because of the complications of their gastrovascular system, scyphomedusae have been enabled to grow to considerable dimensions 
and overshadow the polyp generation as a whole so that the latter frequently gives the impression that it were a larval form only.

It is easy, after everything we have learned about the intestinal system of scyphomedusae, to understand correctly this system as it occurs in hydromedusae. Since there are no sarcosepta in Hydrozoa, there is also no division of their intestine into a central part, the stomach, and into the four gastral pockets. Attempts were made earlier to find something similar in the formation of the medusa buds. This has been an important contribution made by Goette (1907) whose suggestion has been supported by Hadži (1909) and by Kühn (1914). He disproved the older interpretation of the development of the medusae buds in Hydrozoa. The intestinal diverticula of tentacles represent the starting point for the formation of the peripheric gastrovascular system of hydromedusae, a situation which is quite different from the one we have found in scyphomedusae. These diverticula have become elongated when the cup-shaped part has been extended, thus when the umbrella has been developed. This has led to the development of the radial canals. Also here, as in Scyphozoa, the basic number of these diverticula has been four. An additional circular canal has been developed due to the tendency of their intestine to form diverticulae. This has finally, even if not generally, led to a secondary polymerization of these canals which especially took place in larger species either by way of a centrifugal growth of these canals from the stomach, or to a centripetal growth of these canals from the circular canal (in some Trachylinae, etc.).

As a conclusion, we can state that the changes of the digestive system of Cnidaria can be perfectly well understood if viewed from our standpoint: in polyps which have become sessile animals, a simplification of this system can be observed so that only a smooth intestinal wall has been preserved, while at the same time only the intestinal diverticula of tentacles have persisted. In the medusa form which has become secondarily free, 
the intestine has been progressively developed into a rich system of pockets and canals which corresponds to the change of the whole form and to the increased size of their bodies. We have here generally a further development of the intestinal system such as had been evolved in their turbellarian ancestors.

\section{The Skin and Cnidae}

The skin of Cnidaria together with their characteristic cnidae, which have given their name to Cnidaria, offer facts which convincingly support our thesis of the origin of Cnidaria from the Turbellaria and, as a consequence of this, of the evolution in the direction Anthozoa $\rightarrow$ Scyphozoa $\rightarrow$ Hydrozoa. It really is suprising how it has been possible for these facts to have been overlooked for so long. This attitude, which one is hardly able to understand, can be partly attributed to an almost hypnotic influence of Haeckel's gastraea theory and to the authority of Haeckel. Quite similar is the situation about the lore of the germ layers and with everything that is connected with it.

Cnidae have in their evolution reached their climax in Cnidaria. No species of Cnidaria has been known which does not possess cnidae distinctive of this same species. Their size, form, organization, and the way they are distributed, as well as the different places where they are developed and used, are frequently characteristic of various taxons of Cnidaria. Attempts have been made to solve taxonomic problems connected with Cnidaria on the basis of conditions that can be found in their cnidae. It is well known, however, that Cnidaria are not the only animal group which produce cnidae.

When we speak about cnidae, we must first know what a cnida actually is. The most highly developed cnidae are wonderful products of very specialized individual cells of skin gland cells. Their secretion becomes partly solid (the capsule, and the long thin tube which represents a continuation of the 
elastic capsule), and partly remains a viscous fluid (within the capsule); it can gush powerfully out of the cnida and it produces physiological effects. The cell itself can mostly be found in the ectoderm and does not actually belong to it but rather to the middle layer (mesohyl or mesenchyme); it is amoeboid and it can function once only. The cell itself (the cnidoblast or nematoblast) as the producer of the cnida is destroyed when this cnida is used in the explosion.

If we disregard the few and therefore very interesting cases of the so-called kleptocnidae, where a predatory species takes over and uses those weapons of its prey which had not yet been used, we find that there are several animal types which are able to produce either genuine cnidae or some closely similar forms (gland cells with a partly formed secretion). Is it a pure coincidence that it is only in Turbellaria that such forms are produced quite generally and in large quantities, in spite of the fact that they have remained on a lower level in their organization and in their phylesis? This again can be easily understood in view of the fact that Turbellaria have remained freely moving animals while Cnidaria have adopted the sessile way of life. It has not yet been clarified whether Nemertinea possess their own cnidae. The fact, however, seems to be much more important that even among Protozoa species or even larger taxons can be found which produce either genuine cnidae or forms which resemble closely a cnida (cnidoid). Cnidaria can therefore not be considered as animals which have "invented" cnidae or which alone can be found in the possession of cnidae.

Before we begin to study in detail the problem of cnidae, let us first make a critical and comparative study of the skin. Similarly, as the Cnidaria owe their name to cnidae, the Turbellaria have been so named because of the tiny water current produced by the ciliate cover of their skin. The surface of their skin remains bare due to this ciliate cover, by means of which the animal is able to move actively, in the same way as in Ciliata 
(Euciliata) among Protozoa; this property had obviously been inherited by Turbellaria from the latter. This same property has afterwards been inherited from Turbellaria by their numerous descendants among Ameria that live a normal, i.e. a free (and not a parasitic) life. It has even passed on (with few exceptions that can be easily explained) to the Polymeria and Oligomeria and up to Chordata, even if here it has been preserved locally only and in the interior of their bodies, particularly during the transition from a wet to a dry environment.

It may not be out of place to mention that the active organelles of cells that could practically be called undulipodia appear phylogenetically first as single (per individual) flagella which are later developed by way of polymerization into numerous cilia; subsequently they can readopt, by way of oligomerization, the form of flagella. The possibility must also be taken into consideration that such an ability to form undulipodia which had been phylogenetically lost (by way of a minus mutation) can again be restored (by way of a plus mutation), perhaps with the limitation that not too long a period had passed from the time of the minus mutation.

Such minus mutations have also taken place in Turbellaria; they have been supported by the natural selection when the mechanism used for the movements develops in their muscular system due to a change in their way of life. In this case, the epidermal cells cease to form cilia and begin to develop a protective organic cover, the cuticle. Among Turbellaria this has been above all the case in Temnocephala. These are Turbellaria that live as epizoic or even as ectoparasitic animals; they are able to move easily (Matjašič, 1959) and they closely resemble Trematoda so that some scholars have even included them among Trematoda. The ciliation of the body surface has been completely lost in Trematoda and in Cestoda that have certainly developed from Turbellaria.

The ciliation of the body surface has been preserved in all small animals that move freely in water. All the young (larval) freely moving stages of Cnidaria are covered with cilia. There 
are few specialized cases only that appear here as exceptions. Thus planulae of Lucernariidae immediately begin to creep over the substratum after they had abandoned their eggs (Fig. 35). No cilia can again be found in young Hydrae which become free during a very advanced state, etc. The primary larvae which live in water (in opposition to the secon-

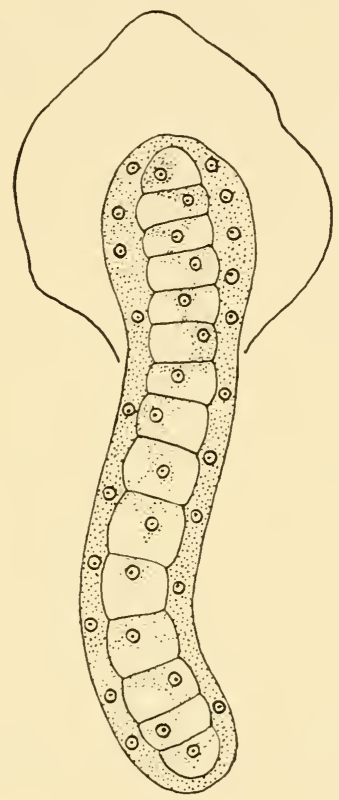

Fig. 35. Hatching planula of Lucernaria sp. (without ciliature). (After Wietrzykowski.)

dary larvae which become free during a much older ontogenetic state) move by means of cilia, regardless of the phylum the adult form belongs to (the branchiostoma larvae). Even in the ciliation we can observe the working of the general rule that polymerization is followed by an oligomerization with a differentiation, here in the form of localizations, formations of bunches of cilia, circlets of cilia, etc.

In Cnidaria we can expect a priori a reduction of the general ciliation due to their transition to a sessile way of life, and 
such a reduction has actually taken place. It is in agreement with our interpretation of the evolution of Cnidaria that this ciliation is best preserved in those Anthozoa which have remained solitary animals, i.e. in Actiniaria and in Ceriantharia. In those species that have become purely sessile and colonial animals, as well as in other sedentary animal types, the external ciliation has been limited more or less to the surface of the tentacle apparatus and to its surroundings, especially to the entrance into the intestinal cavity. Those freely moving (swimming) species have ceased to form cilia whose size has been increased and whose active movement is effected by means of contractions of muscles (medusae, Siphonophora); these cilia can, however, return if the size of the species has been subsequently diminished (Halammobydra, sporosacs of the hydroids, Dicoryne conferta, see Fig. 8).

There is a great need of protection in the sedentary animal types. The protective measures regularly take place on the free surfaces of the animals; these measures are such that they do not prevent the animal from making partial movements (usually contractions). This protection is usually achieved in such a way that part of the free surface of the animal body becomes covered with a firm cuticle, and a smaller intermediate part with an extensile cuticle (Endoprocta, Ectoprocta, Phoronidea, etc.). This is also the situation in Cnidaria. To this protection have additionally come secretions from the cells of the skin glands as a new means of protection. In Cnidaria this has largely been done by cnidae.

As early as in Octocorallia we can see the formation of the so-called periderm to be connected with the formation of colonies (Stolonifera). It is a secretion of an organic substance in the form of a cuticle which must not remain in its entire extent connected with the skin epithelium and which is in spite of this able to increase constantly its thickness. The contractile and extensible part of anthopolyps is covered with a thin extensible cuticle so that in case of need the polyp is able to retract entirely into the broadened part (the theca) of its 
periderm. The same can also be found in Scyphozoa, even if here to a lesser degree because scyphopolyps are less inclined to form cormi. The formation of a periderm is widely developed among the Hydrozoa (Hydroidea), especially in the most primitive Thecata. These have even developed devices in the form of a lid by means of which the animal is enabled to close the opening of its theca so that finally the entire body of the hydranth is protected by its periderm. The Athecata have ceased to form the hydrothecae and they have replaced the personal protection with the so-called renovation of damaged or destroyed hydranthes.

The formation of a periderm is not an invention of Cnidaria; similarly as in the case of cnidae, Cnidaria had inherited this inclination and ability from their turbellarian ancestors. It is natural that in Turbellaria as freely moving animals a general and strongly formed periderm has never been developed. In Turbellaria a thick and firm cuticle can appear locally only and under special circumstances. Thus cuticular thorns (they are probably built of scleroprotein) can be found on the dorsal side of the genus Enantia, among Polycladida. Much more frequently we find firm cuticular formations developed in connection with their genital organs (the male organs of copulation). It should be mentioned in passing that this ability to form a firm cuticle had also been inherited by numerous types of Ameria. The best parallel to Cnidaria can be found in Endoprocta as sessile descendants from Turbellaria which usually form cormi. In these, similarly as in Cnidaria, we can find in one and the same individual all possible transitions from a ciliate and bare epidermis, to such that one has cilia and that is covered by a thin and extensible cuticle, and finally to one that is covered by a thick periderm which can be occasionally equipped with spines. This periderm can serve, as it does in Cnidaria, as a true exoskeleton.

In Anthozoa and in Hydrozoa, especially in the former, the formation of cormi is attended with a secondary impregnation of the organic skeleton matter with mineral salts; this has led 
to the formation of a firm skeleton that can be polished and rubbed. Even in this Cnidaria do not show an originality. The first steps in this direction had again been made by Turbellaria, even if in these this development has preserved a local and moderate character due to the fact that Turbellaria are freely moving animals. As a matter of fact, not even Turbellaria have been the first to have discovered this possibilitysimilar things can be found as early as in Protozoa. Calcium salts have been used as a skeleton material in two different ways, either as a substance which impregnates the periderm, or as biocrystals (spicula). Both these elements can also be found elsewhere in the animal world; Mollusca and Brachiopoda can serve as examples of the former development; Cestoda, Nematoda (male animals), Brachiopoda with their spicula, Tunicata, and others, as examples of the latter. These spicula or calcoblasts have been occasionally cemented together into a firm skeleton (e.g. in Octocorallia, etc.)

In two species of Turbellaria (Turbella klostermanni and Sidonia elegans) L. v. Graff (1882) was able to observe skin inclusions that produced an effervescent effect when treated with the acetic acid; they had therefore been impregnated with a lime-containing carbonic acid. Similar morphological elements have been described by O. Steinböck (1931) as "small calcareous bodies" in connection with the primitive Acoela Nematoderma batbycola; he did not determine, however, the true chemical nature of this substance.

This ability to deposit mineral salts in their own cells or in the products of their cells (traces of which can be found in the freely moving Turbellaria) has undergone further progressive development during the transition to a sessile way of life and to the formation of cormi. The madreporic corals and so-called Hydrocorallia (Milleporidae and Stylasteridae) are some of the most important absorbers of the sea chalk. Frequently it has been maintained (e.g. by Kükenthal, Kästner, etc.) that the calcareous skeleton is formed by the ectoderm only. This is not true for Octocorallia, and probably it is not 
true for other Anthozoa either. Why should we deny the mesenchymal (mesodermal) character to such cells that ontogenetically and phylogenetically arise in the skin layer and which later migrate into the middle layer, when in other animal types the same cells are considered forthwith as mesenchymal cells?

As for the cellular structure of the skin layer it can be stated that from our standpoint we can expect in Anthozoa the conditions to be more primitive than in Hydrozoa (we speak here of the polyp generation only). Similarly as in Turbellaria, where a slightly cellularized epidermal epithelium (ectocyte) can regularly be found in the primitive Acoela and where we therefore speak of a syncytial structure, we find cases with a plasmodial (syncytial) ectoderm (epidermis) first in Anthozoa among Cnidaria. The cellularization, i.e. the morphological-physiological division of the elementary units of Eumetazoa (of the true cells) in the primarily polycaryontic plasmodium becomes more and more complete in the direction Anthozoa $\rightarrow$ Scyphozoa $\rightarrow$ Hydrozoa. This, however, is not true for the skin layer only. In sessile animals we find this cellularization to be connected with an epithelization, and it is especially developed in those animal types that form cormi. In this sense the middle layer (and the nervous system) lags behind. In Cnidaria the middle layer retrogresses more and more until finally the climax of this development is reached in hydromedusae and in the hydranths of the corm-forming $\mathrm{Hyd}$ roidea. I have already stated that this development has never led to a complete loss of the middle layer (it can occur locally only).

Finally we also find the actual conditions of the skin gland cells to agree better with our interpretation of the origin of Cnidaria than with the earlier theory. A mucous membrane can be found in the primarily solitary Anthozoa only. Some Actiniae (e.g. the common red Actinia equina) are able to live in the tidal zone because at ebb-tide they cover their bare and contracted bodies with a thick layer of mucus. This lowest 
form of the activity of skin glands, the secretion of the mucus, has later been developed into various other more specialized secretions. As early as in solitary Anthozoa we can find on one hand a more local (at the flattened sole of the foot) secretion of a glutinous and therefore thicker slime, and on the other hand (especially in Ceriantharia) a glutinous and tubular secretion whose duty is to protect the animal body. The formation of the periderm also belongs here, with the difference that here slime has become solid matter. If we disregard the formation of slime, which is connected with the feeding mechanism of the animal, we find in scypho- and hydro-polyps the gluti ous slime to have an active role in their aboral poles only; this is especially true for those polyps that have become secondarily solitary animals, as is the case with Hydra. The gas gland in the pneumatophore of numerous Siphonophora represents a special case. It is proved by such isolated and specialized cases that mutations in the skin gland cells can reappear again and again.

In Cnidaria we see how the skin progressively loses the character of a mucous membrane, a fact which can be explained as being due to the formation of cormi and, in connection with this, to the sessility and to the formation of a periderm. In Turbellaria, on the other hand, the character of the mucous membrane has been better preserved; it has especially been developed at the anterior ends (the deeply sunk-in front glands) and on the ventral surface; here it has been specialized and the slime has been given an important role which enables the animal to creep over the substratum. This property has later been inherited and further developed, by others (e.g. Gastrotricha), and also by Mollusca. This slime secreted by the skin glands can become so firm that it can be extended into glutinous threads that can be used by the animal for the formation of nets by means of which the animal can catch its prey, and for the formation of thin cords which the animal uses to descend. What else can the colloblasts of Ctenophora be than specialized cells of skin glands which behave like 
cnidae and which can be used by the animal when it catches its living prey? Colloblasts had ultimately been developed from the same source as cnidocytes, i.e. from the cells of the skin glands of the old Turbellaria; in both these cases, however, the development took place independently, and therefore parallel, and this is the reason why it is senseless to combine Ctenophora, as Acnidaria, with Cnidaria into one higher taxonomic unit of Coelenterata. The main difference that can be noticed between colloblasts and cnidae is that colloblasts do not represent a firm and formed unit (glutinous kernels cannot be attributed to it!) and that they function as a glutinous substance without the aid of an explosion. In spite of the fact that they form two types of elastic, or contractile, filaments (a straight and a spiral filament), they have reached a lower level of differentiation than the one that has been reached by cnidae.

Thus we return now to the cnidoblast as the skin gland cell which shows the best developed differentiation and specialization in the entire animal world. Once separated from its "maternal cell" (cnidocyte, stinging cell, nettle cell), or after the death of the maternal cell, the capsule or the nematocyst, though itself not alive, even if it possesses a complex structure, can continue to function in a foreign environment as is proved by the so-called kleptocnidae. The fact has frequently been forgotten or quietly passed over (e.g. in the book by Hyman) that cnidocytes are originally monocellular gland cells and that they did not appear as such, i.e. as fully developed, suddenly in the Cnidaria; they had a long phylogenetic development behind them which leads by way of Turbellaria down to the Protozoa. It has already been mentioned that similar forms had probably been developed polyphyletically as early as in Protozoa. Even a cursory comparison of the "nettle capsule" of the dinoflagellate Polykrikos schwartzi, with the trichocyst from the paramecium and from the so-called polar capsule of the Myxobolus (Cnidosporidia), with cnidae of Cnidaria, and finally with various partly solid secretions of the turbellarian 14* 
gland cells (rhabdites, rhamnites, sagittocysts) shows clearly that the development has gone here into considerable width.

Here we can bypass the problem whether genuine and autonomous cnidae can be found as early as in Infusoria; the socalled cnidotrichocysts of the Prorodon are, according to Krüger (1934, 1936), certainly very close to a genuine cnida. Since we consider that the Turbellaria as the lowermost Eumetazoa, had been developed from the ciliate Protozoa, we accept it as certain that Turbellaria had inherited their tendency to gland cells with a strongly gushing and partly firm secretion from their protozoan ancestors; in Cnidaria this same ability has developed into a real virtuosity (Fig. 36).

It seems, especially on the basis of studies made by Meixner and by Kepner and his school on Microstoma, that no genuine cnidae have been developed anywhere among the Turbellaria. In Turbellaria the highest level reached in the development in this direction seems to be in sagittocysts, a fact which, as has already been mentioned, can easily be understood if we take into consideration the way of life on one hand of Cnidaria and on the other of Turbellaria.

The Nemertinea, too, had inherited from their turbellarian ancestors, even if to a lesser degree, the ability to produce forms similar to cnidae. It may not be a mere chance that, especially in Nemertinea, such secretion products can be found on their unarmed proboscides. Frequently one can find in the epidermis cells of the anterior part of their proboscis rodlike structures which are very similar to rhabdites of Turbellaria. It is improbable that these rod-like structures could be of a foreign origin. Genuine cnidae have been found in the wall of the proboscis of several species belonging to the genera Micrura, Cerebratulus, and Lineus; these cnidae show a regular distribution (in two longitudinal lines); their origin has unfortunately not yet been determined, so that we do not know whether they are autonomous. The cnidoid forms, however, cannot be expected in Nemertinea with an 

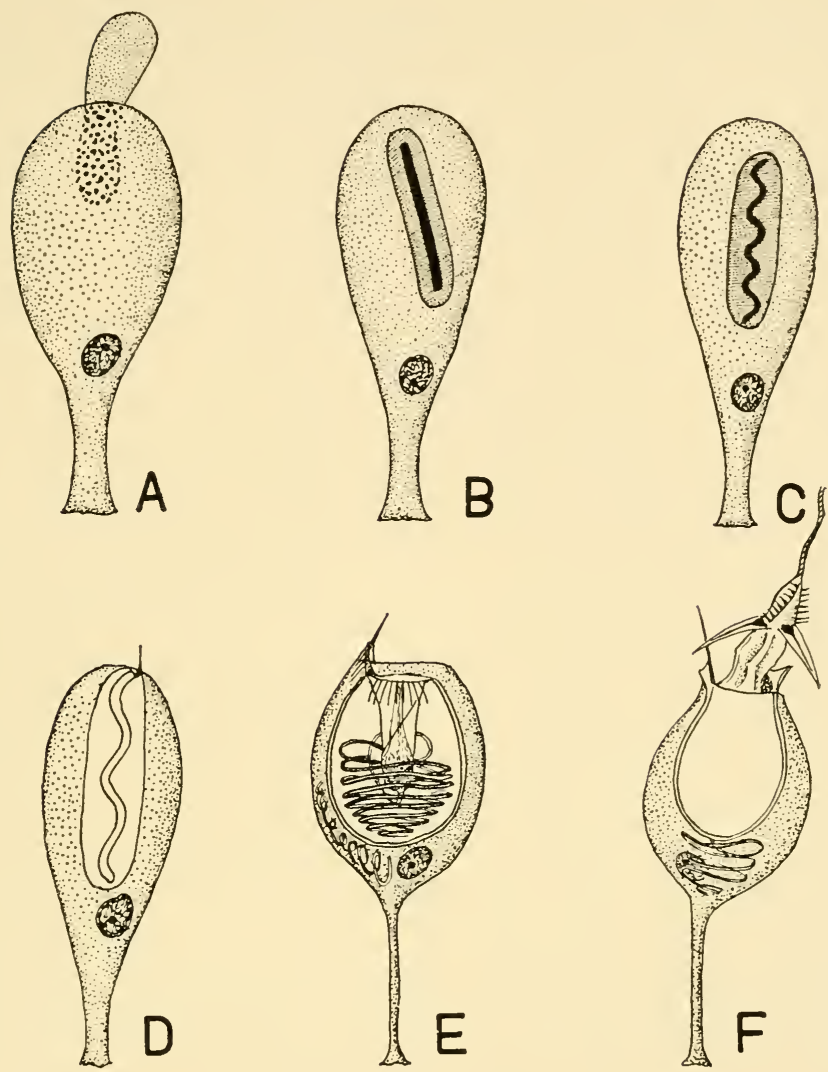

Fig. 36. Hypothetical evolution of cnidae from secretory cell without solid secretion, (A) to fully developed cnidae (B-F).

armed proboscis, where poisonous glands are used, together with stilettos, as an effective weapon.

In the Cnidaria genuine cnidae only appear, as has already been mentioned, quite generally and without a single exception. This should not be considered as surprising, since all levels of evolution in the direction towards a genuine cnida can already be observed in Turbellaria. This fact which must be expected if we accept our standpoint, i.e. a progressive 
evolution of cnidae within the group of Cnidaria, can point to the direction Anthozoa $\rightarrow$ Scyphozoa $\rightarrow$ Hydrozoa. This progressive evolution can be observed in several elements. First, in the general form of the capsule, we find in Anthozoa an oblong or rod-like form to be prevalent. It is only in Scyphozoa, and especially in Hydrozoa, that it becomes differentiated into several other external forms, particularly into a spheric or into a pear-shaped form. Another development can be observed in the wall of the capsule. It is in Anthozoa only that we find cnidae with a thin-walled capsule and with a predilection for acid colouring substances; they have been given a special name, i.e. that of spirocysts. Earlier it was believed to be a sign of the primitive character of these spirocysts that they do not show a continuity between the wall of their capsules and the "thread." Will (1909), Weill (1930), and recently C. E. Cutries (1955), however, have been able to show that we nevertheless have here genuine cnidae, even if the connection between the capsule and the thread still seems to be a tenuous one.

A further progressive evolution can be observed in the equipment and in the form of the thread. It has been used as a basis for the systematization of cnidae by Weill (1930) and by Carlgren (1945). A further subtle morphological and physiological difference can be observed on and around the capsule of the cnidae if we compare the anthozoan and hydrozoan cnidae (cnidocil, lasso, various fibrous forms in the cytoplasmic body of the cnidoblast, the formation of the stem, etc.). A conspicuous phenomenon is a progressive variety of the cnidarian apparatus that can be observed in one and the same species of Cnidaria, a fact which has led to the notion of a "cnidom." Such a differentiation can be further developed in another direction, i.e. to a more regular and therefore to a more effective grouping of cnidae into variously formed batteries. Again this development has reached its climax in Hydrozoa, especially in Siphonophora. In this connection we also find a more and more distinct differentiation of the strictly 
localized place of origin of cnidocytes, combined with an increasingly complicated migration of half-formed cnidocytes to those parts of the animal body where they can be used (this has first been described by Hadži (1907), later confirmed by numerous other authors). From a physiological and ecological standpoint we can notice a further specialization into penetrating, volvent, and glutinating cnidae, a development which again has reached its climax in Hydrozoa.

Finally we can observe that when the climax has been reached in the progressive evolution the subsequent development can turn in a retrogressive direction. This can be seen, first, in the fact that a polycnidom can secondarily be changed into a monocnidom, thus to a simplification in the selection of cnidae, as this is the case with Trachylinae. Another retrogression can be observed in those cases where cnidae degenerate, as can be seen in the so-called rhopalonemes and desmonemes in Siphonophora (Diphyes). Cnidae have lost their primary function in numerous hydromedusae, especially in Trachylinae. Here they remain, half developed, in the middle layer; amassed in the margin of the umbrella and behaving passively, they serve as an elastic and strengthening inner skeleton.

As a conclusion it can be stated that cnidoid forms belong to the category of non-living, even if organic, secretions of the cytoplasm. They first appear, in an acellular state, as early as in Protozoa; they have been continuously progressively developed in several directions and they have reached their climax in Dinoflagellata (Kofoid and Swezy, 1921; Weil, 1925; Chatton, 1914); they have reached another climax in Infusoria (Krüger, 1934). The Turbellaria, as the first Eumetazoa, had inherited their inclination to form cnidoids and passed it on to several of their descendants. An absolute climax has been reached in this development in the sessile Cnidaria. The progressive line of evolution points clearly in the direction Anthozoa $\rightarrow$ Scyphozoa $\rightarrow$ Hydrozoa, reaching its final and general culmination in Hydrozoa. In the latter, a partly retro- 
gressive development can also be observed. It is probably a unique case in the whole animal world that animals that use Cnidaria (this is true, as it seems, for Hydrozoa only) as their food, e.g. Turbellaria, Ctenophora, Gastropoda, are able to use cnidae which have been taken from their prey, as the so-called kleptocnidae (Martin, 1914). It seems that the cnidae of only the Hydrozoa can be used as kleptoknidae because they are of the highest quality.

\section{Ontogeny}

Initially, I gave a general explanation of my interpretation of the relationship between ontogeny and phylogeny. I agree with Garstang, de Beer, Steinböck, Sacarao, and numerous other zoologists who have resolutely rejected as wrong and misleading the so called "fundamental biogenetic law" as it was formulated by Ernst Haeckel. According to this law, ontogeny is supposed to be a shortened and somewhat changed ("falsified!") phylogeny; the phylogeny did not represent some old ontogenies (ontogenetic stages), but rather a chain of adult stages or forms. Even if basically wrong, this interpretation as proposed by Haeckel was originally useful for propagating the idea of Evolution. This quasi-law has now been banished for all times from the demesne of biology; this, however, should not mean that ontogenies cannot serve as a useful material when we are attempting to come to some phylogenetic conclusions by means of a comparative method. It is only necessary that we proceed cautiously in order to avoid the pitfalls of the so called caenogenesis.

We are aware of the fact that we will have to overcome considerable difficulties in the struggle for a new interpretation of the phylogeny of Cnidaria; this is due especially to a widely believed, yet false, combination of two concepts that were proposed by Haeckel; this is, on the one hand, the concept of a general repetition of the phylogeny in individual ontogenies; and, on the other, the concept of a gastraea as the 
primitive form of all Metazoa. The moruloid and blastoid forms have simply been presupposed and taken over from the plant world when they could not be found among the animals. It should be mentioned in passing that in my opinion there have never existed any Gastraeadae. I even maintain that the ontogenetic stage called gastrula does not represent any recapitulation of a "gastraeadic" ancestor.

It is quite surprising that various zoologists could not see that the well-known facts in the field of the ontogeny of the cnidaria do in no way agree with their concept of the origin of Cnidaria from some gastraea-like ancestors, with Hydrozoa as the earliest form. It was within this important sphere that it was necessary to take into consideration the factor of "caenogeneses" from some unknown origins. One could expect, on the basis of this preconceived scheme, that the ontogeny of Hydrozoa as that of the supposedly most primitive Cnidaria should have the most typical course, i.e. that the impregnated and fertilized egg cell should pass, by way of a total and equal segmentation, through the stages of a morula and of a blastula (or coeloblastula) to grow finally by way of an invagination of its vegetative half into a double-layered gastrula. The facts, however, tell a different tale. Such a "typical" recapitulation can be found nowhere in the ontogenies of all Hydrozoa that have been investigated up to this point. Occasionally an invagination-gastrula can be found in Scyphozoa and in Anthozoa; there is also everywhere an Anlage of the intermediate layer, as yet not that of a third "germ layer," as a mesoderm. Here the types of development show an extraordinary variety and they depend strongly and obviously on the quantity of the food substance which has here a purely passive role. It can be therefore easily understood that a sharp opposition to the gastraea theory soon appeared after this theory had been formulated by Haeckel; Ilya Metschnik off, an expert in the ontogenies of Cnidaria, proposed his own theory of a parenchymella, and in this he was followed by several other scholars; Lankester (1877), on the other hand, 
developed simultaneously with Metchnikoff the planula theory.

It is characteristic that so far there has been nobody who prepared to interpret the ontogeny of Hydra as typical in spite of the fact that until recently Hydra has been considered as the most primitive cnidarian (even now we can find in the recent book by Hyman the family Hydridae given the first place in the system of Hydrozoa, a classification which must presuppose these to be the most primitive Hydroidea and therefore also the most primitive forms of Cnidaria). A. A. Sachwatkin (1956), also tried to derive Metazoa (together with sponges) from the colonies of Flagellata. He considers the flagellate blastula to be the primitive form, and correspondingly had to bring forward the "metagenetic Leptolida" in order to demonstrate his ideas in one example of the supposedly primitive Metazoa. The examples selected by Sachwatkin, i.e. the calcareous sponges, the metagenetic Leptolida, the "lower Scyphozoa," and sea urchins represent a poorly selected group because they all belong, without exception, to very specialized types, and because they cannot actually even be compared with each other.

In our analysis and comparison of ontogenetic morphogenies we will consequently begin with the study of conditions as they occur in Anthozoa, above all in those that live a primarily solitary life. These are the Actiniaria and Ceriantharia. We find a "typical" ontogeny with a total cleavage, with a flagellate blastula, and with an invagination gastrula in Actiniaria, among Anthozoa: this in spite of a presence of various vitelline substances. Haeckel himself selected a species of anthopolyp as a typical example (Fig. 37). As early as in Octocorallia we find this situation changed. The segmentation, though total and subequal, is followed by a stereoblastula; gastrulation takes place by way of a secondary delamination. Few species only belonging to Scyphozoa have been studied so far from this point of view (mainly Nausitho: and Chrysaora). Cleavage seems to be more unequal here, and its result is a 
coeloblastula which develops later into an invagination gastrula. It has already been mentioned that no invagination gastrulae can be found in Hydrozoa; their early ontogeny is in reality very plastic and it can be easily changed. This can
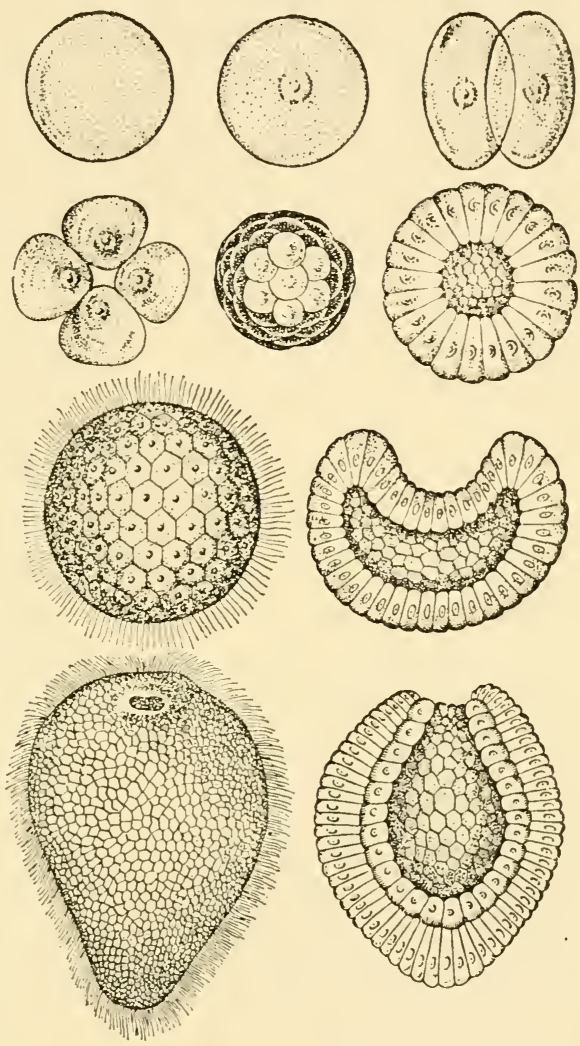

Fig. 37. The early ontogeny of an anthopolyp after E. Haeckel.

be explained as being due to the partial influence of two factors. One of these is the progressive trend to develop a freely living larva, a planula; the second factor is the prevalent role which is given to the formation of cormi. As a consequence of this, the planula does not develop first into a primary 
individual but instead it immediately begins to form cormi, a phenomenon which can be observed above all in Siphonophora.

The conditions that can be observed in the ontogenies of Cnidaria as a whole, show that the phylogenetic development must have followed the direction Anthozoa $\rightarrow$ Scyphozoa $\rightarrow$ Hydrozoa, and not the opposite direction, as has been believed till now. The modes of evolution show considerable variety and they are therefore not uniform. The younger pelagic larva, the planula, seems to be most characteristic in the ontogeny of Cnidaria; it had been evolved parallel to the sessility of the adult form of polyp. It would be misleading if we tried to see in this planula a recapitulation of a common adult ancestral form. This planula does not feed independently, and it can therefore not live long when swimming freely. A slight prolongation of the planula stage can be achieved by means of a reserve food given to the egg before it starts on its way; yet in this way the mode of evolution is changed.

In Cnidaria a significant prolongation of the pelagic stagewithout the usage of a medusoid generation (primary or secondary) - has been reached, as it seems polyphyletically, so that the subsequent development (yet not a real metamorphosis) takes place in the pelagic zone; the larva as such becomes able in this way to feed independently. Such a larva has been called an "actinula;" attempts have been made to suggest that the actinula represents the primitive form of Cnidaria, an equally misleading interpretation. The more progressively developed planktonic larvae have no longer a freely moving medusoid generation.

Actinulae of a very specified form can be found as early as among the primarily solitary Anthozoa, i.e. in Ceriantharia (Fig. 46). They are known as Semper's larvae, while at the same time their relationship to certain adult forms could not be determined. They develop some properties of the adult form preserving simultaneously, as it seems, certain archaic characteristics, e.g. a phase with four tentacles only, with six sarcosepta, and with a well-developed bilateral sym- 
metry. Such a larva is known as a cerinula. The inclination to prolong the pelagic stage can even lead so far, that it is already during the plankton phase that sexual maturity is reached (Dyctylactis benedeni). In these larvae we find a combination of transient larval characteristics and of the definite properties of the grown-up individuals. A higher level of development can be observed above all in their ciliation. "Zoanthellae" with their oblong form, possess a long cilia-line along the ventral side; "zoanthinae" whose form resembles that of a vat, have a circlet of cilia and they swim in a special way by means of pulsations, somewhat similar to the type of swimming that can be observed in medusae. As early as in the Anthozoa we can find cases (Zoantharia) where the planktonic phase of the planula has been given up. This planula itself has a life of short duration only and it soon develops into an oözoöid.

No actinulae can be found among Scyphozoa where medusae are the frevalent generation. In the metagenetic species we find the planula stage to be completed early; in Lucernariidae, as has already been mentioned, planulae do not rise into the pelagic zone, they have no cilia, and they creep for a short while only over the sea bottom (Fig. 35).

Among Hydrozoa, freely swimming larvae which resemble an actinula can again be found in the very specialized Athecata (Myriothelidae, Tubulariidae). They become free only as such and they are certainly of an independent origin. They have been developed parallel to the retrogression of the free medusoid generation. Larvae of Narcomedusae, which have also been called actinulae, have nothing in common either with the larvae of Ceriantharia or with those of the athecate Hydroidea, except if we allow the homology (homotypy) to be completely watered down-something that has often been done in recent researches. The Scyphozoa which have no actinulae appear between Anthozoa which have actinulae and Hydrozoa. The supposed actinulae of Narcomedusae and of Trachomedusae are larvae which are medusae and not polyps: in these 
the polyp generation has been abandoned as completely as in the hypogenetic Scyphozoa. The larval stage has been prolonged (it is only externally polypoid) by means of a transition to a temporarily parasitic way of life, it has been more strongly developed, and it has even become able to reproduce asexually.

It should be mentioned in passing that it has not been proved that the planula either of Scyphozoa or of Hydrozoa has evolved into the adult form of a medusa by way of a prolonged life in plankton. We always have a case of a secondary direct change due to the abolition of the benthonic polyp generation wherever a planula has actually been changed directly into a medusa, a fact which can always be proved if we make comparisons with the closely related species. All hypotheses which suggest that the medusa form, or that the planktonic planula or actinula represent the primary form in the phylogeny of Cnidaria must therefore be rejected.

We can state, when we now compare the ontogenetic development of Cnidaria with those of Turbellaria, that little actual material is available especially about the Acoela. Furthermore, we can see (as was also the case with the genital apparatus) that the ontogenetic morphogeneses show a strong inclination to a specialization which has reached its climax in groups whose eggs represent extreme cases of exolecithality. On the other hand, a trend of evolution can be observed towards a strictly determined and differentiated type of development that can be found as early as among the Acoela (e.g. in the species of the genus Convoluta). This trend, combined with the formation of a duet (in Acoela) and of a quartet (in higher Turbellaria), as well as the formation of an Anlage of a middle body layer which is closely connected with the Anlage of the intestine from a strictly fixed blastomere (4d), had been inherited by Ctenophora by way of Polycladida. Here already the mesohyl is formed during the ontogeny out of two sources, evidently a contrivance of the evolutional technique. 
The conditions that can be observed in the ontogenies of Cnidaria had certainly not been developed from such trends, but rather from others that were more primitive, more plastic, more changeable, and less determined. It appears, in opposition to the previously widely accepted interpretation, that it is the youngest stages in the ontogeny i.e. cleavage that are changed first, and it is therefore necessary to be very careful when attempts are made on this basis to derive conclusions regarding phylogeny.

We must mention here in passing the extremely interesting example of a turbellarian (Prorbynchus stagnalis M. Sch.) where two rather different ontogenetic processes could be observed in one and the same species (O. Steinböck and Sister Bernardina Ausserhofer, 1950). Hyman (1957, II:171) is right when she emphasizes, "Practically nothing is known of the development of these Rhabdocoela and Alloeocoela that have endolecithal eggs." To this it should be added that much depends even in endolecithal eggs, on the quantity and on the type of the distribution of the egg yolk.

As a matter of fact, no larva clearly of a planula type can be found among the Turbellaria. It can, however, be said that it had been prepared in the Turbellaria as a transient stereogastrula which was later developed in the benthonic and sessile Cnidaria into a typical pelagic larva. Turbellaria as a whole show little inclination to develop genuine larvae, and this is particularly true for those species that have begun to live in the fresh water or even on the land. It is only in the Polycladida that planktonic larvae (Goette-Müller's larva) have been developed as a parallel (and nothing more!) to an actinula.

If we disregard the special characteristic of Acoela (that they have lost the second meridian cleavage which has led in micromeres to the formation of a duet instead of a quartet) we see that conditions that can be found in Cnidaria, and especially in Anthozoa, show in reality close resemblance to those that occur in Acoela. In these a stereogastrula which is very similar to a planula is usually formed by way of epiboly. 
The oral opening is developed later by. means of an invagination. The contents of such a stereogastrula is naturally not an entoderm but rather an entomesoderm which was called an archihiston by Steinböck-inasmuch as it still shows the primitive character of a plasmodium (syncytium). It becomes clear that the supposedly primitive formation of the entoderm, which has been considered as typical and which takes place by way of an invagination of the vegetative half of the coeloblastula, had been evolved so to speak in the "technique of embryogenesis" from a combination of an immersion ("passing into the interior") of certain macromeres, e.g., $4 A$ and $4 B$, and of an epiboly (surrounding growth by means of micromeres; cf. conditions in the early ontogeny of the Polychoerus caudatus, according to Gardiner, 1895, and of Convoluta roscoffensis, according to Georgévich, 1899).

It can be given as a direct proof of the origin of Cnidaria from Turbellaria that it is in the Anthozoa only that we find instances of an inclination to spiral cleavage which is typical of Turbellaria and which was passed over from Turbellaria to Mollusca as well as to Annelida. The simplification of the in-determined character of the early development of Cnidaria has been mainly due to the retrogression of the middle body layer which was caused by their sessile way of life. In this connection the role of the blastomere $4 d$ as that of a supplier of the entomesoderm (coeloblast, according to Dawydoff) has been diminished so that what has remained has been the irregular formation of the so-called entomesoderm, the mesenchyme.

It is characteristic, if viewed from the standpoint of a general study of ontogeny (embryology), that no typical formation of the so-called germ layers can be observed either in Turbellaria or in their descendants Cnidaria. Kühn (1914) was obliged to state in connection with Hydrozoa that "... die Keimblätterbildung (takes place) durch Differenzierung aus einem soliden, indifferenten Blastodermhaufen oder einem Syncytium." Is it better not to speak here of any germ layers 
at all? An illuminating example in this connection is the athecate hydroid Turritopsis nutricola whose ontogeny begins with a regular cleavage that soon becomes irregular in order finally to lead to a syncytial state; naturally enough this develops later into a cellular state. Even in other cases in the Cnidaria, a return to a syncytial state is not at all rare.

As a conclusion we can say that the facts that have been known so far about the ontogeny of Cnidaria and Ctenophora do not stand in opposition to our concept. On the contrary, we find these facts if viewed from our standpoint are more understandable than they have been till now. Two trends can be found in ontogeny (as in the morphology) of the adult forms: a retrogressive development, and a progressive trend. The retrogressive evolution which takes place parallel to the simplification of structure leads to a reduction of the middle layer and to the in-determined character of the early development, and the progressive trend leads to the formation of a planula larva typical of Cnidaria. Both these developments depend on the sessile way of life, combined with the formation of cormi.

\section{Asexual Reproduction and Regeneration}

No phylogenetic significance has usually been attributed to asexual reproduction. It is usually considered that where the sessile way of life has been adopted by Eumetazoa it appeared spontaneously. This is generally true even if there are numerous exceptions. It should suffice to mention here, on one hand, Rotatoria, Gastropoda, and Lamellibranchiata which incline to partial sessility, and the completely sessile Brachiopoda: among all these animals not a single case of asexual reproduction has been known so far; and, on the other hand, Annelida which move freely and which show, in spite of this, instances of an asexual reproduction not only by means of division but also by way of budding. In all those cases where freely moving animals reproduce asexually we 15 
have usually (though by no means always) animal groups which developed directly from some related animals that lead a sessile way of life and which as such reproduce asexually. As examples we can mention, among Cnidaria, some species of hydromedusae (above all those that belong to the Hydroidea); hydropolyps that have become secondarily solitary animals; the Thaliacea among Tunicata; two secondarily solitary species of Ectoprocta (Monobryozoon ambulans and $M$. limicola), and perhaps the Loxosoma species among Endoprocta (it is possible that these are primarily solitary animals!).

In agreement with the rule that a tendency to asexual reproduction can be observed in sessile animals the Cnidaria (in clear contrast to Ctenophora!) show asexual reproduction in widely adopted and manifold ways. This has led in all three subclasses to the formation of cormi-these are least developed in Scyphozoa-and in this way to polymorphism (polypmedusa, polypean polymorphism, medusan polymorphism). It is usually supposed, even if it is not always explicitly stated, that in Cnidaria this type of reproduction appeared, so to speak, spontaneously as a consequence of a general plasticity. This has been linked with the regenerative ability. This, however, is an explanation born of a dilemma. Reproduction by means of transverse division can be found in Turbellaria, even if it is not of quite a general distribution and it occurs in spite of the fact that they live as freely-moving animals. Here, too, attempts have been made to bring this property into a causal connection with the well-developed ability to regenerate.

These conditions become clear and understandable if viewed from the point of view of my concept (Cnidaria as descendants from Turbellaria). First it should be mentioned that there are-besides specialized forms of asexual reproduction, laceration, formation of podocysts, cladogony, etc.-mainly two types of asexual reproduction: division and budding. Of these two types, division seems to be the more primitive form, because it appears early in the freely living Protozoa, especially in Flagellata and in Ciliata ; in Flagellata as a longitudinal division, 
and in Euciliata as a transverse division. It is not difficult to imagine that budding had evolved out of division during the sessile way of life; this can be observed as early as in Ciliata (Euciliata on one hand, Suctoria on the other).

We can rightly reverse the situation and can maintain that the facts observed in connection with asexual reproduction give support to our interpretation of the origin of Cnidaria from Turbellaria. In the freely-moving Turbellaria transverse division only appears; they inherited it from their euciliate ancestors both in its primitive form as an architomy as well as in its more progressive form as a paratomy. It is true that this transverse division does not appear equally well developed in all animals that belong to the class Turbellaria because it is coordinated with the way of life and the degree of plasticity; we can find it mainly in their "lower" subgroups: in Acoela (cf. the recent studies by P. Ax and Schulze and O. Steinböck) and Rhadocoela, in Tricladida, and in the terrestrial Planaria. These are animals which reproduce regularly only by means of division. In the terrestrial Planaria, fragmentation can frequently be observed which slightly resembles laceration such as occurs in Actiniae. It can be easily understood that in Turbellaria no budding has been developed because of their active movements. Yet in spite of this we find in some Rhabdocoela that paratomy has reached a state which is so similar to budding that experts in Turbellaria have believed that it really was budding.

Transverse division appears, as can be expected from our standpoint, in the primarily solitary Anthozoa, even if it is not frequent. The best known case is that of Gonactinia prolifera; Carlgren was able to show that here transverse division occurs serially in the form of a paratomy. The case of Fungia which has also been closely studied shows that transverse division can be found among Madreporaria, even if in a somewhat different connection (unequal division and the alternation of generations). This type of division was taken over by the Scyphozoa from their anthozoan ancestors; here it is called 
strobilation even if this word suits those cases only where we have a probably primary multiple transverse division (e.g. the polydisc strobila). The products of this special form of transverse division develop into medusae simultaneously taking over the sexual function. Since scyphomedusae are developed primarily by way of transverse division, it can be understood that they never reproduce asexually (e.g., by way of budding), as can occcasionally be observed in hydromedusae.

It is interesting to notice that in Hydrozoa, which do not include any species that live as primarily solitary animals, transverse division has reappeared in those species that have secondarily adopted a solitary life. This is known for Protobydra (besides a longitudinal division), and apparently abnormally even in Hydra. The transverse division of the coenosarc in the hydrorhiza which can lead to the formation of podocysts can perhaps also be attributed to it as well as the decomposition of the Polypodium chain. Here we can also include the isolated case of hydromedusa (Gastroblata raffaeli) which reproduce asexually by means of longitudinal fission (Fig. 38).

A direct transverse division cannot lead to the formation of cormi. We cannot know how the formation of cormi began during the geological past. Yet if we refer to conditions that exist in Ciliata and above all if we take into consideration those that exist in the primarily solitary Anthozoa we can come to the conclusion that the formation of cormi had begun in all probablity as an imperfect division when budding had developed from division due to the great inequality of its products: in this way the larger "half" of the divided polyp grew into the maternal animal, and the smaller "half" into the bud. The longitudinal division, too, had possibly been secondarily developed from the primary transverse division, such as it occurs in Ciliata and in Turbellaria by way of an oblique division. In the higher sessile animals we find budding preserved as the only form of the asexual reproduction. 
As early as in Actiniaria which possess no skeleton and which we consider to be the most primitive Cnidaria, laceration appears as well as transverse division, as a form of the asexual reproduction: it takes place close to the margin of the foot disc, thus close to the aboral end of the anthopolyp; it represents an intermediate form between an oblique division

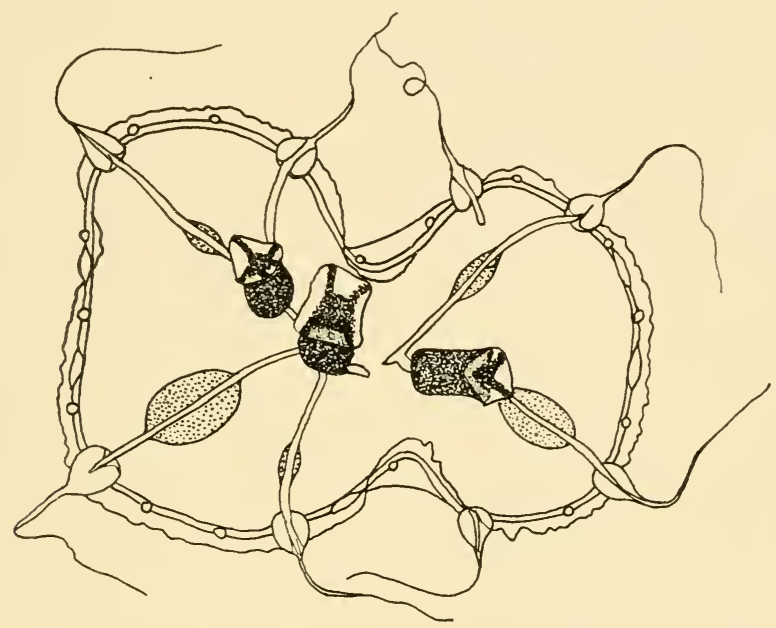

Fig. 38. The leptomeduse Gastroblasta raffaeli with longitudinal scission; (after Babić).

and budding. To this we must add the appearance of the formation of cormi in Actiniaria (family Corrallimorphidae). So far this has been too little taken into consideration; in its form it is connected with conditions that exist in those solitary species that have the ability to lacerate. Processes resembling stolons are formed with the production of blastozooids; they are the forerunners of the coenenchyme. This was also the way that Anthozoa first began to be attached to the substratum, how they became sessile animals.

Only the Ceriantharia are, as it seems, all primarily solitary animals and as such they have remained without asexual reproduction. In all other Hexacorallia, however, the formation of 
colonies had been introduced by means of an imperfect budding or division; frequently it is not easy to distinguish budding from division. Even budding itself does not always take place in such a way that one could distinguish between extratentacular and intratentacular budding. As early as in Anthozoa, i.e. in Octactiniae, development has led to the formation of regularly excrescing and individualized cormi as well as (even if in a moderate degree) to the polymorphism (dimorphism) of the polypoid individuals (subindividuals).

All the three body layers participate, as much as this has been known till now, in the budding of Anthozoa (the so-called typical budding); this may also be true for Scyphozoa. As will be seen later, it is first in the Hydrozoa that socalled atypical budding appears. In this the main role is always played by those cells of the middle layer which are usually considered as un-differentiated cells; an interpretation which was sharply contested by Steinböck (1954). We must mention that in the higher types with a sessile way of life of corms the budding always takes place in the so-called atypical way, without participation of the endoderm. It has already been mentioned that scyphomedusae which have a prevalent role among Scyphozoa do not reproduce asexually. Transverse division appears regularly (even if not generally!) in scyphopolyps, yet here it has become so specialized that normally polyps are not developed by way of transverse division, only medusae. This transverse division which has become multiple can be so profuse and impetuous that it gives the impression of a terminal budding (the formation of the so-called polydiscal strobilae). Lateral budding and the simultaneous formation of colonies becomes rare. The buds of polyps which grow directly on the maternal polyp or on the stolons, become free and finally asexual reproduction is completely abandoned (Lucernariidae, the hypogenetic species). The formation of podocysts develops in the secondarily solitarized scyphopolyps which live in a shallow sea (Hadži, 1912); they represent a spe- 
cialized remnant of the formation of stolons and in this connection also of cormi.

As in the case of budding, regeneration which is well developed in Anthozoa (just as in Turbellaria) regularly takes place from out of the body wall. Tentacles which have been cut off are unable to develop into a whole new animal apparently because of the absence of mesohyl. The species Boloceroides however, represents according to Okada and Komori (1932), an exception. This is a case of specialization.

Even in the evolution of asexual reproduction the climax has been reached in Hydrozoa, both as regards the frequency and variety combined with specialization (the formation of polymorphic and individualized cormi), as well as in the sense of the so-called atypical formation of buds without the participation of the intestinal epithelium. The transverse division recedes completely into the background, and the individuals (hydranths) more and more lose their ability to form buds and to regenerate. The colony prefers to abandon a damaged polyp and to renew it out of the un-differentiated "coenosarc" (Hadži, 1915) than to replace it by means of renovation (Fig. 39).

In Hydrozoa, just as in the case of Scyphozoa, medusae are developed (and they certainly had been developed during the geological past) as special sexual individuals primarily by way of asexual reproduction only, combined with alternation of generations. It is certain that medusae had been evolved by the two groups in two completely different ways, once by means of a transverse division of the maternal polyp, and the second time by way of budding either of the polyp or of the medusa. On the other hand we find that in the two groups, species which had evolved in the same way where the medusa is developed directly from an egg and where the benthonic polyp generation has completely disappeared (the hypogenetic species).

It is in the Hydrozoa only that divisions of whole parts of cormi can serve the purposes of reproduction and of spreading. 

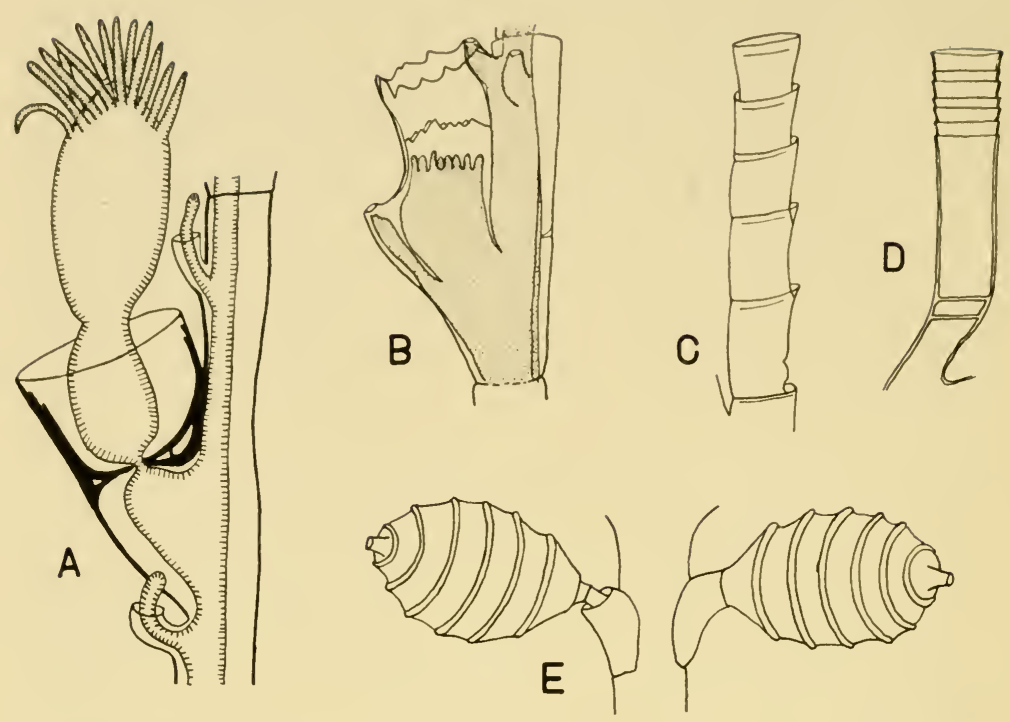

Fig. 39. A few cases of renovation in the thecate hydroids. A, Plumularia sp. with embedded hydrothecae as result of reiterated renovations. B, Aglaophenia sp. with reduplicated hydrotheca; C, Halecium sp. D, Lictorella sp., E, Synthecium sp. with gonangia as renovants. (A-B, after Hadži, C-E, after Broch.)

Such is the case with cladogony which I described (Hadži, 1919 Fig. 40), i.e. the separation of twigs of the colonies of Thecata which are transported by the sea current or which become attached to a substratum in the vicinity of the maternal colony. Something similar can be found in some Siphonophora which belong to the group Calycophora where whole specially organized cormidia are separated from the maternal colony and continue to live independently, producing sexual individuals. Finally, we find only in Hydrozoa an asexual reproduction of cormi as such.

The fact may not be completely irrelevant that, contrary to what has been found in Scyphozoa whose medusae can never reproduce asexually, this kind of reproduction does not appear quite so rarely in hydromedusae where it is more frequent in 


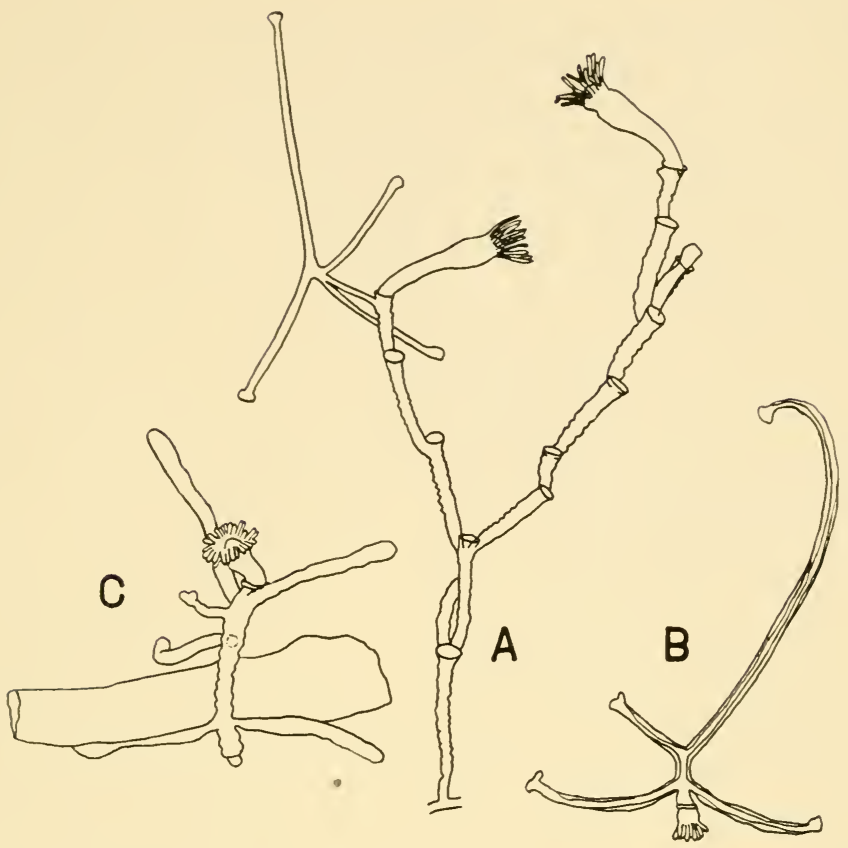

Fig. 40. Cladogony as a special form of asexual reproduction of thecate hydroids (Fam. Haleciidae). A, Halecium pusillum, a cormus in cladogony. B, cladogonium freely floating. C, young cormus of Halecium sp. developed from a cladogonium on Cystosira (after Hadži).

the form of budding than in that of a division (Gastroblasta raffaeli, Fig. 38). The buds can appear in various places, even at the manubrium which corresponds to the oral cone of hydropolyps. In the young parasitic and hypogenetic Narcomedusae, budding can occur even at their aboral ends (e.g. in Pegantha, according to Bigelow Fig. 46 D). It must be emphasized in this connection that, by way of budding, hydromedusae only can be developed from hydromedusae (the one supposed exception is still a matter of dispute). 


\section{Endosymbiotic Monocellular Algae}

The fact that the so-called zooxanthellae and zoochlorellae which live intracellularly can be frequently found in Cnidaria as well as in Turbellaria cannot yet alone be considered as a direct proof of a close relationship between these two animal groups; in particular it does not help us to distinguish which of the two groups is the parent and which the descendant. Nor is it proved that this appearance can be considered as a pure coincidence only. This situation, however, is altered when we notice that in the Turbellaria the mutual relationship between the animal and the alga stands on a more primitive level than the level which has been reached in this connection in Cnidaria. So far the physiological circumstances of this symbiosis have not been clarified (the extent of the mutual dependence as regards breathing and feeding); we will take into considerations those conditions only that can be observed during the transmission of symbionts from one generation to the other.

We can disregard the circumstance that there are at least two types of symbiotic algae, the green coloured zoochlorellae (this is, as a matter of fact, not a taxonomic idea), and the zooxanthellae whose colour is yellow to brown (they are actually Dinoflagellata, according to Chatton). The situation is usually such that zooxanthellae prevail in those animal partners that live in the sea, while zoochlorellae are more numerous in those partners that live in fresh water. It should also be mentioned here that such endosymbioses occur as early as in Protozoa (especially in Radiolaria and in Euciliata). As for the Turbellaria, we can read in the manual written by Hyman (II:143), "Most acoela and some rhabdocoela and alleocoela harbour symbiotic chlorellae and xanthellae;" these live mainly in the mesenchymal cells under the skin muscle tube. It is important in this connection that these algae do not pass over into the egg cells so that the young animals which have just developed from an egg do not possess symbionts and they must be later 
"infected" by food which contains these algae, e.g. by the egg shell whose surface is covered with these algae.

It is characteristic that no case of a symbiosis with these algae is known in Polycladida and that correspondingly zooxanthellae do not occur in the completely transparent pelagic Ctenophora which derive from Goette-Müller's larvae that have adopted neoteny-even if such a symbiosis could be expected.

In Turbellaria the majority of cases of endosymbiosis can be found in Acoela and in other primitive groups. It is not impossible that in Acoela the absence of nephridia could be attributed at least partly as being due to this endosymbiosis since the algae which live in the cytoplasm use up the products of excretion of their partners. The physiological relationship with these algae can be very complex in numerous species of Acoela (e.g. in species belonging to the genus Convoluta). Yet in spite of this we find that the continuity of this symbiosis has not been secured in the most reliable way, i.e. by means of a transition of algae into the egg cells. It could perhaps be said that as early as in Acoela the eggs are better isolated against their surroundings in the body of the partner. The possibility, however, cannot be excluded that in those Turbellaria which regularly possess symbiotic algae, cases will be found where symbionts are directly transferred by means of egg cells. This could be the case in Acoela with a primitive sexual apparatus. Von Graff $(1908,1943)$ admits the possibility that in the species Polychoerus caudatus Mark. the algae are present even in the cleaving egg.

It is well known that in Cnidaria a symbiosis with monocellular algae can be frequently found. We can observe such cases in all the three subdivisions, and most frequently in Anthozoa, among others in Actiniae and in Madreporaria. This symbiosis occurs so regularly in the cliff-building corals, whose most profuse growth takes place in the well-lit upper zone of water, that earlier it was quite generally believed that these corals lived and grew mainly or even exclusively at the expense of their endosymbionts. This endosymbiosis, however, 
does not go so far, as has been shown by recent researches, their animal food is usually consumed during the night and therefore during the day-time we do not find any food of animal origin in the gastrovascular system of polyps belonging to corals. It seems that the sessile way of life favoured the stabilization and further development of this mutual relation-

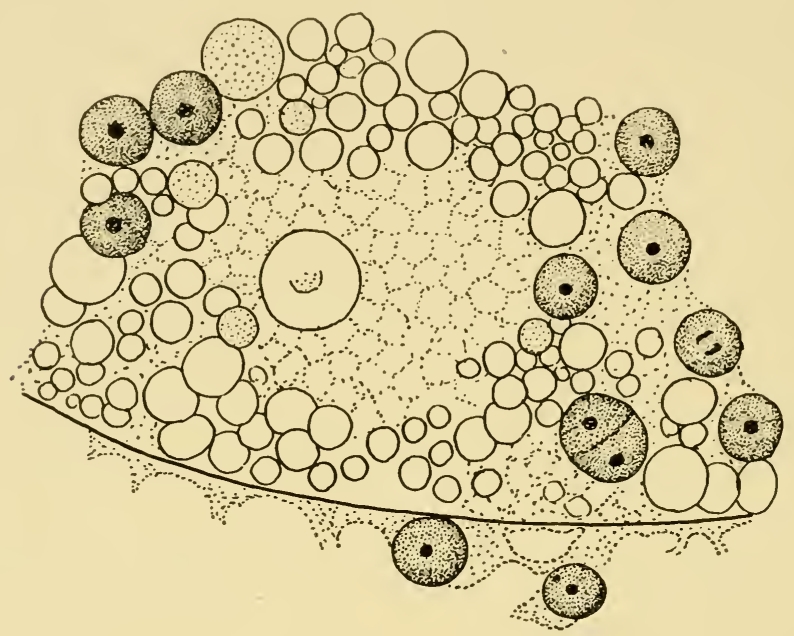

Fig. 41. A section of the egg-cell of Halecium sp. with symbiotic zooxanthellae pervading in the egg (from Hadži).

ship, so that finally these algae could be regularly passed over to the eggs, a development which was facilitated by the position of ovaries and by their approachability. It was more than 40 years ago that I was able to show how zooxanthellae actively penetrate into the young egg cells of a species belonging to the hydroid genus Halecium (Hadži, 1912 Fig. 41). At about the same time (1906) I succeeded at the then established "Biologische Versuchsanstalt in Wien," which was headed by the well-known experimental zoologist Hans Przibram, in making green Hydra colourless, after I had reared the sexually ripe animals in complete darkness (the same experiment 
was repeated later by others with the same result). Not only as a species but also as a genus, the green Hydra (Chlorobydra virridissima) is so closely connected with its green symbiont that it is only with difficulty able to live without it.

The fact may not be completely without significance that in the lower Turbellaria the symbiotic algae appear everywhere in their interior ("entomesoderm," or better archihiston, according to Steinböck), clearly a sign of a lower level of development. In Cnidaria we find, with a few exceptions, these algae in the intestinal cells only.

Thus the facts that can be observed in the symbiosis between animals and the monocellular algae support my thesis that this symbiosis had been developed as early as in Protozoa, that it had been inherited from these by the lowermost Eumetazoa, then by Turbellaria, and that from these it was transferred to Cnidaria where it has been further evolved.

With this we can conclude the second part of our present study. In it I have endeavoured to make it at least more probable that, contrary to the widely accepted opinion, the phylogenetic development of Cnidaria followed the direction Anthozoa-Scyphozoa-Hydrozoa, a direction which also agrees with the generally accepted principle that the sessile way of life leads both to the radial symmetry and to the retrogression of the organization (especially inasmuch this is connected with the free movement) with a simultaneous progressive development of new or of already present properties that are connected with the sessile way of life, and that in this way a new animal type is being developed. No well-warranted facts are known to me which would be incompatible with this new interpretation. On the contrary, I find that many facts which could not be understood or which were obscure, become understandable and clear if viewed from this standpoint. 


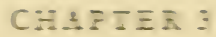

\section{THE CONSEQUENCES \\ OF THE NET INTERPRETATION \\ OF CNIDARIA}

I- uunetuds ouher cases where a simila change ot a sequence of stbgrougs Within the trume cl lurger group had to be pade, the cunsequences of stuch a chinge hare not been imporan. It was simpli uecessar to change the secuence, and itis was all. The larger grovp could still pueserte the position it had prewionslt occtoisd in the rarural znimal spstem. In out cuse, too, we End as the firs consequence of the zew in-

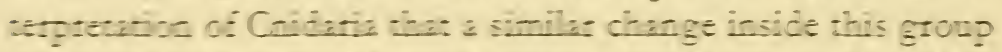
becones necessar. The nust expect ic adrance and reckon

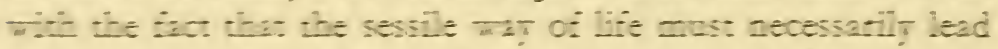
to a tetrogtessuon ot the generi structure, expeciall since we

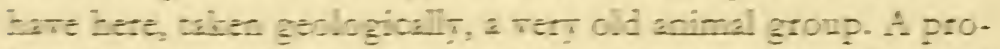

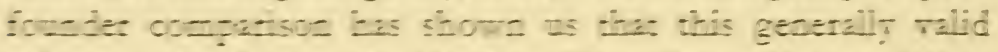
rule zust also be crue for Cridatia. Itus the erolution of Coidaris began in al probabilir with the primarily solitary and cult Bghti uoting anthopolps, and it coutinued within

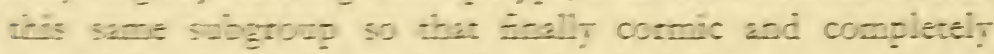
imobie species iare dereloped which hare nerertheless yeswerted, thl the queseco dat, clear remains of an earlier biinerel sy-medr. In adfition to this, the derelopment led Io the puelt adia spmetric sciphopolps which hare a

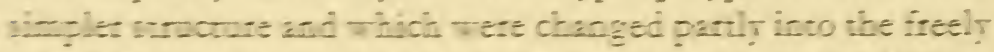
rw..... lerel of orga-ization acd thet hare begun as a whole to preTal oree the primitre prolp iorm. The 2-dropoltp, with its 
eren simpler structure, had act erclred fecu scppioneduse but rather from the more primitire scpphopcips; it had aguin dereloped-as a repetition ot the derelopment mich had al-

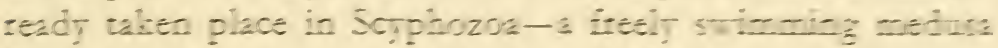
form. Sereral subgrougs of Criciaria which hate in the wean-

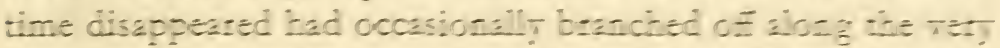
long road that ewolution of Cridaria had vaken. Ithis is bou the most probable reconstruction of the ghtlogent of Coidiris should appear in briet outline.

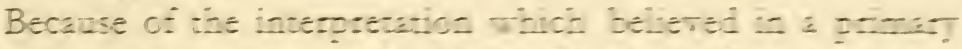

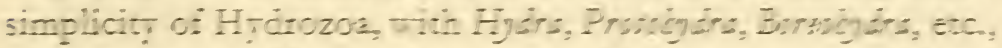
at the begining lan incerpretzion which we are ucto dbiged to abandon), the Cnidaria hare been placed, usuali in conecttion with sponges and with Crecopinoes, at the ret begi- ing. at the root of all poircellelar animals; the hate been bunded as animals with two bodj laters. This group of animals bas also been given a special name, that of Coelecterum, in coumus: to the more highit dereloped polfoelitur znimals, the Coulo.

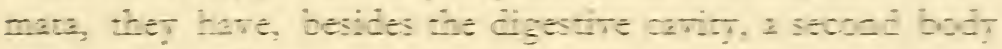
carits, the coelom.

This grouping which has been articially formed has not become senseless. Sponges mere the Ars: animals which had to be excluced from this groug. The are in ze:lig nos Coelenterata at all and the recent attempts wich tr to taite out ine sponges as true Coelenterata are theretore useless. इpouges uete followed br Crenophora which were bormeri, and ereo sumetmes not, simpir called Acnidiria, ie animals telused us C-ids-

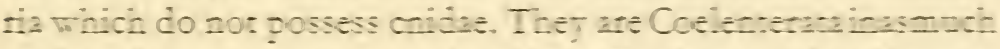
as thet do not possess a coelom lactuall! thet ate Acoelomitr. a notion which is considerably broter and mone sciulie which, howeree, I do no: wisl co incroduce and ewen less $: 0$

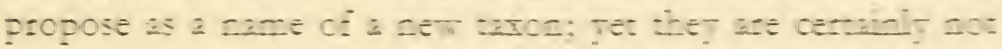
Coelenterata, as I hare shown abore, sicce Coelenterata as such do not exist at all and wht is more, thet hate nerer existed. The Crenophora erolred from some somewhat more hagily de-

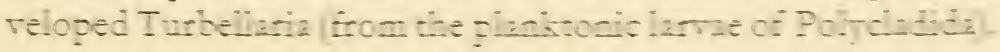


In this way only Cnidaria remain, as the last of the Mohicans, in the large group of Coelenterata. As a matter of fact, we can even now find books on zoology (cf. D'Ancona, Lezioni di Biologia e Zoologia Generale, 1939) where Cnidaria only appear under the heading of Coelenterata. Nevertheless, they have left an impoverished Coelenterata in the old place of the natural system.

Important problems arise as soon as we place Hydrozoa with their secondarily solitary and simplified species at the end of the system of Cnidaria and when we put the primarily solitary Actiniaria in their stead; such a problem is, for example, the question of how to connect Actiniaria "downwards," or where to derive them from? Can we still continue to use the gastraea theory which is irrevocably connected with the hypothesis that Coelenterata consist of two body layers? Or should we rather take into consideration the next "lower" Eumetazoa, the Turbellaria, which scholars have tried again and again somehow to connect with Coelenterata, usually in the same way as they have tried to derive Turbellaria from Coelenterata. If, however, we accept the opposite thesis, which seems a priori to be the more probable one, that Cnidaria had developed from Turbellaria, then suddenly a new difficulty arises; though repeatedly mentioned in passing, nobody (with one exception, O. Steinböck) has ever earnestly attempted to solve the problem of the origin of Turbellaria. This again opens the important question of the origin of the eumetazoan Turbellaria from their protozoan ancestors. Were these Protozoa which stood closest to the present day Euciliata, or colonies of Flagellata?

The zoologist who proposes a new interpretation of the evolution of Cnidaria which he wishes to be generally accepted, is also obliged to give rational answers to all the questions raised above; these answers must be at least more probable than the answers that have been given and defended till now.

In a series of sactions I will endeavour to discuss the most important consequences of the new interpretation of Cnidaria, 
firstly the detailed problems (e.g. the new system of Cnidaria and the position of Cnidaria in the natural animal system, the probable origin of Cnidaria or the turbellarian theory of Cnidaria) and secondly the more general problems as, for example the origin of Metazoa, the origin of Turbellaria, the relationship between Protozoa and Metazoa, the criticism of Haeckel's theory of a blastea and of a gastraea, as well as the idea of germ layers, the problem of mesoderm and of metamerization, the origin of the ontogeny and its further development together with secondary cellularization, the general division of the animal world connected with a reconstruction of the animal classification-all this connected with an objective discussion of the opposite interpretations.

\section{The New System of Cnidaria}

It will be our first task to construct a system of Cnidaria which will be a truly natural system and which will agree with the new interpretation of the origin and of the phyletic development of Cnidaria. Since this is not the right place to discuss in detail various taxonomic problems, we will limit ourselves to an outline of the system as it appears in larger groups or taxons. The division of Cnidaria (the corresponding system category will be later defined) into three main groups remains unchanged since it agrees well with facts; we only have to change the sequence of these three groups into the direction Anthozoa $\rightarrow$ Scyphozoa $\rightarrow$ Hydrozoa. With regard to the relationship connections of these three groups with each other we have to take into consideration two possibilities. It is possible either that both Scyphozoa and Hydrozoa had developed independently from each other, from the Anthozoa; or that Scyphozoa had developed from Anthozoa and that later these Scyphozoa themselves had evolved into Hydrozoa, the youngest representatives of Cnidaria. The second possibility seems to be more probable since Scyphozoa appear, so to speak, 16 
in the middle between Anthozoa and Hydrozoa. Theoretically, a third type of evolution is also possible, i.e. that all the three subtypes of the Cnidaria had developed in parallel, from a hypothetical indifferent state. Such a concept, however, seems to me as completely improbable. As for the classification however, it is irrelevant which of the possibilities be true. It appears as very probable, in view of the great difference which can be found in the way that the medusa form is primarily developed from the primitive polyp form in the two groups of Cnidaria, that the separation of both Scyphozoa as well as of Hydrozoa took place at a time when neither the former nor the latter had yet developed their medusa forms.

The main characteristics of Anthozoa can be enumerated as follows: (1) they have a muscular gullet (pharynx with siphonoglyphs (2) they have primarily solitary individuals (3) they have a polyp form and no medusa form; (4) they have well-developed longitudinal folds in their intestine, the genuine sarcosepta which are orally connected with the gullet; (5) they form intracellular sclerites which are permeated with the calcium carbonate; (6) they still have a well-developed middle layer.

The following elements are characteristic of Scyphozoa: (1) their "septal apparatus" is strongly reduced in the form of usually four short taeniolae with taeniolan muscles and teaniolan funnels; (2) they have developed scyphomedusae as a special sexual generation which has become the predominant form of Scyphozoa or even a form which alone has been preserved (the hypogenetic species); (3) they do not possess an ectodermal gullet; (4) scyphomedusae are developed, with the exception of the hypogenetic species, where they develop directly from the egg and the lanal cells, from polyps by way of a transverse division.

The following characteristics should be mentioned in connection with Hydrozoa: (1) no traces of sarcosepta or pharynx can any longer be found in their polyps; (2) their middle layer is strongly reduced so they give the impression that they con- 
sist of two body layers only; (3) they have developed their own medusa form, the hydromedusa, which is formed primarily by way of budding (and secondarily directly from the egg cell); secondarily the medusa generation is frequently more or less reduced; (4) only they have developed pelagic, polymorphous (also with regard to the medusa form), and strongly individualized cormi (Siphonophora).

Changes that have occurred within the frame of individual groups (subclasses, earlier classes) must also be studied in agreement with this new interpretation of the phylogeny of Cnidaria; they cannot, however, be discussed here in greater detail. As for Anthozoa, we can preserve their present subdivision into two groups of Hexactiniaria (Hexacorallia) and Octactiniaria (Octocorallia), yet with the difference that, in contrast to the present sequence, we give Hexactiniaria the first place, and Octocorallia the second. This is not difficult to justify. The polymerous state with large and not fixed numbers is, according to a very generally valid rule, the primitive state, and the oligomerized state with a fixed and diminished number is secondarily developed from the former. We must therefore reverse the presently valid sequence and put Hexactiniaria into the first place and Octactiniaria into the second place. Other properties of these two groups also agree with this new sequence. Thus we find, for example, the primarily solitary anthopolyps (among these a type with a rounded off aboral end) among the Hexactiniaria only. A dimorphism of polyps and the individualized cormi can be found in Octactiniaria only. Octactiniaria each have (with one exception where we find a different number of septa) eight branched tentacles. Hexacorallia have been given the first place also by some other zoologists (e.g. A Kästner, in his Lebrbuch der speziellen Zoologie, yet he does not give his reasons for such a sequence).

It is clear that the first order within the group Hexacorallia is that of Actiniaria. This has usually been the case in earlier classifications, too. As for the remaining orders, we find the equally primarily solitary Ceriantharia regularly placed at the 16* 
end of Hexacorallia, because of their aberrant septal apparatus; more rarely, however (for example, Beklemischew, 1958), they are considered as a category equal to that of Hexacorallia. It is certain that the Ceriantharia had evolved as a side branch from a root which has remained unknown so far (we cannot expect that we will ever be able to find the corresponding fossil material since we have here animals that possess no skeletons whose development goes far back into geological past). It may be better, however, to place them immediately after the Actiniaria because they have remained, like Actiniaria, primarily solitary animals. They should be followed by the remaining orders: Madreporaria (it is possible that among these there still exist primarily solitary and now already completely immobile species), Antipatharia, and finally Zoantharia. The last two orders are highly specialized, each in its own direction.

Fortunately enough, the subdivision of Octocorallia (Octactiniaria) has already been well made. The most important difference between various systems that have been proposed can be found in the number of subgroups. Some zoologists are satisfied with three orders (Alcyonaria, Gorgonaria, and Pennatularia) while it is believed by others that five orders must be distinguished (Stolonifera, Telestacea, Alcyonacea, Coenothecalia, Gorgonacea, and Pennatulacea). This difference, however, is not important in connection with the present study.

At present, we are hardly able to determine with certainty where and when Scyphozoa had branched off from the Anthozoa. Those Anthozoa which stand closest to the present day Stolonifera among Octocorallia, represent in all probability the starting point for the evolution of Scyphozoa (Fig. 42). This development had probably taken place at a time when their ancestors did not yet of necessity possess branched tentacles and when their polyps, which were protected by a periderm that extended high over the body of the animal, formed the "creeping" (stolonate) cormi. Medusae began to be developed from some intermediate forms which have now become extinct. Viewed from our standpoint we must put into the first 
place of the classification of Scyphozoa those species where the two generations are found equally well developed, where both the periderm as well as the formation of cormi can be observed in the polyp generation, and where the medusa form stands closest to the polyp form. A definite classification of the more primitive Scyphozoa will only be possible when we know the whole life-cycle of a majority of species belonging to them. Now

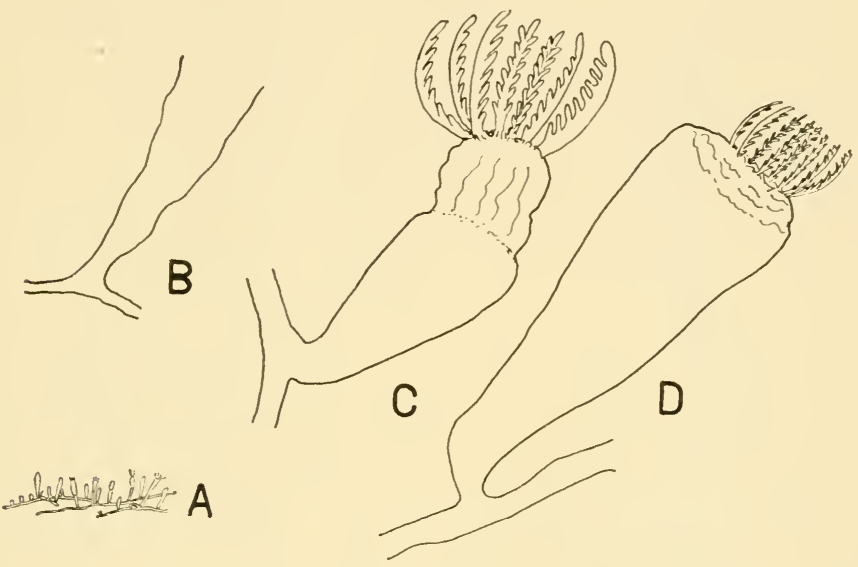

Fig. 42. A stoloniferous octocoral (Cornularia cornucopiae) with peridermal theca. A, a colony (cormus) nat. size (after Delage and Hérouard). B, empty periderm. C-D two polyps (orig.).

we are able to propose a provisional system only. It can be said in connection with the numerous attempts to develop a system of Scyphozoa which have been proposed till now (this group has been usually and improperly called Scyphomedusae) that all these attempts completely disregard the actually primary polyp generation (this is usually mentioned as a polypoid larva, as a "scyphistoma"). Scyphopolyps are not even mentioned among the enumerated characteristic properties of individual subgroups.

Two peculiarities of these systems can be mentioned as common to almost all the attempts which we have not the space to enumerate here. The first is that the order Lucernariidea 
is placed at the beginning and that of Rhizostomae at the end of the system; the second generally prevalent characteristic of this system is that Lucernariidea are followed by Carybdeidea (also called Cubomedusae), and Rhizostomae by Semaeostomata. The fifth order Coronata is placed in the middle (this is the sequence in Krumbach's Handbuch der Zoologie). Frequently, we find three orders only mentioned (e.g. by Claus), and the two orders Semaeostomata and Rhizostomae united in one order (as Discomedusae or Discophora), and the first two orders grouped together as Calycozoa, etc.

It is clear already now that Lucernariidea cannot be placed at the basis of the system of Scyphozoa, even if they give the impression of being more primitive forms. It is completely impossible that scyphomedusae had developed from scyphopolyps during their sessile way of life, as was formerly believed by Goette in connection with hydromedusae. Taking into consideration the well-founded supposition that the polyp is the primary form, we must consider it as possible that the broadened oral half had been separated by means of a transverse division from the aboral half of the polyp which continued to live as a sessile animal. This oral half had simultaneously taken over the gonads and was transformed into a medusa. We must therefore explain the mixture of characteristic properties of the polypoid and medusoid forms that can be observed in Lucernariidea, and that has been correctly emphasized by numerous specialists (especially by Krumbach and by Tochru Uchida, 1929) as being due to the fact that the separation of the medusoid half had been secondarily discontinued (we will meet something similar in Hydrozoa), and that since then the medusoid form has become well enough adjusted to the life in the benthos so that finally something new has been developed as a result of this amalgamation. The transverse division (not the budding) which takes place but once, seems also to be a primary event; it can be repeated after the passage of an interval of time; this can lead finally to a quick series of multiple transverse divisions, a 
development which has been called a strobilation and which can thus be considered as a secondary phenomenon. In the blindly ending trend of the Lucernariidea, the monodiscal strobilation without a separation has appeared as a consequence of a regressive evolution.
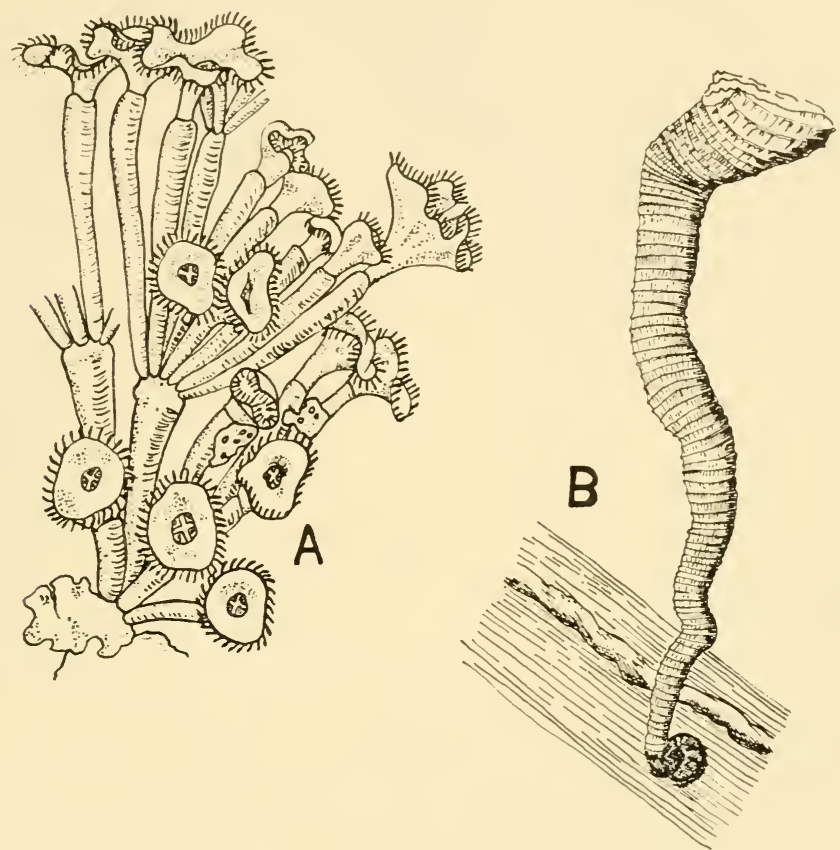

Fig. 43. Two species of scyphopolyps with peridermal thecae. A, Nausitboë sp. (from Komai). B, "Tubularia striata" Vanhöffen a solitary scyphopolyp of unknown systematic position; (after Vanhöffen).

In this way Cubomedusae, or rather Carybdeidea, come into the first place; they should be followed by Lucernariidea (they have also been called, less fortunately, Stauromedusae). The further sequence can remain the same as it now usually appears in these classifications, i.e., Coronata, Semaeostomata, and Rhizostomae. The polyp generation of the Coronata show a few signs of primitivism as has already been mentioned 
(Fig. 43). The ending "-medusae" should rather be avoided since in Scyphozoa there is-as far as we know on the basis of our present research-not a single large systematic group (e.g. an order) which includes the hypogenetic species (i.e., the medusa forms) only. I have discussed this problem at a greater length in another study (Hadži, 1955 a).

No radical changes are necessary because of the new interpretation of Cnidaria in connection with orders that belong to Hydrozoa. The order Hydroidea has been, and still remains, the basis order of Hydrozoa and there can be no doubt that other orders had evolved from it. No primarily solitary hydropolyps exist any longer. All those species that live now as solitary animals, as well as those that do not have any tentacles, are a result of a secondary development. For the present two additional orders, besides that of Hydroidea, are sufficient, i.e., Trachylina, as a group of all the hypogenetic species which have been wrongly interpreted to be highly primitive animals yet which are, in fact, highly specialized forms (just as in the case of the benthonic Hydra which occurs in the polypoid form only); and Siphonophora. As for Trachylina, it seems very probable that they evolved diphyletically, once as Narcomedusae, and the second time as Trachomedusae. It can therefore be expected that sooner or later it will become necessary to make a sharper distinction between these two groups of Hydrozoa if we continue to make taxonomic distinctions between two "sister groups" that had evolved separately even if they look similar, because their evolutions pursued two parallel ways and because their roots were closely related to each other.

With regard to Siphonophora it can now be considered as certain that they had evolved from the benthonic cormi of the polypo-medusoid athecate Hydroidea (Hadži, 1918, 1954). They therefore represent the climax in the progressive evolution within the order Hydrozoa and thus also of Cnidaria as a whole. In these the polymorphism is finally extended to the medusoid form also. The individualization of cormi, the way 
to which had been prepared as early as in Anthozoa, finds its climax in the Siphonophora.

It was in agreement with the old interpretation which believed in a progressive evolution of Cnidaria, that within the Hydroidea, the Athecata were considered quite generally and
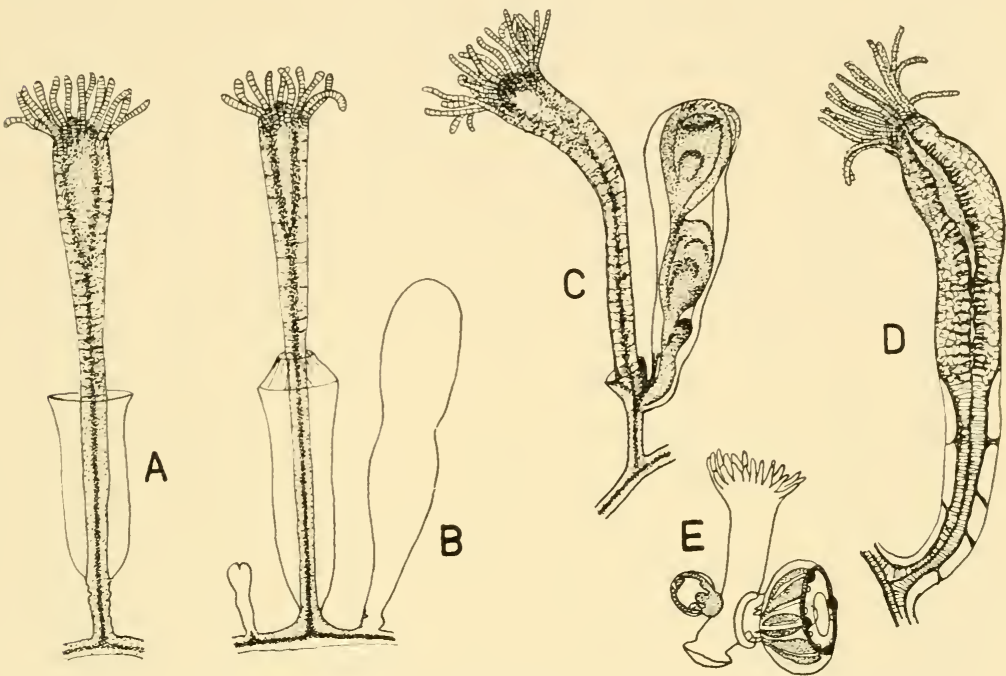

Fig. 44. The progressive and regressive evolution of the hydrothecae. A, Hebella sp. B, Lafoëina vilae-velebiti Hadži, with operculate hydrotheca; C, Haleciella microtheca Hadži. D, Halanthus adriaticus Hadži, the theca has fully disappeared, and remains only as the tonofibrilles. E, Eugymnantha inquilina Palombi, a "thecate hydroid" without the rest of periderm (A-D after Hadži, E, after

Palombi).

without exception in comparison with the Thecata, as the more primitive initial group. It was considered that since Athecata have simpler forms that they must also be the more primitive animals; at the same time solitary species, and above all Hydra, have been known as Athecata. There are no really solitary Thecata, close to this state is only the species Eugymnantha inquilina Palombi (Fig. 44). Furthermore, it is known that the cormi of Thecata show a higher degree of individualization than those of Athecata. Because of the new 
interpretation of the origin and the evolution of Cnidaria, and also of Hydrozoa, we are forced to change the sequence, i.e. we consider Thecata as the more primitive group of Hydroidea. A whole series of facts make it probable that the rich evolution of Hrdroidea had begun with the Thecata. First we must imagine the most probable way in which the Hydrozoa eventually dereloped from Scrphozoa. It may prove useful to trace even further back in phylogeny, back to the origin of Scyphozoa. The reader will remember that I derive the Scrphozoa, especiall! their polyps, from the so-called "creeping" and therefore stolonate cormi of Anthozoa (probably from some forms that stood closest to the ancestors of Stolonifera, among the recent Octactiniaria) that had dereloped a cuticular periderm, (which can be easily distinguished from the skin high orer their anthocodium, the head of the polyp; Fig. 43). It was from the Scyphozoa, which had the polyp form only and which did not ret produce a medusa generation, that the equally stolonate and creeping cormi of Hydroidea was in all probability developed, first in the form of hrdropolrps. The personal part of the hydropolyp had been further divided into the basal and undifferentiated stem and into the differentiated head (hrdranth). The hrdrotheca with its special form had evolred in several directions from the undifferentiated peridermal part, reaching its climax in those species which hare an automatically closing corer lid (Fig. 44). During this phase of the progressire erolution, the Hrdrozoa already began to produce their medusae, a derelopment which was independent from the otherwise similar morphogenetic process of the formation of medusae that can be found in Scrphozoa -as has already been repeatedly emphasized in the present study; in Scrphozoa the medusae formed by means of a transverse division, and in the thecate Hrdroidea from the separated buds of hydranths. This also agrees with the fact that quite generally budding as a consequence of a prolonged sessile way of life prevails orer transterse dirision. 
The species (genera and families) with a more or less cylindrical or with a down-turned conic hydrotheca, with the formation of medusae as individual buds, and without the theca lid, should be placed at the beginning of the system of Hydroidea (e.g. Hebellidae). The subsequent evolution of Thecata diverged into several directions with a great variety in the forms of thecae, with polymorphism, retrogression of the medusa form, and with various forms of cormi which can also include individualized forms (a secondary monopodial growth). A special direction in evolution can be observed in those species whose hydrotheca has been progressively reduced (fam. Haleciidae) so that finally these animals have completely ceased to form a theca (fam. Campanopsidae). The last remains of a former theca can be found in the so-called tonofibrils which in the Thecata had provided an elastic connection between the basis of the theca and the hydranth (the socalled luminous dots over the diaphragm which separate the hydranth from its stem). This is how we can understand the evolution of the Athecata from the Thecata. The possibility cannot be completely excluded that the evolution from a thecate to an athecate state did not occur once only, yet such a polyphyletic development must be first specifically proved.

This is not the right place to discuss all the details. It should suffice that we give here in a broad outline, a new and more suitable system of Hydrozoa, as it appears if we view Hydrozoa as animals that have evolved from Scyphozoa. The old systems which are mainly based on the actinula hypothesis and which try to avoid comparisons between hydromedusae and scyphomedusae must now be abandoned. The classification of Cnidaria must be necessarily based on the morphology of the main primitive form which is common to all Cnidaria, i.e. that of the polyp. The hypogenetic species that can be found both among Hydrozoa as well as among Scyphozoa, are a résult of a secondary development. This development, however, had followed two different lines which can be understood only if we take into consideration the way that medusae had evolved 
in both these cases from their respective polyps. In Scyphozoa again this development had pursued two completely different paths; in the first type of development the medusoid distal half of the polyp had remained attached and this was followed by a reduction and by a change of the medusa form. In the second type the planula continued to exist in the plankton, the polypoid generation has been omitted and it is in this way that the scyphomedusa can develop directly from a planula. This second variant has been repeated in Hydrozoa-we speak here of the hypogenetic species or even groups, using the expression as it was proposed by Haeckel (Trachylina). The Hydromedusae remain attached to their cormi because here medusae are developed from the whole hydranth (and not out of half only), and that by way of budding; these medusae are afterwards changed into a more and more reduced medusoid form which can finally completely disappear, the polyp generation which was earlier sterile is now changed into a sexual and the only existing generation.

The classification of Hydrozoa, and especially that of Hydroidea, which tries to express the evolutionary path these animals had taken, must be based on conditions that can be observed in their polyp generation; yet at the same time it must also take into consideration the younger medusa generation as regards the details of morphology. It is important that we adhere, in our endeavours to work out the details of this system, to the fact that Hydroidea do not include any primarily solitary species, and that in these animals solitarization has been evolved several times, along several lines of evolution (Fig. 45). This is not a theoretical supposition but it can be also easily observed in the recent material (in the so-called Limnomedusae).

It seems that three orders are sufficient for the division of Hydrozoa, i.e. Hydroidea, Trachylinea, Siphonophora. Since we do not know which, taken geologically, of Siphonophora and Trachylinea is the older (it is even possible that both of them had evolved diphyletically), so it is the same if the first 
place is given to the first or to the second group. It was a retrograde step in the classification of Hydrozoa when the accepted rank of order was given to two families by Hyman in spite of the fact that this classification has now been found to be erroneous; to Stylasteridae (they are valued by Stechow only as
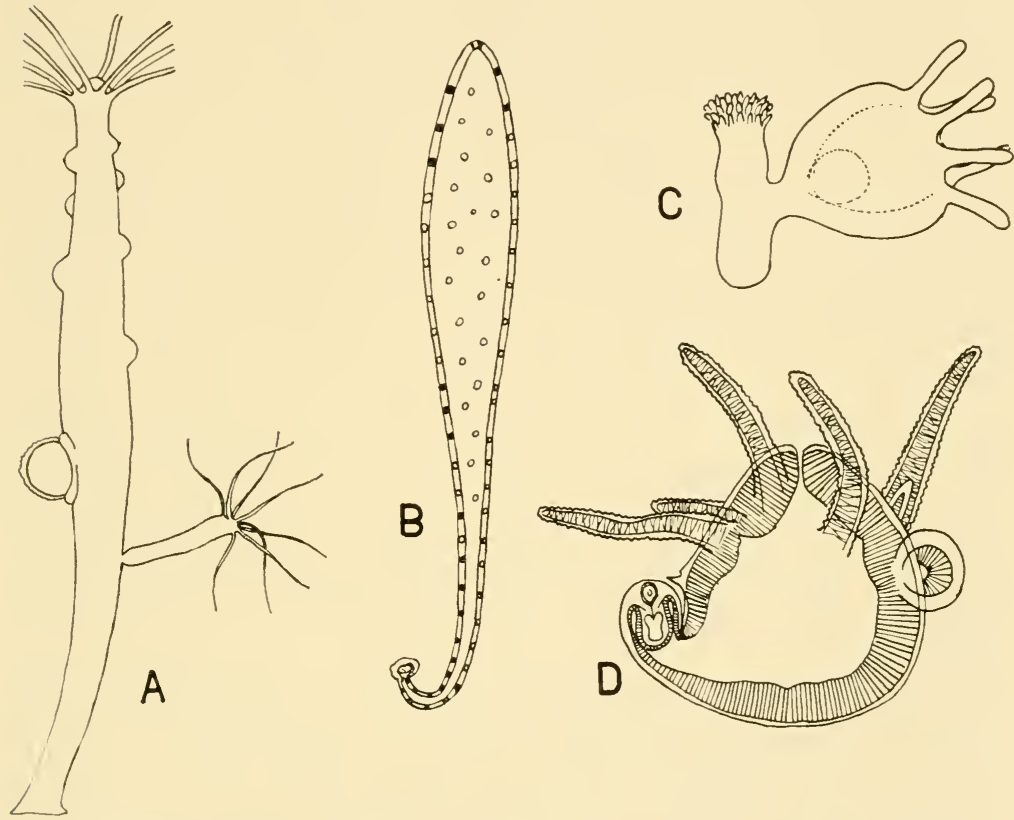

Fig. 45. A few secondarily solitary hydropolyps. A, Hydra sp.

B, Protobydra sp. C, "Microbydra"-polyp of Craspedacusta sp.: D.

"Haleremita"-polyp of Gonionemus sp. (after various authors.).

a subfamily), and to Milleporidae (which have also been degraded by Stechow to the rank of a subfamily).

In spite of the fact that from our viewpoint there can be no doubt that the Hydrozoa had inherited their tendency to alternation of generations from their scyphozoan ancestors, it seems nevertheless more probable that the ancestors of the present Hydrozoa had separated from the ancient Scyphozoa before the latter had developed their own medusa generation. 
Thus the typical alternation of generations, medusa: polyp, had evolved twice, and each time from its own root. In spite of the great similarities that can be observed in the forms and basic organisations of scyphomedusae and hydromedusae-a fact which can be explained as due to the similarities that exist in scyphopolyps and hydropolyps-there exist considerable differences between the two medusa forms especially as regards the way how they are developed. With the exception of the secondarily acquired hypogenesis, we find that all the scyphomedusae develop without any exception from scyphopolyps by way of an unequal transverse division, while all the hydromedusae develop equally, without any exception, by way of budding. The division must be considered as the more primitive form of the asexual reproduction, and budding as its more advanced form. I wish to mention in passing that as early as in the Anthozoa we can find in the higher subtype of the Octactiniaria budding only as the form of their vegetative reproduction while division prevails in the Hexactiniaria. In the scyphozoan polyps budding prevails, yet transverse division has been preserved in the formation of the medusa generation.

It should be mentioned in passing that it is already time that the hydroidologists as well as other zoologists adhere strictly to the names Thecata (after Fleming, 1828), and Athecata (after Hincks, 1868) when they speak of the two groups of Hydroidea; they should avoid the awkward names Calyptoblastea (or even Calyptoblastiques), Thecaphora, or the too narrowly interpreted Campanulariidea (Stechow, 1922), Gymnoblastea (Gymnoblastiques with the addition Hydroida), Tubulariidea (Stechow, 1922). The double names: ThecaphoraeLeptomedusae and Athecatae-Anthomedusae (after Broch, 1924) can also be dispensed with.

Capitata (Kühn, 1914; Coryninea, according to Stechow, 1922) have been placed, within the group Athecata, in front of Filifera or Bougainvillinea; this has been so because it has been believed that the actinula with tentacles that had thickened 
ends represents the starting point not only in the evolution of Hydroidea or Hydrozoa but also of Cnidaria as a whole. It is quite possible that we have here two parallel lines of evolution both of which end in very specialized solitary forms; once with Hydrae, the other time with Branchiocerianthinae.

\section{The Rank and the Position of Cnidaria in the Animal Classification}

According to our interpretation, Cnidaria represents a blindly ending side branch on the main stem of Turbellaria. The Cnidaria assume the position or the category (taxon) of a class within the stem (phylum) of Ameria. Thus the three subgroups, Anthozoa, Scyphozoa, and Hydrozoa, are in reality three subclasses. A graphic representation of this position and of their division will be found in the final chapter of the present study.

The Cnidaria with this, as we believe, well-founded, connection with Turbellaria to which we will later return, lose the isolated position they have enjoyed till now. It seems to us therefore an exaggeration when they are given the rank of a phylum, as has recently been done by Hyman, by Kästner, and by numerous other scholars. I consider that the category (taxon) of a phylum should be used less liberally and that the number of phyla must be limited to the absolute minimum. I will later show that a very small number of recent phyla is in reality fully sufficient (thus the Eumetazoa can be divided well enough into four phyla). Usually we will find the taxon of a "class" to be satisfactory.

As a welcome side effect of the new classification which tries to avoid the high categories of Radiata and Coelenterata (after the system by A. S. Pearson, the editor of the Zoological Names, etc., prepared for the Section of American Association for the Advancement of Science, Durham, N. C., 1949), we find that the whole animal system becomes considerably 
simplified. This has not only a scientific value but also a value in teaching (Hadži, 1952 a). We must discourage the use of the group phylum Coelenterata (Leuckart 1848 after A. S. Pearson) instead of Cnidaria, regardless of priority which is anyhow not internationally binding in connection with the names of the highest categories. I have already emphasized that the notion, and thus also the category, of Coelenterata-and correspondingly the opposite notion of Coelomata-have now become completely superfluous because they do not find support in the natural facts. They must now take their place in the archives. If we have to dismiss the group Coelenterata then we must also necessarily abandon the group which represents the opposite to Cnidaria and which has been given the name of Acnidaria. These are in reality Ctenophora that constitute a class of Ameria, which has the same rank as Cnidaria because they had evolved from Turbellaria parallel to, and independently from, Cnidaria.

The Cnidaria, together with Turbellaria, Ctenophora, Trematoda and Cestoda seem to be somewhat more closely connected because of one important common property, and that is that they all are aproctous animals, i.e. their digestive tract does not possess a primary anal orifice. In spite of this it is unnecessary to establish a special category which would include all of them and to give it a special name. In practice the usual pair Aprocta-Euprocta can be well enough used in comparative morphology.

It is unfortunate that, even recently, attempts have been made to use for systematic purposes such an antiquated notion as that of Radialia (or some similar ones) even if it appears with a somewhat changed scope (e.g. without Echinodermata). This attempt has been made, by Beklemischev (1958) who classifies Ctenophora as Acnidaria among Radialia. In both cases the radial symmetry, which is usually partial only, had evolved, as has been already shown, secondarily from the bilateral symmetry. Moreover, the structure of Ctenophora is in reality bisymmetric and not radially symmetric. Parallel to 
the necessary abolition of the notion Radialia (or Radiata) we must also abandon the opposite notion of Bilateria. The structure of all Eumetazoa was originally bilaterally symmetric and it was secondarily only, usually as a consequence of a prolonged sessile way of life, that they have dereloped a more or less explicit inclination to the radial symmetry.

Each change of the animal system, especially when it inrolves the higher ststematic categories, is unwelcome and scholars will therefore try to avoid it. Yet if we endearour to derelop a natural system we are obliged to change this system until it agrees with facts and with the interpretations that are based on them. It is all the better if a simplification of this classifacition appears as a result of our endeavours.

\section{Discussion of the New Interpretation of the Evolution of Cnidaria}

The division of the taron Cnidaria - regardless of the system category which should be attributed to it-into the next lower units, has been so firmly accepted by expert zoologists and by others, in the sequence Hrdrozoa $\rightarrow$ Scrphozoa $\rightarrow$ Anthozoa that no attempt will be easily accepted which tries to change this sequence. This is, in my opinion, not only a consequence of an otherwise healthy conservatism which suggests caution; it is also due to the fact that the suggested change of a widely accepted sequence has not been supported by a discorery of, for example, a "living fossil," or at least by some special new facts. The majority of zoologists have been influenced even more by the consequences which this new interpretation implies, than by the first two motives.

In spite of the fact that the first publication of my thesis appeared as early as in 1944, the new sequence of the groups of Cnidaria has not found acceptance, as far as I knot; in any manual or text book on neozoology. It should be remembered here that some palaeozoologists (Schindewolf, 1950; 
Kühn, 1939) have emphasized even before the publication of my study, that the Anthozoa with a primary bilaterally symmetric organization should be regarded as the most primitive Cnidaria, even if these scholars did not use quite the same words. To the best of my knowledge there were only a few neo-zoologists who, prior to the publication of my studies, expressed the idea of a primitive character for bilateral symmetry in Anthozoa; thus Haacke (1879 and 1893) who suggested, that the primitive gioup "zoophyta" were bilaterally symmetrical, and that radial symmetry had developed due to the transition to the sessile way of life. F. Pax (1910), an expert in Anthozoa, must also be mentioned; he wrote very cautiously, and limiting himself to Anthozoa, as follows, "Man könnte daher geneigt sein, die Aktinien von fusslosen, kriechenden oder grabenden Formen mit bilateraler Symmetrie abzuleiten, die später mit dem Übergange zur festsitzenden Lebensweise eine radiale Symmetrie erworben haben." Pax believed that the subsequent evolution depended on the type of development of the aboral end of the animal body (whether a foot disc was present or not). In the study on Hexacorallia which was written by Pax and which appeared in the large German work Handbuch der Zoologiz (edited by Kükenthal-Krumbach), Pax did not express this his opinion, clearly because he had to comply with the systematic division that had already been determined by the editors. In 1954 Pax agreed entirely with my suggestion.

Jägersten (1955 and 1959) has accepted my views regarding the priority of Anthozoa. It is true that Jägersten did not do this as a cnidariologist, nor for the reasons which have induced me to consider Anthozoa as the first group of Cnidaria, but rather because he has found my interpretation suits his theory of a bilaterogastraea, a theory which I will return to later. Marcus (1958:26) adopts the interpretation we find in Jägersten's work.

Kästner, even if he is under the influence of the fascinating ideas that had been formulated by Haeckel, has never- 
theless written in his most recent "Lehrbuch der speziellen Zoologie" (1:92) the gratifying note that it is "keineswegs entschieden, ob die traditionelle Reihenfolge Hydrozoa, Scyphozoa, Anthozoa der Stammesgeschichte entspricht, und ob die Coelenterata stammesgeschichtlich die Wurzel der Bilateria bilden oder einen frühen, blind endigenden Seitenast der Eumetazoa."

It was in the form of rhetoric questions only that Ulrich (1951:260) expressed his support of the idea of the primitive character of Anthozoa within the frame of Cnidaria. These questions refer to actual difficulties one meets if one tries to consider the Hydrozoa as the most primitive Cnidaria. Ulrich concludes his discussion of the problem with the observation, "lassen sich alle Schwierigkeiten recht gut beheben, wenn man die Evolution des heutigen Cnidarienmaterials nicht als progressiv, sondern als regressiv beurteilt." Simultaneously Ulrich promises a special work which will discuss this theme; to the best of my knowledge, however, nothing has so far been published.

Remane, too, is undecided when he writes on Cnidaria in the extensive Handbuch der Biologie which is still being published under the editorship of L. von Bertalanffy. In the text Remane preserves the traditional sequence of the three classes of Cnidaria. In the final paragraph ("Phylogenie, Systematik"), however, Remane states as follows, "Den phylogenetischen Zusammenhang der drei Klassen Hydrozoa, Scyphozoa und Anthozoa kennen wir noch nicht... Wer der einfacheren Organisation hohe stammesgeschichtliche Bedeutung zumisst, wird die Hydrozoen als primitivste Gruppe betrachten, doch lassen sich mit gleicher Berechtigung auch andere Auffassungen vertreten." We can see the reverse sequence-whose correctness I have tried to prove-to be more justified in the fact that later Remane himself has made a more positive statement in favour of the new interpretation (Remane, 1960). On the basis of a statement made by Steinböck (1958) and according to which Coelenterata as 
well as the oldest Metazoa as a whole had been bilaterally symmetric animals we can think Steinböck, too, considers the Anthozoa as the most primitive Cnidaria. I think that I am justified to include Sir Gavin de Beer among those prominent zoologists to have repeatedly evaluated positively my suggestions regarding the evolution of the Cnidaria (1948, 1951, 1958).

\section{The Probable Origin of Cnidaria}

We come now to the question of what the origin of Cnidaria could be if we declare the Anthozoa as the most primitive Cnidaria? Is it impossible to change the now prevalent opinion according to which the Cnidaria evolved as the lowest Eumetazoa from some colonies (cormi) of Flagellata by way of the three stages moraea-blastea-gastraea?'This second question also remains if we take into consideration other initial forms, e.g. a placula, a parenchymella, a parenchymula, a planea, a planula, a phagocytella, etc., instead of a gastraea; all these forms are in reality variants only, which do not differ in principle from the classical form even if they are held in high esteem by a number of scholars.

At first, one feels inclined to say that a reversion of the line of evolution of the three subgroups of Cnidaria is incompatible with the traditional derivation of Cnidaria from an ancestral form similar to a gastraea. The difference between such a form and an anthopolyp seems to be too great and a more extensive change of the old interpretation seems to be a necessity. So tar there have been, to my knowledge, two attempts only to overcome these difficulties. The first such attempt was made by Remane (1920), and the second by Jägersten (1955). The motives for such attempts lay in both these cases outside the sphere of the comparative morphology of Cnidaria; both were closely connected with the problem of the formation of the coelom. Remane adheres in his interpretation closer to the old concept. He 
finds the primitive form in an "Urcoelenterate" with four gastral pockets and thus with a radial symmetry; yet at the same time he admits the possibility that this "Ausgangsform der Cnidaria vor ihrer Festsetzung einige bilaterale Züge des Baues schon besass." Jägersten, on the other hand, constructs a completely new initial form which he calls a "bilaterogastraea" (Fig. 55). This, at first sight, original and ingenious construction, however, has all the characteristics of a so-called "suitcase theory" (Koffertheorie). Jägersten places into this constructions all those elements which he afterwards needs in his subsequent derivations. This is clearly a combination of the gastraea theory as it had been proposed by Haeckel (Jägersten [1955:333] himself admits that it is a "modification of Haeckel's theory"), and of the enterocoele theory as it had been proposed by Sedgwick, with the addition of an otherwise sound idea which had already been supported by several other scholars that the structure of the primitive form had after all been bilaterally symmetrical, and that this form as such had crept over the bottom of the sea. The construction as it has been proposed by Jägersten shows therefore numerous advantages over the interpretation as given by Remane. Yet if we look at Jägersten's bilaterogastraea from a somewhat different angle we find in it a similarity to the primitive Turbellaria, a similarity which Jägersten himself failed to notice. Jägersten had to overcome both great and small difficulties when he derived all other larger types of Eumetazoa, including sponges, from this bilaterogastraea. These difficulties force him to take refuge in a very large number of speculations (it is quite true, however, that we cannot avoid speculating to a certain extent). We miss among these large types (Cnidaria, Ctenophora, Coelomata) a direct derivation of the actually coelomless Coelomata which do not possess an anal orifice (by a coelom we understand in reality a perigastrocoele and not a gonocoele). Jägersten, however, mixes up these two notions; he believes the enterocoele theory to be changed now into a gonocoele theory, as he states this himself 
(1955:353) The final result of all this is that the intestinal pockets become at one time an enterocoele (= perigastrocoele), and another time a gonocoele (in a large number and without any actual order in Nemertinea). This, however, is not the right place to make an analysis of the other statcments that have been made by Jägersten.

A criticism of Haeckel's concept of the origin of Cnidaria must naturally be extended to the modifications of the same concept as found in the works by Remane and by Jägersten (together with Naef); they have both preserved Haeckel's basic ideas. The situation becomes even worse, because we find here, in addition to the old mistakes, a new blunder also, i.e. the idea of a colonial origin of Eumetazoa (Coelenterata); moreover, the old idea of enterocoely is here found transferred to an earlier phyletic stage in order to preserve the validity of Haeckel's so-called "fundamental biogenetic law" and of the gastraea theory, while at the same time not a single real argument is given by the author which could support his suggestions. The only progress which can be found in Jägersten's interpretation, if it is compared with similar older combinations, lies in the fact that he believes in an early transition of the "blastea" to a life on the sea bottom and, in connection with this, in a transformation of the form of its body in the sense of a bilateral symmetry (this constructed primitive form has therefore been called by the author a bilaterogastraea*).

The main difference which exists between Jägersten's standpoint and the interpretation proposed in the present study is that we both come to the conclusion that it is necessary to reverse the phylogenetic sequence of the subgroups of Cnidaria while at the same time we have both begun

* Prof. O. Steinböck has kindly called my attention to the fact that as early as in 1874 Haeckel had suggested that some gastraea which had sunk to the sea botton began to creep over the substratum and in this way had developed bilateral symmetry; it was called by Haeckel a gastraea bilateralis (repens). 
our research from two completely different starting points and motivated by tw o different aims. Jägersten wishes to improve and therefore to save the old concept, and in this he finds an obstacle in the old interpretation of the evolution of Cnidaria; this induces him to reverse the direction of the evolution of Cnidaria. I, on the other hand, have started my research with a study of Cnidaria; facts which had already been known have induced me to submit to a revision of the connections that exist first among the Cnidaria themselves, which was followed by a study of the relationship connections among Coelenterata, and finally among Metazoa. Ultimately, this has led me to a new interpretation of the evolution of the animal world. As a result I have found that it has become necessary to make much more radical char ges in the present interpretations (starting with a reform of the system of Cnidaria) than those changes that have been proposed by Jägersten and by other well-known zoologists.

Jägersten, and Remane before him, have only made the situation increasingly worse. 'This has happened in such a way that on one hand the contrast between a primary mesenchyme and a secondary coelothelium (mesoderm s.str.) which was previously quite unpleasant, has now become all the more acute; and, on the other hand, the uncertainty regarding the groups of Ameria which do not possess an actual coelom s. str. (perigastrocoele) and even less a metameric perigastrocoele has become all the more evident. Jägersten could not, as it seems, accept unreservedly a solution, dictated by this situation, of a large scale retrogressive evolution while at the same time Remane finds himself obliged to admit such a possibility. Jägersten remains silent about all this while at the same time he promises a special study; yet to the best of my knowledge no such study has not appeared so far. It would have been better if Jägersten in order to remain consistent, had gone further and connected his bilaterogastraea with the Enterocoela, since it is clear that Annelida are Ecterocoela. It is improbable, because it is unfounded, that a retrogressive 
evolution of freely moving animals could possibly have reached the extent that Remane, and even more so Jägersten, are obliged to construct. The problem of the "mesoderm" becomes complicated beyond any solution (see p. 100 in Jägersten's study, 1959). Jägersten will finally be obliged to admit the existence of a coelenterate type, and this in an even wider scope than was previously done by Haeckel, who even classified Platyhelminthes among Coelenterata. This is more consistent since Platyhelminthes are in reality not Coelomata in the usual sense of the word; see in this connection Jägersten's discussion of Xenoturbella (1959:101). The uncertainty Jägersten finally feels can be seen in the fact that in his diagram (1955: Fig. 8, p. 352) he does not use the notion and the category of Coelenterata in opposition to Coelomata-he only mentions Cnidaria and Ctenophora. In a more recent publication (1959:101) he quite misleadingly classifies Xenoturbella among Coelenterata, yet in the same paragraph he also speaks of the "so-called coelenterates."

It is not important that I have been the first neo-zoologist who has (earlier and independently from Jägersten) come to the conclusion, on the basis of a study of comparative morphology, that the Anthozoa had evolved as the earliest form of Cnidaria. The important thing is that I have come to this conclusion by means of a study of comparative morphology while taking into consideration the ecological conditions. Because of this new situation it has become necessary to try to find the probable origin of Cnidaria: it was in this way that new problems arose and that a whole new sphere of research has been opened.

It was clear from the very beginning that the old concept can be used no longer in this new situation, even if it has been used so long without any reservations in spite of the fact that there have been numerous well-known zoologists who have expressed doubts about the foundations of the same concept, such as is the so-called "fundamental biogenetic 
law" as it was formulated by Haeckel, and the hypothesis that the polycellular animals had evolved from some colonies of monocellular animals. In this situation it was most obvious to think first of the Turbellaria. The Turbellaria have been interpreted by almost all zoologists and above all by experts in Turbellaria, as "Coelomata" with a primarily primitive structure. The zoologists who represent an exception here are those who adhere to the enterocoele theory, i.e., those who, like Sedgwick, try to deduce the metamery of Eucoelomata from the cyclomery of anthopolyps. The typical Turbellaria, however, i.e. the higher Turbellaria which stand above the Acoela and which are usually taken into consideration by zoologists when Turbellaria are mentioned, seem to be more highly developed than Cnidaria because of their organization and above all because of their extremely complex hermaphroditic genital apparatus. For all these reasons it appeared as an almost hopeless task to try to derive the Cnidaria from the Turbellaria.

This situation, however, changes quickly when we take into consideration the frequently noticed fact that here we have a case of a transition from the freely mobile to a sessile way of life. Take for example Endoprocta (Kamptozoa), Ectoprocta (Bryozoa), etc. Even among the Turbellaria themselves the sexual apparatus shows a high degree of variability and there are some recent species of Turbellaria with a very simple genital apparatus. We are therefore fully justified in concluding that the possibilities of negative mutations had existed at the time when the ancestors of Cnidaria first evolved from the old forms of Turbellaria. It should be mentioned in passing that a reduction of the genital apparatus did not take place in individual cases of parasitism (e.g. in Fecampia) that can be observed among the more highly developed Turbellaria, but also in several cases of Ameria whose development from Turbellaria can be considered as more than probable (e.g. Nemertini, Gastrotricha, etc.). Such a reduction took place even while free mobility was 
preserved. It has already been shown that the last traces of the hermaphroditic state can still be found in Anthozoa. It is probable that Cnidaria branched off from their turbellarian ancestors even before the same was done by Nemertinea and by Gastrotricha.

It has already been shown that all the differences that exist between the anthopolyps of the Cnidaria and the Turbellaria can be divided into two groups: the first group includes those changes which had been brought about by the transition to the sessile way of life usually characterized by some kind of reduction, and the second group which, though connected with the sessility, must be interpreted as a result of a progressive evolution (cnidae, tentacles, strong contractility, etc.). All these properties can be found developed earlier than in Cnidaria, even if then only to a lesser degree.

In view of the fact that there is now hardly a single zoologist who could doubt that the Turbellaria represent the starting point in the evolution of many other animal types, it may be possible to accept at least as a possibility that Cnidaria, too, had evolved from some turbellarian ancestors, provided that the anthopolyps are the most primitive among the free and solitary polyps of Cnidaria. There are now few experts in Turbellaria who still support the interpretation which sees in the acoelous Turbellaria as well as in the Turbellaria as a whole, a result of a retrogressive evolution. The most prominent among these scholars is Marcus, a well-known expert in Turbellaria, who goes his own way in his interpretations of the phylogeny of the animal world (E. Marcus, 1958:52, Fig. 1). It would take us too long to investigate his opinions in detail here. He and I speak two different languages, and his line of thought remains unintelligible to me (e.g. when Marcus considers the larvae of Anthozoa to represent a special animal group; or, when he refers, on one hand, to a similarity between the form of cleavage in Ctenophora, and on the other in the ctenostome and cheilostome Ectoprocta, which he prefers to call Bryozoa). Marcus too, it seems, 
considers my interpretation as undiscussable, for he maintains a complete silence about it; it is improbable that my interpretation could have remained unknown to him since he must have read at least my article which appeared in Systematic Zoology.

When we have suceeded in suggesting that it was at least probable that the Cnidaria, with Anthozoa as their first group, had evolved from Turbellaria, we soon meet a new problem, i.e. which subgroup of Turbellaria can be considered as the closest relation of the Cnidaria? In our attempts to solve problems where the theoretical or logical conclusions cannot be avoided (they can also be called speculations) we must be constantly aware of the fact that the animals concerned had long ago ceased to exist, that the information at our disposal covers the recent animals only, that no transitional forms are available, and that nothing is known of a possible existence of some other "side branches" which have now disappeared without leaving any trace. We must ke content with the determination of the deg ees of probability.

Initially we were helped, by the fact that among the recent animal groups we do not find progressive and specialized species only, but also some more primitive forms. The possibility of arriving at a correct solution of such a problem is therefore not quite so small.

Scholars, in their earlier endeavours to make phylogenetic deductions, have tried to go far back and to identify indifferent initial forms or ancestors. The planula hypothesis can be mentioned here as a typical example which is at the same time closely connected with our problem itself. It was concluded, under the influence of the so-called "fundamental biogenetic law" that a polycellular organism had originally existed, similar to a planula of Cnidaria; and that it had evolved from an equally hypothetic gastraea which itself was considered as a possible starting point for the evolution of three groups of Coelenterata: Spongiaria-Cnidaria-Ctenophora. It was the third group only which had formed the basis for further 
progressive evolution in the direction of Coelomata. There are many zoologists who still adhere more or less to this interpretation. In our case we should attempt to derive the Cnidaria from the lowest Turbellaria, thus from Acoela. Yet even a slightly closer comparison shows that this cannot be made. Anthopolyps - taking into consideration the solitary forms only-have a much more highly developed organization than the Acoela, even if there are some recent species of Acoela which already possess a complex genital apparatus.

Among the Turbellaria we must search for forms that had later evolved into Anthozoa among those groups that are already more highly developed than the Acoela. A completely unjustified accusation has been made by Remane (1960) who states that I wish to derive the Cnidaria from the Temnocephala. I have never either mentioned or maintained anything of this kind (cf. Hadži, 1958 a). On the other hand, I see in the Temnocephala with their one-sided specialization, an instructive example, in effect an experiment made by Nature, which shows what can and what must evolve from an animal type when a species that reached a certain level in its organization, abandons the creeping way of life and begins to live as a semisessile animal. The Temnocephala are Turbellaria that are closely related to Rhabdocoela; in all probability they had evolved from the latter. Matjašič even considers that they are still similar enough to the latter so that they can still be classified among Rhabdocoela. The Temnocephala had become specialized as epizoic animals and have therefore ceased to creep by means of their ventral surface covered with cilia; they have assumed an erect position and at the same time they have developed a special disc at their aboral end, by means of which they attach themselves to the substratum; their oral orifice has been transplaced to the anterior end of the animal (now to its upper end). An initially solitary pair of tentacle-like excrescences has been polymerized. The ciliation has been reduced due to the fact that they move actively by means of body muscles, and their skin has become 
protected with a cuticle which serves as a means of defence and support.

In all probability the Temnocephala have preserved their free mobility because of their epizoic way of life, and in this they differ greatly from Cnidaria. The recent researches that have been made by Matjašič (1959) have shown that this free mobility can even be very active. It is due to their free mobility that Temnocephala have also preserved their ability to pair; this is the reason why we can still find in Temnocephala the typical complex turbellarian hermaphroditic genital apparatus, while the same has been lost in Cnidaria which have evolved into purely sessile animals.

I believe that I am not wrong when I conclude, on the basis of well-known facts, that phylogenetically (and also geologically) the Temnocephala must be younger than the Cnidaria. The Temnocephala can still be considered as Tuubellaria; they had branched off from their rhabdocoelan ancestors and have finally developed into a new subtype, into a new order. They are therefore not related to Cnidaria in a direct line; they rather represent a parallel development to the evolution of the latter, a development which can nevertheless prove helpful in our attempts to understand the way the Cnidaria had evolved from their turbellarian ancestors.

The case of Temnocephala can thus serve us as a kind of pattern. In spite of objections made by several zoologists, and above all by Beklemischev and by Reisinger, we will suggest that like a temnocephalian the primitive anthopolyp had raised, first for a briefer period, and later permanently, its posterior end which is now its aboral end. The oral orifice which was originally placed on the ventral side, even if in the anterior part of the body, has been transferred to a purely terminal position when the animal began to be attached to the ground. The Temnocephala had thus repeated the first stage in the older evolution of Cnidaria; yet here they have stopped, or rather they have evolved further in their own direction as epizoic, or let us better say, as ectoparasitic animals. They 
have remained strictly individualized, i.e. they have not adopted asexual reproduction.

There were probably times when the Acoela were the only Turbellaria in existence; at a somewhat later date the Acoela appeared together with the younger (let us call it) eucoelous subtype which had already been equipped with a distinct intestine. There can be no doubt that those recent Turbellaria which stand closest to this younger form are now classified as Rhabdocoela. We will therefore call these hypothetical ancient Turbellaria as "rhabdocoeloid Turbellaria," in order to avoid in this way the accusation that we wish to derive one recent form from other recent forms. We will therefore avoid the problem whether these rhabdocoeloid Turbellaria would already be called Rhabdocoela by an expert in Turbellaria, or whether he would give some other name to them as, for example, Prorhabdocoela; such a problem is not important.

Regardless of whether the Turbellaria have been considered as primarily primitive animals - which is certainly a more probable interpretation-or whether they have been viewed as ar imals with a secondarily simplified organization which is a result of an eventual retrogression, it has nevertheless been usually thought that various other "lower worms" and not only Platyhelminthes had evolved from the older turbellarian forms. A majority of zoologists (let us hope that in the near future they will be followed by the others) consider the Turbellaria to be primarily primitive animals; they can thus easily imagine how ages ago these Turbellaria, together with some Protozoa, were the prevalent animal forms which represent the starting point of the whole subsequent evolution of the eumetazoan animal world. It is therefore quite natural that we also try to derive the Cnidaria from these rhabdocoeloid Turbellaria which have shown such an exceptional ability to evolve. At the same time it has been necessary to dethrone the Cnidaria, with Hydra in the front row, from the position they had in the animal system. The Cnidaria have 
to abandon the first place in the animal system, and thus the starting point of the subsequent progressive evolution; they must yield this place to such unrepresentative worms as the Turbellaria which creep by means of their bellies. The concept which sees in Hydra as well as in all Hydrozoa the most primitive forms of Eumetazoa among all the recent species has been the main obstacle to the reception of our interpretation.

It is quite unimportant what name we give to the supposed turbellarian ancestors of the present Cnidaria; this is even more true since there are still considerable shortcomings in the classification of the recent Turbellaria so that it is constantly necessary to change the classification. It can only be stated with full certainty that the direct ascendants of Cnidaria had already possessed an intestine with an ectodermal gullet and that they had no intestinal diverticula; that instead their intestine had developed a tendency to form longitudinal folds.

\section{The Larvae of Cnidaria}

This may be now the best place to discuss in more detail the formation of larvae in Cnidaria as well as their possible phyletic significance.

According to a generally valid rule, the larval stage is usually developed in the ontogenies of benthonic animals, and above all in animal types that live in the sea, as sessile animals. This larval stage lives regularly in plankton. It is therefore a case of a typical and probably also of the oldest and of the most widely accepted deviation that can be observed in the ontogenetic process. The ecologic significance of this stage is clear and it does not need any further discussion, while at the same time there is even now no unanimity among the zoologists regarding the phyletic significance of larvae. During the flowering of comparative embryology (which should be rather called ontogenetics), and under the fresh 
influence of the Theory of Evolution when everyone was eager to find further proofs of the descendence theory, scholars became convinced that these larvae must represent something more than a transitional stage only in the individual ontogenies. Following the example of Darwin, Müller, and Haeckel, scholars became used to the concept of recapitulations. Yet here they have made a mistake which appears rather insignificant at first sight and which has been afterwards pointed out by several critics, above all by Garstang. On one hand they have confounded the repeated (yet changeable) ontogenetic stages with the adult forms of ancestors, and on the other hand they believed in a recapitulation not only of individual characteristic properties of the ancestral embryos and of larvae but also of whole ontogenies or at least of whole stages as such. These methodological blunders have proved fatal. A large amount of honest and intensive work was made completely useless. Numerous theories and hypotheses were proposed and courageously defended which were built on the basis of the so-called "fundamental biogenetic law." As an example, I wish to mention here the famous trochophore theory as it was formulated by Berthold Hatschek, an ingenious zoologist and my greatly admired teacher. Yet in spite of all this, these theories can still contain some useful elements, they still have some element of truth in them. There can be no doubt that recapitulations take place and that the comparative morphology of larvae can prove as useful and informative for our phylogenetic speculations as is the comparative morphology of embryos; provided that it is taken from the right angle and that the probability of true recapitulations is kept within limits. Mistakes of the old type continue to be made down to the present day as can be seen in the case of Endoprocta (Kamptozoa) that have been united with Ectoprocta into a new group Bryozoa because of certain similarities that can be observed in their larvae.

There have been three main reasons which induced scholars to see in larvae a recapitulation of an ancestral form. 
First, we see that larvae which belong to large, and not infrequently even to very large, animal groups, can have a very similar form and structure. Secondly, the organization of these larvae is almost always simpler (exceptions, however, can be found almost everywhere in the field of biology); larvae are therefore believed to be closer to their supposed ancestors. Thirdly, not a few cases have been known where there can be no doubt that certain characteristic properties can be found in larvae, or in embryos, that are also known to occur in their supposed ancestors; while at the same time these properties can be found no longer, or at least no longer function, in the corresponding recent grown-up forms.

We do not intend to make here a detailed analysis of the problem as to what can be understood as a larval stage. It is certain that the distinction must be made of very different orders of larvae (primary, secondary, possibly even younger), i.e. larvae can be developed from various stages in the development of embryo. This takes place in such a way that any such stage begins to evolve in its own direction, and it adopts its own system of feeding which is usually not parasitic. There are, however, also some parasitic larvae; the problem remains open whether there exist any sessile larvae: and it seems that such larvae can be found among the Echinodermata, the Pentacrinus stage of the Comatula. A necessary transformation by means of which the animal endeavours to reach the organization and the way of life of the adult form is the consequence of such a development. A radical metamorphosis frequently becomes necessary in these cases.

We are finally able, after prolonged and repeated discussions, to make a more uniform evaluation of the morphologic significance of larvae and to use it correctly for phyletic purposes. At all events, it can be stated that similar larvae had been evolved quite independently along several lines of evolution by sessile sea animals. Convergence has played an important role; it was brought about by the adaptation to a planktonic way of life. It can be maintained that one of the 18 
worst mistakes that have been committed in the history of the comparative morphology and of the phylogenetics (and which is, unfortunately enough, still made) has been the attempt to use these changes in the ontogenies as a basis for phylogenetic speculations and simply to transfer these ontogenetic and morphogenetic processes into the phylogeny of the adult forms. This has been done in spite of the numerous cases where it is obviously impossible that an organ or an orifice (e.g. the anal and oral orifices) had originally evolved in the same way as it is now developed in the ontogenies.

I mention here as an example the pilidium of Nemertinea. Who could think even for a moment that the "formation of a worm" as it can be observed in connection with the pilidium could be at the same time a recapitulation of an ancestral form? How can it be certain, or at least probable, that we have a recapitulation when one and the same organ or characteristic property can be found in such widely different forms in the ontogenies of closely related species, or even (as has already been mentioned, see p. 211) in the ontogeny of one and the same species; especially when it occurs in a way which cannot possibly be reconciled with the way of life of the adult ancestral form (e.g. the coalescence of the socalled primitive mouth, and the new formation of an anal or oral orifice in the same place)?

What is then the situation in connection with the larvae of Cnidaria? We can state that no genuine and generally occurring planktonic larva has actually been developed by the Cnidaria (Fig. 46). The Planula which is the most frequent form has usually been interpreted, as well as actinula as the (ontogenetically and phylogenetically) "younger" larva. Besides these there are also some other larval forms, the cerinula, the ratarula, and the siphonula which can be met with within the limits of some smaller groups. In no case can we find among Cnidaria a more highly developed larval type whose subsequent development would be subject to a metamorphosis as this is the case, even if to a small 
degree only, with the larvae of some Polycladida, among the Turbellaria (Goette-Müller's larva).

It is hardly correct to use the word "larva" when we speak about the planula. A planula is in reality only a slightly more accentuated stage of development which is unable to feed
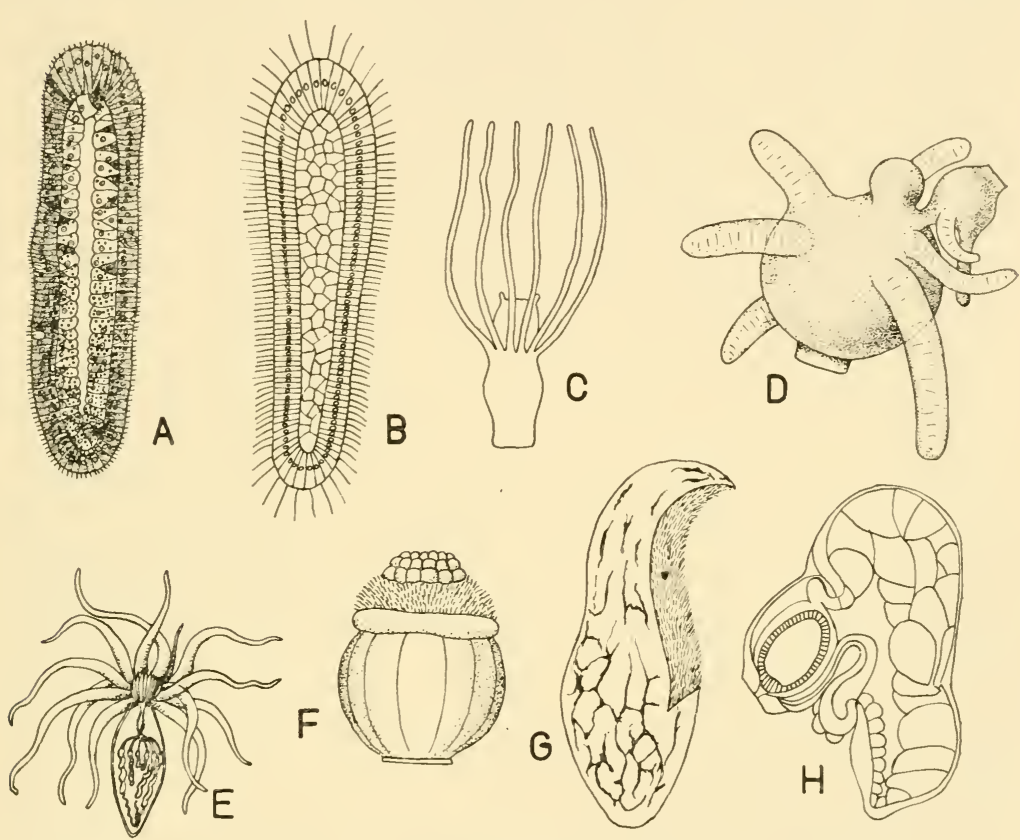

Fig. 46. Some examples of planktonic larvae of the Cnidaria. A, longitudinal section of a planula with gastral cavity (Gonotbyrea sp., after Wulfert). B, a solid planula. C, actinula of Tubularia sp. (after Hyman). D, later actinula of a narcomedusa (Pegantha sp., after Bigelow.) E, cerinula of a ceriantthide larva (Arachnactis albida after Vanhöffen). F-G larvae of unknown zoantharians Zoantbina sp. and Zoanthella sp. (after Senna). G, siphonula, a calycoforean larva (Galeolaria sp.) (after Metschnikoff).

independently and which is therefore not fit to live long in the real plankton. Its function, with a few exceptions, is clearly to settle down not far from the place inhabited by its "parents" and to develop into a new primary polyp. The absence of an oral orifice and of an intestine which can be 
observed in the planula is a primary phenomenon, in comparison with numerous Ectoprocta where the same phenomenon is clearly a result of a secondary development. No metamorphosis is necessary for the further development of the planula. It is well known that in a few aberrant cases the planula does not develop directly into a polyp, but rather into an actinula or into an Anlage of a cormus.

The question finally arises whether this planula could represent a recapitulation of an ancestral state? Our answer must already be negative for reasons of principle alone. Moreover, such a creature is clearly unable to live as an independent organism because it has neither a mouth nor an intestine. We would be obliged, if we tried by all means to see in a typical planula a recapitulated ancestral form, to make further suppositions, and explain above all the absence of a mouth and of an intestine as secondary phenomena. This would finally lead us to the question what had been the reason that such a development had taken place. It should be mentioned in passing that this planula in no way corresponds to a gastrula (nor does it correspond to a gastraea); its contents is not a "pure" entoderm such as is known in a gastrula, but rather a mixture of entoderm and of the mesohyl (mesoderm), and thus an entomesohyl. The planula is therefore not a coelenterate larva.

In the Anthozoa, which we consider to be the most primitive Cnidaria, a trend can be observed which tries to prolong the free life in the plankton of the younger stage of their development. Two main lines of evolution can be identified in this connection, if we disregard a possible third line of evolution where the free stage no longer leaves the benthos, a case that can be observed in some Zoantharia (Rakovec, 1960). In Ceriantharia a real beginning can be observed of the subsequent progressive evolution of the plankton stage which has even induced scholars to give special names to this stage (cerinula, cerianthula, etc.). Unfortunately, little is known of the subsequent development of these already specialized larvae. 
Frequently an "actinula" is developed also by other species of Anthozoa; it represents a more progressive stage in the development towards an anthopolyp whose most important requisites can already be found here during the life in free water (the mouth together with gullet [pharynx], septa, tentacles). Hardly any development can be observed in special larval organs (e.g. in the tuft of sensory cilia that occur at the aboral end of larvae). We have here a prolongation of the plankton phase only, or an early development in the direction of the adult state. Neither can we speak here of a metamorphosis (the primary tentacles are not, as it seems, abandoned).

The occurence of a true actinula is limited to Anthozoa. The form which has been developed by Hydrozoa and which has also been called an actinula is not identical to a genuine actinula and it should be given a different name (perhaps a hydrula). The latter form has neither a gullet nor septa; it has only an oral opening, and intestinal cavity, and few tentacles whose ends are thickened. It seems that larvae similar to an actinula had repeatedly been evolved by Cnidaria, a development which had perhaps even been repeated by Hydrozoa several times (e.g. the actinula of Tubularidae, and the actinula of Narcomedusae).

If we adhere to our interpretation of an evolution of Cnidaria in the direction Anthozoa $\rightarrow$ Hydrozoa we could rightly expect that a progressive evolution of larvae could be observed in Scyphozoa and in Hydrozoa. This, however, is not the case. How can this fact be explained? I believe I have found an acceptable interpretation of this situation in the fact that during the subsequent evolution a completely new generation of individuals that live in plankton had been invented both by Scyphozoa and by Hydrozoa, i.e. medusae in their two "editions." The medusa generation had taken over not only the sexual function but also the "task" of distributing its own species, a development which is especially characteristic in those cases where as a result of a secondary development the species is represented by the medusa genera- 
tion alone. In this way a further general evolution of the larval stage has become completely unnecessary. Ephyrae can occasionally be found mentioned as if they were larvae of scyphozoan medusae; they are in reality their juvenile stages only.

No actinulae have been found among the Scyphozoa; we find instead planulae which, however, have never been observed to reach the real plankton and to live there. Just as we can speak here of a planula larva, we could also speak of an ephyra larva of the metagenetic medusae; the only difference is that the ephyra as a larva of medusae does not develop out of an egg but rather by way of asexual reproduction. In Narcomedusae, on the other hand, we find that a so-called polypoid larva had been developed after the primary polyp generation had been lost; this larva even shows an inclination to a parasitic way of life. It is only superficially similar to an actinula and it must be therefore treated as a formation sui generis.

Among the Siphonophora we find conditions that are somewhat more unusual; they stand in connection with the individualization of the planktonic cormi and resemble slightly those that can be found among the phylactolaematous Ectoprocta. The planula of Siphonophora (e.g. the ratarula of Chondrophora) soon begins to form secondary individuals as well as the impersonal parts of a polymorphous cormus (siphonula). Here there are even provisional individuals (nectocalyces, etc.) which are later abandoned, thus a development which is similar to a metamorphosis. We must not forget that Siphonophora represent the peak in the evolution of Cnidaria!

\section{A Comparison of Cnidaria Larvae with Other Larvae}

A comparison of larval conditions such as they occur in Cnidaria with those that exist in the equally sessile Endoprocta (Kamptozoa), among the Ameria is very illustrative in 
this connection. The Endoprocta are not only an animal group which lives on the sea bottom; they also show a strong inclination to form cormi; they have not, however, developed, a stage which would correspond to a medusa generation and which would be able to live in plankton. Taken geologically, Endoprocta may be younger than Cnidaria because they are euproctous animals. Thus their phylogenetic root had reached a higher level of evolution than the level that has been reached by the aproctous Turbellaria. The Endoprocta, on the other hand, have developed a planktonic larva whose organization is even more highly developed than is the organization of the adult animal form. Moreover, this differentation had pursued its own direction which made it necessary that a radical metamorphosis be introduced. This is a typical example of a special evolution of a larva which leads its own way of life. It would be completely wrong to see a recapitulation in this kind of development, i.e. to conclude on the basis of present conditions that the ancestors of Endoprocta had lived in plankton and that they had the form and the structure of a trochophore. It is even misleading to call the larva of Endoprocta, a trochophore and to compare it in any way with the larva of Ectoprocta in order to justify in this way a systematic union of these two groups, as this was done by Marcus (Hadži, 1956). These are in reality pure convergences only which were caused by very similar ways of life, a fact which can also be observed, for example, in all the sessile animal types.

Since Endoprocta, as euproctous Ameria, are in all probability (as has already been emphasized) phylogenetically younger than Cnidaria, it seems incomprehensible why the older Cnidaria, which are equally sessile animals that form cormi, had not developed a planktonic larva with a higher level of organization. We have already tried to give an answer to this problem, taking into consideration the formation of the medusa generation which had taken over the role of planktonic larvae. A medusa, however, cannot be considered to 
be essentially a larva; it rather represents a special generation, it is an adult individual which belongs to the same species and which can secondarily completely replace the primary polypean individual.

We will add here some observations regarding the conditions that exist among the Turbellaria in order to complete in this way our study of the Cnidaria larvae. It can be stated quite generally that the mobile Turbellaria which live in benthos do not show an inclination to develop larvae. There are only a few and usually bigger species of Polycladida which had evolved their own larvae (the so-called Müller's larva); yet these larvae have not reached a very high level of evolution either in their organization or in the special larval devices. This larva assumes another position, i.e. it hangs in free water with its head turned upwards, and it forms a girdle of body process (usually eight) whose margins are covered with stronger cilia. I cannot agree with Kaestner who tries to connect this margin covered with cilia with the praeoral circlet of cilia that can be found in the trochophore of Annelida, and with the tuft of cilia that occurs on the vortex of larvae. It is much more simple to suppose that larva of Polycladida has phyletically nothing in common with the trochophore (actually we have here quite different larvae which resemble a trochophore!) of Annelida. The circlets of cilia and the terminal tufts of the same are requisites that can be frequently found among the primary planktonic larvae.

Müller's larva represents (this is not true for all the Müller's larvae) in all probability the starting point for a side line of evolution which had finally led to the formation of Ctenophora by way of an early sexual maturity and of a continued life in plankton. Here we can observe a very interesting case of the invention of a larva. The typical Ctenophora, themselves planktonic animals, had no need of a special larva for the distribution of their own species. Let us say that a typical transitional stage only had developed in the ontogenies of Ctenophora; its form and organization resemble closely 
those of an actually existing species (the so-called Cydippestage). Some Ctenophora, however, have become specialized and they have descended to a life in the benthos. It is in connection with these species and subgroups that the earlier Cydippe stage has taken over the role of a true larva which must even go through a metamorphosis during its subsequent development. In this case, we can rightly speak of a recapitulation; yet this is a recapitulation of a transitional ontogenetic stage which is very similar to-even if not exactly the same as, a species in its grown-up state.

It should be mentioned, for comparison, that no larval form had been developed by the Chaetognatha that had also evolved by way of neoteny from the planktonic larvae of Brachiopoda (Hadži, 1959 a, 1959 d). No typical common transitional stage of Chaetognatha has become known either. The possibility cannot be excluded, however, that in future a common ontogenetic transitional stage of Chaetognatha will be found after a detailed study of their ontogenies.

\section{The Origin of Turbellaria}

We will return now to our main problem. We have refused to accept the attempt made by Jägersten who has tried to support the enterocoele theory by means of a supposed initial form which he constructed ad boc and which he called a bilaterogastraea. He equipped this form with all those elements which could prove useful in his subsequent derivation of the Metazoa from it. Our new task will be to show or, rather, to find the most probable place where the origin of Turbellaria, and thus also of all Eumetazoa, has to be sought. I must state at the very beginning that no well-founded reason is known to me which could justify considering Turbellaria as a product of a retrogressive evolution. I share this opinion with the leading experts in Turbellaria and I refer here among others to Otto Steinböck (1958). The same standpoint regarding the Turbellaria 
has also been accepted by a majority of other zoologists who are not experts in this field. It will be therefore sufficient if we mention that Turbellaria are freely moving animals, and that they feed independently, mostly as predatory animals. There is no reason why we should try to derive them from some sessile or parasitic ancestors. It is therefore very surprising to see how Marcus, an expert in Turbellaria, places the Turbellaria in his sketch of the genealogical tree of the animal world (Marcus, 1958:52, Fig. 1) high above the Tentaculata which he locates between the Turbellaria and Cnidaria (Coelenterata $=$ Radiata). Even Chaetognatha appear in this sketch closer to the Coelenterata than to the Platyhelminthes. At the same time Marcus derives both the Platyhelminthes and Nemertinea from the Sipunculoidea. One would expect that Marcus considers Turbellaria to be a progressive type because they are placed (together with other "-helminthes") by him so high on the genealogical tree. I find it completely incomprehensible when Marcus tries to derive all these lower "worms" from the coelomatic Bilateria (Marcus 1958:33) endeavouring to find in them traces of the coelom cavities while at the same time he makes no difference between an actual coelom (our perigastrocoele) and other coelom cavities, above all the gonocoele. Marcus thus believes the Turbellaria to be secondarily simplified animals which have played a subordinate role only in the phyletic development; he even considers Nemertinea to be more primitive than Turbellaria.

Remane (1957), too, does not stand far from the view proposed in Marcus' work. In Remane's sketch of the genealogical tree of the animal world, the Tentaculata can be found placed under the Platyhelminthes whose position is somewhat hesitatingly indicated. Remane defends himself against Steinböck's accusation that he tries to derive Turbellaria from Annelida; at the same time he states that both these groups had a common ancestral form which as an already segmented animal had necessarily been anneloid. It is not important whether complete parapodia and the so-called secondary or somatic segments 
had already been developed. In any case Remane, too, sees in Turbellaria a product of a retrogressive evolution (admitting at the same time that some properties of 'Turbellarians also be a result of a progressive development above all their complex genital apparatus). Remane consequently does not attribute any great phyletic significance to Turbellaria. The Turbellaria must be content to represent a small side twig on the mighty branch of Protostomia.

Let us now pass over to the next problem. What is the situation regarding the line of evolution within the group of Turbellaria itself? Do the Acoela represent the starting point of the evolution of 'Turbellaria, are they not only the simplest but also the most primitive Turbellaria, or perhaps the most highly developed Eucoela? We consider it as certain (the same opinion is shared also by Steinböck, Hanson, Beklemischev, and the majority of other zoologists, beginning with v. Graff) that the Acoela do not only have the simplest structure or organization of all the Turbellaria that we know ; they are also primarily the most primitive Turbellaria. If we disregard here all the problems regarding the origin of Turbellaria, and if we observe Turbellaria as a whole as an independent animal group, we cannot find a reason why we should not place the Acoela at the beginning of a natural system of Turbellaria. The interpretation which sees in a simple phenomenon a primitive element also, is certainly justified here, even if it is not justified in a number of other cases: a fact which I am well aware of and which is repeated unnecessarily by competent zoologists in their criticisms of my thesis. In spite of the fact that the Acoela lead the same way of life as the remaining typical Turbellaria nevertheless their organisation is far behind the others. At the same time a clear trend towards a progressive evolution can be observed in the Acoela. Beklemischev has already shown this trend as particularly evident in their hermaphroditic genital apparatus. We will presuppose all this as a well-known fact and limit ourselves to the statement that in reality there is no justification to consider the Acoela as 
secondarily simplified Turbellaria. The only objection to this that could be mentioned in this connection is the small size of the Acoela. There is a generally valid rule that a general decrease of an animal body, when it passes certain limits, not infrequently leads to an extensive reduction of the animal organization which appears as its simplification. It can lead not only to an abolition of the system of blood vessels and of the respiratory organs (numerous instances in the very small species of the Acarina) but also of the whole feeding and digestive systems (the dwarfed males of numerous Rotatoria). The conditions are clear in these and in many other cases because here the causal relationship between the diminution of the size of the animal and the simplification of the organization can easily be identified. In all these cases organs and systems of organs can be found in certain forms while the same cannot be found in the related forms whose size has been secondarily diminished. In the case of the dwarfed male Rotatoria we find even the female animals that belong to the same form equipped with such organs; these female forms have at the same time also a considerably larger size.

Somewhat less easy to survey are those cases where a larger animal group consists exclusively of very small species, which at the same time also have a simpler structure, this can be observed, for example, in the Rotatoria and in the Tardigrada. A detailed study of these groups, however, shows that there are some facts which prove with great certainty that the size of the species, which belong to these groups, had been secondarily diminished and that a simplification of their organization had taken place as a consequence of such a diminution. One of the elements that can be helpful in this connection is the trend towards a decreased number of cells and their constancy; another element is the loss of ability to regenerate (in Rotatoria the lost parts of cells only are able to regenerate). Thirdly, we can take into consideration a highly developed ecologic specialization, e.g. the adaptation to small recesses in which an animal lives. 
A primary small size of an animal type must be strictly distinguished from a secondary diminution (Hadži, 1956 b). No element which has been mentioned above and which could serve as an indication of a secondary diminution can be found in the Acoela; they must therefore be considered as primarily small animals, and the simplicity of their structure which is everywhere clearly evident as a primitive characteristic. We will repeat here, in order to emphasize this statement the better, that the Acoela are genuine, perfect, freely living animals which are able to make rapid active movements (certainly in the most primitive way, by means of a pulsation of cilia) and which feed as predatory species; they did not develop all these properties de novo but rather had inherited the same-as we will soon try to prove-from some equally small ancestors.

The Acoela, as well as all the Turbellaria, are typical bilaterally symmetric animals. They show no traces of a radial symmetry, unless we agree with Beklemischev who believes he sees an indication of the radial symmetry in the presence of several pairs of longitudinal nerve trunks. This fact, however, is quite unimportant because it did not lead to the development of a general radial symmetry and because the central nervous system of the whole group of Turbellaria had become consolidated in the direction of the bilateral symmetry.

\section{The Origin of the Acoelous Turbellaria}

We have shown that Cnidaria had evolved first as anthopolyps and in all probability from the rhabdocoeloid Turbellaria, and also that the Acoela are the most primitive subgroup of Turbellaria. Our next problem will be the origin of the Acoela, and the question, "how can the origin of the Acoela be explained?" We are well aware of the fact that in an attempt to answer such a question we are on very uncertain ground, that the only solid facts that can be found are in the recent animal types, and that we cannot avoid making con- 
jectures. We must be content with the degrees of probability, and we have no right to declare any attempt to represent or reconstruct another path of evolution as impossible.

We could make our task very easy if we accepted the interpretation that was proposed by v. Graff, and, in a somewhat modernized form, by Beklemischev. According to this interpretation a planuloid being which resembled a gastrula (Grobben) has been the starting point for the, evolution of Turbellaria (and at the same time of Coelenterata and of Eumetazoa generally). Beklemischev (1958:109) modified this widely accepted interpretation stating that the Turbellaria had developed "von einem Zweig der Urcoelenteraten, die durch Neotenie im Planulastadium geschlechtsreif wurden." I consider such a deduction as completely improbable and, moreover, as a purely auxiliary solution. Those who accept this interpretation like to refer to larvae as recapitulations of their adult ancestors. The planula appears in reality among the Cnidaria as a larval form without being at the same time a recapitulation of some ancestral form, as it has been already stated. The latter, however, could not at all be found among the Turbellaria, and especially not among the Acoela where one would expect above all such a recapitulating planula. Yet even if it had been found, this could not be considered as a proof for a planuloid origin of the Acoela, and thus of Turbellaria. Matters being such as they are, we have no real basis of deriving the Acoela from some planuloid ancestors.

G. S. Carter (1954), who agrees with several points of my interpretation, considers that I underrate the facts of ontogeny and that in this I make a mistake. It is true that in my opinion we must place more emphasis in our phylogenetic conclusions and constructions on the conditions of comparative morphology of the adult forms, than on the data that can be obtained by means of a comparative ontogenetics (embryology). I especially insist that we must be more than careful in our evaluations of the freely living larval forms. I support my view not only with the well-founded abrogation 
of the so-called "fundamental biogenetic law" as it was proposed by Haeckel (even if recently an apology was written by Remane (1960) in favour of the same law), but also with facts that can be observed in the ontogenies; these facts show how easily and radically the morphogenetic conditions of the ontogenetic as well as of the embryonic and of postembryonic stages can be changed. Such changes can even take place within one and the same line of evolution (animal group). It should be pointed out that during the development which takes place under the protection of a husk or of the maternal body, thus free of the direct influences of the environment, as well as towards the close of an eventual larval life in another biotope when a metamorphosis has become necessary, morphogenetic processes can frequently be observed which would be impossible during an adult stage which leads an independent life (e.g. the changes of the intestinal openings). Because of these changes, zoologists are now divided into two opposite groups. Unfortunately enough it must be repeated again and again: in our phylogenetic conclusions we must take into consideration the comparative morphology of the adult form (this should above all be considered!) as well as the stages of the ontogenetic development; yet in the latter study we must proceed even more carefully and critically, taking constantly into consideration the peculiarities of the ontogenetic processes.

In our case, i.e. in the study of the origin of Turbellaria we consider that it agrees better with the given facts (we must not forget that this is an opinion only, based on a probable conclusion) when we do not take the easiest road by simply supposing (this, too, is a supposition only, and nothing else) that the most primitive Turbellaria had evolved from some planuloid ancestors. Regardless of the fact that no planuloid forms can be found now among the living animals (this is not decisive because it is possible that such forms had formerly existed and that they have now become extinct) - we cannot consider the so-called Mesozoa to be such animals because they are endoparasites-it is my opinion that no planuloid animal 
type can actually be able to live. I cannot see any likelihood that the supposition that either a planuloid (a "planaea," corresponding to a planula of Cnidaria) or a gastruloid animal (a gastraea, corresponding to a gastrula of numerous animal types) had ever existed, is correct. I therefore do not take into consideration such ancestral forms.

As long as we believe that the Eumetazoa had evolved exclusively as a formation of cormi on the pattern of the early stages of the animal ontogeny, which usually takes place by means of a complete cleavage of the fertilized egg cell, we will hardly be able to find any other solution than to accept the existence of a planuloid form or of a modification of the same. Yet if we succeed in freeing ourselves of this truly fascinating idea we find new possibilities, new probabilities emerging before us. Some older zoologists have already thought along the same lines (Dobell, 1911, and before him Saville Kent, Sedgwick, etc.).

Some zoologists have already called our attention to the extraordinary similarity that exists between the structure of a holotrichous infusorian and of a turbellarian (Fig. 47), at the same time they had no intention of making phylogenetic conclusions on this basis, even if such conclusions have seemed to be tangibly close. We will mention here the detailed comparison only between the Infusoria and the Turbellaria which was made by the well-known Hungarian zoologist V. Gelei, an expert in Infusoria, in Hydra, and in Turbellaria. In this comparison a whole list of striking similarities has come to light which can naturally also be considered as pure parallelisms or as accidental similarities. V. Gelei did not think here of possible homologies because there are also differences between these two animal types that can above all be observed in the extremely specialized nuclear apparatus and in the sexual phase of the Infusoria.

Let us mention here briefly these similarities: (1) the range of the body size; (2) the general form of the body (habitus); (3) the general ciliation of the body surface and the type of 


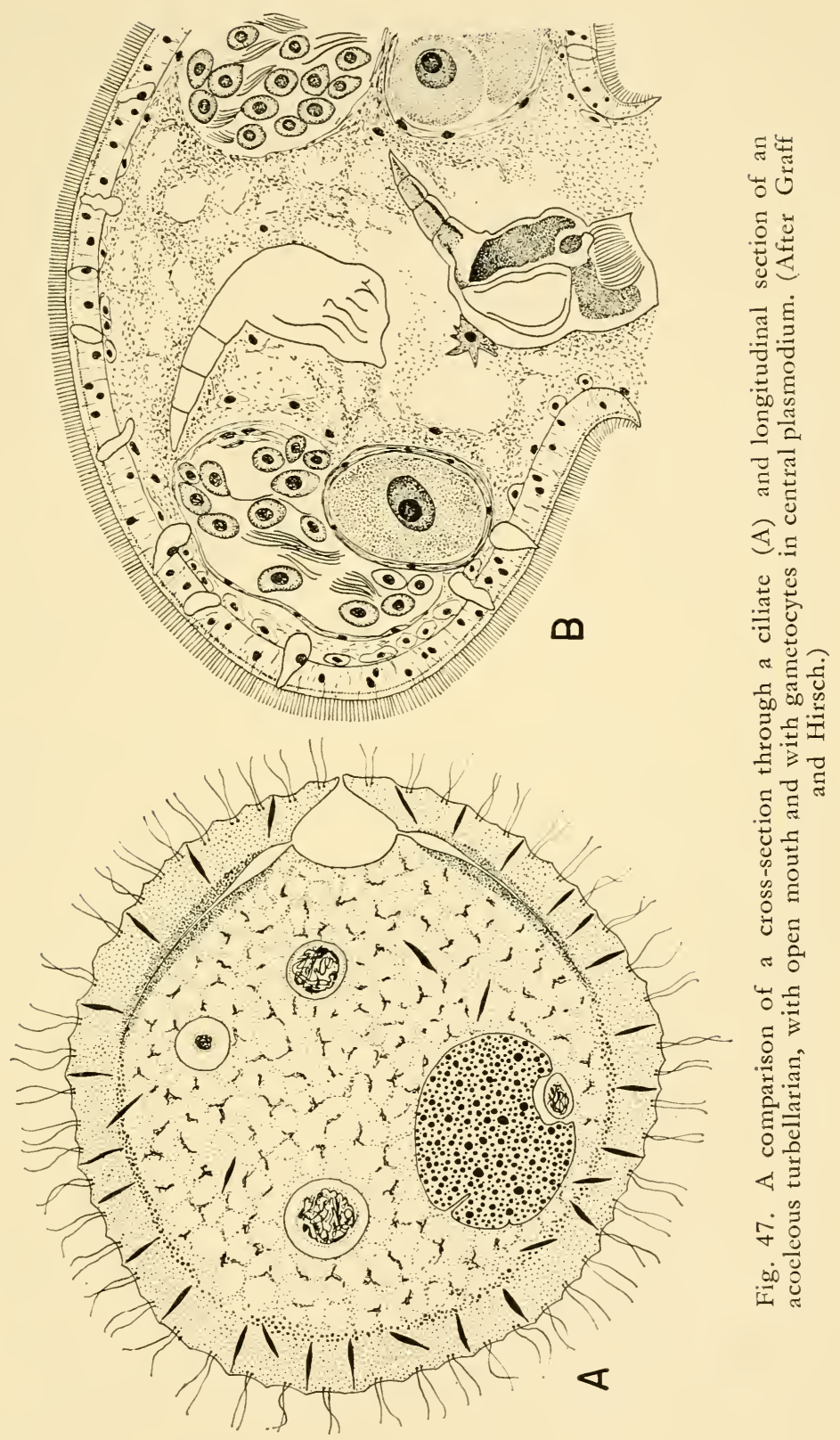


movement which is connected with it; (4) the fact that the interior of their bodies consists of three body layers, with (a) a more or less solidified external layer, with fine variations especially as regards its fibrillar and sculptural nature, (b) a somewhat softer middle layer (without streamings) which contains nuclei, the contractile myonemes, and the contractile emunctories (vacuoles), (c) the streaming digestive internal layer (cyclosis), with the digestive vacuoles; (5) a cytostome; (6) a cytopharynx, and not unfrequently (7) a cytopyge; (8) the trichocysts; (9) a hermaphroditic regime combined with pairing and with an internal fertilization; (10) the asexual reproduction by means of transverse division.

All these, and other similarities which are not mentioned here, should thus be accidental analogies or parallelisms! Can this be probable? The improbability of such a group of analogies becomes even less acceptable if we succeed in clarifying or in rationally solving the most important differences that now seem to exist between these two animal groups.

There is one more important reason why we should consider these similarities as true homologies and why we should accept the derivation of the Acoela, as the lowest Eumetazoa, from some infusorial ancestors as more probable than the derivation of Coelenterata from some colonies of Flagellata. This reason can be found in the continuity of development of the whole polycellular unit which I will return to discuss on a later page. It is quite natural that the similarities that exist between a holotrichous infusorian, as it is now known and a recent acoelan, are explained by those who adhere to the widely accepted colonial theory, as if they were analogies or parallelisms only; they are logically obliged to come to this conclusion. It is easy to understand why these scholars try to bring forward possibly good arguments against the "plasmodium theory" ("Plasmodiumtheorie") as it was called by Steinböck. Thus Remane wished that those scholars who accepted the plasmodium theory could bring forward evidence in the form of "transitional forms," "proved patterns," "certain in- 
termediate forms," "certain special homologies." Steinböck $(1958: 204)$ is right when he emphasizes that Remane is much less strict when he tries to prove his own thesis and that in connection with his thesis he is satisfied mainly with one rather remote analogy of green algae (the frequently misused Volvox) as well as with an (according to our interpretation wrong) application of the misused "fundamental biogenetic law" as it was proposed by Haeckel (cleavage = a recapitulation of the formation of the colony of Flagellata, etc.).

Several scholars have objected in principle against any possibility of deriving the Eumetazoa from some ancestors that resembled the present day Infusoria, stating that the Infusoria are a much too specialized animal group, a blind alley, and that therefore no new animal type could possibly have evolved from them. The fact that they harbour of two nuclei only with a clear dimorphism of the two nuclei, the absence of free gametes, conjugation as an isolated form of the sexual reproduction are all unique properties which can only be a result of a long evolution in their own direction. I must admit the great importance of these differences and that at the first sight these differences really seem to disprove a possible deduction of Eumetazoa from Infusoria. I myself (Hadži, 1944 : 160) was initially under the influence of these facts: I endeavoured to avoid them and tried to find an origin of the Acoela farther back among the Protozoa, i.e. among the polykaryonic Flagellata. This, however, is in principle not so important.

After a thorough analysis of the sexual conditions that can be found both in the Infusoria and in the Acoela and after an intensive comparison of the two (Hadži, 1950) I have finally come to the conclusion that it is possible to overcome these seemingly unbridgeable difficulties, that these differences do not represent a matter of principle, that instead they have only been gradually increased. This has finally led to the widely different forms that can be observed in the extreme cases in the two animal types. In this connection I can also mention the study by Hansen (1958). 
It cannot be emphasized strongly enough that quite naturally the recent Infusoria with their frequently extreme specializations cannot be taken into consideration as ancestors of the equally recent Acoela. The time when the line of Turbellaria had supposedly branched off from its infusorian stem lies far back in geologic time. It is even questionable whether a name like infusorian, or the acoelous turbellarian, could still be given to this common original form, by a modern zoologist. It is quite irrational and beyond understanding to expect or to require that some recent transitional forms should be shown to those who oppose our thesis, as proofs of this development which has been so important for the evolution of the animal world. The phylogeneticists have repeatedly emphasized that such fatal steps in evolution have frequently taken place much faster than did the development of some less important "novelties" and the evolution of large types.

\section{The Relationship Between the Conjugation and the Copulation}

First a problem of terminology. The word "copulation" has unfortunately been used for two entirely different phenomena. On one hand, it is a union of the gametes of the two sexes, or the fertilization itself. On the other hand, it means an act by which the individuals become united which belong to the two sexes, i.e. a pairing, and this is the reason why we speak quite generally of organs of copulation: they play the main role in such a union. The etymology of the word itself shows that it was originally coined for the second interpretation. It would therefore be better if we use the equally well-known word syngamy when we speak of the union of the two gametes.

It has already been mentioned that conjugation is considered as an opposite to syngamy as if it were something entirely different from the latter. This has been the main reason why the Ciliata - as the only Protozoa that have adopted con- 
jugation-all the remaining Protozoa reproduce by means of syngamy-have been considered as basically different from other animals and that a derivation of Eumetazoa, where syngamy regularly occurs, from Ciliata was looked upon as an unacceptable principle. In various manuals and text books conjugation is usually defined in the same way as it appears in the book by Hyman (I:75). " ... conjugation or temporary contact of two protozoans with nuclear change." Almost the same words are used by Kudo $(1946: 154)$ in his Protozoology, and by numerous other authors when they characterize this phenomenon. The emphasis in this sentence is on the "nuclear change." A little later Hyman (I: 173) gives quite a rational interpretation of this "nuclear change" when she writes, "The micronuclei, which play the important role in conjugation all divide twice, and these divisions probably correspond to the maturation divisions of metazoan germ cells, involving a reduction of chromosomes." Even better is the statement made by Hall (1953: 80) who writes, "Typical conjungation involves the change of haploid pronuclei (gametic nuclei) between two paired organisms, the formation of a syncaryon in each, and then nuclear reorganization."

I will now endeavour to show (Hadži, 1950) of this conjugation, a true peculiarity of Ciliata, that its individual elements had been inherited by Eumetazoa via Turbellaria, Acoela. Conjugation does not represent an obstacle to such a derivation, provided that we place the point of separation of Ciliata and Turbellaria, and thus of Eumetazoa, farther back to a time before the Ciliata have become specialized and before they have evolved the conjugation which is now typical of them. Numerous peculiarities of sexual conditions that can be observed in the lowest Eumetazoa, in the Acoela, can be better understood if viewed from our standpoint.

There certainly was a time when, sexuality had not yet been developed by the living organism-only the Protista existed. This sexuality, however, had already appeared when the Protista still wielded an unlimited authority. Why and how 
this has taken place cannot be discussed in the present study. This bisexuality has proved to be of great advantage to organisms in their further development and so it was quite generally accepted. Later it was even combined with propagation and thus with the preservation of the continuity, a function which it did not originally possess. The sexual phase of the life-cycle had been developed by Protozoa along different lines and in very different ways. Very primitive conditions can be found preserved even now in various species of Protista. In these the sexual phase is represented by a special sexual generation which however does not differ morphologically from the usual, the so-called vegetative generation (Dogiel prefers to call it a "neutral" generation). This most primitive type of syngamy has been called, as is well known, a hologamous isogamy. The sexualized generation differs from the vegetative generation in its chemical properties only-sexuality itself was originally a biochemical phenomenon. We are hardly justified to speak of gametes during this primitive stage of the sexuality. The two units are naturally attracted because of the biochemical differentiation into two opposite directions which cover each other in the 0 -point (there is, according to the wellfounded theory by Hartmann, no absolutely + and no absolutely - developed sexuality). This finally leads to a union of the two. This was the basis from which special conditions had evolved parallel to the various lines of evolution of Protozoa (we will omit here the Protophyta). The development went mainly into two directions. On one hand it led to a strong differentation of the two partners in the sexual generation. Two gametes had evolved that differ from each other, in agreement with the function they have: the slender, actively swimming androgametes have also been called microgametes (usually in the form of spermatozoa, resembling flagellates; secondarily, however, they can become atypical and they can lose their free mobility), and the passive gynogametes (macrogametes) which are usually equipped with a reserve food substance. The gametes no longer feed independently; they have 
ceased to be a special generation, and thus complete individuals. They preserve the monokaryonic state, because it is during this state that syngamy, with the act of fertilization can be accomplished most easily. This state has been preserved throughout a mainly progressive evolution, right up to man. This is the reason why this primitive state (the monokaryonic state, i.e. the monocellularity) can be found recapitulated even now at the beginning of the ontogenies. The second line of evolution led (as early as in Protozoa!) to a transference of the biochemical sexualization to the "vegetative" generation which we call the generation of gamonts and which had preceded the earlier generation of gametes; this transference took place in a somewhat different way. This is the generation (later when asexual reproduction is abandoned, it is the only existing generation) which produces gametes. Approximately half of its individuals have developed into gynogamonts (the later females), and another half into the androgamonts (the subsequent males). Similarly as in the case of the primitive gametes, no externally visible morphological differences had been initially developed by the primitive gamonts of the two sexes. Yet they attract and repel each other depending on the fact whether the two gamonts that meet have the same or the opposite values.

As early as in Protozoa the formation of gametes (combined with meiosis) and their differentiation, reaches its climax in oögamy; this was accepted by Metazoa as the only form known to them and it has been preserved throughout phyletic evolution, i.e. up to the formation of man. Sexualization can go even further; as early as in Protozoa it was transferred to the earlier vegetative generation (as the so-called agametes). This has led to the sexualization of the whole life-cycle (e.g. in Cyclospora caryolitica, according to Schaudinn).

It may be useful to point in this connection to the conditions that can be observed in the Gregarinida. Two individuals of the generation of gamonts with the same form, yet sexually (biochemically) differentiated, become united; we can truly 
say that they copulate or pair without developing an intimate contact; they each mutually encyst the other. The gynogametes are produced after multiple divisions by one individual which functions as a female, and androgametes by the other individual which functions as a male. They become "free" even if they remain within the wall of the cyst and are in this way separated from the environment. Here the gametes can meet in masses to copulate and to produce zygotes. Yet in this way the whole mass of the cytoplasm of the gamonts is not used up. A considerable part of it remains as a remnant which is destined to die. The zygotes naturally become finally free. It should also be mentioned that in the case of a typical oögamy, one gynogamete only can be developed by way of meiosis by a gamont or by a gametocyte which can be produced by a gamont (in this case the gamont actually becomes a praegamont), while the number of androgametes can be very large.

The hermaphrodite state emerges at the first sight quite unexpectedly among such animals that had originally adopted the system of two different sexes. It appears, it seems, without a true motivation. This phenomenon, however, can be more easily understood if the generally valid and well-founded theory of a potential bisexuality is taken into consideration (Hartmann et al.). Less easily understood are the immediate reasons which can lead in individual cases to an introduction or to an omission of the hermaphrodite state. It is well known that in each individual which is normally gonochoristic (dioecious) the sexual biochemistry of one sex only is prevalent, while the biochemistry of the opposite sex remains inactive. An initially indifferent gametocyte can become divided by way of heterocyclic (not equivalent) divisions, and under special circumstances it can thus develop into units with two sexually opposed orientations. Such a development must be supposed to have taken place during the transition from the polykaryonic Zooflagellata to the primitive Ciliata. One of the great advantages of the polykaryonic theory, if compared with the colonial theory, an advantage which must not be under- 
rated, is that it makes possible for us to understand the hermaphroditism not only of the Platyhelminthes, as the lowest Eumetazoa, but also of the numerous groups of Ameria (especially of the Mollusca), and of other larger or smaller groups which have reached a higher level of evolution. It can be stated quite generally that almost everywhere among the Eumetazoa an active inclination towards hermaphroditism can be observed.

Even if among the recent Protozoa no transitional forms can be found from the polymastigous and polykaryonic Zooflagellata and the lower Euciliata, there can still be no doubt that the Infusoria had evolved from just such Flagellata. The circumstances have been the reason that the endoparasitic poly- and hyper-mastigous species of Flagellata, which are partly equipped with the polymerized nuclei, have only been preserved till now, or that such species only have become known. They are certainly strongly specialized, and thus greatly modified. The Opalinidae have been considered, wrongly, to be transitional forms; they were therefore placed under the name "Protociliata" at the root of the Ciliata. The Opalinidae are in reality strongly modified Zooflagellata and they lead, moreover, an endoparasitic way of life. In spite of this we find the Opalinidae to be very interesting here; first, kecause they include species that are mutually closely related which contain two, it seems equivalent, nuclei only; the completely round body is covered with cilia; and, secondly, because some of their species contain an increasing number of nuclei, yet their sexual phase is in spite of all this only slightly atypical (Wessenberg, 1961)*. They form freely moving and externally slightly differentiated (in their size) gametes which belong to both sexes and which move by means of cilia. It is certain that the syngamy takes place in the Opalinidae. Untortunately,

* Wessenberg who has recently (1961) made a study of the Opalinidae proposes a special taxon for this group under the name Opalinata which should be placed between Flagellata ("flagellated Protozoa") and Ciliata ("ciliated Protozoa"). 
little is known about the sexual phase of other polykaryonic Flagellata. It was even thought that they have completely lost their sexual phase, a supposition which has now been proved wrong.

It could perhaps be expected that some transitional forms might be found among the Euciliata, a possibility which cannot be completely excluded. Thus the case of the Glaucoma (Dallasia) frontata Stokes was described some time ago by G. N. Calkins and by R. Bowling (1941). It has been reported that a copulation as well as a typical conjugation can be observed in this species of holotrichous Infusoria. Yet the very fact that the copulation and a simultaneous conjugation have been observed makes it improbable that we really have a copulation here. It is supposed that here two individuals which consist of two nuclei each, encyst with each other which finally leads to copulation. The true gametes, however, consist of one nucleus only and they must pass through one or two meioses. I believe that the case of the Glaucoma frontata can be set aside.

The Zooflagellata (with strongly polymerized and equivalent kinetids and nuclei which were gonochoristic and reproduced asexually, yet which propagated during the sexual phase by way of oögamy producing greatly unequal free gametes that copulated mutually, creating one zygote each time) can be imagined as the initial form for the evolution of the typical Euciliata as well as of the primitive Eumetazoa. These gametes were haploid, and the zygote as well as the vegetative generation diploid. It should be mentioned in passing that in this they differed greatly from Volvox which is frequently mentioned as a pattern of the ancestor of Metazoa: the zygote of the Volvox undergoes a reduction, a fact which was particularly stressed by E. D. Hanson (1958:27).

There were in all probability two processes which played a major role during the transition from the polymastigous and polykaryonic Zooflagellata that had thus been developed by way of polymerization: (1) the emergence of the hermaphroditism, and, (2) the internalization of gametogenesis. The inter- 
mediate form, which was equipped with both these new properties, developed in all probability at the point where the two lines of evolution separated. Evolution then pursued two diverging directions, on one hand the line of evolution of Ciliata, and on the other the line of evolution of the Eumetazoa, with the acoelous Turbellaria at its very beginning.

Let us first make a closer study of the lines which were pursued by the Ciliata in their evolution. The following characteristics were decisive in connection with their lines of evolution: (1) the prevalence of oligomerization; it did not influence the vegetative (somatic) nuclei only whose number has thus been reduced to the possible minimum, i.e. to the socalled macronucleus which cannot be considered in spite of its singleness as a homologue to the originally single nucleus; oligomerization has now also been extended to the internal sexual phase and as its consequence we find one sexual karyon preserved. This development has led to (2) a further internalization of the gametes themselves so that finally one andro-and one gyno-gamete only are formed; copulation also becomes internal. Somewhat later, oligomerization was extended to ciliation; this has led, according to the rule, to a differentiation of the cilia into the ciliary organelles. This evolution had also led to a new form of copulation which is externally invisible because of its internalization and which is therefore better protected against the external influence. At the same time, it had determined the destiny of Ciliata since they could never develop into larger species; they have remained microscopic Protozoa with the ability to develop intensive differentiations, particularly if we take into consideration the range of dimensions within which they can change. A part of these properties (which was not really a very small part!) had been inherited by the Eumetazoa, because even before the separation of the two lines of evolution a comparatively high level of organization had already been reached within the frame of their small dimensions.

It is actually not very important to know in connection with our task, the evolution of Infusoria after their 
separation from the line pursued by the Eumetazoa. Still it may prove useful if we make a closer study of their sexual phase; in this way we could make it clear that the form of conjugation of the sexual phase is not essentially different from a typical copulation. This will finally enable us to propose homologies of the two forms without any greater difficulty. We suppose quite naturally that the descriptive part of the process is well enough known. At the end we intend to touch very briefly on a few final stages of the process of conjugation which as extreme cases, diverge so far from oögamy as the typical sexual form, that a proposed homology might appear less probable if we do not take into consideration the whole development.

We will first characterize (define) the Ciliata (Infusoria) as they appear if viewed from our standpoint. This definition will agree, I believe, better with the actual facts than do the currently accepted definitions. Infusoria are hermaphroditic, polykaryonic Protozoa whose two karyons are already differentiated during the vegetative phase, without free gametes during the sexual phase; the generation of gametes is reduced to a possible minimum; it is at the same time also transferred into the interior of the generation of gamonts (praegamonts) which has made a pairing of the equally sexualized gamonts necessary. The so-called micronucleus represents during the vegetative phase a homologue to the sexual potency of the hermaphroditic primitive sex cell; the micronucleus is equally divided during the vegetative reproduction by means of transverse division; during the sexual phase, however, at a certain moment it divides heterocyclically (unequally) which leads to the formation of gametic karyons of the two sexes, the so-called pronuclei, i.e. the stationary and the migrating nuclei. The gynogamete with its cytoplasm merges totally with the cytoplasm (the plasmodium) of the gamont (the paternal animal) which leads to an internalization of the zygote. The androgamete remains moving actively and it can secondarily readopt the form of a sperm cell. A new set is developed 
by way of postgamous divisions which are again heterocyclic; their result is usually a vegetative, complex, or polyploid karyon (macronucleus), and a primitive, sexual, diploid karyon (micronucleus).

Scholars such as, Jennings, (1939) have tried to interpret the Infusoria (Paramecium) as gonochoristic animals. According to such an interpretation, the two so-called pronuclei (actually the gamete karyons) which frequently do not differ externally from each other, are supposed to belong to the same sex, they are supposed to be both androgametes or gynogametes. If this were true, then only two individuals which belong to the opposite sexes would be able to conjugate. Such a case, though theoretically not impossible, is completely improbable. In numerous hermaphroditic species of Eumetazoa with a uniform hermaphroditic gonad (e.g. in the Roman snail Helix pomatius, as well as in some Protozoa, e.g. in the Heliozoa) we can observe that the sexual cells of the two sexes can be developed by way of a heterocyclic division from an "indifferent" primitive sex cell. This can lead in special cases, e.g. in numerous Euciliata that have adopted a sessile way of life, that-under the influence of a strongly developed sexual dimorphism of praegamonts - the microgamont which becomes free begins to function as a male, and the sessile macrogamont as a female; yet in spite of this we can observe in both of them a clearly hermaphroditic nature.

J. P. Turner (1941, in Protozoa in Biol. Research: 617) expressed his opinion that conjugation cannot be considered as a propagation or a reproduction, but rather as a reorganization only. I believe this interpretation to be wrong, it agreeing only with the external superficial appearances. It has already been stressed that conjugation completely corresponds to a propagation or reproduction, combined with the sexual act; the only difference is that it is rather modified and somewhat concealed. Not even the usual consequence of the fertilization which takes place during conjugation is missing; it is manifest in Protozoa with free and numerous agametes in the postgamous divisions 
of zygotes which occur frequently en masse. The postgamous divisions of the still enclosed zygote (the zygote karyon) are strongly reduced in the conjugating Ciliata because of their general oligomerization; yet there still occur regularly, special divisions of the zygote karyon which succeed each other quickly and which are also followed by the divisions of the whole body. Numerous experiments have shown that the exconjugants divide more rapidly. This shows that during the conjugation the animals also become reorganized (or, as we frequently hear, rejuvenated). An essential difference between the genuine Ciliata and all the Eumetazoa lies in the fact that in the latter each zygote is regularly developed by way of divisions into a new polykaryonic individual which finally leads to an embryonic development. It is worth mentioning that much later this situation finally evolves in numerous animal groups, including man himself, into the phenomenon where simultaneously, or even generally, one gynogamete only is developed while the number of androgametes remains very large, a situation which can be easily understood if we take into consideration the function of the spermatozoa.

Other facts that have been observed and described under various names in connection with the sexual karyons of Infusoria, such as endomixis, hemimixis, reorganization, etc., can not only be compared with the similar developments in the "normal" Protozoa if we view the organization of Infusoria from our point of view; they can even be declared as simply homologous to self-fertilization and to parthenogenesis. A detailed study of these problems, however, would pass the limits of our present task.

A sexualization of the generation of gamonts takes place also elsewhere among the Protozoa as this can be seen in the case of Eugregarinida where the syzygy can be observed; from the Protozoa it was clearly transferred to the Eumetazoa. The question arises whether something similar could not also be found among the Acoela? As far as I know no sexualization of the vegetative phase has yet been observed in the Acoela. 
This, however, does not mean that it does not actually occur since we are still far from knowing much about the biology of the Acoela. No extensive and planned experiments in their culture have been made so far. Many more precise investigations have been made in connection with the Infusoria. In these we can find a clearly developed progressive evolution towards a sexualization which has led to an explicit sexual dimorphism. During the early stage of this development the individuals do not show any morphological difference whatever, in spite of the fact that they are already physiologically differentiated. Here we can find an analogy to hologamous isogamy. The behaviour of individuals with different biochemical polarizations is similar to the behaviour of males and females of the gonochoristic or dioecious Eumetazoa. The individuals with the opposite "signs" are mutually attracted, and this leads to a union, to a pairing. In the actual sexual generation, in the gametes, the differentiation can take place in two directions only (bipolar: + and - ), if we disregard the indifferent middle, the 0 -point (e.g. the worker bees, and other similar cases), so that two sexes only can be developed. In the generation of gamonts of Euciliata, however, development has led, as it is well known, to the formation of several "mating types." In the species of Infusoria we could correspondingly expect several morphologically different forms which would answer the several "mating types." It is not known to me whether anything of this kind has been observed till now. Yet we find in the Infusoria, above all in their sessile species, sexual dimorphism further developed, in spite of their hermaphroditic state. On a lower level the difference is mainly of a quantitative nature, as is shown by the frequently quoted example of the Opistbotricbium janus. We speak in this connection of macro- and of micro-conjugants which are developed by means of a division that takes place before the conjugation. This division, however, should not be called-as it usually is - a progamous division; the true progamous division takes place during the conjugation in the gametic karyons 
and it is unequal. In this species the essential difference between the two sexes is not morphological, but rather physiological (biochemical). While its true microconjugants (any macroconjugants) are still able to conjugate mutually, the microconjugants when they meet are unable to do the same.

Sexual differentiation has pursued another direction in the species Metopus sigmoides (according to Noland, 1927). The two sexes do not differ morphologically, yet their destinies are different. One of the conjugants loses its individuality due to conjugation, and it is destroyed. Both these elements, the sexual dimorphism and the unequal destiny of the two conjugants can also be observed in the sessile Peritricha. The macroconjugant remains sessile; it functions mainly as a female even if it shows hermaphroditic inclinations because it is only its gynokaryon which becomes fertilized and which continues to develop. Its androgamete passes over into the free and swimming microconjugant which has taken over the role of a male; it produces one functioning androgamete only while all the rest get lost and they are resorbed by the luckier macroconjugant. This situation reminds us to some extent of some fish species that inhabit the deep sea whose dwarfed males live as parasites on their larger females. Two important differences must naturally not be forgotten in this connection: these fish are gonochoristic animals and their males are not resorbed.

Instead of speaking about the macro- and micro-conjugants we could also speak of the gyno- and andro-conjugants, parallel to the females and males of the gonochoristic Eumetazoa; yet these conjugants of Infusoria are hermaphrodites. The process of conjugation (as a secondary sexual process) becomes so one-sided that during the same process the androconjugant loses his life. This, however, cannot be observed among the Eumetazoa, but we can also mention the Rotatoria whose males can become strongly degenerated, or rather they can evolve one-sidedly and in this way retrogress so that finally they do not even appear! The females, however, which 
have become in this way their only representatives, do not develop into true hermaphrodites; they reproduce parthenogenetically having become unable to reproduce by means of division or of budding.

The consequences of the total internalization of gynogametes (which are changed into karyons) - which forms the main difference between the genuine Infusoria and the Eumetazoahave been radical and remarkable. We must therefore call special attention to them. They had clearly evolved only after the lines of evolution of the Infusoria and of the Eumetazoa had become separated. In opposition to the conditions that can be observed in the Eumetazoa where not only the androgametes but also the gynogametes as such or even mature embryos which have developed into young forms leave the maternal body, we find in the Infusoria that their gynogametes remain in the maternal body, their cytoplasms merge with the cytoplasm of the maternal body without any distinct line left between the two. This phenomenon can be observed in the Infusoria only. The zygote is preserved even after completed insemination and fertilization, and the Anlagen of the prospective infantile individual which had developed by way of divisions, are now merged in the maternal body by means of the cytoplasm; in this way the maternal organism is not only rejuvenated (reorganized), it becomes almost eternal; the maternal form does not die, it continues its existence even if it suffers a partial death due to the loss of its macronucleus. The final result is a new complex animal which consists of the maternal and infantile parts that constitute its whole nuclear apparatus as well as parts of its cytoplasm. Thus it is not the maternal organism which continues to exist here as a whole; we rather have, in effect, a double being, while at the same time there is also no real corpse. These unusual conditions have become possible only in combination with an extreme reduction of karyons.

The evolution of the Eumetazoa has pursued an entirely different direction. The gametes of the two sexes have remained 
free; the polykaryonic state has also been preserved. The rejuvenating sexual process, a warrant of continuity, has remaired connected with the gametes. The materral bodyinasmuch as it is not saved by an asexual reproduction-is exposed to an unavoidable ageing which ends every time with the death of the individual. Yet this has also made possible, as it has been frequently emphasized, the progressive evolution of the transitory sexual phase which has finally led to the formation of important and extremely differentiated species.

There are still other paths that were pursued in the evolution of complex species, i.e. such that lead by way of numerous biological units (in this case of genuine, primary cells), and thus of polycellular beings (Spongiae and frequently also Metaphyta). In many respects they have begun to resemble Eumetazoa, especially as regards their sexual phase and the ontogeny, a fact which we will later return to discuss in more detail. Still these forms have remained far behind the Eumetazoa, a fact that can be observed above all in the level which has been reached in their organizations, and in the uniformity, or in the wholeness, of their individuals.

The instance of the Ophryoscolecida to which Dogiel has called our attention shows that the nuclei of Infusoria had in reality earlier been independent cells, and in certain cases even true gametes. They had adapted themselves to a life in the stomach of the Ruminantia; they have thus become strongly specialized and they can in no way be considered as primitive forms. These remarkable minute animals have developed special armour-like formations which cover the whole surface of their body, with the exception of its terminal oral area and which protects the animal against the effects of gastric juices. The "bridging" during conjugation by way of cytoplasm has thus become impossible, while at the same time conjugation itself has been preserved. The contact of the two conjugants has been modified; the two individuals, which are usually dimorphous, touch each other with their terminal ends which are not covered with the armour- 
like layer. An intermediate space is developed in this way which is surrounded by the terminal ends and which is filled with a fluid. The androgametes (the migrating nuclei) of the two individuals must thus leave their maternal bodies and cross this intermediate space. Not only does a cellularization of these karyons take place, during which the cytoplasm which surrounds a nucleus becomes separated from the common cytoplasm of the conjugant, but also this cytoplasm develops into a spermatozoa which is similar to a flagellate. Thus equipped the androgamete which is now free (it deserves fully this term), crosses by swimming the intermediate space and penetrates actively into the cytoplasm of the partner in order to reach in this way the gynogamete which has kept the form of a karyon.

This androgamete of Entodiniomorpha is not a typical spermatozoan, its cytoplasmatic process which is similar to a flagellum is supposed to be developed at the anterior end of the cell and it does not correspond cytologically to the flagellum of a spermatozoan; yet in spite of this - and contrary to the interpretation proposed by Jägersten - it is an androgamete and it is a homologue to a spermatozoan. Nor can we suppose that it represents a line of evolution which had prepared the way to the formation of a spermatozoan. It is much more probable that we see in this special form of androgametes a new formation which has never led-and which will never lead - to the formation of a typical spermatozoan. According to Dollo's rule of irreversibility, the androgametes of Entodiniomorpha have not been able to recover the characteristic properties of the typical spermatozoa which they had previously lost; their androgametes have now been developed in a new way. In this way it becomes clear that I do not derive the spermatozoa of Eumetazoa from such androgametes that can be found secondarily developed in Ophryoscolecidae: in fact I derive the Eumetazoa from such infusorial ancestors that possessed free and typical androgametes. It is therefore unjust when Jägersten accuses me of $20 *$ 
treating the spermatozoa of the Eumetazoa in a similar way to the spermatozoa of these aberrant species, and when he states, "This is a pure conjecture with no basis in facts, but necessary for Hadži's view" (Jägersten, 1959 : 93).

I refuse to accept this accusation because it is completely unjust; at the same time I wish to emphasize that even if there were no case of Entodiniomorpha, my interpretation would still appear as more probable than the interpretation which was proposed by Jägersten, especially in those cases where he refers to the excellent research that was done by Ake Franzén (1956). The form, the structure of the spermatozoa, and the spermatiogenesis are so widely different not only when we compare different animal groups but also within the frame of one and the same systematic unit; we are therefore completely unable to develop on this basis a clear idea of the phylogeny of animals and we can also oppose on this basis a derivation of the Eumetazoa from some infusorial ancestors. It should suffice if we quote here the statement made by Franzén $(1956: 467)$ in the summary to his work; here he states that the spermatozoa of a "primitive type" can be found in such numerous and widely different animal groups as are, "Cnidaria, Plathelminthes (only Xenothurbella bocki.), Nemertini, Aschelminthes (only Priapuloidea), Annelida, Echiuroidea, Sipunculoidea, Mollusca, Tentaculata (only Brachiopoda), Echinodermata, Enteropneusta, Tunicata, and Acrania."

It should not be taken too tragically that the spermatozoa of the Acoela do not belong to this primitive type. In the Acoela the spermatozoa had been able further to develop equally one-sidedly as their organs of copulation. In these and in their hermaphroditism they differ from the Nemertinea which have also reached a low level of evolution. The internal insemination and fertilization, which we believe that the Turbellaria had inherited from their infusorial ancestors, has been preserved in the side line of Platyhelminthes only (Trematoda, Cestoda). In numerous other side-lines of evolution, however, 
as well as in the main line that evolution had taken and which had finally led to the formation of the next higher phylum of Polymeria, we find that the separation of the two sexes had emerged, pairing had been abandoned, and an external fertilization had been introduced. No generally valid regularities or uniformities can be observed in this development. In the Mollusca, for example, we can find at least occasionally a very strong hermaphroditism reintroduced (species of snails with a uniform hermaphroditic gonad) which appears in combination with pairing, with the new formation of organs of copulation, and with an internal insemination and fertilization.

The example of Ophryoscolecidae with their individualized androgametes is not an isolated case among the higher Infusoria. The androgametes of the hypotrichous infusorian Euplotes patella which has a thickened pellicle assume, according to Turner (1930), during their migrations, the form of small amoebae and move actively by means of lobopodia. This is clearly a secondary phenomenon. This amoeboid androgamete of Euplotes is not a modified spermatozoon, as this is the case, for example, in Nematoidea or in numerous crayfishes; it is rather a new formation which had evolved from a state of a secondary karyon: in Euplotes no plasmatic bridge can be secondarily developed any longer; the "degenerated" (retrograded) karyonic androgamete has thus become reactivated, yet this time in a special way.

The early separation of somatic (vegetative) and sexual karyons is characteristic of the genuine, higher Infusoria from which we do not derive the Acoela. The two gametic karyons which bear the two opposite signs are developed by way of a heterocyclic division. We find also the macroand micronucleus developed from a zygote karyon by way of an unequal division. This represents a very advanced specialization, and it has naturally been inherited as such by the Acoela. It must be assumed that the macronucleus is all powerful in spite of its limited function because it receives the complete 
number of chromosomes which are, moreover, frequently strongly polyploidal; yet a part of them, has become inactive. The experiment which was made by Horváth (1947) has shown that this is really so. This Hungarian zoologist suceeded in separating the micronucleus by means of ultraviolet rays. The animals remained alive. Parts of the old macronucleus which is normally lost, developed during the conjugation into a new micronucleus. I wish to mention in this connection that in the Acoela no early separation of the Anlage of a gonad takes place as far as we know about the early ontogeny of the Acoela. This is also true for other Turbellaria. We must consider it as a result of a secondary development if we find in other groups of Eumetazoa, e.g. in Nematoidea, an early developed "germ path." It seems to be very probable that in the primitive Infusoria - which alone can be taken into consideration as ancestors of the Acoela and which still have gametes that become free-no early separation of the somatic and generative karyons had taken place, a situation that can still be observed in the Opalinidae.

\section{The Point of Separation in the Evolution of Infusoria and Eumetazoa}

This deviation from the straight line of argument which investigates the origin of the Acoela, and thus of all the Eumetazoa from their infusorian ancestors, has led us to the more highly developed Infusoria whose complexity has been the main reason why it is so difficult to get the right interpretation of acoela evolution. It has been necessary to make this detour because with it we are enabled to determine with the greatest probability, the point where the two diverging lines of evolution had separated. I myself wavered initially on how to solve this question. I was inclined to believe-in view of the numerous peculiarities that are limited to the Infusoria only-that the Acoela 
had separated from the line of evolution that had been pursued by Infusoria even before the true Infusoria had developed, i.e. that the separation had taken place on the level of polykaryonic and polymastigous Flagellata (Hadži, 1944:160). I thought that this stage of the phylogenetic development should replace the planula as proposed by v. Graff. Later (Hadži, 1953 : 149) I became convinced that we must place this important point somewhat higher, and so I spoke of the "infusorial-like ancestors" of the Acoela. Due to a recent restudy of the whole problem I have now come to the conclusion-I will soon return to this point-that the separation of these two lines of evolution had taken place on the level of very primitive Infusoria which on the other hand had already ceased to be genuine typical Flagellata. These very primitive Infusoria had later developed on one hand into the typical Infusoria, and on the other into the Eumetazoa. It is much more probable that the definitive and total incorporation and internalization of gametes had taken place after the line of Eumetazoa had already separated from the line Infusoria. In the latter the development had led to conjugation as it is now known. This means that in the Eumetazoa the gametocytes have never lost their independence and that they have never united their own cytoplasm with the cytoplasm of the progamont. No cellularization of gametes has therefore in all probability been necessary. The sexual phase had become internalized; it was just at this point and in its transition to the hermaphroditism that this primitive infusorian - which was the ancestor of Eumetazoa-had differed from its equally polykaryonic and hypermastigous flagellate ancestors.

It should be mentioned in this connection when we refer to the Flagellata as direct antecedents of Eumetazoa that in this way we are easily able to understand, without being obliged to take refuge in the thesis of flagellate colonies, the numerous cytological peculiarities that can also be found in the Eumetazoa, and not only in sponges. 
Yet before I begin an explanation of an outline of the probable organization the animal had at the point of separation of the lines of evolution of the Eumetazoa and of Infusoria (an outline which can now be made with a considerably greater precision) I would like first to call attention to the excellent and instructive work by Earl D. Hanson (Hanson, 1958).

I believe I am right when I state that Hanson has basically accepted my concept, even if he did not accept it at first. In his criticisms, expositions, additions, and ammendations Hanson refers, when he discusses my arguments, to the necessarily brief article only which appeared in the Systematic Zoology (Hadži, 1953). Hanson's leading idea is that my concept cannot be accepted in its present form, that it still needed some essential propping and complementing, above all the point where I discuss the deduction of the Acoela from the Infusoria. In his argument he states that, "The parallels between the Acoela and the Ciliophora may be illusory, in terms of demonstrating a phylogenetic relationship, if it turns out that the acoel has its counterpart only in a fictional amalgam of ciliate characters that has no more reality than the mythical Chimera." I believe that with such a formulation Hanson has gone much too far and that the formulation is inadequate. The fact which is called by Hanson somewhat ironically an "amalgam" or a "Chimera" is in reality a phenomenon which has been earnestly studied by modern phylogenetics where it is known under the name of the Mosaic theory, or Watson's rule, as it has been called (Sir Gavin de Beer, 1954). An "accidental" convergence of several characteristics of an existing type can lead by way of genetic regulations to new combinations (naturally with a simultaneous appearance of new mutations). It is in this way that new animal types which are able to live-and no chimeras-can develop and in all probability also have developed.

Moreover I wish to remind the reader that at the beginning of the present study I already said that I came to my 
concept (naturally not as the first zoologist, yet quite independently) neither by way of a study of Infusoria or of Turbellaria, but rather after a protracted study of problems connected with the Cnidaria. I am therefore happy when I see other zoologists who work in other special fields of interest, such as, Otto Steinböck, a prominent expert in Turbellaria, extend their interest in the direction of the present study. I am also very happy to see that Hanson wrote his study mainly from the point of view of an expert in Protozoa, even if we do not agree in every detail.

There are mainly three points in which Hanson tries to improve and perfect my concept in order to make it more acceptable, after he had submitted it to a detailed criticism.

Hanson wishes first that something more definite, more real, could be found instead of the too general name of the infusorial ancestors of the Acoela which were designated as "infusorian, or ciliate-like" animals. Hanson has selected the "pleurostomatous gymnostomes" from the group Euciliata which is rich in forms, more precisely from the subgroup Holotricha which even now possess the most primitive characteristics. These "pleurostomatous gymnostomes" are on one hand homogenous, and on the other hand they have remained most similar to the Acoela. Hanson selected as their representatives the two genera Remanella and Dileptus. It is quite natural that the two forms which are genuine (as well as recent!) Euciliata have all those similarities with the Acoela that can be generaly found among the Euciliata, even if they belong to the more specialized forms. We are therefore interested in those pecularities of these two genera of Euciliata which could justify us to suppose that they are more closely related to the Acoela than are the remaining Euciliata. Such peculiarities could be the un-differentiated character of cilia, the primitive conditions of the cytostome surrounding, and above all the plurality of nuclei, thus of karyons, whether they be somatic or generative. 
It certainly is welcome and useful for my concept when Hanson called my attention to these forms of Euciliata which show some combined primitive characteristics, provided that the plurality of the two types of karyons be a primary phenomenon. This makes my thesis of the origin of the Acoela, and thus of the Eumetazoa generally, from some infusorialike ancestors even more probable and more easily acceptable. Yet at the same time I do not think that it is of much importance. The two Holotricha are genuine Infusoria, and not some transitional forms. They suit the scheme of the Mosaic theory by way of which the characteristics that can be observed in one descendant can be attributed to different ascendants so that these characteristics become united and combined in a certain descendant which develops thus into a new type. It cannot be understood how our concept which derives the Acoela from the Infusoria could depend on this addition which was made by Hanson.

The second difficulty of the original concept which makes its acceptance hard or even impossible was formulated by Hanson in the following question, "Why is the macronucleus, one of the most important of all ciliate characters, absent in the Acoela?" (Hanson, 1958:33). This has in my opinion not been the most fortunate formulation of this question; its words should rather be, "what corresponds, what in the Acoela is homologous to the macronucleus of the Euciliata?" I was not able to discuss this problem in detail in my very short article which appeared in the Systematic Zoology, yet even from this article, and even more so from my other publications it appears clear that I consider the macronucleus to be homologous to the somatic, or vegetative ("indifferent") karyons. The macronucleus is therefore a result of a secondary oligomerization which succeeded during the evolution of Infusoria, the polymerization that had taken place in the Flagellata, the ascendants of Infusoria. The polymerization, however, was continued in the evolution of the Acoela, it was even further developed not only in the quantitative 
but even more so in the qualitative direction; the karyons had become differentiated in spite of their total potentiality after they had assumed a certain place in the animal body, a development which was of the greatest importance for the prospective destiny of the Eumetazoa. This is the reason why there is no macronucleus in the Acoela, this is one of the key differences between the higher Infusoria and the lowest Acoela. It must necessarily be supposed that formerly there had also existed intermediate forms between the stage with numerous vegetative karyons and the stage which has one karyon only and which is the final stage reached by way of this oligomerization. Not only in such stages was the number of karyons decreased, but even their behaviour was different (the type of origin, the subsequent divisions, etc.). The macronucleus, however, has lost its total potentiality and it has to be newly formed after each conjugation (combined with a copulation) when the final stage of the oligomerization has been reached and when everything has become concentrated in the macronucleus.

The case of Remanella which has been pointed out by Hanson is certainly very interesting because here the macronuclei are not developed by way of a division of themselves, but instead they develop continually from the micronuclei which themselves are also able to multiply. It is quite possible that a more primitive stage is represented in this case, even if a special development cannot be excluded either. The plausibility of my concept again does not depend on all this. Hanson states, "It can be seen that nuclear situation in Remanella provides a transitional form in a possible series going from that typical of the ciliates to the dispersed nuclei of the acoels" (Hanson, 1958:37). At the same time he adds that this series can also be reversed which, however, is not probable for other reasons. I confidently expect that new material evidence will come to light during the future studies of Infusoria which will support our thesis. 
The third addition to my concept which was made by Hanson regards "The transition from ciliated protozoan to acoel." It consists in the suggestion that cellularization did not take place earlier than in the Turbellaria and that it can therefore not be considered as the decisive step in the evolution of Metazoa proper. The essential difference between the Infusoria and the Acoela, and thus the decisive step in the evolution of Metazoa, had been, according to Hanson, the fact that in the Acoela the karyons had become morphologically complex, in comparison with the Infusoria where they are physiologically "compound." A progressive organization had become possible because of this step. The variously placed karyons had become heterogeneous, and this heterogeneity was increased even more by a subsequent formation of cell membranes, by a visible cellularization or compartmentalization.

Hanson is certainly right when he attaches great importance to the differentiation of the somatic karyons. This, however, is not something entirely new because I too have thought of such a differentiation in the form of a regular distribution of karyons in all the three body layers. Neither have I attributed any primary role to the cellularization because it does not appear earlier than in the already "finished" yet primitive Acoela, and even then only partly. Moreover, I believe that it is a mistake to attribute a leading role to a single peculiarity in the creation of the Acoela ("crucial point" or "key step," according to Hanson). It seems to me much more probable that they had emerged because of a convergence of several important characteristics and moments, among which we must mention above all the internalization of the sexual phase combined with hermaphroditism.

After this discussion of Hanson's study on the primitive Infusoria I wish now to refer to the important work by I. B. Rajkov (1957). Rajkov discusses the problem of the origin of the bikaryonic state of Infusoria using in his study the discoveries that have been recently made in connection with 
the primitive Infusoria. Rajkov asserts that even now species can be found among the lower Infusoria which do not have a "nuclear dualism." As an example he mentions Stephanopogon Lwoff (1936) whose body has several equal nuclei which are not polyploid and which contain only little DNA. Quite bizarre is the case of Trachelocerca phoenicoptera (Rajkov) with its complex karyon which develops by way of a union of several macronuclei and which also encloses six micronuclei. This sounds very strange and it can be interpreted only as a lateral development from the main line that evolution had taken in connection with conditions that are typical of the higher Infusoria. In the conjugant of the same species we find a larger number of the so-called pronuclei (gamocyte karyons) from seven to twenty-two. They develop into three to six androgametic karyons (migrating nuclei). The fertilization is multiple, its result, however, is one zygote only which remains alive while all the rest is lost. Rajkov is right when he concludes that this is a remnant of a former multiple formation of gametes and that the conjugation of the Infusoria had therefore evolved from such sexual conditions that are characterized by the gametogenesis and by the copulation, a fact which several years ago I did not only maintain but also tried to prove (Hadži, 1950).

Tracheloctrca belongs to the holotrichous Holophryidae which Rajkov believes to be the most primitive Infusoria. We can expect that many interesting facts will still come to light during the future researches.

\section{The Origin of the Complex Hypercellular Individualities}

We will now return to the problem of the cellularization which was so thoroughly discussed by Hanson. All biologists agree that the monocellular state with one cell nucleus, thus the primary independent monokaryon, represents 
an extraordinarily important stage in the evolution of life (Unicellularia, according to Rotmüller; Eunucleata, according to Moskovskij). The growth in size of an individual is necessarily limited by such a monokaryonik state. This limit was overcome several times during the subsequent phylogenetic evolution which, in agreement with a generally valid regularity, did not involve all the lines of evolution that had developed from one and the same origin. In the meantime a new bifurcation emerged; plants and animals. This bifurcation has also taken place in several lines of evolution. Complex and therefore easily enlarged bionts had evolved along the line which was pursued in the evolution of plants by way of the so-called formation of colonies; its reason was a strongly developed cell wall. The phrase "formation of colonies," however, which is so frequently used is rot completely adequate. The monocellular individuals of one and the same species can also unite into a biological whole even if they belong to different colonies. Coenobia and polycellular algae are regularly developed from descendants of a zygote, more rarely from a vegetative cell (a spore). The products of division do not scatter, they rather remain together and develop into parts of a new unit which are organically connected, they develop into a complex individual. This process can be considered as a parallel to the process of the polymerization. The daughter cells are not only all powerful they are even primarily destined to develop into independent individuals. They lose part of their "sovereignty" by becoming members of a coenobium or of a colonial union, they become subordinated to the new unit or entity. This leads via practice, to a heterogeneity of originally equal and equivalent cells, to their differentiation. It can be observed above all in the development of the generative and of the somatic cells. Tissues, organs, and systems of organs are also developed in this way. The freely moving way of life is relinquished quite early by all the plant bionts (with a few exceptions, Volvocales!). They become permanently sessile beings which 
agrees with their type of feeding. Even androgametes which preserved their free mobility are now only active when they are transferred from place to place. This situation has finally become even more complicated because of an introduction of a remarkable alternation of generations.

In spite of a strong inclination of the plant cells to remain mutually separated they have nevertheless developed plasmatic connections which go through the cell walls, or they had even completely relinquished their cell walls and had developed in this way syncytia. The case of Caulerpa and of the whole group it belongs to is well enough known. Here a total polykaryonic stage had been reached. Among the higher plants it is not seldom that partial syncytia can be found.

The animals certainly pursued several lines in their evolution, yet it was one line only which succeeded in making essential progress while all the remaining lines had already come to a stop at the level of Protozoa. Another primitive line led to the formation of Spongiae. The evolution of animals has been much more vivid because of their heterotrophic type of feeding, because of their free mobility which is connected with the type of feeding, and-which is a very important fact-because of the very thin walls of their cells. The limitations of the monocellular and mononuclear state were overcome along at least three paths. One of these was, so it seems, a development which led to a slight increase of the cytoplasmatic mass which was subsequently divided half-way, first into two and later into several parts; its result had been the formation of considerably larger complex individuals. Yet this division into halves has not led to differentiation, it remained rather mechanical, and its result has only been an increased breathing surface. It was soon combined with a polymerization of the nucleus. This is how the comparatively large Foraminifera, e.g. of the type of Nummulites, evolved. Yet no genuine Metazoa had ever possibly developed along this way. 
The second type of development of the complex individuals was similar to the one we have just described in connection with the evolution of plants. As in the case of plants we find now in the Flagellata an inclination to develop colonies. In the true colonies the products of division of the maternal cell remain only mechanically attached to each other (coenobia, colonies s.str.). This had finally led to the formation of cormi when certain cytoplasmatic connections developed between the members of the same colony. In this way the coordination, and finally the differentiation of partners became possible. This is how I imagine the evolution of Spongiae which begun characteristically from an already more highly developed and therefore more specialized group of Flagellata. I am supported in this suggestion by important factual arguments. These species of Flagellata were characterized by a cytoplasmatic collar which had developed around the basis of the flagellum (Choanoflagellata, Craspedomonadida). During this development the ability has not been lost either to abolish the collar form or even to assume an entirely amoeboid form and type of movement. Even if there were no species of Proterospongia (two such species have actually been described in the meantime) it would still be very probable for reasons that cannot be discussed here that the Spongiae had evolved from some originally freely-living cormi of Choanoflagellata after the latter had adopted the sessile way of life. Nothing can be changed in this connection by the fact that examples can occasionally be found among the Eumetazoa whose cells have a collar formation. The organization of Spongiae is in fact entirely different from that of all the Eumetazoa. Neither can this situation be changed by the fact that a sexual phase with an oögeny can also be found in Spongiae as well as in the Eumetazoa because we know that the same sexual conditions had also evolved in various plant groups. These are properties that had been inherited from the protistic ancestors of all the polycellular forms, in spite of the fact that we have here several lines of evolution. It may be 
emphasized that a formation of syncytia can also be observed in Spongiae, and not only in plants.

The embryonic development, the ontogeny, emerged as a necessary consequence of this state in all these lines of evolution, thus in Volvocidae and in various other polycellular algae, in Cormophyta, and finally also in Spongiae. It is therefore not surprising to see that there is a great difference between these ontogenies as a result of their separate lines of evolution; this is also true for Spongiae in comparison with the Eumetazoa. No other higher animal group has ever evolved from Spongiae. The further evolution of the animalic bionts had begun from another centre.

The most successful attack against the limiting influence of the monocellular state, an attack which has finally led to the formation of the most highly organized beings, of the polycellular animals and man, was made by way of polymerization, the plurality of nuclei in a cytoplasmatic or plasmodial body which preserves its individuality, and by way of a subsequent cellularization (Fig. 48). The way along which this development had taken place and how it occurred has already been partly discussed, but we will still return to this problem. The importance and the significance of the difference which exists between the development of multiple cells by way of a formation of colonies or cormi, and the development of such cells by way of polymerization can easily be understood. The first type of development is characteristic of plants, and the second of animals. The two types of development have in common (1) the growth in size; (2) the possibility of a differentiation; and (3) the sexual phase with the ontogeny at its end. Cellularity can also be considered as one of these common properties even if here there is one important difference. In the polycellular plants the cellularity is a primary element which is obstinately preserved; in the Eumetazoa on the other hand cellularity is a secondary development from a polykaryonic state. Cellularity is almost never completely carried through in the 


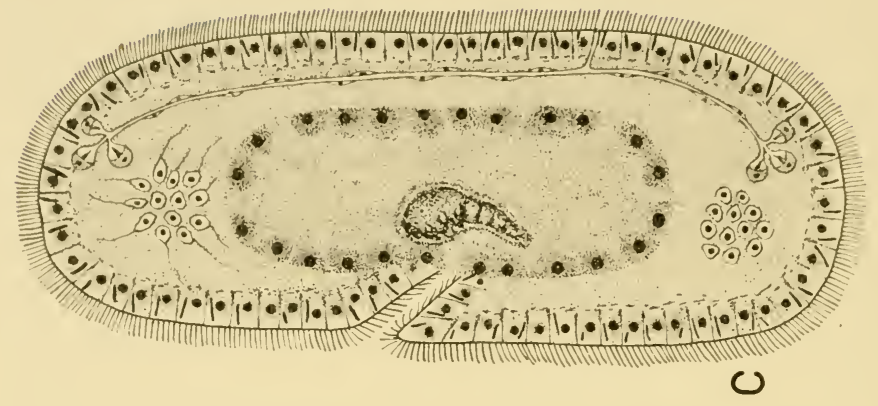

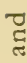

5
0
0
0
0
0
0
0
0
0
0
0

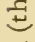

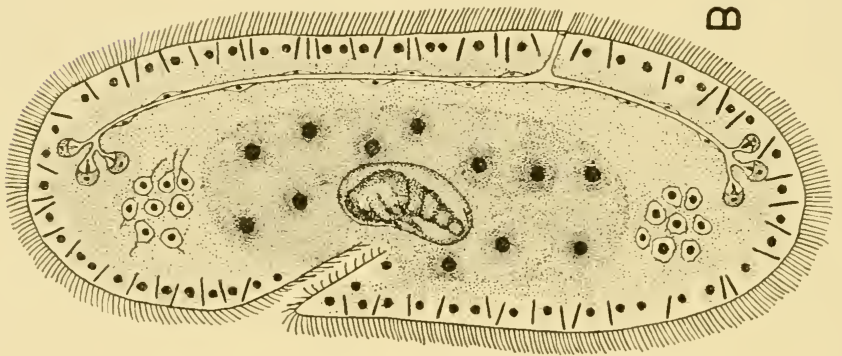

焉

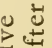

$\stackrel{\Xi}{\Xi}$

อิ

$\stackrel{\circ}{\stackrel{ \pm}{\Xi}} \cdot \stackrel{ \pm}{\Xi}$

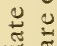

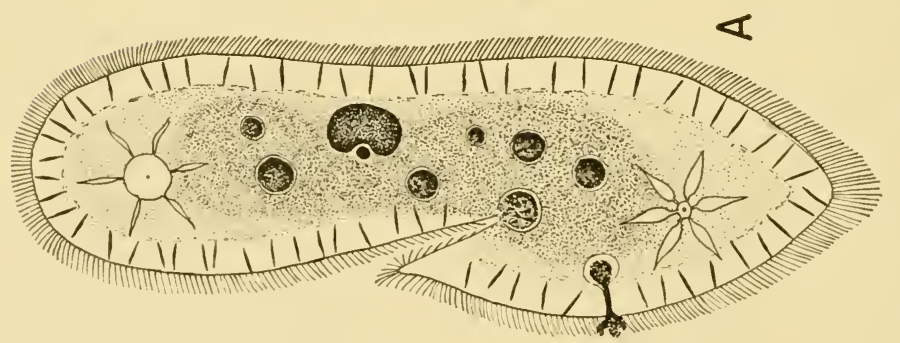


Eumetazoa (which is particularly true for the middle body layer, the mesohyl!); their tissues show a strong inclination to a return into the plasmodial state which we usually call a syncytium.

An even more important difference between the polycellular plants and sponges on one hand and the Eumetazoa on the other, a difference which became evident above all during the subsequent destiny of the phyletic development, is the fact that such a degree of unity can rever be reached by the polycellular bionts by way of a colonial formation, as is the unity that has been reached by the Eumetazoa. The latter had inherited from the very beginning a uniform and already comparatively highly developed organization from their protozoan ancestors, as far as such an organization could be developed within the framework of a mono- or paucinuclear state. The objections made by those scholars who adhere to the old interpretation which considers that all the polycellular organisms had evolved from colonies of cells-among these scholars we mention above all Sachwatkin (1956) and his intensive and extensive work-cannot deny the fact that a great difference exists between the organizations of Spongiae and of the Eumetazoa, even if we take into consideration the sedentary way of life only (e.g. a comparison with the Hydroidea). Neither can they abrogate the difference that exist between a sum of choanoflagellates where each member still continues to exist as an individual which must first become adapted to the "new conditions," and, on the other hand, an infusorian with various organelles and generally fine structures which evolves in a direct line into progressively higher Eumetazoa while at the same time the unity of the animal is constantly preserved.

There can be no doubt that a new form can develop by way a coenobium from originally independent "monocellular" protozoic or protophytic individuals; the development goes from the state of coenobium via a formation of a colony or of a cormus to that of the final form. Such a development can $21 *$ 
be observed in numerous Metaphyta and in Spongiae. Yet at the same time it can also be stated that great progress has never been made along this way towards a definite whole. The circumstances led to a change of exactly those metabionts that had evolved by way of colonial formations to the sessile way of life. This has proved an obstacle to a subsequent progressive development. A "blastuloid" organization (Volvox) represents the highest level reached in the evolution of the freely moving Metaphyta. Bypassing the immobile Metaphyta in which no central organs have been developed we see that the sponges have not come very far in their evolution either. They again have not developed any central organs or any devices which would unite the whole aggregation into a higher organic entity; they represent a very lowly-developed side branch of the animal world.

In the numerous, mainly sessile Eumetazoa which had frequently adopted a freely moving way of life (Siphonophora, Ectoprocta, Taliacea) and which had developed their own cormi ("colonies") higher units can be developed from several originally independent individuals. These new units represent a new organization, they have developed their own individuality, and it is not even easy to identify their original complexity. Siphonophora may serve here as the best and probably also as phylogenetically the oldest example. They have certainly reached the highest level of development in this direction. They excel in a highly developed external homogeneity, they show a considerable polymorphism both in their polypoid and medusoid "persons" (the formerly independent individuals), they have even evolved new impersonal and thus specifically cormic organs, e.g. a pneumatophore. Yet on the other hand they have not developed any centralized nervous system, neither have they been able to overcome the limitations of Hydrozoa and to develop in this way into a new animal type; this in spite of the fact that they have become at least slightly freely moving animals. 
The quicker and higher development of the Eumetazoa was made possible by their animal type of feeding which is connected with a search for the living prey, and by the free mobility which is connected with their type of feeding, and finally by the direct transference to the Eumetazoa (of the type of Turbellaria) of an already plasmodial and polykaryonic unit which had already been enriched by all those elements that could be attained in a monocellular state. It has already been repeatedly stressed that quite a few zoologists already before me had taken into consideration the possibility that the Metazoa had evolved from some infusoria-like ancestors. Yet they were unable to develop a clear concept because they stood under the influence of the now still widely accepted interpretation that the Spongiae, the Cnidaria, and the Ctenophora, thus the so-called Coelenterata, are the lowest Metazoa (we will bypass the Mesozoa which are still very problematic). The only thing these scholars dared to do was make a few brief and vague remarks on the subject. The way to the new interpretation has become open only now after we have first developed a new concept of the evolution of the Cnidaria, after an exclusion of the Spongiae from the sphere of Eumetazoa, and after a removal of Ctenophora from a society with Cnidaria, and finally after we have bypassed the so-called Mesozoa. To this we must also add the recognition that the Acoela have the lowest organization that can be found among the Turbellaria, and that this simplicity is not a consequence of an eventual retrogression but rather a primary phenomenon. The situation became clear when the barrier that had artificially separated the polykaryonic and therefore plasmodial Infusoria from the equally plasmodial and polynuclear Acoela was dropped. 


\section{Is the Plasmodial State of the Acoela a Primary Element?}

The antagonists of the new interpretation soon discovered its critical point; they believed that at this point they had the best chance to attack my concept. This point is the primary nature of the plasmodial state that can be found in the Acoela and in other Turbellaria. This was followed by a cellularization.

Erich Reisinger, endeavours to prove the faults of my thesis of the primary plasmodial state of the primitive Metazoa, the Turbellaria included. He believes that in a polykaryonic state we must always see secondarily developed syncytia. He tries to support his criticism not only by means of facts that he collected in some occasional observations, but also with experiments.

In order to make his interpretation of proposed facts all the more acceptable Reisinger initially makes an unproved statement in which he declares our concept to be "schon auf Grund der total verschiedenen Sexualverhältnisse der gamotogamen Ciliate einerseits und der gametogamen Metazoen andererseits als äusserst problematisch erwiesen."

Let us now consider those facts that were used by Reisinger as a basis of his conclusions, and above all the proof he obtained by way of an experiment. Reisinger pressed through a silk-gauze an already cellularized freshwater triclad Planaria polycbroa O. Schmidt. In the separated rags of tissue Reisinger could see spheric complexes (the so-called "Sphärien") being developed in spots where cells ran together to form syncytia (with the exception of a few cells that remained amoeboid). Many parts became histolized. Thus syncytia similar to the central parenchyma (= endocyte, according to Westblad) of the Acoela (all the syncytia are actually similar!) have been developed from the cellular tissues.

Observations have shown that under the influence of the protozoic parasites (Sporozoa) of the species Eucoccidium monoti the cellular intestine of the species Otomesostoma can change back into a syncytial state. Such a development can 
take place, so to speak, normally in Planariae when they take too much food, a fact which is also mentioned by Reisinger. By means of some not too tender experimental interventions Reisinger succedded in leading a syncytial skin layer into a cellularized state (in Gyratrix and in Koinocystis); he used strong irritations in this experiment. Reisinger also succeeded in artificially stimulating the opposite process to the one first mentioned. He mentions the fact which he believes to be in itself a firm proof, that in all the ontogenies of the lower Turbellaria that have become known up to now, these ontogenies take place by way of a cellular development only ("rein zellig"), thus by way of cleavages.

On the basis of all these facts Reisinger comes to the conclusion that there are no primary plasmodia in the Turbellaria but rather secondary syncytia which show a constant inclination to return under certain conditions into their cellular components. He ends his discussion with the statement, "Die von Steinböck und von Hadži vertretene Ableitung der Metazoen von Ciliaten über polyenergide plasmodiale Stadien erscheint durch unsere Befunde weitgehend erschüttert" (Reisinger, 1959:641).

As regards the "totally different sexual conditions" that are supposed to exist between the Infusoria and the Eumetazoa, we have already shown that in this case we cannot really speak of such a total difference.

We cannot attribute the same conclusive power to the findings made by Reisinger as Reisinger himself would like to give them, in spite of the fact that they are very interesting. Reisinger goes even so far in his endeavours to deny the existence of a primary plasmodial state in the Turbellaria, that he calls Steinböck's opinion a "pure fiction" (Reisinger, 1959: 642) which is certainly unjust in view of the fact that Steinböck (Steinböck, 1954) too tried to prove his thesis of primary plasmodia in the Acoela (which he calls archhiston) by means of experiments.

It is unnecessary to discuss whether there are any true 
syncytia; any experiments in this connection are completely superfluous. This is a phenomenon which can be found scattered in the whole animal world, a fact which has been recognized long ago. We even know that among algae which have reached a low level only in their organization (they are therefore certainly primitive organisms among the polycellular forms) there is a whole group, among the Siphonales, which shows a strong inclination to form syncytia, in spite of the fact that these are plants and thus colonial polycellular forms whose cells had evolved quite generally strong membranes. In the best known case, in Caulerpa, we find cellularity eliminated to such a degree that it appears only slightly during the phase of the formation of gonads while at the same time it has completely disappeared from the ontogeny. In spite of this Caulerpa has remained a lowly organized alga, and therefore a plant.

We are particularly interested in the cases that were observed by Reisinger during his experiments where a cellular state was developed from a syncytial (plasmodial) state. It should be mentioned in this connection that a trend must be taken into consideration by means of which the cellular tissue of the Eumetazoa developed from the plasmodia. Moreover, it is quite difficult, even if not impossible, to decide precisely whether in a given case we have a true primary plasmodium or a secondary syncytium. In the case of the more highly developed Turbellaria it must also be taken into consideration that their plasmodia have already reached a state of readiness to pass over into a cellular state; in these a slightly stronger irritation can be sufficient to produce a segregation of their karyons, and thus the formation of cells. Reisinger did not quote any example of artificial cellularization of the plasmodia Acoela.

As a contrast to all this we can find the very instructive experiments that were made by Steinböck in the most primitive Acoela (Amphiscolops). Steinböck could prove with these experiments that all three parts of the archhiston, all 
the three body layers (which are called by Steinböck ectoplasmodium, mesoplasmodium, and entoplasmodium) are all capable, that they are able to develop a new unit even if cut into the smallest dimensions (a minute-piece measuring $157 \mu$ punched from the posterior end of the animal). They have therefore the character of a primary plasmodium. Where can we find a similar case in the whole animal world of the Eumetazoa that an entoderm can regenerate into a whole new animal even if it exists in a syncytial state?

The most important element among Reisinger's arguments against the existence of a truly primary plasmodium in the Eumetazoa (our thesis of a polykaryonic and plasmodial origin of the Eumetazoa loses every foundation if there are no such plasmodia) is, according to Reisinger, "Die ausnahmslose zellige Primärstruktur früher Embryonalstadien" (Reisinger, 1958: 642); or, in other words: the ontogenies of the Turbellaria. Among these, the Acoela begin with a cleavage, thus with a formation of cells which is only subsequently followed by a formation of syncytia. Steinböck has already given an answer to this observation. Above all, very little is known about the early ontogenies of the most primitive Acoela. We agree with Steinböck who does not think that species of Metazoa will be found with the oldest form of the early ontogeny (i.e. a plasmodial state) preserved. Evolution has led in the inactive sessile polycellular algae (Caulerpa) to a state where a division of karyons takes place as an obviously secondary phenomenon instead of a cleavage or of a division of the zygote.

Scholars have become used, under the influence of the colonial theory and of the blastula-gastrula, to see in the early development of the Eumetazoa by way of cleavage (the division of karyons that can be observed in insects was naturally immediately explained as a secondary caenogenesis) a recapitulation of the phylogeny which is supposed to repeat the primitive formation of colonies by the monocellular Flagellata. Scholars have begun only recently to analyse experimentaly, 
the early ontogeny taking into consideration the physiological viewpoint. On the basis of everything we now know about the very variable ontogenetic morphogenies it appears as improbable that the first, and thus the oldest, steps in the ontogenies-which had evolved regardless of the phyletic antiquity of total divisions of cells, with few exceptions that can be explained-could possibly represent a recapitulation of the oldest state in the evolution of the Eumetazoa. It seems a priori much more probable that physiological factors, above all the greatly increased need of oxygen as a consequence of a very intensive metabolism, had caused the cellularization to be adopted in the early ontogenies. The organism has thus endeavoured to evolve large surfaces for each active karyon for the sake of an increased gaseous interchange (the intake of oxygen, and the delivery of the carbon dioxide). This has caused the earliest cellularization which took place ad boc. This trend had led occasionally to a temporary segregation, to really anarchic states of blastomeres even if the whole as such had not been lost. The state of the fully developed animal, the plasmodial state, has been recovered after this period of "Storm and Stress," and it can therefore be rightly considered as a primitive state.

Thus the initially reversible cellularization began in all probability in the earliest ontogenetic stages with cleavage as its very special form. It was much later that true cellularization combined with the formation of tissue, had evolved; it remained permanently without losing its ability to return under special conditions into the plasmodial state. Such a state is called a syncytium. Actual cellularization did not appear immediately and generally, but rather by degrees; this is proved by conditions that can be observed in Turbellaria. First it took place in the skin layer (the ectodermal epidermis), later in the intestinal wall (the entodermal intestinal epithelium), and finally in the middle layer where it has usually not been complete (the mesoderm s. 1. or mesohyl, usually divided into the mesenchyme and the coelothelium). 


\section{An Attempt to Reconstruct the Initial State of the Eumetazoa}

After all these criticisms, counter-criticisms, and discussions we will now try to reconstruct the initial state or the earliest state of the Eumetazoa. In this attempt I will correct and improve my previous suggestions which were necessarily vague. I am well aware of the hypothetical character of this construction, yet at the same time I believe that it is not simply a product of pure fancy as this has been maintained by some of its critics; it is based on critically selected facts and it can claim at least some probability, at least as much as can be claimed for those attempts that have been made so far by those who adhere to the colonial theory. It should be discussed critically and compared with the theory which is now generally believed to be true. From the very beginning I have expected that this interpretation would not find an easy acceptance because it requires that the old interpretation which is so deeply rooted and which at the first sight appears so bewitchingly clear should be given up. It cannot be expected that the numerous older zoologists could easily relinquish an opinion which they have considered true for such a long time. It is said that such a revolutionary idea will have to wait for the next generation to win its full success. My reconstruction of the origin of the Eumetazoa stands in opposition to the "belief" in the colonial origin of the whole animal world and to a number of other similar "beliefs" (e.g., the question of the relation between ontogeny and phylogeny, the significance of cleavage and of other ontogenetic mechanisms, the idea of germ layers, the "germ path," the general division of the animal world, the problems of the mesoderm and of the coelom, etc.). To this we must add the supposed difficulty which arises because I consider that the Metaphyta and the Spongiae have evolved out of the colonies of Protista, and thus in different ways to that which the evolution of the Eumetazoa had taken. 
Instead of the vague and indefinite statement that the Eumetazoa evolved first as the Acoela, among the Turbellaria, from some Infusoria-like ancestors, we can now state with better justification that the Eumetazoa evolved firstcontrary to the evolution of sponges and of all the polycellular plants-as polymerized Protozoa, thus as Protozoa that were equipped with a larger number of karyons and of kinetids (cilia); during that state they were no longer typical Flagellata, neither had they yet reached the state of the typical Infusoria; yet their ancestors stood closer to the Infusoria than to the Flagellata mainly because of the fact that their diploid and polykaryonic vegetative generation (the "indifferent" generation, according to Dogiel) had also become the bearer of the sexual, i.e. hermaphroditic, generation. These ancestors can therefore be called the "Urciliata" (Protociliata) (the primitive Ciliata).

The gamontized generation therefore also includes the sexual generation which had remained cellularized (it has by now already changed into a phase). This sexual generation develops its maturity in the interior of the maternal animal and it becomes free in the form of gametes after or during pairing. The zygotes which are formed in this way, the fertilized egg cells, develop during ontogeny into a new generation. Thus the progamic subphase of the sexual phase has been internalized and this has led to the formation of gonads. These gonads represent therefore from the very beginning a corpus separatum in the body of the Eumetazoa and they cannot be considered as a genuine gland.

The earliest Eumetazoa have taken over all the organelles as well as the finer and specialized cytoplasmatic structure of their protozoan ancestors. For reasons that are so far unknown, the direction of division changed just at this moment from a longitudinal to a transverse division via an oblique division. This change occurred during a transition which is at the same time one of the most crucial transitions in the whole evolution of the living world. The change may perhaps 
stand in a causal connection with the longitudinal rows of cilia.

From this reconstructed form we can deduce without any difficulty both the line which had been pursued in the evolution of the Infusoria with their characteristic mergings of the cytoplasms, the internalized sexual phase, and the conjugation; and the line which had been pursued by the Eumetazoa in their evolution with their gametes which become free.

\section{The Origin of the Middle Body Layer-of the So-Called Mesoderm}

The very large number of known characteristics common to both the Infusoria and the Turbellaria described by v. Gelei has been increased since the publication of his study. In this connection I would like to call attention to the fact that Remane himself, one of the most active opponents of the interpretation which suggests a direct relationship between the Infusoria and the Turbellaria and of its consequences, has stated that the numerous similarities that exist in the two groups could not be interpreted as a purely incidental phenomenon but rather as a proof of a closer relationship; and that they made any further proof i.e. transitional cases, patterns, etc., unnecessary. To this we could add that no facts have become known till now that disprove this close relationship. Furthermore, we must also mention a fact whose value should not be underrated, i.e. that many phenomena can be much more easily understood and explained if viewed from the standpoint of my thesis of the Prociliata as the ancestors of the Acoela, and therefore of the Eumetazoa, than when they are studied on the basis of the old interpretation which believes in a colonial origin of the Metazoa. I would like to mention in this connection the much discussed problem of the origin of the third, or of the middle, body layer, the mesoderm, and the coelom problem which is connected with it. 
According to the old interpretation, the three body layers evolved successively one after the other, each after a long interval of time. This conclusion was reached mainly on the basis of the formative processes that can be observed during ontogeny. Yet on the other hand, these processes show a perplexing variety so that it was impossible to decide which of them represents the most primitive form. Haeckel proposed a special stage which contained no body layers and which he placed before the stage with one body layer. He believed he found support for this theory in the ontogenetic facts. $\mathrm{He}$ called this stage a morula. It did not actually suit the whole scheme he proposed and it has not been generally accepted. The one-layered stage which had according to his interpretation the form of a hollow sphere was given the name of a blastaea, after the ontogenetic blastula; it was thus believed to be recapitulated in the blastula. All the cells of the epithelium as the most primitive form of the tissue, are identical. The first differentiation had taken place by way of a separation of the vegetative and of the sexual functions, and, in connection with this, of their bearers. This is how the appearance of the "germ path" was also explained. The zoologists have not been too embarassed by the fact that no blastaealike form can be found among the Metazoa. Haeckel, however, who found such an absence unpleasant, tried to track down such an animal form in the sea plankton. Thus a description was made of a certain "Magosphaera" which, however, had soon to be removed from the list of animal species because it was found to be nothing other than a colony of Flagellata. After this failure a blastaea-like form was borrowed from the plant world; this is the now famous Volvox; Volvox still continues to play the same role even in spite of the fact that we know that it has nothing in common with the origin of the Eumetazoa. It should serve as a pattern. Volvox is certainly a genuine green alga, and a particularly specialized fresh water alga. It emerged as a final product of evolution along a sideline. Many forms can be found among algae which show a 
transition from the monocellular species to the colonial polycellular forms. How can we ke certain that the polycellular heterotrophic Eumetazoa evolved in the same way as was "invented" for an autotrophic regime? Scholars should refer in this connection only to developments that can be observed in ontogenies. Yet are these really recapitulations of some adult ancestors? Contrary to the widely accepted opinion which sees in Volvox pattern at least a plan of the primitive ancestor of the Eumetazoa, we consider such a suggestion as completely improbable.

Those who adhere to the old interpretation maintain that during the second stage of the phylogeny of Metazoa, a twolayered animal which was able to digest its food, the so-called gastraea, had evolved from the primitive one-layered hollow sphere whose members were able to perform, as epithelium cells, all functions with the exception of the sexual function. This two-layered form was called a gastraea, after the name of gastrula. Yet again no such animal exists. It was believed that the typical evolution of this diploblastic form had been similar to the development that can be occasionally observed in the ontogenies where the posterior half of the animal (i.e. the part of the animal situated opposite to the direction of swimming) invaginates into its anterior half. Thus with one action the animal succeeded in developing not only its two layers, the ectoderm and the entoderm, but also a digestive cavity and an oral opening which was unfortunately placed at the posterior end of the animal. The gastraea as we find it thus reconstructed is supposed to have lived as a planktonic animal and it was radially symmetrical. This gastraea descended later (partly simultaneously, according to Haeckel) to the sea bottom where it evolved into various "Gastraeadae." The latter were quite early given the unsuitable name of "Coelenterata." No gastraea ever existed among plants; it is typical of the polycellular animals. It possessed a skin and an intestinal layer and showed a corresponding difference in its tissue and in its cells. 
It has later been found that there are no primary twolayered animals, except some forms which show traces of a strong retrogression due to their parasitic way of life. Haeckel himself showed a considerable uncertainty on this point; he wavered when he tried to find the actual division between the two-layered and the three-layered animals (the Coelenterata and the Coelomata, or the Diploblastica and the Triploblastica); he even suggested once that the Platyhelminthes should be classified among the Coelenterata. These animals had not evolved a genuine middle layer, an actual mesoderm, but only the so-called mesenchyme or the mesogloea.

Finally, it was established that a majority of Coelenterata posses a middle (the third) body layer and that they really represent a very motley group. Platyhelminthes were the first to have definitely left this group; they were followed by Spongiae (this, however, has not been accepted by all zoologists). The Cnidaria and the Acnidaria (=Ctenophora) have thus remained the last two groups which have still continued to be classified as Coelenterata. Yet even in connection with the Ctenophora it has become more and more clear that they do not really belong here. The Cnidaria have thus been the last group which have still been considered as Coelenterata, yet even with these it has been found that they possess the third (the middle) body layer even if it is only slightly developed. They are therefore no real Diploblastica either.

Evolution finally led, according to this interpretation, to the formation of the third layer. The difficulties which emerge together with it are even greater. The third body layer (actually its solid part only because the sparse mesenchyme is usually not considered as a part of the actual mesoderm) develops during the ontogenies (which should serve as patterns for the phylogenetic conclusions) in widely different ways. Two such developments are particularly conspicuous because they generally even if not completely, exclude each other. In one case the mesoderm is developed from the ectoderm (the ectomesoderm), or, if we are more precise, from the transitional 
area between the skin and the intestinal layers. In the second case the middle layer develops as a pure derivative of the primitive intestinal wall (the entomesoderm) usually by means of a formation of diverticula growing out of the intestinal wall. This formation of diverticula partly resembles the formation of lodges that are developed in anthopolyps together with their sarcosepta. Attempts have also been made to derive the formation of the ecterocoele from that of the enterocoele. This situation becomes even more complicated in the ontogenies of numerous higher animal groups, above all of the Arthropoda and of the Vertebrata; thus in the human embryo the mesoderm develops from not less than five different Anlagen. The human body is also developed during the ontogeny from four and even more layers.

These differences in the type of development of the mesoderm which lead to the formation of secondary body cavities (coeloms), and the differences that can be observed in the type of development of the intestinal orifices-they all occur in the ontogenies-even if they are not radical, have served as a basis for the general classification of the animal groups (Ecterocoelia or Protostomia, and Enterocoelia or Deuterostomia). We must consider this as an unfortunate solution. The point where this supposed separation or bifurcation of the two large groups had taken place is assigned characteristically by various zoologists into different levels.

The question must also be raised whether it can a priori be probable that the Eumetazoa, which are considered as a homogeneous group whose body layers are believed to be mutually homologous, can all have such a common basic part, as the middle body layer is supposed to be, and at the same time that such a part had evolved not only ontogenetically but also phylogenetically along two essential different ways, so that the same form is not even mutually homologous. It seems to us much more acceptable to consider that the middle body layer is the same everywhere, that it is therefore always a homologous formation, whose ontogenetic development, however, has 
been changed during evolution in the sense of a methodical rationalization or of a "technical" improvement. Such a development need not have been divergent, but rather successive: it was first ecterocoelic (teloblastic) and later enterocoelic; thus the formation of the "mesoderm" itself has never had anything in common with the formation of the intestinal diverticula, nor with the formation of lodges as they can be found in the Anthozoa.

The intestine too evolved according to the old interpretation as a new form along several lines; thus the Eumetazoa with their intestines are supposed to have evolved several times from a form that had no intestine (blastaea)! Can such an interpretation be probable? The development of a uniform intestinal cavity is one of the main characteristics of the Eumetazoa (therefore the name Gastrobionta, according to Rothmüller). We consider that this cavity evolved first in Protozoa as a transitional digestive vacuole; in the earliest Eumetazoa this had led to an ad boc (i.e., for each swallowed piece) formation of a digestive cavity in their internal plasmodium; the final result of this development had been a permanent digestive cavity which is surrounded by a specialized epithelium that contains glands and which is able to resorb food. It had originally one permanent orifice, and finally two that are situated in the two opposite parts of the body. Nothing is changed in this connection by the fact that the ontogenetic type of formation had afterwards been repeatedly changed, similarly as in the case of the development of the Anlage of the middle body layer. The Metaphyta, however, have never been able to develop anything that could resemble an intestinal cavity. No new form has ever been evolved from the hollow sphere of the Volvox. In those cases where some plant species had begun to catch an organic prey (animals) and to digest the same we find this digestion to be purely external, limited to the surface of the plant.

What is then the situation with Spongiae? There can be no doubt that the Spongiae are genuine animals, yet at the same 
time they differ basically from all the other polycellular animal forms (Eumetazoa). Not only had they evolved into their polycellularforms from their protozoan ancestors along a completely different way, i.e. by way of some colonies of Flagellata, they also never developed a true digestive cavity which could be considered as a homologue of the cavity that occurs in the Eumetazoa: their chambers that are surrounded by choanocytes are not digestive cavities, i.e. they are not a multiplied intestine. The choanocytes are not gland cells, or intestinal cells; they digest in their own organelles, in the digestive vacuoles, in the same way as the solitary or colonial Choanoflagellata do. The Spongiae have neither an intestine nor an oral orifice. The choanocytes which are actually similar to the Choanoflagellata can only become amoeboid; as such they had been somewhat differentiated, yet they have never been able to develop a genuine tissue, and even less so an organ or systems of organs.

Under the influence of the old interpretation zoologists have tried to explain the internalization of an originally external digestive surface. It was supposed that the digestive surface had been originally situated externally on the body of the animal because the first colonies (blastaeae) had consisted, according to this interpretation, exclusively of individuals that gathered their food independently, which they digested each in their own vacuoles. These zoologists think that it was only secondarily and after the first differentiation had taken place, that the digestive surfaces were transferred into the interior of the animal body, which was subsequently covered with the skin cells. This transference into the interior was supposed to have taken place in different ways as this is shown by the patterns that can be observed in the ontogenies. The invagination of the posterior half into the anterior half was considered as the most primitive and typical way of the development of the intestine (the invagination gastrula). All this is extremely improbable and it is not supported by facts. These are pure reconstructions made on the basis of facts that can be $22 *$ 
observed in the ontogenies and under the assumption that they represent some kind of recapitulation. The development of a digestive organ by way of internalization, as was suggested by the old interpretation, can in reality be taken into consideration in connection with the Spongiae only; yet even in this case it did not lead to the formation of a genuine intestine.

The old interpretation meets great, insurmountable, difficulties when it tries to explain the subsequent evolution of organs, thus of the protonephridia, of the muscular system, of the nervous system, etc. All these "tasks" were already solved on the level of Protozoa. The way to all these differentiations had been prepared as early as in Infusoria. This is true not only as regards the size of the body, its form, its symmetrical conditions, the ciliation and the type of movement, but also for the type of feeding, the transverse division, the sexual phase together with the hermaphroditism and with the pairing, the triploblastic system and for all the peculiarities of the three layers.

The long list of similarities that can be found both in the Infusoria and in the Turbellaria (a list which was originally made by v. Gelei and which has now been enlarged) shows that these are obviously no accidental similarities, convergences, or parallelisms; they are instead true homologies which exist because they go back to a common line of evolution. These homologies are too numerous for us to consider them as accidental. The progressive evolution which began from a common basis continued especially as regards the middle body layer. We will later return to discuss this problem in more detail. It should suffice to state here that the function of the external or skin layer, which is usually called an ectoderm (the notion and the name have been originally created within the field of the older embryology of the higher Vertebrata), had only been to protect the animal and to maintain the contact between the animal and its environment; while the function of the entoderm had been on the whole to receive and to prepare the food; the middle layer (the mesohyl or the meso- 
derm s. 1.), on the other hand, had taken over a large number of functions and it had later even participated in the formation of the skin and of the intestine (as a cutis layer of the skin or as a muscularis). The final result of this is that in an adult animal we can no longer find any sharply-limited system of layers; we only have a whole animal before us.

\section{Two Difficulties of the Theory of Polykaryons}

There are in the main two facts which seem to be the cause of considerable difficulties in connection with our interpretation, in spite of the fact that such a large number of similarities can be observed in the Infusoria and in the Turbellaria. One difficulty consists in the fact that I consider the plasmodial-polykaryonic thesis as probably more valid for the evolution of the Eumetazoa, while at the same time I admit that the colonial thesis seems to be much more justified for the evolution of the Metaphyta and of Spongiae. The second difficulty consists in the fact that in the ontogenies of the Eumetazoa as well as of Spongiae, the first steps are regularly made by way of divisions of cells or of cleavages and not, as one would expect, by way of a division or polymerization of karyons, which would take place in a permanently uniform cytoplasm, and by way of a subsequent cellularization. Or, in other words, why is the supposedly primary morphogenetic process not recapitulated at least in the lowest Eumetazoa, in the acoelous Turbellaria? I have already partly shown that it is possible to explain rationally both these difficulties.

Above all it is comparatively easy to overcome the first difficulty. I think that it is much more probable-in spite of the recent attempts (A. A. Sachwatkin) to place the Rhizopoda with their amoebae at the root of Protozoa - that the Flagellata are the most primitive Protista (Protophyta + Protozoa). In the recent Protista we can find active two trends to a higher development, i.e. the development by way of a formation of 
colonies, and the development by way of a polymerization of karyons and a differentiation of the cytoplasm. The situation in Protophyta is clear because they depend entirely on a gaseous food, salts dissolved in water and the sun rays that penetrate the exterior of Protophyta. Their units had soon developed firm cell walls and they had shown from the very beginning an inclination to form coenobia and colonies (cormi). It is certainly characteristic that in plants the transition from the monocellular to a polycellular state had occourred several times, as this is now generally believed by botanists. The Volvox-line which has been so frequently misused as a pattern must be mentioned here as a special case. Volvox is characterized by the fact that it has remained a freely-moving form even after it had begun to form special "colonies" and after it had made the first steps towards a differentiation. Something similar had also taken place in the Dinoflagellata.

The subsequent evolution of the polycellular plants had been very slow and it did not make any important progress in the direction of an integration (individualization) and of a differentiation (the formation of tissues and of organs). Still we should not attribute all these facts as being due to the colonial origin of plants. The whole regime of metabolism has also had an important role in this connection, as well as the immobility of plants (the fact that they are attached to their substratum). A local and partial mobility was achieved in a different way than in the case of the Protozoa and of the Eumetazoa; this was due to the fact that no muscles or nerves have ever been developed by plants in spite of their ability to contract and to conduct irritation.

It has already been mentioned that in Metaphyta a significant formation of syncytia can not infrequently be observed (Caulerpa and its relatives, some tissues of higher Metaphyta which function as vessels, etc.). The example of Siphonales shows that no special plant type has ever been developed by Metaphyta by way of secondary syncytia which would include new elements that could be considered progressive. 
As in the case of Metaphyta, nobody (as far I know) has tried to derive the Spongiae from some polykaryonic ancestors; the colonial "spirit" of the Spongiae is much too obvious. The difference which exists in various interpretations of the origin of Spongiae are centred exclusively around the problem whether we can consider that Spongiae have a common origin with other Metazoa (Eumetazoa), or whether they evolved as a special type of polycellular animals from the colonies of specialized Flagellata, the Choanoflagellata. Originally, zoologists, who all came under the influence of ideas of Leuckart and Haeckel, were inclined to believe that the Spongiae do not represent an extra type, that instead they were genuine Gastraeadae. Numerous and important differences that exist between Spongiae and other Metazoa were noticed, yet again those differences were attributed to diverging evolutions which had started from a common origin (the primitive polyp). Scholars soon became perplexed when they found (Metschnikoff, Sollas, Delage, and others) that the greatest differences occur in just the earliest ontogenies; the interpretation has therefore been more and more widely accepted which sees in Spongiae a special case among the polycellular animals. In the system of the animal kingdom they were therefore separated as Parazoa (Sollas) or as Enantiozoa (Delage) not only from the Coelenterata but also from all the other Metazoa, the Eumetazoa. Nobody has found any objection to the fact that a cleavage occurs in the ontogenies of Spongiae in the same way as in the Volvocales and in the Eumetazoa.

This situation is definitely solved, when the derivation of the Eumetazoa from the colonies of Flagellata is generally accepted. Objections, even if not supported by any very important and convincing newly discovered facts (the supposed discovery of nerve cells in Spongiae by O. Tuzet and by her school cannot be considered as a sufficient proof) have suggested that the Spongiae had a common origin with the remaining Metazoa and that the differences which exist between the Spongiae and the remaining Metazoa were not 
absolute and that the same differences could be explained rationally.

As an instructive example I wish to quote here the interpretation that was proposed by Marcus (1958). He discusses the evolution of Spongiae in the following paragraph, "The oldest animals, the Zoomastigina, evidently gave origin to sponges and coelenterates, which are connected at their common root (Heider, 1885). Transitory forms between flagellates and sponges do not exist, as Proterospongia is a regenerating fragment of a sponge (Tuzet, 1945). As sponge larvae have ordinary flagellated cells, the Porifera need not have arisen from the Choanoflagellata. In any case, sponges and Cnidaria must have originated together, because both have germ layers. There exist other parallels between sponges and true Metazoa (Eumetazoa). The definitive layers of the Calcarea and simple Tetractinellida are achieved by a process similar to gastrulation by invagination. What previously had been described as inversion of the germ layers, is understood today as multipolar inwandering (Meewis, 1938). Spongillids show an "accélération embryonaire" (Brien and Meewis, 1938) like many limnic Eumetazoa. The alternations of shape in the blastula (pseudogastrula, stomoblastula, "plissement" of the coeloblastula of Halisarca) are intelligible as the result of the small space available for embryonic expansion in the body of the parent sponge. "The evolutionary line of sponges was divergent from the beginning and ends blindly." (E. Marcus, 1958:26).

All these arguments are supported by rather weak facts and they do not prove to be convincing enough. They could be complemented by two additional arguments which were mentioned by Tuzet; the supposed presence of nerve cells in Spongiae, and the fact that in some Eumetazoa, cells can be found similar to choanocytes. Yet all these objections cannot conceal the basic fact that the Spongiae do not possess an intestine (neither had they secondarily lost such an intestine), and in connection with this they do not have an oral or even less so an anal orifice. It should be mentioned in passing that a 
separate derivation of Spongiae from some cormi of Choanoflagellata can be considered to be more acceptable than a common deduction of all the Metazoa from some colonies of collar-less Zooflagellata. The former interpretation would appear more plausible even if the case that there were no Proterospongia were true, because no transitional form between the Flagellata and the Eumetazoa has become known up till now and in spite of this there are so many zoologists who "believe" nevertheless in the latter type of derivation. In the meantime another species of the Proterospongia has been discovered (we have already mentioned this in our study) and there can be no doubt about its nature.

And what can be said of the argument or even "proof" of the statement that the larvae of Spongiae possess ordinary flagellated cells which are only subsequently, by way of metamorphosis, developed into the choanocytes? This should prove, according to Marcus, that the Spongiae cannot be deduced from the Choanoflagellata! It seems as if the Spongiae were the only animals which had no right to evolve special early phases capable of swimming freely, and all zoologists can see in them an example of recapitulation. The spongulae are new forms like all the freely swimming larvae of the Metazoa; the animal does not need any collars of cytoplasm in order to be able to swim; these collars can rather be considered as a hindrance. The spongulae do not feed independently.

What can then be the value of the equation: germ layers $=$ the same origin? It is even less convincing than in the case of flagella which have been just discussed. All the germ layers are a product of a rationalized ontogeny. There are no, and there never were any, germ layers in the Metazoa, not even in their lowest forms. If, in spite of this, we wish to talk about the germ layers (better, body layers) in analogy to the ontogenetic conditions that can be observed in the higher Vertebrata, we see that it is just at this point that there are radical differences between the Spongiae and the Eumetazoa. These differences cannot be simply dismissed with the nice phrase: 
"the inversion of germ layers" which is so typical of Spongiae and which has been the reason that the name Enantiozoa has been given to them, is simply a "multipolar inwandering." In reality it appears also as an invagination and as a unipolar inwandering, thus in all the forms that we are used to find in a normal "gastrulation," yet it is usually reversely polarized! In the same way we could speak about germ layers in Metaphyta, or at least of something that is similar to this ontogenetic process. We are therefore not yet justified in supposing that there has been a common origin. Yet in spite of this, all the organisms represent a community, even if the complex organisms evolved several times. We must not forget that all the metabionts had originally evolved from the primarily solitary Flagellata. It is therefore not surprising that we find many common elements in them in spite of the fact that they evolved several times and invarious ways from these Flagellata.

There is no species of Spongiae - at least as far as I knowwhich begins its ontogeny with a division of karyons, thus without a simultaneous division of cells or without a cleavage. In the Eumetazoa on the other hand we can find such a development quite frequently even in such groups as the insects. We can rightly consider this type of development as a result of a secondary evolution. The larva (spongula) of Mycale passes subsequently, according to $\mathrm{H}$. V. Wilson (1935), into a syncytial state. A formation of syncytia, however, can also be found elsewhere among the Spongiae, especially in those cases where we have a formation of macroscleres. We are therefore not allowed to conclude immediately that in all those cases there had been a polykaryonic phylogenetic origin where an animal type of a metaphyte can be found which forms syncytia. The well-known case can be mentioned here in passing where under the influence of a very simple saline solution a larva of Polychaeta was changed into a plasmodial animal (Lilly). It would be wrong to conclude on this basis alone that all the Metazoa had evolved from some plasmodial ancestors. 


\section{Can Egg Cleavage Be Really Considered} as a Proof of the Colonial Theory?

We can now return to the question: can we conclude on the basis of the fact that the ontogenies of a majority of Eumetazoa begin with a cleavage, i.e. without a polykaryonic state, that the Eumetazoa also evolved (similarly as Metaphyta and Spongiae) from some colonies of the Flagellata? Those who adhere to the old colonial theory find this situation considerably simpler, especially if they believe in the validity of the "fundamental biogenetic law" and if they consider that the evolution proceeded in the direction Mono-, Di- and Triploblastostoidea. In this case one could expect in advance that the Eumetazoa also develop as postgamous individuals by way of polytomous or repeated divisions of cells, and secondarily and under special conditions plasmodially. This is the case when their zygote is overloaded with a reserve food while at the same time it is possible that a change in the type of breathing also plays an important role in this connection.

I think, in spite of the apparently greater probability of a generally colonial evolution of all the metabionts, that the Eumetazoa evolved from a plasmodial or polykaryonic state and that they adopted their cleavage as a result of a secondary development at a period when the older stages and the adult animal form had already become cellularized, even if we are well aware that we cannot offer any strict proof for this interpretation. We can make our interpretation only probable, and that on the basis of two "indications." The first indication which has already been mentioned could be the physiological necessity of the parcelling or of the cellularization just during the earliest ontogenetic stages because of the great consumption of oxygen and because of the need of large free surfaces which is a consequence of the increased consumption of oxygen.

The following objection can be made against this indication. If the cellularization had appeared at such early stages of the 
ontogeny, how is it then possible that this situation is not preserved and why does the internal structure return into the plasmodial state? To this we can remark that in all probability the physiological factors of the evolution as well as the inherited properties could be responsible for such a development. Moreover, we can call attention to the fact that even in Metaphyta and in (as we have just mentioned) Spongiae which both had certainly evolved from some colonies of cells, we can observe during a certain stage an inclination to form syncytia. This stage is again followed by a period of cellularization.

The second and even more important indication can be found in the long list of similarities that can be observed in the Infusoria on the one hand, and in the acoelous Turbellaria on the other. These similarities make it probable that the Eumetazoa had evolved from the polykaryonic Infusoria. We can therefore conclude that initially the postgamous divisions were limited to the karyons only, that the youngest embryos had a plasmodial character so that no recapitulations take place any longer in the early development.

Zawarzin (1945-1947) was not influenced, by the facts of cleavages, against the colonial theory. He placed the separation of the monocellular and polycellular animals far back into the remote geologic past, to a period when the separation of the animal and plant lines of evolution had taken place. The Metazoa had evolved, according to his interpretation, without any differentiation parallel to the Protozoa from the polyenergid plasmatic masses. This is a very radical concept which lacks probability because it presupposes that the sexual phase evolved in both lines (thus parallel to the plantal line) at least twice independently from each other and that it had led in spite of this to similar conditions. Neither does this concept explain the numerous similarities that exist between the Infusoria and the Turbellaria. How can we then interpret the not rare cases of the polykaryonic Protozoa? 


\section{The Basic Principles That Should Be Used in a Reform of the Whole Animal System}

The necessity, or the unavoidable consequence, of changing the previous interpretations of the phylogenetic processes of the animal world becomes self-evident when we summarize the individual results of our study. They can be enumerated as follows:

(1) The evolution of Cnidaria had been mainly retrogressive (it was partly progressive in a specializing direction) because of their sessile way of life. This sessility of Cnidaria is a secondary phenomenon. The evolution of Cnidaria did not proceed in the direction Hydrozoa $\rightarrow$ Scyphozoa $\rightarrow$ Anthozoa as this has been believed till now, but just in the opposite direction.

(2) The Cnidaria are not the most primitive and thus the lowest Eumetazoa in spite of the fact that they have the simplest structure. The most primitive Eumetazoa are in reality the mainly plasmodial Acoela, among the Turbellaria.

(3) The primitive Eumetazoa had not been radially symmetrical animals; they were instead bilaterally symmetrical which was a consequence of their way of life; they were able to move actively over a solid substratum. The radial symmetry had been developed secondarily as a consequence of their sessile way of life which had been secondarily adopted by them.

(4) The Spongiae are a special group of Metazoa, they show a special structural type, they have their own way of feeding and their own form of development. They must be definitively separated from any connection with the other Metazoa, the Eumetazoa. They stand thus as Parazoa between the Protozoa and the Eumetazoa. They must be therefore excluded from the group of Coelenterata.

(5) The Ctenophora cannot be considered as Coelenterata. Like Cnidaria they had evolved from the Turbellaria, yet starting from their own root and parallel to the evolution 
of Cnidaria. The Cnidaria had evolved from the rhabdocoeloid Turbellaria, and the Ctenophora by way of neoteny from the planktonic larvae of Polycladida.

It has been found that it has become necessary to submit the phylogenetic relationships of other animal groups to a critical revision in spite of the fact that all the findings made by me and the conclusions reached on the basis of these findings have been limited above all to the lowest groups of the Metazoa. This revision must be made from the same standpoint and under the application of the same working methods that have led me to a necessary reform of the interpretation of the evolution of Metazoa from Protozoa, and especially of the phylogenetic evolution of the Cnidaria. Thus a revision has become necessary of the genealogic tree and of the graphic representation of the phylogenetic connections of the large groups, as well as of the systematictaxonomic classification.

First I will make a brief explanation of those principles which I used in my work on a reform of the animal system.

The comparative morphology of recent animals and of those animals that have been preserved as fossils was, is, and remains the main method that can be used in our work when we wish to construct a natural animal system and above all the genealogical tree of the animal world. It can be expected that in the future biochemistry will also make essential contributions to this field. All the facts should be taken into consideration by the comparative morphology, both those that can be observed by way of a general and of a detailed analysis and comparison of the adult forms, and of all the other stages of development, beginning with the gametes; while at the same time we must also take into account the physiological and ecological moments and the changes of the environment. In our morphological comparisons we pay special attention to the adult stage, and only in the second line to the ontogenetic stages; we have been led to this both by the experiences we have had so far and by the theoretical conclusions. 
The historical circumstances have been the main reason why, since the days when the idea of evolution became accepted for the whole field of biology and above all for the field of phylogenetics - and in connection with this into the fields of systematics and taxonomy-scholars have relied too much on facts that can be observed in ontogenies. The wrong interpretation of the mutual relationship between the ontogeny and the phylogeny ("embryos-ancestors," according to de Beer) has played a particularly fatal role. It can never be repeated frequently enough that a basic mistake was made by the so-called "fundamental biogenetic law" as formulated by Ernst Haeckel which proposed that each ontogeny, even if it was secondarily more or less changed, was in principle a recapitulation of a phylogeny in the sense (which does not appear clearly in the formulation) that these were the earlier adult stages being recapitulated. At the first sight this difference does not appear to be too significant because recapitulations do indeed occur during the ontogenetic developments and they are also highly welcome in our phylogenetic operations. Even if we do not wish to discuss the whole problem, we will mention one typical example whose consequences have proved fatal.

This is the phylogenetic evaluation of a characteristic ontogenetic stage which occurs quite frequently, i.e. that of larvae. Any "proof" which could support the idea of evolution was highly welcome during the "storm and stress" period of biology when this idea still had to struggle for its final victory. The planktonic larvae of some sessile or parasitic animal types have not only proved to be of help in endeavours to determine the true nature of the corresponding adult forms which had been considerably changed. Peculiarities that are characteristic of a somewhat broader group of animals are preserved or recapitulated in larvae while at the same time these properties have been lost in their adult forms. Various zoologists, however, have gone further than this by stating that an ancestral form as such is recapitulated in larvae and 
that these ancestors were adult animals which lived in plankton. These scholars have not been satisfied with a recapitulation of individual properties or of whole stages (phases), they have believed instead that, in principle, the whole phylogeny can be recapitulated.

I have been accused of underrating those facts that can be observed in the ontogenies which could be used in the reconstructions of phylogenies. It is not difficult to show that it is the opposition which places so high a value on the same facts that they are actually in this way misinterpreted. As an example we can mention here in passing the development of the mouth in the ontogenies. The functioning oral opening of all the Eumetazoa is certainly a homologous form, i.e. it must have a common origin; yet in the ontogenies the same oral opening is developed during very different stages of development and in different ways (from the so-called primitive mouth, after the closing of this primitive mouth, and finally as a secondary intestinal opening while the primary mouth is changed into an anal orifice). It is true that these types of development have been observed in the ontogenies of several animal groups and that even if they are not a recapitulation of a common adult ancestral form (the old ontogenetic stages must naturally also be considered as ancestors) they can still be a common property which has been supplementarily acquired and they can still serve as a proof of a direct relationship. Should these conclusions be justified or at least probable then there must be no unexplainable exceptions, neither can a single peculiarity be decisive if it appears together with other common characteristics. In such cases the possibility must also be excluded that certain characteristics are not actual parallelisms, i.e. that they are not peculiarities that evolved after the separation of the lines of evolution had taken place, or that they had not emerged in completely different lines of evolution.

In our search for homologies, i.e. recapitulations of the morphological peculiarities of the adult stages, we must be 
very careful. Yet we should be even more careful when in this search we wish to identify the embryonic, post-embryonic, and above all the larval stages. Everything shows that the peculiarities of the not fully adult phases are even more subject to greater and special changes, regardless of the fact whether they live under the protection of the maternal animal or of protective hulls, or finally as free animals that feed independently; these phases can therefore not be considered as recapitulations in the old sense of the word. The changes and new formations which have a purely ontogenetic character have nothing in common with the peculiarities of the adult animal. It is therefore wrong to endeavour to show that such characteristics had already functioned in the ancestors of recent forms. This can be best seen in the attempt made by Boettger (1952) who tried to explain functionally the changes of the oral and anal orifices that can be observed in the ontogenies of the Eumetazoa (Protostomia-Deuterostomia). These and other similar changes can take place during the ontogenetic ontogenies only, thus during the nonfunctioning phase of the evolution.

This shows that generally we must be very cautious when we try to use the ontogenetic facts for our phylogenetic constructions (speculations). Other facts and methods must be also constantly taken into consideration. We must constantly distinguish between the material established in the nonfunctioning phases and that established in the functioning phases yet not in the sense of the adult stage, and between the final stages of development and those stages which directly precede the definitive stage, e.g. metamorphosis and the stage which frequently follows it. Metamorphosis emerged as a necessary phase of the ontogenies; it is a consequence of a widely different development of the larval phase. Such a metamorphosis can have really catastrophic proportions, or in other cases it can be considered as a non-functional ontogenetic event.

Those authors who try, too quickly, to conclude, on the basis of some similarities that can be observed during the larval 
stage, that there are some closer phyletic connections, will come in all probability to the wrong irterpretation of the phylogenetic developments. This is the case with the interpretation proposed by Marcus (1958) regarding the Ectoprocta and Endoprocta. He tries to unite these two groups because of an accidental similarity that can be observed in their larvae (the trochopl.oroid larvae) in spite of the great difference which actually exists between these two animal groups, especially as regards the organization of their adult forms. These are clearly analogies only that occur in the adult forms and in larvae (cf. Hadži, 1958 and P. Brien, 1960).

The more probable interpretation seems to be that special larval stages were developed independently by each larger group of animals. The way of life of these larval stages is completely different from that of the adult animals. We can come to a more plausible conclusion regarding the relationship connection only if we also take into consideration other very important characteristics and peculiarities which are not limited exclusively to the larval stage.

Fortunately enough we find that recently zoologists have become more and more convinced that it is necessary to relinquish the erroneous "fundamental biogenetic law" which sees whole phylogenies repeated in the ontogenies, with a very small number of deviations ("Fälschungen") which cover $10-0$ per cent only of the total number; all the rest are, according to this interpretation, palingeneses, i.e. recapitulations. We get such an impression when we read the reports (both reviews and discussions) given at a special symposium of zoologists from Northern Germany, Ontogenie und Pbylogenie (Zool. Anz., 164, 1960). On the other hand we find scholars who still adhere to this quasi-law and who try to save what can still be saved (Remane and his school). They courageously defend Haeckel's definition, making only occasional retreats, while at the same time they miss the main point which is that this old concept is wrong in principle when it compares the ontogenetic stages of recent animals with the adult 
ancestral forms and when it even equates these two different elements. Remane would like to reach at least a compromise end so he proposes that the word "rule" should be used instead of the word "law" while at the same time he still continues to use the old word. Such a proposition, however, does not touch the main problem, above all because zoologists prefer to avoid the word "law" quite generally in the modern biology, and even more so the phrase "the basic law." Neither has the adjective "biogenetic" been very fortunately selected; it should be avoided because in the general biology the word "biogenesis" signifies the origin of life on our planet. And above all we can see how senseless it is to speak in this connection of a rule if we use the expression "the rule of the relation between the ontogeny and the phylogeny" (or even better: "between the ontogenies and the phylogeny") instead of the phrase "the biogenetic rule." We have actually here a biogenetic phenomenon which we still have to study intensively, and where we are still far from knowing its exact character. Would it not be sufficient if we simply call this biological phenomenon a recapitulation in order to characterize in this way a special case of repetition which probably takes place in each ontogeny and which is apparently a reiteration of characteristics and peculiarities that had originally belonged to the adult stages of the ancestral forms? This would then be recapitulations in a narrower sense of the word which can serve as best proof in our phylogenetic speculations. Yet there are also other recapitulations (s. lat.) which may also prove useful in our phylogenetic constructions which, however, must be used with considerable restraint. These are the ontogenetic repetitions of those characteristics and peculiarities which have nothing in common with the final stages of the ontogenies, with the fully adult functioning stage. Haeckel called them caenogeneses (Fälschungen, falsifications, deviations). If we look upon this problem from the point of view explained here then we must admit that theoretically such cases can exist where the whole 23* 
ontogeny of a certain animal species or of an animal group had been changed so much that it no longer shows any trace of recapitulations s. str. We believe that it is completely improbable that cases could exist where up to 90 per cent of characteristics that can be observed in an ontogeny could be interpreted as true recapitulations. In this connection we can mention the important difference which can be found in the evaluations made by botanists regarding the frequency of recapitulations that can be observed in plants. Broman (1919) who studied this problem as an expert in genetics puts the maximum at 30 per cent, and Zimmermann (1943) even at 80 per cent.

In future the symmetrical conditions should be studied more critically than they have been till now, especially if we wish to use them in our phylogenetic and systematic-taxonomic constructions. Fortunately enough we can find that a considerable progress has now been made in this direction. The notion and the word Radiata and other similar names (Radialia, etc.) should be avoided from now on. We soon find, if we accept this suggestion, that the opposite symmetrical condition, the bilateral symmetry and the corresponding category Bilateria have also become unnecessary and that they should also be abandoned. These symmetrical conditions are very changeable. They depend entirely on the type of movement or on the kind of contact which exists between the animal and its immediate environment. Not infrequently, we can find different symmetries side-by-side in one and the same animal group (taken phylogenetically they naturally occur one after the other); some transitory forms have also become known.

Bilateral symmetry must be considered a priori not only as a characteristic but also as a primary property of animals which are able to move, in opposition to plants which are immobile. Radial symmetry, which in animals occurs only rarely, is therefore a secondary phenomenon. It is certain that a symmetry can be changed much more easily than the whole structure. A transition from a horizontal (parallel to 
the substratum) to a vertical attitude is sufficient to start a trend towards radial symmetry (towards the polypoid form). This change of symmetry can make a rapid progress regardless of the original form of structure when this induction is supported by an attachment of the animal to its substratum. It is obvious that the general type of structure nevertheless plays a certain role in this development. It is enough if we refer in this connection to the case of Aprocta-Euprocta. Radial symmetry can develop much more easily and fully in the aproctous type than in the Euprocta; in the latter the anal opening which was originally situated opposite to the oral opening must be first transplaced, and finally abandoned, before they are able to develop a perfect radial symmetry.

Recently, a significant tendency can be observed among zoologists to use the highest categories, especially that of the phylum, quite frequently when they classify larger groups of animals. Not infrequently, we can find systems which propose even up to forty different phyla, so that finally we could get an impression that there were the same number of completely different types of structures, and that the scholars are probably too subjective when they use the category of a phylum. It seems to me that in this way the true phyletic relationships only become blurred. It may be better for us to use the category "phylum" more carefully as long as we do not dispose of more exact methods of determining the antiquity of a certain structural form. The mistake will probably be less significant if we exaggerate in the opposite direction, even if in this way we expose ourselves to the accusation that we would like to take refuge in the old notion of a "progressive scale of all things," or of "l'échelle animale." Such an accusation is entirely unjustified as we will show later. We must pay our main attention to the classes, to their peculiarities and to their groupings which must not always be made from a purely formalistic point only. We can expect that in future many such uncertain problems will be clarified by means of biochemistry. 


\section{Evolution Generally Had a Progressive Trend}

Quite generally and, as it seems to me, correctly it has been assumed that the evolution has generally taken a progressive course. This, however, should not mean that there had been no retrogressive evolution. Cases of distinctly retrogressive phyletic evolution are well enough known and it is therefore completely unnecessary for us to try to prove them in our study. Whole classes have emerged as a result of a retrogressive evolution; yet at the same time we must be aware of the fact that these retrogressions did not lead to reductions only, but also to some new acquisitions which appeared as a consequence of a specialized way of life. This was the same way of life which had caused a reduction of important organs or systems of organs. An internal parasitism and a completely sessile way of life have been the main cause of such a retrogressive development (cf. Cestoda among the Ameria, Rhizocephala among the Polymeria, and Tunicata among the Chordonia). The question arises here whether any limit can be found in such a development in the sense: what is the highest systematic category where such a generally retrogressive trend can still be observed? When we see that there are not only classes (Dicyemidea, Cestoda) that had evolved by way of a basically retrogressive development, but also subphyla as this is proved by the case of the Tunicata, we cannot exclude the possibility that even a whole phylum had eventually evolved by way of an evolution which shows a mainly retrogressive trend. Later we will return to discuss this problem more in extenso.

Among other factors which must be kept in view when we try to reconstruct the phylogeny of the animal world or its genealogical tree that could serve as a basis of a natural animal system, we must mention the various interpretations which have been proposed regarding the origin of large groups. It is by no means surprising to see how widely different opinions have been adopted by the zoologists on just 
this point. They all waver between the two extreme interpretations because they do not know of any exact method by which to determine the absolute age of individual animal types (frequently we cannot even determine their relative age), nor have they available any evidence in the form of fossils. One of these interpretations believes that there is no difference between the process of specialization, or the evolution of species by way of slight steps in the direction to such a differentiation, and the evolution of very large types of animals. According to this interpretation the "geologic age" can be considered as the only factor; the whole evolution had been uniform and gradual, yet it was not shared by all the species or all the lines of evolution.

The opposite standpoint has been defended above all by the palaeozoologists, and it is connected with the names of several zoologists; it believes in special, i.e. revolutionary, changes which had led to the formation of completely new animal types. We cannot find as many animal types as would be expected if evolution had constantly been strictly slowly evolutive, and never revolutionary, marked by sudden greater changes. The origin of the minor taxons is now already well enough known. Important sudden changes could also be observed in various cultures and experiments. It is therefore almost certain that the new types do not emerge in the way as was formerly thought, so that a young bird could suddenly emerge from an egg of a reptile. It is also clear that in the development and the preservation of a certain type of structure much can also depend on the external factors (the conditions of the environment). It is not my task to discuss here in detail the problem of macro-evolution versus micro-evolution. Still I wish to emphasize that I accept the standpoint as represented by neo-Darwinism combined with that of the genetics. As in the case of numerous other problems of biology we consider the phenomenon of the origin of new animal types to be a complex factor. It can be only approximately conjectured where the limit is between 
micro- and macro-evolutions. It is my opinion that the evolution of new types does not take place in one way only, and that the rapidity of these changes can be widely different, i.e. that there are species which are very old and conservative, and species which are very young, of which we can say they have just been developed.

The fortunate discoveries from the younger geological ages (e.g. that of the Archaeopteryx) can help us to get a clearer idea how the development of a new sub-type, let us say of a class, must be understood. De Beer (1954) has shown how in this case and probably also in many other cases within the frame of the Vertebrata, new mosaic-like combinations of characteristics that had existed individually and scattered within the frame of an older subtype (the peculiarities of a type, however, in our case those of the Vertebrata, are preserved) emerge as mutations parallel to the changes of the environment. They develop into new combinations and as such they become "new" peculiarities which win a predominant role due to the working of natural selection.

This type of formation of new animal forms which should be considered as representatives of higher taxonomic units is now known under the name of Watson's rule. It has certainly not been the only element which influenced evolution. It can be considererd also in connection with the evolution of other animal types and, so it seems to me, also with the evolution of the Cnidaria within the frame work of the turbellarian type. Later we will return to discuss this problem.

There can be no doubt that the descendants which show considerable variety and which therefore represent new animal groups can be developed by way of neoteny. The bestknown case which has also been studied by way of experiments is that of Proteus; it could be established that in a not very remote past, a new subtype of tailed Amphibia had developed by way of neoteny; this subtype was given the rank of a family 
by the taxonomists. The geologically much older Appendiculata, among the Chordonia, were given at least the rank of an order. The Chaetognatha, which are considered by some zoologists as an extremely isolated group and which are therefore considered to represent a higher systematic category (e.g. Beklemischew), have even been given the rank of a special class. I have succeeded in showing (Hadži, 1958 c) that the Chaetognatha evolved by way of neoteny from some Brachiopoda.

In connection with the highest animal types, above all in connection with the high systematic category of phyla, it appears as very probable, (1) that there can be only few such categories; (2) that they must be very old taken from a geological point of view; and, (3) that they evolved as a consequence of a combination of a special genom, the change of the environment, the change in the way of life.

Finally, I must touch another problem even if I do not consider it to be of a very great importance. This is the question whether the phylogeny of animals had been monoor poly-phyletic. I wish to mention this problem because some accusations were made against me that, in my attempt to reconstruct the phylogeny, I approach, the pre-Darwinian concepts ("l'échelle animale," and other similar interpretations). Quite naturally I must reject such an accusation because it is entirely unjustified. There can be in principle no linear or ladder-like evolution if we accept the standpoint of the neoDarwinism. The alternative question of a monophyly versus a polyphyly is completely wrong. We can immediately see when we observe all the riches of the animal forms, and above all if we take into consideration also the palaeozoological forms, that the problem can only be of a monophyly and an oligophyly (yet not in an alternative sense). The few attempts to construct a genealogical bush instead of a genealogical tree have not been received favourably by zoologists. Something very similar has been proposed recently by Lemche (1958) who suggested four main branches or subordinate stems. 
In order to avoid any misunderstandings I wish to mention that I think here of large groups only. We can see, if we make a survey of almost innumerable attempts to construct the genealogical tree of the animal world, that almost all these genealogical trees are based on the principle of the oligophyly. This is also true for my attempt. If we observe the best-known case of the Vertebrata, where we are not obliged to work with special phyla, we can see that in these evolution had proceeded concordantly with the principle of the oligophyly, and not of the polyphyly and there had been probably only one diphyly when the reptilian type evolved on the one hand into birds, and in the other into mammals. Yet even these two highest groups did not evolve in all probability from a common initial point as can be understood if we view the extremely schematized sketches of the genealogical tree.

G. S. Carter who sees in my attempt a similarity to "l'échelle animale" has in his own attempt taken into consideration the diphyletic development only of his superphyla which correspond roughly to my phyla. The "radiation" of his superphylum Annelida, however, does not issue from a single point, or from a single root, but rather from a very prolonged curve.

The example of the evolution of the Protozoa shows that we are not always able to use the concept of monophyly only. Here polyphyly seems to be the most probable form of the evolution. Yet even here the word polyphyly sounds an exaggeration because it seems that there were only two to three separate lines of evolution. The word oligophyly appears therefore to be more suitable also in this case. The same is true for the evolution of the Metazoa from the Protozoa; it is quite improbable that in the evolution of the Metazoa there were more than two separate roots or points of separation, i.e. besides those of the Parazoa and the Eumetazoa, that of the Mesozoa also, as the third root.

The question also arises of how those cases can be evaluated and named where two or several subtypes had probably evolv- 
ed from one type, yet not by way of a radiation which had started from the same centre in the same geological age, nor from the same level reached by the primitive type. Such a development can be observed in the Turbellaria as the initial form for the evolution of Ameria with their numerous "side branches" or subtypes. Their developments had probably begun (this cannot be strictly proved) from separate "Anlagen of buds" (we cannot possibly speak here of roots).

This shows the relative character of the distinction between the "monophyly" and the "polyphyly." It frequently depends on the taxonomic rank we give to a certain animal group, above all whether this is a phylum in a narrower or broader sense of the word. 


\section{THE NEW CLASSIFICATION \\ OF THE ANIMAL WORLD AND \\ THE NEIW GENEALOGICAL TREE}

\section{The General Premises}

\section{The Limit Between the Plant and the Animal Worlds}

A general revision of a system, which is already more than one hundred years old and which has therefore become very stabilized, has now become necessary above all in connection with the earliest Metazoa, because of the discovery that the Cnidaria do not represent the most primitive Metazoa, that their simple structures are a consequence of their transition to the sessile way of life, and that they had evolved from the Turbellaria (the turbellarian theory of the Cnidaria). To these discoveries we must add here also the interpretation which sees in Spongiae a type of Metazoa that had evolved completely separately, and which tries to explain the possible origin of the Ctenophora by way of neoteny from the planktonic larvae of the Turbellarian Polycladida. The notion and the taxon of Coelenterata have now become superfluous because they have lost their meaning. There can no longer be any Coelenterata, rather, they have really never existed. This group was built on erroneous interpretations. We see that the notion opposite to that of the Coelenterata, that of the Coelomata, also loses its justification as a consequence of the abolition of the Coelenterata. What can be done now in this situation? 
I could have satisfied myself by selecting one from the unfortunately numerous animal systems, a system which would seem to me to agree best with the natural facts, and propose those changes which have now become necessary within the framework of the same system. This was done by the palaeozoologist A. H. Müller (1958:3) who has constructed a genealogical tree of the animal world mainly on the basis of the scheme which was originally outlined by L. Cuénot while at the same time Müller took into consideration my theory about the Coelenterata ("unter Zugrundelegung der Coelenteratentheorie von J. Hadži"). Even such a change of the system is sufficiently radical and it can be expected that the zoologists will prefer to oppose it than to accept it favourably. This, however, can only be interpreted as a good sign because a certain amount of conservatism may not be amiss here.

Yet such a solution would be insufficient above all because of the fact that the Coelenterata have usually been considered as identical with the Diblastica. Thus the problem of the middle body layer (mesoderm or mesohyl) together with that of the coelom s. str. appears in a new light, a problem which itself is of great importance because of its significance for the main division of the animal groups. All these reasons have induced me to place my reform of the animal world on a broader basis so that in this way we can come closer to our ideal; to develop a natural, i.e. evolutional, animal system.

The first difficulties we meet with, however, emerge as soon as we try to determine the position of the animal world (regnum animalium, animalia, zoa, Zoaea,) within the framework of the world of living organism (bionta, organisms). It is not my intention to discuss in detail these difficulties. We can distinguish between three main types of organisms if we take into account the way they feed; the same three types also show certain morphological differences. For reasons that are purely theoretical, and in agreement with our interpretation of the first emergence of the organisms ("of life" as we usually say), we must give the first place to the primarily heterotrophic 
organisms. We consider them to be the first and phylogenetically the oldest type. It cannot be proved with certainty that such organisms still exist. In view of the fact, however, that in the main evolution there was regularly one part only that had further evolved while another part had remained on the same old primitive level (certainly with some smaller changes) we must admit the possibility that there still exist essentially unchanged descendants from the primary heterotrophic organisms that were neither plants nor animals. We can think here at once of viruses; it is even possible that not only viruses can be placed into this group, and moreover not all the organisms that are now known as viruses can be classified into this group. In the meantime the viruses have become strongly specialized, they live now mainly as endoparasites with animals and plants. By way of a purely logical thinking we can conclude that in the era of the primary heterotrophic organisms the latter had lived freely, feeding mutually on each other. Such an era, however, could not possibly have been of along duration because under such conditions life would necessarily soon come to an end if the producers of the "living substance" had not evolved in the form of organisms which were capable of chemosynthesis; such forms can already be classified among the bacteria. A new difficulty emerges here of how to distinguish these primitive organisms from the "younger" saprophytes which are usually classified as plants. All these organisms can be considered as a special group, and since it is necessary to name them, we could call them Anucleata after Rothmüller, or Monera after Barkley (the latter name, however, does not mean the same thing here as it was understood by Haeckel).

The Anucleata which were capable of chemosynthesis probably also evolved into the second main type of organisms which feed autotrophically or by means of photosynthesis. These organisms are plants (Phyta) regardless of whether they are mono- or polycellular forms. All plants can be divided practically and roughly into Protophyta and Metaphyta; yet 
such a division does not seem to have much sense if viewed entirely from the phylogenetic standpoint. It is more than probable that the Metaphyta had evolved several times from various protophytic lines. Neither is it sensible to bring together in our system of organisms, all the nucleate (monocellular) plants and animals into one group of Protista (Protobionta, according to Rothmüller, Unicellularia, Thaloidea, Protista, according to Barkley; yet not according to Haeckel). We are not justified in proposing such a group because its members had evolved in all probability polyphyletically and because it is obviously necessary to make a distinction between plants and animals. Rothmüller believes that such a distinction should be made on a higher level only, on the level of the polycellular beings, where he distinguishes between the Cormobionta (i.e. Phyta, according to Barkley) and the Gastrobionta (i.e. Zoaea, according to Barkley; Metazoa s. 1. autorum).

The third main group consists of animal organisms which are secondarily heterotrophic (Zoa, Zoaea, according to Barkley). They feed at the expense of the autotrophic organisms. If we wish to remain consistent we must not classify as animals such Protophyta which feed either "occasionally" heterotrophically (while at the same time they lose their chromoplasts) or permanently in the same way as animals while at the same time they preserve characteristics that can be easily identified and which show that they certainly belong to the Protophyta. The fact alone that they are monocellular forms cannot be considered as sufficient reason for a classification of such a being as an animal. This is the case with the Cystoflagellata (with Noctiluca etc.) within the group of the Dinoflagellata. Because of such species or even whole groups we could think that there is no uniform group of animals. Zoologists should rather agree to exclude these forms from the animal world and thus to leave them to be studied by botanists or by protistologists who specialize in them. This would be the fate of the so-called Phytoflagellata which do not even 
constitute a uniform and taxonomically fixed group, of the so-called Mycetozoa (Myxomycetes, as they are called by botanists), and of the problematic Xenophyophora. If we drop the notion and the name of the systematic group Phytoflagellata then we are logically obliged to do the same with the Zooflagellata (as a name of a group). Zoology as well as the zoologists lose nothing if they transfer into the field of botany (Phytology) a number of Protista that had secondarily adopted the heterotrophic type of feeding. In fact they can win in this way a great deal because by means of such an exclusion a clear limit can be drawn between the world of animals and the world of plants. Moreover, a majority of "attempts" which were made by Nature to cross the limit between the autotrophic and the heterotrophic organisms soon came to a stop so that no new type of organism has ever evolved in this way. This is best illustrated by the example of the Cystoflagellata which consist of three monotypic genera only (among these Noctiluca is best known). Other descendants also have a completely isolated position from the typical Dinoflagellata that adopted the parasitic way of life.

Repeated attempts have been made to construct a system of Protista on the basis of their cellular structures. The Protista have therefore been frequently divided into the subcellular (without distinct cell nuclei: Protocytoidea, according to Naef), primarily cellular (among these are a majority of the monocellular Protophyta and Protozoa), hypercellular (with polymerized parts of cells, the cell nucleus included), and finally the polycellular and secondarily cellular forms. Such a classification does not seem to be very useful in our systematic and taxonomic work. It is not useful in connection with the animal world because the animals do not include any subcellular species; neither is it useful for the plant world because there are no secondarily nuclear plant species. Such a division, however, can be very good from a purely notional point of view, and within the broader frame of a general biology. Even the word "acellular," after Dobell, is usable 
as a notion in spite of the fact that it is formed in a rather negative sense: it is not uniform and it includes various categories (the subcellular, hypercellular, and actually also the primarily monocellular forms).

\section{The System of the Protozoa}

Now when we have drawn a clear limit between the Protophyta and the Protozoa we must also draw a limit between these and the higher forms. This does not seem to be such a difficult task because it has become clear that no corresponding transitional forms have survived till now. We can therefore still continue to use the name which was introduced some time ago, that of the Protozoa (Goldtuss, 1820) even if this word has not been most fortunately selected and in spite of the fact that in the meantime its exact meaning has been changed several times. The first sub-kingdom can therefore preserve its old name of Protozoa until it is changed by an eventual international decision. Few attempts only have been made to construct an animal system which do not include the taxon Protozoa (cf. the system proposed by Ulrich, 1951).

Scholars have usually been satisfied to give the rank of classes to the subdivisions of the Protozoa. Usually there are from four to five classes; this is dependent on the fact of whether we consider the Sporozoa s. lat. as one or as two classes (Telosporidia and Amoebosporidia, according to Doflein and Dogiel, 1937). The first scholar who proposed that the subregnum Protozoa should be divided into two larger subgroups was B. Hatschek (1888). He proposed in his famous Lebrbuch der Zoologie (unfortunately never completely published), that the first three large groups, i.e. Flagellata, Rhizopoda, and Sporozoa, should be included in the phylum Cytomorpha while each of these large groups should be given the category of a cladus (thus a higher category than that of a class). The phylum Cytoidea (i.e. "similar to the cells," now 
the "hypercellular forms") should consist of an independent cladus of Ciliata. Hatschek also united the two phyla into the division Cytozoa. In spite of the fact that Hatschek was the first zoologist who suggested such a classification we now find the same division generally used, yet under other names which were coined (quite unnecessarily) by Doflein (1901) as the Plasmodroma and the Ciliophora.

In many respects the Ciliata represent in fact an isolated group among other groups of Protozoa. The standpoint can also be defended that the climax of the progressive evolution of the Protozoa had been reached in the Ciliata. In spite of this it seems to be rather problematic that we should separate the Ciliata so thoroughly from the Flagellata by means of such a strict division into two large groups because it is quite certain that the Ciliata evolved from the latter. It may be better and more in agreement with the genetic conditions if for the present we avoid dividing the main groups of the Protozoa taxonomically into subgroups. In morphological practice, however, the notional pair Cytoidea and Cytomorpha may prove useful, as is also the case with the notion of the Undulipodia (Hadži, 1944) which includes the Flagellata and the Ciliata because of their undulipodia (flagella and cilia). In the literature we can frequently find that the division of Protozoa into two large subgroups has not been accepted (cf. Hall's Protozoology).

Some protozoologists, e.g. Bělař and others, may be right when they suggest that the taxon "phylum" should be given to those groups of Protozoa which have been given the taxon "class" so far, even if in the Protozoa the morphological differences (the structural forms) are not so conspicuous, owing to their acellularity and small dimensions, as in the geologically younger Metazoa. We must take into consideration here the fact that the Protozoa had at their disposal a much longer period for their evolution than the Metazoa. This circumstance has perhaps also been the reason why protozoologists are still so uncertain regarding the relation- 
ship and connections that exist within the sub-kingdom Protozoa. This uncertainty is met with as soon as we try to solve the question of which group of the Protozoa should be placed at the beginning. Naturally enough this question is also connected with the problem of the origin of the Protozoa.

If we bypass the earliest studies of the Protozoa we see that initially a majority of zoologists had considered the Rhizopoda and the amoebae as the most primitive Protozoa because they do not have a permanent external form and because they develop transitional pseudopodia only. These forms were considered as the initial forms for the evolution of the Flagellata and subsequently also of other types of Protozoa. Since about the time of E. A. Minchin (1912, cf. Hall), however, the Flagellata have usually been given the first place because they have been considered as a form that had developed from the Phytoflagellata. Pascher has later especially brought forward important arguments in favour of this new interpretation. This, however, did not mean that this difficult problem has been definitely solved. On the one hand there appeared Sachwatkin (1956) as an assiduous propagator of the older interpretation which believes in a priority of Rhizopoda and of amoebae. On the other hand Grassé (1948), an excellent and experienced researcher in Protozoa, has recently suggested that the two large groups of Protozoa should become united into a new group which he called Rhizoflagellata. These should represent a large uniform central group which had served as a starting point for the evolution of all the other groups of Protozoa. The main argument Sachwatkin brings forward to support his theory of the priority of the Rhizopoda is-besides the unpolarized character of these animals and a supposedly primary absence of flagella which Sachwatkin derives from the actinopodia of the Heliozoa-above all their primary heterotrophic type of feeding. He brings this fact in connection with Oparin's theory of the origin of organisms. The possibility cannot be 24* 
excluded in principle that the primarily heterotrophic organisms (in all probability micro-organisms) - thus the organisms that had existed earlier than the eutrophic forms - had evolved into the animal Protista, thus into the Protozoa; yet at the same time such a suggestion cannot be made probable either. It must be assumed, if such a deduction be right, that the flagella had evolved at least twice, or we must suppose that the Protophyta had evolved from the Protozoa which does not seem to be probable at all. For all these reasons the interpretation which believes in the priority of the Flagellata appears to be more probable. The suggestion made by Grassé has one purely formal advantage because it avoids the problem which of the two groups should be given the priority. We could justify such a unification from a factual point of view only if we could prove that the common primitive form had both flagella and pseudopodia and that the separation of forms did not take place once only but several times. The suggestion for such a unification has perhaps been made too early and it is possible that in future it will be found that the suggestion has been justified. It seems to us that the Flagellata represent the central type of the Protozoa, that all the other types of Protozoa up to the Ciliata had evolved from the Flagellata, and that the Flagellata had played a role similar to the role of the Turbellaria, among the Metazoa.

Here we are not interested in any further and more detailed taxonomy of the Protozoa inasmuch as it has no connection with the evolution of the Metazoa. We would only like to point to the fact that the polykaryonic (plasmodial) states can be found developed by way of nuclear divisions - thus by way of a polymerization of nuclei (which, however, is not identical with the incomplete division!)-everywhere in all the large groups of the Protozoa, even in such that lead an entirely parasitic way of life; and that a process can afterwards be observed in the personal development cycles which is very similar to a cellularization. This process takes place in such a way that either the "young cell" is, so to speak, excised from the 
plasmodium (as we usually call the polykaryonic state), or so that the whole polykaryonic mass disintegrates into the monocellular, and thus simply cellular, parts or individuals.

On the other hand comparatively few cases can be found among the Protozoa s. str. where permanent unions (colonies, cormi) appear as a consequence of successive divisions (palintomy, according to Sachwatkin). If we exclude the Ciliata, we can find such formations of colonies among the more primitive Flagellata. As far as I know there does not occur even a single case among the Protozoa below the Ciliata where even a slightest trace of a polymorphism can be observed among the members (zooids or persons) of the colonies of Flagellata, neither can we find any differentiation into the purely vegetative and into the sexually active zooids. The Ciliata on the other hand can sometimes be found to develop colonies. In these colonies the zooids had developed mutual organic connections by means of stems, and here we can also find a slight differentiation of zooids even if it appears in their sexual characteristics only. So far nobody has ever thought of deriving the Metazoa from such "colonies" of Ciliata.

\section{The Transition from the Protozoan to the Metazoan State}

Thus it appears that the main line of evolution had in all probablity proceeded, as far as we can establish this on the basis of our present positive knowledge, from the Cryptomonada (according to E. C. Dougherty) by way of Chrysomonadina (Cuénot) to the Zooflagellata (Mastigophora) with the Bodonidea or quite generally Protomonadina (according to Cuénot and Grassé) at the beginning. Here a rich ramification can be observed. It had led to the emergence of the Rhizopoda or the Sarcodina, and the two parasitic groups which are usually united in our classifications under the name Sporozoa in spite of the fact that they had evolved at least diphyletically. 
These Flagellata had afterwards evolved along at least four diverging lines: The first two lines ended "blindly" as Protozoa. The first of these lines is represented by the Flagellata. In this line we can observe a strong inclination to polymerization. The line ends with the Hypermastigina which now only live in other animals. The second line is very short, it consists of a

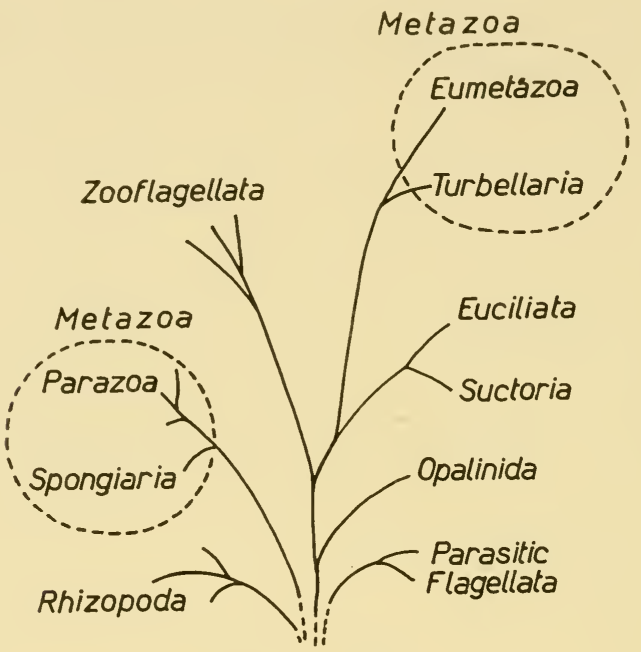

FLAGELLATA

(Protozoa)

Fig. 49. Evolutionary relationship Flagellata-Metazoa; (orig).

few species only which belong to the Opalinidae (Opalinata, according to Wessenberg). These again live as parasites in the bodies of other animals. The third line had actually broken from the narrow frame work of the Protozoa, yet it evolved into a weak branch only of "polycellular" forms or Metazoa, i.e. into the Spongiae whose subsequent development had soon ended blindly (Fig. 49). It was the fourth line which had played a leading role; it had begun with the primitive Ciliata and it had continued to evolve all the way along the main line of the development of the Eumetazoa. 
All these suggestions have a strongly hypothetical character. It is therefore not surprising if we find numerous other interpretations of this development. A majority of zoologists consider that the Ciliata represent a blind side branch of the Protozoa, and that the Metazoa had evolved directly from the colonies or aggregations of the genuine Flagellata. It is believed that this latter evolution had been either monophyletic (e.g. from the Choanoflagellata, according to Lameere) or diphyletic (once as Spongiae and the second time as Eumetazoa). We will not mention here all the other interpretations which are even less probable or which are occasionally quite bizarre. We can give here as an example only the interpretation that was proposed by Awerinzew (1910); he considers that the Metazoa did not evolve from the Protozoa, and that instead the two groups developed simultaneously along two parallel lines of evolution from some even more primitive ancestors. Franz, too, considers that the Protozoa and the Metazoa only had some common ancestors; these ancestors were algae which had already reached a somewhat higher level of development and which afterwards evolved on the one hand into the Protophyta and these into the Protozoa; the higher plants had evolved from the Protophyta parallel to the development of the Protozoa; the other line of evolution had gone from the algae by way of a "blastaea" towards the Metazoa. Fortunately enough, such extravagant interpretations have never been accepted by anybody.

It is generally considered that the Ciliata evolved (mono- or polyphyletically?) from the Zooflagellata; yet no recent transitional forms have ever been found, neither do we know of any transitional forms between the Ciliata and the Metazoa. Zoologists have tried to avoid these difficulties by referring to the pattern that can be observed in plants, yet at the same time they have completely forgotten that this is a pattern only, an analogy, which represents a possibility and which must not be interpreted as an indication and even less so as an evidence. There are a few facts only which take place in the ontogenies 
of Metazoa (cleavage, the "blastaea" stage) and which could eventually be interpreted as indications; yet there are also other possible interpretations of these same facts which are even more probable. It has already been emphasized that the only satisfactory evidence that can be found in the recent Protozoa point to the evolution of the Spongiae from the Choanoflagellata, while there are no facts available which could be used as evidence of the corresponding evolution of the Eumetazoa.

In my opinion there are three more or less probable ways how we can interpret the evolution of the Metazoa from the Flagellata. These three variants are:

(1) Spongiae Eumetazoa

Flagellata $\rightarrow$ Ciliata

(2) Spongiae

Flagellata $\rightarrow$ Ciliata $\rightarrow$ Eumetazoa

(3) Ciliata

Flagellata $\rightarrow$ (blastaea $) \rightarrow$ Mesozoa $\rightarrow$ Spongiaria $\rightarrow$ $\rightarrow$ Eumetazoa

There is no great difference between the first and the third variant. It is only a question of the interpretation of the origin of Spongiae. We have already dealt sufficiently with this problem in our discussions and we have come to the conclusion that the Spongiae represent an isolated group. The diphyletic origin of the Metazoa (the variant No. 2) appears therefore to be more probable than the monophyletic evolution (the variant No. 3). There remain the variants No. 2 and No. 3 only if we disregard the variant No. 1 which seems to us be quite improbable. Here we do not take into account the inessential differences that appear within the framework of the third 
variant, i.e. whether evolution proceeded primarily by way of a blastaea, or a planula, or a parenchymella. Certain characteristics that are common both to the Spongiae and to the "genuine" Metazoa (the Eumetazoa) do not disprove the diphyletic origin of the two groups and the independent evolution of the Spongiae. The same properties (these common characteristics are generally the sexual phase and the ontogeny which follows this sexual phase, with its initial phases, the cleavage, the blastula, the gastrulation) can also be found in the plant world during the transition of plants into the polycellular forms, above all in the Phytomonadida or Volvocales. These similarities are therefore clearly parallelisms.

One more possibility can also be taken into consideration which, however, can also be considered as a variant of the pattern No. 3. This possibility is that all the Metazoa had evolved from the colonial Choanoflagellata. According to such an interpretation the Spongiae were the first which had evolved, while the remaining Metazoa, the Eumetazoa had developed from a very early phase of this phylogeny. We find such a variant, which can hardly be defended, quite unacceptable (Kemna has perhaps thought of some similar kind of development?).

Thus we find that contrary to the situation which exists in plants it is comparatively easy to divide the animal world into the Protozoa and the Metazoa inasmuch as we do not consider that the Protozoa consists exclusively of the heterotrophic monocellular (i.e. mononuclear) animal species. The limit between the Protozoa and the higher forms is also clear and I dare maintain that no transitional forms between the Protozoa and the Metazoa will ever be found among the recent species; such forms had probably died out long ago. 


\section{There Are No Well-Warranted Mesozoa}

Before we begin to deal with the taxon Metazoa it may prove useful if we discuss briefly the problem whether the taxon Mesozoa, which is frequently used by zoologists, be justified or not.

Without any intention to go here into details we wish to state that we understand as "Mesozoa" only the Orthonectoidea (Orthonectida + Dicyemida). In all the other cases which were also considered as Mesozoa it has been possible to establish with considerable certainty that they were not real Mesozoa s. l. We will therefore not discuss them in this connection. So far the Mesozoa have been given various taxonomic evaluations; as a maximum they have been considered as a sub-kingdom (cf. Zoological Names, etc., published by AAAS, edited by A. S. Pearse, 1949); as a minimum they have been classified as an appendage only of the Platyhelminthes (e.g. by Cuénot). Moreover they have also been evaluated as a phylum (e.g. by L. H. Hyman, The Invertebrates, I., 1940) which is a middle-way found between the two extremes. So far the whole problem has remained unsolved. The most extreme solution can be found in Beklemischew (1958) who simply does not mention the Mesozoa at all in his systematic survey of the animal groups because of the uncertainty which exists regarding their systematic position. In this way he is not obliged to accept the auxiliary solution and to represent the Mesozoa as a kind of appendage. Beklemischew's solution is quite naturally purely formalistic. We must decide which interpretation appears at the present moment (on the basis of our present knowledge) as the most probable.

The main problem is whether the simplicity of the body structure which we find in these animals can be considered as a primary phenomenon, or whether it is a result of a secondary development which had taken place as a consequence of their parasitism. It appears as much more probable 
that it is a result of a secondary simplification (so-called "degeneration") because these animals are very specialized endoparasites which pass through a phase, even if short, of a free life. This, however, does not mean that there had been no partially progressive evolution, especially at the point of the development cycle; this can be observed in the Sporozoa among the Protozoa and in the Platyhelminthes (Trematoda). We will leave it undecided whether this was a case of the neoteny with the miracidium larva as the initial form, or something else.

This interpretation will appear a priori more probable to those who accept the theory that the Metazoa evolved from some colonies if they take into consideration the classical interpretation that evolution proceeded along the line blastaea-gastraea. Yet the planula thesis too seems to be now much less firmly rooted (Hatschek suggested the phylum Planularia, with the Planulozoa as the only cladus).

Those who accept the polykaryonic theory could derive the Orthonectidea directly from the Ciliata by way of a polymerization and of a partial cellularization which can be easily identified. There is, however, a large number of facts which completely disprove such a derivation. The equally numerous similarities appear as analogies, as a consequence of a retrogressive development which was caused by their endoparasitism. The Dicyemida seem to be much more reduced forms than the Orthonectida because they are built of a smaller and definite number of skin cells. It cannot be therefore expected (as this has been repeatedly emphasized) that anything essentially new could come to light with a discovery of those parts of the life cycle of these animals which have remained so far unknown. In all probability such a discovery will not help us to get a more precise knowledge of the origin of the Orthonectoidea, even if such a possibility cannot be completely excluded.

We come therefore to the conclusion that there are no Mesozoa in the sense as this word has been used till now. Neither had they ever existed, because both the Spongiae and 
the Acoela had in all probability passed quickly through the intermediate stages of evolution while there had been in all probability no third type of evolution. It is therefore completely improbable that any corresponding transitional forms will be found in the future. Even if such forms had existed in the most remote past they have certainly not left any identifiable traces of their existence.

Otto Steinböck (in his report at the symposium held in California, 1960) has recently made an interesting suggestion of considering the Ciliata as actual Mesozoa. This suggestion is in fact nothing more than an ingenious observation; the actual Ciliata are in reality genuine even if complex Protozoa, similarly as are, for example, the Nummulites, the Hypermastigina, and the Opalinidae. The evolution of the Metazoa had gone parallel to the evolution of these forms and there are the hypothetical Proacoela only which could be considered earnestly as "Mesozoa."

\section{The Classification of the Metazoa}

\section{The Parazo as an Independent Subregnum}

In spite of the fact that the evolution of the complex "polycellular" forms had been so widely different in the plant and animal worlds we find that this difference does not have an absolute character. All the known facts if viewed objectively disprove that the evolution of these complex forms-which had made possible their differentiation and a larger size of their bodies - had been monophyletic within the sphere of animals and that it had been basically different from the evolution of plants. There are the Spongiae which have been given such widely different scientific names and which have been found to be problematic in several respects; in a certain sense they are more similar to plants than are the remaining polycellular animals. Originally they were considered as Zoophyta; the 
founders of the theory of Evolution, however, and above all Ernst Haeckel, had the unfortunate idea of classifying them as Coelenterata. A little later, before scholars succeeded in identifying the completely aberrant ontogeny of Spongiae, they were pronounced by Huxley (1875), Sollas (1884), and by other zoologists to be a form which stands completely apart from other polycellular forms. And what had been the main reason why the Spongiae with their special organisation and way of life have continued to be considered as Coelenterata in spite of the discovery made first by Metschnikoff of the special character of their ontogenies? It was again the fatal gastraea theory as it was proposed by Haeckel! Spongiae occasionally possess (as is also the case with the Eumetazoa) a freely swimming larva which has on the whole the form of a blastula. This form has therefore been considered as a recapitulation of the blastaea. It is followed by a gastrula-like form which again has been considered as a recapitulation of the gastraea. Is there anything else that could be desired?! Haeckel, as a master in this field, has also coined attractive names: Olyntbus and Ascon. In his scheme he placed them possibly close to the Hydra and to the "typical" gastrula and the whole impression reached in this way was quite convincing. This idyll was not disturbed by an actually quite unpleasant fact that the "spongian gastrula" becomes attached by means of its primitive mouth, that it does not possess a digestive intestine, that it subsequently develops a new opening at its "aboral" pole whose function is rather that of an anal orifice than that of a mouth (thus the Spongiae would therefore be a special type of the Deuterostomata), and that the Spongiae show numerous orifices (pores) in their body wall where the sea water is drawn by means of choanocytes (they substitute here the digestive entodermal cells) into the internal system of canals in order to be finally forced out through a larger opening (the osculum) after it had gone through a special filtration system and after its oxygen had been removed. As far as I know there in not a single species of Spongiae which as such would 
consist exclusively of the sexually mature and solitary olynthi or ascons. It may be therefore better to speak of an olynthus stage which appears above all during the ontogeny (perhaps as an already sessile larva or as a kind of a postlarva) of the Calcispongiae. Complex individualities are soon developed by way of a further growth (budding or incomplete divisions) and in these it is frequently difficult to identify the single individuals or persons. A labyrinth of external and internal canals is soon developed. This complex can finally adopt a homogeneous form, it can become an individualized complexus (cormus). Most frequently, however, we find the olynthus stage not repeated at all. Larvae of benthonic or even sessile animals are not recapitulations of any ancestral forms, neither are spongulae anything of this kind. Their structures and forms show an equally rich variety as can be observed in the Cnidaria larvae.

The Spongiae have repeatedly been discussed in the present study, in which I have also tried to explain the phylogeny of the Spongiae as it appears to have taken place. I have also criticized the opposite arguments, above all those that were brought forward by Tuzet, which try to place the Spongiae as members of the large group of Metazoa. I definitely accept the thesis which considers that the Spongiae evolved quite independently of the main body of the actual Metazoa from the cormi of the Choanoflagellata, in spite of the fact that the Spongiae represent a polycellular animal type with a complex structure. Taxonomically the Spongiae must be considered without any doubt as something more than a phylum. We only have to make the decision whether we should place the Spongiae together with other polycellular forms (which evolved in all probability from a common root of protozoan ancestors) into one and the same subregnum of Metazoa, or whether it would be better to consider them as a special megataxon similar to that of a subregnum which could be inserted between the Protozoa and the Metazoa, or the Eumetazoa. In the second case we could 
use the already existing name of Parazoa Sollas 1884. It will be better for us to accept the view that each taxon should include one animal group only which had evolved monophyletically; we should no longer use as a taxon the notion and the name Metazoa, which should represent from now on a morphological notion only. Instead of the Metazoa we should rather use the name Eumetazoa when we speak of a special taxon.

The name Enantiozoa which was proposed by Delage is not only younger, it is also less fortunate; the question is not that in Spongiae the three body layers occur perhaps in an inverted sequence, i.e. that the skin is found in the interior of their bodies and that the intestine occurs externally, but rather that the Spongiae possess an external body layer which is not homologous with the skin of the Eumetazoa, and a nonuniform inner body layer which cannot be considered as homologous to a reversed intestinal layer. These are in reality morphological units which cannot be mutually compared, which are not mutually homologous. It is true that during the ontogeny we could get an impression as if a reversion of the two layers had taken place. Nothing can help here even if we try to save the old interpretation by printing pictures of spongulae, as has frequently been done, with the actual posterior end (posterior as seen when the animal swims, and quite generally during the polarization) turned upwards. This serves as a good example of how even the most difficult problems can easily be solved by means of a deliberate misrepresentation of facts in false pictures. This can lead, naturally enough, to apparent solutions only.

The name Porifera, which was introduced by R. E. Grant is also very characteristic of Spongiae. Nowhere else in the whole animal world (with perhaps the only exception of the Echinodermata) can pores be found which do not serve as openings through which the animal excretes its own products or the water that had been used for breathing, but rather as openings which serve for the intake of water that brings the necessary 
food to the animal. In spite of this we still prefer the name Parazoa because the word itself ends in -zoa; such an ending is much more suitable for the few subregna of the animal world and it should therefore be used in this sense only.

\section{The Eumetazoa and Their Subdivisions}

The Elimination of the terms Coelenterata: Colomata. So far there have been no essential differences between our system of the animal world and the traditional and generally used systems. Yet the whole basis of the subsequent system necessarily becomes changed when we take into consideration the consequences of our interpretation that the real polycellular forms (the Eumetazoa, or the Metazoa s. str.) had evolved from the polynuclear (i.e. polykaryonic) or plasmodial Protociliata. From now on the organization does not develop anew from some independent units, from the protistic individuals which had been previously free, as had been the case with the "Metaphyta" and with the Spongiae. This kind of evolution leads, with a few "initial" exceptions (Volvocales), exclusively to the formation of sessile organisms. The evolution which had led to higher forms followed another principle: and in these the cells gathered to form a new individuality of a higher type, a new whole with a complex structure.

From the very beginning the genuine animal freely-living polycellular forms had taken over the higher organization which had been reached already on the level of the Protozoa (Ciliata). Thus, it was the eumetazoan organism which formed these cells and not conversely that the previously individual cells formed a polycellular organism, as was the case with the Metaphyta and with the Spongiae. This is the only way the development of an internal digestive organ can be rationally explained: thus neither by way of an invagination of a hollow sphere, nor by a wandering in of individual earlier skin cells into the interior of the same sphere. The names Enterozoa, according to Lankester, or Gastrobionta (animals with an 
intestine), according to Ulrich, which have also been suggested for the Eumetazoa are therefore very suitable.

We must not be led astray by the types of formation of the "body layers" which can be observed during the ontogenies; these types are mutually different and they change more and more during their subsequent development. These ontogenetic types of formation can even become identical in the two lines of evolution which are basically different (in the phylogenetic sense!); on the one hand in the evolution of the Metaphyta and of the Spongiae which alone are without an intestine, and on the other hand in the evolution of the Eumetazoa. We must liberate ourselves from the belief that the ontogenetic morphogenesis via blastula-gastrula were identical with the phyletic morphogenesis via blastaea-gastraea, as if the ontogenetic type of development were identical with a recapitulation of the phyletic (i.e. of the fully developed forms) development.

A digestive plasmodium existed even before there was any ontogeny of the Eumetazoa; it had been a substratum of the subsequent intestine in the interior of the proeumetazoon where the cyclosis of the digestive vacuoles had been stopped. There was no need of a gastrulation by way of a transference of the blastula epithelium into the interior of the animal. It was only due to the introduction of the ontogenetic morphogenesis (which finaliy became necessary) combined with an initial cleavage that the gastrulation processes finally began to take place. We can therefore still continue to use the notions gastrula and gastrulation even in this new situation, yet without any parallel idea of a blastaea or a gastraea. No Blastaeadae or Gastraeadae had in reality ever existed and no such forms can be found among the recent animals either, at least as regards the Eumetazoa.

Quite frequently we can see in some Cnidaria and Endoprocta how an intestine (the entoderm) develops from the "skin" (the ectoderm) during the ontogenies or during the so-called atypical budding. Experimental embryology, however, has shown that these processes are an instance only of an 
ontomechanism as this has been suitably named by $O$. Steinböck, or of the "morphogenetic technique" as I called the same process even earlier.

Viewed from our standpoint it appears as self-evident that the principle of a simultaneous emergence of the third, the middle body layer, or of the so-called mesohyl, with the second body layer has the same validity. All the Eumetazoa are without any exception three-layered animals if there is any sense at all of speaking about three layers in the adult animals, because there are frequently more than three layers present even as early as in the ontogenies. The Eumetazoa inherited all these three layers (zones, parts) from their prociliate ancestors, and these three layers had therefore not been newly developed by the Metazoa. The pair Diploblastica: Triploblastica is therefore completely superfluous. All the three body layers evolved continuously and parallelly without an internal struggle of the originally independent elements against the secondary centralization combined with the subordination, as had necessarily been the case with the evolution of the colonies of the Protozoa.

In connection with a general classification of the animal world and with taxonomy it is important that we call attention here to the subsequent evolution of these three layers. This is necessary because one can frequently hear the objection that too great an importance is usually attributed to the conditions of the middle body layer, i.e. of the mesohyl and of the coelom. As a matter of fact, however, we find that during the ontogenetic and phylogenetic developments the morphogeny is much more intensive in the middle layer than in the two remaining body layers. In order to be as brief as possible I wish only to mention that it is above all the duty of the external layer to "serve" as a means of protection (it is used as an organ of movement by means of cilia in the more primitive and small forms only; this property had been inherited from the infusorian ancestors); it also produces gland cells and the cuticular formations. And what is produced by the middle layer? 
Both the skin and the intestine receive a mesohyl layer with muscles, nerves, etc. Later we will return to discuss again in detail the products of the middle body layer and their destinies. We are therefore fully justified in paying special attention to the conditions of the middle body layer in our attempts to construct a natural system of the animal world.

In opposition to the numerous attempts that have been made so far to construct a classification of the animal world on the basis of the colonial theory and in agreement with the hypothesis of an evolution along the line blastaea-gastraea where a bifurcation in the sense Coelenterata: Coelomata becomes necessary at the very beginning of the Eumetazoa, we find the classification of animals considerably simplified if we construct it on the basis of the plasmodium theory of the origin of the Eumetazoa in the sense of a development along the line Prociliata-Acoelia (Turbellaria). The adherents of the colonial theory have been unable to identify any well-founded and therefore probable phyletic relationship between the Coelomata and the Coelenterata. On the other hand it is possible to make a rational and probable interpretation of the phyletic, systematic, and thus also of the taxonomic conditions as they existed at the very beginning of the Eumetazoa and of their subsequent evolution if we view them from the aspect of the plasmodial theory and in combination with the new interpretation of the phylogeny of the Cnidaria. We can express the uniformity of the Eumetazoa both in their system and in their taxonomy if we abandon the opposing pair Coelenterata: Coelomata (which is actually identical with the pairs Diploblastica: Triploblastica, and Radiata: Bilateria) and if we place the Turbellaria at the beginning of the Eumetazoa. The symnetrical condition which as a property of the animal body has played so far an important role (which was unfortunately only a negative one) will lose from now on its importance. We have shown that in the Eumetazoa there has never been any primary radial symmetry; that this symmetry emerged more or less developed only in those animals which $25^{*}$ 
had adopted, for a long time, the sessile way of life. We have also shown that there is no justification in a distinction between the primarily two and three-layered Eumetazoa. We must therefore cease to use this supposed difference in the taxonomy.

There have been still other systematic attempts where a bifurcation was suggested at the very root of the Eumetazoa. Thus, for example, the Metazoa (s. 1.) were divided initially by Hatschek into the Protaxonia and the Heteraxonia. This is thus a classification on the basis of a supposed difference in the axial conditions. Hatschek himself later abandoned this classification because it was soon found that the axial conditions are in reality very unreliable, that they have a role similar to that of the symmetrical conditions in the phylogeny and they are strongly changeable especially during the ontogenies.

A completely unacceptable classification was made by Colosi (1956:505). He divided the Eumetazoa into the Perineura and the Epineura. It is only the Coelenterata that are included among the Perineura while even Ctenophora do not appear in this group. Colosi suggests that the Coelenterata consist exclusively of the Cnidaria. He naturally believed in the primary nature of the nerve-net system without a dorsal brain ganglion. On the other hand, he inclines strongly to a key-like dichotomy in the general classification of the Eumetazoa which he pursues for not less than eight stages up to the category of types (i.e. phyla).

Furthermore, I should like to mention those classifications which use the notion Coelomata while at the same time omitting the opposite notion of Coelenterata. Thus, for example, the groups Porifera, Ctenophora, Cnidaria and Planuloidea remain separate in the schematic survey which was made by J. Meixner and published in the Handbuch der Biologie (edited by L. von Bertalanffy and F. Gessner), Pt. III (The Genealogical Tree of the Animal World). In the text, however, we find nevertheless the word and the category of coelenterata used by A. Remane (ibid: 85 ) and by other collaborators (e.g. by 


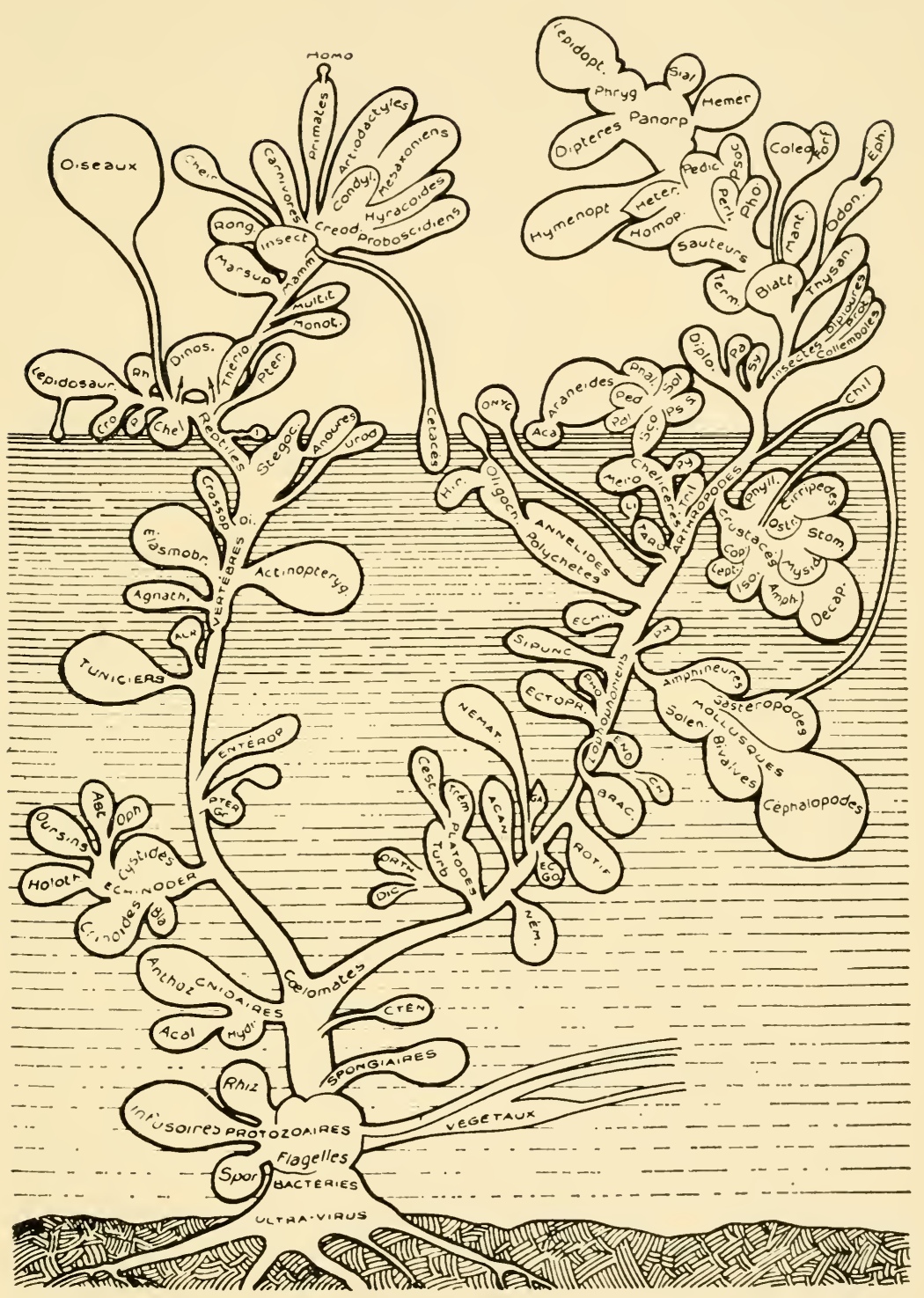

Fig. 50. Genealogical tree (After L. Cuénot.) 
W. Marinelli, ibid: 311). It is possible that in the schematic survey the word Coelenterata was omitted due to a mistake only.

L. Cuénot (Traité de Zoologie, 1., ed. P. Grassé, Phylogénèse du règne animal, Arbre généalogique, p. 2, cf. Fig. 50), too, would like to dispense with the taxon Coelenterata while at the same time he preserves the taxon Coelomata. Cuénot represents this graphically in such a way that he places two independent short branches on a very short common stem; the left branch which is longer represents the Cnidaria while the smaller branch which appears on the right side of the stem embodies the Ctenophora. In this way the scheme as proposed by Cuénot shows some similarities with my genealogical tree. Cuénot speaks of the Neuromyaria instead of the Eumetazoa. In the text, however (p. 25), he mentions the coelenterate stage ("Le Neuromyaire Coélentéré") which shows that he still preserves the notion of the Coelenterata even if it is used purely formalistically only and not taxonomically.

The palaeozoologist A. W. Müller (1958) has accepted to a considerable degree the general division of the animal world as it was proposed by Cuénot (1948), while at the same time he also takes into consideration the coelenterate theory as it has been proposed in my publications ("Unter Zugrundelegung der Coelenteraten-Theorie von J. Hadži” 1944, see Fig. 51). The taxons or notions Coelenterata and Coelomata do not appear in his graphic representation of the genealogical tree. The acoelous Turbellaria appear in the middle of the stem (Fig. 51); the Cnidaria and the Ctenophora are derived from the Turbellaria. A. H. Müller does not follow my suggestions in his subsequent interpretation of the evolution; he prefers to preserve the dichotomy of the Coelomata into the Protostomia and the Deuterostomia. Yet at the same time he is undecided where and how these Deuterostomia should be annexed. We can therefore see in his graphic representation the "left" branch hanging in the air. I think it is very important that A. H. Müller (1958:3) as a palaeozoologist finds that Hadži's theory $(1944,1949)$ does not stand in any opposition 
to palaeontology, that "sich keinerlei Widersprüche zur Paläontologie ergeben und mit deren Hilfe sich auch andere Erscheinungen zwanglos erklären lassen."

Attempts can frequently be found where the Coelenterata (these are usually understood as a group which includes the

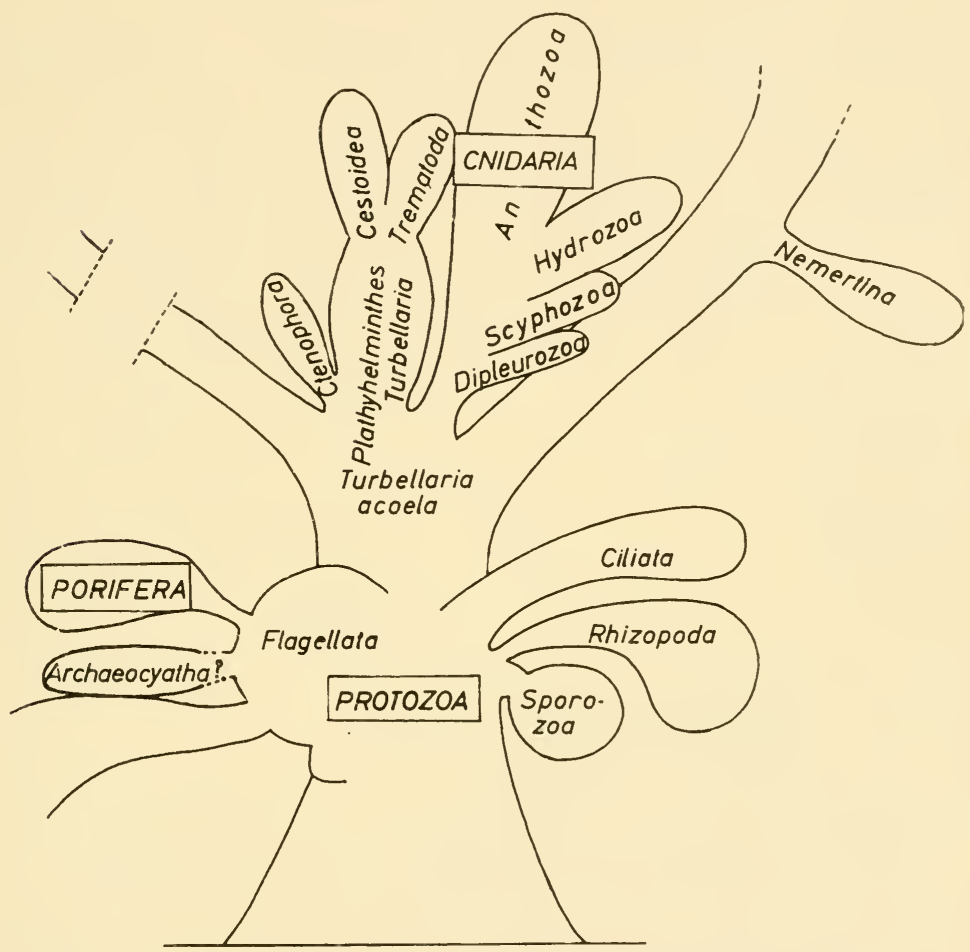

Fig. 51. The lower part of the genealogiel tree (after A.H. Müller), changed to agree with my turbellaria theory of cnidarians.

Cnidaria and the Ctenophora) do not appear on the geneological tree as a counterpart to the Coelomata but rather as an intermediate stage between the colonial Flagellata and the Coelomata (the Bilateria or even Vermes). This is, for example the case with the outline made by Schmalhausen (1947:411); in his graphic (Fig. 52) representation, however, we find 
the groups of Coelenterata indicated as a side branch so that finally we still get the impression of a bifurcation where the actual stem is represented by one of the two branches. The point in question is here a common, very primitive and perhaps planuloid ancestral form. Schmalhausen also goes his own

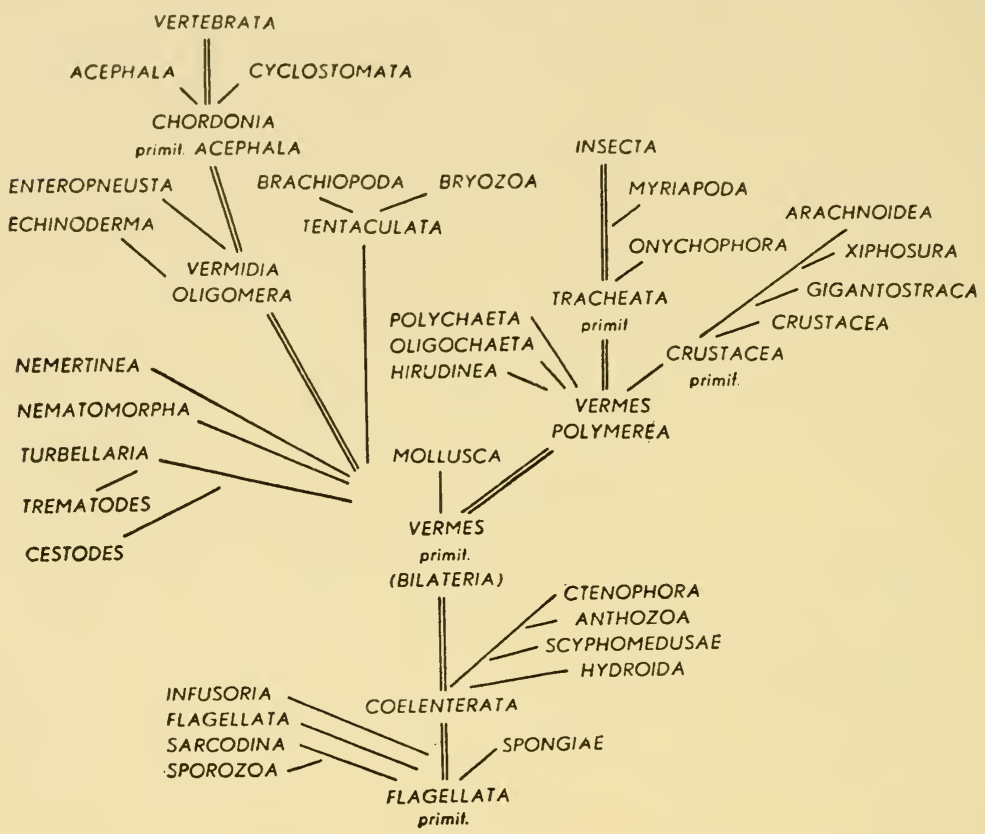

Fig. 52. Genealogical tree of animal kingdom. (After Schmalhausen.)

way in his interpretation of the phyletic conditions of the Bilateria; later we will return to this problem.

A very interesting suggestion was made by $G$. S. Carter (1940:482; cf. Fig. 53). In his construction of the genealogical tree Carter takes into account to a considerable degree the conditions that can be observed in larvae. We have already declared such a method erroneous. The classification of the animal world as it was proposed by Carter shows considerable simplifications which certainly is a welcome sign even 
if Carter goes in my opinion a little too far. Carter divides the Metazoa (these are actually the Eumetazoa, they do not include the' Spongiae as the Parazoa) at their very root ("the ancestral metazoan") into two lines; these two. stems were given the rank of superphyla, they are very

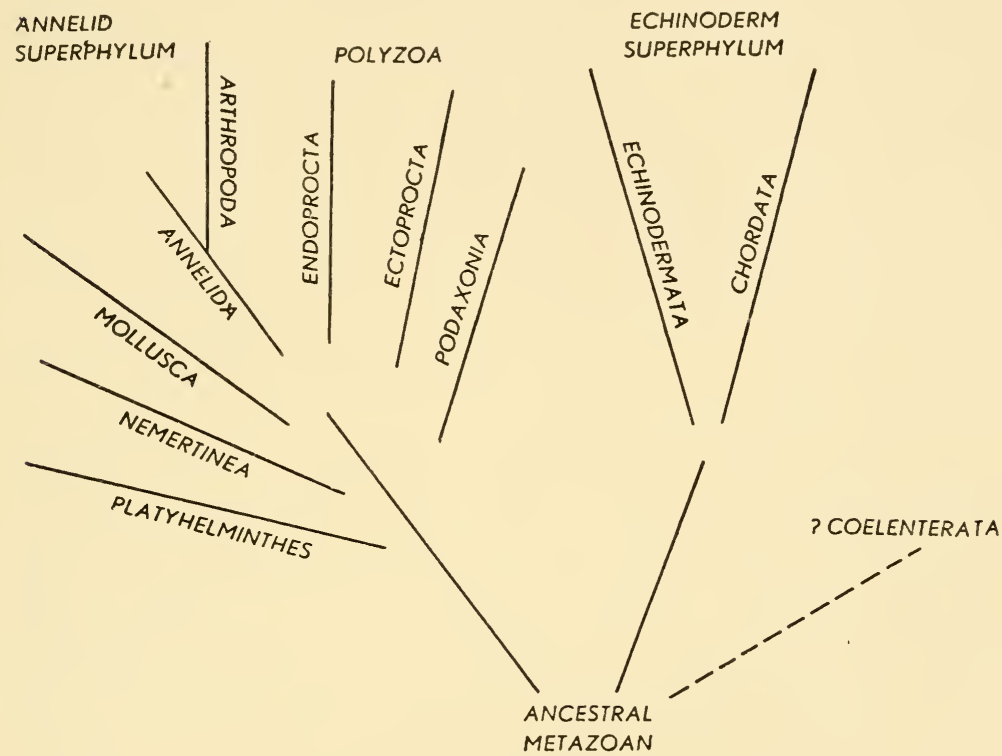

Fig. 53. "Evolutionary relationship of the invertebrate phyla" by G.S. Carter.

unequal, and they corespond roughly to the dichotomy Protostomia : Deuterostomia (the "Annelid superphylum" and the "Echinoderm superphylum"). As regards the Coelenterata Carter remains undecided; nevertheless he shows an inclination to derive them as an independent stem or as a third branch from the same "ancestral metazoan." A definite progress can be seen in his idea of a hypothetical ancestral form which had lived as a benthonic animal and was as such bilaterally symmetric, which had three body layers and did not consist of metameres (the amerous state), which showed a slight 
cephalization, and which possessed a ventral opening and even an anal opening and in all probability also protonephridia. We think that in this way too many requisites have been given to this hypothetical initial form. This can be seen above all when we try to derive the aproctous Platyhelminthes from it, while on the other hand it is not difficult to derive the Coelenterata from it because we can rightly maintain that some of these requisites had been lost due to the transition of these animals to a sessile way of life. As regards the Platyhelminthes, Carter must make some revisions; he must derive them from an even older primarily aproctous primitive form in which case he will be obliged to consider the Platyhelminthes as an independent branch - thus as the fourth branch-or he should go back to an already disqualified interpretation and consider the aproctous state as a secondary phenomenon, as a consequence of a degeneration.

We can easily understand that Carter does not assume a strictly negative attitude towards my interpretation. Carter discussed my suggestions twice. The first time in a very objective report which appeared in the review Science Progress; in this report he gives very adroitly all the essential points of my construction. Carter stresses judiciously that "it is not likely that zoologists will soon reach general agreement on them" (sc. my interpretations in the field of the "high phylogeny"). He mentions correctly the great interest which exists about these problems, and states as follows, "It is refreshing to read a discussion of these questions in which the results of recent zoology are taken into account. For this we have to thank Prof. Hadži."

In another detailed study (Carter, 1954) Carter took a critical attitude towards my suggestions as they appeared in the article which was published in Systematic Zoology. In this study Carter accepted, at least partly, my interpretations and expressed his opinion that it could be expected that my interpretations would not be accepted by many zoologists. Unfortunately enough Carter could not come to any conclusion 
concerning the derivation of the Cnidaria and of the position of the Cnidaria in the animal system; in his humility he considers himself not sufficiently competent to make any conclusions in this respect. Neither does Carter come to any conclusion regarding the origin of the Eumetazoa even if he admits that there are many points which seem to support the plasmodial theory, and that the old interpretation was based on the blastaea theory as it was proposed by Haeckel. I expect that in future Carter will come to some conclusions over these points, and that he will accept the idea that the Cnidaria evolved from some ancestors which they had in common with the Turbellaria, that he will accept the plasmodial theory, and finally that he will find that these interpretations agree best with the natural facts that have become known till now. It is a fortunate sign that such a large number of British zoologists have rejected the wrong interpretation of the relation embryos: ancestors. The only thing which remains to be accepted by them is the idea that the planktonic larvae must be interpreted as ontogenetic stages that had emerged secondarily as new forms which must not be compared with the adult ancestral forms but only mutually with each other. This is a necessary consequence of the right interpretation of recapitulations as they must be understood if they are not looked upon from the easily misleading standpoint of the "fundamental biogenetic law."

Among the more recent attempts to construct the animal system, mention should be made of the genealogical tree as it was proposed by Franz and Heberer (1943:291). From the standpoint of bifurcations this system is certainly the most extreme. The starting point is represented by the completely hypothetical and improbable gastraea. The pair Coelenterata: Coelomata is abandoned. The next lower stage, the Parazoa: the Eumetazoa are also abandoned. All the Metazoa are divided into two groups, the Protostomia and the Deuterostomia. The consequences of such a classification can rightly be considered as "horrible." Among the Coelenterata, 
which do not appear here under this name, the Platyhelminthes and the Bryozoa evolve almost together from one and the same point (we cannot speak here of a root or of a stem) which is also the starting point for the evolution of the Chaetognatha. The Annelida are placed above the Brachiopoda, Mollusca, Crustacea, and Arachnoidea, etc. It is really completely unnecessary to discuss here this system. The point of the bifurcation of the Eumetazoa can be found placed equally deeply in the system which was proposed by Steiner (1956).

This survey does not include all the attempts which have been made so far to find the correct position of the Cnidaria in the animal classification. Yet even this should suffice to show how many different opinions have been proposed so far and how little all these attempts are able to survive earnest examination. I suggest on the basis of the plasmodial theory of the origin of the Eumetazoa that the Cnidaria should not be separated from the remaining lower Eumetazoa. The dichotomy Coelenterata: Coelomata has in this way become superfluous. The same is also true for the two similar dichotomies, Diploblastica: Triplobastica (because there are no primary Diploblastica), and Radiata (Radialia): Bilateria. All the Eumetazoa have a primarily bilaterally symmetric structure, the radial symmetry being a secondary element caused by the transition to, and a longer preservation of, the sessile state. In this way we also achieve a simplification of the general system and of the genealogical tree.

\section{The Coelomata as a Taxonomic Unit}

On the basis of our study of the Cnidaria and of Ctenophora we have come to conclusion that the large taxon of Coelenterata has really no justification. The question necessarily arises in this connection what should be the destiny of its "counterpart," of the Coelomata, as that of a special taxon. The name itself indicates that those animal groups are included in this taxon which show a coelom in their internal 
structures. A new problem which emerges here is what should be understood as a coelom? This problem has already been sufficiently discussed in the present study. The name itself has been given to widely different body cavities which all appear in the middle body layer while at the same time they are of different origins. All these differences, however, can be reduced in the last line to two categories which are divided into several subcategories. We must distinguish between the coeloms in a wider sense of the word which are all the body cavities that are surrounded by the "mesodermal" cells that form their epithelia, and the coeloms in a narrower sense of the word, i.e. the body cavities which are developed around the intestine and which possess their own epithelium. The coelom is subject (which is very important!) to segmentation during a certain phase of the phylogenetic development. Whenever we speak of a coelom we usually think of this coelom s.str. for which I have suggested the word perigastrocoele to be used. This perigastrocoele played the most important role in the phylogeny of the Eumetazoa and it is therefore quite right if we take into consideration the development of this perigastrocoel when we propose larger taxons. Just in the point of this coelom $s$. l. we can observe cleanly the great difference which exists between the two opposing theories about the origin of the Eumetazoa. Those who accept the colonial theory consider that all the body cavities of the Metazoa, the intestinal cavity included (in the last line this could also be considered as a coelom s.l.), are new formations which had evolved completely independently from each other and not all at the same time. The perigastrocoele, and this is mainly the coelom to be discussed, is usually derived from some sack-like outgrowths of the intestine, and the coelom cavity from the intestinal cavity. Such a type of development which can be observed directly and quite frequently during ontogenies would presuppose that a fundamental change takes place in the function of the sacklike outgrowths of the intestine. 
An entirely different situation emerges if we view this problem from the standpoint of the polykaryonic theory, i.e. from the point of view which derives the Eumetazoa (the primitive Turbellaria) from the primitive Ciliata. All the "later" body cavities, the intestinal cavity included, had been developed, more or less clearly, in their initial stages as early as in the Ciliata, and that in the form of some permanent or transitory vacuoles, slits, or similar spaces that had appeared in their cytoplasm and that were filled with the cell fluid. They have not appeared anew in the Eumetazoa, they had only evolved, and differentiated in various ways. If we understand as a coelom s.l. all the body cavities, we can consider that all the Eumetazoa are actually Coelomata, at least primarily, because many coeloms or even all of them can get lost as a result of a secondary development. This is true both if we accept the one or the other theory about the origin of the Eumetazoa, because the coelenteron of the Coelenterata could be considered as the first stage only of the enterocoelic state of the "genuine" Coelomata.

We can therefore see that those who adhere to the enterocoelic version must necessarily meet with great difficulties as soon as they try to propose a major division of the Eumetazoa. They must propose such large taxons as the Acoela and the Pseudocoela, because there are no Eumetazoa earlier than the Annelida which had developed a coelom s. str., (a perigastrocoele). At the same time they are uncertain whether the morphological conditions that correspond to these categories, such as the absence of an actual coelom, be a primary or a secondary phenomenon. This has been the reason why there are so many zoologists who would like to explain all the Eumetazoa of the Bilateria group, which show a simpler structure, as a product of a retrogressive evolution, an interpretation which has not been supported by any convincing arguments.

When we see that there are such widely different body cavities and systems of cavities - which are different not only in their forms but also in the ways that they are developed 
during the ontogenetic processes-even in groups which are otherwise obviously closely related, it becomes evident that it is better not to use the conditions of the coeloms as a basis for classification. Zoologists should therefore avoid such classifications as the Coelomata (because of the untenability of their counterpart, the "Coelenterata"), the Acoelomata, the Pseudocoelomata, etc. As an example, we mention here the lower "Coelomata" or the "Bilateria." The coelom cavities can be found as early as in the Turbellaria which we consider as the earliest Eumetazoa. These cavities occur in three forms if we do not take into consideration the digestive cavity. First of all we can frequently find a whole system of canals, often with a vesicle at its extreme end; this is the socalled protonephridium which functions as an emunctoryexcretory organ. We derive this nephrocoele from the pulsating vacuoles of Protozoa.

There have been some zoologists who would like to consider this nephrocoele as an initial state for the evolution of the "genuine coelom" (the perigastrocoele; the nephrocoele theory which was proposed by Faussek). This is completely wrong because we can find this nephrocoele preserved as such up to the very end of evolution; it is quite natural that during the evolution it had been variously changed, especially after the appearance of the perigastrocoele. This nephrocoele was occasionally lost in the permanently sessile types whose size had been secondarily strongly diminished as has already been mentioned. In some other cases we find this nephrocoele changed beyond recognition (e.g. in numerous Arthropoda with the so-called mixocoele). Cases are also known where a combination of the nephrocoele with the perigastrocoele (which had developed much later), or with other coelom cavities (e.g. the gonocoele) have been observed.

The second coelom which appeared quite early during the phylogeny of the Eumetazoa is the gonocoele. It evolved completely independently and without any phyletic connection with the protozoan ancestors of the Eumetazoa. Earlier 
it was frequently called a "sack gonad" (Hatschek). There is no gonocoele yet in the most primitive Turbellaria which might be also true for the phylogenetically oldest Eumetazoa; these forms do not yet possess a regularly formed gonad but only an irregular aggregation of gametocytes which do not have any gonoducts. The gonocoele can be found highly developed in those animal groups which do not possess a perigastrocoele (our Ameria) as is the case, with the higher Mollusca. This gonocoele can again disappear secondarily, just as is the case with the nephrocoele; this takes place above all in those animal types which possess a perigastrocoele, yet it can also be observed in the Ameria. Not infrequently we can find gonocytes which do not form a hollow or a solid gonad and which become simply attached either to the skin (this can be observed in some Cnidaria), or to the intestinal epithelium (also in some Cnidaria), or even to other coelom cavities, above all to the perigastrocoele. At first sight this fact seems to support the gonocoele theory which maintains that the genuine coelom (the perigastrocoele) evolved from the gonocoele. Such a theory cannot be accepted from our standpoint.

The beginnings of the third coelom, of the haemocoel in the form of a haemocoelom system can also be found as early as in the Turbellaria. In these it appears occasionally as a system of blood vessels and lymphatic ducts; it can be found well developed first in the Nemertinea and in the Mollusca. It had been inherited by the Annelida as the lowermost Polymeria from some ancestors which stood closely to the Nemertinea. A pulsating centre of the haemocoele, a muscular heart, does not appear earlier than in the Mollusca.

There are some other special coeloms. Let us mention two such coeloms only; the rhynchocoele of the Nemertinea, and the cardiocoele (the pericardial cavity) of the Mollusca; these forms thus occur in the Ameria. All these coeloms and systems of coeloms evolved in the middle body layer, usually by way of a continuously progressive evolution from some 
slits and cavities filled with the "cell fluid." This is the case, for example, with the gonocoele of the Turbellaria with its different canals, side cavities, and not infrequently with a secondary polymerization (of gonads, of the terminal organs of nephridia). This is how a rich morphological differentiation became possible. It seems to me, however, that they do not represent a sound basis to be used in classification. These various coeloms and the morphological peculiarities which are connected with them enter into such a variety of combinations that it is impossible to develop on this basis any principle which could be used in the classification. Fortunately enough there have been only few attempts which have tried to construct a system on the basis of the conditions that can be observed in these coeloms. Among these we must mention above all the high taxons the Acoela and the Pseudocoela, or the Acoelomata and the Pseudocoelomata. To these we must add the Eucoelomata as the third subseries (according to Schimkewitsch, 1891). This classification was accepted by Hyman (1940) and by the AAAS(Zoological Names, edited by A. S. Pearse, 1949), obviously under the influence of Hyman. The category Acoelomata has only sense if we consider only the perigastric coelom as a genuine coelom; yet if we accept the broader definition and if we consider the coelom to be any body cavity which can be found in the middle body layer of the Eumetazoa and which is surrounded by its own cells, we see that the category Acoela becomes completely senseless because in this case there are no genuine and primary Acoela among the Eumetazoa. The notion of the Pseudocoelomata is even less justified and useful. There do not exist any Pseudocoelomata at all. A body cavity can either be a primary cavity, i.e. without a coelothelium; or it can be a genuine coelom if it has a coelothelium. The category Pseudocoelomata consists, according to Hyman, of the Aschelminthes and of the Endoprocta only. These Endoprocta are actually Aschelminthes that had adopted the sessile way of life but they do not differ essentially 
as regards coelom conditions from the Acoelomata. It is even more erroneous to consider "the phylum Tardigrada" as a member of the Pseudocoelomata. In connection with this we could speak at the most of a myxocoele because in their ontogenies we can observe pairs of coeloms that are distributed metamerically like the enterocoeloms. The Tardigrada must therefore not be placed anywhere near the Aschelminthes. They certainly stand closer to the primitive Arthropoda.

The suggestion which was made by Colosi (1956:505) should be mentioned here as an example of an extremely onesided systematization based on the conditions that can be observed in the coelom cavities. Among the taxons which are given various ranks we can find, in Colosi's suggestion, the Deuterocoelomata (including Mollusca, Molluscoida, Annelida, and Onychophora); instead of their counterpart the Protocoelomata or the Acoelomata we find the group ("stipite") Protonephridia which includes all the Ameria. A part of these (these are actually the Aschelminthes) can be found under the name Pseudocoelomata. All this refers to the Protostomia. The Deuterostomia are divided into the Archicoelomata (they include the Chaetognatha only!), and the Metacoelomata (the Hydrocoelomata and the Chordata). The Syncoelomata consist of the Tardigrada and the Arthropoda. The name Parenchymatosa appears twice; once it includes the Nemertinea and the Endoprocta, and another time the Gordiacea. I can hardly believe that any zoologist will ever accept such a classification, it is a purely personal affair.

There is according to my interpretation a basic difference between the various coeloms s.l. and the actual coelom s. str., the perigastrocoele. The coeloms s.l. can be found in widely different groups of the Eumetazoa and it seems that they had evolved polyphyletically. They cannot be used as a sound basis of a high taxonomy, except in a negative sense, i.e. that all the Eumetazoa which do not possess primarily a perigastro- 
coele can be grouped together because they show a closer relationship also in other respects. They could therefore be called the Acoelomata; y et this name can be misleading because in all the subgroups of the Acoelomata we can find some kind of a coelom cavity. It will be therefore better to make the classification on the basis of the coelom conditions while at the same time another very important property is also taken into consideration (this in combination with many others!); this is the fact whether the metamerized perigastrocoelic sacks are primarily absent or present. In this way we can get the two groups, the Ameria and the Polymeria. We can see that the special coelom which surrounds the intestinal canal emerges, as it seems suddenly (this at least is the impression we can get now!), and trom the very beginning in a metamerized or polymere form. In this way we meet here with an important gap which must be taken into consideration by the systematists and by the taxonomists. Later we will discuss this problem more in detail. Let us now first turn to the notion and to the taxon Ameria.

\section{The First Phylum of the Eumetazoa: the Ameria}

The Turbellaria are according to our interpretation the earliest Eumetazoa and as such they represent a starting point of an evolution that radiated into several directions. There are not less than fifteen distinct subtypes-we believe that it is sufficient if we give them the taxon of classes - which evolved from these Turbellaria, and these classes again evolved into numerous subgroups (ordines). We can notice some differences in the levels reached in the organization of all these classes of the Ameria which are due to the various ways of life the species belonging to these groups had to adopt. Here we can find forms which live in various environments as freely living animals, as parasites, animals which burrow in the sea bottom, semi-sessile and fully sessile (evolved even several times!) species. The extent of this difference can be $26^{*}$ 
best seen if we juxtapose, for example, on the one hand an acoelan or a hydropolyp, the males of various Rotatoria, the Cestoda, and the solitary Endoprocta, and on the other hand the Cephalopoda, the various higher Turbellaria, the Nematomorpha, etc.

It must be admitted, however, that certain very conspicuous common traits of the general organization and of the level of this organization can be found in all these animal groups which are otherwise so widely'different. This proves that they do not only have in all probability a common origin, but also that they are mutually more closely related. The groups which are thus brought together under the phylum of Ameria show the following common traits if compared with the next higher phylum: (1) Not a single class of all these fifteen classes has a perigastrocoele, thus a coelom s.str. (2) We cannot find anywhere in this phylum a genuine segmentation and a formation of body regions which would appear in combination with this segmentation. (3) None of these classes has ever developed any extremities, and they have therefore not developed any articulated extremities. (4) Their protonephridia had developed into metanephridia only by Molluscs as a consequence of the union with the increased gonocoel. The first three characteristics represent negative values only, yet they can also be expressed in a positive way, i.e. (1) these classes possess only the coeloms s.l.; (2) their body is always homogeneous (the amerous state); and (3) they can move in various ways (by means of cilia or of body muscles), yet never by means of genuine extremities.

As could be expected there appear occasional characteristics, at least as Anlagen, scattered among the classes of Ameria which had played a very important role in the evolution of the Polymeria, the next higher animal type. As an example, we can mention the polymerization of individual organs (gonads, nephridia, etc.) or of whole parts of the body (Cestoda), an increased growth into length combined with a curving movement, etc. 
The notion of Ameria which represents an unsegmented state of body and its use for the taxonomic purposes is not something entirely new. It is only the contents of this notion which are new. Houssay (quotation after Krumbach, 1937:13) came close to this notion by creating the notions and the names Paurometamera and Polymetamera which, however, are limited to the Enterocoelia only. Schneider (1902) seems to have been the first zoologist to have used the word Ameria, yet he used it for an entirely different notion, i.e. for the Echinodermata only which he included in the phylum Coelenteria. Much closer to our notion of Ameria stands the notion which was introduced by Bütschli (1910) under the name Amera and which has as a taxon other contents. Bütschli who was followed in this point by the editors of the Handbuch der Zoologie (Kükenthal-Krumbach) does not include among the Ameria either the Coelenterata or the Mollusca; he and the editors of the Handbuch still adhered firmly to the notion Vermes, which is used for practical reasons only, yet in no way as a taxonomic unit.

As regards the Coelenterata it has been shown that there is no such animal group, and that both the Cnidaria and the Ctenophora belong to the phylum Ameria because they had both evolved from the Turbellaria.

It is in agreement with our definition of the Ameria if we include among them the Mollusca in spite of the discovery of Neopilina by Lemche (1958) and in spite of his interpretations. In another work I have discussed in extenso the case of Neopilina (cf. Hadži, in press). For a long time scholars have endeavoured (and there are some zoologists who try to do this even now), to bring the Mollusca into a closer connection with the Annelida, and thus with the Polymeria; this was done in such a way that they have declared the Mollusca to be secondarily simplified animals (this was suggested especially by the older zoologists), or they have considered them as animals which have just reached the initial stage of the polymerous state. Those who believe in a closer 
relationship between the Mollusca and the Annelida have referred above all to the similarity that can be observed in their planktonic larvae of the trochophore type and to the spiral cleavage. We have already shown that neither an external similarity which can be found in larval forms nor a spiral cleavage can be used as firm proofs of a closer relationship between two animal groups. The situation is perfectly clear as regards the coelom conditions and the segmentation; a polymerization frequently takes place in the primitive Mollusca and in Neopilina (which is not a primitive mollusc but rather a primitive gastropod); this polymerization disappears in the higher subtypes and it is only partly preserved in the Cephalopoda (Tetrabranchiata). This, however, should not be interpreted as a remnant of a genuine segmentation but rather as a minimal polymerization. Zoologists have endeavoured to find by all means at least in the ontogenies a recapitulation of a formation of genuine metameres (of the perigastrocoele); they have even maintained that they have found something of this kind. Yet they actually saw something that does not really exist because that was what they wished to see. We can best see how much we are justified in classifying the Mollusca among the Ameria if we compare the lower and the lowest Mollusca - which differ only slightly from the probable initial form which stood close to the Turbellaria-with the other Ameria, and if we do not include first into such a comparison the highest Mollusca, the Cephalopoda.

H. Lemche went too far in his display of his scientific imagination (which can be quite useful if it is used within proper limits) when he tried to exploit the discovery of Neopilina. My opinion is shared by numerous other zoologists (cf. the discussion by C. Böttger and by others at the XVIth International Congress of Zoologists). There is no factual justification for a derivation of the primitive Arthropoda from the Mollusca, and above all of the articulated extremities from the ctenidia. In the primitive Mollusca we can find a somewhat stronger trend to the polymerization, just as is the 
case with Nemertinea, among the Ameria. This trend towards polymerization and to an organization in the direction of a polymerous type grew weaker and weaker during the subsequent evolution of the Mollusca; an evolution which was generally progressive even if it went in a special direction. The Mollusca must therefore be interpreted as a side branch on the stem of the Ameria. The Cephalopoda not only represent a climax in the evolution of the Mollusca, but also of the evolution of the Ameria as a whole. It does not seem that any new, higher animal type has ever evolved from Mollusca. We will also find very similar conditions in other main groups of the Eumetazoa.

$\begin{array}{ccc}\text { Turbellaria } & \text { Cnidaria } & \text { Gastrotricha } \\ \text { Trematoda } & \text { Ctenophora } & \text { Kinorhyncha } \\ \text { Cestoda } & \text { Nemertinea } & \text { Endoprocta } \\ \text { Rotatoria } & \text { Priapuloidea } \\ \text { Nematoda } & \text { Acanthocephala } \\ \text { Nematomorpha } & \text { Nollusca }\end{array}$

We can see if we make a survey of the fifteen classes* which constitute the phylum Ameria that it is quite impossible to make subdivisions of this phylum where the classes that are more closely related with each other could be brought together into special subphyla or superclasses (Fig. 54). This is also true for the most widely accepted division into the Platyhelminthes and the Coelhelminthes (Aschelminthes), or into the Scolecida and the Aposcolecida, as well as for many other similar classifications which were suggested by various zoologists. Many of these notions can prove quite useful here and in comparative morphology - as is the case with the pair Aprocta : Euprocta-

* In all probability a sixteenth class will have to be added to these, i.e. that of the Orthonectoidea, as a side branch of the Trematoda (which were called Mesozoa by Beklemischew (he divided the class Trematoda into two groups: Monogenea and Digenea; in addition to these he also created an independent class Udonelloidea). 
yet they cannot be used in taxonomy. They only lead to an unnecessary complication of the animal system and to differentiations which are, we can say, personally biased. The case of the antithetical pair Platyhelminthes : Aschelminthes where these two groups are understood as two parallel taxons, or

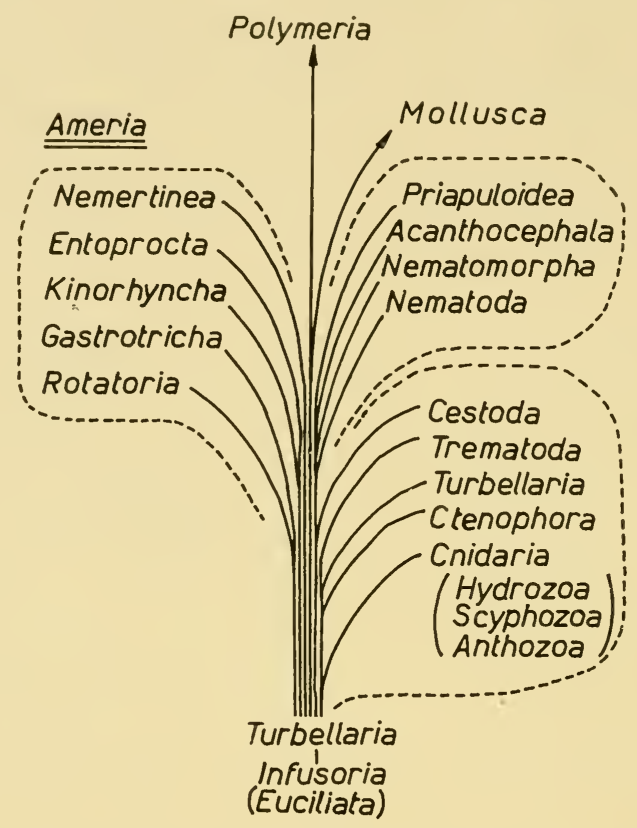

Fig. 54. Genealogical tree of the phylum: Ameria.

as two taxons where one stands above the other, shows the inadequacy of such a division because there is no real limit between these two groups, because the Nemertinea stand in the middle between them, and finally because not all the Turbellaria are really flattened animals. We do not even know which groups should be classified as Aschelminthes. According to Hyman the Aschelminthes consist of the following classes: Rotifera, Gastrotricha, Kinorhyncha, Nematoda, Nematomorpha, and Acanthocephala; yet they do not include 
the Priapuloidea, Nemertina, Endoprocta, and Mollusca (the first and the last classes are placed among the Coelomata).

It should be mentioned in passing that in our study of all these various animal systems we frequently get an impression that the pre-Darwinian custom of using a dichotomic determinative key in the construction of an artificial system still continues to work subconsciously even nowadays (cf. the attempt made by Colosi which has already been discussed above). The systematists must liberate themselves of this influence if they wish to construct a truly natural animal system. At present, it would be sufficient if these classes were placed into such a linear order, or indicated in the genealogical tree in such a way, that certain classes which are supposed to be more closely related appear close to each other. It is possible that after a certain time we will perhaps get a better knowledge of their mutual relationships and it will be only then that we will be able to begin with a more detailed work on these subdivisions. Important progress has recently been made just in this direction; as examples we can mention here the work made by Steinböck (the relationship connections between the Turbellaria and many other groups of the Ameria), Golvan (the closer relationship connections between the Priapuloidea and the Acanthocephala), and my own studies Cnidaria-Ctenophora, Turbellaria).

\section{The Transition from the Amerous to the Polymerous State}

We have seen that the phylum Ameria as the first phylum of the Eumetazoa does not possess a perigastrocoele. When we continue with our construction within the framework of one genealogical tree, we soon meet with the problem of how to explain the emergence of a perigastrocoele (coelom s.str.) and what its functional correlative could be. The problem is whether this perigastrocoele had originally evolved as a uniform cavity which had later become segmented, or alternatively that there had been originally a segmented 
perigastrocoele which had afterwards evolved by way of oligomerization into a uniform cavity. Both these types of evolution are a priori possible and probable.

A comparison of all the methods of coelom formation shows-we exclude here in principle all the tubular and cavernous formations which had evolved either by way of an infolding of the skin, as is the case with the tracheal system of the land-inhabiting Arthropoda, or by way of a folding of the skin layer, as is the case with the peribranchial cavity of the Chordata-that all these "hollow spaces" (tubules or cavities) emerge in those parts of the mesohyl where we can find either streaming of the fluid or rhythmic movements of the contractile organs. The first group includes the phenomena of the blood vessel system and the system of lymphatic and water ducts; the second group includes the rhynchocoele, the cardiocoele and, obviously, the perigastrocoele. A third group consists of the gonocoeles in the gonads together with their gonoduct, and the glands with central cavities and their secretory canals.

Thus the coelom cavities develop above all in combination with the frequently repeated or rhythmical movements of the internal muscular organs. We think that it was the peristalsis of the intestinal tube-after a mesenchymal muscularis had been added to the entodermal tubes-which led to the formation of a perigastrocoele. At the same time the intestine became lengthened as a consequence of a lengthening of the entire body. Another consequence of this lengthening of the body was also a radical change of the type of movement which now took place by means of wave-like zigzag contractions of the skin muscle tube which progressed from the front of the animal backwards. Such an interpretation has already been suggested by various other zoologists and it is therefore not an invention of my own.

We must now study as closely as possible the factual material and try to interpret it correctly in order to be able to determine which of the two possibilities is most feasible. So 
far we are still unable to present a strict proof which would definitely support the one or the other thesis. We cannot completely exclude the possibility that the perigastrocoele did not evolve once only; yet as much as we know and think now about the emergence of new types, this possibility does not seem to be very likely. The standpoint we take again depends heavily on the way we interpret the various phenomena that can be observed during the ontogenies. The interpretation of ontogenetic morphogeny has had a decisive influence on the way scholars have tried to solve the problem of the perigastrocoele and of its segmentation. The transition from the not-segmented to the segmented state must necessarily be recapitulated somehow in the ontogenies, yet this can be either a recapitulation of the ontogenetic development that had taken place during the phyletic past of an ontogeny, or it is partly a recapitulation of states that had existed in the adult (functioning) ancestors.

If now we first take a look at the conditions that exist in the adult recent Ameria we find that here there are no genuine transitory forms. This could actually be expected. Those forms that could eventually be interpreted as transitory forms between the state with a uniform perigastrocoele and that with a more or less segmented perigastrocoele give an impression of a secondary retrogression, of an oligomery combined with a change in the way of life or with a change in the type of movement (to a burrowing or semi-sessile way of life). Later we will again return to this same problem.

Among the recent Ameria, the Nemertinea are "the" group in whose organization we can find the greatest concentration of those elements that had probably led to the emergence of the type Polymeria. The Nemertinea show a strong inclination through their increased length combined with a sinuous type of movement, to a formation of various kinds of coeloms (the rhynchocoele, the gonocoele combined with the gonochoristic state and with a simplification of the auxiliary organs, the haemocoele, and the nephrocoele), to a development of 
planktonic larvae, and to a polymerization of various organs. One could even say that if the Nemertinea had not their characteristic proboscis and only slight indications of a perigastrocoele we could easily consider them as Archiannelida. These are not Polymeria, they are genuine Ameria which had probably evolved from some ancestors which stood close to the Turbellaria and which had served as a starting point for the evolution of the primitive Annelida and therefore of all the Polymeria. The perigastrocoele thus evolved, as did all the coeloms, from some "slits" that had developed in the middle layer, in the mesohyl (mesoderm), and which were filled with the cell fluid or with the body fluid; and not from some sack-like formations of the intestine. All connection (such connections are not numerous) of coeloms with the intestine are secondary phenomena. Those zoologists will therefore oppose my interpretation (and such zoologists are quite numerous) who firmly accept the enterocoele theory, in spite of the fact that they meet with difficulties which cannot be overcome as soon as they try to derive the conditions that can be observed in the Polymera (Ecterocoelia) from those that can be found in the Oligomeria (Enterocoelia).

It must be admitted that it is possible to imagine a state with a uniform perigastrocoele as the first step in the development towards a segmented perigastrocoele. Yet at the same time we see that among the recent groups of the Eumetazoa there is not a single group, at least as far as I know, which would represent such a state. Animals which show a state that is at least rather similar to that with a uniform, general perigastrocoele can be found on the periphery of the Polymeria only, and above all among the Oligomeria. As regards the reduction of segmentation we can find as early as in the Annelida, species which are more or less atypical, and therefore aberrant, and which clearly show a kind of a retrogression of the perigastrocoele system. Such a reduction is even more advanced in the Sipunculoidea and in the Echiuroidea. It is therefore completely wrong to place the Endoprocta close 
to the Ectoprocta as has been done by various zoologists (e.g. by Marcus and by Hyman) as if the "Bryozoa" evolved from the Kamptozoa parallell to a development of an undivided coelom cavity. This is clearly a case of a pure parallelism only, which took place as a consequence of a similar one-sided way of life at two entirely different levels of the general organisation. The polymerous Annelida can be considered as the direct ancestors of the Ectoprocta while at the same time they are genuine Oligomeria which show the extreme level reached in the retrogression of the perigastrocoele.

A clear trend towards a reduction of the segmented coelom system can also be observed in the evolution of the coelom conditions in the second large subdivision of the Polymeria, in the Arthropoda. These adopted movement by means of articulated extremities while at the same time the length of their body was decreased. As a final result of this reduction we find one undivided body cavity, the myxocoele, which is clearly a secondary element and which is also united with the primary body cavity. Something similar had also taken place in the Hirudinea, among the Annelida, where again it appears together with a change in the type of movement.

All the facts that can be found in the field of the comparative morphology of the adult Polymeria therefore support the interpretation that the perigastrocoele evolved first in the form of numerous pairs of small sacks, and thus as a segmented form. It was only due to a retrogression of this system (oligomerization) that the number of segments were decreased, or even that segmentation had completely disappeared. It is therefore quite improbable that the polymerization of the perigastrocoele had taken place step by step, thus by way of an oligomeric state (zoologists have usually thought here of a trimery).

At the beginning of our present study we have already discussed the problem of the relation between the ontogeny and the phylogeny. It is obvious that we must proceed very carefully when we consult the ontogenetic morphogenies as 
regards the conditions of the perigastrocoele. The fact must be taken into account that the destiny of the perigastrocoele had been closely connected with the destiny of the mesohyl (mesoderm). We have already repeatedly emphasized that in all probability the whole mesohyl-which is also a substratum of the perigastrocoele-of the Eumetazoa had been inherited by the Eumetazoa from their infusorian ancestors. Viewed from this standpoint we must reject the suggestion which tries to derive the mesohyl from the ectoderm (Ecterocoelia) or from the entoderm (Enterocoelia). Because of the monocellular initial state of each ontogeny (the fertilized egg cell), and because of the cellularization which takes place during the ontogenies, it became unavoidable for the ontogenies to develop gradually into a definitive three-layered state. This process can be understood quite generally as a recapitulation of the old adult state inasmuch as there is in these ontogenies first a monocellular state which is followed by a polycellular state. The same or even greater diversity that we can observe in the ontogenetic development of the entoderm (of the Anlage of the intestinal tube) can also be found in the development of the third body layer, the mesohyl. It is in principle wrong to try to find special recapitulations of the adult states. We cannot therefore accept a systematic division into the Ectocoelia and the Entocoelia. We could speak on an ecterocoelic and an enterocoelic type of development of the perigastrocoele if we had worked exclusively within the field of the comparative ontogenetics. Yet we must refuse to accept such a use of the type of development of the perigastrocoele during the ontogenies for taxonomic purposes because we know that among the Ecterocoelia we can find species or even smaller groups (e.g. the Tardigrada) which develop their perigastrocoele in the enterocoelic way.

Consequently, we must consider a derivation of the perigastrocoele sacks from the "lodges" of the Anthozoa as wrong. This is obvious already for the very fact that we consider the Anthozoa as the most primitive Cnidaria which evolved only 
into other classes of Cnidaria and not into any other form of the Eumetazoa. We think that the formation of the coelom (we think here always of the perigastrocoele only) had originally nothing in common with the intestine. The cases, however, where the coelom sacks develop during ontogeny as a result of an outgrowth of the intestinal wall (enterocoely) - a phenomenon which can be regularly observed among the higher Eumetazoa and occasionally also among the lower Eumetazoa-must be understood as a type of development which had evolved secondarily, as a rationalization, as a special "mechanism" which had developed from the phylogenetically older ecterocoelic type of development. Frequently, it is neither the ectoderm nor the endoderm which serves as a basis for the development of the perigastrocoele, but rather the transition area between the two. In the Polymeria the perigastrocoele develops, with few exceptions that have already been mentioned, without a participation of the Anlage of the intestine, yet in widely different ways. Very frequently we can find the Anlage of the segmented perigastrocoele developed in the form of a pair of the so-called primitive mesodermal cells which change into a pair of cell strings that finally develop into pairs of sacks that grow frontwards. This can be observed above all in the polychaetous Annelida, among the lowest Polymeria. Thus we cannot find here an intermediate preliminary stage with a uniform perigastrocoele. On the other hand we can frequently find in the derivative Annelida, Anlagen of coelom sacks which later grow together forming a secondary uniform perigastrocoele.

The ontogenetic type of development of coelom sacks had been necessarily strongly modified because of the planktonic larvae, the trochophores, which had been evolved by the benthonic Annelida. This suggests that we cannot make any conclusions regarding the original type of development by means of a comparison between the development of the mesohyl and of the coelom sacks in recent species; in this point there can be no recapitulation in the narrow sense of 
the word. The temporary development of a few pairs of coelom sacks that can be observed in the larvae of Annelida has nothing to do with the tetramerous Cnidaria. We must therefore resolutely reject the idea of a derivation of polymery from oligomery (Ivanov; we usually think here of trimery),
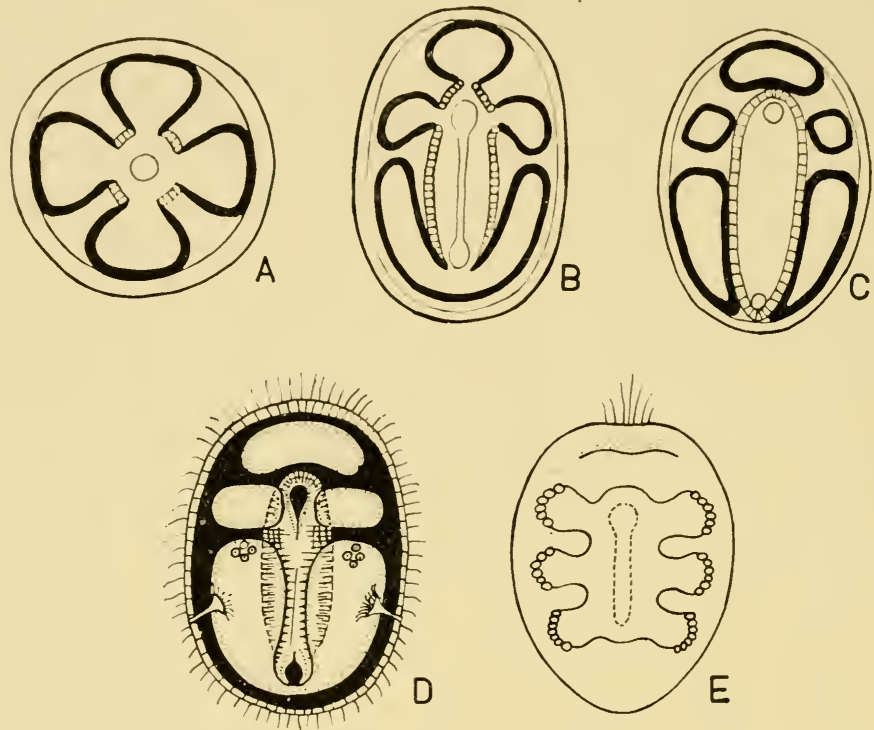

Fig. 55. Derivation of bilateral symmetry with "larval segmentation" from tetraradial symmetry of supposed primitive cnidarian by A. Remane (A-C). D, Remane's construction of a primitive segmented metazoon. E, Jägerstens "bilaterogastrea."

as well as of a derivation of polymery from cyclomery (Sedgwick, and many other zoologists after him, e.g. Remane and Jägersten cf. Fig. 55).

In our system the Polymeria must precede the Oligomeria, and the primitive Oligomeria, the so-called Tentaculata, cannot be interpreted as a transitional form between the unsegmented and the polysegmented Eumetazoa. This is a necessary consequence of the fact that we derive the Polymeria from an amerous state by way of a polymerization of several 
organs, and by way of an intensive growth in length combined with a sinuous type of movement, under the presupposition that the perigastrocoele had consisted from the very beginning of several segments. I wish to emphasize that for ecological and physiological reasons it is improbable that the polychaetous Annelida evolved from such animals as the semisessile or completely sessile Tentaculata. The facts such as the existence of transitional forms between the Annelida and Phoronoidea, suggest that there had been an opposite line of evolution.

Besides the interpretation which has been proposed in the present study, and the interpretation which we have rejected, there is one more theory which tries to explain the origin of the polymerous state. We can mention it only briefly. There have been besides older zoologists such as Haeckel, some modern scholars (e.g. Steinböck) who have believed that the so-called zooidal hypothesis could be the most acceptable interpretation. According to this theory a kind of a chainlike complex develops from an oözoöid, or a primary person, by way of an incomplete transverse division which can easily pass over into a budding. This complex had afterwards been changed by way of a subsequent complete integration into a uniform polymerous state. It must be admitted that many facts can be referred to by the adherents of the zooidal hypothesis. Thus above all the not so rare phenomenon of an initially incomplete repeated division which can be observed among the Turbellaria (the chain-like formation of the microstomous species). This is certainly a case of an only slightly modified asexual reproduction which could never possibly have led to any permanent polysegmented state. The case of the Cestoda which is also frequently mentioned in this connection belongs, I think, to the completely different category of morphogenesis than the development of a polymerous state.

The evolution of the Cestoda stands entirely under the influence of their endoparasitic way of life which has a very 
specific character: here the anterior part of the animal evolved into a scolex by means of which the animal becomes attached to the intestinal wall of its host while the remaining part of its body swims freely in the fluid and nutritive contents of the intestine. It is therefore not surprising to find in these animals an oppulent growth which is followed by a polymerization that can be observed above all in their complex hermaphroditic genital apparatus, especially since we can also quite frequently find a polymerization in the ancestors of the Cestoda, in the Turbellaria. The development of proglottides as a kind of a superstructure, a regulated segmentation of the body where the individuality is preserved in spite of a secondary separation of individual segments or of groups of segments, are all purely results of a further adjustment to such a specialized way of life. Yet even in this way no polymerous animal had ever been able to evolve. The state of "strobila" had evolved in an entirely different way and under completely different circumstances than did the genuine polymery (segmentation). The Cestoda are a blindly ending side branch of the Ameria which could never have been able to evolve into a higher form; they can therefore not be used as a pattern by means of which we could explain the evolution of a new higher type of segmented animals. They represent a very interesting special case only of polymerization; yet it must be mentioned in this connection that not every polymerization led to a segmented state.

As regards the "indications" that can apparently be observed in the ontogenies we can clearly see if we view this problem from our standpoint that they cannot be used as proof in favour of the zoöidal theory. Ontogeny has been considerably changed since the days when the primitive and regulated segmentation first evolved from the primitive Ameria with a scattered polymerization. This change is characterized by the emergence of a larval stage which remains a longer time in plankton. We cannot therefore expect to find recapitulations here any longer. Especially great were the changes which 
emerged as a consequence of the fact that true metamorphosis had been introduced. The institution of teloblasts and the introduction of zones of proliferation are two such new elements of the ontogenetic process.

\section{The Problem of Justification of the High Taxons: Protostomia-Deuterostomia}

Before we continue with our attempt to construct a new animal classification we must first discuss the problem of a justification of a division of the "Coelomata" into the two high taxons, the Protostomia and the Deuterostomia. This dichotomy has been widely accepted since the days of Hatschek and Grobben as a consequence of a belief that there had been a bifurcation of the two lines of evolution. There are few systems only which did not incorporate such a dichotomy. We therefore get an impression that this dichotomy must be supported by solid basic facts and interpretations. As is well known, the name itself has been given due to a phenomenon which can be observed in the ontogenies of numerous Eumetazoa, and even among our Ameria, where a definitive oral opening, i.e. the mouth of the adult form, appears as a continuation of the embryonic primitive mouth (gastrostoma). We must add immediately that among the species or subgroups which are classified as Protostomia there is either no mouth whatever, or a so-called primitive mouth only. During the subsequent evolution the primitive mouth grows together while at the same time a real "definitive" mouth is newly formed, instead of the "primitive mouth" in the same place more or less where the primitive mouth would be expected if it had been preserved. In numerous other Eumetazoa we can find a development which at first sight seems to be rather surprising and where an embryonic primitive mouth does not develop into a definitive mouth but rather into an anal opening, while at the same time the actual mouth is newly developed $27 *$ 
in a place where we would actually expect an anal orifice. Something similar has also been observed in one species of the Ameria, in Gordius (Švabenik, 1925).

These differences are certainly conspicuous and need an interpretation. First it should be stated that all our Ameria and Polymeria are usually considered as Protostomia, and all our Oligomeria and Chordonia as Deuterostomia. There is, however, an uncertainty among the adherents of the dichotomy Protostomia: Deuterostomia (also if these taxons appear under other names) on how they should classify the so-called Tentaculata (a part of our Oligomeria). Hatschek and Grobben placed them resolutely among the Protostomia (i.e. Ecterocoelia, according to Hatschek); the same was done by Hyman (as a part of the Schizocoela), Beklemischew (as Podaxonia which, however, include also the Brachiopoda!), etc. Frequently, the Tentaculata are also considered as an intermediata or transitional group between the Protostomia and the Deuterostomia (e. g. by Cori) because on the one hand they have a definitive mouth which develops from the primitive mouth (yet in the same place if the primitive mouth grows together in due time), and on the other hand because their perigastrocoele develops partly in the enterocoelic way which is quite an inconvenient fact for those who believe in the dichotomy discussed here.

After everything we have said about the evalution of the ontogenetic facts and their exploitation in a natural system of animals it becomes clear that we must proceed very carefully when we wish to use these facts for some taxonomic purposes. We must therefore assume not only a critical but also a negative attitude towards a division of the Eumetazoa as the Coelomata into the Protostomia and the Deuterostomia. We must take this stand not only towards those attempts which seek to represent the various types of oral and anal openings that can be observed during the ontogenies as if they were recapitulations of some adult states (cf. the desperate attempts made by Boettger (1952) and Steiner (1956) who tried to explain these 
differences on a functional basis) but also towards those zoologists who consider correctly that these are morphogenetic processes which are typical of the ontogenies only and which are therefore not recapitulations of some adult ancestral form. I have discussed this problem extensively in another study (Hadži, 1948).

It should be mentioned here that this high division of the Coelomata does not depend exlusively on the destiny of the primitive mouth during the ontogenies. It is usually combined with other pairs of opposite characteristics, above all the type of development of the perigastrocoele (the Ecterocoelia and the Enterocoelia, according to Hatschek, which have already been discussed), and the position of the cerebral process along the body axis (Epineuria-Hyponeuria). Even if there are some differences in special points between all these classifications we must nevertheless admit that there really exist such groupings of characteristics. The origin of these differences, however, can also be interpreted with the same justification in another way by means of which we can create an even more probable construction of the genealogical tree where the "function" of the so-called Tentaculata can be seen even more clearly. Those who accept the thesis of an evolution of the two main branches of the Coelomata from a common root run into great difficulties as soon as they try to explain why the protostomial state on the one hand and the deuterostomial state on the other had evolved with all the other differences which appear together with them. They have therefore been obliged to take refuge in a very hypothetical and therefore improbable construction as has already been shown here.

Both the facts as well as the working method show that it is much more probable that the two taxonomic units which are known under the names of Protostomia and the Deuterostomia had not developed side by side, i. e. parallel or diverging from a common root, but rather one after the other, so that first the Polymeria evolved from the amerous Eucoelomata (they are both grouped together as Protostomia), and the 
Oligomeria afterwards evolved from the Polymeria. It was within the framework of the Oligomeria that a perfect deuterocoelic-enterocoelic state had been reached. A perfect hyponeuria, however, did not develop earlier than in the Chordonia.

It is even easier to pursue the enterocoelic type of development of the greatly reduced system of the perigastrocoele in the Oligomeria than it is to study the deuterostomic state. It is again a clear case of a rationalization of the ontogenetic morphogeny, a simplification of its mechanism. The formation of the perigastrocoele is brought closer to the Anlage of the intestine. This takes place in such a way that first some cells migrate from the epithelium of the primitive intestine into the middle layer where they subsequently gather to form the perigastrocoele sacks. This agrees well with the interpretation which had already been suggested some time ago and which considers the oligomerous state as a secondary phenomenon which had evolved from the polymerous state by way of an oligomerization. The deduction of the perigastrocoele from the "lodges" of the anthopolyps which has been from the very beginning a completely improbable suggestion, becomes herewith superfluous. This is also true for the entirely artificial construction of a bilaterogastrula and for many other similar things.

The fact that various Anlagen of the basic parts of the adult organization begin to develop first in rather different ways while finally they all become considerably uniformized (rationalized) can be considered as a general regularity of the ontogenetic morphogeny. This can be clearly seen in connection with the formation of the intestine (cf. the various modi that occur in the Cnidaria alone), of the perigastrocoele, of the segmentation, etc.

Thus we come to the conclusion that the categories Protostomia-Deuterostomia do not exactly express the actual facts, nor do they stand in agreement with the correct interpretation of the same facts. This result is therefore similar with our findings in connection with the high taxons the 
Coelenterata-the Coelomata, the Radiata-the Bilateria, the Ecterocoelia-the Enterocoelia. The categories Protostomia and Deuterostomia must be therefore abandoned. We find it completely satisfactory if we use the simplified classification of the primarily polymerized animal groups (classes) as members of the phylum Polymeria. We emphasize explicitly that we speak here of the primarily polymerized animal classes only, because there are also secondarily polymerized animal classes which belong, as we shall later see, to the phylum Chordata.

\section{The Classes of the Phylum Polymeria}

Just as in the case of the phylum Ameria we find in the Polymeria that the evolution of their basic structural (Fig. 56) type had spread radiating into several different specialized directions. The system and the taxonomy of the groups of Polymeria which have emerged as a result of these various lines of evolution have been treated variously by zoologists. The study of these various groups of Polymeria has been made difficult by the fact that the same animal groups were placed into various categorical levels by various authors. There is a considerable unanimity among scholars about the general sphere of the high group of the Polymeria; it corresponds roughly to the old notion of the Articulata as it was proposed by Cuvier.* The word Articulata can be met with not infrequently even now as a name of a high taxon, e.g. in Beklemischew. In this case the two notions and high taxons Articulata and Polymeria correspond exactly to each other. Yet 1 prefer the name Polymeria because it agrees better with the names of other groups with a similar rank, the Ameria and the Oligomeria, and because it expresses better what it represents; the Oligomeria are finally also "articulated" animals while at the same time they had evolved separately from the typical

* Not in the sense of the "Articulata" Huxley (1869) as a subgroup of the Brachiopoda! 


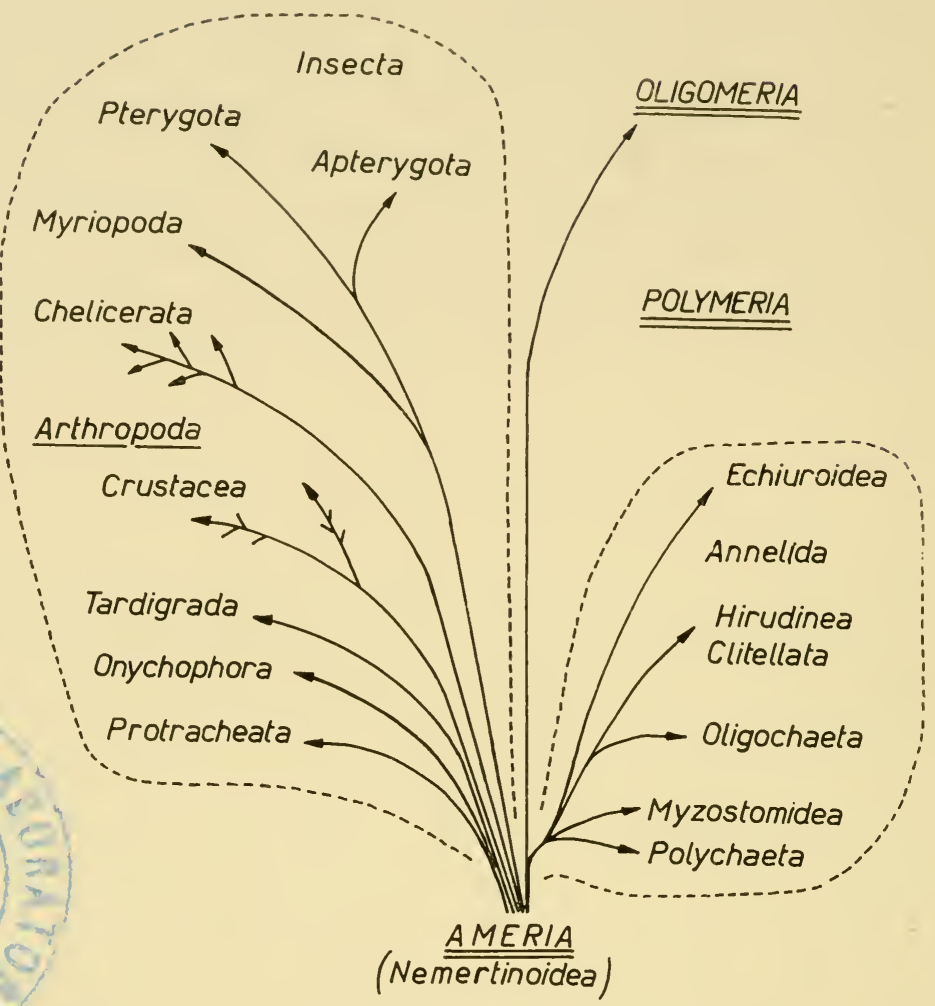

Fig. 56. Genealogical tree of the phylum: Polymeria.

"Articulata," and they must be therefore clearly separated from the latter.

Contrary to the situation which we have found in the geologically much older Ameria, we see that it is quite easy to identify the two main lines of the evolution of the Polymeria which have been used for a long time already as high taxons. We will consider them as subphyla and use the names Annelida and Arthropoda. It is quite easy to classify all the classes of the Polymeria into one of these two subphyla. Beklemischew, on the other hand, distinguished here five subphyla, i.e. Annelida, 
Tardigrada, Pentastomida, Onychophora, Arthropoda. Such a classification can be well defended as well as a classification into three subphyla. In this classification we place an intermediate group which has stumps only as extremities (Tardigrada, Onychophora, Pentastomida) between the Annelida which have no extremities at all, and the Arthropoda which are equipped with the articulated extremities. The question remains open whether we can consider these forms as a primary element or whether they had emerged as a result of a secondary development. We think that it is sufficient to divide the Polymeria into two subphyla, the Annelida and the Arthropoda (this is true at least for the recent Polymeria) because we find that the stump limbed Polymeria do not only show closer relationship connections with the Arthropoda but also because they do possess extremities even if these extremities are very simple or if they are remains only of such extremities (Pentastomidea).

The dichotomy corresponds well, as this has already been mentioned, to the two main lines of the evolution of the Annelida which have remained more primitive animals. From these we derive the Oligomeria, i.e. the next higher phylum which is as such the third phylum of the Eumetazoa. The situation of the Annelida resembles that of the Turbellaria, among the Ameria. In these the polymerization had reached its highest development. On the other hand, we can soon observe a trend to a sessile way of life and its necessary consequences, a shortening of the body length and a reduction of the polymerous state. The Arthropoda on the other hand, the second main branch which had a much more exuberant evolution, played a role similar to that of the Mollusca among the Ameria. At the same time they far surpassed the Mollusca because they solved much better, not only in water but above all in the sea also (Crustacea), the problem of transition to a life on dry land by developing a tracheal system. Just as in the case of the Mollusca, among the Ameria, we find that the Arthropoda have still remained within the sphere of the type 
of the Polymeria in spite of their progressive evolution combined with a strong specialization.

The suggestion to use the Spiralia as a systematic notion and the group of the Protostomia as a part of the same must be resolutely rejected. Such a suggestion was made, for example by Kaestner (1954) and by others. Such a group would include besides some clear Articulata (Annelida, Echiuroidea) also many groups of our Ameria, above all the Mollusca, Nemertinea, and the Platyhelminthes. The spiral type of cleavage appears in widely different animal groups and it is in no way connected with the problem of the closer relationship. It belongs exclusively into the sphere of rationalizations of the mechanism of the early ontogenies. Or in other words, the spiral type of cleavage can appear in all those cases where similar conditions of the egg structure appear together with the mechanism of cleavage which is connected with the former. Attempts have also been made to justify the existence of the Spiralia as a natural group by referring to the similarities that can be observed in their larvae (Kaestner, 1954:13). Such an argument has already been sufficiently discounted in our present study.

It would be in no way difficult to show the unacceptability of the division of the groups which are covered by the notion and the taxon Polymeria into several (six) phyla as we find this in the Zoological Names (edited by A. S. Pearse). The classification which comes closest to the system which we consider as the best one is the system which was proposed by Beklemischew. It has already been mentioned that his characterization of the whole high phylum Articulata (the seventh high phylum, according to Beklemischew) corresponds exactly in its sphere with our Polymeria. The main difference exists in the fact that Beklemischew divides his high phylum into five subphyla (Annelides, Tardigrada, Pentastomida, Onychophora, Arthropoda) while we believe that a completely sufficient subdivision can be made by means of two subphyla (Annelida, Arthropoda). We consider that the three remaining groups 
(Tardigrada, Pentastomida, and Onychophora) do not "deserve" to be given the category of subphyla. All these three groups can be considered as very specialized Arthropoda. Beklemischew has also given the rank of an independent subphylum to the Sipunculoidea (he calls them Prosopygia) as well as to the Kamptozoa. He places these two subphyla after the Arthropoda which is certainly quite unusual. In this connection we can also dispense well enough with the category of superclasses even if it is true that among the classes of the Polymeria there are some groups which are phylogenetically more closely related, similarly as has been the case with the Ameria. It seems that we will probably come to a generally acceptabie scheme of the system of the Polymeria if we abandon these unnecessary subdivisions.

The subphylum Annelida can be well enough subdivided into three classes, the Polychaeta, Clitellata, and the Echiuroidea. The class Polychaeta is the main one whose ancestors had evolved into all the other subtypes as well as into the Arthropoda and into the next phylum Oligomeria. Several zoologists have given the rank of a class to the group Myzostomida; I myself have done this in my proposed genealogical tree of the animal world (Hadži, 1944:179); this, h wever, is really not necessary. The Myzostomida can be considered as specialized Annelida of the type of the Polychaeta; this is also the way how they are treated in the system proposed by Beklemischew. It is almost a question of personal taste whether we unite the Oligochaeta and the Hirudinea, the two groups of the Annelida, into a new group Clitellata, or whether we consider each of them as an independent class. There is no disagreement among zoologists that the Arthropoda evolved from some Annelida-like ancestors. Yet we are still uncertain about the way along which this evolution had proceeded, whether the Arthropoda evolved once only or after repeated similar attempts made by Nature. The systematists of the Arthropoda show in this point a considerable variety of opinions. For the present we can again avoid the obligatory 
intermediate categories in our system of the Arthropoda such as the superclasses. All the recent Arthropoda can therefore be subdivided directly into the corresponding classes. The number of these classes varies, if we take into consideration the recent animals only, between thirteen (e.g. according to Comstock), eleven (e.g. according to Beklemischew), and five. I believe that five classes are sufficient because there are many suggested classes here which can be easily considered as subclasses. These five classes are, the Onychophora (with the Protracheata and the Tardigrada), Crustacea, Chelicerata (among these are also the Xiphosura and the Pentastomida), Myriapoda (here are also included the Symphyla, Pauropoda, Diplopoda, and the Chilopoda with the old root of the Hexapoda), and the Insecta (with the two subclasses, Apterygota and Pterygota).

We can easily see how well-founded is a reduction of the number of classes if we study the classification as it was proposed by Comstock who suggested that there should be thirteen classes of the Arthropoda (yet without the Trilobita; his classification goes back to the year 1925!). He considers the Onychophora and the Tardigrada as two separate classes; separate classes are also the four groups of the Myriapoda (this can be found quite frequently even in modern zoologists because of the differences which exist in the relationship connections between the four groups). The Palaeostraca (Limulus), Pycnogonida, and the Pentastomida are also considered as independent classes which in reality must be grouped together with the Arachnida into the class Chelicerata. Comstock separates, quite unnecessarily, a part of the Apterygota as Myrientomata from the remaining insects as Hexapoda, a classification which is no longer in use. In this way we find that five classes sufficiently represent all the major subdivisions of the Arthropoda. I will not discuss here the still open problem of how the Arthropoda had actually evolved from the Chaetopoda, and how the present classes evolved from the primitive Arthropod, because I 
cannot propose any new ideas here which would clarify these points. The Arthropoda probably evolved on the sea bottom and along several radiating lines. The hypothesis proposed by Giljarov about the origin of the youngest (and in spite of this very old) group Hexapoda from some ancestors similar to the Myriapoda wich had lived in the soil seems to me to be very probable. The Arthropoda left the sea several times to live on land; and this is also true for the Polymeria as a whole.

The number of subtypes is smaller in the Polymeria if we compare them with the situation which can be found in the Ameria. There, in one subclass only, which consists entirely of species that live as parasites, are the Pentastomida among the Chelicerata; on the other hand we find among the Ameria three classes which consist exclusively of the parasitic species. At the same time, however, we can identify parasitic species or even whole subgroups in other classes of the Arthropoda also (with the exception of the Onychophora and the Myriapoda). Among the Cirripedia we can find endoparasites whose form had been simplified beyond recognition so that it is their freely swimming larva only which shows their arthropodal provenance.

Among the Polymeria we cannot identify a single class of fully sessile species while on the other hand there exist two such classes among the Ameria (Cnidaria, Endoprocta). The reason for this is the fact that a fully sessile way of life can exist in a fluid medium only because a majority of the Polymeria are now terrestrial animals. There is one group only among the sea, inhabiting Polymeria (the Cirripedia among the Entomostraca) which includes fully sessile species; yet again these Cirripedia do not form any colonies (cormi) because they have completely lost the ability to reproduce asexually. It is noteworthy that forms similar to cormi have been developed by the Polychaeta which reproduce by way of division and of budding.

There is, however, another point which we find most interesting in connection with the way of life of the Annelida 
and which is important for the correct solution of the problem of the origin of the next "higher" phylum. These are two phenomena which are mutually closely related. One of these phenomena is connected with the way of life, above all with the type of feeding and of movement, and the other phenomenon with the evolution in the direction polymerization $\rightarrow$ $\rightarrow$ oligomerization. We find in this connection an interesting and revealing survey of the development trends such as they appear within the phylum Polymeria which had finally led to the emergence of the next "higher" phylum.

The starting form of the evolution of the Polymeria had been, contrary to the situation which we find in the Ameria, a bilateral richly segmented and therefore elc ngated eumetazoan which had moved over the surface of the sea bottom by means of a sinuous type of locomotion and which fed as a predatory animal. The polymerization took place in a part of their subline of evolution which developed progressively; its consequence are species which can reach a length of several metres, which consist of hundreds of segments, and which are either equipped with parapodia (Polychaeta errantia), or not at all (Oligochaeta). In the shorter side lines of evolution, however, the animals have actually preserved their free mobility while at the same time the size of their body was considerably decreased and the number of their segments reduced. They had thus been subjected to oligomerization. This development had taken place both in the Polychaeta and in the Oligochaeta. There have also been some instances of neoteny.

Another part of the freely moving Polymeria also developed progressively; they changed their type of movement by developing lateral excrescences which evolved into active extremities. Here the number of segments had been reduced (oligomerization) in several sublines of evolution, even in those whose representatives had begun to live on land; this reduction was usually combined with a differentiation of various parts of segments which led to a regionalization of the body, and above all to the cephalization of the foremost 
segments, the acron included. In several sublines we can observe a parallel development towards a stabilization of the number of segments, e.g. in the Crustacea where the number was fixed at twenty. Yet even in these lines the oligomerization can continue to have an important role, above all in the side lines with a specialized way of life (e.g. the Pentastomida), or with a strongly diminished size of the animal body (e.g. the Acarina).

The part of these Polymeria which evolved progressively reached its climax in several groups of the Polymeria that live in water and on land and whose evolution has always ended blindly. It should be mentioned in passing that during this development the conditions of the perigastrocoele and of the other coelom cavities had also been considerably changed and that this development led to the formation of a secondary myxocoele. Among the Arthropoda that live in the atmosphere we find the "worm-like forms" still preserved in the Protracheata (Onychophora) and in the Myriapoda s.l., as well as in the larvae of numerous insects.

Now, we must return to the primitive Polychaeta among the Annelida. A part of these had given up the mobile way of life and predatory macrophagy and had begun to live as sessile and microphagous animals. This transition was made by freely moving species which had developed temporary protective tubules. Such species have been preserved down to the present day. The tubicolous way of life is different from the truly sessile way of life where the animal becomes directly attached to the substratum after metamorphosis had been completed. In this line of evolution we can also find formation of regions (e.g. Chaetopterus) and a diminution of the size of the body which had also led to a reduction of polymery, yet no special new type ever emerged as a consequence of this development. It has always remained a blindly ending side branch.

The radiating lines of evolution which started from the prototype of the Polychaeta proceeded even further. A part of these abandoned life on the free surface of the sea bottom and penetrated partly into its soft sediments where they 
burrow, eating sediments that are rich in organic substances, or they try to find protection in cliff fissures, empty shells of snails, etc. The parapodia and the sinuous type of locomotion became superfluous to these animals; this again led to a considerable reduction of their organization which had even reached the internal polymerization. The consequence of this development had been the loss of the dissepiments and of the
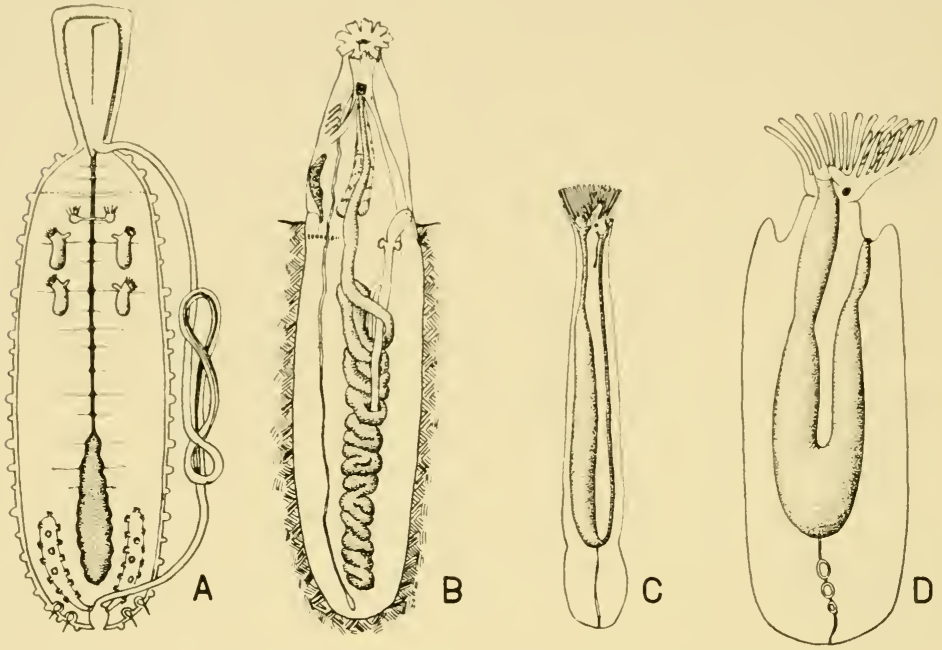

Fig. 57. Schemes illustrating the transition from Polymeria to Oligomeria: A, echiurid; B, sipunculid; C, phoronid; D, ectoprokt.

chaetae. Even among the recent species we can observe all the stages of this reduction which finally led to the emergence of a new animal type. These stages are well represented by the following forms: Arenicola-Sternaspis (with the disintegration of the dissepiments)-Echiurus (the segmented perigastrocoele can be found in their embryos only)-Sipunculus-Phoronis (Fig. 57). We will later return to discuss the Sipunculoidea in more detail. The elongated worm-like form had been preserved by all these animals in spite of the loss of an external and of an internal segmentation. We frequently hear in this connection 
of modified trochophores; this, however, does not have any sense if viewed from the phylogenetic point of view. Greater differences can be observed during the embryonic development.

The most important conclusion we can reach on the basis of our present discussion is that the polymerous state of the perigastrocoele must be considered as a primary, primitive phenomenon, and that the oligomerous state had only evolved secondarily due to a reduction of the segmentation. This reduction had been caused by a changed way of life. This development had finally led to a complete uniformity of the perigastrocoele.

\section{The Phylum Oligomeria and Its Classes}

We come now to a point of the construction of the natural animal system and the formation of the phylogenetic tree. This is considered quite generally as disputable but it can easily be solved if viewed from our standpoint. All zoologists agree that the Arthropoda represent a climax in a generally progressive evolution of the animal world and that for this reason no further evolution had been possible along this line. We must therefore presuppose that the evolution which led to the emergence of the Chordonia and of the Vertebrata, had started from a lower level than that reached by the Arthropoda. It has already been indicated how various zoologists believed they found the point of separation of the two lines of evolution in various parts of the genealogical tree. In the scheme of the genealogical tree we can find this evolution represented by two strong branches, the protostomic and the deuterostomic branches. As a typical example we can mention here the genealogical tree as proposed by Cuénot (see Fig. 50). Without any intention to deal here in detail with all the attempts that have been made so far to represent the phyletic relationship between the Protostomia 
and the Deuterostomia (also when treated under other names which we have already mentioned) I wish only to point here to the suggestion made by palaeozoologist Müller (1958). Here we can find a combination of the scheme as it was proposed by Cuénot-which Müller obviously considers as the best among the now available schemes-and of my diagram. The uncertainty Müller feels regarding the point where the left branch (the Deuterostomia) should begin, according to the interpretation proposed by Cuénot and by a number of other zoologists, is characteristic. A small operation only is needed here to reach a well-founded conclusion. The left branch which is hanging loose in the scheme proposed by Müller can be extended so much that it reaches the right branch at a point above that spot where the Mollusca branch off, and below the point where the Annelida branch off. In this way the two main branches are changed into a stem, and the Annelida-Arthropoda are represented as one of the main branches. It is true that in this way we expose ourselves to the accusation that we try to revive the old, long obliterated idea of an "l'échelle animale" or even of a general stage-like sequence. I consider such an accusation as unjust because in my attempt there is neither a simple gradation, nor only one uniformly progressive line of evolution. My construction represents a true genealogical tree, and not a ladder, with the only difference that this tree has a real trunk (as this is normal in all the trees) and only one main top. The new element which can be found in my attempt, however, is the fact that in my interpretation of natural facts I have come to the conclusion that there has been a long era of a retrogressive evolution. I have endeavoured to make this suggestion as feasible as possible.

During the transition from the phylum Ameria to the phylum Polymeria, as well as from the phylum Oligomeria to the phylum Chordonia, evolution always began at a somewhat lower level of the earlier type, yet it immediately continued in a progressive direction. This evolution led to the emergence of a new type whose organization reached another higher 
level afterwards. Due to various specializations this new type again evolved into various subtypes. Here we can find only a few side lines that led to a simplification of the organization because of a retrogressive trend in their evolutions (Tardigrada, Pentastomida among the Chelicerata, Acarina, the parasitic Cirripeda; among these there may also be some cases of neoteny). Several classes and subclasses have reached a very high level of organization, especially among the Arthropoda, with the Insecta as the highest point of this development. Had there been no further evolution, first in a retrogressive direction (Oligomeria) which was followed by a progressive evolution (Chordonia); the Arthropoda with the Chelicerata and the Insecta would be the two groups representing the climax and the end of all the evolution of animals.

\section{The Sphere of the Phylum Oligomeria}

Those who believe in a dichotomy of the "Coelomata" into the Protostomia and the Deuterostomia meet with great difficulties as soon as they try to draw exact limits between these two main subdivisions of the Coelomata. This could not be the case if these two subdivisions really stood clearly in opposition to each other, as was originally believed. Above all there should be no such combinations as protostomy on the one hand, and enterocoely on the other, or vice versa; yet such combinations do actually exist. We can avoid these and many other difficult and unpleasant facts if we interpret these apparently conflicting phenomena by means of the mosaic theory; if we consider that both the deuterostomatous and the enterocoelic states evolved from the protostomatous and ecterocoelic states. For the sake of a more easy understanding of these processes we will find it helpful, as this has repeatedly been emphasized, if we take into consideration the changes in the way of life and if we exploit more critically the ontogenetic facts. 
The fact has already been known for some time and it has been accepted by many zoologists, that there exist several small groups of worm-like animals which somehow represent the transition from the Annelida to the Eucoelomata which consist of few segments only. These intermediate forms are partly enterocoelic, partly ecterocoelic animals. Earlier this group was frequently called the Gephyrea (the bridging animals). This grouping, however, was out of agreement with the facts because it includes animals which clearly belong to the Annelida; these animals were called now the Gephyrea chaetophora (Echiuroidea). These Echiuroidea have been classified quite generally and rightly among the Annelida, usually as one of their independent groups (as a class or even as a phylum which is certainly an overvaluation).

As regards the system of the remaining small groups which are not at all or only partly segmented, and which possess at the same time a perigastrocoele, we find above all that the group Molluscoidea corresponds least to the natural facts, and that its name has been least fortunately selected. We can see that according to Grobben, the Molluscoidea (which are also called Tentaculata in the parenthesis) consist of three classes, i. e. the Phoronidea, Bryozoa (Ectoprocta), and the Brachiopoda. These Molluscoidea appear immediately after the class Mollusca within the phylum Protostomia (Zygoneura, according to Hatschek). All these three classes have in reality nothing in common with the Mollusca. This is a grouping which has been introduced because of a lack of a better solution. Neither is the correct position given to the Mollusca in this system; the Mollusca are clearly primarily unsegmented animals, while at the same time the unsegmented character of the Molluscoidea must be considered as a secondary phenomenon. Fortunately enough we find that this name "Molluscoidea" has fallen into disuse.

A much better name has been proposed for this animal group by Lang. He called them Prosopygia. The members of the group which have thus become united under this name 
do really show a characteristic phenomenon; in these animals the anal opening was transferred to the anterior part of the bodies as a consequence of their semi-sessile or fully sessile way of life. Lang has therefore classified among his Prosopygia the Sipunculoidea which are usually placed close to the Annelida; the Sipunculoidea are, as a matter of fact, prosopygous "worms." Few zoologists have accepted these suggestions proposed by Lang (e.g. Haeckel). Beklemischew, on the other hand, has gone very far in the opposite direction; he limited Prosopygia to the Sipunculoidea only.

The word Tentaculata has been much more widely accepted as a name of this group. There have also been some other suggested names, e.g. the Podaxonia and the Vermidia (Delage and Hérouard), yet their spheres have been slightly different. All these attempted groupings have one thing in common; they include a part only of the Eumetazoa which possess few segments only and which are therefore classified among the Protostomia. In this way they are sharply separated from the remaining groups with few segments which are classified among the Deuterostomia. Few zoologists have tried to escape this magic circle attempting to reach better solutions on the basis of other concepts.

The attempt which comes closest to my suggestion both as regards its sphere and its name was made by Krumbach within the framework of his "Vermes" (Vermes Polymeria). Yet they do not include either the Sipunculoidea (in spite of the fact that his characterization of the Oligomera suits to the Sipunculoidea, with the exception of the number of their segments) or the Echinodermata which actually belong to the Trimeria. Schneider (1902) unites his Ameria (with the Echinodermata as their only cladus) with the Trimeria (Enteropneusta and Tentaculata) into the type Coelenteria; they are thus "Metazoen, deren Mesoderm vom Entoderm stammt und phylogenetisch als Enterocoel auftritt" which, however, does not correspond with our concept. Lameere united all the groups which we classify as Oligomeria-with the exception 
of the Sipunculoidea-under the name Dérosomes, yet he placed them within the framework of "Les Vers." Ulrich (1950) has also united, as we have done, all these groups (with the exception of the Sipunculoidea) but he used here different names (Tentaculata for three of our classes, and Enteropneusta for two of our classes); at the same time we find in his work the Chaetognatha accompanied by a question mark as a sign that Ulrich could not come to any decision regarding the classification of this truly aberrant group. The Sipunculoidea are also equipped by Ulrich with a question mark. The frame work, however, of this group is in Ulrich entirely different from the sphere of our Oligomeria. All these groups are placed under the name Archicoelomata (as a counterpart to the Neocoelomata which again are equipped with a question mark) at the beginning of the Bilateria (Coelomata). This must be interpreted in such a way that Ulrich believes that all these animal groups have primarily few or no segments; correspondingly we find the Platyhelminthes with the Nemertini, Endoprocta, and the Priapulida placed at the end of the system, together with the Protostomia which are preceded by the Deuterostomia. It is noteworthy to see that Ulrich inclines to judge "die Evolution des heutigen Cnidarenmaterials nicht als progressiv, sondern als regressiv." In his text Ulrich makes a good observation regarding the widely accepted division of the Coelomata into the Protostomia and the Deuterostomia. He states, "sie (sc. this division) wird jedoch der tatsächlichen Mannigfaltigkeiten nicht gerecht, tut den Dingen mancherlei Zwang an und gedenkt die im einzelnen ungeheuer heterogenen, bruchstückartigen Producte einer unermässlichen Evolution bereits mit einem intimen Einzelmerkmal regieren zu können." Ulrich stands clearly too much under the influence of the ideas that had been proposed by Remane.

We have not accepted the division of the Eumetazoa into the Protostomia and the Deuterostomia as such a division could not represent an actual phyletic development. We are therefore not hindered in uniting all the groups of the Eumeta- 
zoa which show an impoverished or reduced segmentation (yet not individual species!) into a large group which we call the Oligomeria. We think that this large group actually represents the natural facts. Here we include those groups which do not show a clear segmentation of the perigastrocoele, as well as those which have three pairs of the perigastrocoele. The following nine classes are therefore included in the phylum Oligomeria:

(1) Sipunculoidea.

(2) Phoronoidea.

(3) Pogonophora.

(4) Brachiopoda.

(5) Chaetognatha.
(6) Ectoprocta.

(7) Echinodermata.

(8) Pterobranchia.

(9) Enteropneusta.

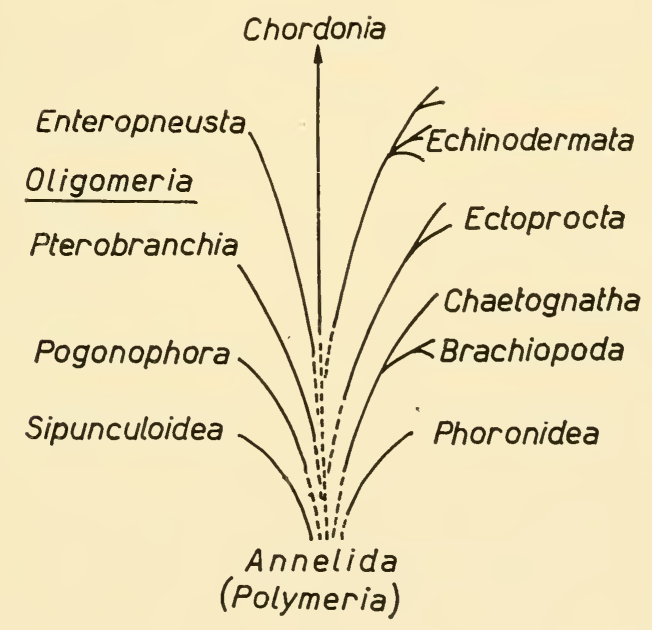

Fig. 58. Genealogical tree of the phylum: Oligomeria.

The phylum Oligomeria consists thus of nine classes which usually represent few species only, in comparison with the fifteen classes that belong to the Ameria, and the eight classes which belong to the Polymeria (Fig. 58). There are two classes only of the Oligomeria which represent a larger number 
of species. They are the Ectoprocta and the Echinodermata. All these species live without any exception in water, and there are few exceptions only (among the Ectoprocta) which do not live in the sea. With the exception of the class Chaetognatha-which is also otherwise an aberrant group-we find that all the groups of the Oligomeria live on the sea bottom. Lameere (1931) and Hadži (1958a) have shown that we can best explain the organization and the place where the Chaetognatha live, which is quite unusual for the Oligomeria-the free water zone-if we consider that the Chaetognatha evolved by way of neoteny from the planktonic larvae of the Brachiopoda. There is no other class or even a lower systematic category among the recent Oligomeria whose species have also become adapted to a life in plankton. Individual species can only be found among the Echinodermata which had also evolved into planktonic animals (Pelagothuria and a few other species), a fact which could be least expected because of the internal skeleton which had also been developed by these same animals. On the other hand we find a rich variety of planktonic larvae developed by these Oligomeria, a fact which could easily be expected; these larvae show widely different forms and structures which are of little use as material used in our phyletic speculations.

The fact that among all the recent nine classes of the Oligomeria there are only two with a richly developed asexual reproduction combined with a formation of cormi (Ectoprocta and Pterobranchia), shows the limited plasticity of the Oligomeria. Polymorphism can be observed quite generally among the Ectoprocta. A very surprising circumstance is the fact that even a dully sessile class such as the Brachiopoda are no longer able to reproduce asexually. We can understand this if we interpret the Oligomeria as secondarily simplified and specialized animals which had evolved from the Polymeria by way of a retrogressive evolution.

On the other hand, widely different trends in evolution can be found within the group Oligomeria itself; the situation here is very similar to the one we have been able to observe in the 
Ameria. In the Echinodermata a secondarily emerging progressive trend is most clearly noticeable; this has been the reason why the Echinodermata have been treated so frequently by the systematists as a special type or as a phylum. The Echinodermata had developed, apart from a hypodermic skeleton, new systems of coeloms and special organs of movement also which are a consequence of their secondarily acquired free mobility. The Echinodermata certainly represent the climax reached in the progressive evolution within the phylum Oligomeria. They have even become indirectly able to reproduce asexually by way of an intensified regenerative ability. They also show a conspicuously rich development of various subtypes, many of these have died long ago.

Among the sessile Oligomeria we notice a progressive evolution in the Ectoprocta too. Yet as regards the condition reached in the organization of their individuals (we can speak here almost of subindividuals) they have preserved a low level, or they had sunk in this respect to an even lower level. A progressive trend can be observed in the formation of their cormi (colonies) which are frequently regularly built and polymorphous.

There are not less than six classes of the Oligomeria, i.e. Sipunculoidea, Phoronidea, Pogonophora, Brachiopoda, Chaetognatha, and Pterobranchia which all include a few species only (only the Brachiopoda possessed numerous species during their geological history), which represent "insignificant" side branches of the evolution that are of little use in our phylogenetic speculations, and which do not play any significant role in the life of the sea.

Special attention must be paid to the class Enteropneusta even if this class does not appear very important from the ecological viewpoint or with regard to the number of species it includes or the living mass. The species which belong to this class (ca. seventy-five) are some of those Oligomeria which had partly won the ability to move freely; they are therefore semi-sessile animals which do not live in tubules built by 
themselves but rather burrow into the soft floor of the sea. They also show a considerable morphological-physiological specialization in this direction which can be observed above all in the anterior end of their bodies. The trend to preserve a "worm-like form" is clear in the Enteropneusta (there are even species which can be several metres long). Even more important, however, is the fact that they show a strong inclination to a polymerization which re-emerges in these forms. This polymerization involves several systems of organs (the sexual, respiratory, digestive, etc., systems) along the third body region which is quite large in these animals. A very important fact also is the circumstance that in the Enteropneusta the maximum number of segments of the perigastrocoele sacks is still preserved. There are still other properties which must be mentioned here such as the presence of a ventral and of a dorsal nerve cord which are equipped with canals, the inclination of these animals to develop an endoskeleton and to form a pair of body folds for the sake of protection, of openings which are used as breathing organs, an inclination of their intestinal tubes to form skeleton-like outgrowths and glands similar to a liver as well as a cavity resembling a pericardium. The absence of metanephridia is conspicuous.

Thus we come to the conclusion, which has actually been also expressed by many other zoologists, that the recent Enteropneusta must be considered as considerably changed (because they are specialized) descendants from an oligomerous ancestral form, which had been at the same time the starting point of the evolution of the next higher phylum Chordonia, that had developed from this ancestral form during a very remote geologic past. This derivation of the Chordonia from some ancestral form, which they had in common with the recent Enteropneusta, is certainly more probable viewed from my standpoint than is the other variant which has also been repeatedly suggested by various zoologists and according to which the Chordonia had evolved from an even more primitive ancestor. 
Finally we must not completely bypass the Graptolitha, an extinct class of the Oligomeria, in spite of the fact that in the present study we take into consideration the recent groups only. There can be no doubt that these Graptolitha must be classified among the Oligomeria and not among the so-called Coelenterata. They obviously represent a case parallel to that of the Siphonophora, among the Hydrozoa, i.e. a type with polymorphic cormi (colonies). We will not further discuss these Graptolitha because they represent a blindly ending side branch of the Oligomeria, and because they are now completely extinct.

\section{The Problem of the Inclusion of the Sipunculida Into the Phylum Oligomeria}

Following the interpretations suggested by many zoologists, and among them many specialists, I considered earlier that the Sipunculida should be classified among the Polymeria and that they should be looked upon as closely related to the Echiuroidea and the Annelida. After a thorough and critical revision of the actual facts, however, I have now come to the conclusion that some older zoologists had suggested correctly - among them especially Arnold Lang who was followed in this respect by Haeckel-that the Sipunculida must be more clearly separated from the Annelida and that they should be classified among the Prosopygia.

As regards the conditions of their perigastrocoele we can find that the Sipunculida show even more oligomerous properties than all the remaining classes of the Oligomeria. They have even lost last traces of an intestinal and of an external segmentation; their perigastrocoele has become a completely uniform organ. They show neither mesenteries nor dissepiments, a fact which is clearly connected with a change in their intestinal conditions. Their anal opening was transferred to the anterior part of the animal body, and their intestinal tube is not only U-shaped as in Phoronidea but it is also coiled. They live as 
semi-sessile animals in canals which they burrow in the sea floor. At the same time, however, they have also preserved some traces of a free mobility; animals have been observed that swam over short distances by means of sinuous movements, a fact which I wish particularly to call attention to.

The standpoint which can be defended here is that the conditions that can be observed in the Sipunculida represent the final stage of an evolution which led from the freely moving Annelida by way of the tubicolous and burrowing Annelida to the Echiuroidea and finally to the Sipunculida. The simple conditions that can be observed in the Sipunculida are therefore not a primary phenomenon. In the Echiuroidea we can still find several peculiarities of the Annelida clearly developed, thus above all the polymery and the neuromery which are temporarily recapitulated in their ontogeny, as well as the metanephridia and the remains of a parapodial apparatus, etc., while at the same time all these peculiarities of the Annelida have been lost in the Sipunculida. There is clearly a sharply-cut limit between the Echiuroidea and the Sipunculida which becomes even more clear if we liberate ourselves definitively from the notion of "worms" ("Vermes") as a systematic category which has now completely lost its significance.

The Sipunculida which have remained to a lesser degree freely moving animals had evolved as such from a simplified structure into a specialized direction; during this evolution they had developed many new properties, and this is the reason why they have been considered, correctly, as an independent higher systematic category (a class, frequently even as a phylum which seems to me an overvaluation). They also show more and more clearly, besides the prosopygy which is a consequence of their burrowing and sessile way of life, another peculiarity which is characteristic of all the Oligomeria; the division of their bodies into two regions (the prosoma and the metasoma). The anterior region which is frequently divided into two parts shows the strongest development in the most primitive groups of the Oligomeria, and 
above all in the Sipunculida. As an introvert it can even be longer, at least in some cases (Phascolion, Aspidosiphon, Onchosoma), than the length of the actual body, longer than the postsoma. This proboscoid introvert had developed in the fully sessile Ectoprocta into the so-called polypid; the postsoma on the other hand is called here a cystid.

A polypoid develops from a "worm;" one of the most remarkable features of this development is a radially-symmetric circle of tentacle-like bodywall extensions which had evolved around the oral opening and which show in some groups of the Oligomeria a more bilateral arrangement (in the shape of a horseshoe, Phoronida, Brachiopoda, a considerable part of the Ectoprocta). A group of tentacles arranged approximately in the shape of a horseshoe can be found already in some species belonging to the Sipunculida.

The fact seems to me to be very important that as early as in the Sipunculida we can find a kind of an initial formation of a calcified exoskeleton as this is best shown by the case of the Aspidosiphon speciosus (according to Hyman). On the one hand we can find here a "posterior shield" secreted at the posterior end of the postsoma which can be compared with an ectocyst, and on the other hand there appears another protective layer at the basis of the introvert, at the place where a protective plate (operculum) can be found in the Ectoprocta, and which corresponds to the collar region, or rather subregion, of many Oligomeria. The introvert of the Sipunculida evolved into a very important organ which is no homologue to the proboscis of the Echiuroidea. To this we must also add the strongly developed retractors that can be frequently found in the Oligomeria. The introvert is frequently covered with various protuberances, even spines, which can be considered as a specialization of the Sipunculida.

A characteristic peculiarity of the Sipunculida is the fact that the place of their anal opening, which had been transferred frontwards, has not been completely stabilized. It usually occurs dorsally at the upper end of the postsoma; in the Onchnesoma 
squamatum, however, it can be found considerably farther forwards, on the introvert itself. Their skin which is generally cuticularized and which is closely connected with the skin muscle tube shows a rich differentiation, it is richly equipped with sensory organs, and it contains numerous skin glands; even coelom canals can be found growing through this skin.

As regards the adult form of the Sipunculida it can be stated that we can notice here some similarities with the Annelida which is by no means a surprising fact, because the Sipunculida had evolved in all probability from some ancestors which they had in common with recent Annelida. They also show some peculiarities that can be observed in the Oligomeria as well as some characteristics that can be found only in the Sipunculida.

As regards the properties the Sipunculida have in common with the Annelida, mention has most frequently been made of the structure of their central nervous system; this is rightly so in spite of the fact that they have preserved the last traces only of a neuromerism as an irregular arrangement of the pairs of side nerves. This circumstance is important because it serves as good evidence that here we do not have an initial state in the development of the neuromerism but rather a discontinuation of the segmentation. The emergence of sensory organs must be evaluated as a progressive and a prospective phenomenon which is closely connected with the cerebral ganglion (the supraoesopheageal ganglion).

Less significance should be attributed to the supposed similarities which the Sipunculida share with the Annelida in their ontogenetic morphogeny because here we find traits which are much more characteristic of the Sipunculida. It is true that the Sipunculida are Protostomia inasmuch as their oral opening coincides locally with the anterior end of a very elongated blastopore, yet here it is formed anew after the blastopore had first grown together. It has repeatedly been emphasized that in my opinion too great a phyletic significance has been attributed to the formation of the oral opening during the ontogeny. 
The question has been widely discussed whether there were at least some temporary traces of a segmentation in the Anlage of the perigastrocoele which develops from a pair of teloblasts. Hatschek (1881) could find no trace of a segmentation in the embryos of Sipunculus nudus. Gerould (1906) on the other hand described and depicted from three to four pairs of coelom sacks which he believed to have found in the embryos of Golfingia vulgaris. Gerould has later revoked these facts; he considered these phenomena later as a consequence of a folding which is due to a strong contraction. Gerould made this statement in a letter only, which is quoted by Hyman. Those who prefer to see in the Sipunculida a preliminary stage of an evolution in the direction towards the Annelida will find the state of things, such as it appears now after Gerould's revocation of his findings, as very important and favourable to their interpretation. I, on the other hand, consider that it is much less important whether in fact a segmented state is still recapitulated in the perigastrocoele of some species belonging to the Sipunculida. It is not impossible that during the future researches forms will be discovered among the more than 250 species of the Sipunculida, which differ considerably from one another, where such recapitulations will be found preserved. Yet even if no such traces of a segmentation are actually found preserved in any of the recent species, there are still other numerous properties which seem to support my thesis that the Sipunculida evolved as a result of a secondary simplification and of a specialization of their way of life.

I wish finally to mention here the urns which are so characteristic of the organization of the Sipunculida. The structure and the ontogenetic development of these urns are well enough known. Yet there exists a considerable uncertainty about their function, and even more so of their origin, of their phylogeny. These urns remind me-by the whole line of their development: the freely swimming urns, the attached urns, the coelothelium cells which are strongly covered with cilia; by the fact that they collect material to be excreted; and finally by 
their visible function as motors of the coelom fluid, of the circulation-of the so-called rosettes that can be found in the Ctenophora which I (Hadži, 1957) have tried to explain as remains of the terminal organs of the protonephridia that had grown together with the gastral epithelium. These could thus be some kind of analogous formations. The ancestors of the Sipunculida possessed many pairs of the metanephridia that evolved from the protonephridia. These metanephridia have generally become superfluous as a consequence of a greatly changed way of life. It is the anterior pair only or a few individual metanephridia (in one species of the Sipunculida we can even see that all the metanephridia have been lost) that have been preserved in a somewhat changed form in order to serve above all as gonoducts. The sexual cells are soon freed in the Sipunculida from their gonads, they circulate in the coelom fluid which functions at the same time as blood, and it was in this way that the metanephridia had been changed into gonoducts. This change had actually taken place as early as in the Annelida. The nephrostomes of the remaining metanephridia had undergone a considerable transformation; they evolved first into the "sitting" and later into the freely swimming urns. At the same time the individual cells have preserved a strong ciliation. This seems to me to be a very probable interpretation which can also be used as a working hypothesis.

The richly developed net of canals are either of the coelomatic or of a schizocoelic origin and which serve as a "hydraulic machine" of these remarkable animals must be considered as a special "invention" of the Sipunculida.

Finally, we must not leave unmentioned the fact that solid forms can be found in their "coelom blood." Baltzer (1931:269) wrote about these forms as follows, "Die Mannigfaltigkeit aller dieser Zölomgebilde gibt dem Leibeshöhleninhalt der Sipunculiden eine erstaunlich hohe Komplikation, eine Kompliziertheit, die von keiner anderen Tier-Gruppe erreicht wird." I mention this circumstance because it again 
supports my thesis of an evolution of the Sipunculida from the ancient Annelida. It does in no way corroborate the opposite thesis which was formulated, for example, by Hyman (1959, Vol. V., 690) with the following words, "The Sipunculida are therefore to be conceived as protostomatous coelomates placed along the main line of the Protostomia that leads to Annelida, Mollusca, and Arthropoda."

\section{The Oligomeria as the Initial Form for the Evolution of the Chordonia}

One of the characteristics of the Oligomeria is the fact that they do not include even a single subgroup (class) whose species would lead a life considered as characteristic of the Eumetazoa. There is one class only which includes exclusively freely moving species (the partly inert Spadella does not disturb this general picture); these are the Chaetognatha. We have already stated at the beginning of the present study that life in free water cannot be considered as a primary and normal way of life. I have tried to solve the problem of the Chaetognatha together with Lameere by attempting to derive them by way of neoteny from the planktonic larvae of the Brachiopoda. This type of derivation corresponds best with the known facts. The Chaetognatha, as strongly specialized Oligomeria, cannot be taken into consideration as a possible initial form for the evolution of the Chordonia. The similarities that can be observed between the Chaetognatha and some small fishes are a purely external phenomenon. The structures, the ontogenies, and the types of movement, however, are entirely different in these two animal groups.

Free mobility has been attained secondarily by the Oligomeria in two other instances. We find, however, that in spite of a prolonged evolution the Echinodermata have still preserved traces of an original radial symmetry that evolved from an even older bilateral symmetry. This can be seen 
even in the burrowing Echinoida where new signs of a bilateral symmetry can be observed re-emerging. The adult forms of the Echinodermata (we state this explicitly), however, can hardly be taken into consideration as possible ancestors of the Chordonia as this is the case with the Chaetognatha. We have emphasized the statement "the adult Echinodermata" because attempts have been made, even by scholars who must be taken seriously, to represent the planktonic and at the same time bilaterally symmetric larvae of the Echinodermata as the initial forms for the evolution of the Chordonia type.

The second attempt which was made by the subtypes of the Oligomeria to achieve secondarily a free mobility can be observed in the small group Pterobranchia. The case is interesting because these are clearly primarily tubicolous animals. No type of the Eumetazoa could or did ever develop from these forms. Their organization had been strongly and irreversibly reduced. Quite remarkably we can find in the Pterobranchia - in these only and in the Enteropneusta - an open connection between the anterior intestine and the external world. This connection, however, which is supposed to be used by the animal in its respiration, was not invented by the Pterobranchia; it can also be found in some older types, even among the Ameria (Gastrotricha). This branchiotrema has been the main reason why zoologists have tried to bring the Pterobranchia into a closer connection with the Enteropneusta which are even better equipped with the gill slits. I believe that zoologists have gone too far with these attempts and that these two groups of the Oligomeria developed independently from each other in a very remote geological past. It seems to me that the Enteropneusta had never really lived as tubicolous animals and that they are therefore the only group of the Oligomeria which possess a primary free mobility that has been preserved by them down to the present day. The possibility cannot be completely excluded, however, that the Pterobranchia-which are now represented by few remaining forms only-had evolved from some burrowing 
ancestors which they had in common with the Enteropneusta. Their evolution proceeded from this ancestral form towards a tubicolous way of life and it had therefore been separate from that of other tubicolous Oligomeria.

A derivation of the freely moving Chordonia from such tubicolous Oligomeria as the Phoronida and the Pogonophora seems to be rather improbable. These two subtypes are clearly specialized, the Pogonophora eren more so than the Phoronida. A complete loss of the digestive system in a non-parasitic animal type can certainly be considered as a unique case. It is clear that nothing could ever have possibly evolved from such specialists; they represent blind side allers of erolution which are so numerous in the animal world. It is unnecessary to argue and prove that no erolution in the direction towards the Chordonia with the Brachiopoda and the Ectoprocta, two completely sessile classes of the Oligomeria, as initial forms had been possible. The situation as it appears from our standpoint-which we consider as the best founded point of viewis such that the Enteropneusta only can be taken into consideration as a possible initial form for the evolution of the Chordonia. Even if it is unnecessary, I nerertheless wish to state explicitly that quite naturally I do not see in the recent Enteropneusta the ancestral forms of the Chordonia; these ancestral forms were in reality at the same time ancestors of the present-day Enteropneusta and lived as such in the remotest geological past. From these ancestors had evolved divergingly (or I could also say in parallel) on the one hand the burrowing Enteropneusta, and on the other hand the Chordonia which had returned to a freely moving life. From these freely moving Chordonia a new subtype (class) had again evolved, in no way accidentally, of the sessile Chordonia which are represented by the blindly ending side branch of the Tunicata.

When we try to derive the Chordonia from the primitive Enteropneusta, among the Oligomeria, we meet with apparently the greatest difficulty-besides some other problems 
which can be solved more easily-of how to explain the evolution of the polymerous state of the Chordonia from an oligomerous state with three segments at the most and with the same number of pairs of the enterocoele (the anterior pair can also be found grown together).

We must include here first a general observation before we try to solve this difficult problem. The number of the main types of life and of the corresponding main forms of life is very limited. We can therefore see them repeated again and again within the framework of all the higher groups. The products of evolution, however, show as parallelisms considerable differences in spite of frequent analogies and external similarities; this is due to the fact that they always start their evolution from different initial points (levels of organization). Because of these similarities and analogies it is frequently difficult to identify the initial point of evolution. In our attempt to solve the problem of the origin of the Chordata we meet with one of the most important repetitions, the repetition of the body segmentation. It first took place by way of an irregular polymerization finally, to reach a regular polymery during the transition from the Ameria to the Polymeria; it was a consequence of a rapid type of movement by means of a curving of the entire body. Polymery was subsequently reduced to an oligomery within the framework of the Oligomeria. During the transition from the Oligomeria to the Chordonia we can again observe an irregular polymerization which led to polymery. And again this had been caused by an active type of movement. In this case, however, we can hardly speak of a sinuous type of movement; here the waves of contractions are very low, and the segments of muscles involved in this movement very short, following each other closely; they are limited to the dorsal side. The effect of these contractions had therefore been greatly increased. Later we can again observe in the Chordonia a secondary reduction of the number of segments, above all among the Tetrapoda where the reduction was caused especially by the 
evolution of their extremities. This had again been followed by a (third) polymerization and by a more sinuous type of movement which is typical of snakes. Among other changes and innovations that can be found in the Chordonia we must mention above all the formation of an internal axial skeleton, with the chorda dorsalis at the beginning.

In the recent animals we unfortunately find a deep gap between the Oligomeria and the Chordonia. This transition had taken place in the remotest geological past. The transition was made by animals whose soft consistency had little chance of preservation as a fossilized form. The speed itself of the evolution was also in all probability increased. Finally, it also seems that it was just this transitional stage which had lived not in a pure sea but, at least partly, in a brackish or fresh water and this again is an unfavourable circumstance for the preservation and for the discovery of such fossilized remains. We have therefore no other alternative but to take refuge in the comparative morphology of the recent adult forms and of the developing stages and at the same time to use our imagination within proper limits.

\section{The Fourth and the Last Phylum of the Eumetazoa; the Chordonia}

It is in no way surprising in view of the available material to see how many different ideas, hypotheses, and theories have been proposed so far in various treatises and even books about the origin of the Chordata, and thus also of the Vertebrata. Only little additional material has recently come to light, such as the discovery of the fossilized remains of the extraordinarily old chordate Jamoytius. It would transcend the limits of the present book if we discuss in detail this problem here. It does not even belong to the sphere of my own research. I will therefore limit myself to show only how this problem could be solved if studied from our standpoint and 
under the application of our working method; I will therefore exclude from this account all those elements which could be too hypothetical.

The situation has become slightly better now because there is hardly any living zoologist who would still be willing to accept a suggestion to derive the Chordonia either from the Nemertinea (Hubrecht, 1883), or from the Arthropoda of the type of the Limulus (Gaskell, 1895-1910, and Patten, 1912). The interpretation which derives the Chordonia directly from the Annelida (Dohrn, 1875; Semper, 1875-6; Minot, 1897; Delsman, 1922; and many others) finds recently less and less acceptance, though earlier it was frequently put forward; the interpretation itself was actually based on a fantastic idea that had been proposed by Hilaire (1818). The same interpretation (it is in reality only a minor hypothesis) has recently been revived in a somewhat changed form, when the Oligomeria have been suggested as a possible starting point of the evolution of the Chordonia. The Oligomeria themselves, however, can easily be derived, as we have already shown, from the polymerous Annelida. Yet even within the limits of a general theory which derives the Chordonia from the Oligomeria there exist several possibilities which can claim various degrees of probability. There exist therefore several interpretations of this kind which were suggested, e.g. by Bateson (1884-6), Brooks (1893), Willey (1894), and Garstang (1894-8) whose interpretation has been accepted by Sir Gavin de Beer and recently by N. J. Berrill (1955) in a special book he wrote on this subject.

I do not intend to begin here with a detailed analysis of these hypotheses about the supposed annelidan-arthropodan ancestors of the Chordonia; we will agree on this point with the critical discussion written by Berrill (Berrill, 1955:2-5). I wish only to state that the peculiarities which the Chordonia have in common with the Annelida (for example the solenocytes, the close connection between the sexual organs and the nephridia). These peculiarities have 
been discussed above all by Boveri (1892) and by Goodrich (1902) and do not seem to disprove the interpretation that the Chordonia did not developed directly from the ancestors of the recent Annelida and that instead this evolution was indirect, by way of the Oligomeria. This is how we can explain the reactivation of certain elements in the Chordonia while the same elements have been lost in the recent Oligomeria.

We can divide all the hypotheses which derive the Chordonia from the Oligomeria into two groups. According to one group of these hypotheses the Chordonia evolved from the larvae of the Oligomeria, thus by way of neoteny; the second group of these interpretations derives the Chordonia from the adult forms of the Oligomeria. It is by no means rare to meet the former type of interpretation. It corresponds to the widely accepted method of solving various phylogenetic problems by means of larval forms. Personally, I am not a priori opposed to use neoteny in various phylogenetic speculations. I myself have used the phenomenon of neoteny twice when I tried to explain the origin of new animal types. First, in connection with the suggested derivation of the Ctenophora from the planktonic larvae of the polyclad Turbellaria, and secondly, in connection with the derivation or the Chaetognatha from the planktonic larvae of the Brachiopoda. I am also firmly convinced that in several other cases new forms, which emerged during the phylogenetic processes, evolved by way of a neotenization. This type of development has to be taken into consideration in connection with the evolution of the Larvacea or Appendiculata, among the Tunicata, and with the Proteidae, among the Urodela, etc.

Yet at the same time I think that we must not go too far in this direction, and that we should use an explanation by means of a neoteny in those cases where all the other types of evolution appear as completely improbable. It has already been mentioned how the numerous attempts that have been made to interpret the origin of all possible groups by means 
of neoteny (or paedomorphosis) have been looked upon by many zoologists with a considerable degree of, partly justified, suspicion.

In connection with a derivation of the Chordata from some larvae of the Oligomeria or even of the Tunicata, I again think that such a derivation does not appear to be well founded and that it can therefore claim little probability even if it is not in principle impossible.

First, we will mention here the attempt made by Berrill (1955), whose views from our standpoint must be considered as completely erroneous. Berrill's derivation starts with the planktonic larvae of the Tunicata which he calls tadpoles. We must above all reject the usage of this word for the planktonic larvae of the Tunicata. These larvae are a very specific form which had been developed by the Tunicata and they have nothing in common with the tadpoles of the Anura, with the exception that they are both larval forms that occur in the Chordata. The remote external similarities can be considered as pure parallelisms.

In the Tunicata we can observe a process which we have already frequently met in the Invertebrata, i. e. because of their secondary transition to a sessile way of life, the Tunicata developed in their ontogeny a planktonic juvenile form, a larva. This certainly is a very illuminating phenomenon. Berrill (1955:11) also considers that the Ascidia are "primarily a primitive sessile, marine group of organisms." The Ascidia, however, are considered quite generally and correctly, together with the Tunicata, as lower Chordata which evolved into secondarily sessile animals. The important problem of the origin of the Tunicata is solved by Berrill very simply with the following words, "... which may or may not be related to the hemichordates." It is quite natural that Berrill who derives the Vertebrata from the planktonic "tadpoles" of the Tunicata does not find it interesting to try to solve the problem of the origin of these Tunicata. Yet what can be done if we find that it is quite improbable that any new form had 
ever been able to evolve from the larvae of the Tunicata? The important discovery made by Kowalewski who succeeded in proving that the 'Tunicata, which nobody knew how' to place into the animal system, are in reality genuine even if retrograded Chordonia, has again shown the phyletic significance of these larvae. These larvae have been used as a representative example which could prove the theory of recapitulation. These larvae do indeed recapitulate or repeat something which is not, however, the adult ancestral form but only its ontogenetic stage that has also preserved numerous characteristics of the adult ancestral form.

The whole organization of the Tunicata larvae is specific and one-sided-we can mention here the fact that in them the notochord occurs in the tail only, a body region which had been newly developed by the Chordata-that there is one form only which had evolved from them by way of neoteny (similarly as has been the case with the Ctenophora and the Chaetognatha), i. e. the Larvacea, and nothing else. This subtype of the Chordonia which exists as an ontogenetic stage only points to an early existence of a general type of the Chordata. Berrill (1955:3) good-humouredly mentions a statement regarding the book by Delsman that the book should be confiscated and burnt; we can say on the other hand about Berrill's book that it would be better if it had not been published at all in spite of a number of sound interpretations which appear in it, because of its basic idea which is certainly wrong. The Vertebrata certainly did not evolve from the planktonic tadpoles but rather from the benthonic "worm-like" Oligomeria.

Neither can we consider as fortunate those suggestions which try to derive the Chordonia from the planktonic larvae of the Oligomeria, or directly from the so-called Ambulacralia (Echinodermata and Enteropneusta). Like the larvae of the Tunicata, these are again very specialized larvae of the Oligomeria which could serve, at the most, as a starting point for the evolution of some other planktonic form 
of the Oligomeria, a development which in all probability never actually took place. It could not be considered as a case of neoteny if this larval form evolved into a new type after its descent to the sea bottom, thus after a metamorphosis. The Chordonia, if they evolved in this way, would have a planktonic larva similar to the so-called dipleura. Something of this kind, however, is unknown to us. The interesting proposition made by Garstang to derive the central nervous system and the endostyle of the Chordonia from an immersion of ciliated bands of the Echinodermata larvae must be considered as an entirely erroneous concept, as a purely artificial construction. Where is here the origin of a secondary metamerization, of the notochord, of gill slits, etc.? Such a suggestion could perhaps be taken into consideration if there were no longer any such animals as the Enteropneusta. The adult Enteropneusta are comparatively less specialized than the planktonic larvae of the Ambulacralia. It is therefore easier to derive the Chordonia from the Enteropneusta than from the planktonic larvae of the Echinodermata. Such a derivation is also, therefore, much more probable. The suggestion that the hypothetical immersion of ciliated bands could be the starting point for the evolution of new organs is somewhat similar to Krumbach's attempt to derive the protonephridia from an immersion of the swimming plates of the Ctenophora.

Even less probable than a derivation of the Chordonia from the planktonic larvae of the Echinodermata seems to be a derivation of the Chordonia from some very ancient, armoured, adult forms of the Echinodermata. Such a suggestion was made by Gieslén (1930) on the basis of a comparison between the carpoidal echinoderm Cothurnocystis elizae and the Ostracodermata in which he took into consideration above all their armour. Such a derivation does not seem to be much better than is a derivation from Limulus. These are clearly cases of parallelisms or similarities which are not based on any closer relationships. Berrill (1955:5) has correctly 
criticized such interpretations, adding at the same time a very proper observation that we must search for the origin of the Chordonia much farther back in the geological past than in the now extinct carpoidal Echinodermata and in the equally extinct Ostracodermi, i.e. much earlier than in the Lower Silurian. In my opinion the Echinodermata cannot be taken into consideration as ancestors of the Chordonia either in their larval forms or as adult animals. The Echinodermata represent the largest and the most ramified branch that evolved from the stem of the Oligomeria. Yet this branch ends blind$1 y$, it did not lead to the evolution of a new subtype, and above all it did not lead to the emergence of a new type: the Chordata.

\section{The Probable Origin of the Chordonia}

It is most probable that the Chordonia evolved as the most recent and highest animal type from the Oligomeria. The recent subtypes of the Oligomeria, however, in which we can observe a greater or smaller degree of specialization cannot be taken into consideration as possible ancestors of the Chordonia; they had in the meantime too long a geological period at their disposal for their own evolution. It must nevertheless be admitted that the Oligomeria which evolved during this period in several directions, reached various levels of organization, while at the same time the Chordonia also went through a rich evolution which finally reached its climax in the human species. There still exist some forms of the Oligomeria which are similar to this early form. Animals with radial symmetry which adopted the sessile way of life became strongly simplified and their evolutions have ended blindly, even in those cases when they secondarily readopted free mobility, e.g. several groups of the Echinodermata and several other groups of the Oligomeria. We find then that there remain few slowly moving groups only, and even among these we must exclude the tubicolous forms, especially 
the very specialized Pogonophora (together with the extinct Graptolitha) as well as the Phoronidea. The only group which therefore remains to be taken into consideration in connection with our problem are the Enteropneusta. And even now we can really find in the Enteropneusta the greatest number of the so-called prophetic characteristics in spite of the repeated specializations which they underwent "in the meantime." The Enteropneusta move slowly yet they are nevertheless freely moving animals, they usually burrow in the ground and they have therefore an anterior part of the body which had evolved much later in the Chordonia into the head of the Vertebrata by way of a cephalization.

It is possible that the process which had taken place in the evolution of the Chordonia was similar to the process we have presupposed in connection with the evolution of the Polymeria from the Ameria, and of the Oligomeria from the Polymeria. The evolution of the new type started in all probability somewhere close to the root of the next lower type. In its evolution it had followed Watson's rule; it was due to a "fortunate" combination of several characteristics that had existed scattered in the next lower type and to the emergence of new characteristics which led to the formation of the new type under a simultaneous radical change in the environment and in the way of life.

We must suppose (and we have good enough reasons to) that something unusual had taken place in the period of transition from the Oligomeria - from a primitive form of the Oligomeria which is no longer preserved in recent animals and which it seems had resembled most closely the presentday Enteropneusta of all the recent Oligomeria-to the Chordonia. The transition of an oligomerous animal similar, perhaps, to a Balanoglossus into a new animal form resembling approximately the recent Amphioxus (Branchiostoma), or the fossil Jamoytius, must have taken place in all probability in the soft shallow ground of estuaries, or even in fresh water. This supposition is based on the fact that Jamoytius, the most 
ancient fossil remains belonging to the Chordonia, has been found in the sediments which had been deposited by fresh water. The second necessary presupposition is that during the phase of transition the ancestors of the Enteropneusta which had evolved into the Chordonia had begun to move more intensively as freely moving animals. This change was probably connected with an adaptation of these animals to a life in firmer and coarse-grained sand sediments after they had left the soft mud and the fine sand. The inclination to swim which had never been completely lost, now became reactivated. Simultaneously, we can observe a rich secondary segmentation in the dorsal side of their oblong bodies which had become strengthened due to these changes; this segmentation had taken place above all in the body muscles and in the nerve trunk which had already become extended along the dorsomedian line. Other organs placed more or less dorsally, as the previously uniform perigastrocoele, the gonads, and the nephridia afterwards also participated in this segmentation.

The consequence of the special type of swimming which had been developed more and more progressively by the freely moving Chordonia-with the exception of the Tunicata which had secondarily returned to a sessile way of life-had been a special new formation which had developed at the end of their bodies. Here a new zone of growth emerged whose consequence was a new body region, that of the tail. The more active type of movement led to a progressive development of the two systems which had been inherited from the enteropneustan ancestors, the breathing system and the system of blood circulation. The trend to develop folds in the region of the breathing organs, which can also be observed in the Enteropneusta, had been continued in the lower Chordonia (the atrial cavity for the protection of the gill apparatus); yet this cavity later became more and more retrograded in fishes, etc., because of the completely freely swimming way of life which these animals had adopted. 
The new type of movement of the primitive Chordonia led to a formation of an elastic inner skeleton staff, the chorda dorsalis. Numerous discussions have been conducted on whether the so-called cephalochord of the Enteropneusta can be considered as homologous to the genuine chorda dorsalis, or not. I think that these discussions are completely superfluous. The anterior outgrowth of the intestine which can be observed in the Enteropneusta is certainly not a "notochord" s.str., it is certainly not a chorda dorsalis. It belongs to a group of forms that had been developed by the intestine and which appear repeatedly even if in various ways in the Eumetazoa. These are the intestinal diverticulae which originally have a lumen and which function as a periphery of the intestine. The lumen is later lost, the diverticulae become solid, and they change at the same time their function by their development towards an inner skeleton. The best known instance of this development are the tentacles of the Cnidaria which had secondarily evolved into solid forms. In some species we can even find that some other parts of the intestine also evolve into an elastic inner skeleton (the Tubularia species). Forms similar to the "cephalochord" of the Enteropneusta can occasionally be found in the Turbellaria (Ax, 1957), etc. (Fig. 33). During the ontogenies we can see the chorda dorsalis developed from the "plafond" of the intestinal Anlage, and this can be clearly observed in the lower Chordonia. The "notochord" of the Enteropneusta and the chorda dorsalis of the Chordonia originate therefore in one and the same trend of the intestinal tube to develop first some "hollow" outgrowths, i.e. outgrowths which have a lumen that later becomes solidified. Here we are not interested in the subsequent destiny of the chorda dorsalis in spite of its interesting character. This chorda is stubbornly preserved and it is recapitulated down to the end of the evolution even if it does not have any function in the adult animals. Its Anlage must clearly have an important morphogenetic function during the ontogenetic development. 
This may be the best place to call attention to a fact which has been, it seems to us, too much neglected till now. It is well known that a planktonic larva appears during the ontogeny of the Enteropneusta which is typical of these animals; this is the so-called tornaria, one of those larvae that had been developed by the Echinodermata and which have been given various names. Zoologists have therefore considered that there is a probability of a closer relationship between these two groups of the Oligomeria. All these larvae, as well as the various planktonic larvae of the Ameria and the Polymeria belong to the category of the primary larvae, as has already been emphasized, i.e. they appear in a form with a lower level of organization. Very frequently we can find the phase of these larval forms transmuted from a freely living phase of the development of an animal and incorporated into an embryonic phase of the ontogeny. It should be sufficient if we call attention here to conditions that can be observed in those crustaceans that live in fresh water.

This tornaria can now be identified as the last primary planktonic form of its kind. The institution of a primary planktonic larva had been abandoned during the comparatively short phase of the phylogenetic evolution when the first chordate evolved from its oligomerous ancestor. It was changed into an embryonic stage, a development similar to that of the nauplius in the evolution of the crayfish (Potamobius), among the decapod crustaceans. It seems easy to attribute this change to a transition to a life in the fresh water; it is considered that a reduction of a freely living larval stage regularly takes place when the animal relinquishes its life in the sea.

There are, however, some exception where this rule does not seem to be valid (e.g. in connection with the Dreissensia polymorpha, a species of bivalve molluscs which had probably penetrated late into the fresh water, and with the phylactolaematous Ectoprocta with their considerably changed larvae that develop forms similar to cormi). 
The planktonic larvae had in all probability again been developed after the animal had returned to a life in the sea. Yet this planktonic phase of the ontogeny now reached a much higher level of organization, and we are therefore fully justified to speak here of a secondary larval form. Such a larva shows the structure of a chordate, and not of an amerous animal as is the case with the trochophores and with the dipleurulae. It is wrong to see in the primary larvae (the various trochophores and dipleurulae) a recapitulation of an adult ancestral form; the same is also true for those larvae of the Chordata that can be justly called tadpoles. Viewed from this standpoint we must reject any attempt (e.g. the attempt made by Berrill) to derive the Chordonia from some kind of planktonic larvae. The evolution of the Larvacea from the "tadpole" of the Ascidia belongs as a clear case of neoteny in a completely different chapter.

A Comparison of the present Attempt to Derive the Chordonia from an Ancestral Form Resembling the Present Day Enteropneusta with the Similar Attempt Made by Hans Steiner

Hans Steiner has also tried to derive the Chordonia from the Enteropneusta in a very interesting study, Gescbicbte der Initialgestaltung der Chordaten (1956) where we can find numerous correct observations. In this study he takes into consideration the comparative morphology and the way of life of these animals. As regards the derivation itself there is a considerable agreement between his and my interpretation; and in this point Steiner therefore does not show in principle anything new. There is, on the other hand, a great difference in the way we interpret the evolution which led to the emergence of the Echinodermata and of the Enteropneusta. Steiner tries to suggest something which I must consider as completely impossible, he correctly rejects the opinions proposed by 
Remane as well as all the other attempts to derive bilateral symmetry from a radial symmetry with the "Coelenterata" as the initial form of this evolution, and above all the attempts to derive by means of the enterocoelic theory, a trimetamerism from the gastral diverticula of a tetraradial polyp of the Cnidaria. At the same time he wishes to preserve the division of the Eumetazoa into the Protostomia and the Deuterostomia, and in this respect he goes so far that he tries to place this unfortunate bifurcation back into the "gastraea" stage (he completely disregards here the Spongiae as well as the Cnidaria and the Ctenophora). Steiner has made here, I think, two basic mistakes; first, when he describes the youngest Metazoa as animals that live in plankton, and, secondly, when he interprets the larval forms as representatives of their adult ancestors. Steiner took great pains to make probable the early bifurcation of the two lines of evolution; the Protostomia and the Deuterostomia, and to provide this thesis with a solid foundation. This attempt, however, must be considered as completely unsuccessful, similarly as has also been the case with a not very different attempt made by Boettger. His construction, the Neurenterica, is, I think, unable to live.

Even the apparently fortunately suggested line of evolution, plankton-benthos-nekton is unable to stand an objective analysis. The benthos represents the starting point and the main type of life from which both plankton and nekton evolved.

It is also wrong to use the ecotypes, even as an auxiliary method, in high taxonomy. All the main types of life (of feeding), e.g. the predatores, turbellores, and the fossores (according to Steiner), can be found as early as in the Protozoa (the numerous benthonic Rhizopoda are fossores). In the Metazoa they do not follow each other in the way suggested by Steiner. We can find in the Cnidaria all these types of life represented perhaps with the exception of the burrowing animals (ceriantharia) if we do not wish to consider as 30 
such the species that stick in the soft sea bottom, e.g. Brancbiocerianthus giganteus. There also exist, however, other types of life which can be frequently met with and which have not been taken into consideration by Steiner. We can mention here the parasitic way of life (which can hardly be found among the "Deuterostomia"), and the various types of movement and feeding which can be observed in the benthonic animals (from good swimmers such as the Cephalopoda and down to the fully sessile animals that can be found above all among the Oligomeria).

Steiner takes a correct attitude regarding the relation, ontogeny versus phylogeny. He considers that the ontogenies of the ancestral forms are only recapitulated in the ontogenies of the recent species; yet at the same time we can frequently find in his scheme of the genealogical tree pictures of larvae instead of the pictures of the corresponding imago forms; this gives an impression that the ancestors could be recapitulated in these larvae, as it these larvae could be a kind of representatives of some ancestral forms. We also get such an impression because Steiner simply transfers some purely ontogenetic processes - such as the morphogeny of the spinal chord by way of a secondarily closed "feeding canal" which finally leads to the formation of the canalis neurentericus and neuroporus-into changes that take place during the adult stage.

We cannot accept Steiner's interpretation when he suggests that the enterocoely and the triple metamery evolved as new forms because of the burrowing way of life of the Enteropneusta. If Steiner had found a correct interpretation, then there could be no homologies in the conditions of the coeloms in the Annelida and in the Echinodermata (the Protostomia and the Deuterostomia). We must conclude that in spite of all the endeavours made by Steiner to explain rationally these differences, the idea of a branching of the Eumetazoa into the two main lines of the Protostomia and the Deuterostomia still remains highly improbable. 
Steiner does not mention my works which had appeared before the publication of his treatise; it is possible that either he did not know them or that he did not consider them as worthy of consideration.

\section{The Genealogical Tree of the Chordonia}

The mutual relationship connections that exist within the phylum Chordonia among the tetrapod Vertebrata can now be considered as sufficiently clarified; the same, however, can not be said for the numerous lower groups of the Chordata, from the Acrania and up to the bony fishes. Since I have not made my own researches in this field, I preserve here the system of the Chordata that was suggested by Vandebroek (1949) at the XIXth International Congress of Zoologists at Paris while at the same time I take into consideration the progress which has recently been made in the field of the paleozoology. If Tunicata had not existed we could divide all the Chordonia into two superclasses, Pisces and Tetrapoda; at the same time, however, we cannot recommend a division of the Chordonia into three greatly unequal superclasses, the Tunicata, Pisces, and the Tetrapoda. The term Vertebrata which is generally so widely used, and which will certainly continue to be used even if not as a taxonomic notion, finds no place in the system proposed by Vandebroek; neither can we find in this system the group Pisces as a name of a class, or the Acrania and the Cyclostomata (the former at least not as an independent class).

The Tunicata should definitively follow the primitive Chordonia, the Agnatha. There can be no doubt that the Tunicata must be considered as Chordonia which secondarily adopted the sessile way of life. It can be said that this was the last opportunity the animals have had to develop a sessile group with all the consequences which we have so frequently observed in the whole animal world, beginning with the 30 * 
Protozoa. The starting point of this evolution were the free living primitive Chordonia which, however, had already lived partly as sessile animals that burrowed slowly in the sea bottom making stops from time to time, just as can still be seen in Ampbioxus. The Tunicata evolved in all probability in the sea; they had developed their own larval form which is slightly similar, as this has already been emphasized, to the tertiary larva, to the tetrapodic tadpole. The entirely planktonic Larvacea have been the only form which had ever evolved from these larvae; they represent a small side branch whose evolution led no further, just as in the case of the Ctenophora.

The Ostracodermata represent a blindly ending side branch of fish-like Vertebrata which, however, remained in the benthos and became very specialized; they are now extinct. Because of them the displaced comparison with L_imulus has been attempted. It is very probable that the class Agnatha (here in a very wide sense) was followed by the Protichtya with their two subclasses, the Acanthodea and the Arthrodira. We know less about the sequence of the Chondrichthya and the Osteichthya, whether they evolved in two parallel lines, or one after the other.

The phylum Chordonia would thus include nine classes (Fig. 59), the same number as the phylum Oligomeria and the phylum Polymeria (quite "accidentally"); only the first phylum of the Eumetazoa, the Ameria, must be subdivided into fifteen classes. The question remains open whether all these groups which have been given the rank of classes are really mutually equivalent. Thus, for example, such classes that evolved in all probability by way of neoteny, as such the Ctenophora, Chaetognatha, and the Larvacea, and other classes as the Turbellaria, Mollusca, Polychaeta, Insecta, Echinodermata, Mammalia, etc., to mention a few only.

The main emphasis, however, must not be placed on the size of each group. A smaller number of subordinate categories, down to the species, can be reached along widely different 
ways. The small size of a group can be due to the fact that it evolved by way of neoteny (this is true for the Chaetognatha, Ctenophora and the Larvacea), or it is a consequence of an ageing of the line of evolution and thus an indication of a decaying phase which can finally lead to a complete extinction.

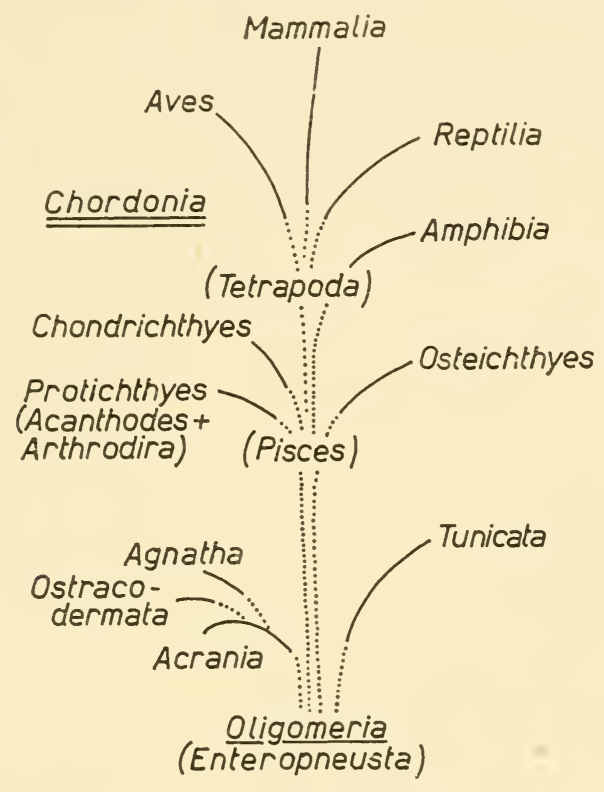

Fig. 59. Genealogical tree of the phylum; Chordonia.

Such a case is probably represented by the Brachiopoda. The small size of a group can also be a consequence of a hyperspecialization, as is the case, for example, with the Pogonophora.

\section{The Genealogical Tree of the Entire Animal World}

After we have constructed partial genealogical trees which show the individual phases in the phylogeny of the animal world we still have to bring them together and to construct 
a general genealogical tree (Fig. 60). The following special characteristics can be mentioned as typical of our construction if compared with the numerous other similar attemps:

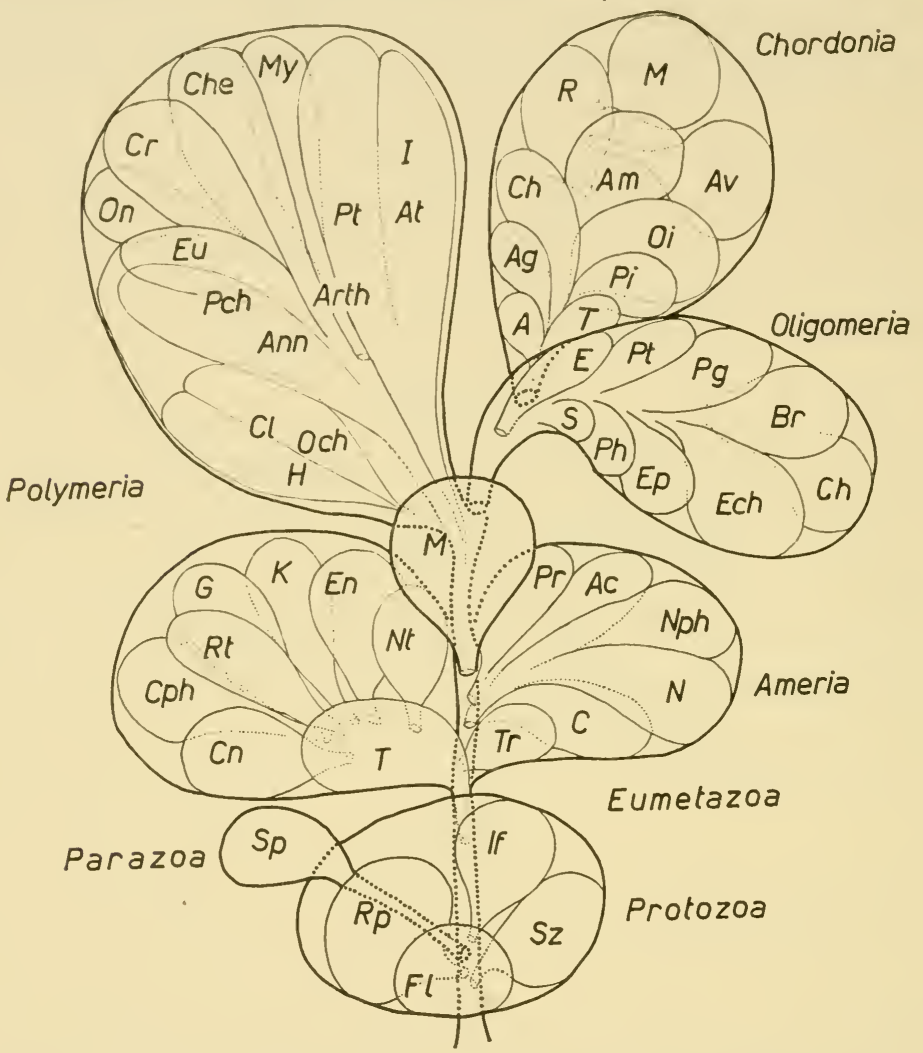

Fig. 60. Genealogical tree of the entire animal kingdom; the monograms used in these diagrams are explained in the list of phyla and classes (see p. 460-61)

(1) Our genealogical tree begins with the animal Flagellata which evolved on the one hand into the Rhizopoda, Sporozoa, and the Ciliata, as well as (by way of the Choanoflagellata) into the Parazoa (Spongiaria), and on the other hand (by way of the Ciliata) into the Eumetazoa. The Parazoa evolved as individualized colonies of the Choanoflagellata. 
(2) The Eumetazoa do not begin, as has been generally believed so far, with the two-layered Coelenterata, but rather with the three-layered Turbellaria and are connected downwards with the primitive polynuclear Ciliata (the plasmodium theory or the polykaryonic theory).

(3) The evolution of the Eumetazoa took place without a bifurcation, thus in a direct line and by way of four main phases or stages which should be classified as phyla: the Ameria, Polymeria, Oligomeria, and the Chordonia (actually: secondary Polymeria). This whole evolution shows clearly a progressive trend which, however, had gone through a stage of retrogression during the phase of the Oligomeria. The scheme of our genealogical tree shows therefore one top only, and not two; in spite of this it must not be considered as a revival of the old type of "l'échelle animale."

(4) The Mollusca have been treated in our study without any hesitation as a class of the Ameria.

(5) The Cnidaria and the Ctenophora are derived from the Turbellaria; they are therefore treated as classes of the Ameria.

(6) The Sipunculoida have been separated from the Annelida, and thus from the Polymeria; they are now classified among the Oligomeria.

(7) It has been suggested that the Chaetognatha had evolved by way of neoteny from the Brachiopoda.

Our first suggestion of the genealogical tree of the animal world (Hadži, 1944:179) is thus slightly modified now (Fig. 60 and the list of the phyla and classes of Eumetazoa; monograms in parenthesis). A mistake was made by the person who made the drawing of my suggested scheme of the genealogical tree as it was reproduced in my article published in the Systematic Zoology (the starting point of the Oligomeria line occurs at the top of the Polymeria line together with the Insecta, and not, as it should, in the Annelida line). This mistake was later corrected (Hadži, 1959b). It can be clearly seen that this is a mistake of the draftsman only if we compare this scheme with the sense of the text. 
Herewith we have also reached a welcome simplification of the whole animal system and of the scheme of the genealogical tree. This simplification can be clearly seen if we compare our scheme with other schemes which are now usually proposed. This simplification is purely a side result of our research and it was not explicitly intended.

\section{A List of the Phyla and Classes of Eumetazoa}

\begin{tabular}{|c|c|}
\hline PHYLUM & CLASSES \\
\hline & Turbellaria ('T) \\
\hline & CNIDARIA (Cn) \\
\hline & Ctenophora (Cph) \\
\hline & TREmatoda $(' \mathrm{Tz})$ \\
\hline & Cestoda (C) \\
\hline & Nemertinea (Nt) \\
\hline I. Ameria (I 5) & Rotatoria (Rz) \\
\hline & GASTROTRICHA (G) \\
\hline & KINORHYNCHA $(\mathrm{K})$ \\
\hline & ENTOPROCTA (En) \\
\hline & Nematoda (N) \\
\hline & Nematomorpha (Nph) \\
\hline & Priapulida $(\mathrm{Pz})$ \\
\hline & ACANTHOCEPHALA (Ac) \\
\hline & Mollusca (M) \\
\hline & Polychaeta (Pch) \\
\hline AnNELida (Ann) & C.titellata (Cl) \\
\hline & ECHIUROIDA (En) \\
\hline II. Polymeria (8) & ONYCHOPHORA (On) \\
\hline & Crustacea (Cr) \\
\hline Arthropoda (ARTH) & Chelicerata (Ch) \\
\hline & MYRIAPODA (My) \\
\hline & INSECTA (I) \\
\hline
\end{tabular}




$\begin{array}{ll} & \text { Sipunculida (S) } \\ & \text { Phoronida (Ph) } \\ & \text { Pogonophora (Pg) } \\ \text { Ectoprocta (Ep) } & \text { Brachiopoda (Br) } \\ \text { III. Oligomeria (9) } & \text { Chattognatha (Ch) } \\ & \text { Echinodermata (Ech) } \\ & \text { Pterobranchia (Pt) } \\ & \text { Enteropneusta }(\text { E) }\end{array}$

IV. Chordonia (io)

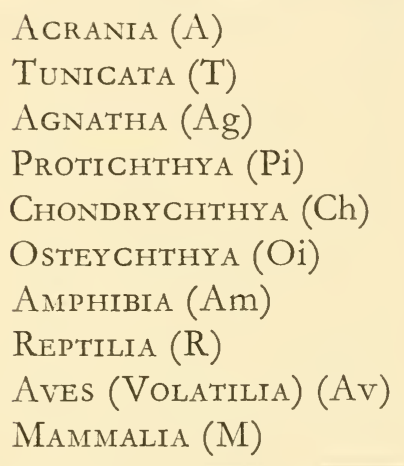

(42) CLASSES

\section{An Attempt to Unify the Animal System}

There still remain numerous suggested systems even if we disregard those suggestions which are now completely antiquated or clearly aberrant. There can be, however, one only truly natural system. We still have a long way to go to reach this ideal solution, even if we are coming closer to this ideal. We can be helped here by new discoveries of some recent species which have remained unknown till now, or of some corresponding fossilized remains. New facts and ideas can 
also be obtained by way of more detailed investigations of the already known animal species and of their ontogenies. Finally new viewpoints and methods of research can also be found useful, especially those offered by the biochemistry. All these elements combined can help us essentially to improve our present animal system. Even now we can see the important progress made within this sphere if we divide all the available systematic suggestions into two main groups, i.e. a larger group of systems which proposes a bifurcation in the evolution of the Eumetazoa, and a smaller group of systems where such a bifurcation cannot be observed.

This state of affairs has been the main reason why I have tried to make a corresponding suggestion before a competent forum, the XIVth International Congress of Zoologists. At this congress I (Hadži, 1956a) proposed that a uniform systematic scheme should be worked out which will be necessarily a result of a compromise and which could serve well enough in our practical didactic and operative work. Such a provisional scheme reached as a result of a compromise must quite naturally not prove to be an obstacle to our subsequent attempts to establish a definitive natural system. The subsequent destiny of my suggestion was entrusted to a special international committee which is headed by a well known zoologist-yet for reasons unknown to me this has been all that has been done in this connection. A standardized system could also serve as a basis for a review of all the valid animal names which was scheduled for publication at the occasion of the $X V$ th International Congress of Zoologists, to take place in London (1958) simultaneously with a bicentenary of Linneus' work Systema naturae.

Let us compare now two selected systems which can represent the two previously mentioned main groups. I choose in this connection on one hand the system proposed by L. H. Hyman (1948, part I:38, cf. Fig. 61)-this system was made, according to a statement by Hyman, partly with the collaboration of Professor W. K. Fischer-and on the other 


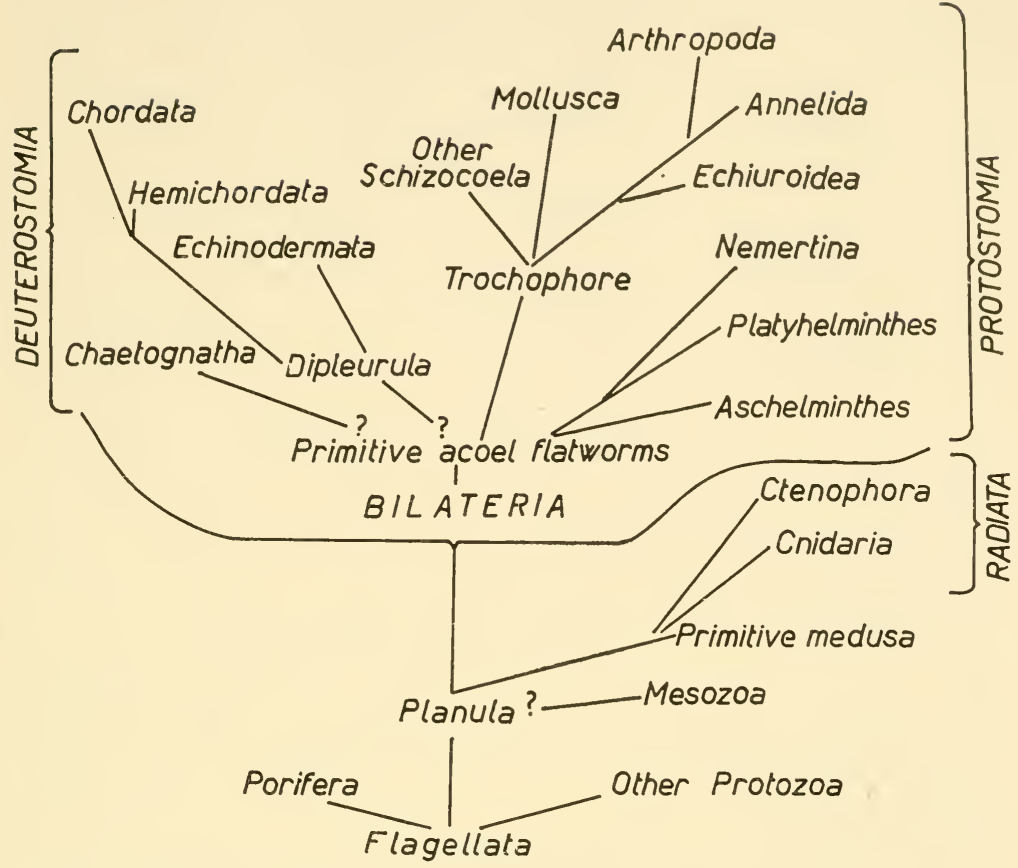

Fig. 61. Evolutionary relationships of the animal kingdom. (After Hyman.)

hand my own system. At first sight the differences between these two systems appear to be very great. We will now try to show that these differences are not actually quite so great, and that they can easily be overcome if only we have a little tolerance and good will. I will therefore enumerate here all those changes which can help us to overcome these differences. Such changes are actually not quite so numerous.

In order to solve first major problems we must abandon above all the two large groups of the Bilateria and Radiata; all the Eumetazoa are actually bilaterally symmetric animals, and there remain the Cnidaria and the Ctenophora only as members of the Radiata. The Echinodermata are quite correctly excluded from this group; there are few purely radially 
symmetric Cnidaria whose radial symmetry is actually a secondary phenomenon; the Ctenophora finally do not show a radially symmetric structure at all. For reasons which we do not wish to repeat here we should also abandon the division of the "Bilateria" into the Protostomia and the Deuterostomia. This is the change which will be least easily accepted and which is at the same time also the most important one that has to be made. All the remaining changes that must be made in the diagram as proposed by Hyman have a minor character only and they are actually "quite painless." First of all we must cancel in this system larval names (planula, dipleurula, trochophore) because they do not belong in such a diagram for reasons which we have sufficiently discussed. Furthermore we must also omit the Mesozoa, a group which is already now accompanied by a question mark. This especially must be done because we do not find indicated in the same diagram the adequate categories of the Protozoa, Metazoa, and the Eumetazoa, even if these groups are used in the linear enumerations.

The "primitive medusa" which has really no justification, must be abandoned as the initial form of the Coelenterata. Neither should the Coelenterata be indicated as such (in the enumeration they can appear together with the name Cnidaria, thus with the exclusion of the Ctenophora!). We must also cease to divide (this is actually not even done in the diagram here discussed) the Eumetazoa (they are indicated here as a "branch") into the Acoelomata, Pseudocoelomata, and the Eucoelomata (they are all written with capital letters only); the same should also be made with the division of the Eucoelomata into the Schizocoela and the Enterocoela, a division which is indicated once only ("other Schizocoela") and which is represented in the linear survey with numbers only. Such a division does not correspond to the well known relationship connections, a fact which is particularly true for the group Schizocoela, yet it is also valid for the group Enterocoela (e.g. the separation of the Brachiopoda from the Chaetognatha, 


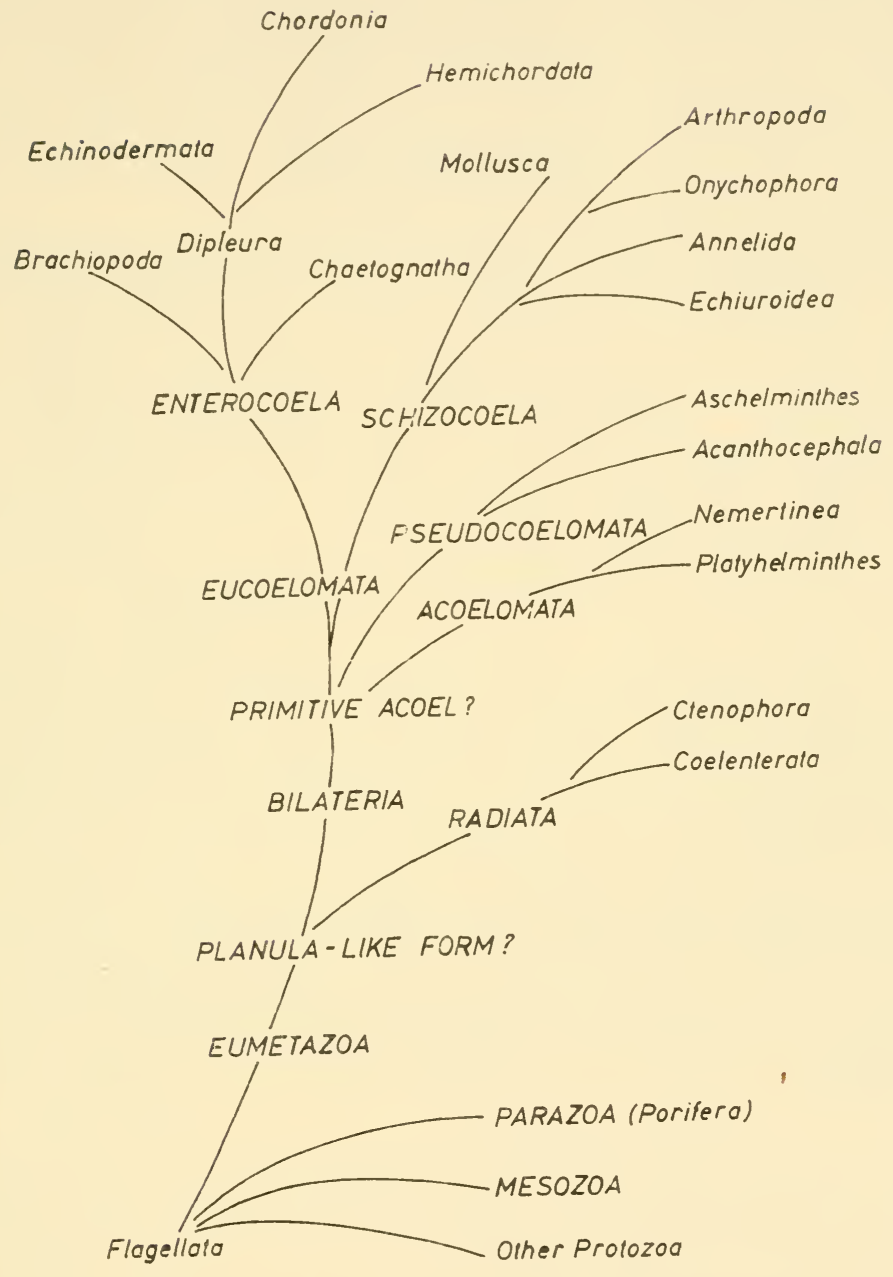

Fig. 62. Genealogical tree constructed by M.J. Guthrie and J.M. Anderson, following the ideas of Hyman.

mise. 
the position of the Mollusca, etc.). If we represent the lefthand main branch as a continuation of the right-hand main branch-and this is actually the main change we have to make-we must necessarily also make some other minor changes in order to reach finally a clear separation of the Ameria, Polymeria, and the Oligomeria.

All these differences appear even slighter if we simplify the diagram proposed by Hyman in the way this was done, for example, by Guthrie and Anderson (1957:219, Fig. 73, Fig. 62), and if we do something similar with my attempt to construct a genealogical tree of the animal world.

Thus it appears to me that in spite of the apparently great differences of opinion which presently still exist regarding the true course of the evolution of animals we will nevertheless reach a general accord between all the zoologists in a not very distant future. The spectrum has certainly become now considerably narrowed. The main difference can be found in the fact-which appears clearly in my discussion and in my systematic survey-first, that I consider the Turbellaria as the starting point of the whole evolution of the Eumetazoa, and secondly, that I suggest that we must discontinue to use in our taxonomic concepts the difference which is found at the basis of the two groups, the Protostomia and the Deuterostomia; these two parallel lines of evolution must now be abandoned. It will not be difficult to solve all the remaining problems. 


\section{BIBLIOGRAPHY}

Alvarado R. (1953). On the origin and evolution of the coelomatous

Metazoa. Proc. XIVth Internat. Congress of Zool., Copenhagen.

Alvarado R. (1960) La evolucion morfologica del reino animal. Rev. Univ. Madrid 8.

Awerinzew S. (1910) Über die Stellung im System u. d. Klassifikation d. Protozoa. Biol. Z bl. 30.

Ax P. (1957) Ein chordoides Stützorgan bei Turbellarien. Z.f. Morph. Ökol. Tiere 46.

Baker J. R. (1948) The status of the Protozoa. Nature, Lond. 161.

Baltzer F. S. (1931) Sipunculida. In Handbuch der Zoologie, edited by KüKENTHAL-KRUMBACH.

Batham E. J., Pantin C. F. A. and Robson E. (1960) The nerve-net of the sea anemone Metridium senile. Quart. J. micr. Sci. 101.

Beer de G. (1954) Archaeopteryx and evolution, Advanc. Sci., Lond. 42.

BEER DE G. (1954 a) The evolution of Metazoa. Evolution as a process (edited by Huxley et al.). Allen and Unwin, London.

Beer De G. (1958) Embryos and Ancestors, - 4th ed. Clarendon Press, Oxford.

Beer de G. (1959) Paedomorphosis. Proc. XVth Intern. Congress of Zooi. London, 1958.

Berg S. E. (1941) Die Entwicklung u. Koloniebildung bei Funiculina quadrangularis (Pall.). Zool. bidrag, Uppsala 20.

Beklemischew IW. N. (1958) Grundlagen d. vergl. Anat. d. Wirbellosen. I. VEB, Berlin.

Berrill N. J. (1955) The Origin of Vertebrata. Clarendon Press, Oxford. Bertalanffy V. L. Handbuch d. Biologie.

Beuerlein K. (1955) Biolog. Systematik u. Phylogenie Zuol. Jabrb. Anat. 74.

Brocea E. (1956) Schema di classificazione dei Protozoi e proposta di una nuova classe. R.C. Acad. Lincei 21, No 6.

Boettger C. R. (1952) Die Stämme des Tierreiches in ihrer systematischen Gliederung (The classification of the different branches of the animal kingdom). Abb. Braunschweig. wiss. Ges. 4.

Boettger C. R. (1955) Beiträge zur Systematik der Urmollusken (Amphineura). Verh. Deut. Zool. Ges., Erlangen. 
Boettger C. R. (1959) Discussion upon lecture of H. Lemche, Molluscan phylogeny in the light of Neopilina. Proc. XVtb Internat. Congress of Zoology, London, 1958.

Börner C. (1925) Die nat. Scböpfungsgescbicbte, als Tokontologie. Ein Entwurf. Leipzig.

Bresslau E. (1909) Die Entwicklung d. Acoelen. Verb. dtsch. zool. Ges. Bresslau E. and Reisinger E. (1933) Turbellaria. Handbuch. der Zoologie (edited by KüKENTHAL-KRUMBACII)

Brien P., and Reniers-Decoen M. (1950) Étude d'Hydra viridis (L.). Ann. Soc, roy. zool. Belg. 81.

Broch H. (1924) Hydroida. - Handbuch der Zoologie (edited by KüKenthalKRUMBACH)

Broman I. (1919) Das sogenannte Biogenetiscbe Grundgesetz und die moderne Erblicbkeitslebre, München and Wiesbaden.

Brooks I. L. (1954) Evolution as a process. Syst. Zool. 3.

Burnet A. L. and Leutz T. (1960) The nematocysts of Hydra. III. Ann. Soc, roy. zool. Belg. 90.

Bürschli O. (1921) Vorlesungen ïber vergl. Anatomie. Berlin.

Carn A. J. (1959) The postlinnean development of taxonomy. Proc. Linn. Soc., Lond. 170.

Calkins G. N. and Bowling R. (1928/9) Studies on Dallasia frontata Stokes.

I-II. Arch. Protistenk. 66.

Calkins G. N. and Summers F. M. (1941) Protozoa in biological researcl. Columbia Univ. Press, New York.

Carlgren C. (1904) Kurze Mitteilungen über Anthozoen. Zool. Anz. 27.

Carlgren C. (1918) Die Mesenterienanordnung d. Haleuriiden. Lunds univ. Arskr. NF, 2, 14. No 29.

CARLgren C. (1945) Further contribution to the knowledge of the cnidom in the Anthozoa especially in the Actinaria. Lunds univ. Arskr., NF. 2.

Carter G. S. (1940) A General Zoology of the Invertebrates. Sidgwick and Jackson, London.

Carter G. S. (1949) The turbellarian theory of the Cnidaria, Sci.progr. 14.

Carter G. S. (1954) On Hadzi's interpretation of phylogeny, Syst. Zool. 3.

Chatton E. (1914) Le cnidocystes du peridinidien Polykrikos schuartzi

Bütschli. Arch. zool. expér. génér. 52.

Chester W. M. (1913) The structure of the gorgonian coral Pseudopleura crassa Wright and Studer. Proc. Amer. Acad. Sci. 48.

Chun C. (1883) Verwandtschaftsbeziehungen zwischen Würmern und Coelenteraten. Biol. Zbl. 2.

Chun C. (1892) Die Dissogonie. Festschr. Leuckart, Leipzig.

Claus C. (1864) Bemerkungen über Ctenophoren und Medusen. Z. wiss. Zool. 14. 
Claus C. (1878) Über Halistemma tergestinum n. sp., nebst Bemerkungen über d. feineren Bau d. Physophoriden. Arb. Zool. Inst. Wien-Triest. Colosi G. (1956) Zoologia e biologia generale. Ed. Unione tipogr. Torino. Сомsтоск, J. M. (1925.) An Introduction to Entomology. Ithaca.

Cori C. I. (1937) Tentaculata. Handbuch der Zoologie (edited by KükenthalKRUMBACH).

Cutries E. E. (1955) An interpretation on the structure and distribution of cnidae in Anthozoa. Syst. Zool., 4.

D’Ancona U. (1939) Lezioni di Biologia e Zoologia Generale. Milano.

D’Ancona U. (1955) Considerazioni critiche sul sistema di classificazione zoologica. Bol. lab. zool. gen. agr. "Filippo Silvestri". Portici, 33.

Dawydoff, C. (1903) Hydroctena Salenskii etc. Mém. Acad.imp. St. Pétersb. (8) 14 , No 9.

Dawydoff C. (1928) Traité d'embrylogie comparé des invertébrés. Masson, Paris.

DAwydoff C. (1953) Contribution à nos connaissances de l'Hydroctena. C. R. Acad. Sci. Paris. 257.

Delage Y. and Hérouard E. (1896-1903) Traité de Zoologie Concrète. Paris. Delsman H. C. (1913) Der Ursprung der Vertebraten. Mitt. Zool. Stat. Neapel.

Delsman H. C. (1922) The Ancestry of Vertebrates. Amerfort.

Dobell C. C. (1911) The principles of protistology. Arch. f. Protistenk. 23.

Dogiel V. (1925) Über d. geschlechtl. Prozesse bei Infusorien (speziell bei Ophryoscolecidae). Arch. Protistenk. 50.

Dogiel V. (1947) Zoologia bespozvonoinib (Zoology of Invertebrates). Moscow.

Dogiel V. (1954) Oligomerizacija homologičnyh organov (The oligomerization of the homologous organs). Izd. Leningr. Univ., Leningrad.

Dohrn A. (1875) Der Ursprung der Wirbeltiere und das Prinzip des Funktionswechsels. Leipzig.

Dollo L. (1893) Les lois de l'èvolution. Bull. soc. geol. III.

Dollo L. (1922) Les Cephalopodes deroulés et l'irréversibilité de l'évolution. Bijdrag tot de dierk. 22.

Dougherty E. C. and Allen M. B. (1959) Speculation on the position of the Cryptomonads in protistan phylogeny. Proc. XVth Internat. Congress of Zool., London, 1958.

Dubinin V. B. (1959) Heliceronosnije životnije (Chalicerate animals). Zool. zh. 38.

Dudich E. (1957) Ein System des rezenten Tierreiches Opus. Zool. Budapest. II. 12.

Eberl-Rothe G. (1950) Über das Zwischengewebe der wirbellosen Tiere. I. Spongien. Thalassia jugoslavica, II. 1.

FAurot L. (1913) Développement et symétrie des polypiers coralliers. Proc. IXth Congess Internat. Zool., Monaco, Rennes. 
Franz V. (1924) Gescbicbte der Organismen. Jena.

Franz V. (1943) Geschichte der Tiere. (Edited by Heberer G. Die Evolutiond. Organismen. Jena.)

Franzén $\AA$. (1956) On spermogenesis, morphology of the sperm., and biology of fertilization among Invertebrates. Zool. bidrag, Uppsala 33.

Franzén $\AA$. (1960) Monobryozoon limicola n. sp., a stenost. bryozoon from detritus layer of soft sediments Zool. bidrag, Uppsala 33.

Gardiner E. G. (1895) Early development of Polychoerus caudatus. J. Morph. 11.

GARSTANG W. (1922) The theory of recapitulation: a critical restatement of the Biogenetical law. J. Linn. Sor., London 35.

Garstang W. (1946) The morphology and relations of the Siphonophora. Quart. J. Miir. Sci. 87. II.

GASKELL W. H. (1908) The origin of Vertebrates. London.

GÉLEI V. J. (1939) Ein Vergleich d. Ciliaten und d. Strudelwürmer. Sbornike praci vyd. k 90. naroz. F. Vejdovskébo. Praha.

Georgevich J. (1898) Étude sur le développement da la Convoluta roscoffensis Graff. Arch. zool. expér. génér. 7.

Gerould J. (1906) The development of Pbascolosoma. Zool., Jabrb. Anat. 23. GHilarov M. S. (1956) Soil and the environment of the invertebrate transition from aquatic to the terrestrial life. Proc. VIl Congrès de la Soc. du sol, Paris, III, 51.

Ghilarov M. S. (1960) Nekatorije obščije zadači evolucionoj morfologiji bespozvonočnih (Some general tasks of the evolut. morphology of in'vertebrates). Uspebi sovrem. biol. 49.

GislÉn T. (1930) Affinities between the Echinodermata, Enteropneusta and Chordonia, Zool. bidrag from Uppsala 12.

Goette A. (1887) Entwicklungsgeschichte d. Aurelia aurita u. Cotylorbiza tuberculata. Hamburg und Leipzig.

Goette A. (1907) Vergl. EntwGesch. d. Geschlechtsindividuen d. Hydropolypen. Z. wiss. Zool. 87.

Goette A. (1912) Entwicklungsgeschicbte d. Tiere. Berlin-Leipzig.

Golvan Y. J. (1958) Acanthocephala. Ann. parasit. bumaine et compar. 33.

GrafF v. L. (1878) Berichte über fortgesetzte Turbellarienstudien. Z. wiss. Zool., Suppl. 21, 3.

Graff v. L. (1882) Monograpbie der Turbellarien. I. Rhabdocoelida. Leipzig. Grassé P. (Ed.) (1948). Traité de zoologie. Masson, Paris.

Grobben C. (1908) Die systematische Einteilung des Tierreiches. Verb. Zool.-bot. Ges. Wien 60.

Grobben C. and Heider C. Das zoologische System. Verb. d. Zool.-bot. Ges. Wien 61.

Grobien C. (1923) Theoretische Erörterungen betreffend d. phylogen. Ableitung d. Echinodermen. S. B. Akad. Wiss. Wien, Abt. I. 132. 
Grobben C. and Kühn A. (1932) Lebrbuch d. Zoologie. Marburg/L.

Gröntved J. (1956) Planctological contributions. II. Meded. Danmarks fis.-og bavinden 1, No 12.

Grošelj P. (1909) Über das Nervensystem d. Aktinien. Arb. d. Zool. Inst. Wien-Triest 17.

Guthrie M. and Anderson J. M. (1957) General Zoology John Wiley, New York.

HaAcke W. (1893) Die Scböpfung d. Tierwelt. Leipzig and Wien.

Hadžr J. (1906) Vorversuche zur Biologie von Hydra. Arch. Entw. Mech. Org. 22.

HADžI J. (1907) Einige Kapitel aus der Entwicklungsgeschichte der Chrysaora. Arb. d. Zool. Inst. Wien-Triest 17.

HADži J. (1907a) Über die Nesselzellwanderung bei d. Hydropolypen. Ibid. 17.

HADži J. (1908) Über das Nervensystem von Hydra. Ibid. 18.

Hadžı J. (1909) Die Entstehung d. Knospe bei Hydra. Ibid. 18.

HADŽI J. (1909a) Bemerkungen zur Onto-und Phylogenie d. Hydromedusen Zool. Anz. 35.

HADŽi J. (1909 b) Ontogeneza i filogeneza bidromeduze (Ontogenesis and phylogenesis of the bydromedusa) 179. knjiga "Rada" Jugoslav. akademije znanosti i umjetnosti, Zagreb.

HADži J. (1909 c) Rückgängig gemachte Entwicklung einer Scyphomeduse. Zool. Anz. 34

HADži J. (1911) Haben die Scyphomedusen einen ektodermalen Schlund? Zool. Anz. 37.

Hadži J. (1911 a) Bemerkungen über d. Knospenbildung bei Hydra. Biol. Zbl. 31.

HADžı J. (1911 b) Razmeštaj $i$ selidba knidocita u bidromeduza $i$ bidroida. (Distribution and migration of the cnidocyts of Hydromedusae and Hydroida) 158. knjiga "Rada" JAZU, Zagreb.

HADŽI J. (1911 c) Über die Nesselzellverhältnisse bei den Hydromedusen. Zool. Anz. 37

Hadži J. (1911 d) Die Reduktion d. Scyphopolypen u. d. Ephyra von Chrysaora. Verb. d. VIII. internat. Zoologenkongresses in Graz 1910.

Hadžr J. (1912) Über die Stellung der Acraspeden (Scyphozoa s. str.) im Systeme. Zool. Anz. 39.

HaDži J. (1912a) Über die Symbiose von Xanthellen und Halecium ophiodes. Biol. Zbl. 51.

HADŽI J. (1912 b) O podocistama u skifopolipa (Clrysaora) (The podocysts of the siyphopolyp Chrysaora) 190. knjiga "Rada" JAZU, Zagreb.

HADžI J. (1912 c) Über die Podozysten der Scyphopolypen. Biol. Zbl. 32. HADži J. (1912 d.) Još o ontogenezi $i$ filogenezi bidromeduze (Once more on the ontogeny and phylogeny of the bydromedusae) 190. knjiga "Rada" JAZU. 
HADžı J. (1913) Allgemeines über die Knospung bei Hydroiden. C. R. $d u$ IXe Congrès internat. de Zool. à Monaco, 1913; Rennes, 1913.

HADžI J. (1913 a) Poredbena bidroidska istraživanja. I. Hebella parasitica (Ciam.) s dodatkom: Hebellopsis brochi g.n., sp. n., Hebella gigas Pieper. (Comp. studies on Hydroids. I. H.g. (Ciam.), with addendum (H.b. g.n., sp. n. H. gigas Pieper) 198. knjiga "RADA" JAZU.

HadžI J. (1913 b) Poredb. istraž. bidroid. II. Perigonimus corii sp. n., P. georginae sp. n. 200. knjiga "Rada" JAZU.

HaDžI J. (1914) Poredb. bidroid. istraž. III. Haleciella microtheca g.n., sp. n., Georginella diaphana g.n., sp. n., Halantbus adriaticus g.n., sp. n. Campanopsis clausi (Hadži) $i$ o porodici Campanopsidae. 202. knjiga "Rada" JAZU.

HADžI J. (1915) O regeneraciji (renovaciji) bidranata u tekatnib bidroida (On the regeneration (renovation) of the bydranths in bydroids) 208. knjiga "Rada". JAZU.

HADŽI J. (1915a) Rezultati biol. istraživanja Jadranskoga mora. Hidroidi. I. Camella vilae-velebiti g.n., sp. n. (Results of biol. researches of the Adriatic sea. I. C. v.-v.). Prirodoslouna istraživanja Hrvatske $i$ Slavonije, 7.

HaDžI J. (1917) Rezult. biol. istraž. Jadr. mora. Porifera, Calcarea. I. Clathrina blanca (Mikl.-Makl.),gradja i razvoj s osobitim obzirom na opća pitanja o spužvama (Results of biol. researches of the Adriatic sea. Spongiae, Calcarea. I. Cl. bl. M.-M., Study on morphol. and development with special regard of the general problems of the sponges). Prirodosl. istraž. Hrv. i Slav., 9/10.

HADŽI J. (1918) Shvaćanje sifonofora (The status of the Siphonophora) 219. knjiga "Rada", JAZU, Zagreb.

HADžI J. (1919) Rezult. biol. istraž. Jadr. mora. Hidroidi. II. Halocoryne epizoica g.n., sp. n., Lefoëina vilae-velebiti sp. n. (Results of biol. research. of the Adriatic sea. Hydroida. II. H. e. g. n. sp. n., L. v-v. sp. n.). Prirodosl istraž. Hrv. i Slav., 11/12.

HADŽI J. (1919 a) Rezult. biol. istraživ. Jadr. mora. Hidroidi. III. Monogonija s pomoću kompleksnih propagula (kladogonija) u nekih halecija, uz opća razmatranja o stologoniji nižih životinja (Cladogony of the Hydroids and the stologony of the lower animals in general). Prirodosl. istraživ. Hrv. i Slav. 14

HADžI J. (1922) Teorija gdje Moser o prameduzi (Theory of the primordial medusa of Mme Moser). Spomenica o 50-god. rada S.M. Lozanića. Beograd.

HADžI J. (1923) Prividna pobočna neomorfoza na stabaocetu tubularije (The apparent lateral neomorphosis on the hydrocaulus of Tuburaria) 108. knjiga "Glasa" SAN, Beograd.

HADŽI J. (1923 a) O podrijetlu, stodstvenim odnosima is sistematsko poziciji ktenofora (On the origin, relationship and systematic position of the Ctenophorae) 228. knjiga "Rada" JAZU, Zagreb. 
HADŽI J. (1925) Variation des Gattungscharakters bei eincm thekaten Hydroiden. Z. wiss. Zool. 125.

HADžI J. (1928) Knidariji bučate i slatke vode The Cnidarians of brackish and fresh waters) 234. knjiga "Rada" SAZU, Zagreb.

HADžr J. (1929) Einige allgemein wichtige Resultate meiner Untersuchungen über Coelenteraten. Proc. of the Xth Internat. congress of zool., Budapest, 1927.

HADžI J. (1944) Turbeiarijska teorija knidarijev. Filogenija knidarijev in njibov položaj v živalskem sistemu ( $T$ bo turbellarian theory of the Cnidaria. The Phylogeny of the Cnidaria and their position in the animal system). SAZU (Slov. acad. of sci. and arts), Ljubljana.

HaDžı J. (1946) Pripombe $\mathrm{k}$ van Beneden-Lameerovi inačici enterocelne teorije (Remarks on van Beneden-Lameere variant of the enterocoelic theory). Zbornik Prirodosl. dr. v Ljubljani, IV.

Hadžr J. (1948) Problem celoma v luči turbelarijske teorije knidarijev. (The problem of the coelom and mesoderm in the light of the turbellarian theory of the Cnidaria). Razpr. IV. raz. SAZU; knj. 4., Ljubljana.

HADžr J. (1949) Die Ableitung der Knidarien von den Turbellarien und einige Folgerungen dieser Ableitung. (C.R. du XIIIe congrès de zool., Paris, 1948 .

HADŽI J. (1950) Uporedjivanje spolne faze infuzorija sa spolnim plodjenjem kod turbelarija ( $A$ comparison of the sexual phase of Infusorians with the sexual reproduction of the Turbellaria) 280. knjiga "Rada", JAZU.

HADžI, J. (1951) Ali imajo ktenofore lastne ožigalke (Have the Ctenophores their own cnidae). Razprave IV. raz. SAZU, knj. 4.

HADžI J. (1951 a) Izvajanje knidarijev iz turbelarijev in nekatere posledice tega izvajanja (The derivation of the Cnidaria from the Turbellaria and some consequences of this derivation). Razpr. IV. raz. SAZU, knj. 4.

HaDžI J. (1952) Nnvi pogledi u filogeniju metazoa (New views regarding the phylogeny of the Metazoa). JAZU, Zagreb.

HADŽI J. (1953) An attempt to reconstruct the system of animal classification. Syst. Zool. 2., No 2.

HADžI J. (1953 a) Bemerkungen zur Hardy's Hypothese über die Abstammung der Metazoen. Bu'l. sci., Yougoslav. I. 4.

HADžI J. (1954) Morfološki značaj pnevmatofora pri sifonoforah (The morphol. significance of the pneumatophor of Siphonophora). Razprave IV. razr. SAZU, knj. 2.

HaDžI J. (1955) Eine Hypothese über die morphologische Bedeutung der sogenannten Wimperrosetten der Ctenophoren. Bull. sci. Yougoslav. II, 3 .

HADžr J. (1955 a) Odnos med scifomeduzo in scifopolipom (The relation between the Scyphomedusa and Scyphopolyp). Razpr. IV. razr. $S A Z U, I I I$. 
HADŽI J. (1955 b) Kritične pripombe s stališča turbelarijske tcorije knidarijev, zlasti Remanijevim izvajanjem o razvoju živalstva (Critical remarks from the standpoint of the turbellarian theory of the Cnidaria and some considerations on the evolution of the animal kingdom with special regard to the Remane's view). Razpr. $1 \mathrm{~V}$. razr. $S A Z U, \mathrm{III}$.

HADžı J. (1955 c) K diskusiji o novi sistematiki živalstva (Remarks to the discussion of a new system of the animal kingdom). Razpr. IV. raz. $S A Z U$, III.

HADŽI J. (1956) Zur Abschaffung der zoologisch-systematischen Gruppe: Bryozoa Ehrenberg, 1823. Bull. sci. Yougoslay. III, 1.

HADžI J. (1956 a) Vorschlag zur Ausarbeitung eines Standardsystems des Tierreiches. Proc. XIV-th Congress Internat. of Zoology, Copenbagen, 1956.

HADŽI J. (1956 b) Das Kleinsein und Kleinwerden im Tierreiche. Ein weiterer Beitrag zu meiner Turbellarientheorie der Cnidarien. Proc. XIVth Internat. Congress of Zoology, Copenbagen.

HaDŽI J. (1957) Die morphologische Bedeutung der Wimperrosetten der

Ktenophoren. J. Fac. sci. Hokkaido Univ., ser. VI., zool., 13. Prof. T. Uchida jubilee vol., VIII.

HADŽI J. (1957 a) Zur Diskussion über die Abstammung der Eumetazoen. Verb. dtsch. zool. Ges. Graz.

HADŽI J. (1958) Za opustitev sistematske zoološke skupine Bryozoa Ehrenberg 1823. (For the abolition of the zool. syst. group of Bryozoa Ehrbg 1823). Razpr. IV. razr. SAZU, IV.

HADžr J. (1958 a) Über die Verwandtschaftsverhältnisse der Chaetognathen. Bull. sci. Yougoslav. IV, 2/3.

HADŽI J. (1958 b) Hydroctena salenskyi Dawydoff 1902. Bull. sci. Yougoslav. IV., 2.

Hadži J. (1959) Zum Problem der Knidarienabstammung. Bull. sci., Yougoslav. IV., 4.

HADŽI J. (1959 a) Izvor in sorodniški odnosi hetognatov (The origin and relationship of the Chaetognatha). Razpr. IV.razr. SAZU, V.

HADŽI J. (1959 b) Štiri knidariološke študije (Four cnidariological studies). Razpr. IV. razr. SAZU, V.

Haeckel E. (1874) Die Gastrea-Theorie, die phylogenetische Classification des Tierreiches und die Homologie der Keimblätter. Jen. Natwiss. 8. Haeckel E. (1896) Systematisibe Phylogenie. Berlin.

Hall R. P. (1953) Protozoology. New York.

Hanson E. D. (1958) On the origin of the Metazoa. Syst. Zool. 7.

Hanström B. (1928) Vergleichende Anatomie d. Nervensystems d. wirbellosen Tiere. Berlin.

HARDY A. C. (1954) Escape from specialisation. In Evolution as a process. London. 
Harms J. (1924) Individualcyclen als Grundlage f.d. Erscheinung d. biol.

Geschehens. Scbrift. Königsberg. geol. Ges., I. I.

HatscheK B. (1884) Entwicklungsgeschichte von Ecbiurus u. d. system.

Stellung d. Echiuriden. Arb. Zool. Inst. Wien-Triest. 3.

НАтsснек B. (1888) Lebrbuch der Zoologie. Jena.

Hatscheк B. (1911) Das neue zoologische System. Leipzig.

Heberer G. (Ed.) (1943) Die Evolution der Organismen. Jena.

Heider K. (1914) Phylogenie d. wirbellosen Tiere. In Kultur d. Gegenwart, 1 Art, 3, Abt. 4.

Heric M. (1907) Zur Kenntnis der polydisken Strobilation von Chrysaora. Arb. d. Zool. Inst. Wien-Triest 17.

Hertwig O. and R. (1878) Der Organismus der Medusen. Denkschr. Med. natwiss. Ges., Jena.

Hertwig O. and R. (1878) Das Nervensystem u.d. Sinnesorgane der Medusen.

Leipzig

Hertwig O. and R. (1879) Die Aktinien... Jena.

Hertwig O. and R. (1882) Die Coelomtheorie. Jena Z. Natwiss.

Hoffman, H. (1937) Die Stammesgeschichte d. Weichtiere. Verh. Zool.bot. Ges.

Horridge G. A. (1956) The nervous system of the ephyra larva of Aurelia aurita. - Quart. J. Micr. Sci. 97.

Horváth J. (1947) The question of the equality of somatic and germ nuclei in respect to heredity and survival on the basis of studies in soil protozoons. Arch. Biol. Hung., 5, II., 17. Tihany.

Hubrecht A. A. W. (1883) On the ancestral forms of the Chordata. Quart. J. Micr. Sci. 23.

Huxley T. H. (1875) On the classification of the animal kingdom. Quart. J. Micr. Sci. 15 (N.S.).

Huxley J. Evolution, the Modern Synthesis. Allen and Unwin, London.

Huxley J., Hardy A. C. and Ford E. B. (1954) Evolution as a procers. London.

Hyman L. H. (1940) The Invertebrates. McGraw-Hill, New York and London.

Imms A. D. (1957) A General Textbook of Entomology, 3th ed. Methuen, London.

InONE Iwas (1958) Studies on the life history od Chordodes japonensis... I. Japan. J. Zool. 12. No 2.

Iwanoff P. P. (1928) Die Entwicklung d. Larvalsegmente bei d. Anneliden. Z. Morph. Ökol. Tiere 10.

Ivanov A. V. (1957) Materialy po embrion. razvitiyu pogonofor (Materials on the ontogeny of the Pogonophora). Zool. Zb. 36.

Ivanov A. V. (1955) O prinadlezhnosti klassa Pogonophora k osobemu tipu vtorichnortikh - Brachiata (On relationship of the class. Pogonophora to a peculiar type of Deuterostomia - Brachiata). Dokl. Akad. Nauk. SSSR. 100, 1-3. 
Ivanova-Kazas O. M. (1959) K voprosu o proizkhozhdenii i evolutsii spiralnego drobleniya (To the question of the origin and evolution of the spiral cleavage). Vestnik Leningr. Univ. 9.

JäGersten G. (1955) On the carly phylogeny of the Metazoa. The bilatcrogastrea theory. Zool. bidrag. Uppsala 30.

JäGERSteN G. (1957) On the larva of Siboglinum. With some remarks on the nutrition problem of Pogonophora. Zool. bidrag. Uppsala 32.

JäGERSTEN G. (1959) Further remarks on the early phylogeny of the Metazoa. Zool bidrag. Uppsala 33.

JoDEAux J. (1958) Contribution à la connaissance des Thaliaces (Pyrosoma et Doliolum). Ann. Soc. Zool. Belg. 88.

Katsnelson Z. S. (1948) Gastrulatsiya i obrazovanie endodermy (Gastrulation and formation of the endoderm). Uspekhi sovrem. biol. 25.

Kaestner A. (1954) Lebrbuch d. speziellen Zoologie. G. Fischer, Jena.

Kenna A. (1908-9). Morphologie des Coelentaires. Ann. Soc. roy. zool et malacol. Belg. 43-44.

Kepner W. A. (1911) Nematocysts of Microstoma. Biol, Bull. 20.

Kofoid C. A. (1940) Cell and organism. In : The cell and protoplasm. Lancaster, $\mathrm{Pa}$.

Koford C. A. and Swezy O. (1921) The free-living unarmored Dinoflagellata. Mem. Univ. Califo. 5.

Komai T. (1935) Stephanoscyphus and Nausithoë. Mem. Coll. Sci. Kyoto Univ., B., 10.

Komai T. (1951) The nematocysts in the ctenophore Euchlora rubra. Amer. Nat. 85.

Korschelt E. and HeIDer K. (1936) Vergleichende Entwicklungsgeschichted. Tiere. Jena.

Krasiv́ska S. (1914) Beiträge zur Histologie d. Medusen. Z. wiss. Zool. 100.

Krumbach Th. (1928-1937) Scyphozoa, Ctenophora, Oligomera. In Handbuch der Zoologie (Edited by Kükenthal-Krumbach).

KRÜGER F. (1934) Untersuchungen über Trichozysten einiger ProrodonArten. Arch. Protistenk. 83.

Krüger F. (1936) Die Trichozysten d. Ciliaten im Dunkelfelde. Zoologica 34. Kudo R. R. (1946) Protozoology, 3th ed. Springfield.

Kürn A. (1910) Die Entwicklung d. Geschlechtsindividuen d. Hydromedusen. Zool. Jabrb. Anat. 30.

KüHN A. (1909) Sprosswachstum u. Polypenknospung b. d. Thecaphoren. Zool. Jabrb. Anat. 28.

KüHN A. (1914) Entwicklungsgeschichte u. Verwandschaftsverháltnisse d. Hydrozoen I. Die Hydroiden. Ergebn. Fortschr. Zool. 4.

Kunn O. (1939) Die Stammesgeschichte d. wirbellosen Tiere im Lichte d. Paläontologie. Jena. 
Kükenthal W. Anthozoa u. Octocorallia. In Handbuch der Zoologie. Edited by KüKeNTHAL-KRUMBACH.

Kükenthal W. and Krumbach Th. (Edit.) Handbuch der Zoologie. de Gruyter, Berlin.

Lackey J. B. (1959) Morphology and biology of a species of Protospongia. Trans. Amer. Microsc. Soc. 78. No. 2.

Lameere A. (1931) Précis de Zoologie. Bruxelles.

Lang A. (1880) Die Polycladen (Seeplanarien) d. Golfes von Neapel. XI. Monograph.

LANKESTER E. R. (1873) On the primitive cell-layers of the embryo as the basis of genealogical classification of animals. Ann. Mag. Nat. hist. 11.

Laubenfels de M. V. (1955) Are Coelenterates degenerate? Syst. Zool. 4. Leloup P. E. (1929) Recherches sur l'anatomie et le développement de Vellella spirans Forsk. Arch. biol. 39.

Lemche H. (1858) Protostomian interrelations in the light of Neopilina. Proc. XVth Internat. Congress of zool., London.

Lemche H. and Wingstrand K. G. (1959) The comparative anatomy of Neopilina galatheae Lemche 1957 (Mollusca, Monoplacophora). Galathea Reports 3.

Lenhoff H. M. (1959) Migration of 14 C-labelled cnidoblasts. Exp. Cell. Res.

Levi C. (1956) Étude des Halisarca de Roscoff. Embryologie et systématique des Demonsponges. Arch. zool. expér. génér. 93.

Lrvanov L. (1931) Puti evolutsii zhivotnogo mira (The ways of evolution of the animal kingdom). Moscow.

Löhner L. (1911) Zum Exkretionsproblem d. Acoelen. Z. allg. Pbysiolog. XII, 4.

Mackie G. O. (1960) The structure of the nervous system in Velleia. Quart. J. Micr. Sci. 101

Marcus E. (1958) On the evolution of the animal phyla. Rev. Biol. 33.

Martin C. H. (1914) A note on the occurence of nematocysts and similar structure in the various groups of the animal kingdom. Biol. Zbl. 34 .

MATJAšrč J. (1959) Morfologija, biologija in zoogeografija evropskih temnocefalov (Morph., biol. and zoogeogr. of the European Temnocephala). Razprave IV. raz. SAZU, knj. 4.

Matveyev B. S. (1947) Rol' embriologii v izucheniyu zakonitosti evolutsii. (The role of the embryology in the study of evolutionary laws). 2ool. Zh. 26.

MEČNIKov I. (1886) Embryologische Studien an Medusen. Wien.

Merxner J. (1923) Über die Kleptokniden von Microstomum lineare (Müll.) Biol. Zbl. 43.

Merxner J. Baupläne der Tiere. In Handbuch der. Biologie (edited by BerTALANFFY). 
Mikinosuke Mryasima (1898) Úber das Nervensystem von Hydra. Zool. Magaz. 10 (Japan)

Mortensen Th. (1912) A Sessille Stenophore, Tjalfiella tristoma (Mortensen) and its Bearing in Phylogeny. British Association, sect. D., Dundee.

Moser F. (1925) Siphonophora. In Handbucb der Zoologie (edited by KüKENtIIAL-KRUMBACH).

Müller A. H. (1958) Lebrbuib der Paläozoologie, II. 1. Berlin.

Naef A. (1931) Phylogenie d. Tiere. In Handb. d. Vererbungswiss., Berlin.

Noland (1927) Conjugation in the ciliate Metopus sigmoides. C. J. morph. Pbysiol. 44.

OKADA Y. K. and Komori S. (1932) Reproduction asexuelle d'un actinic Boloceroides, Bull. biol. France 66.

Palombi A. (1935) Eugymnantha inquilina nuova leptomedusa etc. Pubbl. Staz. Zool. Napoli. 15.

Pantin C.F. (1956) Origin of the nervous system. Pubbl. Staz.zool., Napoli. 28.

Pax F. Hexacorallia In Handbuch der Zoologie (edited by Kükenthal-KrumBACII.)

PAx F. (1954) Die Abstammung der Coelenteraten nach der Theorie von Jovan Hadži. Nat-wiss. Rundscbau.

Pears A. S. (Ed.) (1949) Zoological Names. Durham, N. C.

Perrier E. Traité de Zoologie. Paris.

Picard J. (1955) Les nematocystes du Euchlora rubra (Köll.)., Rec. Trav. Stat. mar. d'Endoum. 15.

PrCken L. (1953) A note on the nematocysts of Corynactis viridis. Quart. J. Micr. Sci. 94.

Polejaeff N. (1893) Sur la signification systématique du feullet moyen et da la cavité du corps. Proc. Congrès Internat. d. Zool., Moscow.

RaAbe Z. (1948) An attempt of a revision of the system of Protozoa. Ann. Univ. M. Curie-Sklodowska, Lublin, 3.

RAY Kov J. B. (1957) Yadernyi apparat i yego reorganizatsiya $v$ tsikle deleniya $\mathrm{u}$ infuzorii (Nuclear apparatus and his reorganisation in the division cyclus of the infusorians). Zool. $\mathrm{Zb} .36$.

Rakovec R. (1960) Beiträge zur Kenntnis der Art Parazoanthus axinellae Schmidt. Biol vestnik 7.

Reisinger E. (1928) Amera. In: Handbuch der Zoologie (edited by KüKenTHAL-KRUMBACH).

Reisinger E. (1931) Polymera. In Handbuib der Zoologie (edited by KüKeNTHAL-KRUMBACH).

REISINGER E. (1957) Zur Entwicklungsgeschichte u. Entwicklungsmechanik v. Craspadocusta... Z. Morph. Ökol. Tiere 45.

Reisinger E. (1959) Anormogenetische u. parasitogene Syncytienbildung bei Turbellarien. Protoplasma 44. 
Remane A. (1936) Monobryozoon ambulans n. g., n. sp., ein eigenartiges Bryozoon d. Meeressandes. Zool. Anz. 113.

Remane A. (1937) Halammobydra, ein eigenartiges Hydrozoon d. Nord-u. Ostsee. Z. Anat., Morph. Ökol. Tiere, A. 7.

Remane A. (1944) Die Bedeutung d. Lebensformentypen für die Oekologie, Biol. Gen. 17.

Remane A. (1950) Die Entstehung d. Metamerie d. Wirbeltiere. Verh. dtsch. zool. Ges., Mainz, 1949.

Remane A. (1950 a) Theorie d. Coelomentstehung. Verh. disch. zool. Ges., Mainz, 1949.

Remane A. (1954) Die Geschichte der Tiere. In Evolution d. Organismen, 2. Aufl., Jena.

Remane A. (1956) Die Grundlagen d. natürlichen Systems, der vergl. Anatomie und der Pbylogenetik 2. Ausg., Leipzig.

Remane A. (1957) Zur Verwandtschaft u. Ableitung d. niederen Metazoen. Verb. dtsch. zool. Ges., Graz, 1957.

Remane A. (1960) Die Beziehungen zwischen Phylogenie und Ontogenie. Zool. Anz. 164.

Romer A. (1959) The Vertebrate Body, 2nd ed., Saunders, Philadelphia and London.

Rotmüller W. (1948) Über das natürliche System der Organismen. Biol. Zbl. 67.

SACARRÃO, DA FonseCA (1952) Remarks on gastrulation in Cephalopoda. Arquiv. do Museo Bocage 23.

SACARrÃo, DA Fonseca (1952 a) The meaning of gastrulation. Arquiv. do Mus. Boiage.

SACARrÃ̃o, DA Fonseca (1952 b) La conception du stade gastrula et la gastrulation. Rev. Fac. cienc., Lisboa, 2 ser., C-II, 1.

SACARRÃo, DA FonsecA (1953) Sur la formation des feullets germinales des Cephalopodes et les incertitudes de leur interprétation. Rev. Fac. cieni., Lisboa 3.

Sachwatkin A. A. (1956) Vergleichende Embryologie d. niederen Wirbellosen. VEB Deutsch. Verl. d. Wiss., Berlin.

SALENSKy W. (1908) Radiata und Bilateria. Biol. Zbl. 28.

Sato T. (1936) Über Atubaria heterolopha. Zool. /nz. 115.

SAVARSIN A. A. (1945-47) Ocherki evolutsionnoi gistologii krovi i soyedinitelno thani (Sketches on evolutionary bistology of the blood and of the connective tissue). Moscow.

SEDGWICK A. (1884) On the origin of metameric segmentation. Quart. J. Micr. Sci. 24.

Seidel F. (1960) Körpergrundgestalt und Keimstruktur. Zool. Anz. 164.

Seilern-Aspang F. (1957) Die Entwicklung von Mairostomum appendiculatum (Fabr.). Zool. Jber. Anat. 76.

Severcov A. N. (1935) Modusi filembriogeneze. Zool. Zh. 14. 
Siarp L. W. (1943) Fundaments of Cytology. New York, and London.

SNodgrass R. S. (1938) Evolution of the Annclida, Onychophora and Arthropoda. Smithson. Misc. Coll. 17.

Stammer H. J. (1959) Trends in der Phylogenic der Tiere; Ektogenese u. Autogenese. Zool. Anz. 162.

Stechow E. (1923) Zur Kenntnis der Hydroidenfauna d. Mittelmeeres... II. Zool. Jabrb., Syst. 47.

Steinböck O. (1924) Untersuchungen über Geschlechtstrakt-Darmverbindungen bei Turbellarien. Z. Morph. Ökol. Tiere, 2.

Steinböck O. (1931) Nemertoderma batbycola n. g., n. sp. In Ergeb. ciner Reise nach Grönland. Vidensk. Medd. Dansk natbist. foren. 90.

Sternböck O. (1936) Eine Theorie über den plasmodialen Ursprung der Vielzeller (Metazoa). Reading at the IV. internat. congress of cytology, Copenhagen, 10-15. VIII. Only the title was published and a summary in Arch. exp. Zellforsch 19, 2/4, 1937, Jena.

Steinböck O. (1952) Keimblätterlehre und Gastreatheorie. Pyramide, 2. Graz.

Steinböck O. (1954) Regeneration azoeler Turbellarien, Verh. disch. zool. Ges., Tübingen.

Steinböck O. (1957) Zur Phylogenie d. Gastrotrichen. Verh. dtsch. zool. Ges., Graz.

Steinböck O. (1957 a) Schlusswort zur Diskussion Remane-Steinböck. Verh. dtsch. zool. Ges., Graz.

Steinböck O. (1958) Zur Theorie der Regeneration beim Menschen. Forschung. u. Fortscbr. 4.

Steinböck O. and Aussenhoffer B. (1950) Zwei grundverschiedene Entwicklungsabläufe bei einer Art (Prorbyncbus stagnalis M. Sch. Turb.). Arch. Entw. Mech. 144.

Stepanov D. L. (1957) Neotenicheskiye yavleniya i yikh znacheniye dlya evolutsii (Occurrence of neoteny and its bearing on evolution). Vestnik Leningr. Univ. 18.

Steiner H. (1956) Gedanken zur Initialgestaltung der Chordaten. Rev. suisse de Zool., 63.

Swedmark B. and Teissier G. (1958) Otobydra vagans n.g., n. sp., hydrozoaire des sables... C. R. Acad. Sci. Paris 247.

Swedmark B. and Teissier (1958) Armohydra janowiczi, n. g., n. sp. Hydromeduse benthonique. C. R. Acad. Sci. Paris. 247.

Švábení K J. (1925) Parasitismus a metamorfoza druhu Gordius tolosanus Duj. (Parasitism and metamorphosis of the species G. t.). Publik. Fak. Sci. Univ. Masaryk, Brno 58.

Sснӓ P PI T. (1898) Untersuchungen über das Nervensystem der Siphonophoren. - Jen. Z. Natwiss. 32. 
Schimkewitsch M. (1908) Über die Bezichungen zwischen den Bilateralia u. Radiata. Biol. Zbl. 28.

Schindewolf O. H. (1930) Über die Symmetrieverhältnisse der Steinkorallen. Z. Pal. 12.

Schindewolf O. H. (1950) Grundfragen der Paläontologie. Stuttgart.

Schneider K. C. (1890) Histologie von Hydra fusca... Arch micr. Anat. 35.

SCHNeIDER K. C. (1896-1898) Mitteilungen über Siphonophoren. Zool. Jabrb., Ont. 9, und Zool. Anz. 21.

SCHNEIDER K. C. (1902) Lebrbuch der vergl. Histologie. Jena.

Shmalhausen I. I. (1947) Osnovy sravnitel'noi anat. pozvonochnykh zhivotnylkh (Principles of the comp. anatomy of the Vertebrata). Moscow.

Thiele J. (1902) Zur Coelomfrage. Zool. Anz. 25.

Tоттом A. K. (1954) Siphonophora of the Indian Ocean. 'Discovery' Reports. XXVII. Cambridge.

Tuzet O. (1948) La place d. Spongiaires dans la classification. C. R. XIIIe Congrès Internat. de Zool., Paris.

Tuzet O. and Pavans de Ceccatty M. (1952) Les cellules nerveuses de Grantis compressa penniger Haeckel (Eponge calcaire). C. R. Acad. Sci. Paris.

Tuzet O. and Pavans M. (1956) Les cellules nerveuses des éponges. $C . R$. $X I V_{e}$ Congrès Internat. de zool., Copenhagen.

Uchida T. (1929) Studies on the Stauromedusae and Cubomedusae, with special reference to their metamorphose. Japan. J. Zool. II.

Ulrici W. (1951) Vorschläge zu einer Revision der Grosseinteilung d. Tierreiches. Verh. Dsch. zool. Ges., Marburg.

Us P. (1932) Embrionalni razvoj ktenofor (Development of Ctenophora). Razpr. prir. sekc. prirod. društva, Ljubljana, I.

VANDerbroek G. (1949) Revision des classes et souclasses de Chordonias inférieurs. C. R. XIIle Congrès Internat. de Zool., Paris.

Watson D. M. S. (1951) Palaeontology and Modern Biology. Yale University Press, New Haven.

Weber H. (1954) Stellung und Aufgaben der Morphologie in der Zoologie der Gegenwart. Verh. Dtsch. zool. Ges., Tübingen.

Weygold P. (1960) Embryologische Untersuchungen an Ostracoden. Die Entwicklung von Cypridina litoralis (G.S. Brady). Zool. Jabrb., Anat. 78.

Werll R. (1925) Les nematocystes et spirocystes des Cnidaires. C. R. Acad. Sci., Paris 180.

Weill R. (1930) Essai d'une classification du nématocystes des Cnidaires. Bull. Biol. France-Belg. 64.

Weill R. (1934) Contribution à l'étude des Cnidaires et de leur nématocystes. Trav. Stat. zool. Wimereux, 10-11.

Weissman A. (1883) Die Entstebung der Sexualzellen bei den Hydromedusen. Jena. 
Wessenberg H. (1961) Studies on the life cycle and morphogenesis of Opalina. Univ. Calif. publ. in Zool. 61, No 6.

Westblad E. (1948) Studien über skandinavische Turbellarien. Ark. zool. 4.

Westblad E. (1949) Xenoturbella bocki g. n., sp., n., a peculiar primitive turbellarian type. Ark. Zool. 41.

Will L. (1909) Die Klebkapseln der Aktinien und der Mechanismus ihrer Entladung. S. B. Schrift. natfor. Ges., Rostock, N. F., 1. MacMillan London. Willey A. (1894) Amplioxus and the Ancestry of Vertebrata.

Willmer E. N. (1960) Cytology and Evolution. Academic Press, New York and London.

Wrlson H. V. (1935) Some criticical points in the metamorphosis of the halichondrine sponge. J. Morph. 58.

Wolf H. (1903) Das Nervensystem der polypoiden Scyphozoa und Hydrozoa. Z. allg. Pbysiol. 3.

Woltereck R. (1940) Grundzïge einer allgemeinen Biologie. I.

Zalokar M. (1955) "Degenerate" Coelenterates. System. Zool. 4.

Zenkevich L. A. (1945) The evolution of animal locomotion. J. morph. 77.

Zimmermann (1943) Die Methoden der Phylogenetik. In Evolution der Organismen (edited by Heberer). Jena. 


\section{INDEX}

(Numbers in italic refer to illustrations.)

Acalepha 79

Acanthocephala 78, 141, 147, 168, $395,396,397,460$

Acanthodea 456, 457

Acarina 272, 419, 423

Acellular state 356

Acnidaria 9, 19, 21, 78, 199, 227, 244, 324

Acoela 71, 139, 148, 172, 196, 210, $211,223,256,271-277,279,281$, 290, 291, 296-298, 301-304, 314, $315,320,321,337,368,386$

Acoelomata 78, 130, 227, 387, 390, $391,464,465$

Acontiae 182

Acrania 296, 455, 457, 461

Actinea 89

Actiniae 73, 99, 168, 178, 183, 197, 223, 246

Actiniaria 101, 108, 117, 148, 149 , $178,179,194,206,217,228$

Actinodactylus 70

Actinula 265

Aeginidae 152

Aequorea 64, 119

Agametes 283, 289

Aglaophenia 110, 220

Aglaopheniidae 159

Agnatha 455, 456, 457, 461

Alcyonaria 232

Allman 53, 111

Alloeocoela 148, 211

Alternation of generationes 153, 154, $215,219,242,307$
Alvarado 2, 26

Ambulacralia 445

Ameria 14, 21, 27, 40, 41, 52, 72, 86, $192,195,253,266,285,346,388$, $390,391,393,399,400,408,412$ $414,417,427,429,440,448,451$, 458,459

Amerous state 452

Amoebae 32

Amoebocytes 7

Amoeboid androgamet 297

Amoebosporidia 357

Amphibia 37, 457, 461

Amphioxus 56, 448, 456

Amphiscolops 316

An der Lahn 146

Anderson 465

Androconjugant 292

Androgametes 282, 284, 287, 288, 290, 292, 295, 307

Androgamont 283

Annelida 27, 32, 40, 41, 68, 123, 142 , $212,213,251,270,296,350,384$, $386,390,400,401,403,405,412$, $415,420,424,431,432,434,437$, $442,454,460,463$

Anoplocepbala 58

Anthocaulus 154

Anthocodium 154, 238

Anthocyathus 154

Anthomedusa 10, 11, 67, 242

Anthophyta 49

Anthopolyp 98, 105, 112, 115, 116, $125,147,154,164,166,180,207$, 226,410 
Anthozoa $31,71,76,87,88,90,95-$ $97,100,101-108,110,113,122$, $123,129,130,133,149,163,166$, $171,172,179,183,185,190,195$, 197, 198, 202-206, 208, 209, 211, $212,215-219,223,229,237,238$, 243, 252, 254, 255

Antimeres $10,59,79,186$

Antipatharia 122, 183, 232

Anucleata 354

Apolemia 121

Aposcolecida 395

Appendiculata 349, 443

Aprocta 78, 241, 224

Apterygota 416

Arachnactis 263

Arachnoidea 384

Archaeocyathidae 31,379

Archaeopteryx 68, 69, 348

Archiannelida 400

Archicoelomata 426

Archihiston 212, 315, 317

Architomy 215

Aristotle 13, 73

Armobydra 51

Arthrodira 456, 457

Arthropoda 387, 412-417, 419, 437, $460,463,465$

Articulata 411, 412, 414

Artiozoaires 87

Aschelminthes 296, 389, 395, 396, 463,465

Ascidia 68, 174, 444, 452

Ascon 7, 369

Asexual reproduction 153, 154, 213 $220,258,266,278,283,294,429$

Aspidosiphon 433

Assymetry 134

Athecata 53, 237, 239

Atypical budding 218,

Ausserhoffer 211

Autotrophy 355, 356

Aves 457, 461
Awerinzew 363

Ax 174, 215, 450

Babić 217

Balanoglossus 174, 448

Balanopbylia 170

Balfour 25

Baltzer 436

Barkley 354, 355

Bateson 442

Bathypelagic animals 141

De Beer, Sir Gavin 21, 23, 24, 33, $68,82,248,300,348,442$

Beklemischew 24, 78, 232, 244, 257 , $271,273,274,349,366,408,411-$ $412,414,416,425$

Bèlař 358

Benthonic life 10, 36, 48, 152

Berrill 174, 442, 444, 445, 446, 452

Bertalanffy v. 247

Bigelow 221, 263

Bilateral symmetry $10,14,19,22,55$, $102,273,344,437,453$

Bilateria $85,245,270,375,380,386$, $411,426,463-465$

Bilaterogastraea 103, 249, 250, 269 , 404

Biogenesis 343

Bisexuality 282, 284

Bisymmetry 10, 102

Blastaea 26, 229, 248, 250, 322, 326, $327,363,364,375$

Blastaeadae 373

Blastoderm 1

Blastopore 434

Blastostyl 159

Blastozooid 217

Blastula 322

Boas 40

Bodonidea 361

Bocttger 341, 394, 408, 453

Boloreroides 149, 219 
Boreobydra 151, 227

Börner 76

Bougainvillea 64

Bougainvillinea 242

Boveri 443

Bowling 286

Brachiopoda 60, 196, 213, 296, 384, $408,424,427,428,457,459,461$, 464, 465

Branchioceriantbus 243, 454

Brancbiostoma 56, 123, 448

Branchiotrema 438

Bresslau 81, 119, 120, 148, 169

Brien 122

Broch 93, 161, 220, 242

Broman 344

Brooks 21, 160, 442

Bryozoa 32, 253, 254, 260, 384, 401, 424

Bubalocerus 70

Bucal diverticulum 173

Bud communities 162

Bütschli 79, 87, 393

Caenogenesis 35, 38, 204, 205, 317

Calcarea 332,

Calceola 184

Calcispongiae 3, 370

Calcoblast 196

Calkins 286

Calycophorae 161, 220

Calycozoa 234

Calyptoblastea 242

Campanopsidae 239

Campanularia 31,92

Campanulariidea 242

Canalis neurentericus 454

Capitata 242

Carcinonemertes 147

Cardiocoele 388, 398

Carlgren 168

Carmarina 123
Carpobotbrium 58

Carter 24, 41, 274, 350, 380, 383

Carybdaeidea 234

Cassiopea 157

Catenulidae 169

Caulerpa 307, 316, 330

Caulus 117

Cellularity 309

Cellularization 197, 229, 295, 299 , $305,309,367$

Cephalochord 450

Cephalization 132, 172, 418, 448

Cephalopoda 49, 392, 394, 454

Cerebratulus 200

Ceriantharia 101, 108, 148, 184, 194, 198, 206, 208, 209, 217, 231, 232, 264,453

Cerianthula 264

Cerianthus 184

Cerinula 209, 263, 264

Cestoda 57, 72, 147, 192, 244, 346, $392,395,405,460$

Chaetognatha $60,269,270,349,384$, $390,426-429,437,438,443,445$, $456,459,461,463-465$

Chaetopterus 419

Chatton 203, 222

Chelicerata 412, 416, 417, 423, 460

Chester 115

Chilopoda 416

Chlorella 222

Chlorobydra 224

Choanocyte 7, 327, 369

Choanoderm 3

Choanoflagellata 5, 6, 7, 308, 311, $331-333,363-365,370,458$

Chondrichthya 456, 457, 461

Chondrophora 67, 141, 160, 266

Chorda dorsalis 13, 173, 174, 180, 441,450

Chordata (Chordonia) 14, 56, 67, 68, 107, 142, 167, 173, 192, 346, 349, $390,437-459,461,463,465$ 
Chordonoid tissue 174

Chrysaora 108, 206

Chrysomonadida 361

Chun 18, 161

Cilia 52-54, 273

Ciliata $62,277,281,284,285,287$, $288,290,303,315,358,360-364$, 367,368

Ciliate rosette 143,144 ,

Ciliation 276, 280

Ciliophora 300

Cirripedia 417, 423

Cladocoryne 63

Cladogony 214, 221

Cladonema 63

Clark 124

Claus 25, 121, 168

Clava 92

Cleavage 7, 35, 206, 207, 279

Clitellata 412, 415, 460

Cnidae 9, 12, 66, 190, $191 \mathrm{ff}$.

Cnidaria 1, 8, 12-16, 19-21, 23-27, $30,31,38,39,44-47,50,51,56$, $57,61-63,66,68,70-75,77-81$ ff.

Cnidoblast 191, 202

Cnidocil 202

Cnidocyte 112, 114, 121, 199, 203

Cnidoid 203

Cnidom 202

Cnidosporidia 72, 199

Cnidotrichoapte 200

Coelenterata $1,2,4,5,8,9,21-23$, 25-27, 46, 75-79, 86, 100, 145 ff.

Coelenteria 393, 425

Coelhelminthes 395

Coeloblast 12, 212

Coeloblastula 205, 207, 322

Coelocoela 26

Coelom 25, 270, 319, 325, 374, 385, $386,392,454$

Coelom blood 436

Coelomata 23, 26, 76, 78, 85, 102, $137,227,244,249,252,253,256$,
$372,375,376,380,383,384,386$, $387,408,409,411 \mathrm{ff}$.

Coeloplana 85,143

Coelothelium 251

Cocnenchynme 217

Coenobium 306, 308, 311

Coenosarc 219

Coenothecalia 232

Colonial theory 284, $335 \mathrm{ff}$.

Colony 308, 330, 387

Colosi 174, 376, 390, 397

Colloblast 12

Comatula 261

Compartmentalization 304

Comstock 416

Concha 170

Conjugant 295

Conjugation 280, 281, 286, 288, 289, 291, 294, 305

Copulation 146, 280, 286, 287, 303

Copulatory organs $145 \mathrm{ff}$.

Corbula 161

Cori 41, 141, 408

Cormic organs 111

Cormidium 110

Cormobiouta 355

Cormogony 116, 234

Cormophyta 309

Cormus 104, 109, 195, 219330 ff.

Cornularia 158, 233

Coronal muscles 118, 124

Coronata 235

Coryninae 242

Cotburnocystis 446

Craspedacusta 241

Craspedomonadida 308

Craspedotella 66, 67

Crustacea 44, 413, 416, 419, 451, 460

Cryptomedusoid 92

Ctenaria 10, 11, 80, 165

Ctenophora 8-22, 25-27, 44, 57, $59,60,63,66,70,75-86$ ff.

Cubomedusae 188, 234, 235 
Cuénot 95, 353, 361, 366, 377, 378 421,422

Cutries 202

Cuvier 56, 74, 86, 411

Cyclomery 253

Cyclosis 278, 373

Cyclospora 283

Cyclostomata 455

Cydippe stage 269

Cystoflagellata 356

Cytoidea 357, 358

Cytomorpha 357, 358

Cytopharynx 138

Cytostome 138, 278

Cytozoa 358

D’Ancona 87, 228

Darwin 260

Darwinism 74, 349

Dawydoff 9, 17, 18, 134, 212

Degeneration 45, 97, 367

Delage 1, 3, 78, 91, 233, 331, 371, 425

Delamination 206

Delsman 442, 445

Deltoid muscle 218

Derosomes 426

Deuterocoelomata 390

Deuterostomia 78, 325, 341, 369, $378,381,390,408-411,422,423$, $425,426,453,454,463$

Diaphragma 239

Diblastica 26, 112, 353

Dichotomy 408, 413

Dicoryne 53, 194

Dicyemida 346, 366, 367

Didermique 26

Digestive system 163, 189

Dileptus 301

Dimorphism 109, 218, 279, 289, 291, Dinoflagellata $65,199,203,336,355$, 356

Dipbyes 203
Dipleurula 452, 463, 464

Diploblastica $75,78,324,374$, 384

Diplopoda 416

Discophora 234

Discomedusae 234

Disconanthes 161

Diskyneta 77

Dissipiment 420

Dissogony 44

Dobell 276, 356

Doflein 357, 358

Dogiel 62, 78, 139, 282, 294, 320, 357

Dohrn 442

Dollo's rule 51, 53, 94, 95, 115, 141 , 144, 295

Dougherty 361

Dreissensia 451

Dudich 78

Dyctylactis 209

Eberl 3

Echinodermata 10, 56, 61, 63, 74, 87, $104,127,184,244,261,296,393$, $425,427-429,437,445-447,451$, $461,463,465$

Echinoidea 438

Echiuroidea 41, 296, 400, 414, 415, $420,424,431,433,460,463,465$

Ecterocoelia 251, 325, 400, 402

Ecterocoelic state 423 type 403

Ectocarpa 112, 182

Ectocyte 140, 197

Ectocytium 128

Ectoderm 116, 117, 323, 324, 328, 375

Ectoepithelium 140

Ectoparasitic animals 257

Ectoplasmodium 317

Ectoprocta 123, 194, 214, 253, 254 $264,312,420,424,427,428,433$ 439,461 


\section{Edwardsia 168}

Eimer 125

Eleutheria 62, 150, 152

Emunctorial organ 137, 278, 387

Enantia 195

Enantiozoa 1, 3, 78, 331, 334

Endocarpous gonad 184

Endocyt 314

Endomyxis 290

Endoparasitism 275, 285, 354, 367, 405, 417

Endoprocta 123, 141, 147, 194, 195, $253,260,266,342,373,389,390$, $395,400,417,460$

Endosymbiosis 223

Endosymbiotic algae 222

Enterocoel theory 249, 250, 253, 400

Enterocoelia 103, 325, 400, 409, 464, 465

Enterocoelic state 386, 402, 410, 423, $425,440,454$

Enteropneusta 173, 296, 425, 429, $430,438,439,445,446,448-452$, $454,457,461$

Enterozoa 78, 372

Entocarpa 112, 181, 184, 185

Entodiniomorpha 295

Entomesoderm 39, 212

Entomostraca 417

Entoplasmodium 317

Entoprocta 14, 68

Ephyra 149, 157, 260

Ephyropsidia 149

Epineura 376

Eucblora 9, 12, 66

Euciliata 97, 138, 192, 215, 222, 228, $286,301,302,362$

Eucoccidium 314

Eucoela 271

Eucoelom 41

Eucoelomata 253, 389, 409, 424, 464, 465

Eudendrium 92, 160
Eugregarinida 290

Eug'mnantbea 91, 237

Eumedusoid 92

Eu metazoa 1-4, 21, 23, 41, 78, 128, $129,138,143,144,149 \mathrm{ff}$.

Euplotes 297

Euprocta 244, 395

Evolutionary mechanics 35

Exconjugant 290

Excretory system 137

Excretory-emunctory organelle 138

Extremities 413, 441

Exumbrella 15

Faurot 178

Faussek 387

Fecampia 168, 253

Filifera 242

Fischer W. K. 462

Fission 50

Flagella 52

Flagellata 4, 7, 26, 32, 56, 62, 96, $144,206,214,228,248,278,285$, $286,299,302,317,322,331,333$, $357-364,458,463,465$

Flemming 212

Foraminifera 307

Fossores 453

Fragmentation 215

Franz 383

Franzén 296

Fundamental biogenetic law 25, 33, $37,38,204,250,252,255,260$, $275,279,335,339,342,383$

Fungia $71,105,106,154,155,156$, 215

Galeolaria (larva) 263

Gametes 33, 151, 282, 283, 285-288, 299,305

Gametocytes 150, 277, 284, 299, 305 
Gametogenesis 33, 286, 305

Gamont 283, 284, 290, 291

Gardiner 212

Garstang 21, 34, 162, 204, 260, 442, 446

\section{Gaskell 442}

Gastraca 5, 7, 25, 38, 80, 96, 104, 111 , $183,205,229,248,264,323,375$, 453

bilateralis 250 theory 249,250

Gastraeadae 26, 78, 87, 323, 331, 373

Gastrobionta 326, 355, 372

Gastroblasta 216, 217, 221

Gastrocoel 402

Gastropoda 56, 65, 204, 213, 394

Gastrotricha 147, 198, 253, 254, 395, $396,438,460$

Gastro-vascular system 164, 179, $182 \mathrm{ff}$.

Gastrozooid 162

Gastrula 206, 207, 327, 369, 373

Gastrulation 373

Gelei 276, 321, 328

Gemmatio 50

Genealogical tree 270-338, 376, 377, $380-383,384,412,422,454,455$, $457,458,465$,

Genital system 145 ff.

Georgevitch 212

Gephyrea chaetophora 424

Germarium 147

Gerould 435

Gessner 376

Gieslén 446

Giljarov 417

Glaucoma 286

Goctte 80, 93, 96, 167, 183, 189, 234

Goette-Müller's larva 210, 263

Goldfuss 357

Golfingia 435

Golian 397
Gonactinia 105, 153, 215

Gonads 44, 117, 149, 150, 181, 184, 389, 392

Gonangia 160

Gonionemus 109, 241

Gonochorism 147, 149, 284, 286, 292

Gonocoel 387-389, 398, 399

theory 249,388

Gonocyte 112

Gonophore 160

Gonosoma 153, 161

Gonothyrea 92, 160, 263

Goodrich 443

Gordiacea 390, 406

Gorgonaria 232

Graff 25, 140, 167, 168, 196, 223, 271, 274, 277, 299

Grammaria 92

Grant 371

Graptolitha 431, 448

Grassé 359-361, 378

Gregarinida 283

Grobben 274, 408, 424

Gröntved 6

Grošelj 125, 130, 135

Guthrie 465

Gymnoblastea 242

Gymnoblastiques 242

Gynocaryon 292

Gynogametes 282, 284, 285, 287, 290, 293, 295

Gynogamont 283

Gyratrix 315

Haacke 99, 246

Hadži 5, 12, 18, 22, 23, 29, 52, 53, 65, $81,82 \mathrm{ff}$.

Haeckel 7, 25, 33, 34, 37, 75, 80, 103, $165 \mathrm{ff}$.

Haeckelia 18, 66

Haemocoel 388, 399

Haimea 108 
Halammobydra 53, 54, 94, 194

Halantbus 237

Halcampia 168

Halcampogeton 168

Haleciella 237

Haleciidae 221, 239

Halecium 220, 221, 226

Haleremita 241

Haliclistus 124

Halicornaria 110

Halistemma 121

Hall 281, 358, 359

Halocoryne 63

Hanson 24, 271, 279, 286, 300-305

Hanström 128, 129

Hardy 21, 85

Harms 7, 78

Hartmann 282, 284

Haswell 70

Hatschek 25, 41, 260, 357, 367, 376, $387,408,409,424,436$

Hebella 187, 237

Hebellidae 160, 239

Heberer 383

Heider 80, 176, 322

Heliozoa 289, 359

Helix 289

Hemichordata 178, 444, 463, 465

Hemimyxis 290

Heric 167

Hermaphroditism 145-149, 167, 271, 284-286, 288, 289, 291-293, 296$299,304,320,328$

Hérouard 91, 233, 425

Hertwig's 25, 112, 125, 126, 168, 184

Heteraxonia 376

Heterocyclic division 280, 289, 297

Heteromedusoid 92

Heterotrophy 353, 356, 360

Hexacorallia 122, 217, 231, 232, 246

Hexactiniaria 100, 109, 122, 231, 242

Hexapoda 416, 417

Hilair 442
Hincks 242

Hirsch 277

Hirudinea 116, 401, 415

Hofstenia 148

Hologamous isogamy 222

Holophryidae 305

Holotricha 302

Horridge 134

Horváth 298

Houssai 393

Hubrecht 442

Huxley Th. 112, 369, 411

Hydra 2, 7, 9, 44, 92, 96, 107, 114$116,124,126$, ff.

Hydranth 150, 187, 219, 238

Hydrocaulus 117

Hydrocoelomata 390

Hydrocorallia 196

Hydroctena 9, 15, 17, 18, 81, 134

Hydroidea 53, 89, 93, 101, 107,161, 195, 236, $312 \mathrm{ff}$.

Hydromedusa 10, 17, 64, 78, 81-83, $89,90,92,93 \mathrm{ff}$.

Hydropolyp 118, 160, 166, 175, 178, 226

Hydrorhiza 123, 131, 133, 136

Hydrosoma 117, 121

Hydrotheca 220, 238

Hydrozoa 8, 31, 52, 71, 72, 75, 87, 88, 91, 93, 95-97 ff.

Hydrula 265

Hyman 24, 25, 74, 78, 81-83, 89, 90 , 92, 93, 100, $101 \mathrm{ff}$.

Hypercellular state 305, 356, 357, 358

Hypermastigida 62, 299, 362, 368

Hypogenetic development 17, 44, $210,218,219,230,236$

Hypolitus 108

Hyponeuria 409

Infusoria 52, 54, 57, 140, 169, 274, 276, 278, $279 \mathrm{ff}$. 
Insecta 456, 460

Internalization of gametogenesis 286 , 293, 328

Interstitial cells 12,112

Intestinal diverticules $12,13,14$

Invertebrata 444

Isogamy 282

Ivanov 404

Jägersten 2, 5, 26, 101, 103, 246, 248-252 ff.

Jamoytius 441, 448

Jennings 289

Kaestner 100, 196, 231, 243, 246, 414

Kamptozoa 123, 141, 253, 260, 264, 401,415

Kemna 365

Kepner 200

Kinetids 286

Kinorhyncha 395, 396, 460

Kleinenberg 115, 125

Kleptocnide 12, 66, 191, 199, 204

Kofoid 203

Koinocystis 315

Komai 143, 149, 235

Komori 219

Korschelt 176

Kowalewski 36, 178, 445

Krasinska 123, 187

Krüger 200, 203

Krumbach 79, 81, 137, 234, 246, 393, 425,446

Kudo 281

Kühn A. 189, 212, 242

Kuhn O. 98, 100

Kükenthal 81, 100, 175, 196, 246, 393

Laceration 215

Lackey 6
Lafoëa 160

Lafoëina 237

Lamarck 74, 86

Lamarckism 34

Lameere 363, 425, 428, 437

Lamellibranchiata 213

Lang 82, 85, 140, 424, 431

Lankester 25, 26, 112, 205, 372

Lar 63

Larvacea $443,452,456,457$

Laubenfels 24, 110

L'echelle animal 345, 349, 380, 459

Leloup 162

Lemche 42, 349, 393, 394

Lemna 49

Lendenfeld 2

Lengerich 152

Lenhoff 122

Leptodiscus 66, 67

Leptolida 206

Leuckart 7, 8, 25, 73, 103, 161, 164, 244,331

Lévi 3

Lictorella 160, 161, 220

Lilly 334

Limnomedusae 240

Limulus 416, 442, 446, 456

Lineus 200

Living fossil 30

Löhner 140

Loxosoma 214

Lucernaria 124, 193

Lucernariidae $88,91,123,149,158$, 193, 218, 233, 234, 235

Luther 178

Lwoff 305

MacBride 82

Macroconjugant 291, 292

Macroevolution 45

Macrogamete 282

Macrogamont 289 
Macronucleus 287, 289, 293, 297, 298, 302, 303

Macrophagy 169, 419

Madrcporaria 31, 32, 98, 105, 154, $170,215,223,232$

Magospbaera 322

Mammalia 456, 457, 461

Manubrium 114, 124, 151, 171, 172

Marcus 41, 246, 254, 267, 270, 332, $333,342,401$

Marinelli 378

Martin 204

Mastigophora 361

Mating type 291

Matjašič 192, 256, 257

Medusae 50, 52, 71, 73, 89, 127, 133, 189, 194, 209

Medusocbloris 66, 67

Medusoid 92, 103, 131, 156, 161

Medusom theory 93

Meiosis 283, 284, 286

Meixner 200, 376

Mesenchyme $112,128,181,251,368$, 398

Mesenteries 148, 175

Mesoderm 117, 118, 121, 252, 264, $318,321,324,328,353,385,403$, 425

Mesogloea 112, 117

Mesohyl 13, 112, 150, 198, 219, 264 $311,328,353,375$

Mesoplasmodium 317

Mesozoa 87, 275, 313, 366-368, 463-465

Metabionta 312

Metagastraea 89

Metagenesis 88, 151, 155, 266

Metamerism 41, 64, 229, 251, 391, Metamorphosis 37, 39, 208, 261, 262, $264,266,269,311,341,419,446$ Metanephridia 142, 392, 430, 436

Metaphyta 294, 319, 326, 329, 331, $334,354,355,372,373$
Metasoma 432

Metazoa 5, 7, 25, 38, 49, 54, 55, 87, $102,103,142,205,229,251,269$, $307,323,331,334,350,358$, $391 \mathrm{ff}$.

Metschnikoff $1,25,26,82,85,205$, 206, 263, 331, 369

Microconjugant 291, 292

Microgamete 282

Microgamont 289

Microbydra 241

Micronucleus 288, 289, 297, 298, 303

Microphagy 169, 170, 188, 419

Microstoma 200

Microstomidae 169

Micrura 200

Migration of cnidocytes 203

Miles 107

Millepora 91

Milleporidae 196, 241

Minchin 358, 359

Minot 442

Minyidae 104

Miyashima 125

Mollusca 21, 41, 54, 65, 66, 72, 134, $137,148,168,196,198,212,285$, $296,384,388,390,392-395,413$ ff.

Molluscoida 390, 424

Monera 354

Monobracbium 63

Monobryozoon 107, 214

Monocaryonic state 283, 305

Monocellular state 283, 307, 330, $357,365,402$

Monodiscal strobilation 157

Monophylly 349, 351

Monopodium 162

Monoxenia 108

Morea 248

Morphogenetic technique 374

Morula 208, 322

Mosaic theory 300,302 
Mosaic-like evolution 68

Moser 93, 94

Moskovski 306

Müller H. A. 24, 353, 378, 379, 422

Müller's larva 20, 21, 82, 83, 269

Mycale 334

Mycetozoa 38

Myoneme 278

Myrientomata 416

Myriopoda 412, 416, 417, 419, 460

Myriothelidae 209

Myxobolus 199

Myxocoel 387, 390, 401, 419

Myxomycetes 356

Myzostomidea 412, 415

Naef $89,250,356$

Narcomedusae 12, 15, 17, 18, 54, 66, 72, 88, 92-95, 152, 209, 221, 263, 265,266

Natural selection 135, 140

Nauplius 36

Nausithoë 108, 117, 149, 151, 185 , $186,206,235$

Nectocalyx 266

Necton 453

Nematoblast 191

Nematobotbrium 58

Nematoda 52, 57, 196, 395, 396, 460

Nematoderma 129, 196

Nematoidea 142, 297, 298

Nematomorpha $392,395,396,460$

Nemertinea 65, 66, 141, 191, 200, $250,253,254 \mathrm{ff}$.

Neocoelomata 426

Neodarwinism 28, 347 ff.

Neopilina 42, 393, 394

Neoteny 19, 21, 23, 49, 94, 274, 348, $349,418,443 \mathrm{ff}$.

Neoturris 123

Nephridial organ 139, 141, 142, 144, $389,392,449$
Nephrocoel 387, 388, 399

Nephrocytes 143

Nerve-net 126

Nervous system 126 ff.

Neurenterica 453

Neuromerism 432, 434

Neuro-muscular-epithelial cell 115

Neuromyaria 378

Neuroporus 453

Noctiluca 355, 356

Notochord 450

Nuclear dimorphism 305, 385

Nummulites 307, 368

Nutation 111, 116

Obelia 64, 133, 187

Ocellatae 134

Octactiniaria 108, 109, 122, 218, 231, $232,238,242$

Octocorallia 158, 194, 196, 206, 231, 232

Oesophagostomum 58

Okada 219

Oligochaeta 415, 418

Oligomeria 14, 41, 64, 87, 142, $167 \mathrm{ff}$.

Oligomerization $10,62,64,122$, $139 \mathrm{ff}$.

Oligophyly 349,350

Olynthus 7, 369, 370

Ontogenetic mechanism 319

Ontogenetic technique 35

Ontogeny $209 \mathrm{ff}$.

Onychophora 390, 412, 413, 415-419, 460

Oögamy 284, 286

Oözooid 108, 162

Opalinata 362

Opalinidae 285, 298, 362, 368

Oparin 359

Ophiuroidea 57, 87

Ophryoscolecidae 294, 295, 297 
Opistotricbium 291

Ordovicium 185

Orthogon 128

Orthonectoida 366, 367

Osteichthyes 456, 457, 461

Ostracodermata 446, 456, 457

Ostroumovia 108

Otobydra 51, 95

Otomesostoma 314

Paedogenesis 39

Paedomorphosis 444

Palaeostraca 416

Palintomy 361

Palmenia 169

Palombi 91, 237

Pantin 127, 130

Parabronema 58

Parallelism 65, 440

Parapbanostoma 140

Parapodia 270, 420

Parasitism 46, 192, 253, 339, 366, 454, 391

Paratomy 215

Parazoa 1, 2, 4, 8, 331, 337, 350, 362, $368,371 \mathrm{ff}$.

Parenchyma 314

Parenchymatosa 390

Parenchymella 25, 26, 205, 365

Parthenogenesis 290, 293

Patten 442

Paucinucleata 312

Paurometamera 393

Pauropoda 416

Pax 24, 99, 100, 149, 175, 246

Pears 243, 244, 366, 389, 414

Pecten 135

Pegantba 221, 263 (larva)

Pelagia 137

Pelagic zone 48

Pelagotburia 428

Pennaria 63, 91, 110
Pennatulacea 109, 232

Pentacrinus 261

Pentamery 184

Pentastomida 413, 417, 419, 423

Pericardium 430

Periderm 107, 109, 195, 199, 233

Perigastrocoel 25, 26, 41, 130, 164, 249, 270, 392, ff.

Perineura 376

Perrier 78, 86, 87

Person (subindividuum) 116, 154, $312,370,405$

Petrunkewitch 24

Peysonell 74

Phallonemertes 147

Pharynx 163, $183 \mathrm{ff}$.

Phascolion 433

Phoronoidea 405, 427, 429, 431, 433, $439,448,461$

Photosynthesis 354

Phylembryogenesis 42, 44

Phylogeny 29, 35, 44, 52, 114, 185 , $204 \mathrm{ff}$.

Phyta 354

Phytoflagellata 49, 355, 356, 359

Phytology 356

Phytomonadida 365

Phytoplancton 48

Phytozoaires 86,87

Pilidium 43

Pinacocyte 7

Pinacoderm 3

Pisces 455, 457

Placula 25

Planaria 215, 314

Planctonic way of life 10,49 , 60,261 ff.

Planea 276

Planula 25, 38, 39, 80, 89, 158, 193, 206, $207 \mathrm{ff}$.

Planularia 367

Planulozoa 367

Plasmodial state $309,311,314-318$ 
Plasmodial theory 278, 384, 459 ff. Polypodium 216

Plasmodium 197, 212, 276, 288, 313, Polypomedusa 123 361

Plasmodroma 358

Platyctena 85

Platyctenea 22

Platyhelminthes 78, 82, 137, 252, 258, 270, $285 \mathrm{ff}$.

Pleurostomous Gymnostomes 301

Plumularia 220

Plumulariidae 110

Pneumatophorae 161

Pneumatophore 161, 162, 198, 312

Podaxonia 408, 425

Podoeoryne 64, 159, 160

Podocyst 107, 158, 214, 218

Pogonophora 4, 168, 427, 429, 439, 461

Polycellular state $330,368,370,402$

Polychaeta 135, 334, 403, 412, 413, $417,456,460$

Polychoerus 212, 223

Polycladida $14,22,54,71,81,82$, $85,140,148,195,263,338,352 \mathrm{ff}$.

Polycnidom 203

Polydiscal strobilation 218

Polyenergetic 72

Polygordius 40

Polykaryonic state 294, 307, 309, 361

Polykaryonic theory 284, 290, 313, $329,335,386,459$

Polykrikos 65

Polymastigida 62

Polymeria 27, 52, 137, 257, 346, 388, 391-393, 399, ff.

Polymeric state 397,405 , ff.

Polymerization 9, 11, 63, 64, 142, 148,170 , ff.

Polymetamera 393

Polymorphismus $70,109,110,153$, $161,214,218,236,312$, f.

Polyp 189, 209

Polyphyly 349, 350, 351, 354

Polysegmented state 405

Polystylophora 174

Porifera 78, 79, 322, 371, 463

Porus excretorius 138

Postgamous division 289, 290, 336

Potamobius 451

Pouchetia 65

Praedatores 453

Praegamont 284, 287, 289, 294

Praesoma 432

Priapuloidea 296, 396, 397, 426, 460

Proacoela 308

Prochorda 180

Prociliata 321, 375

Poeumetazöon 273

Progamous division 291

Progressive evolutior $145,75,98$, $103,111,167,259,271,329,414$, 449

Pronucleus 288, 289, 305

Prorhabdocoela 258

Prorbyncbus 120

Prorodon 200

Prosopygia 415, 424, 425

Protandry 148

Protaxonia 376

Proterospongia 6, 7, 308, 322, 333

Proteidae 443

Protichtya 456, 461

Protista 33, 65, 66, 103, 138, 281, 282, 319, 329, 355

Proteus 348

Protobionta 355

Protociliata 285, 320, 372, 375

Protocoelomata 390

Protocytoidea 356

Protobydra 151, 216, 211

Protomonadina 361

Protonephridia 22, 137-144, $328,387,390 \mathrm{ff}$.

Protophyta 65, 227, 354, 360, 363 
Protosepta 98,

Protostomia 78, 271, 390, 378, 381, 408, $4 i 0$ ff., 463

Protozoa 5, 23, 49, 52, 54, 56, 67 $96,149,222,258 \mathrm{ff}$.

Protracheata 412, 416, 419

Protrochophora 38,

Protrochula 81

Przibran 224

Pseudocerops 386

Pseudocoela 386, 389

Pseudocoelomata 64, 389, 465

Pseudogastrula 322

Pseudometamerization 64

Pseudoplexaura 115

Pseudopodia 359, 360

Pterobranchia 173, 427-429, 438, 461

Pterocorallia 32, 98, 185

Pycnogonida 416

Pycnothrix 159

Pyrosoma 66, 67

\section{Quinet 95}

Radial symmetry 10, 19, 99-101, $135,182,273,344 \mathrm{ff}$.

Radialia 78,100, 222, 244, 245, 344, 463 Radiata 56, 73, 87, 185, 273, 344, 437, $447 \mathrm{ff}$.

Radiolaria 49, 60, 61

Rajkov 304, 305

Rakovec 264

Ratarula 162, 266

Recapitulation 25, 34, 96, 186, 205, 260, 264 ff.

Regeneration 272, 429

Regressive evolution 45, 110, 112 , 116,122

Reisinger 6, 81, 88, 120, 128, 257

Remane 2, 5, 24, 25, 26, 53, 66, 94, 107, 166, 247 ff.
Remanella 301, 303

Reniers-Decoin 122

Renovation 195, 219, 220

Repetition 440

Reptilia 457, 461

Retrogressive development 126, 132 , 136, 140, 148, 149, $159 \mathrm{ff}$.

Rhabdites 66, 200

Rhabdocoela 10, 71, 172, 210, 222, 256, 258

Rhamnites 200

Rhiza 117

Rhizocephala 346

Rhizoflagellata 359

Rhizopoda 329, 351, 358, 361, 362, 453,458

Rhopalium 133, 136

Rhynchocoel 398, 399

Rotatoria 147, 213, 272, 292, 395, 460

Rotmüller 306, 354, 355

Rugosa 185

Ruminantia 294

\section{Sacarão 35}

Sachwatkin 25, 26, 77, 206, 311, $329,359,361$

Sack gonade 150

Saprophyta 354

Sarcosepta 31, 32, 102, 118, 122, 149, $150,151 \mathrm{ff}$.

Saville-Kent 6, 276

Scappus 160

Schaudinn 283

Schimkewitch 389

Schindewolf 31, 32, 98, 245

Schizocoela 408, 463

Schmalhausen 379,380

Schneider K. C. 119, 121, 125, 131, 393,425

Schulze 215

Sclerosepta 31, 32, 180, 182, 184 
Scolecida 395

Scolex 57, 58

Scyphopolyp 118, 124, 166, 198

Scyphomedusae 16, 31, 67, 88, 118, 124, 131, $133 \mathrm{ff}$.

Scyphostoma 90, 99, 156, 233

Scyphozoa 32, 39, 71, 72, 76, 90, 91, $95,96,97, \mathrm{ff}$.

Scyphyla 80

Sedgwick 103, 249, 253, 276, 404

Segmentation 385, 392, 399, 420, $427,435,449$

Self fertilization 290

Semaeostomata 284

Semper 442

Semper' s larvae 208

Senna 263

Septa 231

Septal apparatus 122, 230

Sessil way of life 339

Sidonia 190

Siphonales 316, 330

Siphonanthes 161

Siphonoglyphe 102, 170, 230

Siphonophora $61,67,72,73,89,90$, 92-94, $101 \mathrm{ff}$.

Siphonosome 161

Siphonula 162, 262, 263, 266

Siphozooid 109

Sipunculoidea (Sipunculus) 271, 296, $400,415,420,425,426,429-437 \mathrm{ff}$. Solenia 117, 164

Solenocyte 442

Sollas 1, 331, 369, 371

Spadella 433, 437

Spermatozoa 282, 290, 295-297

Sphaeries 314

Spiralia 35, 414

Spirocyte 202

Spongiae 1-3, 5-8, 21, 25, 52, 67, 124, 144, $164 \mathrm{ff}$.

Spongiaria 25, 255, 319, 333, 391

Spongicola 183
Spongilidae 322

Spongula 3, 7, 333, 370

Spora 306

Sporosac 53

Sporozoa 53, 314, 357, 458

Statocyst 133

Stauromedusae 123, 235

Stechow 241, 242

Steenstrupia 64

Steinböck 24, 35, 120, 129, 181, 196, 210, 212 ff.

Steiner 384, 408, 452-455

Stephanoscyplus 108, 117, 149, 183

Stephanopogon 305

Stereoblastula 206

Stereogastrula 211

Sternaspis 420

Stolon 218

Stolonifera 194, 232

Stomodaeum 167

Stomotoca 64

Strobilation 128, 149, 156, 216

Stylasteridae 196

Styloid 92

Subcellular state 357

Subepithelium 115

Suctoria 97, 215, 362

Suitcase theory 80,249

Svabenik 406

Swedmark 95

Swezi 203

Symbionts 72

Symbiosis 225

Symphyla 416

Syncaryon 281

Syncoelomata 390

Syncoryne 63

Syncytial state 315,317

Syncytium 197, 212, 213, 307, 309, $312,314 \mathrm{ff}$.

Syngamy 280, 282, 285, 290

Synthetium 220

Syzygy 290 
Tadpole 452, 456

Taeniolae 96, 117, 118, 180, 182, 188,230

Taku Komai 12

Tardigrada 272, 390, 402, 412-414, 416,423

\section{Teissier 95}

Telestacea 232

Teloblasts 326

Telosporidia 357

Temnocehala $14,20,70,140$, 192, 256

Temnocephala sp. 70

Tentaculata $270,296,405,408,409$, 425,426

Terminal budding 218

Tetrabranchiata 42,394

Tetracorallia 184

Tetramery 184, 186, 404

Tetrapoda 440, 455, 457

Tetrapostbis 146

Thaliacea 214, 312

Thaloidea 355

Theca 194

Thecata $110,159,195,220,222,237$, 239, 242

Thysanoplana 179

Thysanozoon 181

Tialfiella 85

Tiaropsis 134

Tornaria 451

Totton 160

Trachelocerca 305

Trachomedusae 92, 209, 236

Trachylina 90, 92, 123, 159, 189, 203, 236, 240

Trematoda 72, 147, 192, 244, 296, $367,395,460$

Trichocysts 66, 199

Tricladida 71, 180

Trilobita 416

Trimeria 425

Trimetry 401, 404, 453
Triploblastic system 328

Triploblastica $75,79,324,374,375$, 384

Trochophora 38, 39, 40, 60, 342, 421, $452,463,464$

Tubularia 92, 174, 263, (larva)

Tubulariidac 89, 107, 172, 209, 242, 265

Tunicata $14,36,123,196,214,296$, $346,439 \mathrm{ff}$.

Turbella 196

Turbellaria 9, 13-19, 22, 23, 31, 37, $41,52,62,70,71,81,85,115,119$, $122,123,126,128,132-137 \mathrm{ff}$.

Turbellores 453

Turner 289, 297

Turritopsis 213

Tuzet 1-4, 124, 331, 332, 370

Typical budding 218

Uchida 108, 234

Udonelloidea 395

Ulrich 247, 357, 426

Umbrella 119, 133, 135, 188

Undulipodia 48, 52, 120, 142, 358

Unicellularia 30,355

Urcoelenterata 83

Urna 436

Urodela 443

Us 82,85

Vanderbroeck 455

Vanhöffen 235, 263

Veliger larva 41

Velum 119, 134

Vermes 379, 393, 432

Vermidia 425

Vertebrata 37, 49, 68, 69, 75, 145, $325,328,333,348,350,421 \mathrm{ff}$.

Vesiculatae 134

Vitellarium 147 
Volvocales 306, 365

Volvocidae 168

Volvox 2, 4, 7, 49, 279, 286, 322, 323, $325,330,372$

Vorticidae 168

\section{Wacke 70}

Wandering of cnidocyts 15

$$
\text { of sex cells } 151
$$

Wattson's rule 68, 69, 70, 106, 300,

$$
348,448
$$

Weill 202, 203

Weismann 114, 151

Wessenberg 285, 362

Westblad 140, 314

Wietrzkowski 193

Will 202

Wilson 334

Willey 442

Wolf 115,125

Wulfert 263
Xanthellae 222

Xenophyophora 356

Xenoturbella 252, 296

Xiphosura 416

Zalokar 110

Zawarsin 336

Zenkewitch 52

Zimmermann 344

Zoa 355

Zoaea 355

Zoantharia 209, 232, 263, 264

Zoanthella 209

Zoanthina 209, 263

Zoochlorellae 72, 222

Zooflagellata 49, 284, 285, 286, 333, 364

Zoomastigina 392

Zoophyta 74, 99, 246

Zooxanthellae 72, 222, 223, 224

Zygoneura 424

Zygote 284, 293, 305, 306, 320, 335 




